\title{
DISCLAIMER
}

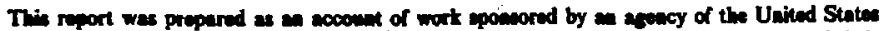

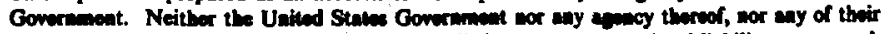

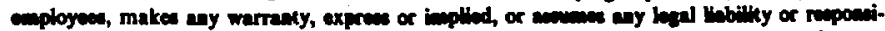

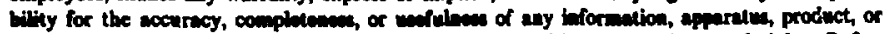

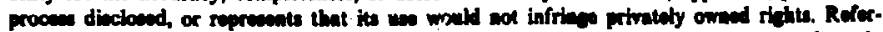

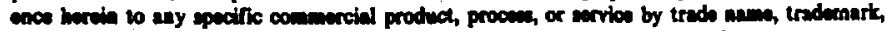

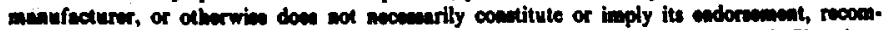
meadation, or favories by the Unted Stave Cowecament or any apucy thereof. The viowa

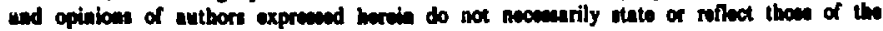
United States Gownmanet or eny apeacy thereof.

\section{Mirror Advanced Reactor Study Interim Design Report}

Lawrence Livermore National Laboratory

TRW Energy Development Group

General Dynamics/Convair Division

EBASCO Services, Inc.

Science Applications, Inc.

University of Wisconsin-Madison

Grumman Aerospace Corporation

Energy Technology Engineering Center

Manuscript date: April 1983

\section{LAWRENCE LIVERMORE LABORATORY} University of California $\bullet$ Livermore, California 994550 


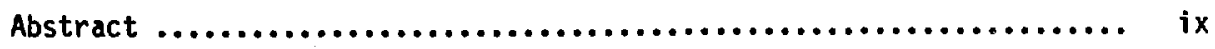

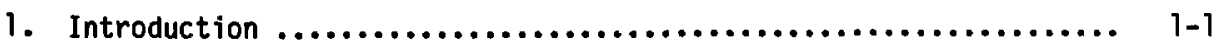

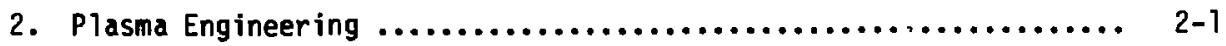

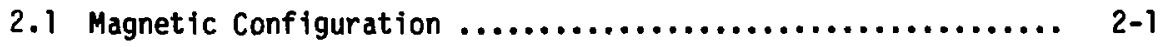

2.1.1 Central-Cel1 Design .......................... 2-1

2.1.1.1 Magnet Description .................... 2-1

2.1.1.2 Design Considerations .................. 2-1

2.1 .2 End Plug Design ............................. 2-6

2.1.2.1 End Plug Configuration $\ldots \ldots \ldots \ldots \ldots \ldots \ldots . . . .62-6$

2.1.2.2 Axicell Coils ......................... 2-8

2.1.2.3 Transition Col1s ....................... 2-10

2.1.2.4 Anchor Colls ........................ 2-12

2.2 Physics Constraints of the Magnetic Configuration ......... 2-13

2.2.1 Radial Transport ............................. 2-14

2.2.2 Parallel Current $\ldots \ldots \ldots \ldots \ldots \ldots \ldots \ldots \ldots \ldots \ldots \ldots \ldots, 2-16$

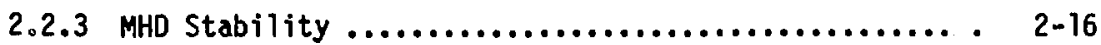

2.2.4 Central-Cell Plasma Rotation ................... 2-21

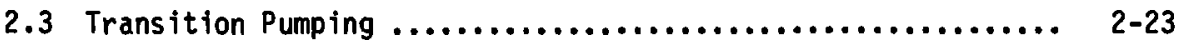

2.3.1 Trapping Rates ............................... 2-23

2.3.2 Charge-Exchange Pumping ...................... 2-31

2.3.2.1 Overview ........................... 2-31

2.3.2.2 Pumping Current ...................... 2-31

2.3.2.3 Pump Beam Energy .................... 2-32

2.3.3 Alternative Pumping Methods ................... 2-33

2.3.3.1 Introduction ......................... 2-33

2.3.3.2 Description of the AC Drift

Pumping Concept ....................... 2-35

2.3.3.3 Estimate of $A C$ Perturbation

Amplitudes $. . \ldots \ldots \ldots \ldots \ldots \ldots \ldots \ldots \ldots \ldots . .2-37$

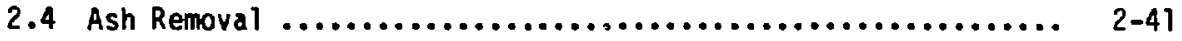

2.4.1 Transition Region Drift Pumping ................. 2-41

2.4.1.1 Stochasticity ....................... 2-42

2.4.1.2 Diffusion Coefficients ................ 2-43

2.4.1.3 Diffusion Equation ................... 2-45

2.4.1.4 Drift Computations .................... 2-49

2.4.1.5 Drift Pumping of Trapped Ions

in the Transition .................... 2-50 
2.4.2 Alternative Ash Removal Methods ................. 2-50 2.4.2.1 Enhanced Drift Pumping of Trapped Alphas by AC Perturbations .............. 2-51 2.4.2.2 Radial Transport Due to Magnetic Perturbations at the Ends of the Central Cell ............................

2.4.2.3 Charge-Exchange Pumping of Alphas

in the Transition ........................

2.5 Anchor Sloshing Ions and Pumping ....................... $2-54$

2.5.1 Ion Distribution Function $2-54$

2.5.2 Adiabaticity

2-63

2.5.3 Beam Trapping

2.6 ECRH Physics 2-66

2.6.1 General Considerations

2-66

2.6.2 Relativistic ECRH Frequency Shifts

2-67

2.6.3 ECRH at the Second Harmonic $2-68$

2.7 Axicell Ion Cyclotron Heating 2-69

2.8 Plasma Startup ...

2.8.1 Initial Startup Phase $2-71$

2.8.2 Final Startup Phase 2-71 $2-73$

2.9 End Leakage Physics 2-77

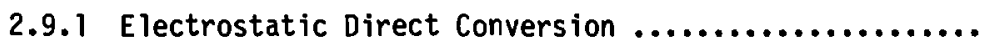
2-77

2.9.2 Distribution of Electrical Potential ................ 2-77

2.9.3 Selective Leakage ............................ $2-79$

2.9.4 The Halo Plasma .............................. 2-81

2.9.5 Power Balance ................................ $2-82$

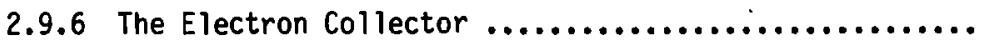
$2-83$

2.9.7 The Ion Collector End

2.9.8 Cold Plasma

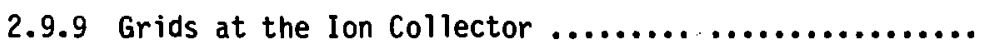

2.9.10 Direct Conversion Efficiency

2.9.11 Haio Plasma Physics

2.10 Plasma Performance

2.10.1 Plasma Parameters

2.10.2 Particle and Energy Injection 


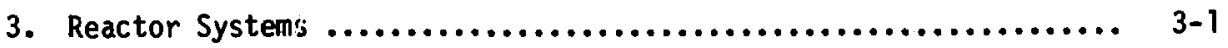

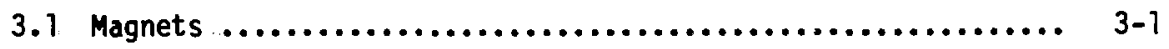

3.1.1 Central Cell Coils .............................. 3-3

3.1.1.1 Design Considerations ................. 3-3

3.1.1.2 Engineering Trade-Off Studies ............ 3-4

3.1.1.3 Magnet Design ........................ 3-5

3.1.1.4 Engineering Analysis ................. 3-9

3.1.2 Axicel1 Superconducting Magnet ................. 3-15

3.1.2.1 Design Considerations .................. 3-15

3.1.2.2 Engineering Trade-0ff Studies ............ 3-16

3.1.2.3 Magnet Design ........................ 3-18

3.1.2.4 Engineering Analysis ................... 3-23

3.1.2.5 Axicell Shield ........................ 3-24

3.1.2.6 Conductor Deve lopment for Axicell

Superconducting Magnets ................ 3-27

3.1.3 Axicell Resistive Magnets ...................... 3-27

3.1.3.1 Design Considerations ................. 3-27

3.1.3.2 Engineering Trade-0ff Studies ............ 3-28

3.1.3.3 Magnet. Design ......................... 3-35

3.1.3.4 Engineering Analysis ................... 3-39

3.1.4 Transition and Anchor Coils ................... 3-42

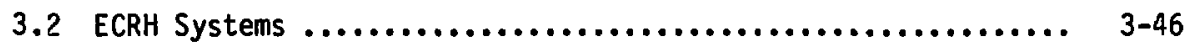

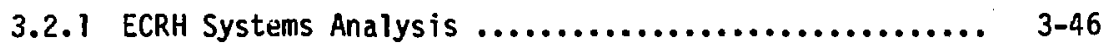

3.2 .2 ECRH Power Supply .......................... 3-48

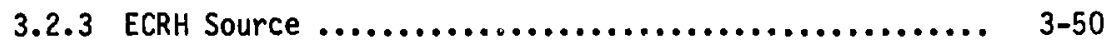

3.2.4 Quasi-Optical Launching System .................. 3-50

3.2.5 ECRH Systems Layout $\ldots \ldots \ldots \ldots \ldots \ldots \ldots \ldots \ldots \ldots \ldots \ldots . . \ldots \ldots$

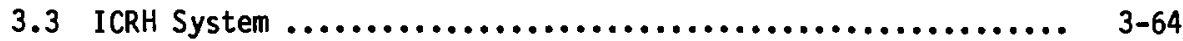

3.3.1 ICRH Systems Analys is ........................ 3-64

3.3.2 ICRH Amplifier and Power Supply ................ 3-66

3.3.3 ICRH Power Feed ............................. 3-67

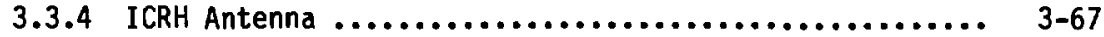

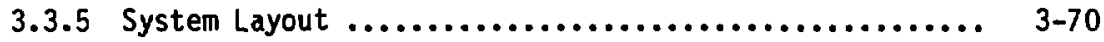

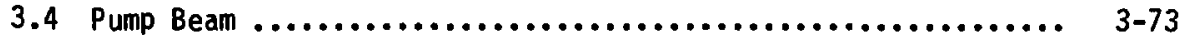

3.4.1 Pump Beam System Analysis ..................... 3-73

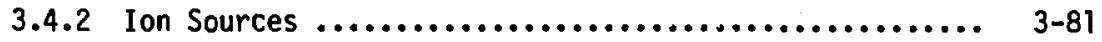

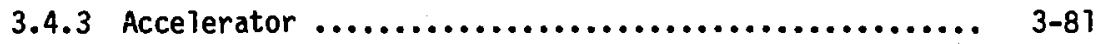


3.4.4 Neutralizer $. \ldots \ldots \ldots \ldots \ldots \ldots \ldots \ldots \ldots \ldots \ldots \ldots \ldots \ldots \ldots ., 3-82$

3.4 .5 System Layout ............................. 3-82

3.5 Sloshing Ion Beam ................................ 3-84

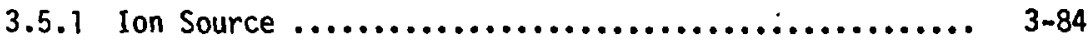

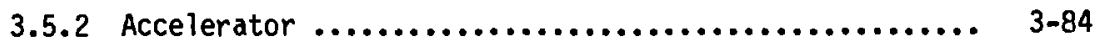

3.5.3 Neutralizer ................................ 3-85

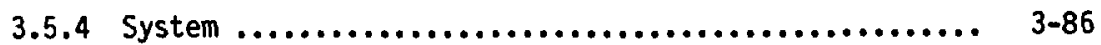

3.6 End-Plasma Technology ............................... 3-89

3.6.1 Direct Converter ............................ 3-89)

3.6.1.1 Grid System .......................... 3-93

3.6 .1 .2 Collector Plate ...................... 3-112

3.6.1.3 Electron Collector ................... 3-119

3.6.2 Halo Scraper ............................... 3-124

3.6.2.1 Requirements ........................ 3-124

3.6 .2 .2 Design ............................. 3-124

3.6.2.3 Performance ......................... 3-126

3.6.3 Continuous Cryopumping System ................. 3-129

3.6.3.1 Requirements ......................... 3-129

3.6.3.2 Performance ........................ 3-129

3.6.3.3 System Description ................... 3-132

3.6.3.4 Design Options ....................... 3-136

3.6.3.5 Helium Refrigeration Load ................ 3-144

3.6.4 Vacuum Tanks and Support Structure ............... 3-144

3.6.4.1 Irm-End Tank ........................ 3-146

3.6.4.2 Electron-End Tank ....................... 3-149

3.6.5 Tritium Considerations ........................ 3-149

3.6.5.1 Tritium Permeation Into Cooling Water ........................ 3-149

3.6.5.2 Tritium Diffusion Mode1 ................. 3-151

3.6.5.3 Results .......................... 3-154

3.6.5.4 Permeation Barriers .................. 3-157

3.6.5.5 Tritium Extraction from

Cooling Water ....................... 3-157

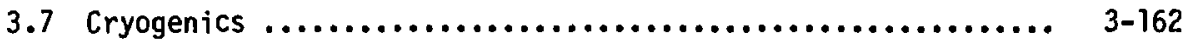

3.7.1 Central Cell Magnets ........................... 3-163

3.7 .2 Axicel1 Magnets ............................. 3-163

3.7.3 Yin-Yang and Transition Magnets ................. 3-164 
3.7.4 Vacuum System .............................. 3-165

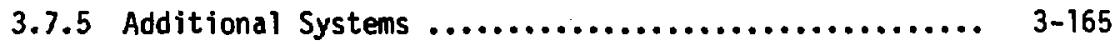

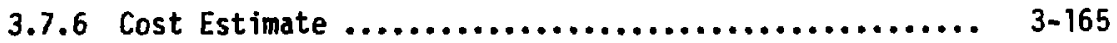

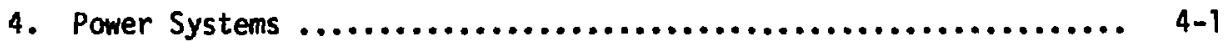

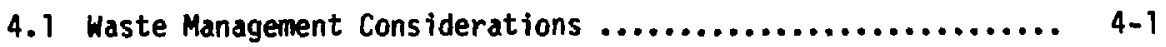

4.2 Lithium-Lead $B 1$ anket ................................ 4-16

4.2.1 Blanket Design .............................. 4-16

4.2.1.1 Design Sunmary ....................... 4-16

4.2.1.2 Structural Design ..................... 4-20

4.2.1.3 Neutronics ......................... 4-22

4.2.1.4 Thermal Hydraulics $. \ldots \ldots \ldots \ldots \ldots \ldots \ldots \ldots \ldots . .4$ 4-27

4.2.1.5 MHD Considerations .................... 4-28

4.2.1.6 LiPb Corrosion ........................ 4-29

4.2.1.7 Lithium-Lead/Water Interactions ........... 4-34

4.2.1.8 Emergency Cooling ...................... 4-36

4.2.1.9 Radiation Effects on Central Cell

Magnets ............................... 4-43

4.2.1.10 Magnetic Effects on Structural

Materials .............................. 4-46

4.2.2. Shield and Radioactivity Considerations ........... 4-53

4.2.2.1 Design Criteria ...................... 4-53

4.2.2.2 Neutronics .......................... 4-54

4.2.2.3 Structural Design ...................... 4-56

4.2.2.4 Thermal/Hydraulics .................... 4-58

4.2.2.5 Radioactivity ...................... 4-58

4.2.3 Blanket Design Optimization ................... 4-62

4.2.3.1. Design Philosophy ................... 4-62

4.2.3.2. Design Optimization Procedure ........... 4-62

4.2.3.3 Figure-of-Merit Parameter .............. 4-64

4.2.3.4 Figure-of-Merit Application .............. 4-66

4.2.4 Tritium Removal $\ldots \ldots \ldots \ldots \ldots \ldots \ldots \ldots \ldots \ldots \ldots \ldots \ldots . . \ldots$ 4-73

4.2.4.1 Tritium Extraction from LiPb ............ 4-73

4.2.4.2 Tritium Isolation ...................... 4-80

4.2.5 Energy Conversion System ...................... 4-82

4.2.5.1 Heat Exchanger Arrangement and

Power Cycle ............................ 4-82

4.2.5.2. Double-Walled Steam Generator ........... 4-85 
4.2.5.3 Tritium Isolation .................... 4-90

4.2.5.4 Removal of Corrosion Products ............ 4-92

4.2.6 Blanket Maintenance ............................ 4-96

4.2.6.1 Maintenance Philosophy ................. 4-96

4.2.6.2 Maintenance Procedure ................... 4-102

4.3 High Temperature Blanket ............................ 4-108

4.3.1 Blanket Design ................................. 4-108

4.3.1.1 Performance Summary .................... 4-108

4.3.1.2 Module Description ....................... 4-111

4.3.1.3 Structural Analysis .................... 4-120

4.3.1.4 Neutronics ........................... 4-122

4.3.1.5 Thermal Hydrautics ...................... 4-132

4.3.1.6 MHD Pressure Drop ....................... 4-140

4.3.1.7 Lithium-Lead Compatibility ................ 4-141

4.3.1.6 Radiation Effects on Structural

Materials ............................... 4-141

4.3.1.9 Solid Breeder Performance ............... 4-152

4.3.1.10 Pod Rupture Analysis .................... 4-160

4.3.2 Shield ...................................... 4-163

4.3.2.1 Shield Design Criteria ................... 4-163

4.3.2.2 General Structural Description ............ 4-163

4.3.2.3 Shield Neutronics ...................... 4-165

4.3.2.4 Shield Thermal Hydraulics ................ 4-167

4.3.3 Seals ..................................... 4-168

4.3.3.1 Vacuum Seals ......................... 4-168

4.3.3.2 Remote Actuated-Piping

Lithium-Lead Connections ................ 4-168

4.3.4 Tritium Removal .............................. 4-171

4.3.4.1 Solid Breeder Tritium Inventory ........... 4-171

4.3.4.2 $\mathrm{T}_{2} 0$ Formation Rate .................... 4-173

4.3.4.3 Tritium Extraction From Helium ............ 4-176

4.3.4.4 Tritium Extraction from LiPb ............ 4-179

4.3.4.5 Tritium Control in the Reactor Hall ........ 4-180

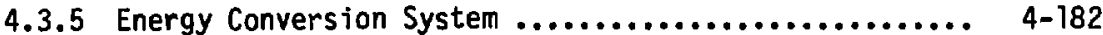

4.3.5.1 System Analysis ...................... 4-183

4.3.5.2 Component Description ................... 4-187

4.3.5.3 Double-Walled Steam Generator ............ 4-188 
4.3.5.4 Tritium Isolation .................... 4-188

4.3.5.5 Corrosion Product Removal ............... 4-189

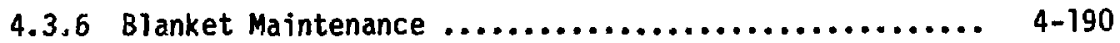

4.3.6.1 Maintenance Phi losophy .................. 4-190

4.3.6.2 B Banket Module Changeout Procedure ........ 4-192

4.3.6.3 Equipment Requirements ............... 4-195

4.3.6.4 Transhauler ......................... 4-196

4.3.7 Blanket Module Manufacturing ................... 4-198

4.4 Solid Breeder Blanket ............................. 4-213

4.4.1 Blanket Design ............................. 4-213

4.4.1.1 Design Summary ...................... 4-213

4.4.1.2 Structural Design ..................... 4-217

4.4.1.3 Neutronics .......................... 4-224

4.4.1.4 Thermal/Hydraulics ................... 4-231

4.4.1.5 Radiation Effects on Structural

Materials ........................... 4-239

4.4.1.6 Solid Breeder Performance ............... 4-240

4.4 .2 Shield ....................................... 4-240

4.4.2.1 Design Criteria ...................... 4-240

4.4.2.2 Structural Design ..................... 4-241

4.4.2.3 Neutronics ............................ 4-243

4.4.2.4 Thermal/Hydraulics .................... 4-245

4.4.3 Vacuum Seals ............................... 4-246

4:4.4 Energy Conversion System ..................... 4-246

4.4.4.1 System Analysis ..................... 4-246

4.4.4.2 Component Description .................. 4-251

4.4.5 Blanket Maintenance .......................... 4-251

4.4.5.1 Maintenance Philosophy ................ 4-251

4.4.5.2 Module Changeout Procedure ............. 4-254

4.4.6 Blanket Module Manufacturability ................ 4-258 


\section{ABSTRACT}

The status of the design of a tenth-of -a-kind commercial tandem-mirror fusion reactor is described at the midpoint of a two-year study. When completed, the design is to serve as a strategic goal for the mirror fusion program. The main objectives of the Mirror Advanced Reactor Study (MARS) are: (1) to design an attractive tandem-mirror fusion reactor producing electricity and synfuels (in alcernate versions), (2) to identify key development and technology needs, and (3) to exploit the potential of fusion for safety, low activation, and simple disposal of radioactive waste. In the first year we have emphasized physics and engineering of the central cell and physics of the end cell. Design optimization and trade studies are continuing, and we expect additional modifications in the end cells to further improve the performance of the final design. 


\section{INTRODUCTION}

The Mirror Advanced Reactor Study (MARS) is a major two-year study of a commercial tandem mirror reactor, conducted by the Lawrence Livermore National Laboratory (LLNL) for the U.S. Department of Energy, with TRW as a prime industrial partner, General Dynamics, EBASCO, and SAI as subcontractors, and the University of Wisconsin as a university partner. Grumman Aerospace is also contributing to the engineering effort. The main objectives of MARS are: (1) to design an attractive fusion reactor producing electricity and synfuels in a tenth-of-a-kind commercial stage of development; (2) to identify key development and technology needs for the commercial tandem mirror reactor and its forerunner, the Fusion Power Demonstration reactor (FPD); and (3), to exploit the potential of fusion for safety, low activation, and simple disposal of radioactive waste. Currently at the midpoint of this study, we describe the general characteristics of the MARS reactor; however, some design trade studies are still being explored. Many MARS features have been part of past mirror experiments or can be tested in near-term mirror facilities such as TARA, the Tandem Mirror facility at MIT, TMX-U, the Tandem Mirror Experiment-Upgrade at LLNL, and MFTF-B, the Magnetic Fusion Test Facility at LLNL.

MARS (Fig. 1.1-1) is a linear magnetic-mirror fusion device using electrostatic plugs to confine a steady-state fusion plasma in a long solenoid (typically $150 \mathrm{~m}$ long to produce 1500 we net). Mirror devices with the topology of the MARS plugs have been part of fusion research since the beginning of the program in the early 1950s. High-beta quasi-steady-state mirror plasmas were first demonstrated at LLNL in 1975 in the 2XIIB singlemirror device. The tandem mirror concept began in 1976 as an improvement over single-mirror concepts. It has two mirrors, one at each end of a solenoid. The basic principles of electrostatic confinement in tandem mirrors were first demonstrated at the proof-of-principle level in the TMX and Gamma-6 experiments in 1979. The main fusion plasma in the central cell of MARS is selfsustained by alpha heating (ignition), while continuous injection of neutral beams and electron cyclotron resonant heating (ECRH) are required to maintain the plug electrostatic confining potentials of $150 \mathrm{kV}$ for the ions and $200 \mathrm{kV}$ for the electrons. The plug injection power is reduced by the use of thermal barriers. Thermal barrier formation and microstability will be tested in the TMX-U experiment during the next two years and at near reactor-levei energies 


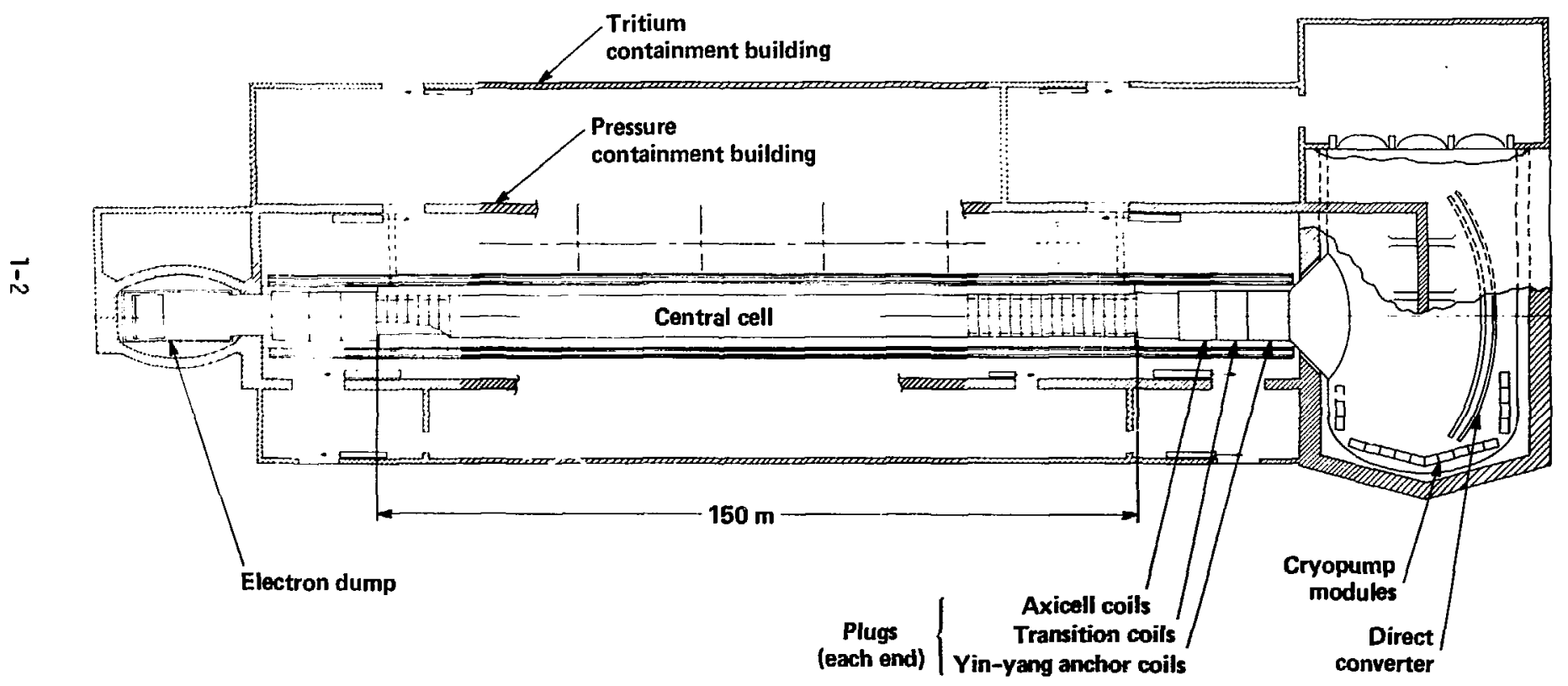

Fig. i.1-1. MARS reactor design. 
in MFTF-B beginning in 1986. Plasma exhaust and vacuum pumping are accomplished in large vacuum tanks at the ends, where direct corversion of escaping ion and electron energy recovers about as piuch electricity as is consumed by the plug beams and ECRH. A small direct-converter module was successfully operated as part of TMX in 1980.

The present MARS end plugs (Fig. 1.1-2), which are the same axicell type that will be used in TARA and MFTF-t, rely on proven yin-yang magnets for high-beta magnetohydrodynamic (MHD) stability. The MARS yin-yang size and field are comparable to the MFTF-B yin-yang recently tested at LLNL. All reactor magnets are steady-state because no plasma current needs to be driven to provide plasma equilibrium or stability. The transition coils are the same generic double-ellipse-type that were recently designed for MFTF-B to cance parallel components of plasma diamagnetic current in the central cell and to control radial transport in the transition regions.

Steady-state fueling in the tandem mirror reactor most likely will be accomplished by neutral beams in the plugs and by injection of high velocity $\left(10^{4}-\mathrm{m} / \mathrm{sec}\right)$ pellets near the ends of the central cell. Impurities and thermal alpha ash are kept to tolerable concentrations $(<10 \%)$ by radial transport. While the axicell is designed to $r$ juce neoclassical (resonant) diffusion for most central-cell ions, residual transport due to the douole-ellipse transition coils can be desioned to induce radial ion flux comparable to end losses, sufficient for $n_{\alpha} / n_{D T} \lesssim 10 \%$. The aipha ash and impurities diffuse into an outer radial plasma zone, or halo, which is not electrostaticilly plugged. This natural diverter sweeps the impurities into the large direct converter tanks, thus minimizing piasma bombardment and surface heating of the first wall in the central cell.

Characteristic MARS parameters are listed in Table 1.1-1. Two particular features stand out. First, the plasma radius $(0.43 \mathrm{~m})$ is small because of high bata, which permits relatively small-bore magnets $(2.4-\mathrm{m}$ inner radius central solenoijis, 30-cm iuner radius 24-T hybrid coils, 3-m major radius yinyangs). Thus, the intermediate step FPD can be constructed with full-scale reactor plug magnets at reasonable coil cost and development. Second, significant recirculating electric power is required to drive the plugs, 


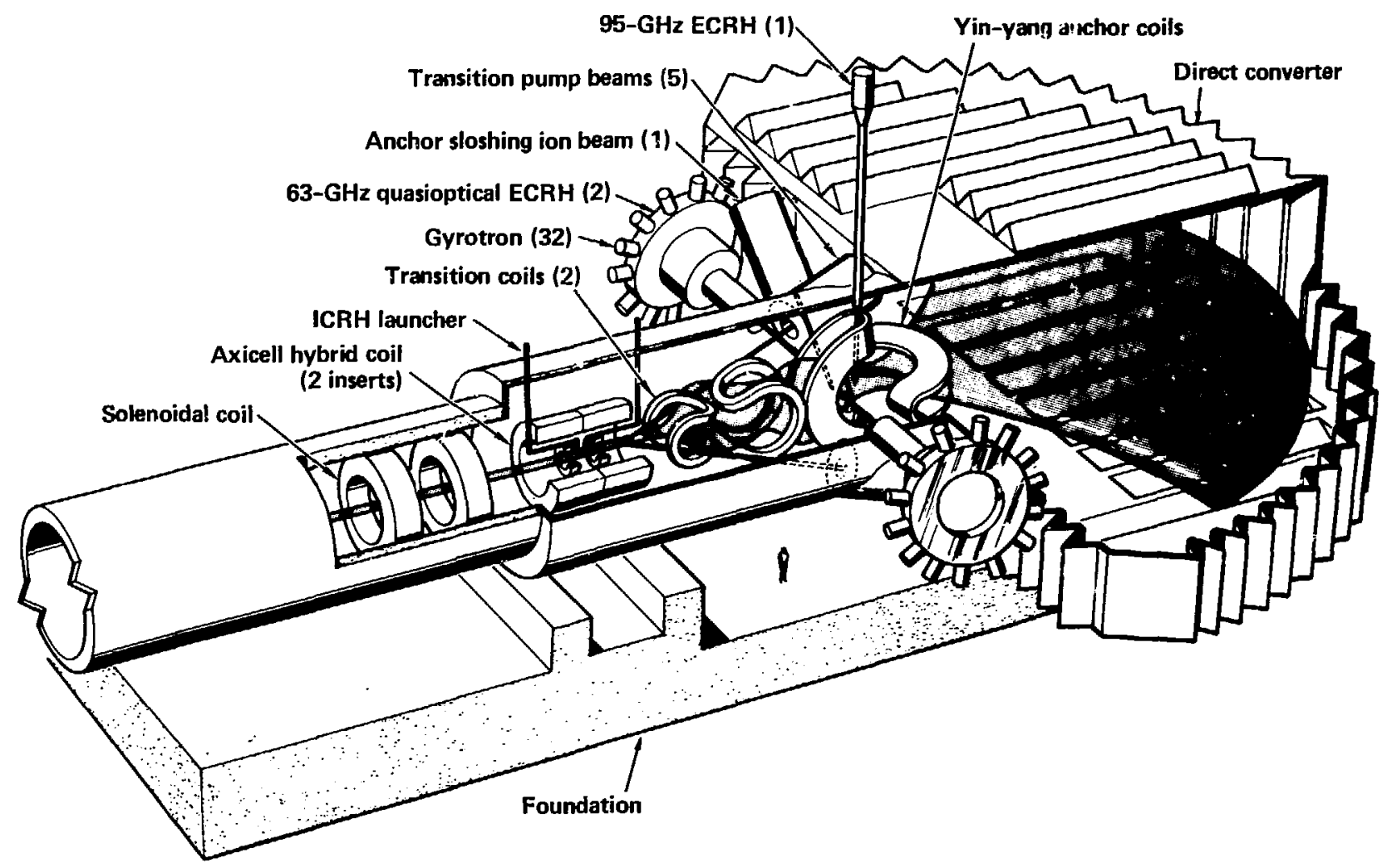

Fig. 1.1-2. MARS axicell configuration. 
Table 1.1-1 Characteristic MARS parameters.

Central cell length

Plasma radius

Solenoid field

Peak axicell mirror field

Yin-yang mirror field

Average central beta

Average central density

First wall loading

Fusion power

Plug neutral beam power absorbed

Plug ECRH power absorbed

Plug ICRH power absorbed

Normal insert coil power

Total recirculating power

Direct converter power

Net electric power produced

Recirculating power fraction

Magnet stored energy

Magnet + shield + blanket mass
$150 \mathrm{~m}$

$0.43 \mathrm{~m}$

$4.7 \mathrm{~T}$

$24 \mathrm{~T}$ (16 T SC $+8 \mathrm{~T}$ Cu insert)

$6 \mathrm{~T}$ (8.5 $\mathrm{T}$ on the conductor)

$40 \%$

$3 \times 10^{20} \mathrm{~m}^{-3}$

$5 \mathrm{~m} / \mathrm{m}^{2}\left(\max\right.$ at $\left.r_{\min } w a l 1=0.6 \mathrm{~m}\right)$ $3500 \mathrm{iw}$

$76 \mathrm{NW}$ each plug (4 MW) $)^{a}$

28 w each plug

$20 \mathrm{Ww}$ each plug

63 each plug

590 Mile $(380 \text { MWe })^{a}$

445 We

1360 MWe $(1570 \text { MWe })^{a}$

$0.30(0.19)^{a}$

$50 \mathrm{GJ}$

20,000 tons

\section{Assumed Efficiencies}

Direct converter

0.55

475-kV sloshing ion beams

0.66

97-kV pump neutral beams

0.69

ECRH

0.65

Axicell ICRH

0.50

Thermal cycle

0.39

Overall system (plant)

0.30

assuming improved transition pumping using radial transport (drifts) instead of $97-\mathrm{kV}$ pump beams. The only remaining neutral beams are the $475-\mathrm{kV}$ sloshing ion beams. 
particuiarly the transition neutral beam pump power. The direct converter output keeps $P_{\text {net }}$ and plant efficiency respectable, but recirculating power is high, which will make reactor cost/kwe (net) sensitive to the neutral beam and rf heating costs. However, with improved transition pumping such as the use of radial drifts, the recirculating power might be cut nearly in half (quantities that change have an asterisk in Table 1,1-1). An attractive current scheme of employing $A C$ perturbations in the transition regions is being evaluated for the engineering requirements of frequency, bandwidth, and amplitude. Assuming that the engineering requirements look feasible and that the scheme can be tested in TMX-U or MFTF-B, AC drift pumping could qualify as the MARS baseline pumping method early in FY 1983.

At this point in the MARS study, we can already identify several key development and technology ttems that will be needed: Negative-ion beams (at least for the 475-kV sloshing beams), high-field 24-T hybrid solenoid coils with unshielded normal inserts, direct converters, and high-velocity $\left(10^{4}\right.$ $\mathrm{m} / \mathrm{sec}$ ) pellet injectors. Improved transition pumping may require new hardware, but that cannot be specified as yet. Among the long-lead-time items necessary to enable a MARS-type FPD to be built, the negative-ion beams are most crucial. New materials for blankets and other structures are also longlead-time items for development, but we mainly need to select and screen promising candidates for final testing in the later phases of the FPD (end of the 1990 's).

During the past nine months of the MARS study, we have made significant advances in designing higher temperature, higher efficiency blankets with low tritium inventory and reduced activation. The University of Wisconsin's $\mathrm{Li}_{17^{2}}{ }_{83}$ blanket, building on extensive previous work from the UW-Witamir design, has been improved and optimized for lowest-cost electricity production for MARS (Fig. i.1-3). With a novel, high performance double-wall heatexchanger, this design could have an overall steam-cycle efficiency of $40 \%$. Combining this efficiency with a high blanket energy multiplication (1.39), a thir blanket (38 cm thick), and smaller-radius solenoid coils $(210 \mathrm{~cm}$ inner conductor radius), this design is expected to minimize the capital cost of the reactor per $\mathrm{kW}$ of net electricity produced. Although costing, reliability, safety, and remote maintenance are detailed subjects to be addressed more 


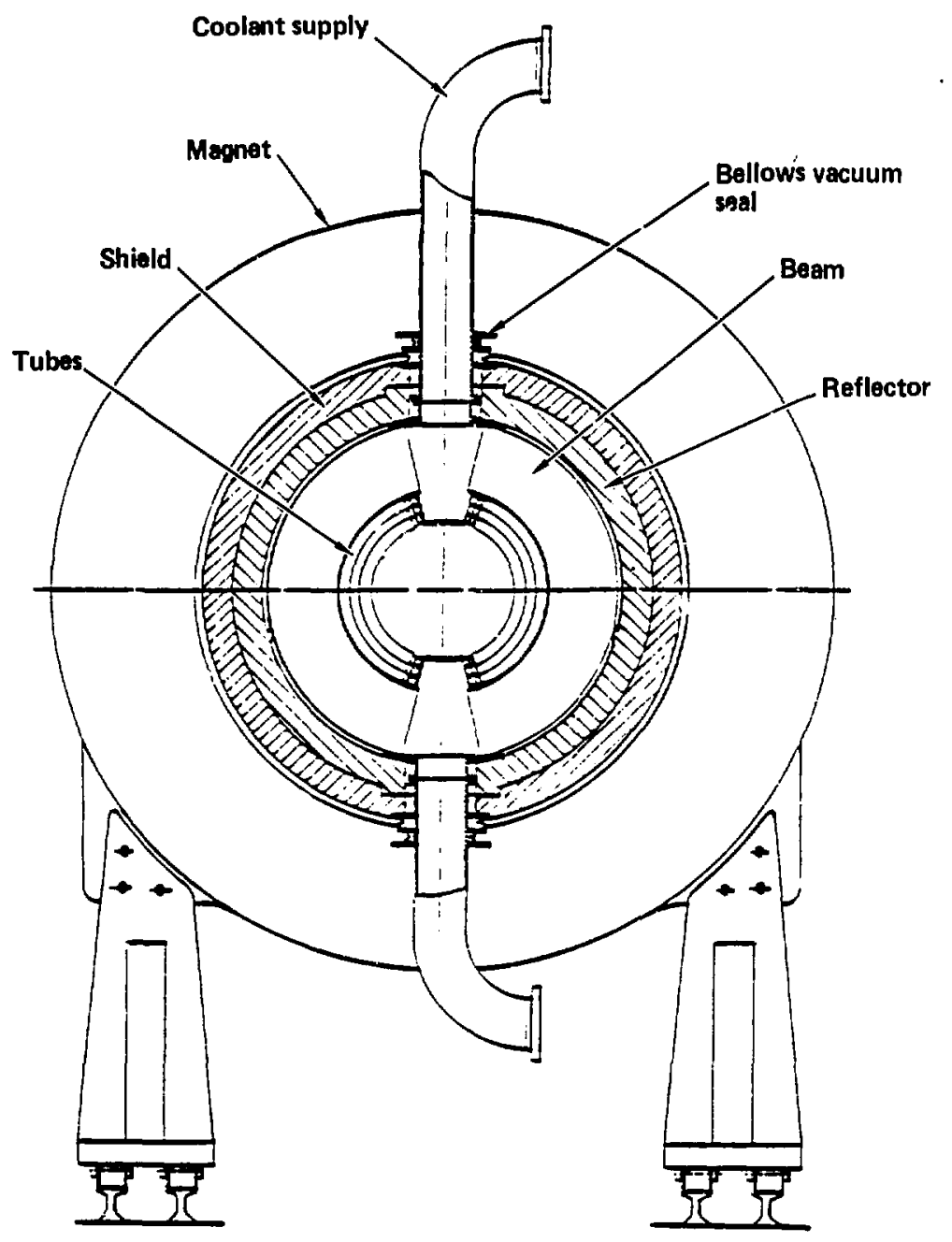

Central cell

(normal portion)

Fig. 1.1-3. University of Wisconsin's lithium lead blanket design. 
during FY 1983, the simplicity and ruggedness of this design is expected to set the lowest-cost standard for electricity production in MARS. The primary critical feasibility issue for this design is the deposition of the corrosion products in the flowing $\mathrm{Li}_{17} \mathrm{~Pb}_{83}$ liquid metal $700 \mathrm{p}$ at $500^{\circ} \mathrm{C}$. Data on this critical materials and liquid-metals-handling issue for $\mathrm{Li}_{17}{ }^{\mathrm{Pb}} 83$ are being addressed by laboratory-scale experiments over the next few years.

Beginning as a proposal concept for achieving higher temperatures for more efficient electricity and/or synfuels production, the TRW blanket design (Fig. 1.1-4) has rapidly evolved into a technically credible blanket capable of delivering $250 \%$ of the blanket heat in the form of $900^{\circ} \mathrm{C}, 80 \mathrm{~atm}$ helium. Using $\mathrm{Li}_{17} \mathrm{~Pb}_{83}$ as a first wall coolant, neutron multiplier, and supplemental tritium breeder, this design has good breeding at low tritium inventory, with overall performance $\left(n_{t h}=44 \%\right.$ at $M=1.16$ blanket multiplication) for electricity production comparable to the $\mathrm{UW} \mathrm{Li}_{17} \mathrm{~Pb}_{83}$ design. It also generates more than enough high-temperature helium to drive a thermal $\mathrm{SO}_{3}$ decomposer in a thermochemical synfuel process. Critical issues for this design are the reliability, complexity, and cost for the blanket and associated balance of plant, and for synfuels, tritium isolation from the synfuel product.

In both the UW and TRW blanket designs, the use of $\mathrm{Li}_{17}{ }^{\mathrm{Pb}}{ }_{83}$ helps keep tritium inventory low, Dut tritium permeation is instead the issue to address in the heat exchanger design. Ferritic steels have been shown to be compatible for use in the magnetic fields of the central ce?l.

Because lithium-oxide has been the solid breeding material most often studied in the worldwide fusion effort, and because liquid-ineial technology may itself become an issue, Grumman has investigated a dry, He-cooled solid $\mathrm{Li}_{2} \mathrm{O}$ blanket design to see if this type of blanket could be compatible with a MARS central cell. They have developed a canister-type $\mathrm{Li}_{2} \mathrm{O}$ blanket (Fig. $1.1-5)$ that can operate at the high wall loadings $\left(\Gamma_{\text {wall }}=5 \mathrm{MW} / \mathrm{m}^{2}\right)$ required for MARS. Like the UW and TRW designs, this design can use ferritic steels for longer life and vanadium alloys for lower activation. The critical issue for this design, as with all previous $\mathrm{Li}_{2} \mathrm{O}$ designs, is sintering of the high temperature $\mathrm{Li}_{2} \mathrm{O}$, resulting in unknown, but perhaps large, inventories of tritium. Connected with this problem is the overall thermal cycle efficiency at the lower He temperatures and higher He pumping power required to cool the 


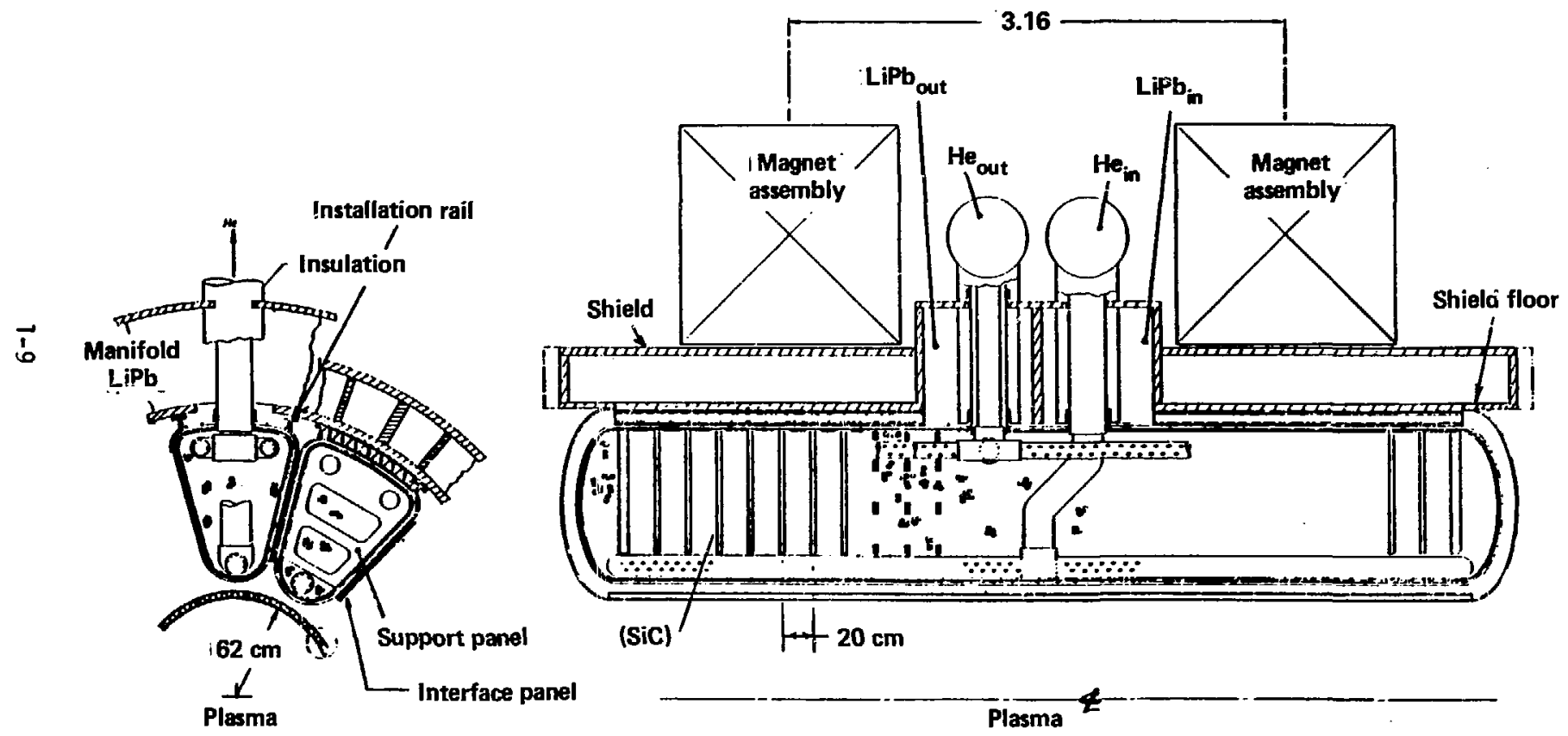

Fig. 1.1-4. High-temperature blanket design. 


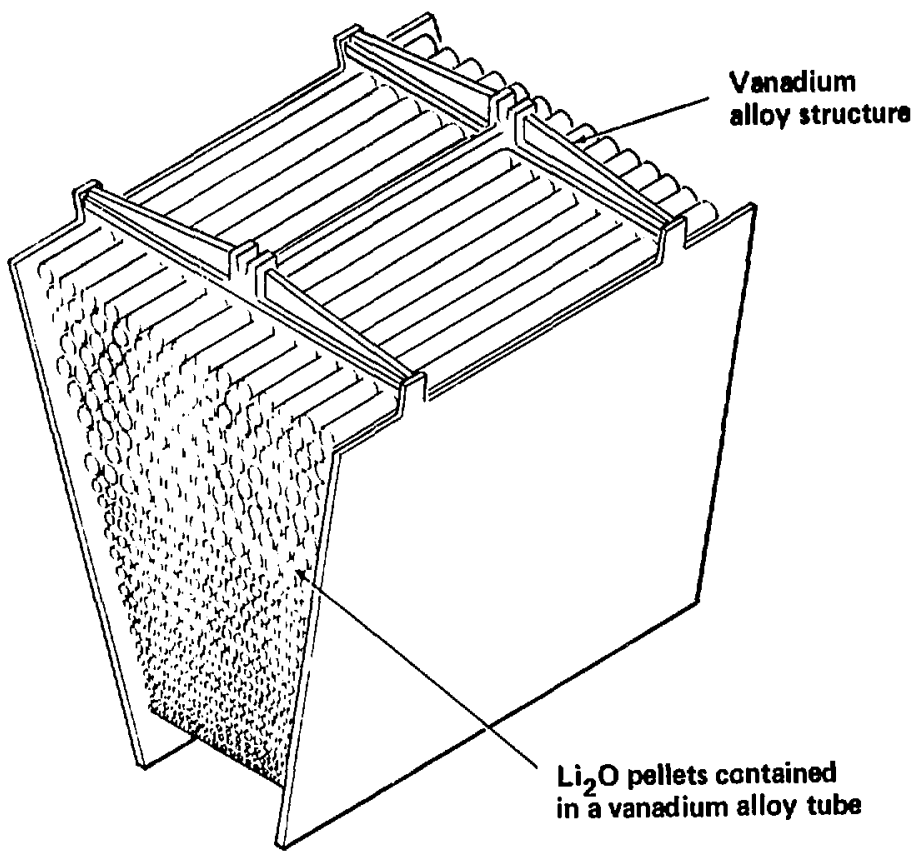

Fig. 1.1-5. Li $i_{2} \mathrm{O}$ blanket design. 
first wall. It is essential to obtain more experimental data on tritium release and sintering of $\mathrm{Li}_{2} \mathrm{O}$ at higher temperatures to further advance this type of blanket.

To focus more effort in FY 1983 on the design of end plug heating systems, remote maintenance of blankets and end plug components, and the design of facilities for rad waste handling and disposal, it will be necessary to select a reference case blanket for electricity production, based on comparisons of performance, cost, and simplicity of remote handling.

In addition to the primary task of designing and costing a reference case MARS plant that produces electricity, the MARS team intends to modify the MARS design in FY 1983 to produce synfuels. As the prime industrial partner and systems integrator for MARS, TRW has responsibility for the synfuel task, with assistance from other synfuel groups. TRW has reviewed existing blanket and synfuel processes and has made the following recommendations to the MARS executive committee and DOE:

1. MARS can and should pursue a synfuel plant design in FY 1983.

2. The synfuel process should be thermochemical water-splitting using the sulfur-iodine process with a thermal $\mathrm{SO}_{3}$ decomposer.

3. The TRW/GD high temperature blanket should be used.

The high temperature electrolysis (HTE) process is not recommended for MARS because the blanket concepts for use with high temperature steam are inadequately developed. However, because of the simplicity and potential performance of HTE, we recommend that research on a suitable blanket for high temperature steam continue independently.

To date, we have devoted a modest effort to the generic fusion problems of safety, activation, remote maintenance, and waste disposal. Once blanket concepts are selected for electricity and synfuels production, we can devote more effort to these issues. The UW and TRW blankets use nonflammable blanket coolants and have low tritium inventories, which should reduce the severity of possible accidents. Afterheat and loss-of-coolant accidents are just now being assessed to determine if backup coolant loops will be required. An important accomplishment in the waste area is the preliminary assessment of how well various structural steel alloys would satisfy NRC rules (10CFR61) for 
Table 1.1-2 Waste disposal ratings (WDR) for the NRC's 10CFR61 rules.

First wall: MARS (TRW) $5 \mathrm{MW} / \mathrm{m}^{2}$ and $3 \mathrm{yr}$ (parent elements in parentheses).

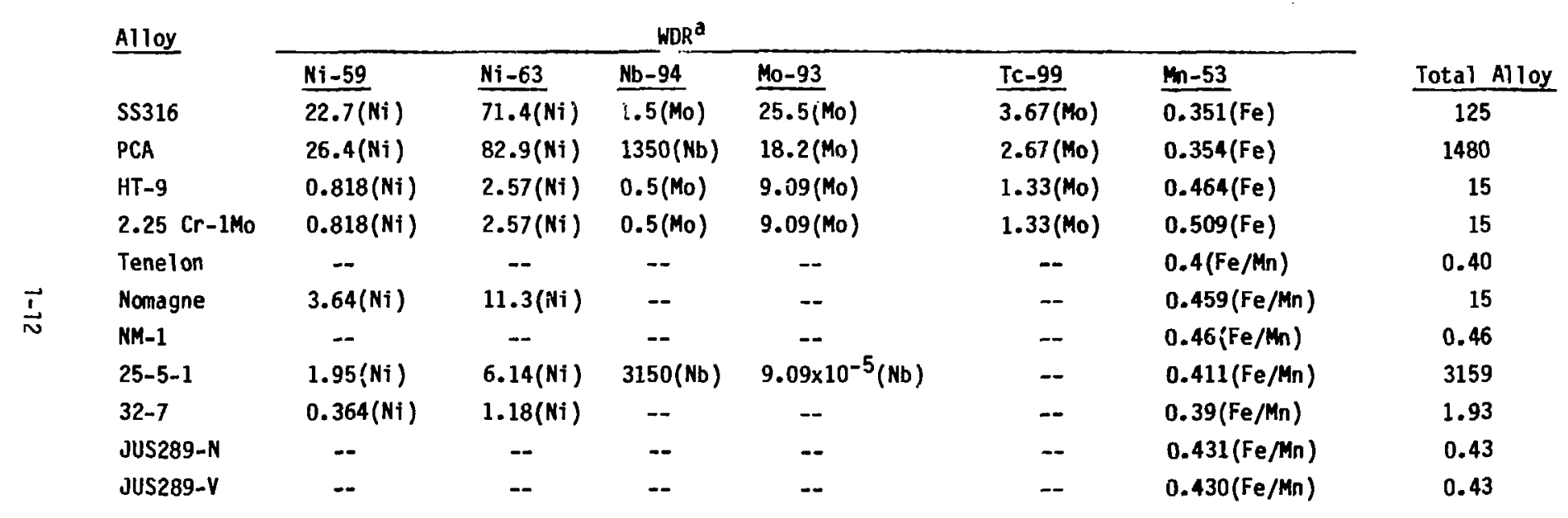

$a_{W D R}=\sum_{i} A(i) / L(i)$,

$A(i)=$ activity of $i^{\text {th }}$ isotope in $\mathrm{Ci} / \mathrm{m}^{3}$, and

$L(i)=$ maximum permissable activity of $i^{\text {th }}$ isotope $\left(\mathrm{Ci} / \mathrm{m}^{3}\right)$ from 10CFR61.

If WOR $<1$, material can be disposed of by near-surface burial without processing. 
near-surface burial (Table 1.1-2). Satisfying such rules would greatly simplify remote replacement, reprocessing, and disposal of activated commercial reactor components. One of our goals with our fusion reactor design is to satisfy such rules while at the same time streamlining the required remote maintenance procedures, reducing the required number of hot-cell facilities, and minimizing the risk of accidents to the plant workers and to the public. All three current blanket designs use low-nickel ferritic steels (such as HT-9), which come closer to near-surface burial guidelines than does stainless steel, but which still exceed those guidelines by a factor of 10 to 20 . We have identified three choices for consideration: (1) Processing the material for suitable dilution in alloys or concrete. This would entail more hot-cell facilities and would generate larger volumes of waste to transport and bury. (2) Isotopic tailoring of the molybdenum component in the alloy, which may be expensive. (3) Developing new alloys to meet the NRC criteria without having to process the blanket material. This choice will require the longest time to develop materials and therefore may be impractical for a nearer-term device such as FPD. However, for the long term, new alloys would benefit a large number of commercial reactors like MARS because the use of these alloys could eliminate reprocessing steps and reduce on-site facilities for such reprocessing. A major limitation with choice 3 is that the alloys identified in Table 1.1-2 lack sufficient data on neutron damage properties to permit redesign now of any of the MARS blankets. Therefore, prudence dictates that remote maintenance and waste processing facilities be designed for the MARS reference case blanket according to choice (1) above; i.e., 20x dilution of HT-9. However, to illustrate the advantages of new alloys that meet NRC criteria without post-irradiation processing, a simpler, direct-disposal scheme, such as that sketched in Fig. 1.1-6, will also be designed for comparison. This simpler remote-handling and disposal scheme would also be available if choice 2 turned out to be cost-effective. 


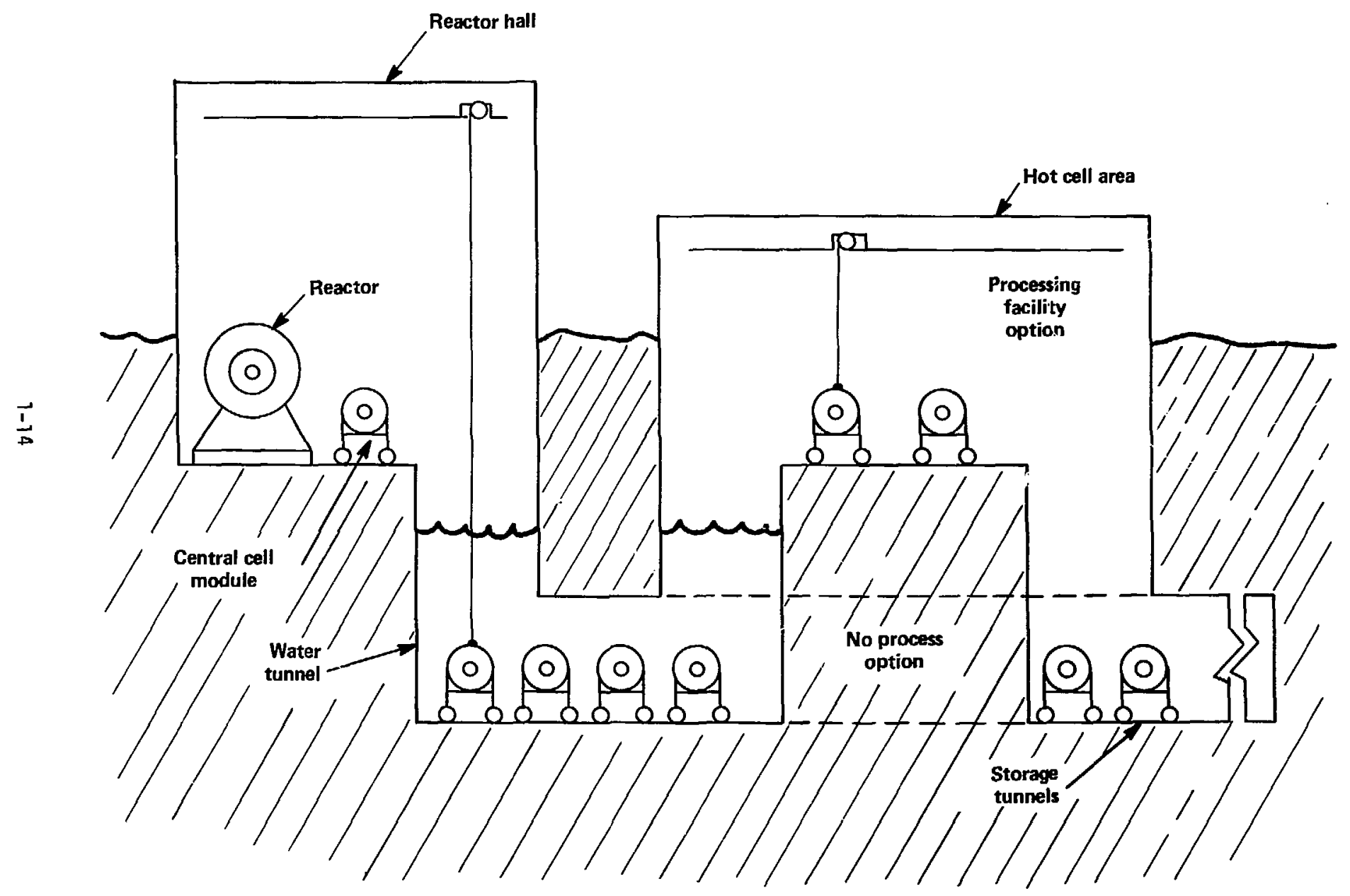

Fig. 1.1-6. Radioactive waste direct disposal scheme. 


\section{PLASMA ENGINEERING}

\subsection{MAGNETIC CONF IGURATION}

\subsubsection{Central-Cell Design \\ 2.1.1.1 Magnet Description}

The central cell magnet set consist of 48 identical solenoidal coils with axial spacing (coil midplane to midplane) of $3.16 \mathrm{~m}$. Conductor bundles for each coil are $0.884 \mathrm{~m}$ wide and $0.932 \mathrm{~m}$ thick with an inner radius of $2.44 \mathrm{~m}$. The conductor bundle has three layers, as show in Fig. 2.1-1, each with a different bulk current density. The total current in each central cell magnet is 12.1 MA. The nominal, on-axis central-cell magnetic field is $4.7 \mathrm{~T}$.

\subsubsection{Design Considerations}

To permit the use of niobium-titanium superconductor in the central cell magnets, the maximum conductor field strength was specified to be less than 8 T. Figure 2.1-2 shows contours of constant magnetic field strength superimposed on the cross section of a central-cell conductor bundle, spaced $0.25 \mathrm{~T}$ apart. The figure shows the peak field strength at the inner radius and midplane of the coil to be about $7.1 \mathrm{~T}$.

For a self-supporting conductor in a solenoidal coil, the conductor hoop siress is $\sigma=\mathrm{JBr} / \mathrm{f}$, where $J$ is the bulk current density, $B$ is the local axial field strength, $r$ is the local radius, and $f$ is the conductor packing fraction. For the central cell coils, $\sigma$ was specified to be less than $280 \mathrm{MPa}$ (40 ksi). Table 2.1-1 gives the calculated hoop stress at the inner surface of each conductor bundle layer, using the current densities from Fig. 2.1-1, the field values from Fig. 2.1-2, and a conductor packing fraction of 0.8 .

Because the central-cell magnet, set comprises axially separated coils, there is an axial ripple superimposed on the nominal 4.7-T solenoidal field. For the magnet set described above, the minimum and maximum on-axis field strengths are 4.67 and $4.85 \mathrm{~T}$, respectively, giving a field ripple of $3.8 \%$. 


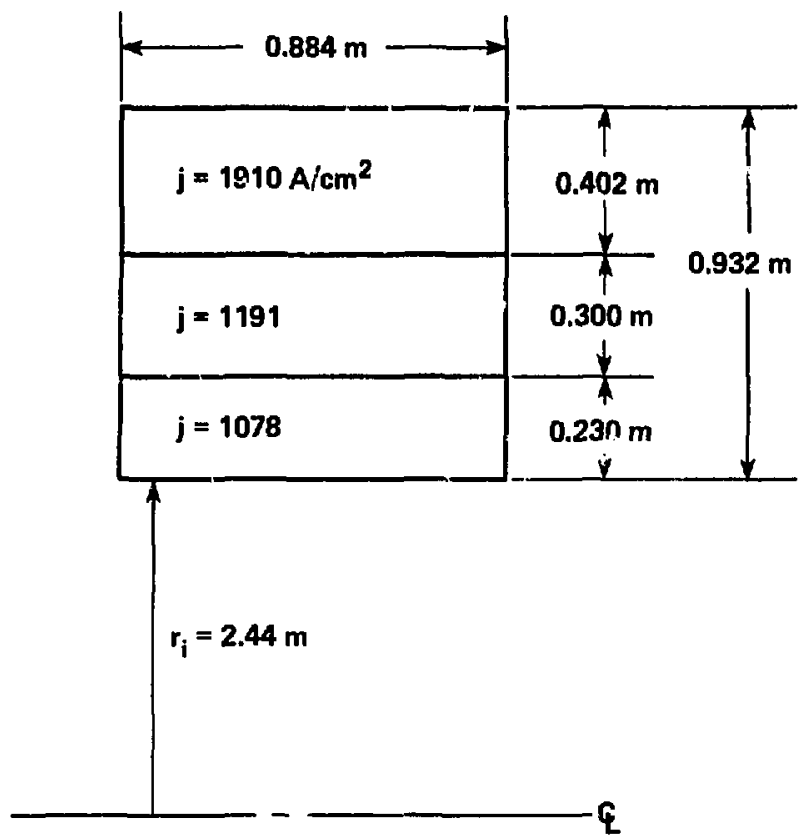

Fig. 2.1-1. Central-celi coil conductor bundle. 


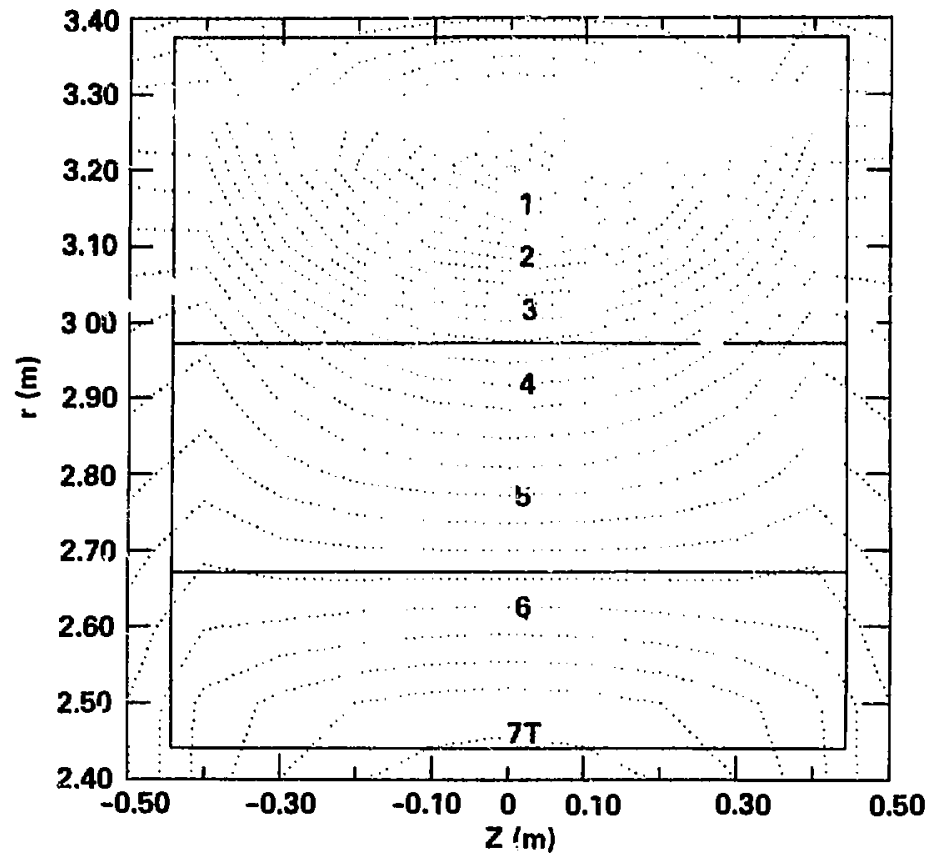

Fig. 2.1-2. Magnetic field contours in central cell coil. 
Table 2.1-1. Hoop stress at inner surface of each conductor bundle layer of central cell coil.

$J\left(M A / m^{2}\right)$

10.78

11.91

19.10
$B(T)$

.7 .1

5.7

3.5
$\sigma(\mathrm{MPa})$

230

230

250

Magnetic field ripple in the central cell causes a reduction in the MHD stability limit for $\beta$ in the central cell. This is because piasma pressure $p$ is axially uniform, and $B$ is minimum where normal curvature is unfavorable and maximum where it is favorable. Thus, the $p / B$-weighted unfavorable curvature dominates, and the central-cell makes a small but negative contribution to stability. (See Section 2.2 for a more complete discussion of MHD stability.) Stability calculations for two values of central-cell field ripple yielded a central-cell $\beta$ limit, $\beta_{c}=0.7$ for no ripple, and $\beta_{c}=0.65$ at $7 \%$ ripple. We were guided by these calculations to choose $4 \%$ as a design value for ripple, with the expectation that such ripple will not degrade the stability more than a few percent.

A study of axial coil spacing vs conductor bundle inner radius for constant field ripple is show in Fig. 2.1-3. For this study, the nominal central-cell field was $4.7 \mathrm{~T}$, the field ripple was $\sim 4 \%$, the conductor bundle cross section was square, and the cross section dimensions and current densicy were chosen to limit the maximum conductor field to $<8 \mathrm{~T}$ and the hoop stress to $<280 \mathrm{MPa}$. The MARS baseline design point, $r_{f}=2.44 \mathrm{~m}$ and $l=3.16 \mathrm{~m}$, is circled on Fig. 2.1-3. Note that the MARS baseline design for central-cell magnets is dimensionally consistent with the high-temperature blanket design (Section 4.3) but not with the LiPb blanket (Section 4.2). For the LiPb blanket, the coil axial spacing remains at $3.16 \mathrm{~m}$ but the inner radius is reduced. This will increase the ripple somewhat above $3.8 \%$, but the additional degradation of stability is expected to be small. 


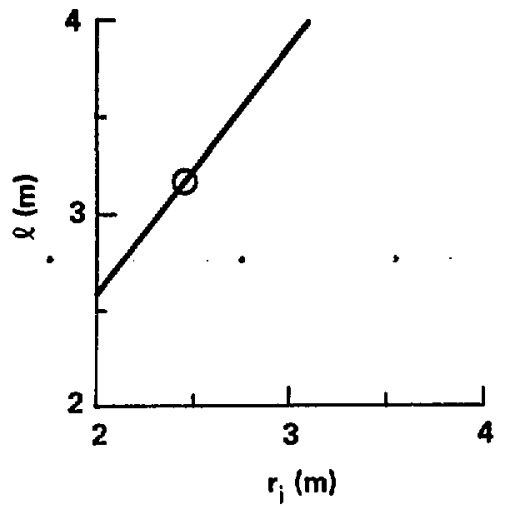

Fig. 2.1-3. Axial coil spacing vs inner radius for constant field ripple. 
There is a mutual attraction between any two solenoidal coils on the same axis with like current directions. A central-cell coil immersed in a line of many other coils has no net magnetic body force acting upon it. However, depending on the energizing/deenergizing sequence for the MARS coils, large net body forces can be expected on central-cell coils. As an upper limit, we have calculated the net axial body force on an energized central-cell coil at one end of a long string of identical energized coils. The forces are given in Table 2.1-2 for each conductor bundle layer. The face pressure is the axial force divided by the area of the end of the annular conductor bundle layer.

Table 2.1-2. Central cell coil forces.

\begin{tabular}{lll}
\hline & $\begin{array}{c}\text { Axial force } \\
(\mathrm{N})\end{array}$ & $\begin{array}{c}\text { Face pressure } \\
(\mathrm{MPa})\end{array}$ \\
\cline { 2 - 2 } & $\begin{array}{l}1.85 \times 10^{7} \\
\text { Inner layer }\end{array}$ & $5.0(720 \mathrm{psi})$ \\
Middle layer & $3.03 \times 10^{7}$ & 5.8 \\
Outer layer & $7.36 \times 10^{7}$ & 9.2 \\
\hline
\end{tabular}

2.1.2 End Plug Design

\subsubsection{End Plug Configuration}

The MARS end plug colls, along with one quadrant of the magnetic flux bundle, are shown in Fig. 2.1-4. The solenoid at the ieft of the figure is the axicell. The resistive insert coils, which produce a mirror region in the axicell, are not visible in the figure.

The two coils following the axicell are transition coils of the doubleellipse type, as evidenced by the flaring of the flux bundle, first in the horizontal plane, then in the vertical plane. This feature is important for limiting the radial transport of passing ions (see Section 2.2). 


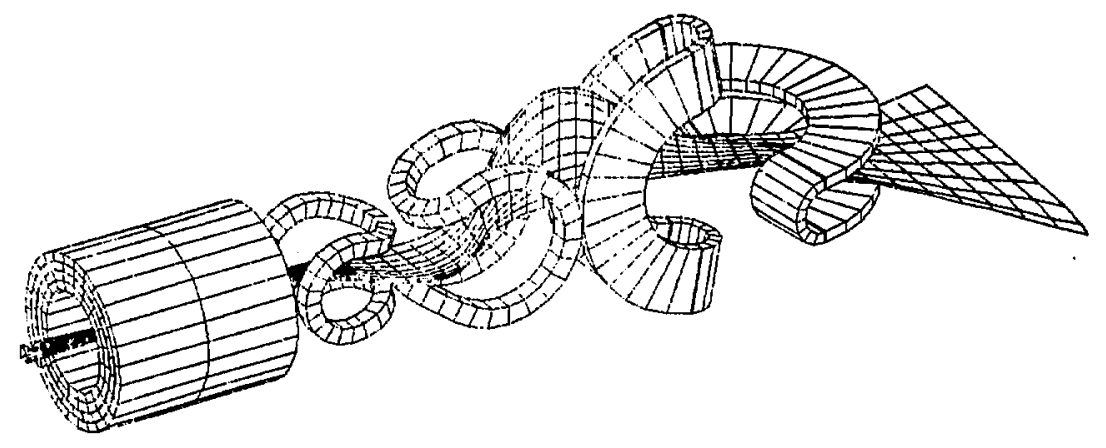

Fig. 2.1-4. MARS end plug. 
The final coil pair is the yin-yang anchor, wich maps the elliptical flux bundle of the second transition coll through a minimum-B mirror cell. This cell provides the primary good magnetic curvature for MHD stabilifty (hence the name "anchor"), and is also the location of the thermal barrier and the confining potential peak.

The variation of magnetic field along the axis of the MARS reactor, as calculated by the magnetic field code EFFl, is shown in Fig. 2.1-5. The axicell mirror fields are 21.1 and $24.3 \mathrm{~T}$; the axicell midplane field is $17.4 \mathrm{~T}$. The minimum transition region field is 1.44 T. The anchor mirror fields are 6.8 and $6.1 \mathrm{~T}$; the anchor midplane field is $2.1 \mathrm{~T}$. Note that tilese fields are slightly different from the nominal values used in the plasma performance analysis (see Secition 2.10). In the final MARS design, these two sets of field values will be made consistent.

\subsubsection{Axicell Coils}

The axicell is an axisymetric mirror cell immediately adjacent to the end of the central cell. The purpose of the axicell is to limit the velocity distribution of central cell ions that can pass to the end plug, thus reducing the requirement for trapped particle pumping in the transition and barrier regions. It does this in two ways: the high mirror field is a magnetic reflector and the potential of the mirror-confined axicell plasma is an electrostatic reflector.

The high magnetic field strengths of the axicell are produced by a hybrid coil arrangement consisting of a superconducting outer coil and normal-conducting insert coils, all simple solenolds. The design of these coils has evolved with the axicell physics scenario. An earlier MARS design, using neutral beam injection to sustain the axicell plasma, had an axicell coil set consisting of four separate coils: two insert coils and two superconducting outer coils. The axial gap between these colls was used for neutral beam injection. The present MARS design, with an ICRH-sustained axicell, does not need an axial gap in the outer colls. An axially continuous superconducting coil provides a background magnetic field slightly above 15 T (nearly uniform over the volume of the axicell plasma) and the insert coils provide the incremental mirror fields. 


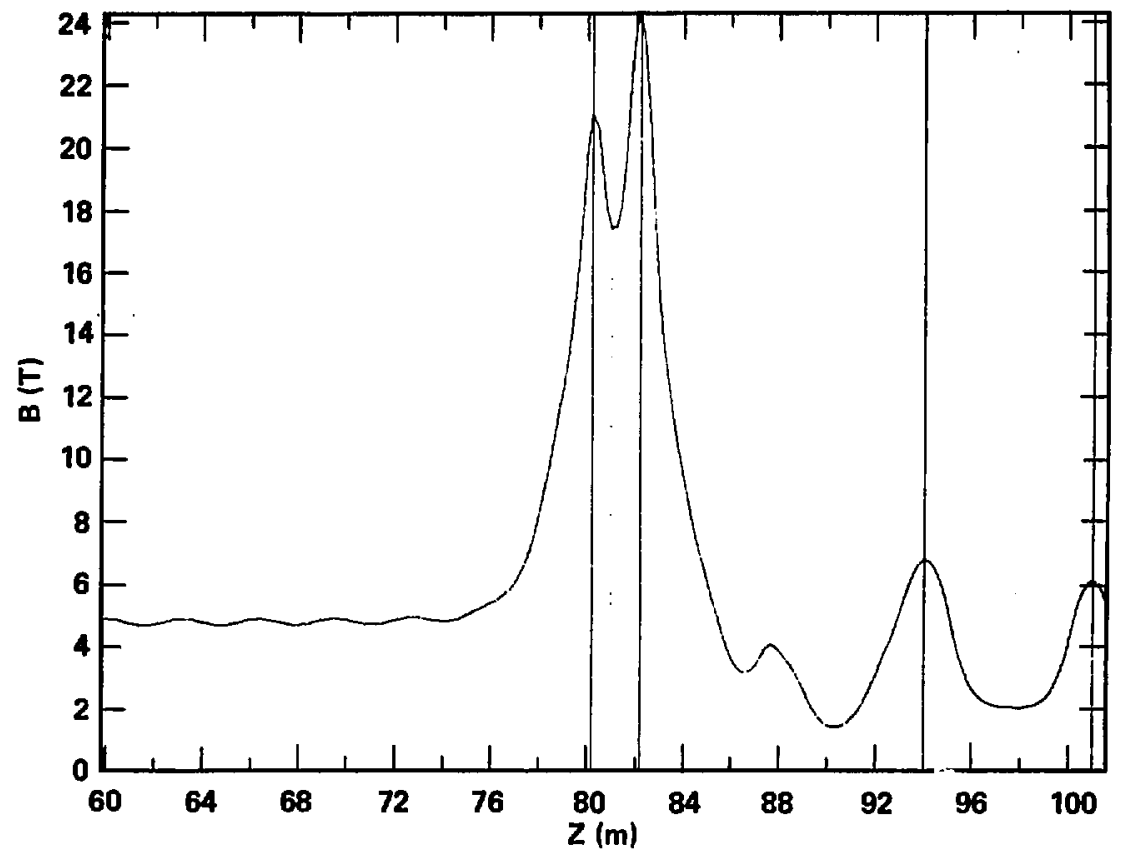

Fig. 2.1-5. On-axis magnetic field strength in MARS. 
Figure 2.1-6 shows the conductor bundle cross sections of the axicell magnets and the contours of constant magnetic field strength. The axicell is centered at $z=81.2 \mathrm{~m}$ and the two insert coils are centered at $z=80.2$ and $82.2 \mathrm{~m}$. The minimum inner radif are $0.32 \mathrm{~m}$ for the $21-T$ insert coil, $0.30 \mathrm{~m}$ for the 24-T insert coil, and $1.4 \mathrm{~m}$ for the superconducting coill. The cross sections for the insert colls are $0.282 \times 0.698 \mathrm{~m}$ and $0.420 \times 0.858 \mathrm{~m}$, and the bulk current density is $2800 \mathrm{~A} / \mathrm{cm}^{2}$. These cross sections and current densities are for an externaliy cooled conductor design for the insert coils.

In revised design using an internally cooled conductor, now chosen as the MARS baseline, the cross sections are $0.317 \times 0.720 \mathrm{~m}$ and $0.471 \times 0.896 \mathrm{~m}$, and bulk current density is $2500 \mathrm{~A} / \mathrm{cm}^{2}$. The constant-B contours for the internally cooled design are very similar to those in Fig. 2.1-6. The internolly cooled design, although having higher resistive losses, was selected to avoid dielectric breakdown in the high field and radiation environments. The total coil currents for the insert coils are 5.5 and 10.1 MA. The cross section for the superconducting cotl is $1.03 \times 5.30 \mathrm{~m}$ and is divided into four equal thickness layers with bulk current densittes of 1050 (inner layer), 1050, 1428 , and $2222 \mathrm{~A} / \mathrm{cm}^{2}$. The total coil current for the superconducting coil is $78.5 \mathrm{MA}$.

As shown in Fig. 2.1-6, the peak conductor field strength in the axicell superconductor is between 15 and $16 \mathrm{~T}$, and occurs at the inner surface and axial midplane of the coll. With the insert colls not energized, the peak conductor field strength increases to $16.0 \mathrm{~T}$.

\subsubsection{Transition Coils}

The transition coils are located at $z=86.1$ and $90.6 \mathrm{~m}$ (centers of curvature of major arcs). The first transition coil has equal major and minor arc radi $i$ of $1.25 \mathrm{~m}$. Major arcs are $210 \mathrm{deg}$ in sweep angle; minor arcs are $180 \mathrm{deg}$. The cross section of the conductor bundle is $0.46 \times 0.46 \mathrm{~m}$, and the bulk current density is $3000 \mathrm{~A} / \mathrm{cm}^{2}$, for a total coil current of $6.4 \mathrm{MA}$.

The second transition coll has a major arc radif of $2.0 \mathrm{~m}$ and a minor arc radii of $1.25 \mathrm{~m}$. Major arcs are $240 \mathrm{deg}$ in sweep angle; minor arcs are 180 deg. The cross section of the conductor bundle is $0.53 \times 0.53 \mathrm{~m}$, and the bulk current density is $2900 \mathrm{~A} / \mathrm{cm}^{2}$, for a total coll current of $8.1 \mathrm{MA}$. The peak conductor field in each of the two transition coils is $6.8 \mathrm{~T}$. 


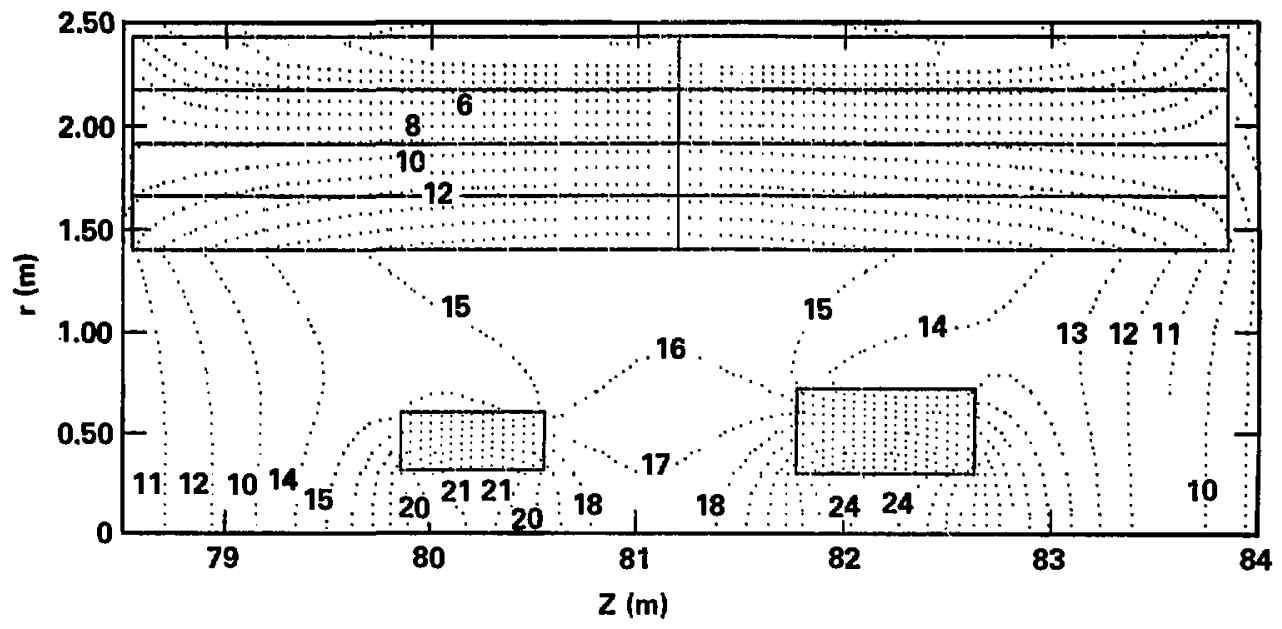

Fig. 2.1-6. Axicell coils and magnetic field contours. 


\subsubsection{Anchor Coils}

The yin-yang anchor colls are located at $z=97.1$ and $98.1 \mathrm{~m}$ (the centers of curvature of the major arcs). The two colls are identical, with major arc radii of $3.0 \mathrm{~m}$ and minor arc radif of $0.8 \mathrm{~m}$. Major arcs are $160 \mathrm{deg}$ in sweep angle, and minor arcs are $180 \mathrm{deg}$. The cross section of the conductor bundles is $0.32 \times 1.85 \mathrm{~m}$, and the bulk current density is $2750 \mathrm{~A} / \mathrm{cm}^{2}$, for a total current in each coil of 16.3 MA. The peak conductor field in each anchor coil is $8.5 \mathrm{~T}$ at the inner surface of the minor arcs. 
Identifying the physics constraints of axicell magnetic configurations and developing methods to satisfy those constraints has been an area of intense effort. For MARS, we have not yet satisfied all of the constraints, and our continuing work will concentrate on the remaining problems. In this section we discuss the nature of these constraints and the status of our efforts to satisfy them. A more detalled discussion of the constraints and a demonstration of a satisfactory solution for MFTF-B has been completed. ${ }^{1}$

We last discussed the physics constraints of the magnetic configuration for an axicell reactor in Ref. 2. Since then, the problem has changed in a number of ways:

1. The relocation of the thermal barrier and confining potential peak to the anchor changed the plasma pressure function. Most significantly, the pressure in the transition region becomes non-negligible because of the passing fons there. The pressure in the transition region (normalized to that in the central cell) is now estimated to be $0.5 \mathrm{~B} / \mathrm{B}_{\mathrm{m} 2}$, where $B$ is the local field in the transition region and $B_{m 2}$ is the outboard axicell mirror ffeld (24 $\mathrm{T}$ in MARS). Because the transition region has mostly bad curvature, this new pressure function makes the MHD stability criterion more difficult to satisfy.

2. The work of Cohen and Byers ${ }^{3}$ has shown that to avoid large stochastic radial transport, we must balance positive and negative geodesic curvature ( $H O$ in the transition region) so that it nearly nulls the line integral of 10 from the axicell to the location of the potential peak. This constraint is the reason for the introduction of the double-ellipse transition coll design. Satisfying this constraint will also nearly null the parallel current through the central cell, which is the geodesic curvature-related criterion previously considered. ${ }^{2}$

3. The $m=1$ ballooning criterion for MHD stability 4 has been shom to be more severe than the flute interchange criterion. However, we have seen that the $m=1$ criterion approaches the interchange criterion if two conditions are met: (a) the magnetic flux between the first wall and the 
plasma is not large compared to that contained within the plasma, permitting effective wall stabilization (true for MARS); and (b) the good curvature on a 45-deg field line in the anchor is symmetric about the anchor midplane. The latter condition ensures that stabilization on all field lines, in particular the 0 - and 90-deg field lines, is provided locally at each end of the machine, important for z-dependent ballooning. In the MARS study, we have continued to use the interchange criterion as a measure of MHD stability, but with the above condition as an additional constraint.

\subsubsection{Radial Transport}

The MARS double-ellipse transition coll design has been adjusted to null the line integral of the geodesic curvature up to the location of the potential peak,

$$
\int_{\text {axicell }}^{\text {potential }} \stackrel{\text { peak }}{\mathrm{LO} d s=0 .}
$$

Figure 2.2-1(a) shows the axial profile and Fig. 2.2-1(b) shows the progressive integral of the geodesic curvature function. Note the balancing of positive and negative contributions in both the transition ( $z=82.2$ to $94 \mathrm{~m}$ ) and anchor $(z=94$ to $101 \mathrm{~m}$ ) regions.

Byers $^{3}$ has shown that large stochastic radial transport would occur in MARS even with $\int H^{0} d s=0$, unless the radial electric field is negative in the transition region. The negative electric field causes a cancellation between the electric- and curvature-driven azimuthal drifts, so that an ion remains on a given field line and experiences the balanced geodesic curvature. Using a model electric field profile, Byers showed good (nonstochastic) particle behavior in MARS with a centerline transition potential of $-50 \mathrm{kV}$ and a grounded halo. Fortunately, this is consistent with the potential biasing of the plasma specified for rotation control in the central cell (see Section 2.2.4).

Recent calculations with Byers's code have shown that good particle behavior is possible in MARS even with an incomplete nulling of the geodesic curvature integral as long as the transition electric field is negative. We will use this latitude to make design revisions that will increase the MAD stability of the system (see Section 2.2.3). 

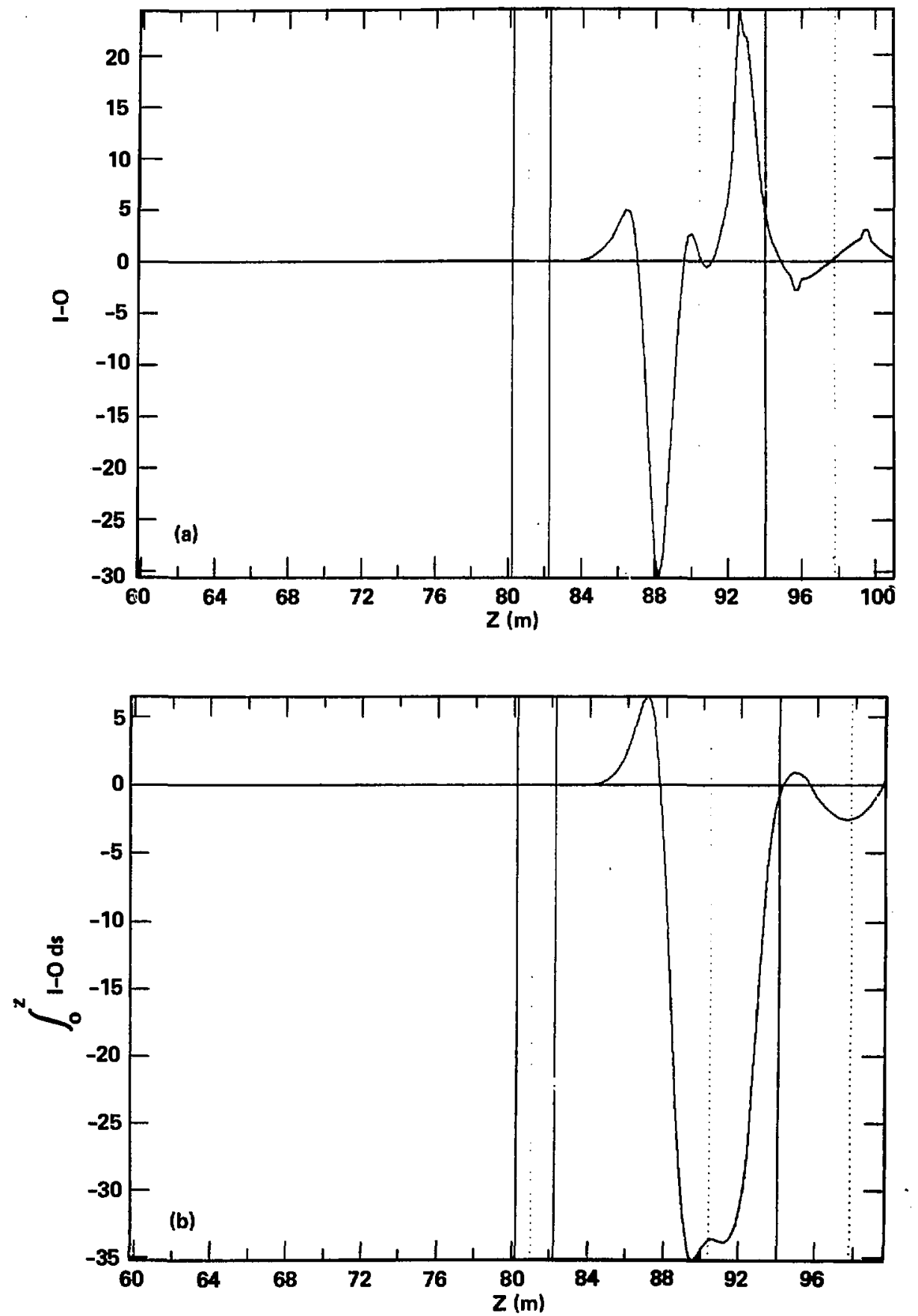

Fig. 2.2-1. (a) Geodesic curvature; (b) progressive integral of geodesic curvature. 


\subsubsection{Parallel Current}

Mear-nulling of the central-cell parallel current is inportant to prevent distortion of the otherwise circular flux surface. How nearly the parallel current must be nulled to prevents damiging flux distortions, especially with the annealing effects of finite larmor radit, is an area of ongoing investigation. Because the plasma pressure is proportional to $B$ in the transition region and nearly symmetric about the anchor midplane (see Fig. 2.2-2), and because the anchor geodesic curvature is much less than that in the transition, it follows that satisfying Eq. (2.2-1) will also nearly null the central-cell parallel current, given by

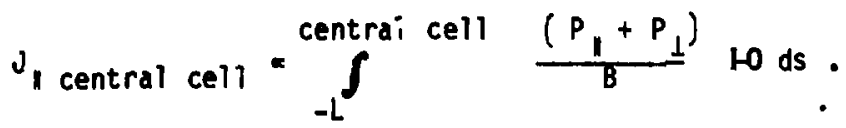

Figure 2.2-3 shows the near-nulling of the central-cell parallel current in MARS.

\subsubsection{MHD Stability}

The interchange stability criterion is given by

$$
\text { - } \int_{-L}^{L} \frac{\left(P_{1}+P_{1}\right)}{B} \text { Ads } \leqslant 0 \text {, }
$$

where is the normal curvature of the field line. In practice, we integrate this function on a 45-deg field line from $z=0$ to L. In addition to satisfying Eq. (2.2-2), we require that be nearly symmetrir about the anchor midplane to ensure that the $m=1$ ballooning 1 imit will approach the interchange limit.

Figure 2.2-4 nows the normal curvature aleng the length of the machine. Note the small positive and negative contributions due to the central-cell field ripple, the small negative contribution due to the simple mirror axicell, the mostly negative contribution of the transition region, and the double-humped positive contribution of the anchor. Figure 2.2-5(a) shows the 


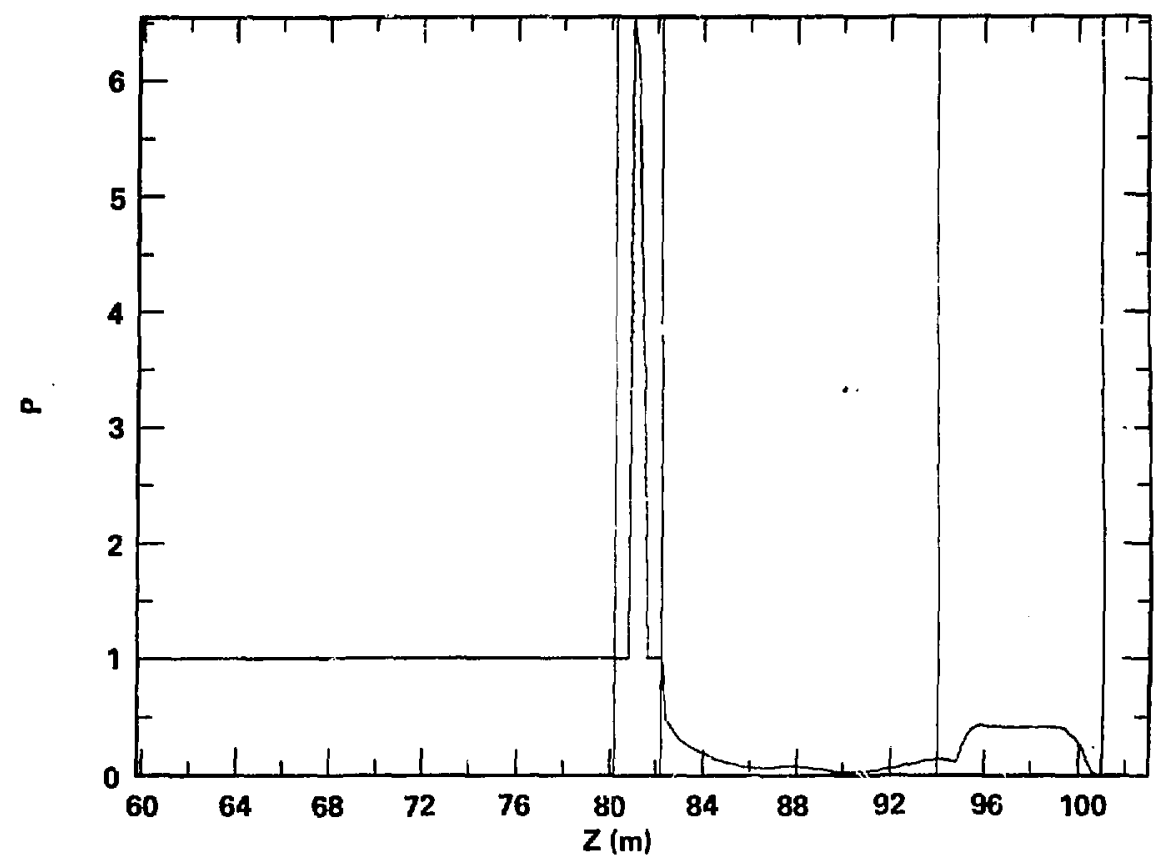

Fig. 2.2-2. Plasma pressure normalized to 1.0 in the central cell. 


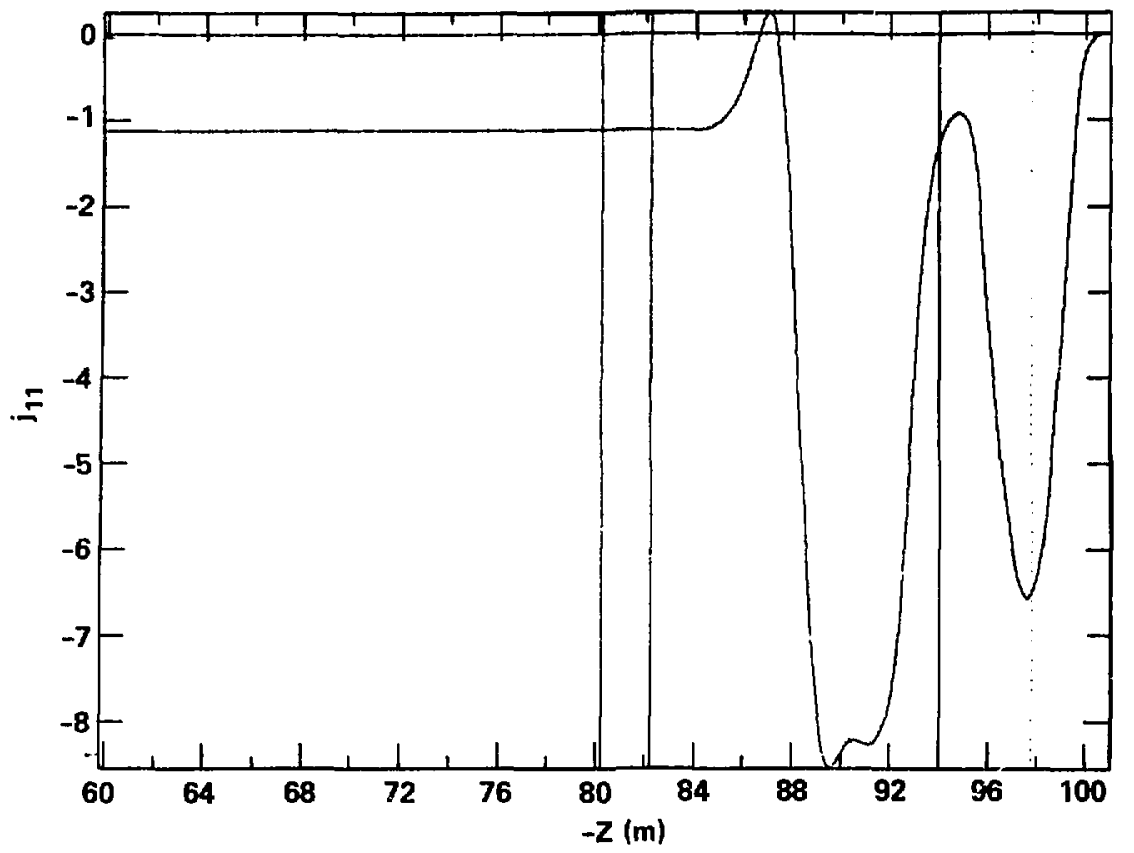

Fig. 2.2-3. Parallel current. 


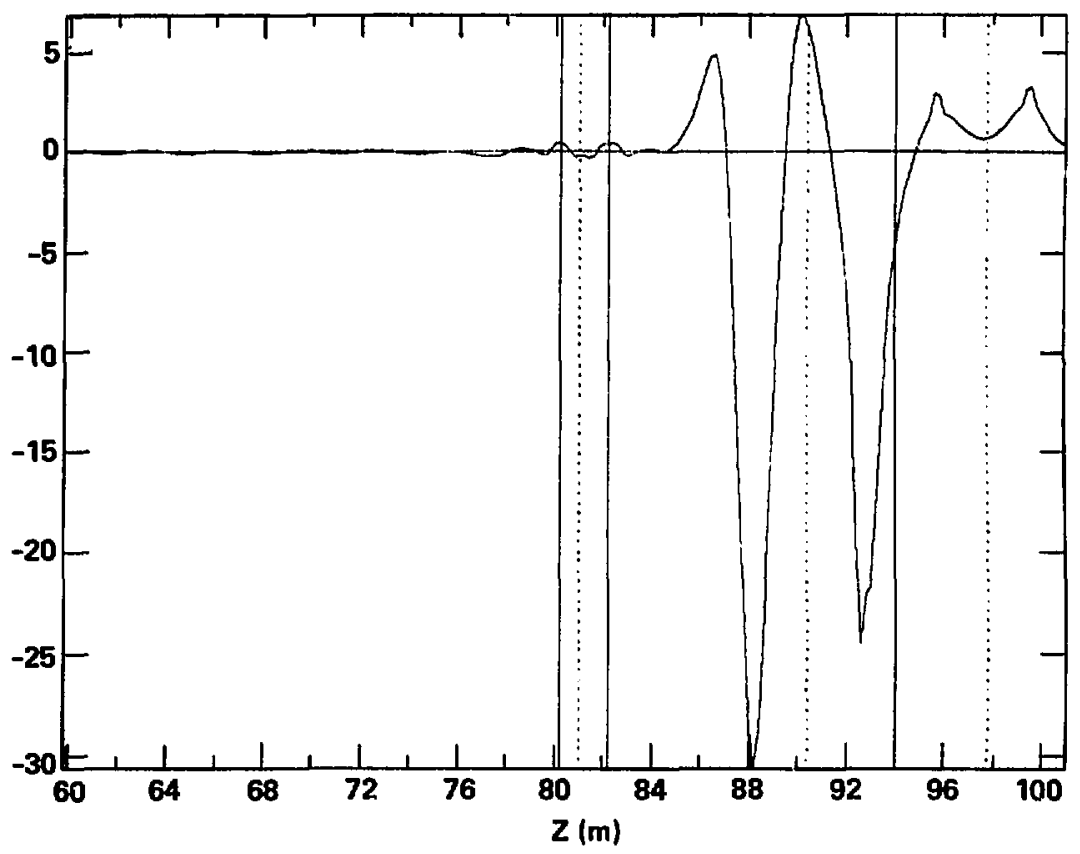

Fig. 2.2-4. Normal curvature. 

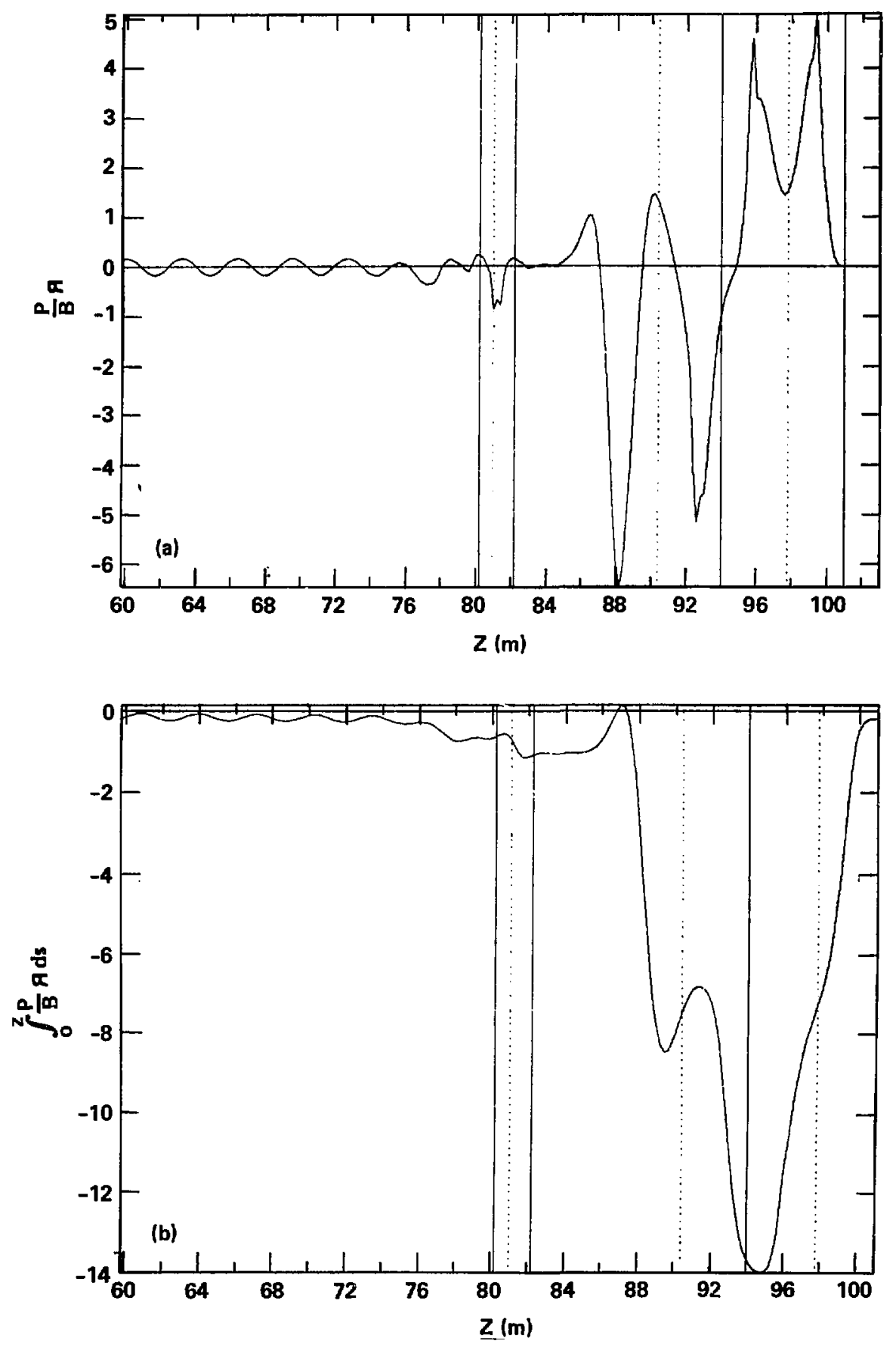

Fig. 2.2-5. (a) $P / B$-weighted normal curvature; (b) progressive integral of $P / B$-weighted normal curvature. 
axial profile and Fig. 2.2-5(b) shows the progressive integral of the $p / B$ weighted normal curvature. Balancing the positive and negative contributions in Fig. 2.2-5(a) results in a mulled integral at the end of the machine, thus signifying stability for the $\beta$ values chosen. The $\beta$ values used in this calculation (all peak, on-axis $\beta_{1}$ values) were central cell $\beta=0.23$, axicell $\beta=0.4$, and anchor $\beta=0.65$ (consistent with the mirror mode limit). The central-cell $\beta_{c}$ value is considerably less than the specifled MARS design value of 0.7 .

Our primary goal in satisfying the physics constraints of the magnetic configuration is to demonstrate MHD stability at $\beta_{c}=0.7$ while simultaneously satisfying the requirements for radial transport and parallel current. We are encouraged that satisfactory radial transport does not seem to require a complete nulling of the geodesic curvature. We have, in fact, al ready developed a magnet design (very similar to the present MARS design) with interchange stability at $\beta_{c}=0.7$ that gives satisfactory radial transport despite its noncancelling geodesic curvature. However, this design has nonsymmetric 8 in the anchor, and $m=1$ ballooning is suspected (although not yet verified). Another approach to improved MHD stability is to sustain a high- $\beta$ mirrorconfined species (probably by $\mathrm{ECAH}_{\text {) }}$ in the good curvature magnetic well of the transition (at $z=90 \mathrm{~m}$, see Figs. 2.1-5 and 2.2-5a). Sample calculations have shown stability with $\beta_{c}=0.5$. In this report we assume that we will be successful in finding a satisfactory design solution at $\beta_{c}=0.7$, and we use this value in our plasma performance calculations.

\subsubsection{Central-Ceil Plasma Rotation}

A mirror machine plasma becomes MHD unstable if the radial electric field $E_{r}$ is too large. The effect is derived by considering net azimuthal electric currents through flute perturbation; these currents come from the guiding center drifts (aithough the currents are modified by the conservation of magnetic flux through the flute). The guiding center drifts are produced by centrifugal, electric, and gradient $B$ (normal curvature) forces. The net azimuthal currents can charge up the sides of the flute, causing a secondary electric field $E_{f}$ which makes the flute $E_{f} \times B$ drift either outward (growth) or inward (damping), depending on the direction of the azimuthal current. Thus, to ensure that the current is in the stabilizing direction, the following criterion must be satisfied: 


$$
\int_{-L}^{L} \quad \frac{p_{1}+p_{1}}{B} \text { Ads }<-\frac{m_{j} n_{j} L_{c}}{B_{c}^{4} r_{p}^{2}} E_{r}^{2} \text {, }
$$

where $m_{i}$ is ion mass, $n_{j}$ is ion density, $L_{c}$ is central cell length, $B_{c}$ is central-cell magnetic field, $r_{p}$ is plasma radius, and $E_{r}$ is the central-cell radial electric field. Note that if $E_{r}=0$, then the criterion becomes Eq. (2.2-2). MARS would violate the criterion with an uncontrolled central-cell radial electric field. The solution proposed for this design is to bias the plasma potential with respect to ground by lowering the potential at both ends of the machine. For MARS, the central-cell potential (on-axis) is reduced to $29 \mathrm{keV}$ with respect to ground. 5

\section{REFERENCES FOR SECTION 2.2}

1. D. E. Baldwin and R. H. Bulmer, A Physics Conceptual Design for the MFTFB Transition Coil, Lawrence Livermore National Laboratory, Livermore, CA, UCID-19562 (T982).

2. G. A. Carlson et al., Comparative End-Plug Study for Tandem Mirror Reactors, Lawrence Livermore National Laboratory, Livermore, CA, JCID-19271 (1981).

3. R. H. Cohen and J. A. Byers, Lawrence Livermore National Laboratory, Livermore, CA, personal communication.

4. L. D. Pearlstein, T. B. Kaiser, and W. M. Nevins, Lawrence Livermore National Laboratory, personal communication.

5. W. N. Kumai, Why Central Cell Electric Potential Control is Necessary, Lawrence Livermore National Laboratory, Livermore, CA, MARS Memorandum LLNL-MARS-82-048 (1982). 


\subsubsection{Trapping Rates}

As center-cell ions pass through the transition region, collisions will cause some of them to scatter in a sufficient pitch angle to become trapped in this region. Without a provision for removing or pumping these trapped ions, the density will increase and desired plasma conditions will be destroyed. The high density of trapped lons will cause the potential in the transition region to increase, and, because many of the trapped ions have turning points in the anchor, they will also increase the density and potential at the thermal barrier. The decreased thermal insulation between center-cell and warm electrons will cool these electrons and lower the confining potential of the center-cell ions. An additional effect, which may occur first, is to raise the pressure in the transition region high enough to cause MHD instability.

Several schemes have been suggested for pumping the trapped ions. In the case of TMK-U and MFTF-B, charge exchange off a pump beam is used. The pump beam energy and direction are chosen so that the charge-exchanged beam atcms, now lons, pass into the central cell while the trapped ions, now neutral, pass radially out of the plasma to the halo or wall. Other schemes under consideration for MARS involve the use of $r f$ magnetic fields to remove the ions radially.

To specify the neutral beam current or rf pump parameters, we must compute the trapping current of passing ions. To do so, we must solve for the distribution functions of the relevant ions from the Fokker-Planck equation. Futch and LoDestro ${ }^{1}$ have used a numerical code to compute trapping rates in square-well magnetic and potential fields for a variety of mirror ratios and trapped $\left(n_{\dot{i}}\right)$ and passing $\left(n_{p}\right)$ ion densities. The relationship between the densities is generally expressed with the quantity

$$
g_{t}=1+\frac{n_{t}}{n_{p}}
$$

The Futch-LoDestro results are well-represented by the expression

$$
\left.\frac{d n}{d t}\right|_{t}=\frac{n_{p}^{2}\left[0.935[1+0.0441 R] g_{t}\right\}^{10 / 3}}{n \tau_{p}\left(g_{t}-1\right)^{7 / 3}},
$$


where $R$ is the mirror ratio, $n \tau_{p}=6.27 \times 10^{11} T^{3 / 2} / 1 n \Lambda, \ln \Lambda=34.9-$ $\ln \left[n / T_{p} E\right]^{1 / 2}$ with $E=$ ion energy at the botton of the well, $T_{p}$ is the passing ion temperature, and $n=n_{p}+n_{t}$.

Futch and LoDestro also studied the effect of magnetic field shapes on the trapping rates with the aid of the bource-averaged Fokker-Planck code. ${ }^{2}$ In this work they assumed uniform pumping throughout the trapping region. To fit their results, we can multiply Eq. (2.3-1) by the correction factor $H$, which is a function of the mirror ratio, the field shape, and $g_{t}$. For a transition mirror ratio of 16, typical of MARS, their results indicate an $H$ factor of about 2.7. However, the field from MARS coils is much more like a square well, so we would expect a much smaller $H$ factor. In addition, their assumption of uniform pumping is not appropriate to a real machine where one would generally pump at certain selected locations. Indeed, charge-exchange pumping at the bottom of the transition section is not at all desirable since it requires the highest energy beam to overcome the potential of the axicell and allow the charge-exchanged beam particles to pass into the central cell. Pumping near the mirror is advantageous from that standpoint since a relatively much smaller potential must be overcome and a lower energy neutral beam can be used. However, it is not clear that a single pump near the mirror can pump the ions in the transition section. The deeply trapped ions, i.e., those with turning points near the bottom of the magnetic field, are not "seen" by a beam (or other pumping device). Thus, these trapped fons cannot be directly charge-exchanged out of the transition section, but must first suffer enough deflection in pitch angle via Coulomb collisions to bring them into the part of phase space with which the pump intersects. Strong enough pumping could distort the ion distributrion function enough to remove these deeply trapped ions, but the required current might be impractical.

To study the effect of pumping at selected locations on the trapping rate, we use the Fokker-Planck code. ${ }^{2}$ The term - vf, where $f$ is the distribution function, is included in the equations to represent pumping and is a function of axial position. In this case, $v$ is adjusted to obtain a steady-state solution with the specified mirror and axicell potentials, passing ion density and temperature, mirror ratio, etc. In its present version, the Fokker-Planck code assumes monotonic magnetic field and potential profiles, and symmetry about the midplane. We can approximate the actual 
situation by neglecting the potential peaks at the inner anchor mirror and at point $A$. In addition, we replace the magnetic field profile with a nearly flat but slightly decreasing field of value almost equal to the transition field (see Fig. 2.3-1). This appears to be a gross simplification of the actual field, but it should be noted that most of the ion trapping is expected to occur near the axicell mirror where the passing and trapped densities are highest. The center-cell passing ions are assumed to be Maxwellian and are launched from the center of the axicell.

We have run several cases coy-responding to different pumping locations with or without an axicell. Only a case that closely corresponds to the current MARS parameters will be presented. Input parameters are collected in Table 2.3-1.

The pump is localized to a range of magnetic fields between 7.8 and $10 \mathrm{~T}$, as shown in Fig. 2.3-1. Results for the distribution function at steady state, as well as the trapped density and potential dependence on $z$, are shown in Figs. 2.3-2 through 2.3-4. The two different boundaries in Fig. 2.3-2 correspond to the center of the axicell, indicated by the number 2, and the highfield axicell mirror, indicated by the number 1. The Maxwellian passing ions are enclosed by the lower of curves 1 and 2 and are indicated by the contours parallel to the $\theta$ axis.

The trapping rate for this case was found to be $2.419 \times 10^{14} \mathrm{~cm}^{-3} \mathrm{~s}^{-1}$. Other quantities of interest are $g_{t}=3.148, E=155 \mathrm{keV}, n=5.43 \times$ $10^{12} \mathrm{~cm}^{-3}$, and $R=16.47$. With the aid of these numbers, we find the trapping rate from Eq. $(2.3-1)$ to be $2.046 \times 10^{14} \mathrm{~cm}^{-3} \mathrm{~s}^{-1}$. The equivalent $H$ factor is 1.18, only a small amount above the value of unity for a square-well field and potential. For the baseline MARS case, an $H$ factor of unity was used, leading to a trapping rate of $295 \mathrm{~A}$ at each end. 


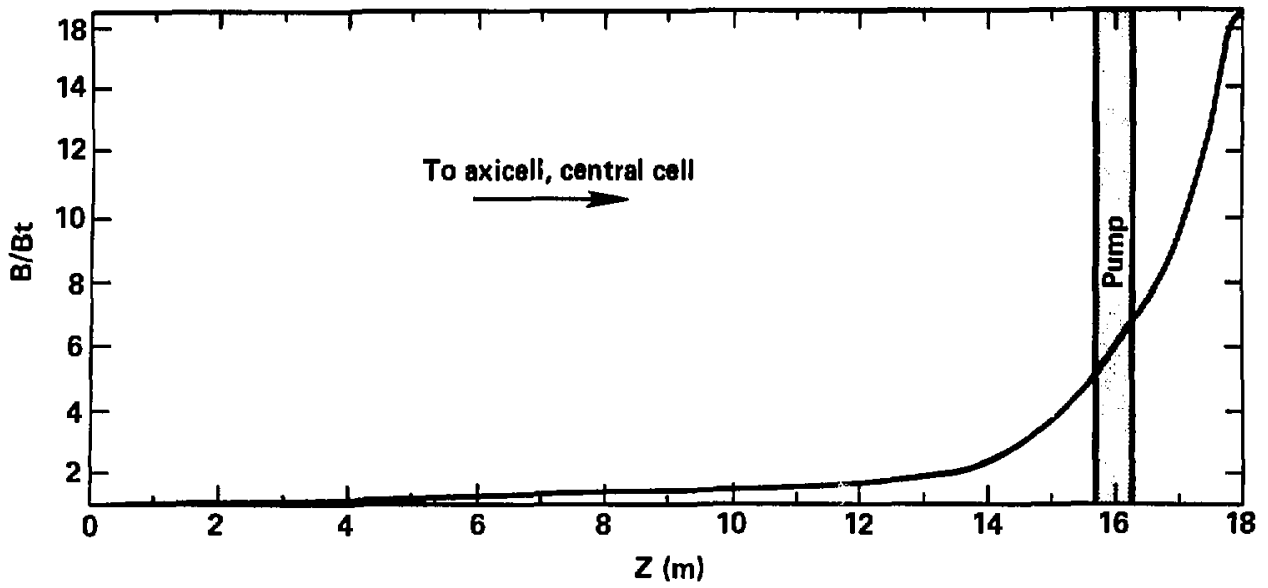

Fig. 2.3-1. Magnetic field used for trapping-rate calculations. 
Table 2.3-1. Parameters for calculating trapping rates in the transition section.

Axicell vacuum magnetic field $(T)$

17.3

Axicell beta

0.40

Axicell mirror field (T)

24.0

Transition magnetic field ( $T$ )

1.477

Equivalent transition length (m)

18

Passing ion density at axicell midplane $\left(\mathrm{cm}^{-3}\right)$

$2.155 \times 10^{14}$

Passing ion temperature (keV)

35

Electron temperature (keV)

24

Potential at axicell midplane (kV)

126.2

Potential at axicell mirror (kV)

82.1

Potential at bottom of transition section (kV)

0.0 


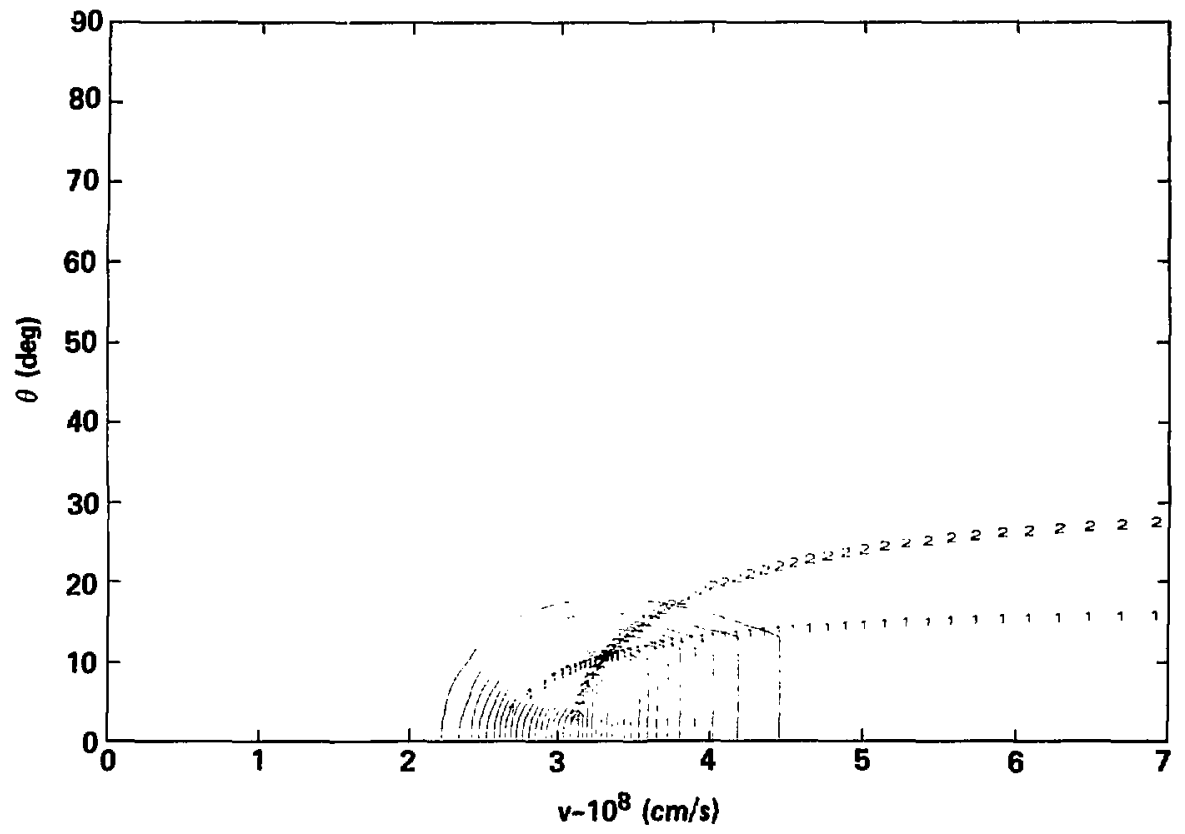

Fig. 2.3-2. Distribution function of ions at transition section. 


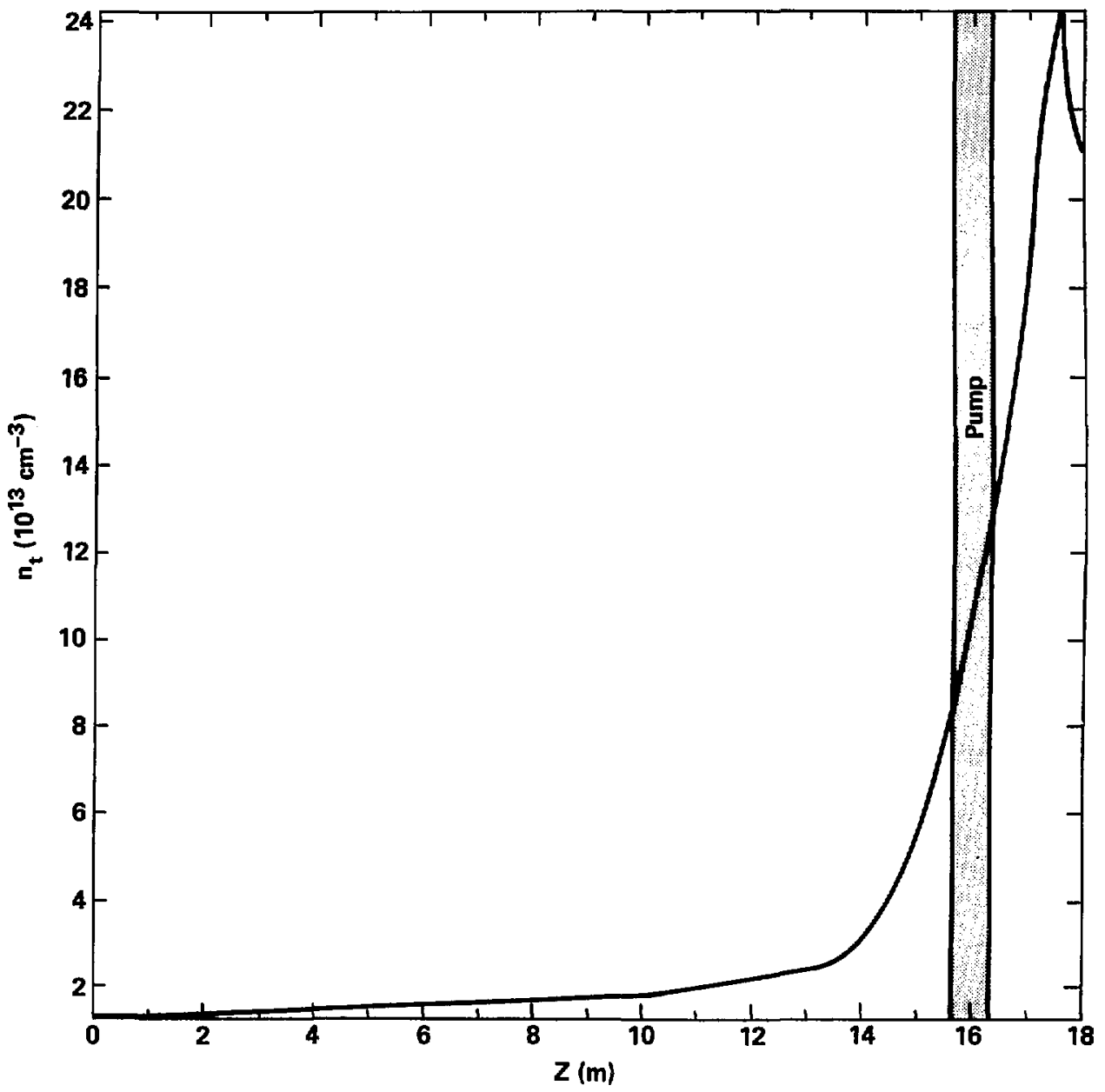

Fig. 2.3-3. Trapped density in transition section. 


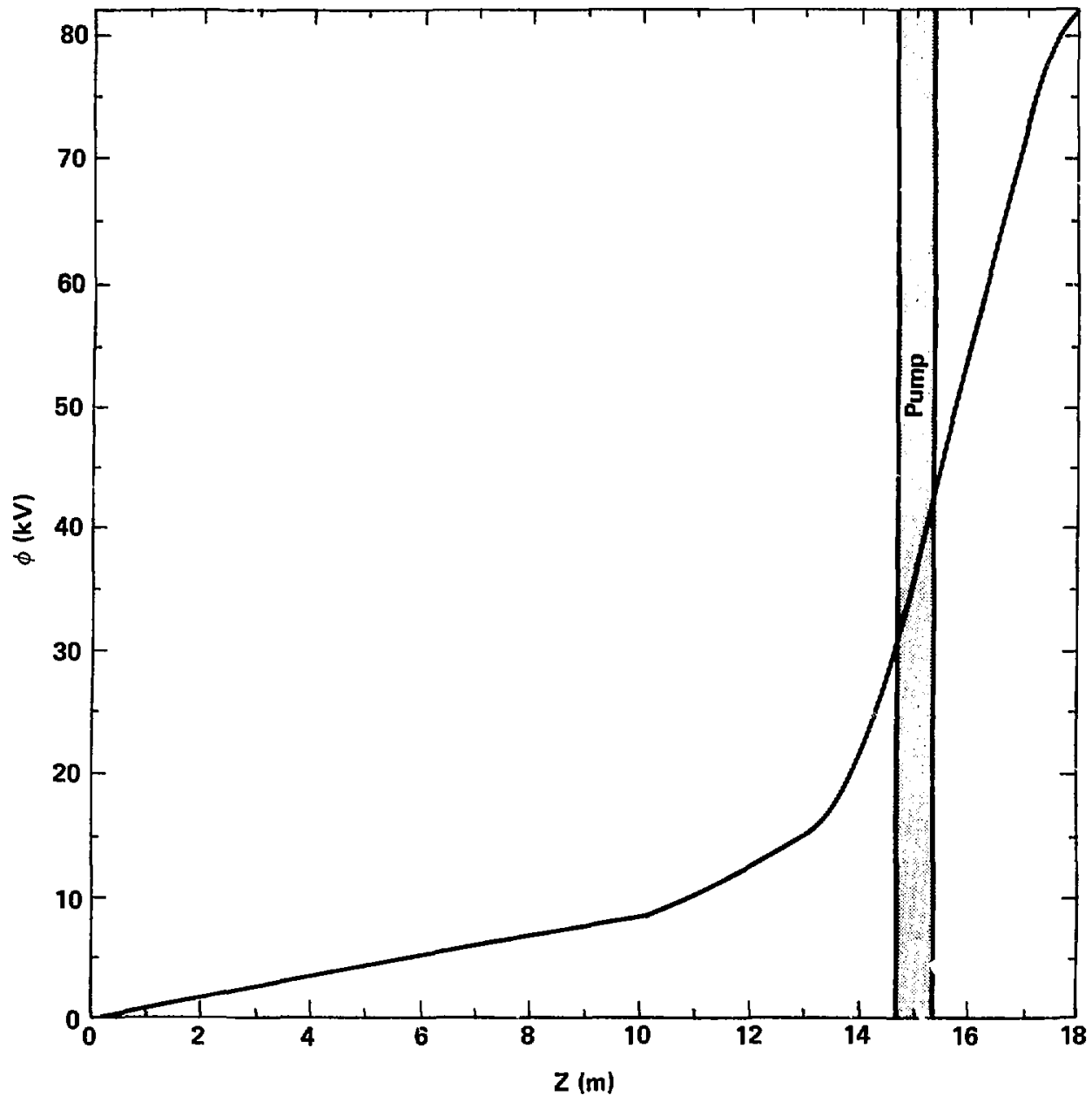

Fig. 2.3-4. Electrostatic potential in transition section. 


\subsubsection{Charge-Exchange Pumping}

\subsubsection{Overview}

Once the current of passing ions trapped in the transition region $I_{\text {trap }}$ has been determined by the method outlined in Section 2.3.1, additional ana is in two specific areas must be undertaken to compute the actual beam power required for charge-exchange pumping. The first is the calculation of the actual neutral beam current $I_{\text {pump }}$ that must be supplied to the plasma to pump a given $I_{\text {trap }}$ (the ratio of $I_{\text {pump }}$ to $I_{\text {trap }}$ is approximately 2.5 io 1 ); the second is the beam energy required to allow the injected beam to fuel the central cell. The required beam power is $P_{\text {nump }}=E_{\text {pump }} I_{\text {pump }}$ *

\subsubsection{Purping Current}

The pump beam current that must be supplied to the plasma for chargeexchange pumping is greater than the trapped ion current for three reasons: (1) a beam particle has a finite probability of ionization as well as charge exchange; (2) both trapped and passing ions can undergo charge-exchange events with the beam; and (3) a charge-exchange neutral that was a trapped ion can be reionized before it escapes the plasma. The relationship between I pump and $I_{\text {trap }}$ can be written as

$$
I_{\text {pump }}=\frac{g_{p}}{\left(g_{p}-1\right)} \frac{1}{p_{c x}} f_{\text {inn }} I_{\text {trap }} \text {, }
$$

where

$$
\begin{aligned}
& \left.g_{p}=\text { (n } n_{\text {pass }}+n_{\text {trap }}\right) / n_{\text {pass }} \text { at the pump beam target location, } \\
& p_{c x}=\text { probability of a beam particle undergoing charge exchange, and } \\
& f_{\text {ion }}=\text { reionization factor, }>1 .
\end{aligned}
$$

The combination $g_{p} /\left(g_{p}-1\right)$ is just $\left(n_{\text {pass }}+n_{\text {trap }}\right) / n_{\text {trap }}$, which increases the current as a result of unavoidable passing ion pumping. For the MARS baseline, this factor is about 1.5. From estimates of or for co- and counterstreaming ion distributions, the value of $p_{c x}$ has been taken to be approximately 0.7 . From previous work on charge-exchange pumping, ${ }^{3}$ the reionization factor has been taken to be about 1.2. This number was based on a simple 
calculation accounting for the charge-exchange neutral traveling the short way through an elliptical flux bundle. Here, our pump beam is aimed at a circular plasma, which has a longer chord length for a neutral to escape. This

suggests that $f_{i o n}$ may be larger than 1.2. A detailed calculation should be done to determine a more accurate value for $f_{i o n}$. Combining these correction factors, we see that $I_{\text {pump }}$ exceeds $I_{\text {trap }}$ by a factor of about $\mathbf{2 . 6}$.

\subsubsection{Pump Beam Energy}

To compute the required pump beam energy, we must know the magnetic field and potential at the pump bean injection point. Once an axial position and injection angle have been chosen consistent with good pump beam access, the magnetic field design gives us the field at that position. Quast-neutrality at that point is used to calculate the potential. Knowing this, the injection energy is then defined by the requirement that the parallel velocity be greater than zero at all positions between the pump team injection point and the central cell. Once this injection energy has been computed, a calculation of beam penetration tells us whether the axial location of the pump beam allows for adequate pumping of trapped particles near the axis. If the penetration is inadequate, the axial position is adjusted (usually to larger $z$ values) until the condition is remedied.

The requirement that the beam particles pass into the central cell reduces to the most restrictive of two criteria: (1) that the particles pass the $24 \mathrm{~T}$ mirror: and (2) that the particles pass the midplane of the axicell. The injection energy is then taken to be the larger of the energy to pass the 24-T peak

$$
E_{i n j, 1}=\frac{\left(\phi_{p i}-\phi_{p b}\right)}{\left(1-\frac{B_{b a r}}{B_{p i}} \sin ^{2} \theta_{i n j}\right)} \text {. }
$$

or the energy to pass the axicell midplane

$$
E_{i n j, 2}=\frac{\phi_{p i}}{\left(1-\frac{B_{m i d}}{B_{p i}} \sin ^{2} \theta_{i n j}\right)} \text {, }
$$


where

$B_{b a r}=$ peak mirror field in axicell,
$B_{m i d}=$ midplane plasma field in axicell,
$B_{p i}=$ plasma field at punp beam location,
$\theta_{i n j}=$ injection angle of pump beam,
$\phi_{p i}=$ potential difference $(>0)$ between axicell midplane
$\phi_{p b}=\begin{aligned} & \text { and pump beam location, and } \\ & \text { axicell mirror. }\end{aligned}$

2.3.3 Alternative Pumping Methods

\subsubsection{Introduction}

Because the neutral beam power in the transition pump contributes the largest amount of recirculating electric power in MARS, we are now in the process of designing a practical means of inducing sufficient controlled amounts of radial diffusion in the transition regions to eliminate or reduce the 140-MN (trapped) neutral beam requirement. We are working on a promising adaptation of Baldwin's original drift-pump scheme ${ }^{4}$ to the double-ellipse type of transitions that have been incorporated in the MFTF-B and MARS axicell plug configurations shown in Figs. 2.3-5(a) and (b). This idea is currently being tested in the LLNL "alpha-beta" drift code, which uses EFFI-generated magnetic fields and various model electric fields as functions of field $B(z)$ and flux $\alpha=8 r^{2} / 2$ (see Ref. 5). These calculations will calibrate estimates, derived here, of the radial diffusion coefficient as a function of the AC field perturbation amplitude and frequency, as well as the conditions for driver frequency bandwidth necessary to ensure that the perturbation-induced kicks are random and not superadiabatic. These calculations are made for MARS parameters such that the equilibrium radial electric fields do not by themselves cause stochastic behavior of passing ions. Assuming suitable resolution of these issues, we can then specify the key engineering requirements for $A C$ drift pumping and assess the practicality of testing this scheme in TwX-U and MFTF-B. If the engineering design appears to be reasonably credible, this scheme would then qualify for adoption as the MARS baseline pumping method. 


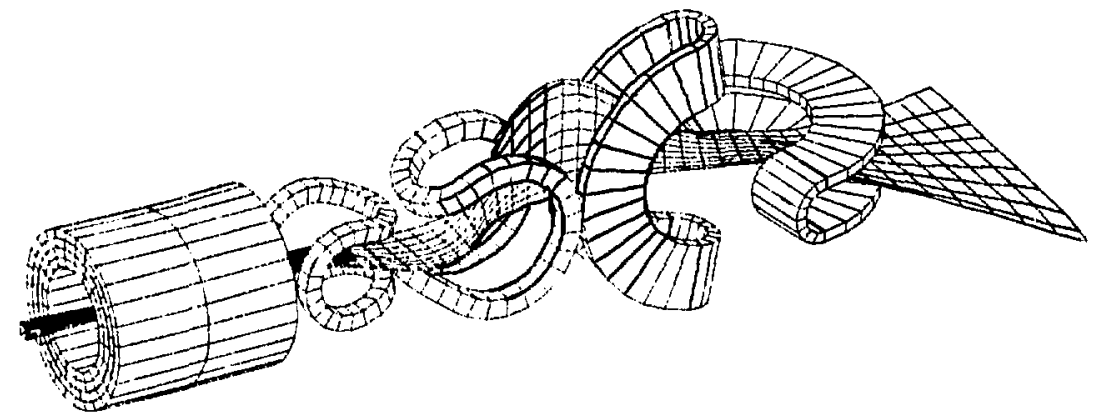

(a)

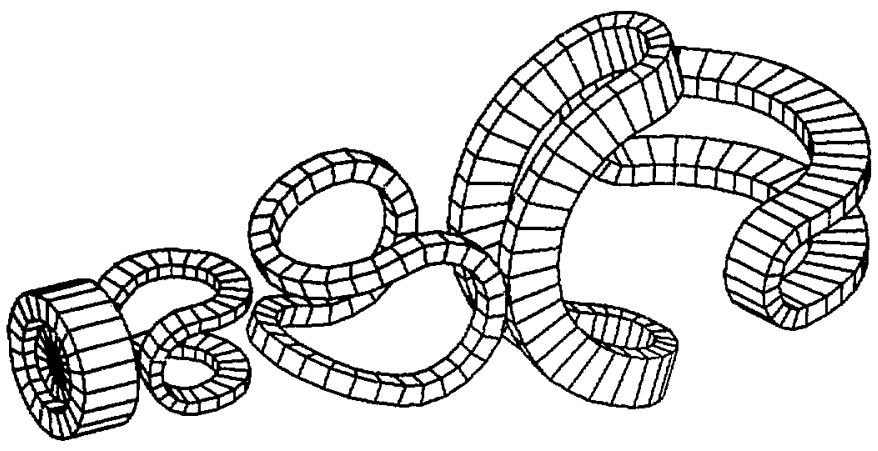

(b)

Fig. 2.3-5. (a) MARS end plug magnets (double ellipse transition with one-quarter flux bundle); (b) MFTF-B end plug magnets. 
Figures 2.3-6(a-d) show axial profiles of field B, potential $\phi$, geodesic curvature 10, and normal curyature, respectively, for one plug of the MARS reactor, corresponding to the magnet set shown in Fig. 2.3-5(a). This driftpumping scheme exploits the unique design feature of double ellipse transitions, namely, that the radial drifts of both passing and trapped ions are nearly canceled when they pass through two large, but opposite-sign, geodesic curvature $(1-0)$ peaks associated with the double transition fans [see Fig. 2.3-6(c)]. Each geodesic curvature spike causes the deuterons that pass through with characteristic parallel energy $\left(\delta \phi+T_{1 c} \approx 140 \mathrm{keV}\right)$ to drift normal to the flux surface by approximately

$$
\frac{1}{2} \frac{\delta \alpha}{\alpha}=\frac{\delta r}{r} \times \frac{M_{1} v_{1}}{2 q B r^{2}} \int 10 \mathrm{ds}=0.28 \text { (one spike), }
$$

with each charge in flux or equivalent radius mapped to the central cell corresponding to the sign of each 10 peak. Because these 10 curvatures have an azimuthal cos $(2 \psi)$ amplitude variation, any drift in azimuth while an ion transits between the opposing 10 peaks tends to break the cancellation and triggers net radial steps for ions passing through. This is why large radialequilibrium electric fields must be avoided to have tolerable radial transport. 6 However, this is also why small, random, azimuthal kicks resulting from a stochastic AC perturbation field strategically located between the peaks would also cause greatly amplified radial step sizes compared with those arising directly from the $A C$ perturbations alone. This is the essential leverage that permits Baldwin's AC coll to operate in double ellipse transitions with much smaller coil currents than had been previously estimated. Equation (2.3-2) leads one to expect comparable step sizes and induced radial transport for both passing and trapped ions (with little or no selectivity, as was the case for charge-exchange pumping), as well as comparable diffusion rates for equivalent $Z / M_{f}$ impurities and thermal alphas (in contrast to charge-exchange pumping, wich cannot renove $Z>1$ ions in the MARS configuration). In Section 2.4.1, we discuss the requirements for achieving steady state by pumping alphas in the transitions, wich implies a relationship between relative concentrations of DT fuel ions and thermal alphas and their 

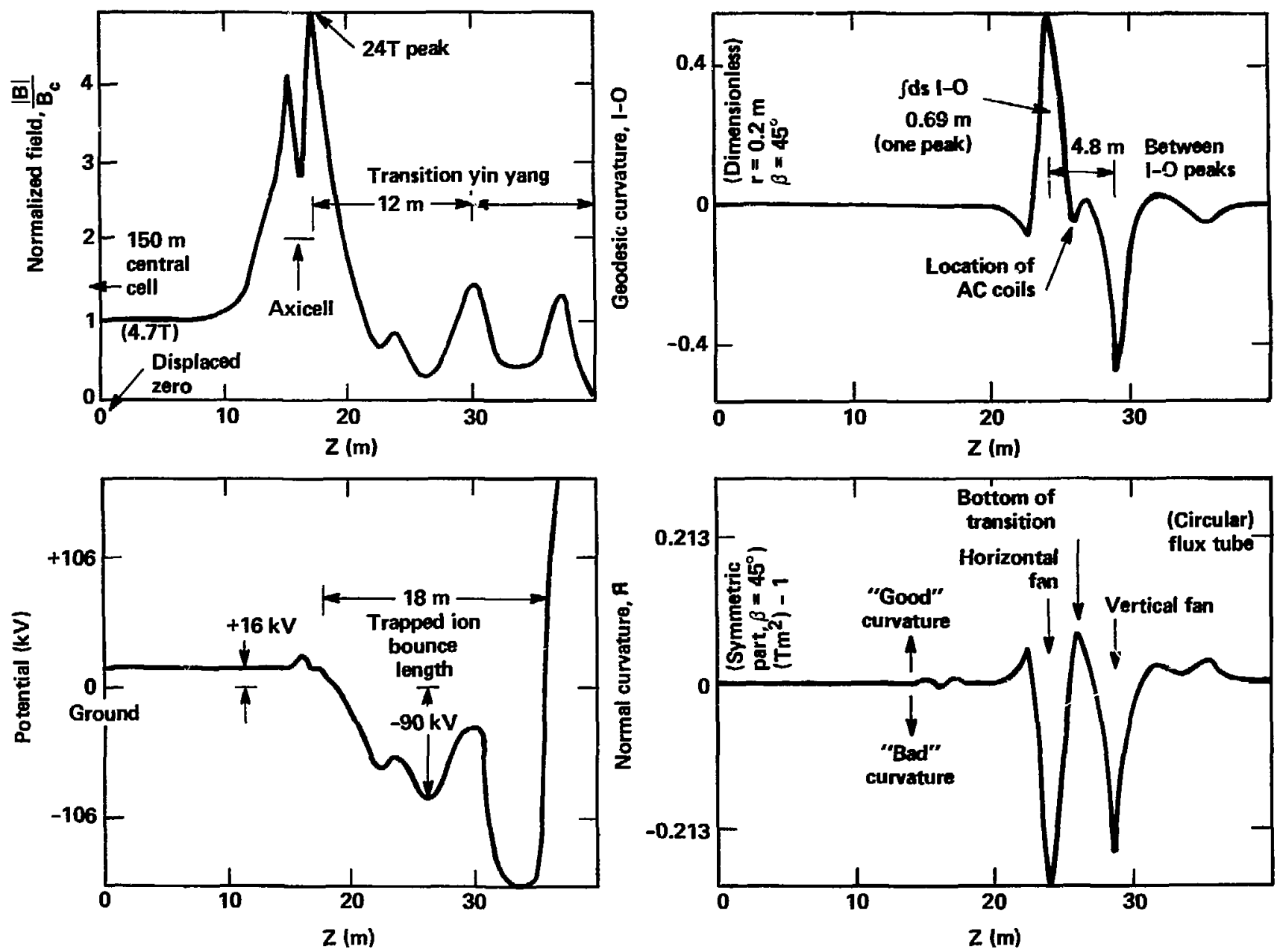

Fig. 2.3-6. Axial profiles of: (a) field $B$; (b) potential $\phi ;$ (c) geodesic curvature 
respective radial diffusion coefficients. The main uncertainty is the number, bandwidth, or sweep rate of the AC perturbation frequencies required to ensure random-phase successive encounters of an ion with the perturbation.

\subsubsection{Estimate of AC Perturbation Amplitudes}

We now estimate the $R C$ perturbation amplitude required to transport a trapped deuteron in a colliston-filling time, cump, of approximately $0.077 \mathrm{~s}$ for IARS parameters. The coll configuration shown in Fig. 2.3-7(a) is similar to the Nagoya Type III coil proposed by Baldwin, but with radial feed to avoid any induced $B_{2}$ components that might cause parasitic heating of ions and electrons by transit-time magnetic pumping. It has been shown that such heating is small, and that the induced $B_{\perp}$ (plasma) $\propto B_{1}$ (vacuum). ${ }^{7}$ Assuming that polarized electron currents short out $E_{z}$ at low frequencies, we expect to induce a $m=1$ transverse electric field with comparable $\tilde{E}_{r}$ and $\tilde{E}_{\text {components }}$ over the plasma cross section. We are interested here in the $\tilde{E}_{r}$ component that gives rise to an azimuthal $\tilde{E}_{r} \times B_{z}$ drift. To maximize the azimuthal kicks $\delta \beta$ in the vicinity between the peaks, we prefer the bucking coil configuration in Fig. 2.3-7(b), centered between the peaks in the 45 deg plane, with $l_{a} \simeq L_{H_{0}} \approx 4.8 \mathrm{~m}$, the distance between peaks [Fig. 2.3-6(c)]. We then pick a characteristic coil frequency corresponding to an ion transit time between the 10 peaks, of $\approx L_{L_{0}} / Y \approx 7 \times 10^{5} s^{-1}\left(f_{0} \approx 100 \mathrm{kHz}\right)$. The random azimuthal kick generated by the perturbation

$$
\delta \beta=\left(\frac{\tilde{E}_{r}}{B_{t} r_{t}}\right) \quad\left(\frac{L_{L}}{v_{t}}\right)
$$

is set equal to the azfmuthal kick required to transport the fon in a time $\tau_{\text {pump }}$

$$
\delta \beta=\left(\frac{\text { bounce }_{\text {pump }}}{{ }_{\text {pum }}}\right)^{1 / 2}\left(\frac{\delta r}{r}\right)^{-1} \begin{gathered}
\text { single } \\
\text { spike }
\end{gathered} .
$$

This yields the required perturbation electric field (MKS unfts) 
(a)
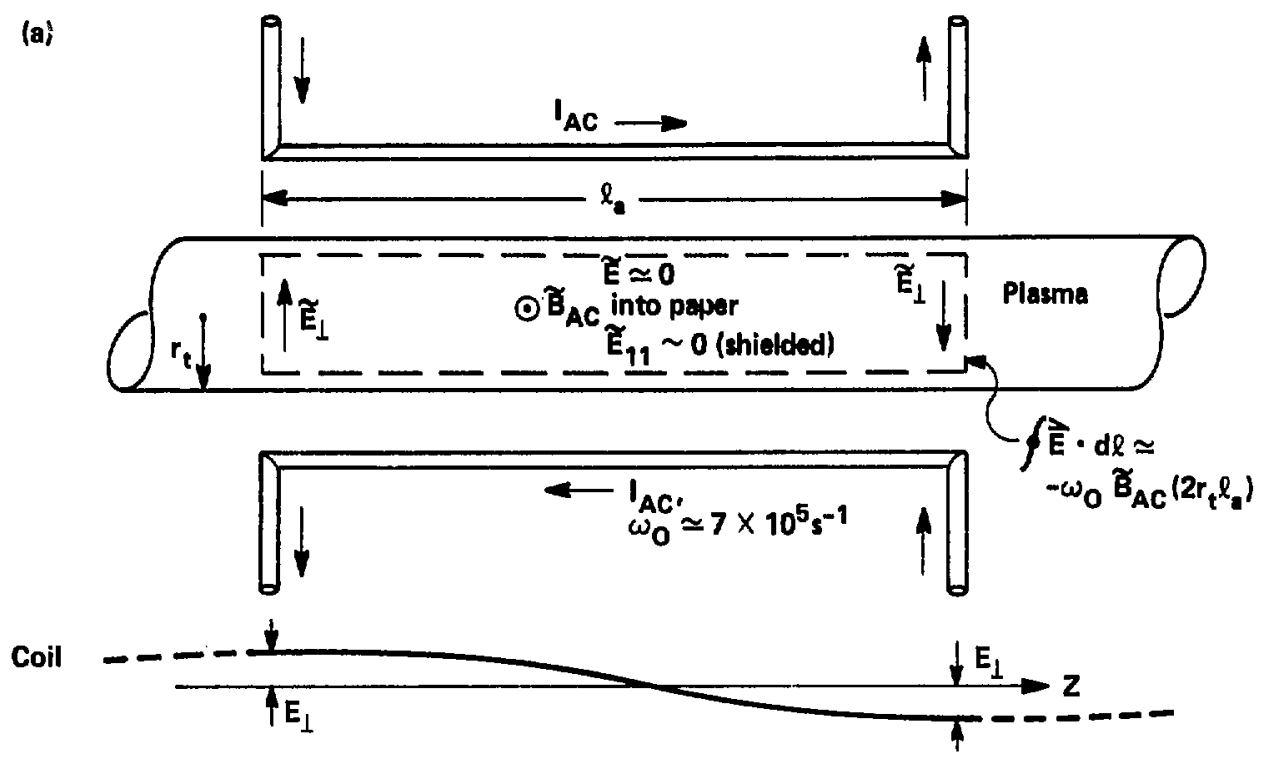

(b)

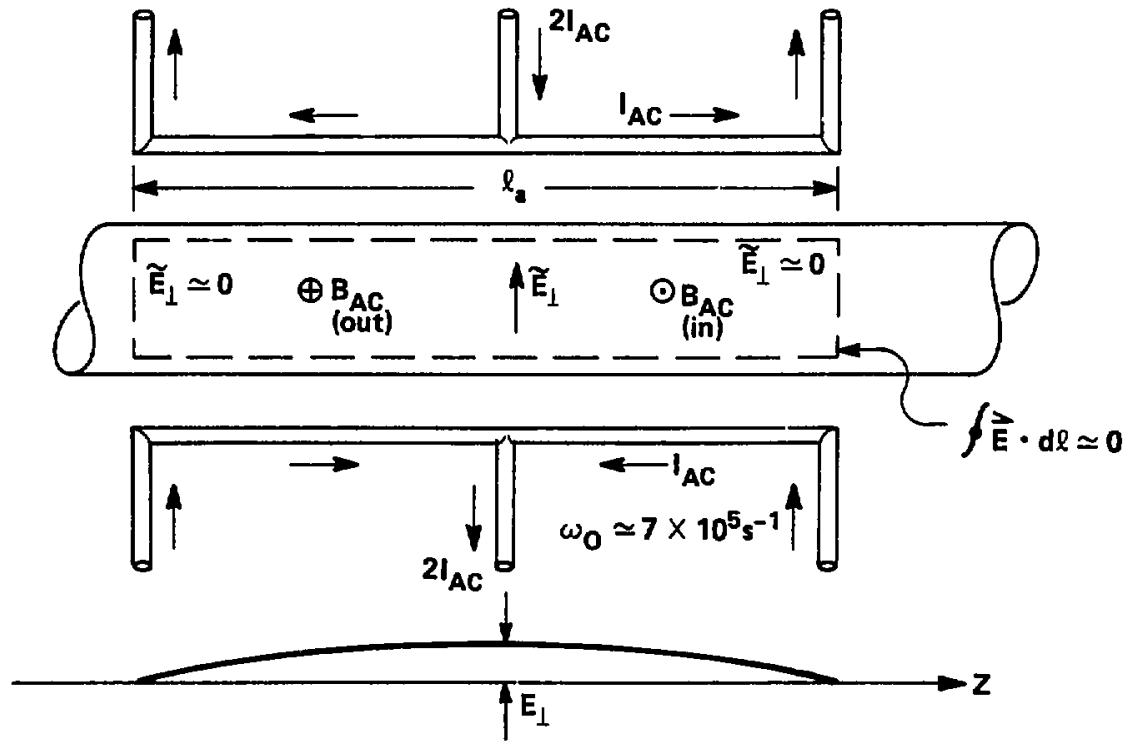

Fig. 2.3-7. Schematic of single (a) and double (b) AC perturbation coils, showing induced $\mathrm{E}$. 


$$
\tilde{E}_{r}=B_{t} r_{t}\left(\frac{v}{L_{L-0}}\right)\left(\frac{\tau_{\text {bounce }}}{\tau_{\text {pump }}}\right)^{1 / 2}\left(\frac{\delta r}{r}\right)^{-1} \begin{gathered}
\text { single } \\
\text { spike }
\end{gathered},
$$

where $B_{t}$ and $r_{t}$ are the field and radius, respectively, of the transition region between the peaks, t bounce is the bounce time of a trapped ion (time between successive encounters with the perturbation), and the $(\delta r / r)$ single spike is given by Eq. (2.3-2). Relating the induced $\tilde{B}$ and $\tilde{E}$ fields to Faraday's law gives

$$
\tilde{E}_{r}-\psi_{0} \tilde{B}_{\perp} L_{H_{0}} / 2 \text {. }
$$

Equating Eq. (2.3-3) and (2.3-4) then gives

$$
\frac{\tilde{B}_{1}}{B_{t}}=\left(\frac{2 r_{t}}{L_{j 0}}\right)\left(\frac{v_{1}}{\omega_{0} L_{10}}\right)\left(\frac{\tau_{\text {bounce }}}{\tau_{\text {pump }}}\right)^{1 / 2}\left(\frac{\delta r}{r}\right)^{-1} \begin{gathered}
\text { single } \\
\text { spike }
\end{gathered} .
$$

For MARS parameters, $r_{t}=0.64 \mathrm{~m}, L_{\mu_{0}}=4.8 \mathrm{~m}, v_{1}=3.6 \times 10^{6} \mathrm{~m} / \mathrm{s}, \tau_{\text {bounce }}=$ $18 / v_{1}=5.1 \times 10^{-6} \mathrm{~s}, \tau_{\text {pump }}=0.077 \mathrm{~s}$, and $\left.(\delta r / r)_{\text {single spike }}=0.28\right)$, Eq. (2.3-5) yields $\tilde{B}_{1} / B_{t}=0.0078$. In contrast, without the aid of the geodesic curvature, the perturbation field amplitude required to pump the ions with radial steps induced by the perturbation alone would have to be

$$
\begin{aligned}
& \frac{\tilde{\beta}}{B_{t}}=\frac{\text { bounce }}{l_{a}}\left(\frac{5 \Delta \omega}{t_{\text {pump }} w_{b}^{2}}\right)^{1 / 2} \\
& \approx \frac{18}{4.8}\left[\frac{5}{0.077\left(1.5 \times 10^{5} \mathrm{~s}^{-1}\right)}\right]^{1 / 2} \approx 0.078
\end{aligned}
$$

or about one order of magnitude greater. Here we assume a $100 \%$ bandwidth requirement, $\Delta w / w_{b}$, and an aztmuthal drift frequency, up, for the parameters shown in Fig. 2.3-6. In this case, negative transition eiectric fields cause $E \times B$ drifts opposite to the normal curvature drift that is dominated by the bad 10 curvature associated with the transition fans. In addition to the 
advantage of reduced perturbation amplitude, the use of $\mathrm{AC}$ drift pumps in double ellipse transitions may need less than $\Delta \omega \sim \omega$ bandwidth to ensure stochasticity in the induced kicks. One could guess that a frequency spread on the order of

$$
\frac{\Delta f}{f}<\frac{L_{H O}}{L_{\text {bounce }}}=25 \%
$$

might suffice, but this, of course, is one of the main subjects to be examined with the LLML apha-beta drift code.

REFERENCES FOR SECTION 2.3

1. A. H. Futch and L. L. LoDestro, Lawrence Livermore National Laboratory, Livermore, CA, UCRL-87249 (1982).

2. T. A. Cutler, L. D. Pearlstein, and M. E. Rensink, Lawrence Livermore Netional Laboratory, Livermore, CA, UCRL-52233 (1977).

3. G. A. Carlson et al., Tandem Mirror Reactor with Thermal Barriers, Lawrence Livermore NatTonal Laboratory, Lfvermore, CA, UCRL-52836 (1 979).

4. D. E. Baldwin, Drift Pumping of Thermal Barrlers, Invited Paper at the American Physical Soctety meeting, Division of PTasma Physics, New York, 1981 .

5. J. Byers, Lawrence Livermore National Laboratory, Livermore, CA, private comunication (1982).

6. D. E. Baldwin and B. G. Logan, Editors, Physics Basis for an Axicell Design for the End Plugs of MFTF-B, Lawrence Livermore National Taboratory, Livermore, CA, UCID-19359, Appendix $H$, page 207, by R. $H$. Cohen and J.M. Gilmore.

7. W. Cummins, Lawrence Livermore National Laboratory, Livermore, CA, private communication (1982). 
To obtain good reactor performance (ignition), most of the 3.5-MeV alphas produced by DT fusion must be magnetically trapped in the central cell until they slow down to thermalized energies of about $40 \mathrm{keV}$. After they slow down, they must be disposed of so they do not dilute the reacting plasma and waste the available plasma beta. Computations indicate that the tolerance of thermalized alphas and impurities is only about $10 \%$ of the central-cell plasma density.

For a reactor generating 3,500 of fusion power, the rate of alpha production is $200 \mathrm{k}$. Of these, the fraction magnetically trapped in the central cell is cos $\theta_{L C}$, about $94 \%$. The loss-cone angle $\theta_{L C}$ is determined by the magnetic field ratio

$$
\sin \theta_{\mathrm{L} C}=\left[\frac{{ }_{C}{ }_{C}\left(1-\beta_{C}\right)^{1 / 2}}{{ }^{B}{ }_{\max }}\right]^{1 / 2}=0.33 .
$$

If an alpha scatters into the loss cone at an energy larger than $2 \phi_{c}=$ $292 \mathrm{keV}$, it may be lost. Fokkcr-Planck computations indicate about 25\% additional alpha losses from coulomb scattering. The rate of production of thermalized alphas is therefore $200 \mathrm{~A} \times 70 \%=140 \mathrm{~A}$.

Thus, the allowable lifetime of a thermalized alpha is

$$
\tau_{\alpha}<\frac{0.1 \% n_{c i} \pi_{c}^{2} L_{c}^{q}}{140 \AA}=3.5 \mathrm{~s} .
$$

Thermalized alphas will be very well contained electrostatically because of their double charge and low energy compared with the confining potential $\phi_{c}$. The only removal technique appears to be some type of radial transport, which should not unduly dispose of efther the fast alphas or the reacting DT ions. We have chosen drift pumping of alphas and impurities passing through and trapped in the transition cell as our baseline model for ash removal.

\subsubsection{Transition Region Drift Pumping}

Under certain conditions, radial transport in the transition cell is more effective than radial transport in the central cell because of the large geodesic curvature in the transition, wheh induces transverse grad-B drifts. 
Using two different drift codes, we have computed radial drifts up to a maximum of several centimeters with each bounce, depending on conditions. If these radial drifts are fully stochastic, this leads to a large rate of radial diffusion. Some control over the diffusion rate is possible by controlling the stochasticity of the motion.

The fraction of central-cell ions if the region of velocity space that allows them to pass through the transition cell is

$$
\begin{aligned}
F=\left[1-\cos \theta_{\mathrm{LC}}\right] \exp \left(-Z \Delta \phi_{\mathrm{pc}} / T_{\mathrm{ic}}\right) & =3.85 \% \text { ior } 35-\mathrm{keV} \text { DT ions, } \\
\text { or } & =1.9 \% \text { for } 35-\mathrm{keV} \text { alphas. }
\end{aligned}
$$

As a result of coulomb scattering, each thermalized central-cell ion visits this region of velocity space several times during its trapped lifetime. If the radial diffusion coefficient is appropriate, the lons will be transported radially at the desired rate.

On the other hand, fast alphas (above $1 \mathrm{MeV}$ ) have small-pitch-angle coulomb collisions and do not enter this region of velocity space, except for the 30\% loss mentioned above. of this $30 \%$, only $6 \%$ are alphas at the full energy of $3.5 \mathrm{MeV}$ and born with pitch angles smaller than $19 \mathrm{deg}$. Under MARS conditions, coulomb scattering is significant only for alphas of energy below 1 MeV.

Although it is not essential, we are hoping to discover a set of conditions under which the radial transport of thermalized alphas and impurities is faster than the radial transport of OT ions. It is sufficient for power balance that these transport coefficients merely be equal, such that about 10 DT ions are pumped out with each alpha. There is some hope that alphas can be pumped selectively compared with or ions by taking advantage of differences in the stochasticity caused by differences in the azimuthal drift speeds.

\subsubsection{Stochasticity}

The azimuthal drift $\Delta \psi$ of a passing ion will be several radians per bounce, depending on radial electric fields and other conditions. The direction and magnitude of the radial displacement $\Delta r=\int v_{r} d t$ will depend on the azimuthal angle \& roughly in proportion to $\sin 2 \psi$. If the azimuthal drift is 
very slow, the motion will be periodic, and no net radial transport will occur. On the other hand, if the azimuthal drift is fast enough, the motion will be stochastic. In this case, the radial displacement per bounce $\Delta r$ will be a random variable and cause radial diffusion. The criterion for collisionless stochasticity is

$$
\Delta r \frac{\partial}{\partial r}(\Delta \psi)>\frac{1}{m},
$$

where: $m=2$ for the quadrupole field of the transition. 1

Several factors will make it necessary to control the radial plasma potential gradient and thereby control tiie $E \times B$ drift speed. In theory, it is possible to adjust the plasma potential gradient so that the motion of the alphas is stochastic and the motion of the OT ions is mostly periodic. The following two facts make this possible:

- The azimuthal grad-B drift due to the non-uniform plasma $\beta$ is in the direction opposite to the direction of the azimuthal $E \times B$ drift. Consequently, it is theoretically possible to adjust the drift speeds to partially cancel each other.

- The grad-B drift speed is proportional to $W_{1} / Z$, while the $E \times B$ drift speed is the same for all particles. Therefore, the condition that tends to cancel the total drift for OT ions will not cancel the drift for alphas at equal energy. This raises the possibility that the fraction $F_{2}$ of 3 lphas moving stochastically will be larger than the corresponding fraction of DT tons, depending on the distributions of plasma potential, plasma $\beta$, and $W_{\perp^{\circ}}$.

\subsubsection{Diffusion Coefficients}

We are working to discover a set of conditions under which the average diffuition coefficient for radial transport will be

$$
\left.\left\langle D_{\alpha}\right\rangle=r_{c}^{2} / \tau_{\alpha}\right\rangle 460 \mathrm{~cm}^{2} / \mathrm{s} \text { for thermalized alphas }
$$

but 


$$
=\left\langle D_{D T}\right\rangle=r_{C}^{2} / \tau_{D T}\left\langle 1600 \mathrm{~cm}^{2} / \mathrm{s} \text { for } D T\right. \text { ions. }
$$

The ?^al diffusion coefficient $D=\Delta r^{2} / \tau$ is a function of several

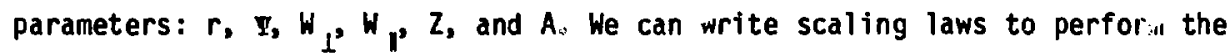
averaging so that

$$
\Delta r=\int v_{\text {grad-B }} d t a \frac{v_{\text {grad }-B}}{v_{1}} \alpha \frac{\frac{W}{Z}}{\left(\frac{W}{A}\right)^{1 / 2}}=W^{1 / 2} \frac{A^{2 / 2}}{Z} \text {. }
$$

Also, $\Delta r \alpha r \cos 2 \Psi$ if the long-thin approximation is valid.

The time $\tau$ is the interval between stochastic bounces. Ints is related to the bounce period $\tau_{b}$, but is longer for two reasons: (1) Only a fraction $\left(F_{1}\right)$--defined by Eq. (2.4-1)--of the central-cell ions is in the passing region of velocity space. (2) Only a fraction $\left(F_{2}\right)$ satisfies Eq. (2.4-2), and therefore, undergoes stochastic bounces, i.e., $\tau=\tau_{b}, \bar{F}_{1} F_{2}$,

where

$$
\tau_{\mathrm{b}}={\frac{\mathrm{L}_{\mathrm{C}}}{\mathrm{v}_{\mathrm{I}}}} \propto \frac{\mathrm{A}}{\mathrm{W}}^{1 / 2} .
$$

By combining Eqs. (2.4-3) and (2.4-4), we have a scaling law

$$
D \propto W^{3 / 2} A^{1 / 2} r^{2} \cos 2 \psi \frac{F_{1} F_{2}}{Z^{2}} \text {. }
$$

We are searching for a condition such that $D_{\alpha} / D_{D T}>460 / 1600$. From Eq. (2.4-5) we find that this requires that

$$
\left(\frac{\alpha}{W_{D T}}\right) \quad\left(\frac{2 \alpha}{F_{2 D T}}\right) \gtrsim 1.83 \text {. }
$$

Here we have cancelled the factor $r_{1}^{2} \cos 2 \psi$, which is the same for all species, and have used the appropriate masses $A$ and charges $Z$ for alphas and DT ions. The factor $F_{1}$ is given by Eq. $(2.4-1)$ for alphas and DT ions. 
Equation (2.4-6) shows that we have two ways of achieving this requirement:

- We take advantage of the fact that some of the radial diffusion occurs as the alpha is slowing down. At energies below $2 \phi_{C}$ $\approx 300 \mathrm{keV}$, some of tie alphas scatter into the passing velocity space where radial transport occurs. Also, all alphas in the transition have an energy larger than the DT energy by the amount of transition potential drop $\phi_{t}=100 \mathrm{kV}$. Therefore, on the average $W_{\alpha}>W_{D T} \cdot$

- The factor $F_{2}$ quantifies the effect of stochasticity. As mentioned in Section 2.4.1.1, there are ways of enhancing the stochasticity of alphas while reducing the stochasticity of $D T$ ions.

From the above considerations, we feel assured that the required ratio of diffusion coefficients is attainable. Now we: must evaluate the magnitude of the diffusion rate.

\subsubsection{Diffusion Equation}

To compute quantitatively the diffusion requirements, we must solve the diffusion equation for the case in which the diffusion coefficient is a function of $r$. Under the long-thin approximation, $\Delta r=k r$ and $D_{t r a n s}=\Delta r^{2} / \tau$ $=k^{2} r^{2} / \tau$, where $k$ is a constant of proportionality that will be determined by drift computations. Here, $D_{\text {trans }}$ is the diffusion coefficient due to radial transport in the transition cell and $D_{c}$ lass is the classical diffusion coefficient. Near the z-axis, $D_{\text {trans }}$ becomes very small and it is necessary to consider other types of diffusion as well: $D_{\text {total }}=D_{\text {trans }}+D_{c l a s s}$.

The radial diffusion flux vector $\underline{\Gamma}$ is given by $\underline{\Gamma}=-D_{\text {total }} \mathrm{\nabla n}$ ions $/ \mathrm{cm}^{2}-\mathrm{s}$, and the required rate of particle injection per unit voiume is

$$
J=-\nabla \cdot \underline{\Gamma}=-\frac{1}{r} \frac{\partial}{\partial r}(r \Gamma) \quad \text { ions } / \mathrm{cm}^{3}-\mathrm{s}
$$

Equation (2.4-7) can be integrated two times, with the assumption that $\mathrm{J}$ is independent of $r$. This assumption is required only to attain an analytic 
solution of the diffusion equation. Actually, $J$ will depend on $r$ for both DT ions and alphas. The solution is the following, subject to the boundary conditions dn/dr $=0$ at $r=0$ and $n=0$ at $r=r_{c}$ :

$$
n(r)=\frac{J \tau}{4 k^{2}} \ln \left[\frac{\frac{k^{2} r_{c}^{2}}{\tau}+D_{c l a s s}}{\frac{k^{2} r^{2}}{\tau}+D_{c l a s s}}\right] .
$$

The containment time for efther alphas or DT fons is

$$
\begin{aligned}
\tau_{\text {con }}=n_{c} / J & >1 \mathrm{~s} \text { for DT lons } \\
& <3.5 \mathrm{~s} \text { for alphas. }
\end{aligned}
$$

Equations (2.4-8) and (2.4-9) can be combined to give the radial density profile $n(r) / n_{c}$, which is shown in Eq. (2.4-1C; and Fig. 2.4-1. The shape (;) this profile is realistic for alphas, for wich tiansverse diffusion is dominant. However, the shape of the DT density proftie will be more flattopped than Fig. 2.4-1 indicates because end losses will probably be more important than transverse diffusion, so that

$$
\frac{n(r)}{n_{c}}=\frac{\tau}{4 k^{2} \tau_{c o n}} \ln \left[\frac{k^{2} r_{c}^{2}}{\frac{\tau}{\tau}+D_{c l a s s}}\right] \text {. }
$$

Whe can solve Eq. (2.4-10) for $\tau_{\text {con }}$ with $r=0$ and $\tau=\tau_{b} / F_{1} F_{2}$, thereby ascertaining the requirements for the stochasticity parameter $F_{2}$. This is shown in Fig. 2.4-2:

$$
\tau_{\text {con }}=\frac{\tau_{b}}{4 k^{2} F_{1} F_{2}} \ln \left[1+\frac{k^{2} r_{c}^{2}}{\tau D_{c l a s s}}\right] \text {. }
$$




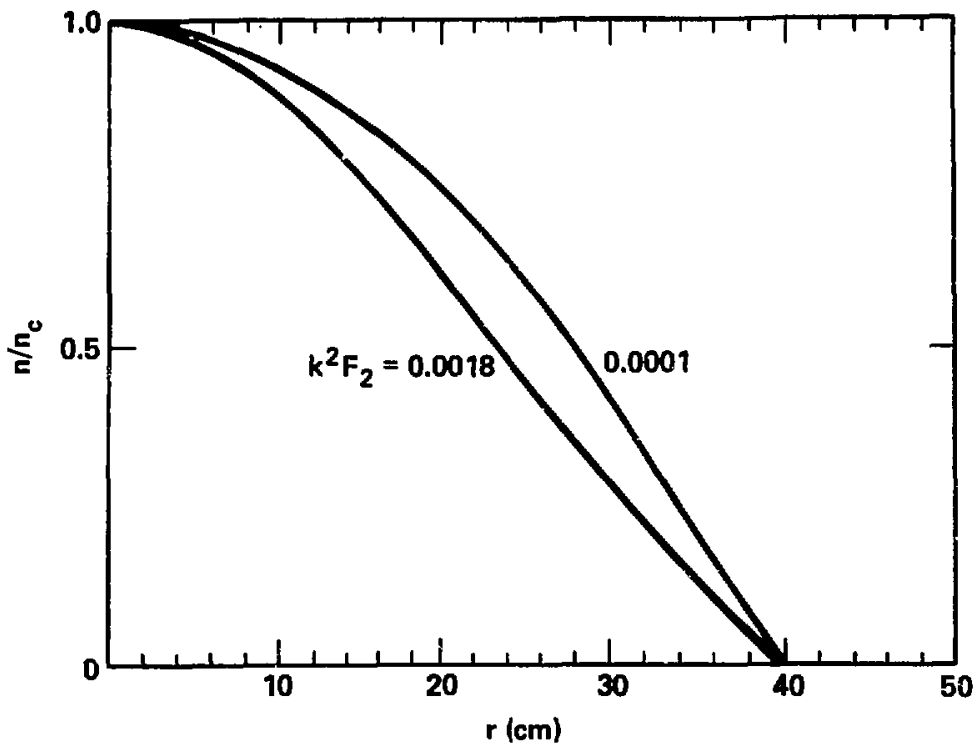

Fig. 2,4-1. Radial density profiles $n(r) / n_{c}$ under the following assumptions: (1) radial transport is the dominant loss mechanism for thermalized alphas and/or D-T ions; (2) under the long-thin approximation, $\Delta r_{r m s}=\mathrm{Kr}$; (3) particle injection is in dependent of the radius for convenierice of an analytic solution to the diffusion equation. 


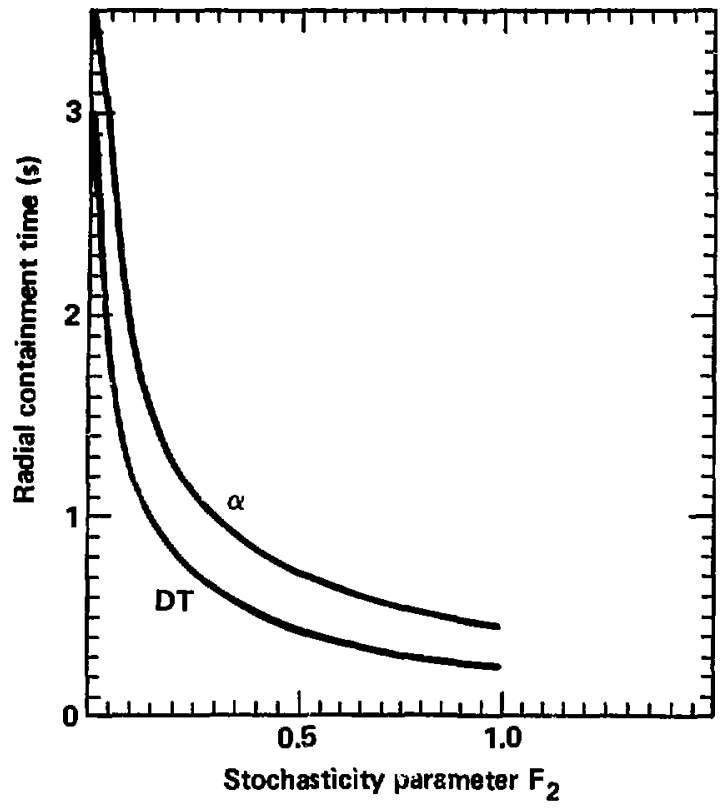

Fig. 2.4-2. Dependence of radial confinement time $J$ of $D-T$ ions and alphas on the stochasticity parameter $F_{2}$. 
In Figs. 2.4-1 and 2.4-2 we use the following values, which are realistic for passing alphas under MARS conditions:

\begin{tabular}{|c|c|c|}
\hline & DT Ions & Alphas \\
\hline$\tau_{\text {con }}$ & $>1 \mathrm{~s}$ & $<3.5 \mathrm{~s}$ \\
\hline Dciass & $100 \mathrm{~cm}^{2} / \mathrm{s}$ & $100 \mathrm{~cm}^{2} / \mathrm{s}$ \\
\hline$F_{1}$ & 0.0385 & 0.0191 \\
\hline$r_{c}$ & $40 \mathrm{~cm}$ & $40 \mathrm{~cm}$ \\
\hline$k=\Delta r / r$ & 0.1 & 0.1 \\
\hline
\end{tabular}

(preliminary result from drift computations)

\$

$10^{-4} \mathrm{~s} \quad 10^{-4} \mathrm{~s}$

Under these conditions, the stochasticity parameter $F_{2}$ must be no greater than 0.15 for DT ions and not less than 0.02 for disposal of alpha ash. It appears that these conditions will be easy to achieve for alpha ash disposal. Some study will be required to avoid excessive radial transport of DT ions. The parameters $k$ and $F_{2}$ are not precisely known at this time.

\subsubsection{Drift Computations}

We are conducting a series of drift computations to attain a better understanding of the dependence of the transverse drifts $\Delta r$ and $\Delta \phi$ on magnetic

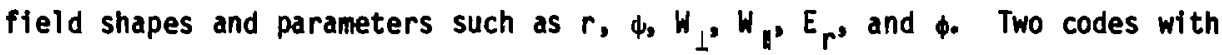
guiding-center drift approximations are being used. In both cases ions are started in the center ceil with a pitch angle small enough to penetrate the 24-T axicell field and are reflected by the electrostatic potential at point $A$ after passing through the transition cell. After one reflection, the ion is stopped and another is launched. The transverse displacements $\Delta r$ and $\Delta \phi$ are measured at the plane of launching the lons in the center cell. Both codes agree on the order of magnitude of $\Delta r$ and $\Delta \phi$ as functions of $r$. 
GCDRIFT is an updated and more accurate version of the old MAFCO-II drift code. ${ }^{2}$ Its main limitation is that three-dimensional electric fields are very crudely modeled in the present version.

A newer code ${ }^{3}$ includes self-consistent electric fields, but it is based on the long-thin approximation, which may not be valid far fron the z-axis in the transition cell. We are now determining the range of validity of this approximation.

\subsubsection{Drift Pumping of Trapped Ions in the Transition}

After thermalization, alphas tend to diffuse from the central cell, first into the region of velocity space occupied by passing lons and then into the region occupied by trapped ions. It probably will be necessary to pump both regions of velocity space. The theory outlined in Section 2.4 .1 is relevant to both populations, with several differences.

Because of the shorter bounce period $\tau_{b}$ for the trapped fons, and therefore smaller azimuthal drift per bounce $\Delta \psi$, it is more difficult to satisfy the stochasticity condition in Eq. (2.4-2) for trapped ions than for passing ions. On the other hand, if the motion is stochastic, the shorter bounce period has a tendency to increase the diffusion coefficient $D$.

\subsubsection{Alternative Ash Removal Methods}

Several other methods of ash removal have been investigated. These can be divided into three categories, which are listed below in order of importance :

1. Enhanced drift pumping of trapped alphas in the transition by $A C$ magnetic field perturbations.

2. Radial transport due to magnetic perturbations at the ends of the central cell.

3. Charge-exchange pumping of alphas passing through the barrier. 4 


\subsubsection{Enhanced Drift Pumping of Trapped Alphas by AC Perturbations}

Pumping of trapped ions by $A C$ perturbations at frequencies near the drift frequency will work in principle with alphas and impurities as well as with DT ions. This technique for DT ions is described in Section 2.3.3. If trapped alphas and impurities are effectively pumped from the transition, the central cell alphas and impurities will diffuse into the transition rapidly enough to maintain the alpha density in the central cell at the required $10 \% .{ }^{4}$ However, the grad-B drift speeds, proportional to $1 / 2$, will be different for the various ion species. Consequently, it may be necessary to broaden the frequency range at which the $A C$ perturbations are excited.

\subsubsection{Radtal Transport Due to Magnetic Perturbations at the Ends of the Central Cell}

The theory of this technique is almost identical to the theory discussed in Section 2.4.1. It would be possible to install some type of magnetic perturbations at the end of the central cell to enhance the radial diffusion of central cell ions with turning points near the perturbations. Several versions of perturbation coils have been investigated for this purpose, as shown in Fig. 2.4-3. We investigated both relatively small resistive coils close to the plasma that are exposed to neutron bombardment and large superconducting coils outside the neutron shield.

To avoid premature pumping of fast alphas trapped in the central cell, we have considered installing the perturbations at the point where the local mirror ratio $R$ is about 2.5. Therefore, only about $23 \%$ of the fast alphas would be affected by the perturbations, and the fast alpha loss would not be severe. In the notation in Section 2.4.1, the pitch angle of ions affected by the perturbation would be a maximum of $F_{1}=1-\cos \theta \times \sin ^{-1} R^{-1 / 2}=39.2 \mathrm{deg}$ and the fraction of alphas affected would be $F_{1}=1-\cos \theta=23 \%$.

However, this requires a rather large value of $B_{2}(6.75 \mathrm{~T})$ and a proportionately high perturbation field. He found radial displacements $\Delta r$ of only a few millimeters per bounce. For these reasons we concluded that enhanced radial diffusion by perturbations in the central cell is less effective than enhanced diffusion of ions passing through the transition, in spite of the larger value of $F_{1}$. Other possible liabilities, not yet investigated, 

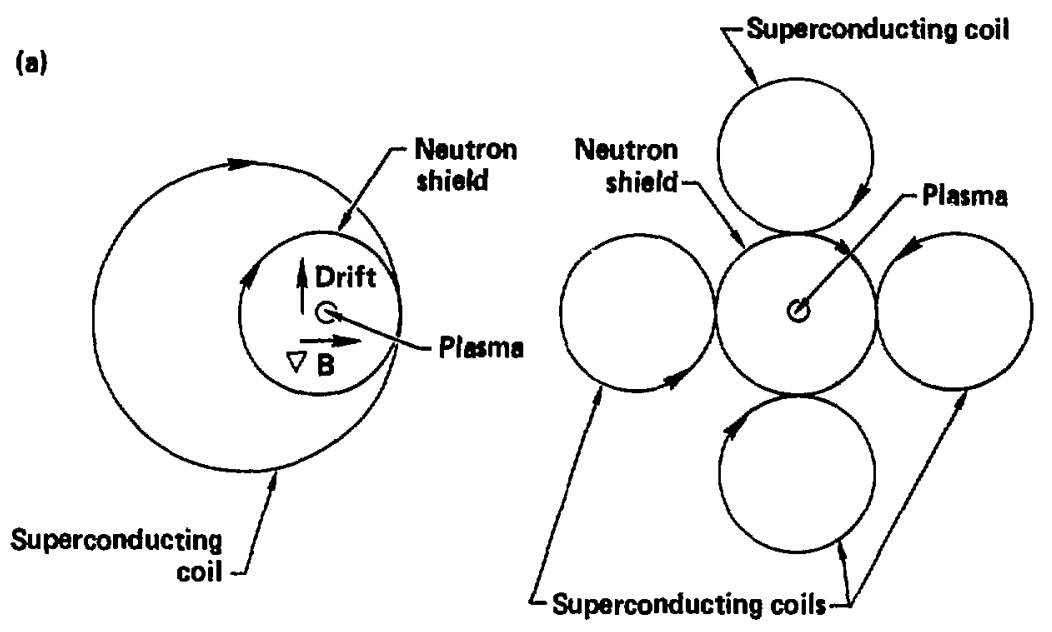

Copper coils inside the neutron shield (radius $\mathbf{0 . 6} \mathbf{~ m}$ )

(b)
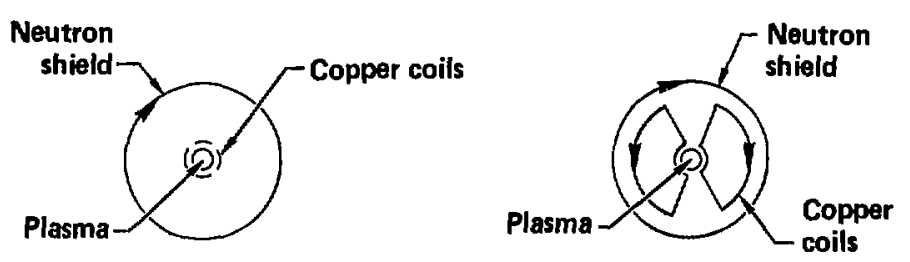

Fig. 2.4-3. Four examples of perturbation coils for pumping thermalized alphas: (a) two versions of large-diameter superconducting coils outside the neutron shield; (b) two versions of resistive coils close to the plasma within the neutron shield. 
are the effects of these perturbations on plasma stability. We consider it worthwhile to preserve this technique as a standby. The order of magnitude of $\Delta r$ was sufficient for alpha disposal in the examples investigated.

\subsubsection{Charge-Exchange Pumping of Alphas in the Transition}

We have shown that under certain conditions it is possible to transfer an electron from a neutral beam atom to a $\mathrm{He}^{++}$in the transition such that the restilting $\mathrm{He}^{+}$is able to escape over the potential $\mathrm{h}+11 .{ }^{4}$ The electron capture must occur after the He ${ }^{++}$has been accelerated by $2 \Delta \phi$, which must be sufficient to permit the escape of the $\mathrm{He}^{+}$.

The conditions required for this do not apply to the MARS baseline model. One of the conditions is that the charge-exchange neutral beam must be it rected outward, toward the end of the tandem mirror. This does not apply to MARS, but is compatible with certain other tandem mirror designs. Once again, such an alternative should be retained as a backup.

\section{REFERENCES FOR SECTION 2.4}

I. R. H. Cohen, "Stochastic Motion of Particles in Mirror Machines," in Proc. of Intrinsic Stochasticity in Plasmas, (Editions de Physique, Orsay, France, 1979) pp. 181-191.

2. W. A. Perkins and D. Fuss, MAFC()-II--a Code for Calculating a GuidingCenter Trajectory of a PartTcle in Magnetic and Electric Fields, Lawrence Livermore kational Laboratory, Livermore, CA, UCRL-50438 (1968).

3. J. A. Byers, "Drift Orbits in the THA and MFTF-B Tandem Mirrors," Muclear Fustion 22, 49 (1982).

4. G. W. Hamilton and B. G. Logan, "Charge-Exchange Pumping of Thermalized Alpha Particles in Steady-State Tandem-Mirror Reactors," Comments on Plasma Physics and Controlled Fusion 6, 139 (1981). 


\subsubsection{Ion Distribution Function}

To calibrate models of ion confinement and pumping power in the anchor, it is necessary to calculate the ion distribution function. These calculations of the are carried out with the bounce-averaged Fokker-Planck code, 1 which assumes symmetric potential and magnetic field profiles. Although the anchor field is symetric, the potential is not. Also, the sloshing ions are injected only on the inboard side of the anchor, at $A^{\prime}$, which adds another asymmetry to the problem. Nonetheless, we can model each half of the anchor, taking the appropriate potentials from the point model plasma code, as described in Section 2.10. The potentials and magnetic fields that we used in this Fokker-Planck study differ slightly from the parameters given in Section 2.10, and are collected in Table 2.5-1.

The several classes of ions that must be considered can be explained with the aid of Fig. 2.5-1, which shows the $v_{1}-v_{1}$ space of ions at the anchor midplane. Four regions of velocity space are apparent: (1) the part that confines the high-energy sloshing lons and low-energy trapped lons in the anchor; (2) a region containing fons that span the space from the transition section to the anchor; (3) a region containing ions that span the space from the axicell through the transition section to the anchor; and (4) a region containing lons that span all regions from the center cell to the anchor. The latter ions are generally known as passing ions. We see from Fig. 2.5-1 that these passing ions cannot scatter directly into the anchor via small-angle collisions, but must first be trapped in the axicell or transition spanning class. Figure 2.5-1 also shows that the ion distribution function in the anchor will be most strongly influenced by the distribution function in the adjacent transition-anchor spanning region. However, the distribution function in the latter region is essentially determined by collisions in the transition region, since the density and length there are so much larger. Thus, we can impose as a boundary condition on the anchor ions the previously computed transition ion distribution function (see Section 2.3.1). One such function mapped to the anchor inboard mirror is show in Fig. 2.5-2. It is evident that the distribution function can be represented by a product of Gaussians in $v_{n}$ and $v_{\perp}$, which is done in the calculations for the anchor ions. 


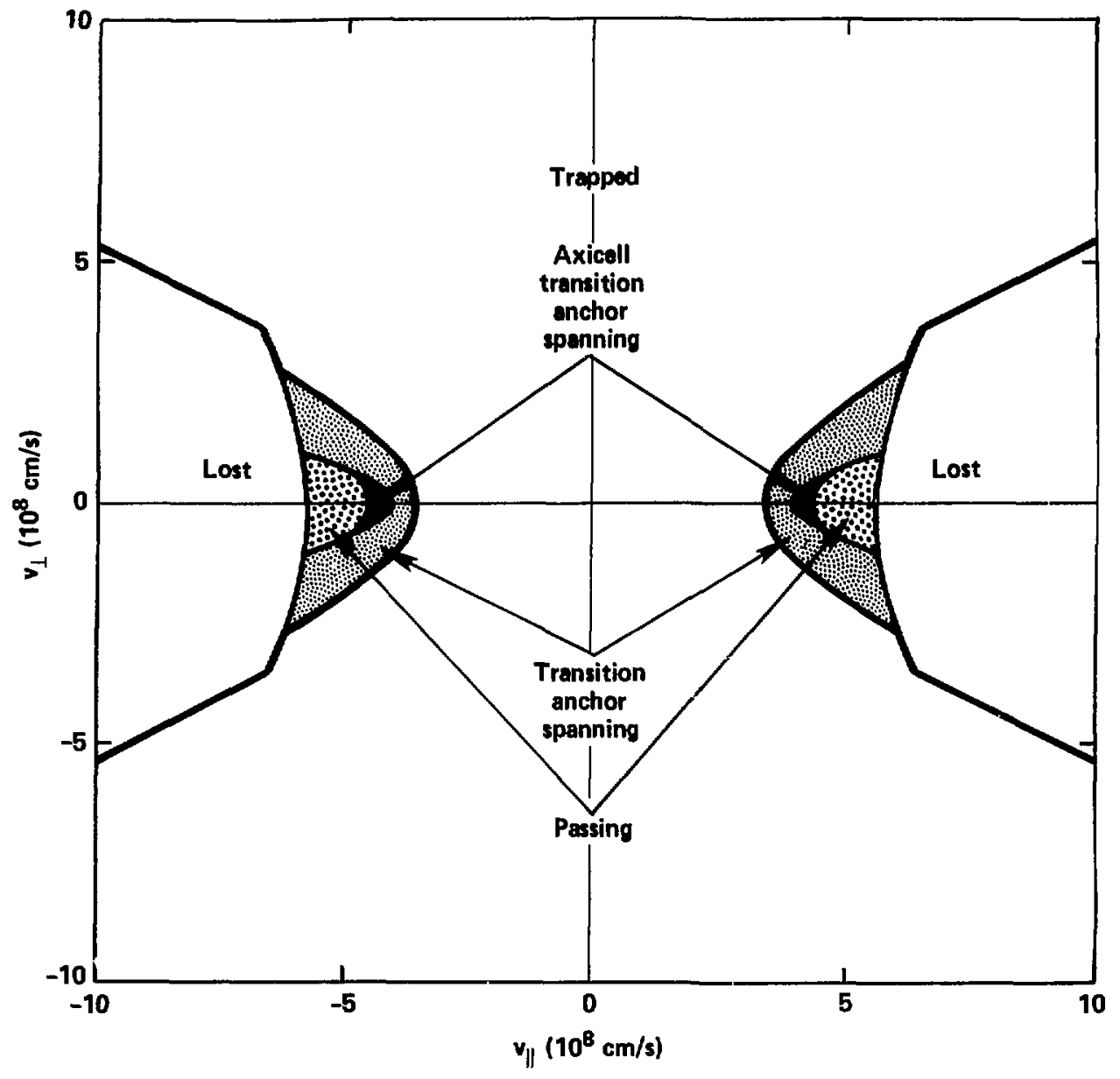

Fig. 2.5-1. Anchor midplane. 


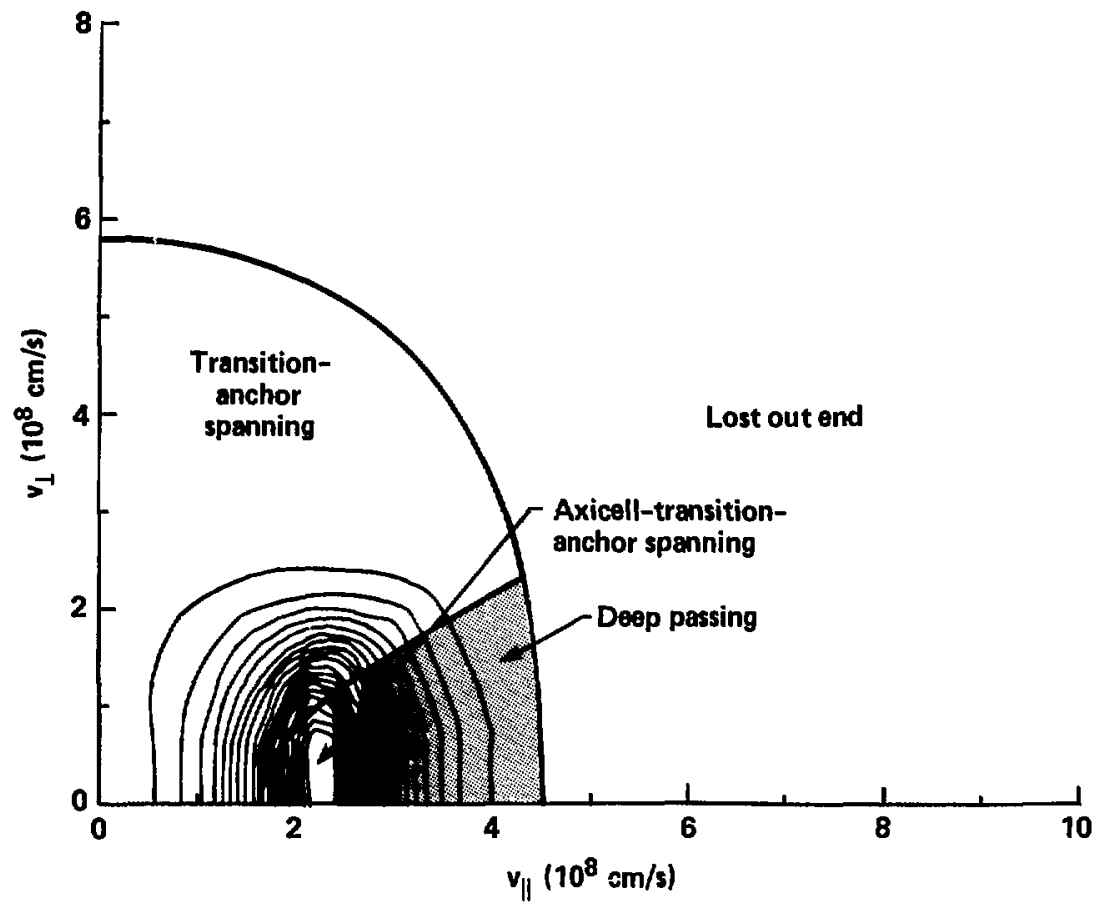

Fig. 2.5-2. $\quad V_{1}-V_{\text {space }}$ at anchor midplane showing boundaries between ions lost out end, central-cell-axicell-transition-anchor spanning (or passing) ions, axicel1-transition-anchor spanning ions, transition-anchor spanning ions, and anchor trapped ions. 
The solution for the anchor ions depends critically oil the sloshing-ion injection current and on the pumping strength and location. To pick out the desired solution, it is necessary to impose constraints on the steady-state result. The constraints imposed correspond to one set of parameters for a MARS case: midplane deep passing uensity $=3.57 \times 10^{12} \mathrm{~cm}^{-3}$, midplane sloshing ion + trapped and spanning ion density $=1.34 \times 10^{13} \mathrm{~cm}^{-3}$ and the ratio of peak (at $A$ ) to midplane density of 3.29. Injection and pumping rates are then adjusted until these values are attained.

Results obtained in this way are collected in Table 2.5-2. Cases 4 and 5 include no transition spanning fons; we see that the pumping and bear c'urrents are about half of the corresponding values with transition-spanning ions (cases 1 and 2). Evidently, the presence of these ions enhances the collision rates, thus causing higher loss rates of ions. Pumping extending over $3 \mathrm{~m}$ (case 1) is also clearly superior to pumping over $8 \mathrm{~m}$ (case 3) since the latter removes more of the sloshing ions before they have scattered to large pitch angles, thus requiring a higher sloshing ion current. The tota? absorbed beam power with case 1 or 2 is about 1.7 times the estimate with the point-model calculations (Section 2.10), a discrepancy that will be corrected in future calculations.

Anchor midplane distribution functions for case 1, as anclyzed with inboard and outboard parameters, are shown in Figs. 2.5-3(a and $b$ ). The density distribution as a function of $z$ for case 1 is shown in Fig. 2.5-4. Of importance for analysis of miscostability to the drift cyclotron loss-cone (DCLC) and two-stream modes are the perpendicular and parallel distribution functions defined by

$$
f_{\perp}\left(v_{1}\right)=\int f\left(v_{1}, v_{1}\right) d v_{1} \quad f_{1}\left(v_{1}\right)=2 \pi \int v_{1} f\left(v_{1}, v_{1}\right) d v_{1}
$$

[f $f_{\perp}$ and $f_{1}$ are shown in Figs. 2.5-5(a and $b$ ) at the anchor midplane]. One criterion for microstability is that $f_{\perp}$ al ways decrease monotonically. We see in Fig. 2.5-5(a) that this criterion is not met for the anchor icn distribution function at the midplane. However, the warm plasma in the distribution function can stabilize the drift cyclotron modes if the temperature of the warm component is large enough, even if the distribution function is not monotonic. We have not tested this distribution for stability, but we can apply 

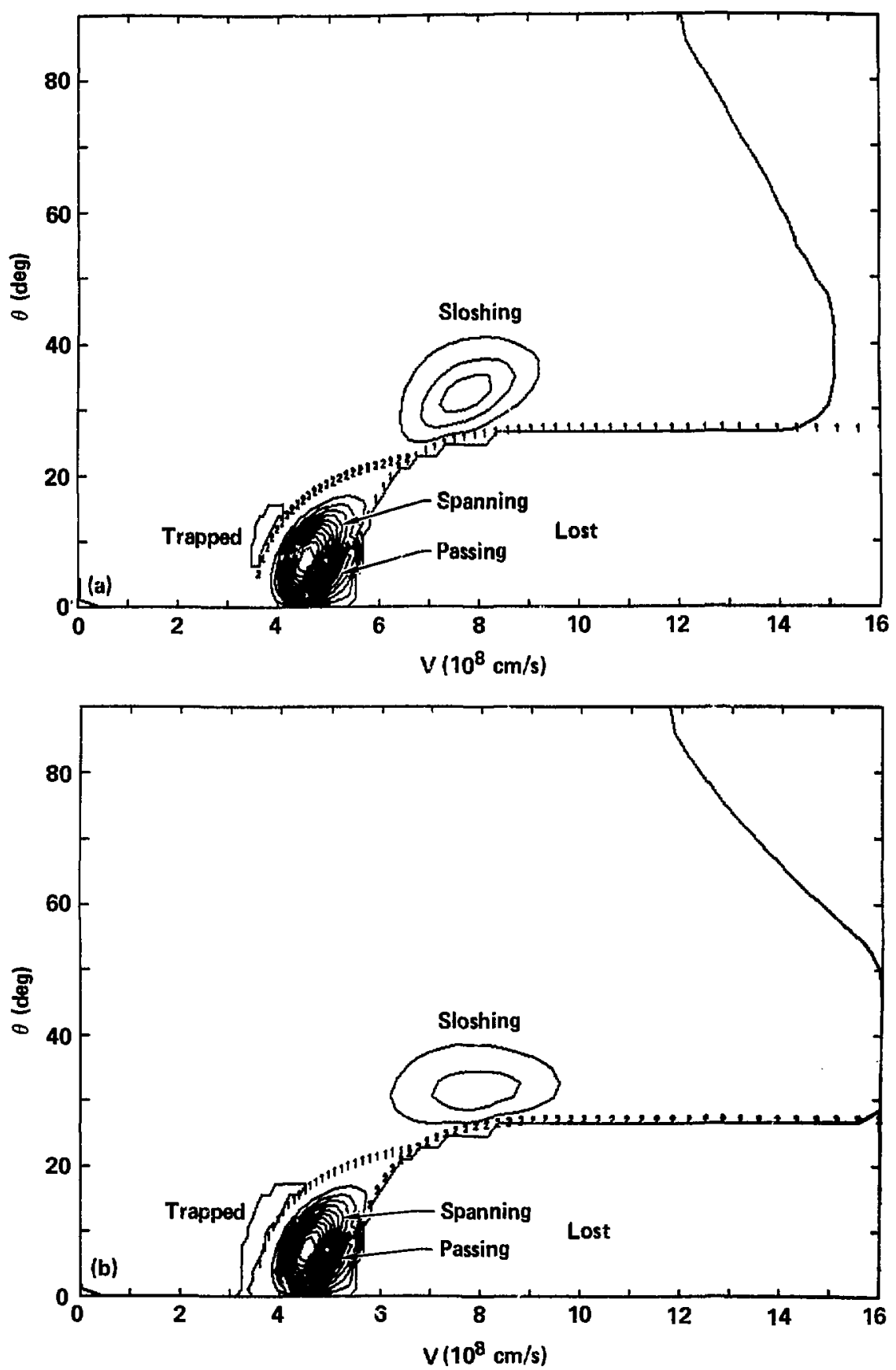

Fig. 2.5-3. Distribution function $2 \pi v^{2} \operatorname{sim} \theta f(v, \theta)$ at anchor midplane for (a) outer and (b) inner half anchor. 


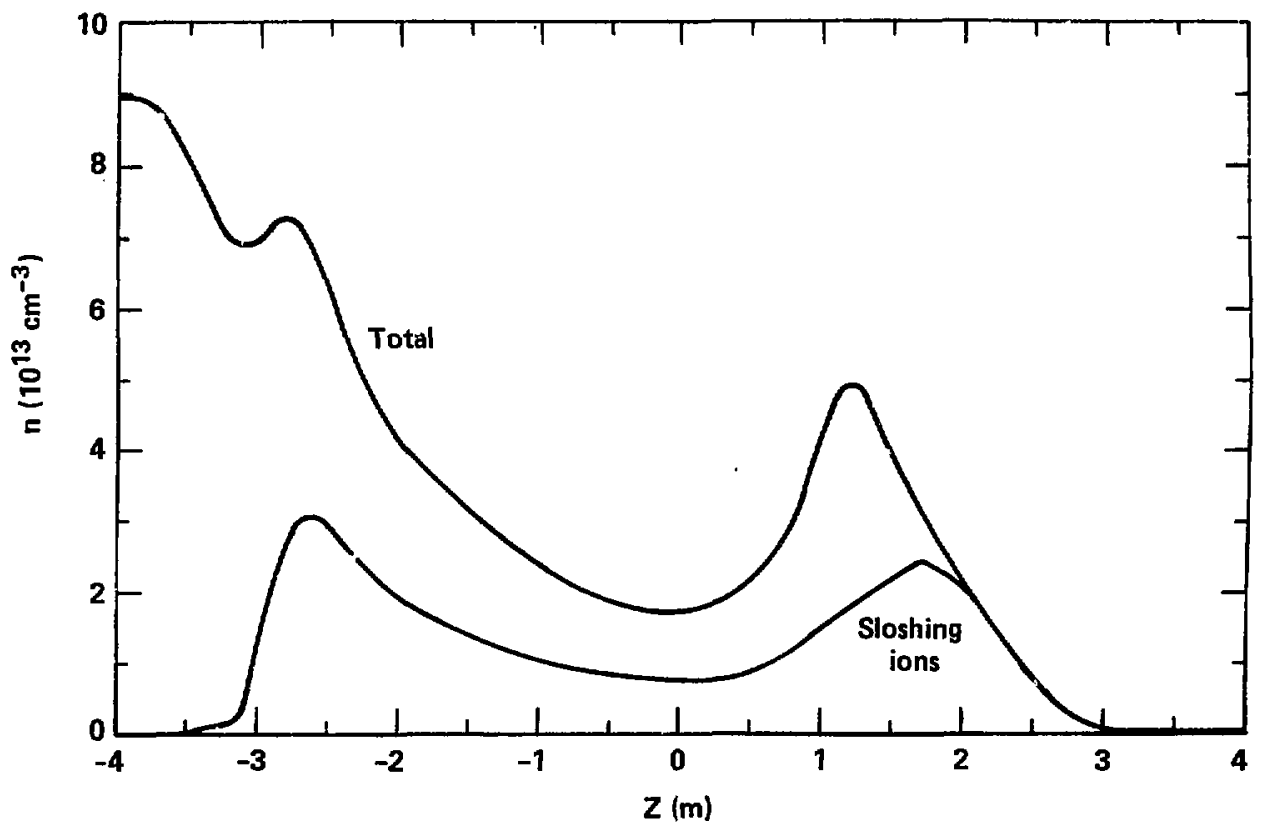

Fig. 2.5-4. Density distribution in anchor. 

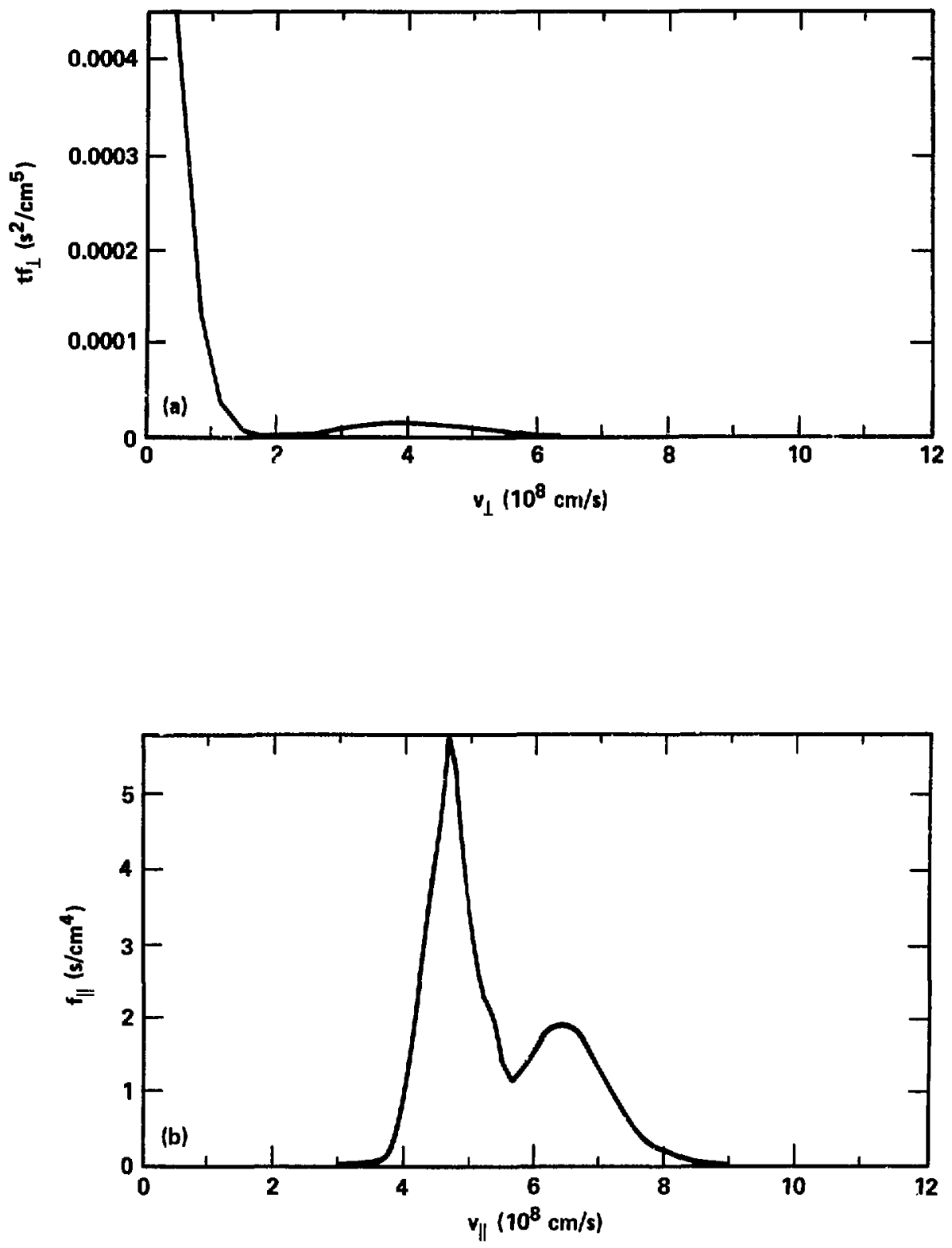

Fig. 2.5-5. (a) Perpendicular $f_{1}$ and (b) parallel $f_{! !}$distribution function at anchor midplane. 
Table 2.5-1. Potentials and magnetic fields used in Fokker-Planck study of anchors.

Location

Center cell

Axicell mirror

Axicell midplane

Axicell inirror

Pump beam location

Transition section

Inner mirror

Injection point $A^{\prime}$ (475-keV ions)

Anchor midplane

Point A

Outer mirror (kv) $\beta$-reduced field (T)

198

2.574

$198 \quad 21.000$

$232 \quad 7.668$

$182 \quad 24.000$

$170 \quad 11.8$

$106 \quad 1.981$

$152 \quad 6.000$

$144 \quad 4.027$

$21 \quad 1.283$

333

2.359

0.00

6.000 
Table 2.5-2 Beam and pumping currents in anchor. A cubic density prafile is assumed. Anchor cross sectional area at midplane $=1.223 \times 10^{4} \mathrm{~cm}^{2}$.

\begin{tabular}{|c|c|c|c|c|c|c|}
\hline Case & Pumping & Passing ions & $\begin{array}{c}\text { Anchor-transition } \\
\text { spanning ions }\end{array}$ & $\begin{array}{c}\text { Requi red } \\
\text { pump current } \\
\text { (A/end) }\end{array}$ & $\begin{array}{c}\begin{array}{c}\text { Total required } \\
\text { absorbed beam current } \\
\text { (A/end) }\end{array} \\
\end{array}$ & $\begin{array}{l}\text { Absorbed beam } \\
\text { power anchor } \\
\text { (MW/end) }\end{array}$ \\
\hline 1 & -150 to $150 \mathrm{~cm}$ & Maxwellian & $\begin{array}{l}\text { From transition } \\
\text { calculation }\end{array}$ & 6.5 & $\begin{array}{l}12.1 \text { (outboard) } \\
12.0 \text { (inboard) }\end{array}$ & 5.7 \\
\hline 2 & -50 to $+5 n \mathrm{~cm}$ & Maxwellian & $\begin{array}{l}\text { From transition } \\
\text { calculation }\end{array}$ & 6.2 & 11.8 & 5.6 \\
\hline 3 & $\begin{array}{l}-400 \text { to }+400 \mathrm{~cm} \\
\text { (uniform) }\end{array}$ & Maxwellian & $\begin{array}{l}\text { From transition } \\
\text { calculation }\end{array}$ & 8.1 & 14.7 & 7.0 \\
\hline 4 & $\begin{array}{l}-150 \text { to }+150 \mathrm{~cm} \\
\text { (uniform) }\end{array}$ & None & None & 2.1 & 4.1 & 1.9 \\
\hline 5 & $\begin{array}{l}-150 \text { to }+150 \mathrm{~cm} \\
\text { (uniform) }\end{array}$ & Maxwellian & None & 3.7 & 7.4 & 3.5 \\
\hline
\end{tabular}


the results for the anchor of MFTF-B (Ref. 2, Fig. F-2), which gives stability boundaries as functions of the ratios of warm-to-hot densities and temperatures. From examination of Fig. 2.5-5(a), we find that there is more than enough warm plasma but its temperature is too low, thus leading to an instability known as the two-component mode. However, we have observed that unstable distributions such as this will relax to a marginally stable state, i.e., enough $r f$-induced diffusion will take place to heat the warm ions sufficiently to achieve stability. Some additional beam current must be supplied to make up for the concomitant loss of hot ions.

\subsubsection{Adiabaticity}

To produce a sloshing ion distribution with a peak-to-midplane density ratio of about 3:1, which is sufficient for anchor microstability, a yin-yang with about a 3:1 vacuum mirror ratio is required. A yin-yang using a NbTi superconductor and LHe I cooling cannot have a mirror field above about $6 \mathrm{~T}$, thus giving a 2-T midplane vacuum field. This is the lowest field in the MARS magnetic configuration that will confine high energy particles.

There is a lower limit for the anchor midplane field below which the adiabatic invariant $\mu=E_{\perp} / B$ of a given plasma particle is no longer a constant of the motion. Under these circumstances, the particle undergoes random changes in $\mu$ that have the same effect as enhanced pitch angle scattering. This enhanced scattering increases the losses of the particle in question. Such departure from adiabatic behavior must be avoided.

The more energetic and massive a particle is, the more restrictive the lower limit on the anchor midplane field will be. This is why the sloshing ions have been singled out for careful study. The injection energy at the inboard turning point of the sloshing ions is $475 \mathrm{keV}$. At the anchor midplane, after falling through a potential difference of about $130 \mathrm{keV}$, these ions have an energy of slightly more than $600 \mathrm{keV}$. In the plasma performance code, the anchor midplane field is computed to keep these ions adiabatic, given an injection energy, plasma beta, anchor length, and yin-yang field shape and mirror field. The formula for adiabaticity has been derived from relevant analytic and numerical studies of ion adiabaticity. ${ }^{3}$ This formula for the sloshing-ion injection energy may be written 


$$
E_{i n j, A^{\prime}}<3.1 \times 10^{-3} \frac{B_{b, v a c}^{3}}{B_{m p}}\left(1-\beta_{p}\right)^{3 / 2}\left(L_{A}^{2}\right)\left(\frac{2}{A}\right)-\left(\phi_{b^{-}}-\phi_{A}\right),
$$

where

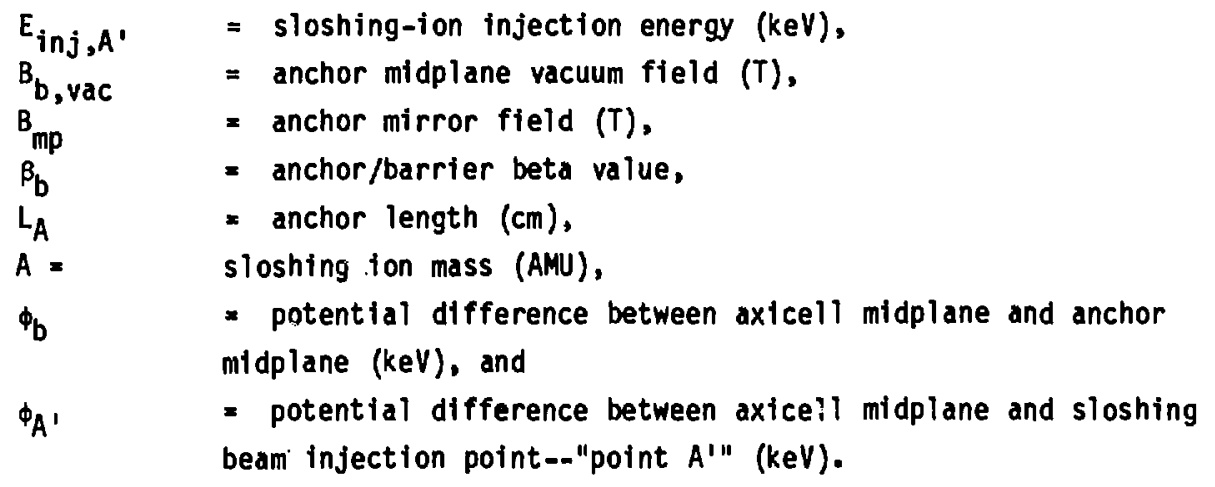

For a sloshing deuterium injection energy of $475 \mathrm{keV}$, an anchor beta of $65 \%$, a length of $7 \mathrm{~m}$, and a mirror field of $6 \mathrm{~T}$, the required anchor midplane field is $2.26 \mathrm{~T}$. This is the value specified for MARS.

\subsubsection{Beam Trapping}

The efficiency of trapping the sloshing beam in the plasma is the lowest of all beams in MARS, about $45 \%$. The trapping efficiency $f_{T}$ is computed by the formula

$$
f_{T}=1-\exp \left[-\int_{0}^{d_{d}}{ }^{f f f} d r \frac{n(r)}{v_{\text {beam }}}\left(\langle o v\rangle_{\text {ion }}+\langle o v\rangle_{C x}\right)\right] \text {, }
$$

where

$$
\begin{array}{ll}
d_{\text {eff }} & =\text { the effective path length the beam traverses }(\mathrm{cm}), \\
n(r) & =\text { the (radially dependent) plasma density }\left(\mathrm{cm}^{-3}\right), \\
v_{\text {beam }} & =\text { the beam speed }(\mathrm{cm} / \mathrm{s}), \\
\langle o v\rangle_{\text {ion }} & =\text { the reaction rate parameter for ionization, both from the } \\
& \text { ions and electrons }(\mathrm{cm} / \mathrm{s}), \text { and } \\
\langle o v\rangle_{c x} & =\text { the reaction rate parameter for charge exchange }\left(\mathrm{cm}^{3} / \mathrm{s}\right) .
\end{array}
$$


The reaction rate parameter shuld be the result of averaging ov over the sloshing distribution and the background distributions, neither of which can be strictly modeled as a beam distribution (delta function) or a Maxwellian. We do not know the detalled forms of the distribution, so we assume that an average between a beam and a mirror-confined distribution models the process to sufficient accuracy. When we perform this average and replace the radial average by the major radius of the elliptical plasma times the volume-averaged density at the injection point, the formula for $f_{T}$ becomes

$$
f_{T}=1-\exp \left[-1.85 \times 10^{-14}\left(\frac{\gamma}{Y+2}\right) n_{A^{\prime}} \cdot r_{A^{\prime}}\left(\frac{A}{E_{\text {inj }, A}}\right)^{0.91}\right],
$$

where

$$
\begin{array}{ll}
r= & \text { radial profile factor for..sity, } i, e,, n(r), \\
n_{A^{\prime}} & =\text { total density at injection point } A^{\prime}, n_{A^{\prime}}=n_{s l o s h, A^{\prime}} \\
& +g_{A^{\prime}} n_{\text {pass, }} A^{\prime}\left(\mathrm{cm}^{-3}\right), \\
r_{A^{\prime}} & =\text { major radius of ellipse at injection point mapped through } \\
& \text { magnetic flux conservation from central cell (cm), } \\
E_{\text {inj, }}= & \text { mass of sloshing ion (AMU), and }
\end{array}
$$

\section{REFERENCES FOR SECTION 2.5}

1. T.A. Cutler, L.D. Pearlstein, and ME. Rensink, Lawrence Livermore National Laboratory, Livermore, CA, UCRL-52233 (1977).

2. D.E. Baldwin and B.G. Logan, eds., Lawrence Livermore National Laboratory, Livermore, CA, UCID-1359 (1982).

3. R.H. Cohen, J.H. Foote, and G. Rowlands, Phys. Fluids 21,627 (1978) and erratum, Phys. Fluids 22, 1006 (1979). 
2.6 ECRU PHYSICS

2.6.1 General Considerations

The field in the anchor has been modelled using a paraxial yin-yang model with the following parameters:

- Vacuum mirror ratio

- Mirror-to-mirror distance

- Ellipticity

- Minimum field

$$
\begin{aligned}
R_{\text {vac }} & =2.7 ; \\
\ell_{p} & =8.0 \mathrm{~m} ; \\
B_{p} & =2.2 \mathrm{~T} .
\end{aligned}
$$

With these values, the other parameters in the model become:

- $\gamma=16.4$;

- $k_{p}=2.01 \times 10^{-3} \mathrm{~cm}^{-1}$;

- $k_{\mathrm{m}}=5.06 \times 10^{-3} \mathrm{~cm}^{-1}$.

A scale length of $20 \mathrm{~cm}$ was introduced to model the density decay in the radial direction.

The field is described by

$$
\begin{aligned}
& B_{x}=\left[-k_{p} f(z)-\frac{1}{2} \frac{\partial f}{\partial z}\right] x, \\
& B_{y}=\left[k_{p} f(z)-\frac{1}{2} \frac{\partial f}{\partial z}\right] y, \text { and } \\
& B_{z}=f(z),
\end{aligned}
$$

where

$$
f(z)=\frac{1}{2} B_{p}\left(1+\frac{y}{4}\right)\left[\frac{1}{1+k_{m}^{2}\left(z+\frac{1}{2} \ell_{p}\right)^{2}}+\frac{1}{1+k_{m}^{2}\left(z-\frac{1}{2} l_{p}\right)^{2}}\right] .
$$

$2-66$ 
The peak electron density was set at $n_{e, 0}=2.8 \times 10^{13} \mathrm{~cm}^{-3}$, corresponding to a plasma frequency of $f_{p}=47.5 \mathrm{GHz}$.

Such a field has on-axis values of:

- $2.2 \Gamma$ at $z=0$ (midplane of yin-yang);

- $2.32 \mathrm{~T}$ at $z= \pm 200 \mathrm{~cm}$ (point A);

- $6.0 \mathrm{~T}$ at $z= \pm 400 \mathrm{~cm}$ (yin -.yang boundary).

The midplane of the yin-yang (point B) coincldes with a minimum of the potential, while point $A$ is situated at the potential peak.

As a preliminary step, a ray-tracing and absorption calculation was performed at point $A$. The absorbing electrons have a temperature of $89 \mathrm{keV}$ and the beta-corrected field is $2.3 \mathrm{~T}$ at $\beta=0.5$. At these temperatures, the relativistic shift and broadening of the resonance is estimated to be $40 \%$ of the nominal resonance frequency, resulting in a launching fundamental frequency of about $40 \mathrm{GHz}$. Furthermore, the results of these preliminary calculations showed that, with this combination of parameters, absorption and penetration of the extraordinary mode will be fair. This can be axplained by the innite density effects on the absorption, which tend to considerably reduce the absorptivity close to the plasma frequency.

A similar study at point B--the midplane of the anchor--is not feasible because the mean energy of the electrons is $460 \mathrm{keV}$, and at this energy level no rellable absorption model is avallable, at least not for frequencies at or below the fundamental cyclotron frequency $(28 \mathrm{GHz}$ with beta correction and an estimated $40 \%$ reduction for relativistic shift).

\subsubsection{Relativistic ECRH Frequency Shifts}

The preliminary calculations lead us to believe that, in steady-state operation, a combination of parameters may be found that provides both acceptable penetration and absorption. The spatial distribution of the absorbed energy rematins a major problem, however, as excessive heating of the plasma surface may cause temperature profile inversion and possible MID instability. "Bum in" due to increased plasma transparency at relativistic energies may alleviate this problem. 
To achieve penetration to the plasma core, it is necessary to heat at a much lower frequency than the nominal resonance. This implies that the heated electrons are heavily Doppler (or relativistically) shifted, and this in turn can drastically alter the distribution function, possibly leading to microinstabilities.

\subsubsection{ECRH at the Second Hamonic}

To minimize losses and avoid the use of metallic parts and transparent windows as much as possible, a quasi-optical energy transfer system can be used to transport the wave energy from its source to the plasma (see Section 3.2.4). Lowering the applied frequency to $28 \mathrm{GHz}$ in the midplane of the anchor and $40 \mathrm{GHz}$ at the potential peak brings one dangerously close to the lower $11 \mathrm{mit}$ of the range where quasi-optical techniques can be successfully applied. We are investigating the use of second harmonic absorption as the principal heating mechanism. This implies the use of gyrotrons operating at higher frequencies that, however, are either avallable $(60 \mathrm{GHz})$ or are under development $(120 \mathrm{GHz})$. The main advantage in adopting these higher frequencies will be operation farther in the optical regime and the accompanying reduction of the launching system dimensions. The consequences for the power density in this system remain to be investigated. 
Until very recently, we had planned to fuel and heat the MARS axicell using 200-keV neutral beams. Because of the space requited for the magnet support structure and neutron shielding of the axicell superconductor, sufficient duct space for the neutral beams could not be maintained. We therefore decided to switch to ICRH of the axicell plasma. The fuel for the axicell is then the cold passing lons that are heated by the radio frequency (RF) fields and trapped in the mirrors.

A number of authors have already considered RF heating in tokamaks and mirrors. Stix ${ }^{1}$ and Kesner ${ }^{2}$ have analyzed the use of the MHD or fast wave for second-harmonic ICRH of the ions with a view toward two-component operation of tokamaks. Kesner has further looked at bulk heating of lons by the fundamental frequency in a single-cell mirror with potential application to the plugs of the Phaedrus tandem mirror. Experiments have been carried out on Phaedrus with fundamental ${ }^{3}$ and harmonic ${ }^{4}$ heating of the plugs, as well as fundamental heating of the center-cell plasma. 5

The harmonics of the ion cyclatron frequency preferentially heat higher energy ions, while the fundamental heats the bulk ions. This result becomes apparent using the approximate velocity-space diffusion coefficient which scales as $\left(\rho_{i} k_{1}\right)^{2 n-2}$, where $\rho_{1}$ is the ion Larmor radius, $k_{\perp}$ is the perpendicular wave number, and $n=1,2,3, \ldots$ is the number of the harmonic $(n=1$ is fundamental). Thus, the higher the harmonic, the higher the energy of the ions that will be heated. To effectively trap the passing lons, we could use heating by the fundamental. However, this could cause unwanted heating of the passing central-cell ions that are already adequately heated by the alpha particles. Thus, the initial plan is for heating at the second harmonic of tritium, or about $180 \mathrm{MHz}$ in the vacuum field and $136 \mathrm{MHz}$ in the plasma field. Tritium is chosen over deuterium because of its better confinement time against Couiomb scattering. If Fokker-Planck studies indicate insufficient trapping to feed the axicell, it may be necessary to add heating at the fundamental irequency. 
Several problem areas must be analyzed in connection with RF heating. These include antenna design, wave propagation, wave conversion (e.g., to ion Bernstein modes), absorption by electrons via transit-time effects and Landau damping, and the effect of the RF fields on the ion distribution function.

Previous theoretical studies of ICRH of fusion plasmas have used quasilinear theory to describe the effect of the RF field on the distribution function. ${ }^{1,2}$ This theory applies when the plasma is sufficiently collisional to destroy the correlations of particles with the RF fiald. The very high density of the axicell should render this approximation valid. We can then use quasi-linear diffusion coefficients in velocity space in a numerical code that solves the Fokker-Planck equation. ${ }^{6}$ Considerable work has already been done concerning the use of quasi-line:- approximations with this code to study ECRH. 7

\section{REFERENCES FOR SECTION 2.7}

1. T. H. Stix, Nucl. Fusion 15, 737 (1975).

2. J. Kesner, Muclear Fusion 18, 6 (1978).

3. D. Smith, Ph.D. Dissertation, University of Wisconsin (1980).

4. S. N. Golovato et al., Nuclear Fusion 22, 741 (1982).

5. B. D. McVey et al., MIT, PFC/JA-82-16 (1982).

6. T. A. Cutler, L. D. Pearlstein, and M. E. Rens ink, Lawrence Livermore National Laboratory, Livermore, CA, UCRL-52233 (1977).

7. B. W. Stallard, Y. Matsuda, and W. M. Nevins, Lawrence Livermore National Laboratory, Livermore, CA, UCRL-87674 (1982). 
The discussion of the physics aspects of MARS has so far been concerned with steady-state operating conditions. Obviously, we have to consider the problem of initiating the plasma and, by heating and fueling, bringing it to the desired operating condition. We have not yet fully evaluated the startup process, but some initial work on both the beginning and final phases of startup has been done.

\subsubsection{Initial Startup Phase.}

The startup siquence envisioned for MARS is similar to that planned for TMX-Ul and the axicell version of MFTF-B. ${ }^{2}$ Plasma stream guns in the end walls create a low-density $\left(n=10^{12} \mathrm{~cm}^{-3}\right.$ ) stream plasma in both anchors. Startup neutral beams are then injected into each anchor to provide a hot, mirror-confined anchor plasma ( $\approx \approx 1.6 \times 10^{12} \mathrm{~cm}^{-3}, E \approx 15 \mathrm{keV}$ ). The anchor plasma density is deliberately kept low at this phase so that the electrons can be heated with about $10 \mathrm{NO}$ O ECRH power. The required startup neutral beams are 20 to $25 \mathrm{keV}$ and $200 \mathrm{~A}$ at each end. The anchor plasma provides MHD stability for the mar ife and some potential for confinement of the central cell fons so that the central cell is microstable:

Microstability of the anchor plasma is inftially assured by the plasma stream. To maintain microstability when the stream guns are turned off, we first create a central cell plasma whose axial loss is sufficient to streamstabilize the anchors. This requires gas puffing and ICRH into the centrai cel1. Once the central cell loss is large enough to stream-stabilize the anchors, the plasma stream guns are turned off.

The central-cell plasma must be heated at low density to reduce the trapping rate in the thermal barriers before the thermal barriers can be established. This is because of the limit on the available barrier pumping current. The combination of reduced collisionality to establish the thermal barriers and sufficient loss-rate for siream-stabilizing the anchor provides an "operating window" for the central cell plasma. This window is illustrated in Fig. 2.8- 1 in the parameter space of central cell density and fon temperature for a given electron temperature. The shaded region defines the central- 


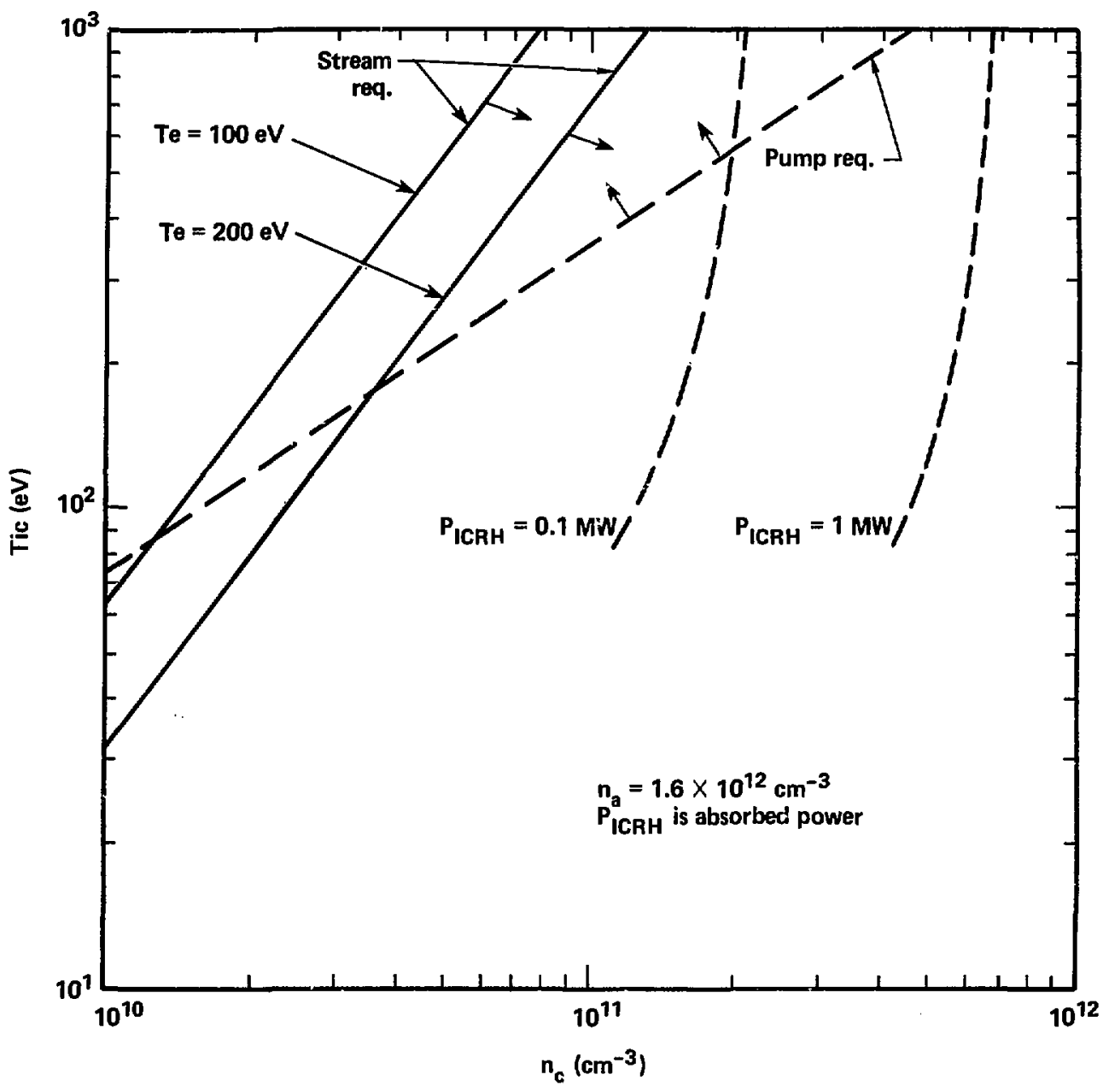

Fig. 2.8-1. Operating window in the central cell for creation of the thermal barrier. 
cell operating window before the thermal barriers can be established. Also shown are the lines of constant ICRH power required to stistain the centralcell plasma at that density and temperature against end loss.

Once the central-cell plasma is in the operating window, ECRH in the anchor can be turned on to heat the electrons. As the electron temperature rises, the anchor density will rise as a result of reduced ion drag. Once the anchor is at a sufficient density to trap the higher-energy sloshing ion beam, this beam can be turned on, the transition region pump beams can be started, and ECRH can be applied to begin thermal barrier operation. The axicell plasma is begun when required for reducing the trapping rate in the thermal barrier. The plasma now has all the elements of the desired configuration; the final step is to bring the plasma to the design operating point. This phise will likely require more ICRH power than Fig. 2.8-1 shows, although the required power has not yet been evaluated.

\subsubsection{Final Startup Phase}

In this subsection, we examine the requirements for startup of the MARS plasma after the thermal barriers have been established--the so-called final startup phase. At first, the axicell and barrier plasmas exist at their full beta values, but the central-cell plasma is initially of lower beta, density, and temperature than the operating point. The goal is to keep the barriers at roughly constant parameters while bringing the central cell up to the rinal conditions. To study startup, the steady-state plasma performance code is exercised at intermediate operating points through which a plausible startup path might pass. For each intermediate operatins point, the sustaining end cell powers are computed. For a particular scenarto to be deemed "successful," these powers should not exceed the operating point powers.

Table 2.8-1 shows the cases run using the physics model. Table 2.8-2 defines the parameters of Table 2.8-1. Note that the final reactor performance $\left(i . e_{-}, Q\right.$ ) is higher than the baseline reported elsewhere in this volume. This is because at the time these cases were run, we did not appreciate the importance of achieving adequate pump beam penetration. This tends to increase both axicell power and pumping power, reducing performance. The chosen values of central-cell beta slown were determined to keep the centralcell plasma radius constant for the given $P_{\text {fus }}$ and ion temperature. The cases 
Table 2.8-1. Physics cases used to model the final startup phase.

\begin{tabular}{lllllllllll}
\hline$P_{\text {fus }}$ & $T_{C}$ & $\beta_{C}$ & $I_{C C}$ & $P_{\text {axi }}$ & $P_{\text {slosh }}\left(f_{\text {trap }}\right)$ & $P_{E C R H, A} P_{E C R H, B}$ & $P_{\text {pump }}$ & $Q$ \\
\hline 600 & 25 & 0.29 & 0.4 & 14.2 & 2.3 & $(0.36)$ & 0.83 & 60 & 11.7 & 6.7 \\
800 & 25 & 0.33 & 0.5 & 13.5 & 2.9 & $(0.39)$ & 1.4 & 60 & 17 & 8.5 \\
1000 & 30 & 0.37 & 0.6 & 14.4 & 4.31 & $(0.43)$ & 2. & 70 & 18 & 9.2 \\
1500 & 32 & 0.46 & 0.7 & 14.1 & 6.6 & $(0.47)$ & 3.4 & 80 & 27 & 11.4 \\
2000 & 35 & 0.53 & 0.9 & 14.4 & 11.1 & $(0.51)$ & 5.3 & 97 & 33 & 12.4 \\
2500 & 35 & 0.59 & 1.1 & 13.9 & 12. & $(0.52)$ & 5.8 & 97 & 45 & 14.1 \\
3000 & 35 & 0.65 & 1.2 & 13.2 & 13.5 & $(0.52)$ & 6.3 & 97.4 & 50 & 15.7 \\
3500 & 35 & 0.7 & 1.4 & 12.8 & 13.8 & $(0.52)$ & 6.1 & 92.3 & 80 & 17.1 \\
\hline
\end{tabular}


Table 2.8-2. Startup parameter definitions.

\begin{tabular}{|c|c|}
\hline Parameter & Definition \\
\hline$P_{\text {fus }}$ (Nw) & D-T fusion power produced in central cell \\
\hline$T_{c}(k \in V)$ & Central-cell ion temperature \\
\hline$\beta_{c}$ & Central-cell beta value \\
\hline$I_{c c}(k A)$ & Cold refueling current required in central cell \\
\hline$P_{\text {axi }}$ (MW) & Sustaining power requitred in axicell \\
\hline$P_{\text {slosh }}($ MW $)$ & $\begin{array}{l}\text { Sustaining power required to produce the sloshing ions } \\
\text { in the barrier region }\end{array}$ \\
\hline$f_{\text {trap }}$ & Trapping fraction of the sloshing neutral beam \\
\hline$P_{E C R H, A}$ (MM) & $\begin{array}{l}\text { ECRH power required to sustain the warm electrons at } \\
\text { the confining potential peak for lons }\end{array}$ \\
\hline$P_{E C R H, B}$ (MW) & $\begin{array}{l}\text { ECRH power required to sustain the hot electrons at the } \\
\text { thermal barrier }\end{array}$ \\
\hline Pesmp (mw) & Charge-exchange pumping power in transition region \\
\hline $\mathbf{Q}$ & $\begin{array}{l}\text { Plasma gain factor } P_{\text {fus }} / P_{\text {inj }} \text {, where } P_{\text {inj }} \text { is the sum of } \\
\text { the above sustaining powers }\end{array}$ \\
\hline
\end{tabular}


were run down to about 600 of fusion power at which poin: solutions became difficult to obtain with the code. This could have been caused by one of two things: (1) the starting guesses for computed plasma parameters were not good cholces for powers below $600 \mathrm{MN}$; or (2) some kind of supplementary heating in the central cell is needed below this power level. More work is necessary to resolve this question. If the second possibility turns out to be the correct one, the results presented here suggest that the reactor can be "throttled" by backing off on injected fuel and end cell power so that it puts out as 1ittle as 1/6 of its rated output, in the saine operating mode as full power operation. This should be a desirable feature in establishing the utility acceptability of the tandem mirror.

Examining the cases presented in Table 2.8-2, wee that the sustaining fueling current and powers are elther monotonically increasing functions or independent of $P_{\text {fus }}$. There is a slight increase $(\sim 10 \%)$ of both the axicell power and the ECRH power used to produce the barrier hot electrons. We feel that these bumps can be removed with better programming of $\beta_{c}$ and the ratios of total barrier density to the central-cell density passing into the barrier region. Considerably more work is necessary to bridge the gap between the initial and the final startup phases.

\section{REFERENCES FOR SECTION 2.8}

1. A. W. Molvik and S. Falabella, Use of ICRH for Startup and Initlal Heating of the TMX-U Central CeTI, Lawrence Livermore National Laboratory, Livermore, CA, UCID-19342 (1982).

2. D. E. Baldwin and B. G. Logan, eds., Physics Basis for an Axicell Design for the End Plugs of MFTF-B, Lawrence LTvermore National Laboratory, Livermore, CA, UCID-19359 (1982). 


\subsubsection{Electrostatic Direct Conversion}

Almost all of the trapped injected power plus the alpha power streams out the ends of a tandem mirror reactor in the form of energetic charged particles. The total current of lost ions must exactly equal the current of electrons for the plasma to remain neutral. By separating the positive and negative currents, we can directly recover electric power and improve the efficiency of the reactor.

if $P_{F}$ is the fusion power and $P_{\text {in }}=P_{F} / Q$ is the total trapped injected power, the power carried out by charged particles is

$$
P_{\text {chg }}=P_{F}(1 / Q+0.2)-P_{\text {rad }}-P_{c x} \cdot
$$

A small amount of power, $P_{\text {rad }}$ is radiated to the walls--mostly as bremsstrahlung from the central cell and as synchrotron radiation from the barriers. Also, the pump beams result in $P_{c x}$ of fast neutrals striking the walls in the vicinity of those beams. The remainder of $P_{c h g}$ is carried out by charged particles, either to the ion collector at one end, to the electron collector at the other end, or radially to the halo plasma.

\subsubsection{Distribution of Electrical Potential}

The potential of the central cell is positive by an amount e relative to the end walls. This positive potential difference results from the fact that electrons tend to escape faster than the more massive ions that are confined

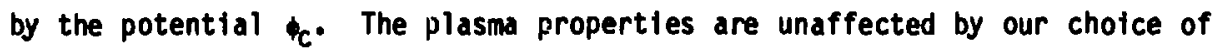
reference potential as long as the differences in potential are unaffected. We have chosen to ground the vacuum vessel and the halo dumps. Because the halo plasma is a very good electrical conductor, this effectively grounds the entire halo. Then, to avoid strong radial electric fields in the central cell, we have adjusted the potentfal of the central cell plasma to near that of the halo by lowering the potential of the end walls as shown in Fig. 2.9-1. The end walls in this case consist of the electron collector at one end and the first grid before the ion collector at the other end. These are the parts of the end walls that affect the electron transport. 


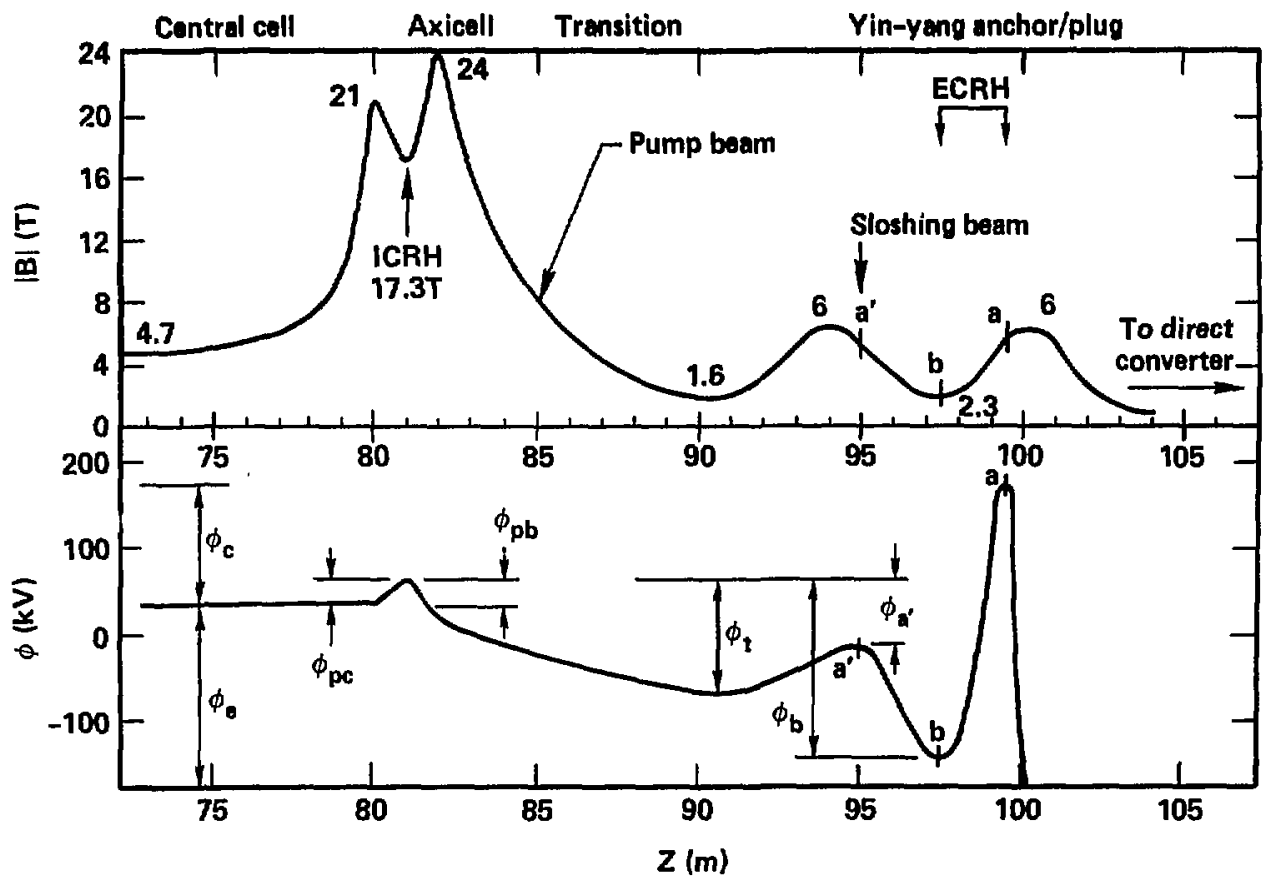

Fig. 2.9-3. Magnetic field and potential in the end cells. Relative to the grounded halo plasma, the central cell potential is positive by about $\mathrm{T}_{\mathrm{ec}} / \mathrm{e}$. 
Some radial variation in the central cell potential still exists, partly because of the radial variation of potential in the end cell plasmas, which are also enclosed by the grounded halo. We rather arbitrarily assume a potential difference of $\mathrm{T}_{\mathrm{ec}} / \mathrm{e}$ between the axis in the central cell plasma and the halo. There is therefore a radial electric field in the central cell plasma on the order of $T_{e c} / e r_{p}$, where $r_{p}$ is the plasma radius. This electric field, in the presence of the longitudinal magnetic field, causes the central cell plasma to rotate and produce an outward centrifugal force on the particles. The destabilizing effect of this outward force is negligible as long as the electric field is as small as $T_{e c} / r_{p}$. However, if we had not reduced the potential in the central cell, the electric field would have been on the order of $\phi_{e} / r_{p}$ and would have had a serious destabilizing effect (see Section 2.2.4). Stability of the central cell plasma therefore requires that the potential of the electron collector and the first grid at the fon collector be about negative relative to the vacuum vessel and the halo plasma. We have chosen to set them at $\mathrm{T}_{\mathrm{ec}} / \mathrm{e}-\phi_{\mathrm{e}}$.

\subsubsection{Selective Leakage}

Central cell lons are confined axially by the positfve potential peaks in the end plugs (see Fig. 2.9-1). They escape by diffusing up in energy until they can pass over the peaks. We can cause the selective leakage of ions out one end by raising the potential peak at the other end. The fractional increase in potential $\Delta \phi_{c} / \phi_{C}$ must be a few times the probable fractional increase in ion energy in one transit through the central cell. That is,

$$
\Delta \phi_{c} \gg \phi_{c} \sqrt{\frac{L_{c}}{v_{n} \tau}},
$$

where $L_{c}$ is the length of the central cell, $v_{1}$ is the mean longitudinal velocity of the lons, and $\tau$ is their average confinement time. In MARS, this only requires $\Delta \phi_{C} \gg 1 \mathrm{kV}$. The practical condition for $\Delta \phi_{C}$ is, therefore, that $\Delta \phi_{c}$ be held larger than any likely accidental variation in $\phi_{c}$ that could selectively leak the fons out the wrong end.

The suppressor grid at the fon collector reflects electrons and causes the selective leakage of central cell electrons out the other end. As Fig. 2.9-2 shows, any electron energetic enough to reach the grids at the fon 


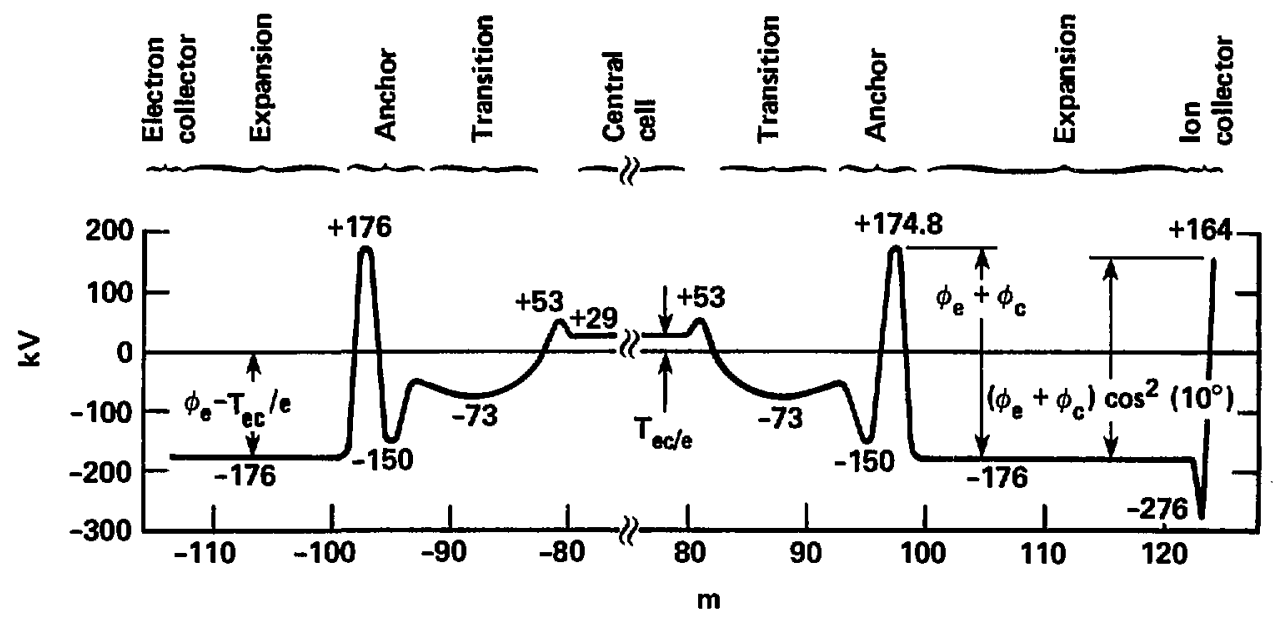

Fig. 2.9-2. Comparing the potentiai variation in the two ends: $V e c=\phi e$ $+\mathrm{T}_{\mathrm{ec}} / \mathrm{e}, \mathrm{V}_{\mathrm{g} l}=\mathrm{V}_{\mathrm{ec}}, \mathrm{Vg} 2_{\mathrm{g}}=\mathrm{V}_{\mathrm{ec}}-4 \mathrm{~T}_{\mathrm{ec}} / \mathrm{e}$. 
collector is also energetic enough to reach the electron collector at the other end. Once an electron gains enough energy to reach the ends, its kinetic energy as it passes through the central cell will be at least $e \phi_{e}$ and its scattering rate will be very low. It can then easily pass from one end of the machine to the other and be collected at the electron collector before it can penetrate the suppressor grid.

The first grid at the ion collector is at the same potential as the electron collector, while the suppressor grid is more negative by about $4 \mathrm{~T}_{\mathrm{e}} / \mathrm{e}$. This rather large potential difference is required to reflect all but about $e^{-4}$ of the electrons, because the reflection takes place where the magnetic field is weak. The perpendicular energy $\left(\sim T_{e}\right)$, which otherwise would not affect the reflection, is converted to directed energy by the decrease in magnetic field.

The selective leakage of lons out one end and electrons out the other results in a net longitudinal current of more than $2000 \mathrm{~A}$. This current produces an azimuthal $B_{\theta} \sim 10^{-3} \mathrm{~T}$, which is negligible.

\subsubsection{The Halo Plasma}

A halo plasma is necessary to prevent high-Z impurities from entering the core plasma. Because the confinement time of electrostatically confined ions varies approximately as $e^{Z \phi / T}$, high-Z ions are confined better than fuel ions. If allowed to enter, they would accumulate, diluting and cooling the plasma.

For the same reason, alpha particles tend to accumulate in the central cell. In the present design, alphas are removed by grad-B-induced radial diffusion in the transition regions (see Section 2.4). When they reach the radius of the halo plasma, they are on flux lines that pass outside of the confining potential. They can then flow along the flux lines to the halo dumps in the end tanks.

About $70 \%$ of the alpha particles must be removed by radial diffusion into the halo; about $5 \%$ are born in the magnetic loss cone and are lost immediately out the ends, while another $25 \%$ scatter into the loss cone after partially thermalizing with the central cell plasma. Also, because radial diffusion is not selective, some fuel ions are removed radially. About 10 fuel ions are 
removed for each alpha since the density ratio is about 10:1. The production rate of alphas is $I_{\alpha}=2 P_{F} / E_{F}=400 \mathrm{~A}$ (electrical). Therefore $280 \mathrm{~A}$ flows into the halo accompanied by about $1400 \mathrm{~A}$ of fuel ions. Each alpha and each fuel ion carries $3 / 2 T_{i}$ of energy into the halo where $T_{i}$ is the ion temperature at the surface of the core plasma. Here we assume $T_{i}=T_{i c}$. The total power flow out of the core plasma and into the halo is therefore $80 \mathrm{~W}$ if $T_{i c}=35 \mathrm{keV}$. In general, the radial current of alpha particles is $I_{\alpha, r}$ $=f_{r} I_{\alpha}$, where $I_{\alpha}=2 P_{F} / E_{F}$ and $f_{r}$ is the fraction of the alphas that are removed radially. The total radial current is then $I_{r}=I_{\alpha, r}\left(1+\frac{1}{2 c_{\alpha}}\right)$, where $c_{\alpha}=n_{\alpha} / n_{D T}$ is the alpha density ratio in the central cell. Also, the radial alpha power is $H_{\alpha_{s} r}=I_{\alpha_{s} r} E_{\alpha_{i} r} / 2$, where $E_{\alpha_{s} r}$ is the average energy carrfed out by each radial alpha. The total radial power is $H_{r}=I_{\alpha, r}\left(E_{\alpha, r}+3 T_{f c} / 2 c_{\alpha}\right) / 2$. Our preliminary estimates are $f_{r}=0.7, c_{\alpha}=0.1$, and $E_{\alpha, r}=3 / 2 T_{i c}$.

Because the halo dumps are at ground potential, no electrical power is recovered from the halo. Most of the $80 \mathrm{MW}$ appears as heat at the halo dumps, while a small fraction of it adds heat to the first wall.

\subsubsection{Power Balance}

Steady-state charge neutrality requires that the net electrical current out of the reactor be zero. Furthermore, $P_{\text {chg }}$, the power carried out by charged particles, is known. These two facts allow us to solve for the particle currents in terms of the different potentials, particle energies, and input powers. Setting the electron current equal to the total elecirical ion current gives

$$
I_{e, t h}+I_{e, b}=I_{i, t h}+I_{s 1}+f_{r_{\alpha}}\left[1+\frac{1}{2 c \alpha}\right]+\left(1-f_{r}\right) I_{\alpha},
$$

where $I_{e}$, th is the current of escaping central-cell electrons, $I_{e, b}$ is the current of ECRH-heated superthermal electrons, $I_{i, \text { th }}$ is the end-loss current of fuel ions from the central cell, $I_{s l}$ is the current of the sloshing ions from the anchor, and the last two terms represent the total radial current and the end loss alpha current.

We can write $P_{\text {out }}$, the total power carried out by charged particles, by considering the case where all particles are collected at ground potential. Then, assuming $E_{\alpha, r}=1.5 \mathrm{~T}_{\mathrm{ic}}$, 


$$
\begin{aligned}
P_{\text {out }} & =\left[\left(1-f_{r}\right) I_{\alpha}\left(\frac{E_{\alpha, e n d}}{2}+T_{e c}\right)+f_{r} I_{\alpha}\left(1+\frac{1}{c_{\alpha}}\right) \frac{3 T_{i c}}{4}\right. \\
& +I_{s 1}\left(E_{s 1}+e \phi_{c}+T_{e c}\right)+I_{e, b}\left(E_{e, b}+e \phi_{b}-e \phi_{p c}-T_{e c}\right) \\
& \left.+I_{e, t h} e \phi_{e}+I_{i, t h}\left(e \phi_{c}+T_{i c}+T_{e c}\right)\right] \frac{1}{e} .
\end{aligned}
$$

When the particles are collected at nonzero potentials, the power flowing out of the reactor is of course not changed. The algebraic sum of the heat produced plus the electrical, wer recovered remains equal to $P_{\text {out }}$.

Because $P_{\text {out }}=P_{\text {chg }}$ is known, can solve these two equations for $I_{i, t h}$ and $I_{e, t h}$ in terms of the potentials, energies, and currents that are calculated by the physics code. (Alternatively, we could obtain $I_{\mathcal{i}, \text { th }}$, and therefore $I_{e, t h}$, from the $n \tau$ values calculated by the physics codes.) The values of the currents $I_{i}$,th and $I_{e}$, th are needed to calculate the heat and electrical power produced when the charged particles are recovered.

\subsubsection{The Electron Collector}

We assume that selective leakage allows all escaping thermal electrons to be collected at the electron collector. However, the loss currents $I_{s 1}$ of sloshing fons, $\left(1-f_{r}\right) I_{\alpha}$ of end-loss alphas, and $-I_{e, b}$ of ECRH-heated superthermal electrons are non-selective. These currents are divided equally between the two ends. The total net current $I_{\text {ec }}$ to the electron collector is therefore

$$
I_{e c}=-I_{e, t h}+\frac{1}{2} I_{s 1}+\frac{1}{2}\left(1-f_{r}\right) I_{\alpha}-\frac{1}{2} I_{e, b} .
$$

The current $-I_{e}$, th of escaping thermal electrons is evaluated from the power and particle balance. For $I_{s l}$ we take all of the injected and trapped sloshing-ion current, since these ions have a much higher probability of escaping out the end than they have of entering and being trapped in the plasma. That is, $I_{s 1}=P_{s 1} / E_{s 1}$, where $P_{s 1}$ is the trapped sloshing ion power and $E_{s 1}$ is the injection energy. We tentatively assume that $10 \%$ of the hot, ECRH-heated barrier electrons escape out the ends, wille the other $90 \%$ thermalize before 
escaping. We take $I_{e, b}=0.1 \times e_{E C R H, b} / E_{e, b}$, where $P_{E C R H, b}$ is the ECRH power absorbed by barrier electrons, and $E_{e, b}$ is the mean energy of the hot electrons in the barrier.

The electric power recovered from the electron collector is $\mathrm{P}_{\mathrm{ec}}=\mathrm{I}_{\mathrm{ec}}$ $x V_{e c}$, where $V_{e c}=-\phi_{e}+T_{e c} / e$ (see Fig. 2.9-2). The heat generated at the electron collector $\mathrm{H}_{\mathrm{ec}}$ is obtained from the currents and impact energies of each of the species. The heat is given by

$$
\begin{aligned}
H_{e c} & =I_{e, t h} \frac{T_{e c}}{e}+\frac{1}{2} I_{s l}\left(\phi_{e}+\phi_{c}+\frac{E_{s l}}{e}\right)+\frac{1}{2}\left(1-f_{r}\right) I_{\alpha}\left(\phi_{e}+\frac{E_{\alpha, e n d}}{2 e}\right) \\
& +\frac{1}{2} I_{e, b}\left(-\phi_{e}-\phi_{p c}+\phi_{b}+\frac{E_{e, b}}{e}\right),
\end{aligned}
$$

where $E_{\alpha, \text { end }}$ is the mean energy of the alphas as they escape out the magnetic loss cone. It is calculated from

$$
E_{\alpha, \text { end }}=\frac{f_{1 c^{2}{ }_{0}}+\left(1-f_{r}-f_{1 c} E_{\alpha, 1 c}\right.}{1-f_{r}},
$$

where $E_{\alpha, 1 c}$ is the average energy of the alphas as they scatter into the loss cone and $E_{\alpha 0}=3520 \mathrm{keV}$ is the energy of those alphas that are born in the loss cone. We have assumed that $f_{r}=0.7$ and $E_{\alpha, l c}=400 \mathrm{keV}$. Here, $f_{1 c}$ is the fraction of the alphas that are born in the loss cone, and $f_{1 c}$ $=1-(1-1 / R)^{1 / 2}$, where $R$ is the $\beta$-increased mirror ratio for the central cell. For MARS, where $B_{c}=4.7 \mathrm{~T}$, and $B_{m 2}=24 \mathrm{~T}$, and $\beta_{c}=0.7$, the mirror ratio is $R=9.32$ and $f_{1 c}=0.055$. In that case, $E_{\alpha \text {,end }}=972 \mathrm{keV}$.

A single grid is required at the electron collector to suppress the emission of secondary electrons from the collector. The grid must therefore depress the potential by at least $20 \mathrm{~V}$ (i.e., a few times $T_{\text {es/e }}$ for secondary electrons) everywhere in front of the collector. The potential of the cold plasma in the end tank will adjust until the loss rate for cold electrons is equal to that for cold ions. Its value will therefore depend on the probability that a cold ion can strike either the grid or the collector plate, 
compared to the probability that a cold electron is lost to the collector. These probabilities depend on the geometry and potential of the grid as well as on the strength of the magnetic field. A sheath that is a few Debye lengths thick forms on the plasma side of the grid. It is the drop in potential across this sheath that adjusts to equalize the loss rates. A sketch of the variation in potential with distance out from the collector is shown in Fig. 2.9-3(a).

Figure 2.9-3(b) shows a sketch of some equipotential contours near the grid and defines the dimensions $r, a$, and $d$ that determine a grid's performance. The grid plane is spaced a distance $d=30 \mathrm{~cm}$ from the collector plate to allow the spacing between tubes, $a=20 \mathrm{~cm}$, to be less than $d$ but much greater than the tube diameter $2 r=1 \mathrm{~cm}$. This provides high grid transparency with tubes that are large enough for convective cooling. By applying 300 $\checkmark$ between the grid and the collector, essentially all secondary electrons can be turned back, even between grid tubes and in the presence of the cold plasma. He model the cold plasma by applying an electric field equal in magnitude to $T_{e} / e \lambda_{D e}$ to represent the sheath on the plasma side of the grid. Here, $\lambda_{D e}=3 \mathrm{~mm}$ is the Debye length and $T_{e}=10 \mathrm{eV}$ is the temperature of the cold electrons. The interface between plasma and sheath is then given approximately by the equipotential contnur where the potential is equal to the plasma potential. Because the plasma potential can differ from that of the collector by only a few times $\mathrm{T}_{e} / e$, this technique should locate the sheath to within a few $\lambda_{\text {De }}$ of its true location. The cold plasma density is determined by the balance between production and loss rates and is estimated in Section 2.9.8.

The cold plasma results in a net current flow from collector to grid, and is therefore a drain on the 300- $V$ power supply. The power consumed is completely negligible, and for power flow analysis, the collector and the grid can be treated as a single unit. For this reason and because the primary electrons are poor ionizers, the vacuum requirement at the electron collector end is less stringent than at the ion collector end where the pressure is $2 \times 10^{-5}$ Torr. 

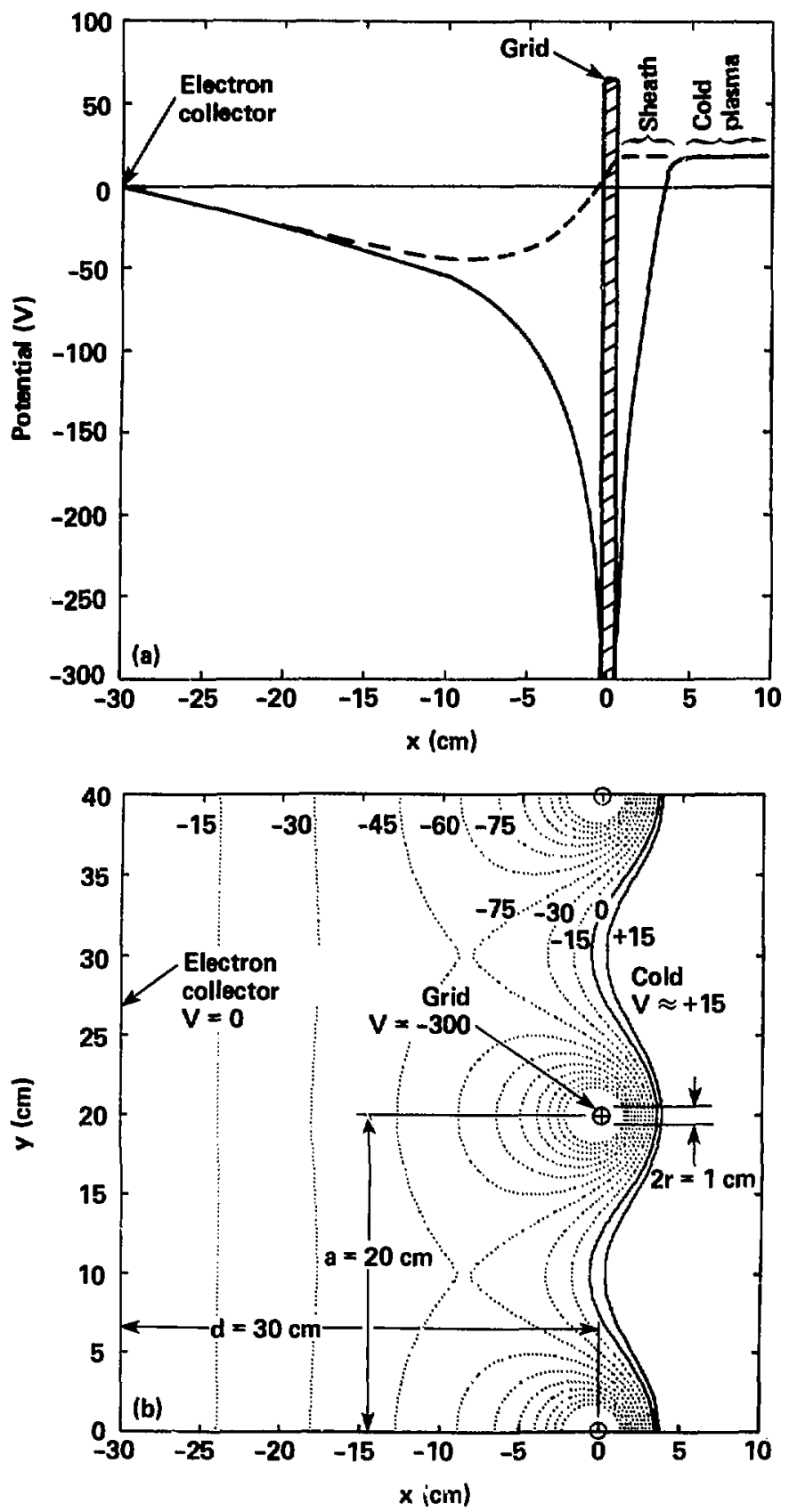

Fig. 2.9-3. (a) The variation in potential near the electron calculator and its single grid; (b) grid configuration with equipotential contours. Potential is given in volts relative to that of the electron collector $\left(V_{\mathrm{ec}}=0.176 \mathrm{kV}\right)$. 
The currents of primary particles arriving at the entrance grid to the ion collector can be written analogously to those at the electron collector. The net incident current is

$$
I_{\text {inc }}=I_{1, t h}+\frac{1}{2} I_{s 1}+\frac{1}{2}\left(1-f_{r}\right) I_{\alpha}-\frac{1}{2} I_{e, b} \text {, }
$$

where $I_{1}$,th is the current of end loss thermal fuel lons, all of which are selectively leaked out the one end. The other currents are as defined for the electron collector.

The entrance grid (gl) intercepts a fraction $1-T_{g l}$ of $I_{\text {inc' }}$ and the suppressor grid (g2) intercepts $T_{g 1}\left(1-T_{g 2}\right) I_{i n c}$, where $T_{g l}$ and $T_{g 2}$ are the transparencies of the two grids. The large negative potential of g2 depresses the potential around each element of $g l$ and prevents the release of secondary electrons from gl. Secondary electrons from g2 all flow to the fon collector.

In addition to the intercepted fractions of $I_{\text {inc }}$, the grids also collect all of the currents of cold tons and electrons produced by tonization and charge exchange in the end tank. The cold electrons all go to $\mathrm{gl}$, and, because of deflection and trapping in the potential well near g2, most of the cold ions go to g2. Because they impact 92 with about $100 \mathrm{keV}$ of energy, the cold lons also release secondary electrons that flow from g2 to the fon collector. We assume that the bombardment keeps the grid material free of oxides and absorbed gas, and therefore take a rather optimistic value of $\gamma=2$ for the secondary electron emission coefficient for the "cold" ions as well as for the primary ones.

As can be seen in Fig. 2.9-2, gl is held at the potential of the electron collector, while 92 is $100 \mathrm{kV}$ more negative. The potential of the ion collector is set as high as possible without reflecting any of the primary ions. Figure 2.9-2 also shows that all primary fons are accelerated by the difference in potential $b_{c}+$ before reaching $\mathrm{gl}$. To allow for 10-deg scattering of the incident ions by the grids, we set the ion collector potential at (he $\left.+\phi_{C}\right) \cos ^{2}(10 \mathrm{deg})$ above that of $\mathrm{gl}$. The grid parameters in MARS are chosen to linit the scattering to less than $10 \mathrm{deg}$. Relative to ground, $V_{g l}$ of $g l$, $v_{g 2}$ of $g 2$, and $v_{f c}$ of the fon collector are 


$$
\begin{aligned}
& v_{g 1}=-\phi_{e}+\frac{T_{e c}}{e}, \\
& v_{g 2}=-\phi_{e}-\frac{3 T_{e c}}{e} \text {, and } \\
& v_{f c}=\left(\phi_{e}+\phi_{c}\right) \cos ^{2}(10 \mathrm{deg})-\phi_{e}+\frac{T_{e c}}{e} .
\end{aligned}
$$

The net currents collected by each of these three electrodes are

$$
\begin{aligned}
& I_{g 1}=\left(1-T_{g 1}\right) I_{i n c}-\frac{I_{c}}{2}-\frac{I_{c}^{\prime}}{12}, \\
& I_{g 2}=T_{g 1}\left(1-T_{g 2}\right)\left[\left(I_{i n c}+\frac{I_{e, b}}{2}\right)(1+\gamma)-\frac{I_{e, b}}{2}\right]+\left(\frac{I_{c}}{2}+\frac{3 I_{c}^{\prime}}{4}\right)(1+\gamma) \text {, and } \\
& I_{i c}=T_{g 1} T_{g 2} I_{i n c}-T_{g 1}\left(1-T_{g 2}\right) \gamma\left(I_{i n c}+\frac{I_{e, b}}{2}\right)-\gamma\left(\frac{I_{c}}{2}+\frac{3 I_{c}}{3}\right)-\frac{2 I^{\prime} c}{3} \text {. }
\end{aligned}
$$

Here, $r$ is the secondary electron emission coefficient and $I_{c}$ and $I_{c}$ are currents of cold ions (and electrons) that result from the lonization of gas in the end tank. The difference between $I_{c}$ and $I_{c}$ is caused by the regions where the ionization occurs, and is discussed later. Notice that $\mathrm{I}_{\mathrm{gl}} \mathrm{I}_{\mathrm{gl}} \mathrm{I}_{\mathrm{ic}} \mathrm{ic}$ $=I_{\text {inc }}$, as it should, since both secondary emission and cold plasma result only in currents from one electrode to another, with no net output current.

The electrical power recovered $(+)$ or consumed $(-)$ at each electrode is obtained by multiplying the current collected by the electrode voltage

$$
P_{g 1}=I_{g 1} V_{g 1}, P_{g 2}=I_{g 2} V_{g 2,} P=I_{i c} V_{1 c} \cdot
$$

We can determine the heating of each electrode from the currents and impact energies of each species at each electrode such that

$$
\begin{aligned}
H_{g 1} & =\left(1-T_{g 1}\right)\left[I_{i, t h}\left(\phi_{e}+\phi_{c}+\frac{T_{i c}}{e}\right)+I_{s 1}\left(\frac{\phi_{a}+\phi_{c}+\frac{E_{s 1}}{e}}{2}\right)\right. \\
& \left.+\left(1-f_{c}\right) I_{\alpha}\left(\frac{e+\frac{E, e n d}{2 e}}{2}\right)+I_{e, b}\left(\frac{\phi_{b}-\phi_{e}-\frac{\phi_{e b}}{2}}{2}\right)\right]+
\end{aligned}
$$




$$
\begin{aligned}
& I_{c}^{\prime}\left[\frac{T_{e c}}{g e}+\left(\frac{V_{1 c}+e-T_{e c} / e}{12}\right)\right] \text {, } \\
& H_{g 2}=T_{g 1}\left(1-T_{g 2}\right)\left[I_{1, t h}\left(\phi_{e}+\phi_{c}+\frac{T_{1 c}}{e}+\frac{4 T_{e c}}{e}\right)\right. \\
& +I_{s 1}\left(\frac{\phi_{e}+\phi_{c}+\frac{4 T_{e c}}{e}+\frac{E_{s l}}{e}}{2}\right) \\
& +\left(1-f_{r}\right) I_{\alpha}\left(\frac{e+\frac{E}{E_{\alpha_{e} e n d}} \frac{4 T_{e c}}{2 e}+\frac{e_{e c}}{e}}{2}\right) \\
& \left.+I_{e, b}\left(\frac{\phi_{b}-\phi_{e}-\phi_{p c}+\frac{E_{e, b}}{e}-\frac{4 T_{e c}}{e}}{2}\right)\right]
\end{aligned}
$$

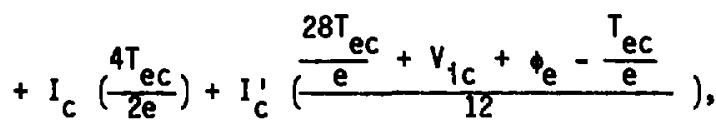

$$
\begin{aligned}
& H_{i c}=T_{g 1} T_{g 2}\left[I_{i, t h}\left(\phi_{c}+\frac{T_{i c}}{e}+\frac{T_{e c}}{e}-V_{i c}\right)\right. \\
& +I_{s}\left(\phi_{c}+\frac{T_{e c}}{e}+\frac{E_{s l}}{e}-V_{i c}\right) \\
& +\left(1-f_{r}\right) I_{\alpha}\left(\frac{T}{e}+\frac{E \alpha, e n d}{2 e}-V_{i c}\right) \\
& \left.+I_{e, b}\left(\phi_{b}-\phi_{p c}-\frac{T_{e c}}{e}+\frac{E_{e, b}}{e}+v_{j},\right)\right] \\
& +\gamma_{g 1}{ }_{1}\left(1-T_{g 2}\right)\left[I_{f, t h}+\left(1-f_{r}\right) \frac{I_{\alpha}}{2}+\frac{I_{s 1}}{2}\right]\left(\frac{3 T_{e c}}{e}+\phi+V_{f c}\right)
\end{aligned}
$$

$2-89$ 


$$
+\left[\frac{\gamma I_{c}}{2}+(2+3 \gamma) \frac{I_{c}^{\prime}}{4}\right]\left(V_{i c}+t^{3 T}+\frac{3 T^{e c}}{e}\right)-(-g) I_{c}^{\prime}\left(\frac{T^{T} c}{e}\right) .
$$

We have assumed here that 92 is $4 \mathrm{~T}$ ec/e more negative than $\mathrm{gl}$, that no centralcell electrons reach the ion collector, and that all primary ions not intercepted by a grid are collected on the ion collector.

\subsubsection{Cold Plasma}

Cold ions and electrons are produced in the end tanks as the primary fons and electrons pass through the background gas. The plugging potential prevents the cold lons from entering the confined plasma, and the huge difference in the magnetic field restricts the flow of the cold electrons into the confined plasma. Therefore, most of the cold electrons and all of the cold fons must flow to the electrodes at the ends.

First, constder the current $I_{c}$ of cold plasma produced before the first grid (gl) at the ion collector end. The cold electrons are reflected by $g 2$, while the cold ions are accelerated through $\mathrm{g} 2$ and reflected by the ion collector. As a result, $-I_{c}$ of cold electrons flows to $\mathrm{gl}$ and $+I_{c} / 2$ of cold ions flows to both $\mathrm{gl}$ and $\mathrm{g} 2$. Each ion is assumed to release $\gamma=2$ secondary electrons that flow to the ion collector because the electric field on the collector side of the grid is strong.

Another smaller current $I^{\prime}{ }_{c}$ of cold ions and electrons is produced between $\mathrm{gl}$ and the fon collector. The electrode to which an fon or electron goes depends on where it is created.? The result is that $\mathrm{gl}$ receives a net $-{ }^{\prime}{ }_{c} / 12, \mathrm{~g} 2$ receives $+3 I{ }^{\prime}{ }_{c} / 4$ and relases $y$ times that current of secondaries, and the ion collector receives the $3 \mathrm{\gamma I}^{1}{ }_{\mathrm{c}} / 4 \mathrm{plus}$ another $2 \mathrm{I}^{\prime}{ }_{\mathrm{c}} / 3$ of electron current. The energies that these particles have and the heat produced where they are collected is determined by averaging the potential differences through which they fall.

The magnitude of $I_{C}$ is given by

$$
I_{c}=n_{0} \sigma_{c} L_{c}\left(I_{\text {inc }}+\frac{I_{e, b}}{2}\right) \text {, }
$$

where $n_{0}$ is the gas density, $\sigma_{c}$ is the total cross section for the production of positive ions and free electrons, and $L_{c}$ is the path length. We are assuming that the mean energy of the cold plasma electrons is low, and that 
their contribution to $I_{c}$ can be neglected. Ionization by fast electrons from the central cell as well as from the barrier is also negligible. Therefore, we take $\sigma_{c}=1.7 \times 10^{-16} \mathrm{~cm}^{2}$, appropriate for the 385-keV $\mathrm{D}^{+}$and $\mathrm{T}^{+}$, and multiply it by the fast ion current. ${ }^{2}$ The effective length $L_{c}$ is somewhat shorter than the distance from the anchor to the first grid because $n_{0}$ is small in the beam near the anchor. We take $L_{c} \approx 20 \mathrm{~m}$. Then $I_{c}=1.2 \times 10^{4}$ $x p\left(I_{\text {inc }}+I_{e, b} / 2\right)$, where $p$ is the background gas pressure.

The current I' ${ }_{c}$ of cold tons produced between $g l$ and the fon collector is due to charge exchange near the collector. As the fast lons are decelerated $\sigma^{\prime}{ }^{\prime}$, the cross section for charge exchange (of $\mathrm{D}^{+}$and $\mathrm{T}^{+}$on $\mathrm{DT}$ gas) increases toward a maximum value of $8.5 \times 10^{-16} \mathrm{~cm}^{2}$ at about $20 \mathrm{keV}$. The distance L'c that fons move at low energy (where $\sigma^{\prime}{ }_{c}$ is large) is only about $0.2 \mathrm{~m}$, so that $I^{\prime}{ }_{c}=n_{0} \partial \sigma^{\prime}{ }_{c}{ }^{\prime}{ }_{c}\left(I_{i n c}+I_{e, b} / 2\right)$ has a value of only about $I^{\prime}{ }_{c}-I_{c} / 20$.

In our calculations, we set

$$
I_{c}=1.2 \times 10^{4} p\left(I_{\text {inc }}+\frac{I_{e, b}}{2}\right)
$$

and

$$
I_{c}^{\prime}=6 \times 10^{2} p\left(I_{\text {inc }}+\frac{I_{e, b}}{2}\right) .
$$

In MARS, the effect of $I^{\prime}{ }_{c}$ is negligible, but that of $I_{c}$ is not. At $p=2 \times 10^{-5}$ Torr, the total heating of $\mathrm{g} 2$ is $30 \mathrm{w}$, and 10 of that is due to $I_{c}$. Because the size of the direct converter is determined by grid heating, it could be reduced by only one-third even if $I_{c}$ could be reduced to near zero. Reducing $I_{c}$ by very much can only $b$; done by reducing $p$, which implies increasing the vacuum pumping speed. Economics, therefore, determine the best pressure $p$, and it appears from preliminary cost estimates that the best value is $p=2 \times 10^{-5}$ Torr in the fon collector end.

At the electron collector end, the production rate for cold plasma is much less. Because there are no fuel ions from the central cell, most of the cold plasma results from the ionization of gas by the alplia particles that scatter into the loss cone and arrive in the ends with moderate energies. In MARS, about $I_{\alpha, e c}=50 \mathrm{~A}$ of alphas (particle current) go out each end with about $750 \mathrm{keV}$ average energy. The electron collector in MARS also receives about $I_{e, t h}=2600 \mathrm{~A}$ of $29-\mathrm{keV}$ electrons. The cross sections ${ }^{2}$ for tonization by these two species are $\sigma_{\alpha}=5.4 \times 10^{-16} \mathrm{~cm}^{2}\left(\right.$ per He${ }^{++}$) and $\sigma_{e}=1 \times 10^{-18} \mathrm{~cm}^{2}$ 
(per $\mathrm{e}^{-}$). We take $\mathrm{L}-15 \mathrm{~m}$ and calculate the cold fon production rate in the electron collector end, I $c, e c$, so that

$$
I_{C, e C}=n_{0} L\left(I_{\alpha, e C} \sigma_{\alpha}+I_{e, t h} \sigma_{t}\right) \sim 18\left(\frac{p}{2 \times 10^{-5}}\right),
$$

where $p$ is now the pressure (in Torr) in the electron end tank. Even if $p$ is the same, the cold plasma production rate is much smaller in the electron end than in the fon end.

Cold plasma current causes little loss of power directly in the electron end, but the derisity of cold plasma determines the Debye length. It is therefore important for the design of the grid. We can estimate the cold electron density $n_{e c}$ from

$$
I_{e, e c}-n_{e c} \approx v_{i c} A_{e c},
$$

where $v_{i c} \sim 2 \times 10^{4} \mathrm{~m} / \mathrm{s}$ is the average speed of the cold ions $\left(5 \mathrm{eV}, 2.5 \mathrm{M}_{\mathrm{H}}\right)$ and $A_{e c}=100 \mathrm{~m}^{2}$ is the area of the beam at the collector. This gives $n_{e c}$ $=6 \times 10^{13} \mathrm{~m}^{-3}$ if $p=2 \times 10^{-5}$ Torr ir the electron end and $\lambda_{\mathrm{De}}=3 \mathrm{~mm}$ if $T_{e}=10 \mathrm{eV}$. This value of $\lambda_{D e}$ was used in Section 2.9 .6 in estimating the voltage required for the single grid at the electron collector. The high negative voltage applied to the suppressor grid at the fon collector makes it less sensitive to $\lambda_{\mathrm{De}}{ }^{\circ}$

\subsubsection{Grids at the Ion Collector}

The grids at the ion collector have two purposes: to turn back the primary electrons and to provide sinks for the cold plasma. Oul grid design is intended to minimize the interception of primary fons while still performing these two functions.

Figure 2.9-4 shows a section through the direct converter and defines the notation. This is the configuration of a plane triode vacuum tube, with our gl taking the place of the cathode. If ignore space charge temporarily, we can write the vacuum potential inside the triode as 3

$$
V(x, y)=A \ln \left(e^{2 E x}-2 e^{E x} \cos E y+1\right)+B\left(d_{g g}+x\right)+C .
$$




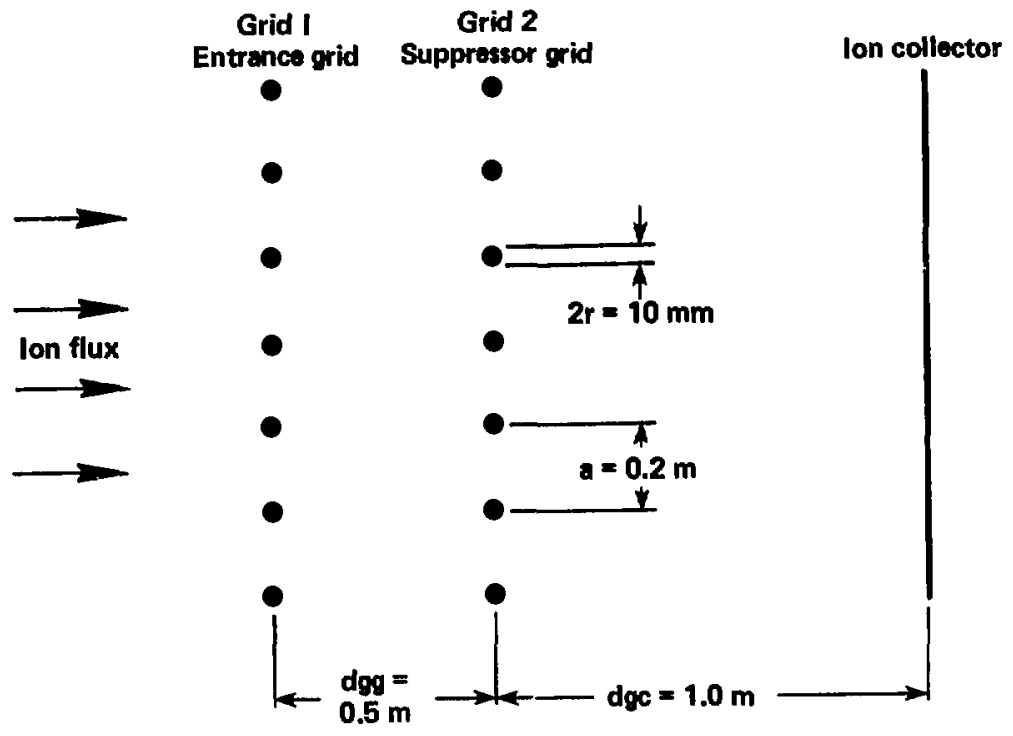

Fig. 2.9-4. Plane triode vacuum tube configuration of the ion collector and its two grids. 
Here, the origin is at the center of a grid tube in $\mathrm{g} 2$ and the $x$-coordinate is normal to the g2-plane, $E=2 \pi / a$, and $A, B$, and $C$ are chosen to satisfy the boundary conditions

$$
V\left(-d_{g g}, 0\right)=V_{g 1}, V(r, 0)=V_{g 2}, V\left(d_{g c}, 0\right)=V_{i c} \cdot
$$

The effect of $g 2$ is weakest in the plane defined by $y=a / 2$, and the minimum value of $V(x, a / 2)$ occurs at $x=x^{\prime}=-(a / 2 \pi) \ln [-(1+4 \pi A / a B)]$. We require that $V\left(x^{\prime}, a / 2\right)$ be sufficiently negative to turn back nearly all of the primary electrons. The electrons from the central cell arrive at gl with a mean energy $T_{e c}$ and are well-directed along the magnetic field because of the decrease in that field. Fokker-Planck (and Monte Carlo) calculations have shown that the primary electrons arriving at gl have nearly a Maxwellian distribution, and therefore, the fraction that penetrates past $\mathrm{g} 2$ is approximately $\exp \left[e\left(V^{\prime}-v_{g]}\right) / k T_{e c}\right]$. Here, $V^{\prime}$ is the average of the potential minima in all $y=$ constant planes. Therefore, $V_{g 2}<V^{\prime}<V\left(x^{\prime}, a / 2\right)$. For MARS we have chosen the grid parameters shown in Fig. 2.9-4, with $V_{g 1}=-176 \mathrm{kV}, V_{g 2}$ $=-276 \mathrm{kV}$, and $V_{i c}=+164 \mathrm{kV}$. This gives $V\left(x^{\prime}, a / 2\right)=-234 \mathrm{kV}$ and $V^{\prime}=-244 \mathrm{kV}$. The value of the exponential, with $T_{e c}=28.9 \mathrm{keV}$, is 0.09 , which is too high. However, the distribution deviates from a Maxwellian at high energy, and the actual penetration is much less than 0.09 . We assume, therefore, that the 100-kV difference between the $\mathrm{gl}$ and $\mathrm{g} 2$ potential is sufficient. To increase that difference would increase the heat $\mathrm{H}_{\mathrm{g} 2}$ and therefore the size of the direct converter.

Space-charge effects modify these results in three ways. First, the cold plasma produces a sheath that tends to shield the plasma from the grid potential. However, the cold electron temperature is less than $100 \mathrm{ej}$ and the electric field just inside $\mathrm{gl}$ is about $1.35 \mathrm{kV} / \mathrm{cm}$. The cold plasma sheath therefore changes the effective $d_{g g}(=50 \mathrm{~cm})$ by about $2 \mathrm{~mm}$ and changes the 100-kV difference in grid potentials by a few hundred volts. The effect is negligible. Second, the space charge of the primary electrons modifies the electric field between the two grid planes. The $50-\mathrm{cm}$ spacing between planes and the $68-\mathrm{kV}$ average difference in potential would allow $450 \mathrm{~A} / \mathrm{m}^{2}$ of $29-\mathrm{keV}$ electrons before the potential were seriously affected by space-charge. ${ }^{4}$ In MARS, the current density of $29-\mathrm{keV}$ electrons arriving at gl is only about 
charge effect is also negligible. The third space-charge effect is that of the fast ions where they slow down between g2 and the ion collector. Here again the effect is negligible. The current density of $D^{+}$and $T^{+}$ions is about $3.5 \mathrm{~A} / \mathrm{m}^{2}$, while the $1-\mathrm{m}$ spacing and the $408-\mathrm{kV}$ average difference in potential would require $16 \mathrm{~A} / \mathrm{m}^{2}$ of $T_{i c}=35-\mathrm{keV}$ ions to be space-chargelimited. 4

Therefore, the high potentials applied to the electrodes dominate over sheath potentials and other space-charge effects. The potential distribution calculated for the vacuum case is a good approximation to the true distribution.

The power density on any small area of the direct converter is proportional to the production rate of power in the magnetic flux tube intercepted by that area. And, because most of the direct converter power comes from the central cell, the production rate is proportional to the square of the density at the radius where the flux tube passes through the central cell. The mapping of magnetic flux is complicated by the diamagnetic effect of the central-cell plasma. We assume that both $n_{i c}$ and $\beta_{c}$ vary with radius $r$ in the central cell as $1-x^{\gamma}$, where $x-r / r_{p}$ and $\gamma$ is a constant. The long-thin approximation then gives the radial variation of $B$ in the central cell as

$$
B=B_{c} \sqrt{1-\beta_{c}\left(1-x^{\gamma}\right)}
$$

For any position on the direct converter that maps from radius $r=r_{p} x$ in the central cell, the power density $d P / d A$ is

$$
\frac{d P}{d A}=B_{d c} \frac{d P}{d \phi}=\left(\frac{P}{A_{d c}}\right) \frac{f\left(\beta_{c}, \gamma, 1\right)(1+\gamma)(2+\gamma)\left(1-x^{\gamma}\right)^{2}}{\gamma^{2} \sqrt{1-\beta_{c}\left(1-x^{\gamma}\right)}} .
$$

Here, $P$ is the total power to the direct converter from the central cell, $A_{d c}$ is the area of the direct converter, $B_{d c}$ is the magnetic induction at the direct converter, $d \Phi$ is the magnetic flux intercepted by area $d A$ at the direct converter, and $f\left(\beta_{c}, \gamma, x\right)$ is defined by

$$
f\left(\beta_{c}, \gamma, x\right) \equiv 2 \quad \int_{0}^{x} \sqrt{1-\beta_{c}\left(1-x^{\gamma}\right)} x d x .
$$


The MARS parameters $\beta_{c}=0.7$ and $\gamma=3$ give $f(0.7,3,1)=0.749$ and

$$
\left(\frac{d P}{d A}\right)_{x=0}=\left(\frac{P}{A d c}\right) 3.04
$$

Therefore, the power density at the center of the direct converter is about three times the average power density over the direct converter. Without the effect of $\beta_{C}$ on $B$, the peak value would be only $20 / 9$ times the average.

\subsubsection{Direct Conversion Efficiency}

Table 2.9-1 shows the MARS parameters, and Table 2.9-2 shows the resulting current, heat, and electric power recovered at each electrode. In Table 2.9-3 we itemize the currents and energies of the different species of charge particles leaving the reactor. Notice that these are electrical, not particle, currents. Because of the se?ertive leakage of electrons out one end and ions out the other, the electron collector is a major component in the direct recovery system. The total electrical power recovered is $P=445 M_{e}$, and the total heat generated at the electrodes (including the grounded halo dumps) is $H=418 \mathrm{w}_{\text {th }}$. Notice that $P+H=P_{\text {chg }}=863 \mathrm{~W}$, so that all of the charged particle power is accounted for. The net efficiency of direct recovery $\eta_{O C}$ is therefore

$$
\eta_{D C}=\frac{P}{P_{\text {chg }}}=0.515 \text {. }
$$

Note that in the overall reactor power balance (Section 2.10), we used a slightly different definition of $\eta_{D C}$, namely, $\eta_{D C}=P / P$ end, where $P_{\text {end }}$ is less than $P_{\text {chg }}$ by the halo power. Most of the 418 of heat could conceivably be used in a thermal cycle to generate more electricity.

Figure 2.9-5 shows a schematic electric circuit that makes use of the electric power and passively generates all of the required electrode voltages except $V_{g 2^{*}}$ A $100-k V, 380-A$ dc power supply is required to maintain $V_{g 2^{*}}$ The load resistors $R_{1}$ and $R_{2}$ can be combinations of gyrotrons and neutral beam injectors. 


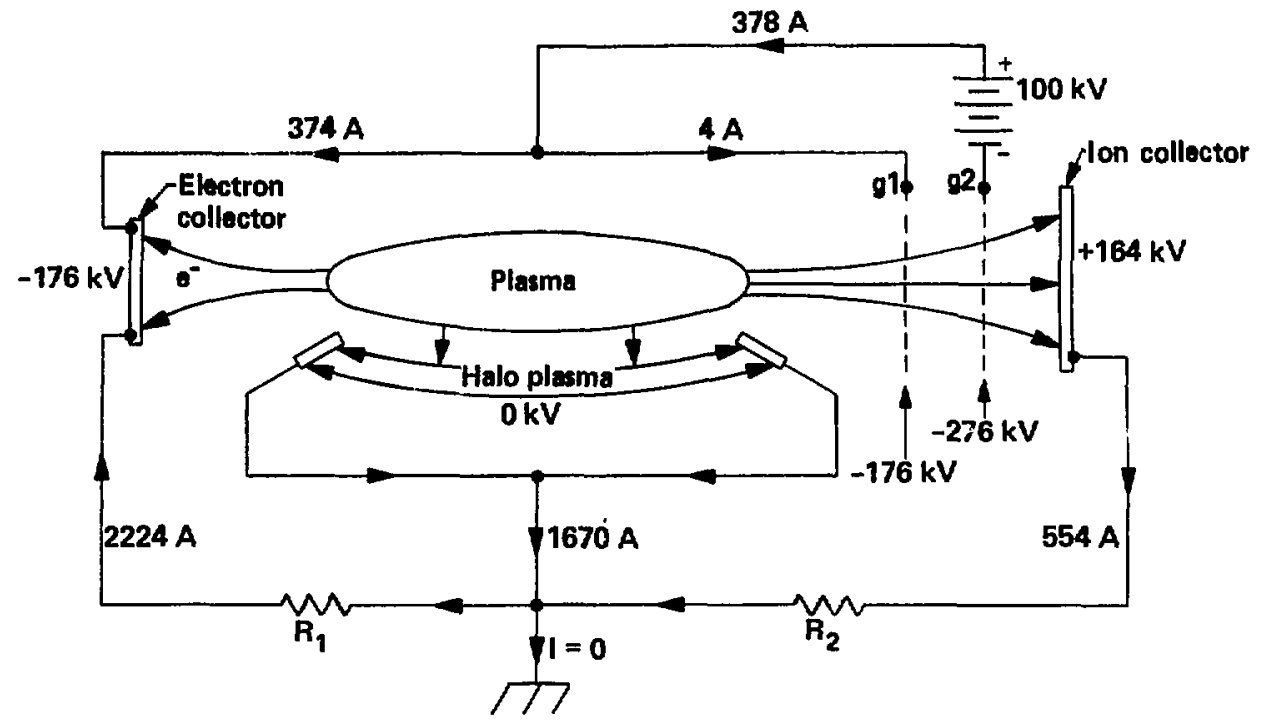

Fig. 2.9-5. Schematic of an electrical circuit that uses the directly recovered power. The load resistances $R_{1}$ and $R_{2}$ could be gyrotrons or
neutral beam injectors. 
Table 2.9-1. MARS parameters used to evaluate the direct recovery of power.

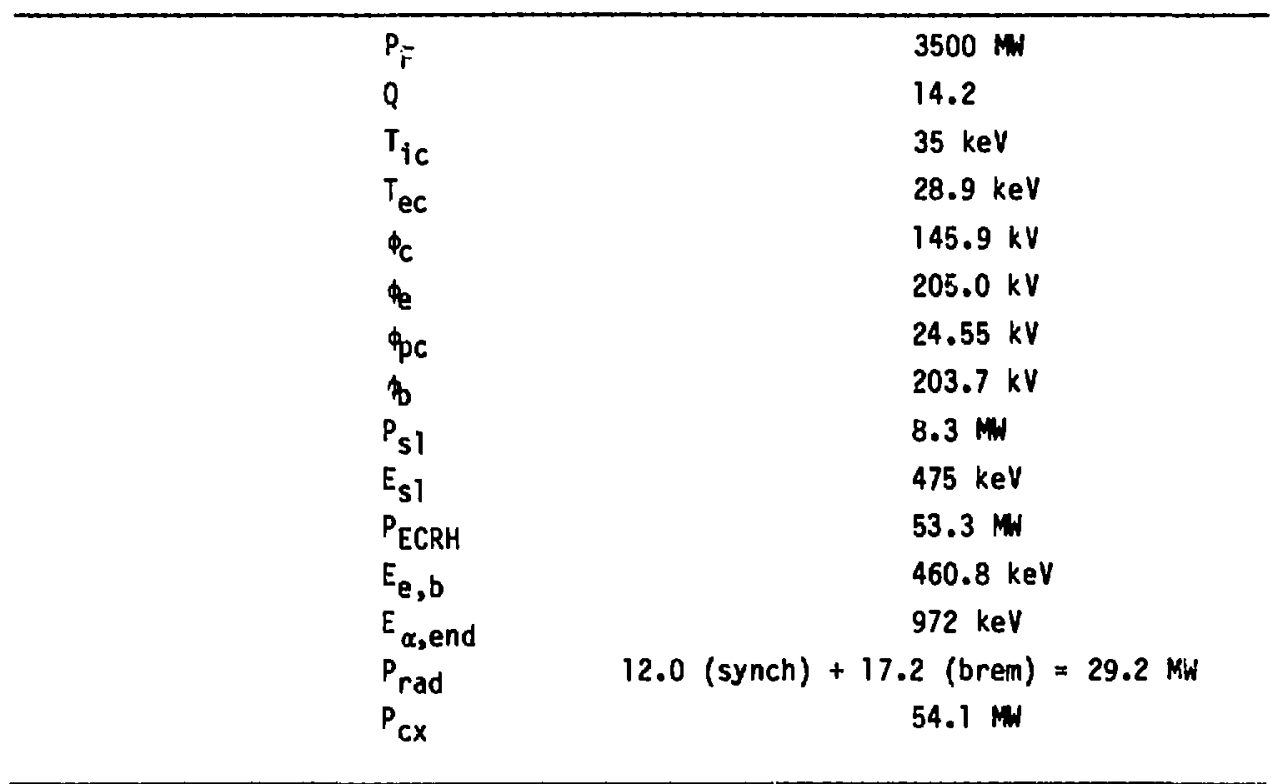

Table 2.9-2. The voltage, current, heat and electrical power generated at each electrode.

\begin{tabular}{|c|c|c|c|c|c|c|}
\hline & Halo & Grid 1 & Grid 2 & $\begin{array}{l}\text { Ion } \\
\text { collector }\end{array}$ & $\begin{array}{l}\text { Electron } \\
\text { collector }\end{array}$ & Total \\
\hline$v(k V)$ & 0 & -176 & -276 & 164 & -176 & -- \\
\hline$I(A)$ & 1670 & -4 & 378 & 554 & -2598 & 0 \\
\hline$H\left(M_{t h}\right)$ & 80.3 & 20.4 & 30.8 & 170.6 & 115.8 & 418.2 \\
\hline$P\left(M_{e}\right)$ & 0 & 0.7 & $-104 \cdot 2$ & 90.9 & 457.5 & 444.9 \\
\hline
\end{tabular}


Table 2.9-3. The electrical current and kinetic energy of sach species of charged particles leaving the reactor.

\begin{tabular}{|c|c|c|c|}
\hline$\frac{\text { Regton }}{\text { Spectes }}$ & $\begin{array}{c}H a 10, \\
V=0\end{array}$ & $\begin{array}{l}\text { Ion end }(g 1) \\
V=-i 76 \mathrm{kV} \\
\end{array}$ & $\begin{array}{l}\text { Electron end, } \\
V=-176 \mathrm{kV}\end{array}$ \\
\hline $\begin{array}{r}\text { Central-cell } \\
\text { Electrons }\end{array}$ & 0 & 0 & $\begin{array}{l}-? .660 \mathrm{~A} \\
28.9 \mathrm{keV}\end{array}$ \\
\hline $\begin{array}{l}\text { Barrier } \\
\text { Electrons }\end{array}$ & $\begin{array}{l}0 \\
435 \mathrm{keV}\end{array}$ & $\begin{array}{l}-5.7 \mathrm{~A} \\
435 \mathrm{keV}\end{array}$ & $-5.7 A$ \\
\hline $\begin{array}{c}\text { Central-csll } \\
0^{+}, T^{+}\end{array}$ & $\begin{array}{l}1392 \mathrm{~A} \\
52.5 \mathrm{keV}\end{array}$ & $\begin{array}{l}865 \mathrm{~A} \\
386 \mathrm{keV}\end{array}$ & 0 \\
\hline $\begin{array}{c}\text { Sloshing (anchor) } \\
\mathrm{D}^{+}\end{array}$ & 0 & $\begin{array}{l}8.7 \mathrm{~A} \\
982 \mathrm{keV}\end{array}$ & $\begin{array}{l}8.7 \mathrm{~A} \\
982 \mathrm{keV}\end{array}$ \\
\hline a particles & $\begin{array}{l}278 \mathrm{~A} \\
52.5 \mathrm{keV}\end{array}$ & $\begin{array}{l}59.6 \mathrm{~A} \\
1382 \mathrm{keV}\end{array}$ & $\begin{array}{l}59.6 \mathrm{~A} \\
1382 \mathrm{keV}\end{array}$ \\
\hline
\end{tabular}




\subsubsection{Halo Plasma Physics}

Although the radial diffusion of $i 500 \mathrm{~A}$ and $80 \mathrm{~m}$ of fons from the transition regions produces a thin halo plasma in the ends, this halo does little to protect the core plasma. However, the halo produced by the absorption of the synchrotron radiation from the central cell ( $\sim 12$ in MARS) can protect the core plasma.

The outside boundary of the core plasma is determined by the radial extent of the plugging potential in the anchors. Immediately outside of the confined plasma, a halo plasma must be maintained to protect the core plasma from recycled gas and impurities coming from the walls. Incoming high-2 impurity atoms must be fonized while they are on field lines that pass outside of the confining potential. If allowed to enter the core plasma, they would be confined better thian fuel ions and would accumulate, because to escape over the confining potentfal o requires that they be heated to an energy greater than $Z_{e} \phi_{c}$. Radial transport in the transition regions would only remove them relatively slowly because of the (energy) ( $^{3 / 2} / Z^{2}$ dependence of the diffusion coefficient (Section 2.4), and because they first would have to become passing ions. He feel, therefore, that the halo plasma must attenuate the incident impurities by at least three orders of magnitude.

A thin halo is created by the radial transport of most of the alphas plus 10 times as many fuel lons out of the transition regions. Once these fons drift out far enough to pass the confining potential, they stream freely out either end. They are in the halo for only one transit time to an end, and transfer only a small part of their energy to the halo electrons. In MARS, about $1600 \mathrm{~A}$ and $80 \mathrm{~W}$ are carried out by these ions, and only about $0.3 \mathrm{~W}$ is transferred (by drag) to the electrons. This sheet of ion current is at least two ion-gyroradii thick. The actual thickness depends on the radial profile of the confining potential, but it is clear that the complete halo must be much thicker and more dense than this ion sheet to give the required attenuation.

The transition regions are vulnerable to the trapping of cold ions. Trapped ions are removed by pump beams, which also release gas at the beam dumps and at the walls where the charge-exchange products strike. When these gas molecules enter the halo, fast Franck-Condon $(F-C)$ atons are produced that penetrate much deeper into the halo than the molecules would. It is the 
attenuation of these fast atoms in the transition regions that sets the most severe requirenent on the halo density. By flux mapping from the 17-cm-thick halo in the central cell, the thickness of the halo is $w=13 \mathrm{~cm}$ at the 9-T pump beam in the transition. The current of F-C neutrals that penetrates the halo must be less than $10 \%$ of the pump beam current that produces charge exchange. If, on the aveiage, half of the incident molecules results in F-C's, and half of those F-C.'s are directed toward the core plasma, then the total current of F-C's toward the core is $1 / 3$ of the incident current of gas atoms (after suming over the recycled F-C's). We therefore require that

$$
\frac{1}{3} I_{p}(1-f+f \times 0.7) e^{-W / \lambda}<0.1 \times I_{p} \times 0.7
$$

where $I_{p}$ is the pump beam current, $f=1$ is the trapping fraction, and 0.7 of the trapped beam results in charge exchange. The mean free path for F-C's, $\lambda$, is given by

$$
\lambda=\frac{v_{F C}}{n_{h}\langle 0 \mid\rangle}
$$

and, from the above requirement, must satisfy $\lambda<w / 1.2$. Here, $v_{F C}=2 \times 10^{6} \mathrm{~cm} / \mathrm{s}$ is the speed of 5-eV F-C's, $n_{h}$ is the halo density, and $\left\langle\sigma_{\mathrm{e}}\right\rangle=3 \times 10^{-8} \mathrm{~cm}^{-3} / \mathrm{s}$ is the rate coefficient 5 for the ionization of $D$ and $T$ atoms by $50-e V$ electrons. The required halo density is therefore $n_{h}>6 \times 10^{12} \mathrm{~cm}^{-3}$.

The primary ioss of power from the electrons is at the halo dumps in the ends, and, if $T_{e_{3}}<100 \mathrm{eV}$, it is limited by conduction. At $T_{e}=50 \mathrm{eV}$ and $n_{e}=6 \times 10^{18} \mathrm{~m}^{-3}$, about $8 \mathrm{~m}$ is conducted out each end by electrons. 6 Even at $1 \%$ concentration of high-Z impurities (such as iron) in this halo, the line radiation is only about $1 \mathrm{w}$, less than $2 \mathrm{~kW} / \mathrm{m}^{2}$ on the first wall. The power required to ionize 4000 A (equivalent) of DT gas (from the pellet injectors and neutral beaw injectors) is negligible by comparison. Therefore, the power required to maintain $T_{e}=50 \mathrm{eV}$ in the halo is about 17 . The central cell electrons radiate about 12 of synchrotron radiation, and most of this cones fran the outer layers where the fundamental frequency is the same as in the halo. The absorption coefficient ${ }^{7}$ in the central cell halo ( $w=0.17 \mathrm{~m}, \mathrm{n}_{\mathrm{h}}$ $=6 \times 10^{18} \mathrm{~m}^{-3}, \mathrm{~T}_{\mathrm{e}}=50 \mathrm{eV}$ ) is 0.84 , so that 10 of the $12 \mathrm{~m}$ is absorbed on the first pass through. Since the first wall is a good reflector, most of the $12 \mathrm{~N}$ is absorbed in the halo. Because of the steep $\mathrm{T}_{e}^{3.5}$ scaling law for 
electron thermal conduction, 12 w absorbed by the halo electrons w111 maintain their temperature at $T_{e}=45 \mathrm{eV}$. We therefore assume that the power required to maintain the halo is supplied by the synchrotron radiation from the central cell and does not appear in the overall power balance.

Any complete physics model of the halo will require a knowledge of the boundary region between the core and outer halo. Not only is the radial variation of involved, but also the potentials of the outer regions of the ion collector and the inner regions of the halo dumps where this boundary layer is collected. Also, the electron confining potential depends on the electron temperature, which is different in the boundary region than in the core plasma.

\section{REFERENCES FOR SECTION 2.9}

1. W. L. Barr and R. W. Moir, Test Results on Plasma Direct Converters, Lawrence Livermore National Laboratory, accepted for pubtication in Nuclear Technology/Fusion.

2. C. F. Barnett, et al., Atomic Data for Controlled Fusion Research, Oak Ridge Nationai Laboratory: Oak RTdge, TN, ORNL-5206 and ORNL-5207 (1977).

3. K.R. Spangenberg, Vacuum Tubes, McGraw-H111, New York, p. 125 (1948).

4. P. T. Kirstein, G. S. Kino, and W. E. Waters, Space-Charge Flow, McGraw Hill, New York, p. 273 (1967).

5. R. L. Freeman and E. M. Jones, Atomic Collision Processes in Plasma Physics Experiments, Culham Laboratory, CDM-RT37 (1974).

6. Lyman Spitzer, Jr., Physics of Fully Ionized Gases, Interscience Publishers, New York. P. 87 (1956).

7. M. Porkolab, Electron Cyclotron Resonance Heating of Tandem Mirrors, Lawrence Li vermore National Laboratory, Livermore, CA, Rept. UCRL-52634 (1978). 


\subsubsection{Plasma Paraneters}

We used a zero-dimensional, multi-region fluid code to self-consistently determine the parameters of the MARS plasma. The plasma model is an outgrowth of the model developed for MFTF-B analysis, 1 and is essentially the same as the model we used in our comparative end plug study last year. ${ }^{2}$ since the model has been described previously, we will simply present the results for MARS.

An essential featura of a tandem mirror with thermal barriers is the axial variation of plasma potential which, along with the magnetic configuration, provides the confinement for the central-cell fusion plasma. Calculation of the axial potential profile, consistent with the particle and energy ialances, is the cornerstone of the plasma model. Figure 2.9-1 shows the axial potential and magnetic field profiles for MARS. The figure shows the nomenclature used in the plasma model for the various potential differences. Table 2.10-1 gives the z-position, vacuum magnetic field strength, and plasma potential at each of the cardinal points in MARS. The magnetic field values in the table and figure are those that were used in the plasma model, and, as mentioned previously, are not exactly the same as those calculated for the MARS magnetic configuration (plotted in Fig. 2.1-5). We bias the potential to near zero in the central cell to satisfy the constraints imposed by centralcell plasma rotation (see Section 2.2.4) and radial transport (see Section 2.2.1). The on-axis central-cell plasma potential is $29 \mathrm{keV}$ above ground and the potential at the minimum field point in the transition region is $72 \mathrm{keV}$ below ground. The $\beta$ and density values are peak, on-axis values; the plasma model assumes a cubic radial density profile. The ion temperature $T_{f}$ is a code input; $35 \mathrm{keV}$ has been determined to be near-optimum for maximizing the plasma performance parameter $Q$. The electron temperature $T_{e}$ is a calculated value. The hot plasma radius, where the hot fuel density goes to zero, defines the inner boundary of the halo plasma, and the first wall location allows for a halo thickness of 3 hot alpha (3.5 MeV) gyroradif. The cold fueling current is that portion of the DT fueling of the central cell that is to be provided by pellet injection near one end of the central cell. Most of the deuterium fuel is provided by the charge-exchange pump beam, and ionized 
Table 2.10-1. MARS magnetic field strength and plasma potential.

\begin{tabular}{lcll}
\hline \multicolumn{1}{c}{ Location } & $z(\mathrm{~m})$ & $B(T)$ & $($ (keV) \\
\hline Central cell & 75 to 75 & 4.7 & $29\left(\phi_{\mathrm{e}}=205\right)$ \\
Inboard axicell mirror & 80.2 & 21 & 29 \\
Axicell midplane & 81.2 & 17.3 & $54\left(\phi_{\mathrm{pc}}=25\right)$ \\
Outboard axicell mirror & 82.2 & 24 & $10\left(\phi_{\mathrm{pb}}=44\right)$ \\
Pump beam location & 84.5 & 7.8 & -13 \\
Transition field minimum & 90.5 & 1.6 & $-72\left(\phi_{\mathrm{t}}=126\right)$ \\
Inboard anchor mirror & 94.0 & 6.0 & $\ldots$ \\
Point A' & 95.0 & 5.4 & $-21\left(\phi_{A^{\prime}}=75\right)$ \\
Anchor midplane (barrier) & 97.5 & 2.3 & $\left.-150\left(\phi_{\mathrm{b}}\right)=204\right)$ \\
Point A (potential peak) & 99.5 & 3.5 & $175\left(\phi_{\mathrm{C}}=146\right)$ \\
Outboard anchor mirror & 101.0 & 6.0 & 0 \\
\end{tabular}

Table 2.10-2. MARS central cell parameters.

\begin{tabular}{|c|c|}
\hline$P_{\text {fusion }}$ & $3500 \mathrm{NW}$ \\
\hline B & $4.7 \mathrm{~T}$ \\
\hline$\beta$ & 0.7 \\
\hline$n_{0, D T}$ & $4.2 \times 10^{14} \mathrm{~cm}^{-3}$ \\
\hline$n_{0, \alpha}$ & $4.2 \times 10^{13} \mathrm{~cm}^{-3}$ \\
\hline$n \tau_{\text {ion }}$ & $5.5 \times 10^{14} \mathrm{~s} / \mathrm{cm}^{-3}$ \\
\hline$n \tau_{e}$ & $6.6 \times 10^{14} \mathrm{~s} / \mathrm{cm}^{-3}$ \\
\hline$n \tau_{\alpha}$ & $1.0 \times 10^{14} \mathrm{~s} / \mathrm{cm}^{-3}$ \\
\hline$T_{1}$ & $35 \mathrm{keV}$ \\
\hline $\mathrm{T}_{\mathbf{e}}$ & $29 \mathrm{keV}$ \\
\hline$L_{c}$ & $150 \mathrm{~m}$ \\
\hline Hot plasma radius & $0.43 \mathrm{~m}$ \\
\hline First wall radius & $0.60 \mathrm{~m}$ \\
\hline Neutron wall loading $\left(I_{F W}\right)$ & $5.0 \mathrm{mw} / \mathrm{m}^{2}$ \\
\hline Cold fueling current & $1.9 \mathrm{kA}$ \\
\hline
\end{tabular}


pump beam neutrals are in central-cell passing ion velocity space. The parameters of the central cell plasma, confined by the potential peak $\phi_{C}$ = $146 \mathrm{keV}$, are given in Table 2.10-2. Table 2.10-3 1ists the various plasma density and energy parameters of the MARS and end plug parameters.

\subsubsection{Particle and Energy Injection}

Table 2.10-4 gives the physics parnmeters of the neutral beam and $\mathrm{rf}$ heating systems for MARS.

The charge-exchange pump beams inject $740 \mathrm{~A}$ of $97-\mathrm{keV}$ deuterium into the transition region at each end. The pump beam aiming point is $z=84.5 \mathrm{~m}$, where the magnetic field strength is $7.8 \mathrm{~T}$. The angle of injection is 25 deg from the exis. More than $99 \%$ of this beam is fonized in the plasma (there are five e-folds of beam attenuation through the plasma). Beam neutrals that are fonized by charge-exchange reactions with transition-trapped lons perform the destred pumping function--the new neutral is lost to the wall while the new ion is in passing, not trapped, velocity space. Ionization reactions by beam neutrals contribute to central-cell fueling, but not to pumping. Chargeexchange reactions with passing ions contribute to neither pumping nor fueling.

The anchor beams inject $20 \mathrm{~A}$ of $475-\mathrm{keV}$ deuterium into each anchor to sustain the sloshing ion distribution. The injection point is chosen to be point $A^{\prime}(z=95 \mathrm{~m})$ instead of point $A(z=99.5$, the potential peak location) to avoid the introduction of cold electrons at the location of the warm electron population. Points $A$ and $A^{\prime}$ are the turning points for the sloshing fons. Because of the high beam energy and modest anchor plasma density, only 45\% of the anchor beam is trapped in the plasma.

The axicell mirror-confined plasma is sustained by ICRH. This represents a recent change in the MARS baseline design and only limited analysis has been performed. The earlier MARS design called for 200-keV neutral beam injection into the axicell. This feature necessitated an axial gap in the superconducting axicell magnet, which lowered the magnetic effictency (on-axis field/conductor field) and introduced difficult neutron shielding and magnet structural support problems. These problems are avoided with ICRH power feedthroughs that enter the axicell region from one end of the superconducting 
Table 2.10-3. MARS end plug parameters.

Axicell

Midplane plasma radius

$0.21 \mathrm{~m}$

Plasma length

$2.0 \mathrm{~m}$

$\beta$

0.4

Hat ion density

$9.5 \times 10^{14} \mathrm{~cm}^{-3}$

Total ion density

$1.16 \times 10^{15} \mathrm{~cm}^{-3}$

Average confined-ion energy

$300 \mathrm{keV}$

$n \tau$

$8.2 \times 10^{13} \mathrm{~s} / \mathrm{cm}^{3}$

Transition region

Length

$12 \mathrm{~m}$

$\beta$

0.15

Passing ion density at $B$ minimum

$5.2 \times 10^{12} \mathrm{~cm}^{-3}$

Passing ion density at pump point

$3.5 \times 10^{13} \mathrm{~cm}^{-3}$

DT particle trapping current

$295 A$ at each end

Anchor

Length

$7 \mathrm{~m}$

$\beta$

0.65

Passing ion density at barrier

$3.9 \times 10^{12} \mathrm{~cm}^{-3}$

Warm electron temperature at

potential peak

$89 \mathrm{keV}$

Hot electron energy at barrier

$460 \mathrm{keV}$ 
Table 2.10-4. MaRS neutral beams and rf.a

\begin{tabular}{|c|c|c|c|c|c|}
\hline & $\begin{array}{l}\text { Energy } \\
(\mathrm{keV})\end{array}$ & $\begin{array}{l}\text { Injected } \\
\text { current/end } \\
\text { (A) }\end{array}$ & $\begin{array}{l}\text { Injected } \\
\text { power/end } \\
\text { (MW) }\end{array}$ & $F_{\text {trap }}$ & Frequency \\
\hline
\end{tabular}

Neutral

Beams

$\begin{array}{lllllll}\text { Pump } & \text { D } & 97 & 740 & 72 & 0.993 & - \\ \text { Anchor } & \text { D } & 475 & 20 & 9.3 & 0.446 & -\end{array}$

ICRH

Axicell

- n -

23

0.85

$180 \mathrm{MHz}$

ECRH

\begin{tabular}{|c|c|c|c|c|c|c|}
\hline \multirow{3}{*}{$\begin{array}{l}\text { Barrier } \\
\text { Potential }\end{array}$} & - & - & - & 27 & 1.0 & $64 \mathrm{GHz}$ \\
\hline & & & & & & \\
\hline & - & - & - & 1 & 1.0 & $96 \mathrm{GHz}$ \\
\hline
\end{tabular}

a Total trapped injected power $=246 \mathrm{mH}$

$Q=3500 / 246=14.2$ 
magnet. Our preliminary choice for the ICRH frequency, $180 \mathrm{MHz}$, is the second harmonic for tritium at the axicell minimum field. The injected power, $23 \mathrm{mw}$ per end, is assumed to be $85 \%$ absorbed in the mirror-confined tritium species.

ECRH is required to sustain the hot mirror-confined electrons at the barrier location and the warm electron population at the location of the potential peak (point A). The frequencies listed in Table 2.10-4 are the fundamentals at the vacuum field values of 2.3 and $3.5 \mathrm{~T}$, respectively. Finite $\beta$ and relativistic effects will tend to lower the required frequencies. We estimate $100 \%$ absorption of this power.

Fueling of the centrel-se!l plasma requires the pellet injection of $1.9 \mathrm{kA}$ of mostly tritium (deuterium fueling is provided by the charge-exchange pump beam). Injection is at the 9.4-T point at one end of the central cell, where the reduced plasma radius improves pellet penetration. For injection of $10 \%$ of the central-r.sll plasma ion population per pellet, the fuel pellets are about $4 \mathrm{~mm}$ in radius. We used Park and Turnbull 's ${ }^{3}$ pellet ablation model (with density profile corrections) to calculate required pellet velocities. For penetration to the plasma axis we would require a pellet velocity of about $43 \mathrm{~km} / \mathrm{s}$. If radial diffusion of injested fuel will permit penetration to only $1 / 5$ the plasma radius, as suggested by some Tokamak studies ${ }^{4}$, then the pellet velocity need be only $1.2 \mathrm{~km} / \mathrm{s}$. The required pellet velocity for MARS has not yet been determined, but it appears that it will be in the range from 1 to $10 \mathrm{~km} / \mathrm{s}$.

\subsubsection{Overall Reactor Power Balance}

Figure 2.10-1 gives an overall MARS power balance consistent with the plasma parameters discussed in this section and the baseline systems described in other sections. Two nunibers are presented for terms affected by the blanket choice: the first refe:'s to the LiPb blanket, the second refers to the high-temperature blanket. The injected power and trapping fractions for the neutral beam and $\mathrm{rf}$ systems are from Table 2.10-4; the electrical efficiencies of these systems have been separately estimated. The thermal conversion efficiencies have been adjusted to include coolant pumping power. The copper coil power is for the baseline-design axicell insert coils with internal conductor cooling. The auxiliary system power is primarily for cryogenic refrigeration. The end-loss charged-particle power is reduced by $80 \mathrm{WW}$ of power to the halo plasma and 57 of charge-exchange power to the wall in the transition region. 


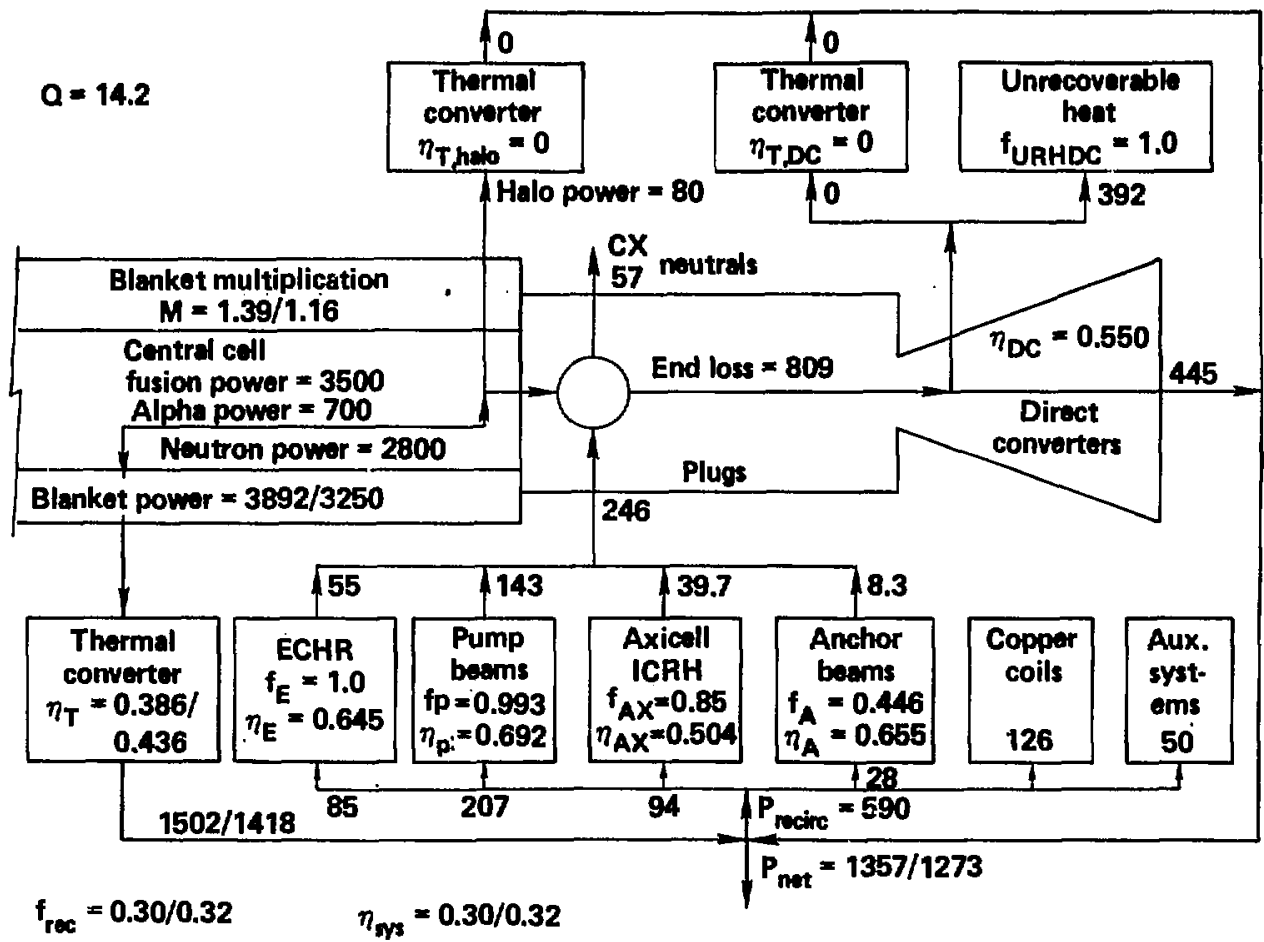

Fig. 2.10-1. Overall power balance. 
The net electrical output of MARS is 1357 or 1273 m, depending on slanket choice, and the 590 of recirculated electrical power represents 30 to $32 \%$ of the gross electrical power. Note that the baseline design does not include thermal conversion of the halo and direct converter heat. Future work will investigate the recovery of this power. If $35 \%$ conversion of the halo and direct converter heat can be realized, the net electrical output will increase by 165 and the recirculating power fraction will decrease to about 0.28 to 0.29 . The other high-leverage area for future improvement of the MARS power balance is in the design of the trapped-particle pump system. Note that the charge-exchange pump beams consume $50 \%$ of the total neutral beam and $r f$ input power, which in turn makes up $70 \%$ of the total recirculated electrical power. Our future work in this area will investigate radial drift pumping, as discussed in Section 2.3.3.

\section{REFERENCES FOR SECTION 2.10}

1. D. W. Baldwin, B. G. Logan, and T. C. Simonen, Eds., Physics Basis for MFTF-B, Lawrence Livermore Mational Laboratory, Livermore, CA, UCID-18496-Part 1 (1980).

2. G. A. Carlson et al., Comparative End-Plug Study for Tandem Mirror Reactors, Lawrence Livermore National Laboratory, Livermore, CA, UCID-1927 (1981).

3. P. B. Parks and R. J. Turnbul1, Phys. Fluids 21, 1735 (1978).

4. D. R. Dobrott, SAI, personal comunication (1982). 


\section{REACTOR SYSTEMS}

\subsection{MAGNETS}

The MARS magnet system includes four principal magnet types. The central-cell magnets are located in the central region of the reactor and generate the axial magnetic field to confine the plasma. An axicell is located at each end of the central cell and is followed by transition coils that shape the plasma and anchor coils to provide plasma stability. Figure 3.1-1 shows the magnet configuration and summarizes major characteristics of each magnet system. For simplicity, only a portion of the central cell with the end cell of the machine is shown.

The central cell incorporates 48 superconducting solenoids to produce an axial field of $4.7 \mathrm{~T}$. Magnet design is based on a reasonable extrapolation of state-of-the-art superconductor technology; the coil can be manufactured by using fabrication methods similar to those used to construct MFTF-B solenoids.

Each axicell magnet group consists of a superconducting solenoid that provides a background magnetic field of approximately $15 \mathrm{~T}$ and two resistive insert coils that increase the magnetic field to 21 and $24 \mathrm{~T}$, respectively. The design of this system is based on advanced magnet concepts requiring further technological development.

The transition and anchor coils are similar to the transition and anchor coil of MFTF-B. The overall dimensions, magnetic field, and location of these coils may present a helium cooling problem. The yin-yang for MFTF-B was designed for a 45-deg-from-horizontal orientation to accommodate bubble ventilation. Additional scoping and design work is scheduled in FY 1983 to complete the MARS design.

The design work performed in Fy 1982 concentrated on resolving key design issues of the central cell and axicell magnats and scoping alternatives to identify the best design approaches. We have proposed a number of innovative solutions to some challenging design problems, including:

- An integrated central-cell coil-blanket design using coll-blanket modules to facilitate machine assembly and maintenance. 


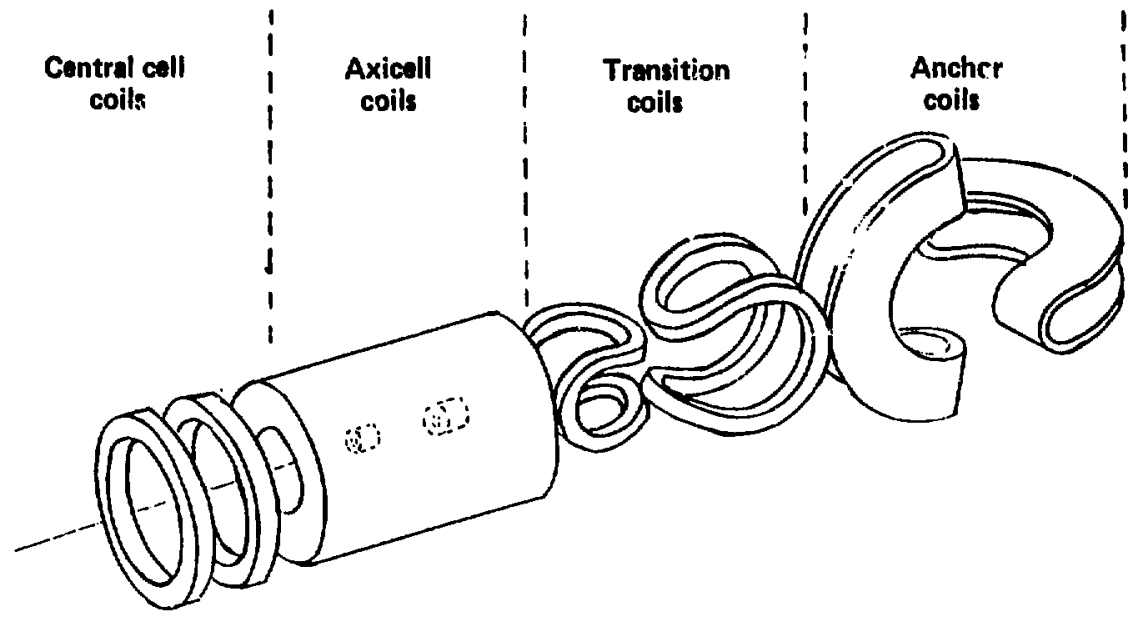

\begin{tabular}{|c|c|c|c|c|c|}
\hline Coil & Quantity & $\begin{array}{c}\text { mean } \\
\text { diameter }(m)\end{array}$ & $\begin{array}{l}\text { Central } \\
\text { field }\end{array}$ & $\begin{array}{l}\text { Poak } \\
\text { fiold }\end{array}$ & $\underset{\text { (t) }}{\text { Weight }}$ \\
\hline Central coll & 48 & 58 & 4.7 & 7.4 & 180 \\
\hline Axicell & 2 & 38 & $21,17.3,24$ & $16^{b}$ & 616 \\
\hline Transition & 4 & TBD & 1.6 & 6.8 & TBD \\
\hline Anchor & $2 Y Y^{\circ}$ & 8 & $6,2.3,6$ & 8.5 & 315 \\
\hline
\end{tabular}

Fig. 3.1-1. MARS magnet configuration. 
- High-performance, internally cooled resistive-.nsert magnets to provide more than 1 full power year (FPY) magnet lifetime in the axicells.

- High-field superconducting coils using graded regions of NbTi, $\mathrm{Nb}_{3} \mathrm{Sn}$, and titanium-doped $\mathrm{Nb}_{3} \mathrm{Sn}$ superconductors cooled with LHe II at $1.8 \mathrm{~K}$ to achieve higher fields with smaller cotls.

- Reinforced conductors for application at the higher fields and stress levels, to reduce coll volume and cost.

- Radiation-resistant polyimide and spiriel insulators to increase radiation tolerance of superco.lducting and resistive magnets.

\subsubsection{Central Cell Coils}

\subsubsection{Design Considerations}

The central-cell magnet requirements are showr. in Table 3.1-1. Major requirements are an axial magnetic field of $4.7 \mathrm{~T}$, an inner radius of the solenoid cryostat of $2.24 \mathrm{~m}$, and an intercoil axiai spacing of $3.16 \mathrm{~m}$.

Table 3.1-1. Requirements for the central cell solenoids.

Central field

Peak conductor field

Operational ?ife

Cryostat clear bore radius

Axial spacing

LHe and vaccum vessel 1 design
$4.7 \mathrm{~T}$

$<8.0 \mathrm{~T}$

$24 \mathrm{FPY}_{3} 30$ yr total

$$
\leq 2.24 \mathrm{~m}
$$

$3.16 \mathrm{~m}$

ASME pressure vessel code 
Based on a design alternative study, a NbTi/Cu conductor cooled with LHe $I$ at $4.5 \mathrm{~K}$ was selected as the baseline design. Existing superconductor technology with demonstrated performance in large colls is based on this combination.

Magnetic field and Forces. The basic magnetic field was calculated with LLNL's EFFI computer code. Peak fields are within NbTi/Cu, pool bofling LHe at $4.5 \mathrm{~K}$ technology. The hoop stresses in the coll were evaluated with the STANSOL computer code. Large hoop forces led to incorporation of steel reinforced conductors.

Radiation Effects. Radiation affects the magnets in several ways. It increases the resistivity of the stabilizer, decreases the mechanical and dielectric strengths of the insulators, and reduces the critical parameters of the superconductor. In addftion, the neutron heat input in the magnets increases the load of the refrigeration system. These effects reduce cryostability, and decrease the peak magnetic fleld and lifetime of the magnets. The central-cell magnets, therefore, are provided with adequate shielding to keep radiation damage within acceptable limits for full performance of the magnets over their design life of 24 FPY.

Radiation damage to the stabilizer increases electrical resistivity, approaches a saturation limit, and is mostly recoverable by room-temperature annealing. Similarly, damage to the superconductor decreases the critical current and is recoverable. On the other hand, the radiation damage to the insulation is cumulative and non-recoverable. Detalled radiation limits for the central cell magnets are discussed later.

\subsubsection{Engineering Trace-off Studies}

Choice of Conductor and Insulation. Several types of conductors have been considered for application to the central cell magnets. A steelreinforced monolithic conductor was chosen to resist the high hoop stresses. Detailed conductor manufacturing techniques and costs were investigated and compared to an alternate design conductor supported with separate stainless steel strips. The alternate was rejected based on cost. 
Polyimide glass and epoxy glass have been considered for the insulating material. Polyimide was chosen for the insulation in high radiation areas since it tolerates high radiation doses $\left(5 \times 10^{9} \mathrm{rad}\right)$. Epoxy glass will be considered if a detailed shielding study shows that adequate margin is maintained for the 24-FPY lifetime.

Choice of Cooling. A cost comparison was made between cooling a central-cell coil with LHe I or with LHe II. The comparison included the cost of the magnet, the cost of the refrigeration system, and the present value of the operating costs. A magnet cooled with LHe II uses less conductor, but requires greater refrigeration capacity and operating costs. Table 3.1-2 shows that the two costs are almost identical. The use of LHe II would require considerable technological development of coil and cryogenic equipment. Also, the refrigeration system would need more space and the service stack and cryogenic plumbing would be more complex with LHe II. Because the design requirements of the central solenoids are met with proven LHe I technology, pool-boiling helium at $4.5 \mathrm{~K}$ is the preferred coolant. A cost comparison was also made for a central solenold with a $2.06-m$ inner radius; the same conclusion was reached.

\subsubsection{Magnet Design}

The central-cell solenoid magnets provide the central magnetic field iecessary to contain the plasma. There are 48 coils, spaced $3.16 \mathrm{~m}$ along the $z$-axis, that produce a central field of $4.7 T$ with a riple of $3.8 \%$. The magnet design is illustrated in Fig. 3.1-2; key features are given in Table 3.1-3. Each solenoid is a complete magnet including case, radiation shield, vacuum vessel, and service stack.

The NDTi conductor is graded to take advantage of the reduction in field and allow higher current densities. Three graded sections are used in the radial direction, with the same operating current of 7535 A flowing in series through each section of graded conductor.

The solenoid case is made of heavy sections of 304LN stainless steel. The insulation is polyimide fiberglass in high radiation areas. The conductors are layer-wound to take advantage of grading. A single service stack at the top provides for the vapor-cooled power leads, LHe, $\mathrm{LN}_{2}$, gaseous exits, 


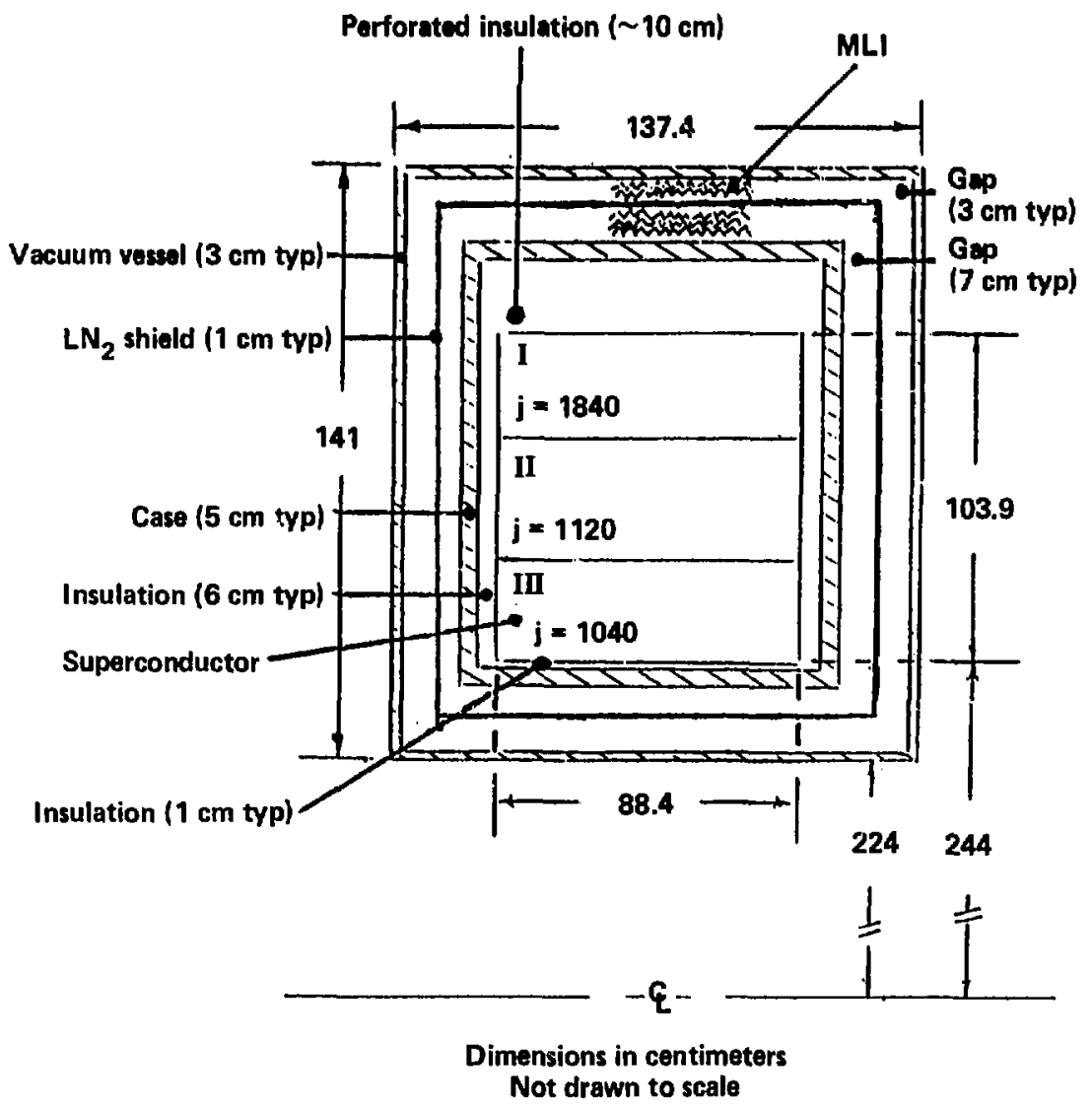

Fig. 3.1-2. Central-cell solenoid cross section. 
Table 3.1-2. Magnet cooling alternatives.

\section{Costs}

LHe I (pool bolling)

LHe II (superfluid)

Cost of coll Including case at $\$ 25 / 1 \mathrm{~b}$ avg cost for

$\$ 11.58 \mathrm{M}$

$\$ 9.06 \mathrm{M}$

first reactor

Cost of LHe thermgl radiation

shield at $\$ 140 / \mathrm{ft}^{2}$

N/A

$0.19 M$

Cost of cryogentc plant

LHe I at \$1750/W

LHe II at $\$ 4550 / \mathrm{W}$

$1.13 \mathrm{M}$

$0.44 M$

N/A

$1.44 \mathrm{~N}$

Comparative capital cost

$\$ 12.71 \mathrm{M}$

$\$ 11.13 \mathrm{M}$

Annual cost to operated based on $80 \%$ usage, $\$ 0.10 / \mathrm{kH}-\mathrm{hr}$

$\$ 0.304 \mathrm{M}$

$\$ 0.505 \mathrm{M}$

Present value of $30-y r$

$2.46 \mathrm{M}$

$4.06 M$

operation at 127 interest

Total 1ifecycle cost ${ }^{a}$

(capital plus present value

$\$ 15.17 \mathrm{M}$

$\$ 15.19 \mathrm{M}$

of operating cost)

${ }^{a}$ Cost estimates do not include stack, Li., shield, vacuum vessel, cryogentc plumbing, power and control system, or maintenance. 
Table 3.1-3. Key features of the central cell solenoids.

LHe I-cooled (pool boiling, $4.5 \mathrm{~K}$ )

Coil spacing $3.16 \mathrm{~m}$

Graded current density (three sections)

Peak field $7.4 \mathrm{~T}$

6-cm side insulation for splices and leads

Conductors react own hoop stresses ( $40 \mathrm{ks} 1 \mathrm{max}$. allowable)

Heat loads

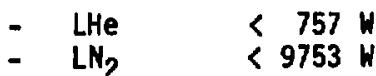

- Vapor-cooled leads--28 liters LHe/hr

Neutronic shlelding requitrements

- $1.1 \times 10^{-4} \mathrm{dpa}$ in copper stablizer before annealing

- $0.06 \mathrm{~m} / \mathrm{cm}^{3}$ peak neutron heat deposition in winding

- $5 \times 10^{9}$ rad dose max in insulation

Approximate weight (including vacuum vessel) 200 tons 
and instrumentation. An $\mathrm{LN}_{2}$-cooled radiation shield is used between the magnet and vacuum vessel. The inner radtus of the vacuum vessel is $2.24 \mathrm{~m}$, the width of the vessel is $1.37 \mathrm{~m}$, and the outer radius is $3.94 \mathrm{~m}$.

Conductor and Insulation. Figure 3.1-3 shows the design of the layerwound conductor. The superconductor may be either cable or monolithic. The Inner two regions of the coll have an OFHC copper-stabilized NbTi superconductor reinforced with a stainless steel core to sustain the larger hoop forces occurring in the high field regions. The outer, low-field region of the coll uses a $\mathrm{BDTI}$ superconductor stabilized with OFHC copper without the steel core. The peak stress is $40 \mathrm{ksI}$ in regions I and II and $28 \mathrm{ksi}$ in region III. Reinforcing the superconductor with a high-strength core material is a new concept presently being studied.

The insulation is made of polyimide fiberglass, a relatively radiationresistant insulator. Turn-to-turn insulation uses the "button-on-a-string" concept; snow-fence perforated sheets are used for the layer-to-layer insulation. Conductor-to-ground insulation consists of multi-thicknesses of polyimide fiberglass sized according to location.

Conductor cooling. The conductor is cooled with pool boiling LHe I at $4.5 K$ and $1 \mathrm{~atm}$. The design heat flux is $0.25 \mathrm{~W} / \mathrm{cm}^{2}$. This value is based on test data obtained for pool boiling heat transfer in liquid helium within simulated conductor packs. Proper interconductor spacing and insulation geometries yield minimum film botling fluxes of $0.25 \mathrm{~W} / \mathrm{cm}^{2}$. Since the design flux is the minimum film boiling flux, the central-cell conductors are designed to be unconditionally cryostable and do not rely on the collapse of a normal zone due to end conduction for recovery. The conductor recovery current analysis is presented later.

\subsubsection{Engineering Analysis}

Magnetics. One of the primary goals of the design was to minimize magnet size consistent with the central field, ripple, and the inner radius requirements. We optinized the design using graded current densities to take advantage of the reduction in field with increasing radius. Peak flelds for configurations did not exceed the $8 \mathrm{~T}$ allowed $\mathrm{fo}^{\circ}$ a $\mathrm{MbTi}$ superconductor, indicating that pool-boiling LHe I was a satisfactory coolant. 


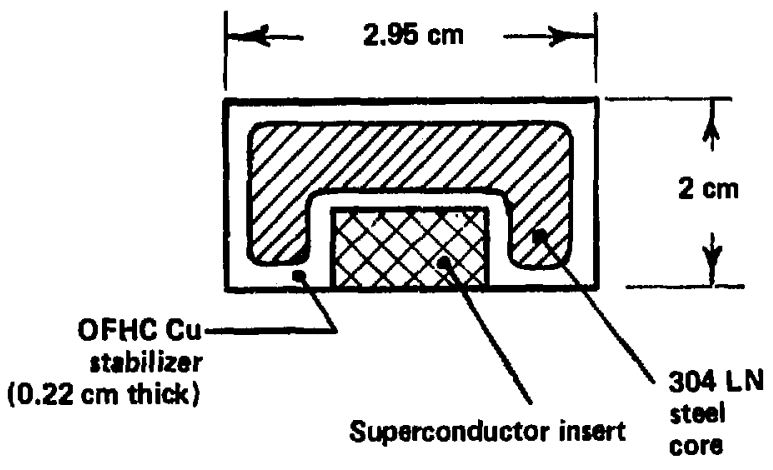

Grades I and II

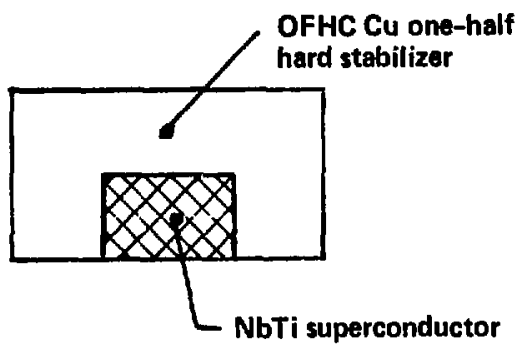

Grade III

Fig. 3.1-3. Central-cell magnet conductor design. 
Stress calculations showed that for reasonable current densities, the stress level of the inner layers exceeded the capability of a copperstabilized conductor. Therefore, we considered a steel-reinforced LHe Icooled conductor. We designed cryostable, steel-reinforced conductors that withstand a 276-MPa (40-ksi) hoop stress as compared with a 193-MPa (28 ksi) allowable stress for a copper-stabilized conductor. The use of high-stress conductors that react their own hoop stresses allowed us to minimize the size of the magnets.

We graded the current density by using cryostability curves giving allowable current density vs field strength for the conducturs under consideration (discussed later). Because field strength decreases with larger radius, the allowable current density rises. The current density was increased until the maximum allowable stress level was reached.

Subsequent EFFI runs using optimized coil geometry verifed that we met the central fields without exceeding the peak field of $8 \mathrm{~T}$. The latest configuration is show in Fig. 3.1-2. The key features of the winding are the three sections of graded current density, the use of LHe I for the coolant, the self-supporting conductors, and the use of a steel-reinforced conductor in the first two grades.

Force and Stress Analysis. Initial analyses of conductor stresses were made assuming self-supporting conductors. After a coll had been sized on this basis, several STANSOL-II computer code ${ }^{l}$ runs were made using varying amounts of substructure in the winding. The STANSOL program takes into account the amount of insulation and gaps; it showed that the conductor stresses matched closely those calculated for free-standing conductors. We concluded that for initial sizing of these magnets, the use of a self-supporting conductor is justifiable and no substructure is required.

In addition to radial forces acting on a conductor, during normal operation there are axial forces that act on the magnets at the ends of the central cell. Under certain fault conditions, some large additional axial forces are also generated. The magnitude of these forces depends on the fault condition; the number of possible fault conditions is large. The EFFI and MAGF codes can quickly calculate these forces for any given fault condition. $A$ worst-case condition is one where all end cell magnets are de-energized, which causes a force of $11.5 \times 10^{7} N\left(25.9 \times 10^{6} 1 \mathrm{~b}\right)$. Fault analysis is also 
linked to the powering of the 48 solenoids, but this aspect of the study has not yet been addressed. In the meantime, the support for the magnets is based on the worst-case load given above.

Conductor Cryostability. To support the central-cell conductor design, cryostability analyses were performed to determine the maximum cryostable current density as a function of field strength for saveral candidate conductors. To facilitate the field calculations, each stability analysis was done In terms of overall current density, packing factor, and conductor unit cell size. The conductor design determines the unit cell area and packing factor. The turn-to-turn and layer-to..1 ayer insulation covers 50\% of the conductor surface; this then determines the wetted parameter. The copper-tononcopper ratio is approximately 10 to 15 for the unreinforced conductors; and approximately one for the steel-reinforced conductors.

The magnetoresistivity of the copper stabilizer is computed from

$$
\frac{P_{D}(B)-P_{D}(0)}{P_{D}(0)}=4.37\left[\frac{B}{P_{D}(0) \times 10^{9}}\right]^{1.115},
$$

where $p_{D}(B)=$ degraded resistivity at field $B($ ohm- $\mathrm{cm})$,

$P_{D}(0)=$ degraded resistivity at zero field $(0 \mathrm{hm}-\mathrm{cm})$, and

$B \quad=$ field strength (T).

Equation (3.1-1) is similar to an equation presented by Kohler ${ }^{2}$ and gives the magnetoresistance of copper damaged by neutron radiation. ${ }^{3}$ For half-hard copper--the material selected for the central-cell solenoid stabilizer--the resistivity at $4.5 \mathrm{~K}$ is $1.9 \times 10^{-8} \mathrm{ohm}-\mathrm{cm}$.

The design heat flux $q$ is limited by the conductor's ability to transfer heat to Lhe I by pool boiling. For an uncondftional stability criteria, the design flux is the minimum film botling, or recovery flux. We conducted a series of tests to measure heat transfer to LHe I from conductors arranged in bundles (to simulate actual winding packs). Through these tests we established correlations between the minimum film boiling flux and the average gap dimension between conductors. 4 Using these data, we chose a design flux of $0.25 \mathrm{~W} / \mathrm{cm}^{2}$ on the conductor-wetted surface. 
The magnetoresistivity of radiation-damaged copper was determined for several typical conductors, including both conventional copper and steelreinforced stabilizer designs, so that we could obtain plots of current density vs field. These plots were used for the first sizing of the colls; an iteration was then performed. Figure 3.1-4 shows the final data for the baseline central-cell conductors. Also indicated on the curves are the actual operating points for the three grades of conductor. The fact that the points fall on the allowable curves fllustrates that the current densities are linited by cryostability.

Radiation Limits. The magnet components most affected by radiation are the stabilizer, insulation, and superconductor. We set the following radiation limits and guidelines to ensure adequate service performance over a 24FPY lifetime:

1. $5 \times 10^{9}$ rad for polyimide insuiation over $24 \mathrm{FPY}$.

2. < $10 \%$ decrease in critical current density of the NbTi superconductor over 24 FPY. This corresponds to approximately $4 \times 10^{18}$ $\mathrm{n} / \mathrm{cm}^{2}, E_{n}>0.1 \mathrm{MeV}$, neutron fluence for $24 \mathrm{FPY}$.

3. $1.1 \times 10^{-4} \mathrm{dpa}$ in the copper stabllizer before annealing.

4. $0.06 \mathrm{mH} / \mathrm{cm}^{3}$ peak nuclear heat deposition in winding.

Our rationale for setting these limits was because the poiyimide-glass insulation retains more than $80 \%$ of its pre-irradiation compression strength after a dose of $5 \times 10^{9}$ rad. This dose is about an order of magnitude higher than that for epoxy glass insulation for equivalent strength loss. The polyimide glass insulation costs about four to six times more than epoxy glass. Epoxy glass insulators are used in low-dose areas of the magnet.

The radiation 1 imit for a NbTI superconductor is within a factor of two of that for polyinide-glass insulation. Neutronic calculations for determining the absorbed dose in the insulation took into account contributions from both gammas and neutrons (which are roughly equal in magnitude). The gammas have little effect on the critical properties of a BbTi superconductor.

For our magnet cryostability analysis we allowed a $20 \%$ increase, due to irradiation, in electrical resistivity of the copper stabilizer. The increase for half-hard OFHC copper at $8 \mathrm{~T}$ anounts to approximately $13 \times 10^{-9} \mathrm{ohm}-\mathrm{Cm}$ at 


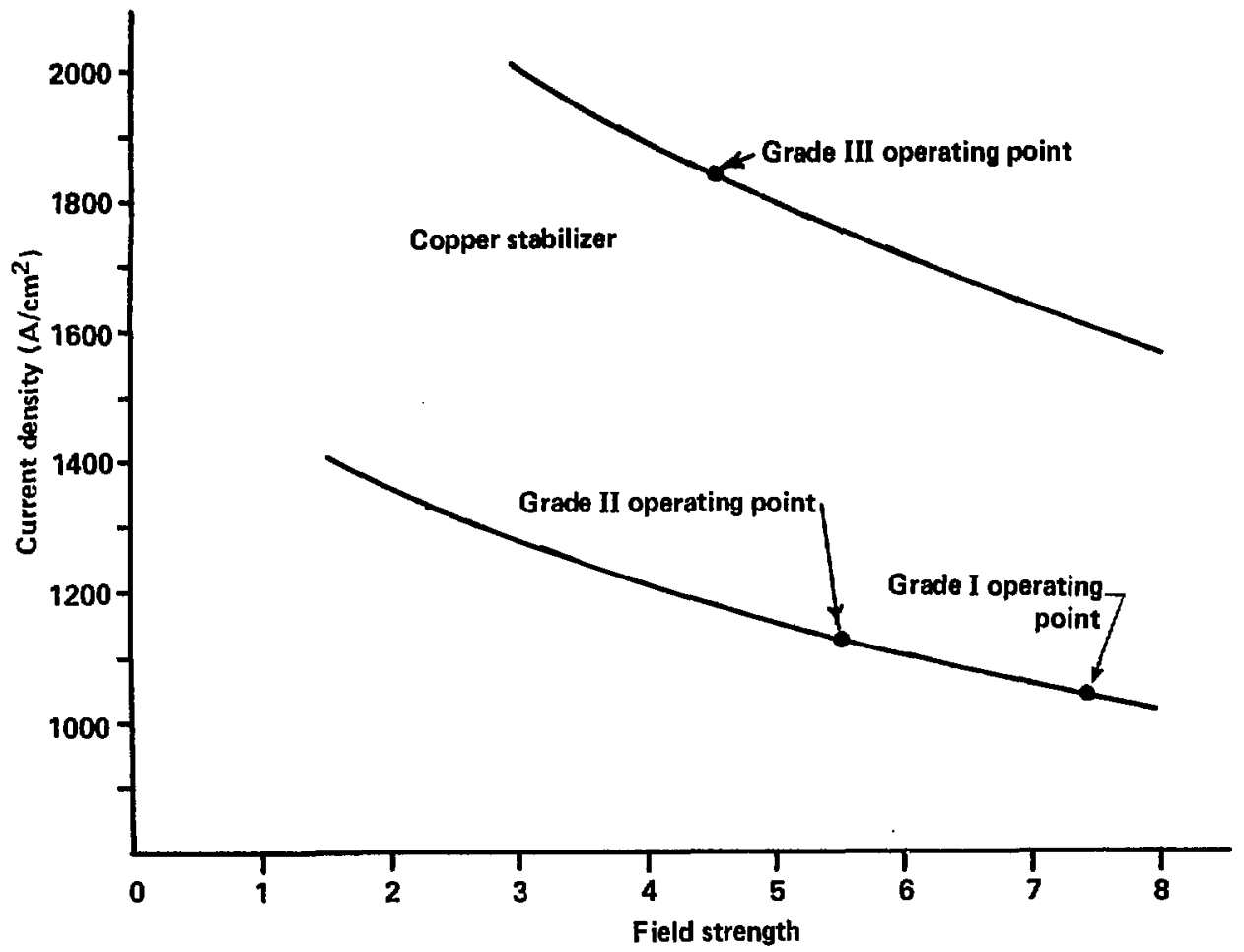

Fig. 3.1-4. Maximum cryostable current density for central cell solenoids. 
4.5 $\mathrm{K}$. The increase in electrical resistivity $\Delta \rho_{i r r}$ of OFHC copper at $4 \mathrm{~K}$ for a fast-fission neutron spectrum has been experimentally determined ${ }^{5}$ as

$$
\Delta p_{i r r}=400\left(1-e^{-1620}\right) \times 10^{-9} \text { ohm-cm, }
$$

where $D=$ radiation damage in $\mathrm{dpa}$. For $D=1.1 \times 10^{-4} \mathrm{dpa}$, this yields $\Delta p_{\text {irr }}$ $=7.06 \times 10^{-9} \mathrm{ohm}-\mathrm{cm}$. In a subsequent room temperature anneal, about 80 to 85\% of this radiation-induced resistivity anneals out, leaving about $1 \times 10^{-9}$ ohm-cm as residual resistivity in the stabilizer. Thus, the allowed increase in electrical resistivity of $13 \times 10^{-9}$ ohm-cm can be net by having the first anneal after more than 5 FPY and always having more than 1 FPY between anneals.

The rationale for arriving at $0.06 \mathrm{mw} / \mathrm{cm}^{3}$ peak nuclear heat deposition in the winding is analogous to that discussed in Section 3.1.2.2. This heat deposition is low enough to satisfy all radiation limits of the materials and also minimizes the total cost of magnet refrigeration equipment.

\subsubsection{Axicell Superconducting Magnet}

\subsubsection{Destgn Considerations}

Table 3.1-4 1ists the basic requirements for the superconducting background coll of the axicell. The 16-T peak field has dictated the use of $\mathrm{Mb}_{3} \mathrm{Sn}(\mathrm{Ti})$ as a superconductor cooled with LHe II as well as the use of steelreinforced conductors.

The magnetic field produced by the superconducting coil should be as high as possible to limit the power dissipation from the resistive insert cofls. This has led to the design of a superconducting coll employing advanced concepts. We have chosen a peak field 1 imit of $16 \mathrm{~T}$ in the superconducting coll because this appears to be the limit attainable using $\mathrm{St}_{3} \mathrm{Sn}$ (Ti) and LHe II. The baseline design with the above assumptions gives a central field slightly above $15 \mathrm{~T}$. This is sufficient to reduce the power dissipation of the four resistive coils to 125 MW.

The large magnetic field in the windings interacting with the conductor current creates large hoop loads so that reinforced conductors are necessary. Additional large magnetic loads are the axial compressive load and the 
Table 3.1-4: Requirements for axicelī superconducting background magnet.
Peale field
$<16.0 \mathrm{~T}$
Operating IIfe
24 FPY (30 yr total)
Cryostat clear bore radius
$<1.12 \mathrm{~m}$

LHe II case and vacuum vesse?

ASME pressure vessel code

axial net force on the coill. The effect of these loads on the detailed stress distribution in the conductor pack and on the casing sizing will be addressed in the future.

\subsubsection{Engineering Trade-0ff Stucies}

Coil Alternatives. Several coil configurations were considered for the axicell. Originally, the magnets were grouped to form a single barrier consisting of nested supercond ting coils and a resistive insert. Trade-off studies of 20-, 24-, and io-T coils with various amounts of shielding showed that a peak field of $24 T$ is an optimum design pcint based on plasma $Q$, resistive coil power, and coll cost.

As the plasma physics requirements were lipdated, the magnet configuration also changed, with the barrier coil evolving into an ixicell wich two sets hybrid of coils rather than a single one. The most recent change in the axicell resulted from combining the two superconducting coi?s while still retaining the two resistive inserts as shown in Fig. 3.1-1. This change was made because furces betweeri the cwo superconducting coils required a large intercoil support and shieid structures that did not leave enough space to accormodate neutral beam injection between the coils.

Choice of Shield Thickness. He conducted an optimization study to compare shield and magnet costs with cryogenic system costs for the axicell. Our resuits are presented in Fig. 3.1-5. The cost of the shield is small, but 


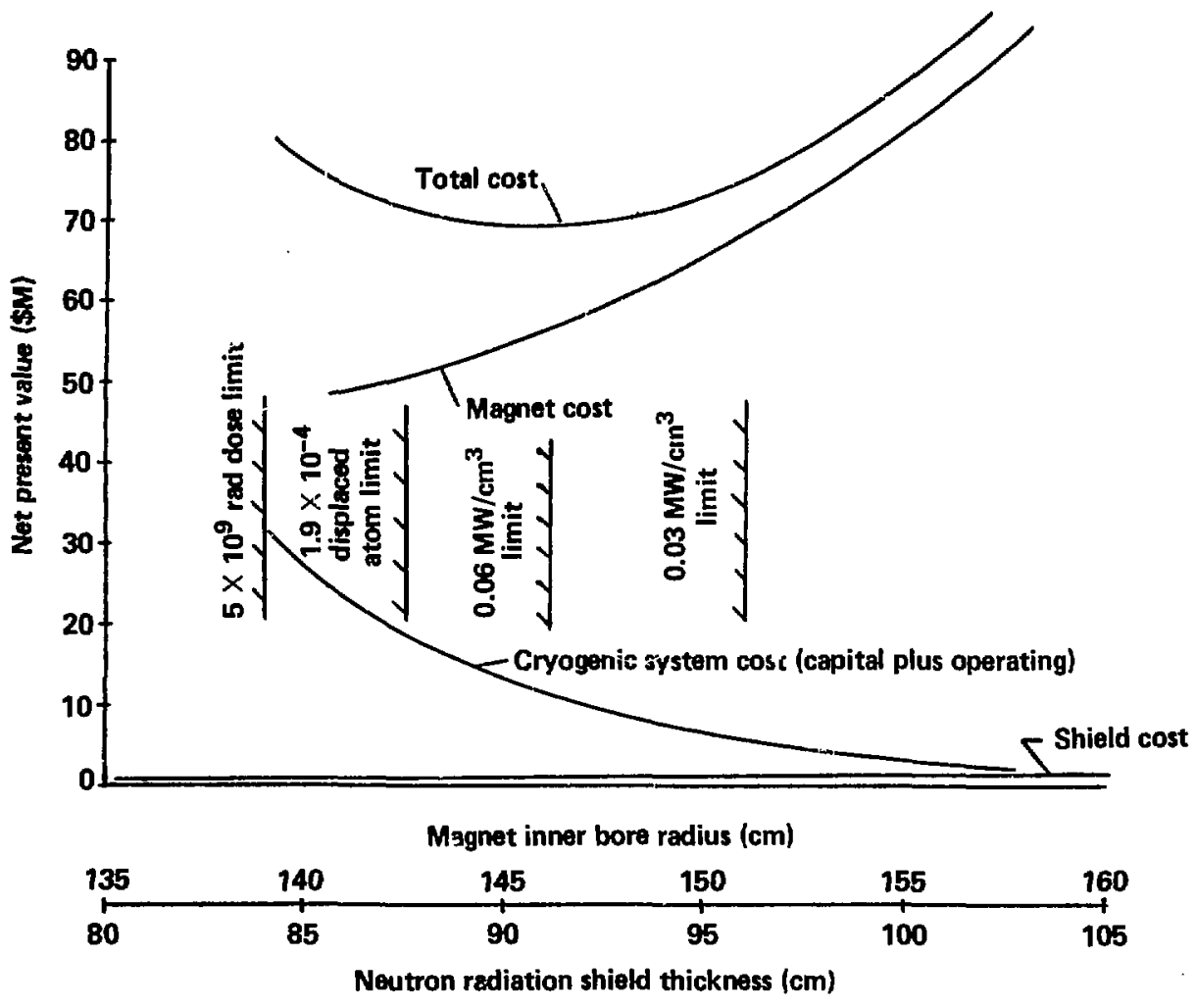

Fig. 3.1-5. Trade analysis of the axicell superconductor shield. 
the magnet cost increases with increasing shield thickness because of the larger size and higher stresses necessary to achieve the required central field. The cryogenic system cost decreases with increasing shield thickness because the heat load decreases. The minimum cost is based on an iron shield approximately $90 \mathrm{~cm}$ thick. As shown in the figure, the $90-\mathrm{cm}$ shield keeps the magnet within all radiation limits. Similar conclusions are reached for a higher-cost tungsten shield.

Cholce of Cooling. Initially, we considered both LHe I and LHe II as the coolant for the superconducting axicell background coil. LHe I was considered because of proven cryogenic technology and LHe II because it allows operation with higher current densities to produce higher fields. Preliminary estimates of maximum design heat fluxes were $0.25 \mathrm{~W} / \mathrm{cm}^{2}$ for LHe 1 and $2.0 \mathrm{~W} / \mathrm{cm}^{2}$ for LHe 11 . Because the maximum cryostable current density is proportional to the square root of heat flux, LHe II allows a current density 2.8 times higher than that of LHe 1. However, in later estimates, the LHe II heat flux was reduced to $1.0 \mathrm{~W} / \mathrm{cm}^{2}$. Therefore, LHe II current density is about twice that of LHe 1 . LHe II requires a much smaller and less expensive magnet than LHe 1 . This benefit outweighs the cryogenic plant capital and operating cost penalties of LHe II over LHe I. For these reasons, we chose LHe Ii as the coolant for the superconducting axicell.

\subsubsection{Magnet Design}

Each axtcell provides two high-field points along the z-axis. The field strengths are $21 \mathrm{~T}$ at $z= \pm 80.2 \mathrm{~m}$ and $24 \mathrm{~T}$ at $z= \pm 82.2 \mathrm{~m}$. These field strengths are obtained by using a superconducting magnet that encircles two smaller resistive magnets. Figure 3,1-6 shows the layout of the system. To minimize the ohmic power loss in the resistive colls, the encircling superconducting magnet is designed for the highest achievable peak fields. As we have already stated, we have chosen a 16-T limit because it is the practical upper bound for $\mathrm{Nb}_{3} \mathrm{Sn}$ superconductor doped with $\mathrm{Ti}$. Because of the high peak field, $16 \mathrm{~T}$, and the high conductor hoop stress of $276 \mathrm{MPa}$ (40 $\mathrm{ksi}$ ), we are using a steel-reinforced conductor and LHe II for cooling. A LHe I-cooled coil using a copper-stabilized conductor would require a much larger bulld.

As in the central cells, the same current will flow in the layer-wound conductors; they are graded to provide varying current densities. The first 


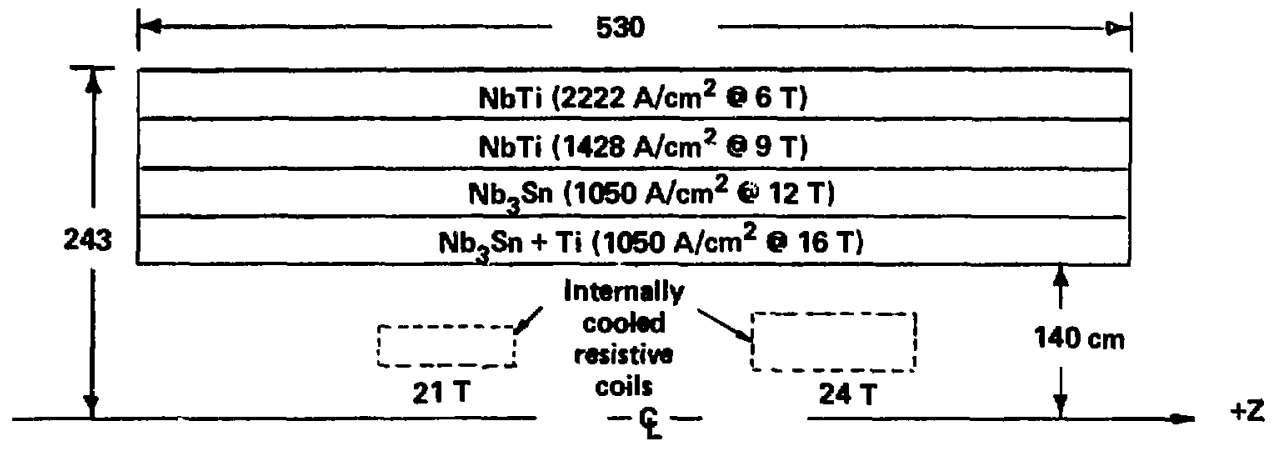

Fig. 3.1-6. Axicell magnet layout. 
section uses $W_{3} \mathrm{Sn}$ doped with $\mathrm{TI}$. for the superconductor. The stabilizer is copper used in conjunction with a 304LN stainless steel reinforcing substrate. The second section uses the same stabilizer and reinforcement, but the superconductor is $\mathrm{m}_{3} \mathrm{Sn}$ without the $\mathrm{Ti}$ doping. This section starts where the field is $12 \mathrm{~T}$. The third section starts where the field is $9 \mathrm{~T}$. The superconductor is $\mathrm{NbTH}$ and the steel-reinforced conductor is reduced in size to increase the allowable stress level to $276 \mathrm{mpa}$. The fourth section again uses $\mathrm{NbTf}$ as the superconductor; the field is fust under $6 \mathrm{~T}$ and the stabilizer is again graded to give a stress approaching $276 \mathrm{MPa}$.

The coll is encased in a Lhe II ressel fabricated from 304 LN stainless steel which is, in turn, surrounded by a LHe I-cooled radiation shield and an outer $\mathrm{LN}_{2}$-cooled radiation shield. Each superconducting magnet has a vacuum vessel. Polyimide insulation is used because of its resistance to radiation degradation. Each superconducting coil has a service stack for all cryogenics, current leads, instrumentation, and exiting gases.

Conductor and Insulation. Figure 3.1-7 shows the basic conductor concept for the axicell superconducting magnet. The conductor carries $7848 \mathrm{~A}$ and has an OFHC copper stabilizer. Transverse and longitudinal grooves increase the cooling area of the stabilizer so that critical heat flux does not exceed $1 \mathrm{~W} / \mathrm{cm}^{2}$. The conductor is supported by a stainless steel backing strip with a rib fitting in the stablilizer. The effective cross-sectional area of the conductor vartes from one graded winding zone to the next to accomodate the current densities shown in Fig. 3.1-6. Figure 3.1-8 shows an alternate conductor concept that incorporates a high-strength core for reacting the electromagnetic hoop forces. This conductor can achieve higher current densities than the baseline, but requires more development.

The insulation is polyimide fiberglass similar to that used in the central-cell solenoids. Turn-to-turn insulation consists of "buttons on a string" placed at regular intervals along the conauctor. Snow-fence perforated polyimide sheets are used for the layer-to-layer insulation. Conductorto-ground insulation is also polyimide fiberglass that is sized according to location.

Conductor cooling. LHe II is unique in that heat is transferred via a complex heat conduction mechanism, and not by the normal convection mode of typical fluids. Numerous experimental studies show that the maximum heat flux 


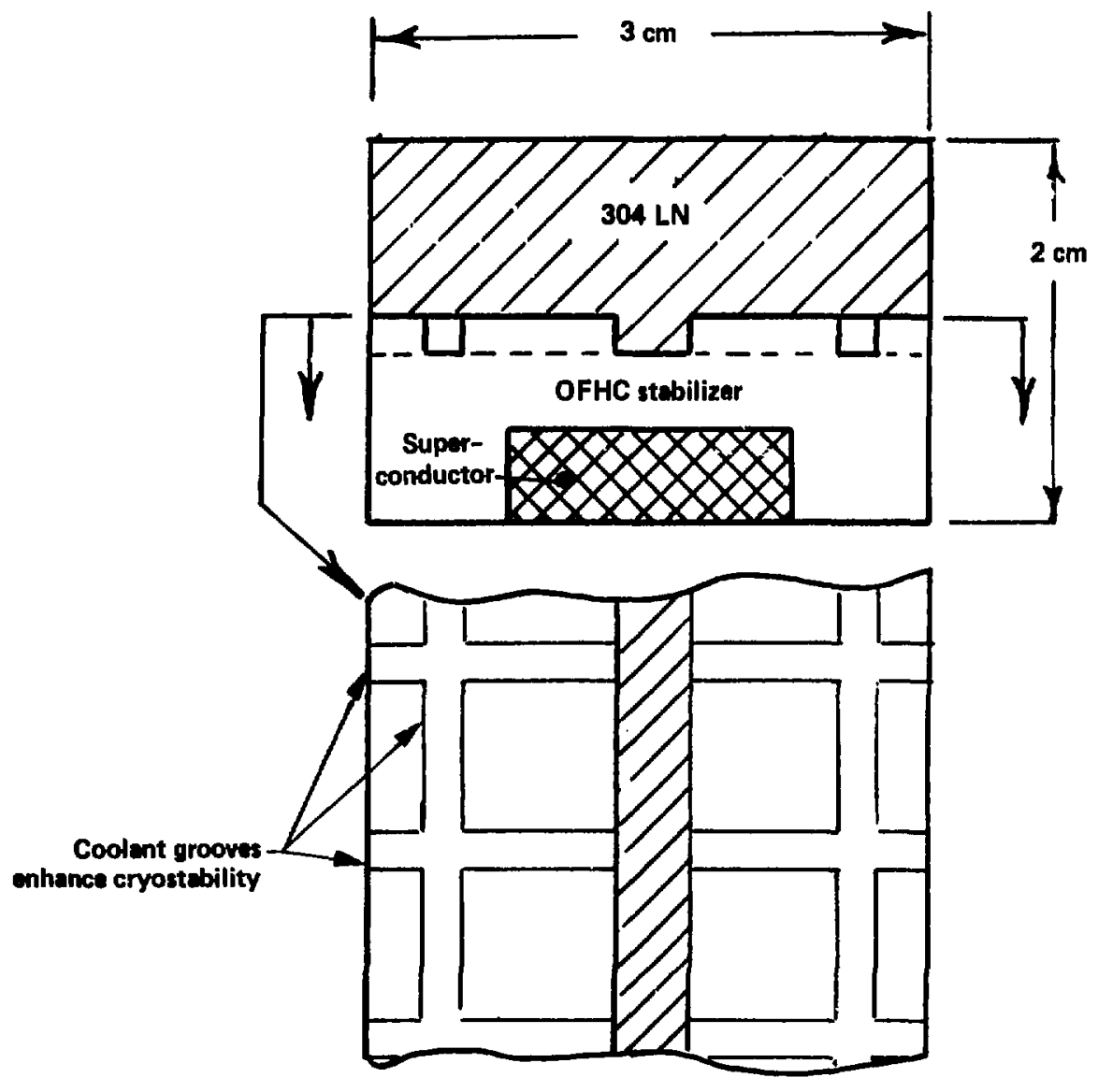

Fig. 3.1-7. Baseline design of the axicell magnet conductor. 


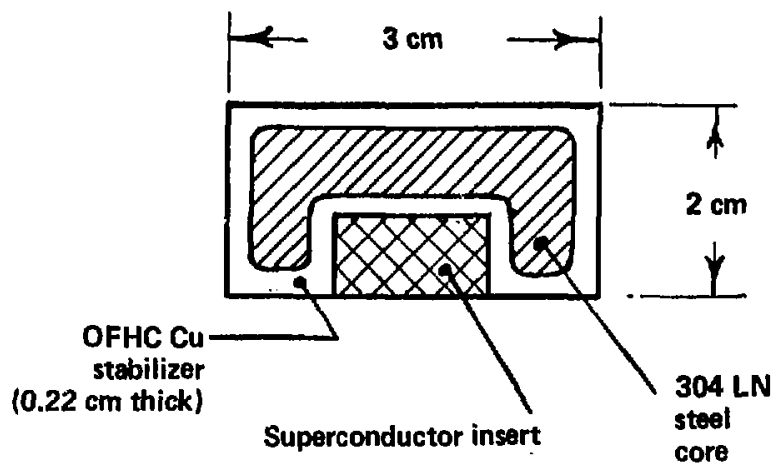

Fig. 3.1-8. Alternate design for the axicell magnet conductor. 
in a channel of Lhe II is inversely proportional to the cube root of the channel length. Hoard ${ }^{6}$ has shown that eight channels are avallable to cool a nomal conductor within a winding. Each channel has an equivalent length equal to 1.5 times the conductor unit cell dimension in the parallel direction. This theory gives an expression for maximum heat flux on the conductor as a function of conductor size, insulation thickness, and insulation coverage. We can equate the maximum heat flux to the joule heat flux generated by a normal conductor, such that

$$
J^{2} \text { 次 }\left(\frac{1+\phi}{\lambda \phi}\right)=29.6\left[\left(\frac{B}{L_{N}^{1 / 3}}\right)+\left(\frac{C}{L_{V}^{1 / 3}}\right)\right] \text {. }
$$

where

$J=$ current density over unit cell $\left(A / \mathrm{cm}^{2}\right)$,
$p=$ stabilizer electrical resistivity $(\mathrm{ohm}-\mathrm{cm})$,
$A=$ area of unit cell $\left(\mathrm{cm}^{2}\right)$,
$\lambda=$ copper/non-copper ratio in conductor,
$\lambda=$ packing factor (conductor area/unit cell area),
$\mathrm{C}=$ cross sectional area of radial channels between conductors,
$\mathrm{L}_{N}=$ cross sectional area of axial channels between layers,
$L_{V}=1.5$ times unit cell axial dimension $(\mathrm{cm})$, and

Equation (3.1-2) is used to perform cryostability analyses and conductor sizing iterations to optinize the conductors.

He initially chose $2.0 \mathrm{~W} / \mathrm{cm}^{2}$ as the maximum heat flux at the surface of the conductor. Subsequent conductor and pack sizing iterations showed that $1.0 \mathrm{~W} / \mathrm{cm}^{2}$ was a more reasonable and iractical level; this value ensured cryostability at the design current densities of each grade of conductor.

\subsubsection{Engineering Analysis}

The engineering analysis of the axicell superconducting coil includes magnetics, forces, stresses, cryostability, and radiation environment considerations. The methods of analysis are essentially the same as those used for the central-cell magnets described in Section 3.1.1.4. 
The cryostability analyses of the conductors, which allow us to derive curves of $J$ vs $B$, differ from the analyses of the central-cell conductors because Eq. (3.1-2) is used to calculate the maximum heat flux. The new curves are presented in Fig. 3.1-9. The actual operating points fall below the curve because the conductors are stress-limited.

The radiation limits for the axicell differ from those of the central cell in two respects. The radiation limit, before annealing at a $20 \%$ increase in resistivity in the stabilizer at $16 \mathrm{~T}$, is increased to $1.9 \times 10^{-4}$ dpa because copper has a higher magnetoresistance at this field. The radiation linit for the superconductor is decreased to $2 \times 10^{18} \mathrm{n} / \mathrm{cm}^{2}$ because $\mathrm{Nb}_{3} \mathrm{Sn}$ is more radiation-sensitive that NoT1.

\subsubsection{Axtcell Shield}

The radiation source in the axicell region of the reactor is high enough so that shielding of the superconducting coil must be provided to meet the design radiation limits. We concluded a one-dimensional analysis of three paths: (1) through the inboard side of the superconductor where there is no resistive insert; (2) through the 21-T (M1) insert; and (3) through the 24-T (M2) insert.

We conducted a study of alternate materials and configurations and selected an option for path 1 with a layer of tungsten $59 \mathrm{~cm}$ thick covered by an iron shield $\left(90 \% \mathrm{Fe}-1422,10 \% \mathrm{H}_{2} \mathrm{O}\right) 14 \mathrm{~cm}$ thick, and a layer of boron carbide $2 \mathrm{~cm}$ thick. For paths 2 and 3, the thicknesses of the regions were reduced proportionately with the shield thickness. The material compositions were unchanged. The results of the calculations for these three sections are given in Table 3.1-5. The design linit for the spinel insulation in the resistive insert is presently $3 \%$ volumetric swelling. This corresponds to about 1.5 FPY life to replacement. If $5 \%$ volume swelling can be tolerated, the life of the insert colls would be 2 FPY from this effect. However, the increase in resistivity from transmutations dominates the lifetime and is discussed in Section 3.1.3. The design limits for the superconducting coil are all met except for peak heating. When the heating rates are averaged over the volume of the coil, the cost of cooling is acceptable. 


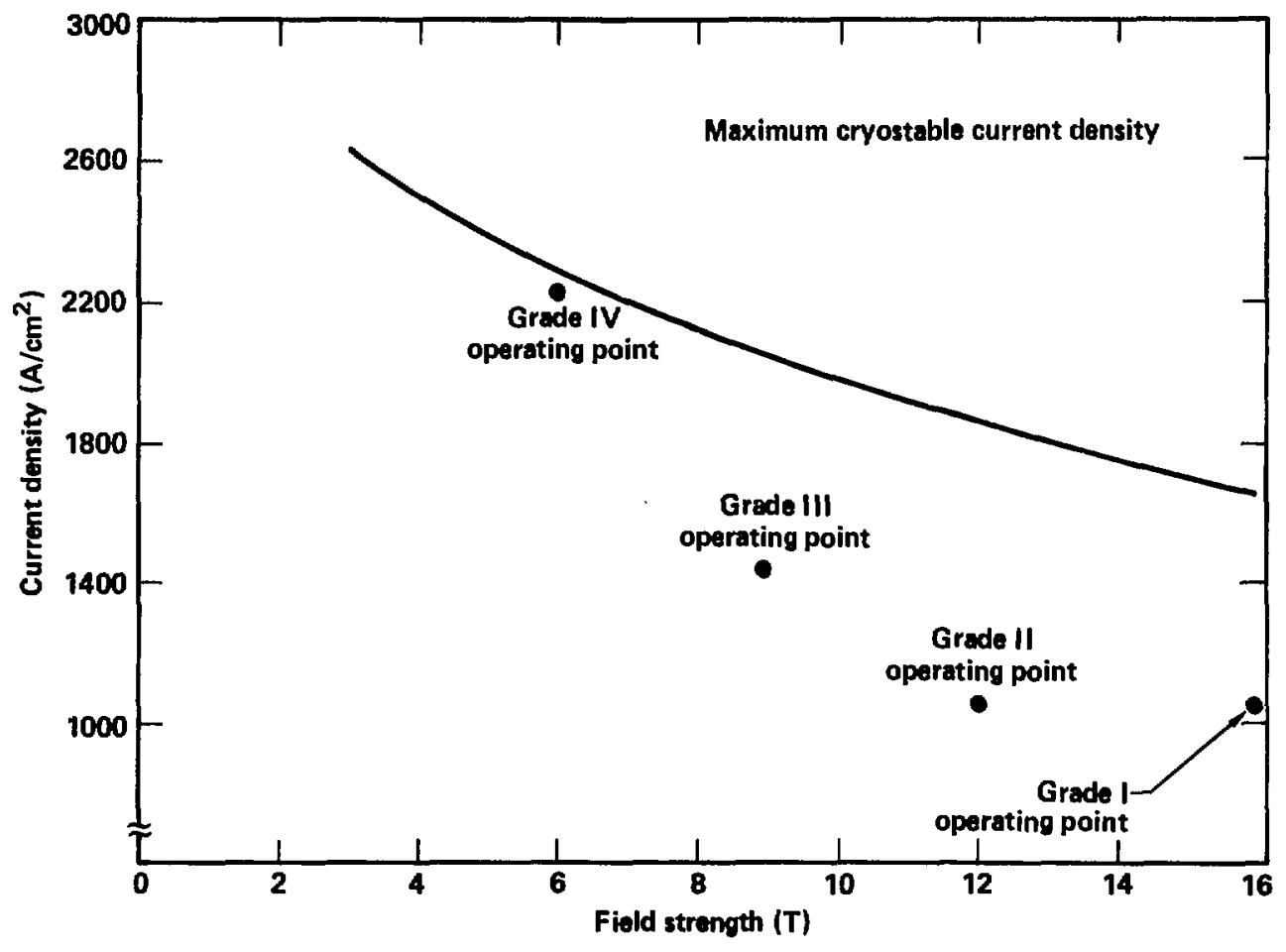

Fig. 3.1-9. Axicell cryostability limit. 
Table 3.1-5. Axicell radiation shielding.

\begin{tabular}{|c|c|c|c|c|c|c|c|}
\hline $\begin{array}{l}\text { Coil } \\
\text { path }\end{array}$ & $\begin{array}{c}\text { Wall } \\
\text { loading } \\
\left(\mathrm{MW} / \mathrm{m}^{2}\right)\end{array}$ & $\begin{array}{l}\text { Wall radius/ } \\
\text { shield thickness } \\
(\mathrm{cm})\end{array}$ & $\begin{array}{c}\text { Spinel peak } \\
\text { fluence } \\
\mathrm{E}_{\mathrm{n}}>0,1 \mathrm{MeV} \\
\mathrm{n} / \mathrm{cm}^{2} \mathrm{FPY}\end{array}$ & $\begin{array}{l}\text { Polyimide peak } \\
\text { dose } \\
\text { (rad/24 FPY) }\end{array}$ & $\begin{array}{l}\text { Peak } \\
\text { nuclear } \\
\text { heating } \\
\left(\mathrm{mW} / \mathrm{cm}^{3}\right)\end{array}$ & $\begin{array}{c}\text { Peak iu } \\
\text { damage } \\
\text { (dpa/FPY) }\end{array}$ & $\begin{array}{c}S / C \text { peak } \\
\text { fluence } \\
E>0.2 \mathrm{MeV} \\
\mathrm{n} / \mathrm{cm}^{2}\end{array}$ \\
\hline 1 & 3.6 & $\begin{array}{l}34.5 / \\
75 \text { shield }\end{array}$ & -- & $2.56+9$ & 0.0746 & $3.76-5$ & $9.70+17$ \\
\hline 2 & 4.8 & $\begin{array}{l}27.5 / 23 \mathrm{Cu} \\
51 \text { shield }\end{array}$ & $3.28+22^{a}$ & $1.76+9$ & 0.0635 & $2.18-5$ & $7.14+17$ \\
\hline \multirow[t]{2}{*}{3} & 4.8 & $25.8 / 49.3 \mathrm{Cu}$ & $3.49+22$ & $2.40+9$ & 0.0387 & $2.46-5$ & $8.00+17$ \\
\hline & $\begin{array}{l}\text { Design } \\
\text { limits }\end{array}$ & & $\begin{array}{l}4.13+22 \text { for } \\
\text { for } 3 \text { v/o } \\
\text { swelling }\end{array}$ & $5.0+9$ & 0.06 & $\begin{array}{l}1.90-4 \\
\text { after } 5 \mathrm{FPY}\end{array}$ & $3.0+18$ \\
\hline
\end{tabular}

a Read as $3.28 \times 10^{22}$. 


\subsubsection{Conductor Development for Axicell Superconducting Magnets}

The design of the axicell superconducting magnet is based on advanced superconductor concepts. Major developments still needed include:

- Fabrication and verification testing of conductors using titaniumdoped $\mathrm{Nb}_{3} \mathrm{Sn}$ for application at the higher magnetic field levels.

- Verification of cryostability of the l.He II-cooled advanced conductors.

- Development of detail designs and fabrication techniques for steelreinforced conductors to evaluate conductor performance and cost.

- Detalled characterization of polyimide insulation properties with regard to radiation tolerance.

- Use of LHe II in large system applications.

\subsubsection{Axicell Resistive Magnets}

\subsubsection{Design Considerations}

Low power dissipation, small coll build, and long operational life in the radiation environment are the most desirable coil features. Design simplicity and easy coil replacement are equally important from construction and maintenance points of view. Minimizing the power requirement is possible by selecting proper coll geometry and current distribution in the windings. However, colls with constant current distribution and appropriate geometry can approximate the ideal coll, and selected them for the MARS design because they minimize coll size and are easier to manufacture. The basic design limitations of these colls are stresses and cooling. Electrical integrity is also a limitation, but its causes are difficult to identify because they depend on individual manufacturing details as well as on design parameters.

Encircled by a 16-T peak-field superconducting coil, the resistive inserts must generate additional central fields to meet the 21- and 24-T requirements and provide the clear bore necessary for the plasma. In addition, the colls should minimize radial build and onmic power loss. We have met these requirements using a uniform current density coil. Future design changes may further reduce the size and power of the coils over the baseline design. 
Because the stress level within the resistive coils is quite high, we selected a magnesium-zirconium-chromium (MZC) copper alloy. Cooling is a limitation for internally cooled MZC magnets because of their relatively long and small water paths, but we chose this option because if offers high confidence in long expected lifetimes.

Of all the magnets in the MARS magnet set, the axicell resistive magnets sustain the most severe radiation exposure. The peak radiation dose rate is approximately $10^{6} \mathrm{rad} / \mathrm{s}$. Space 1 imitations on these magnets do not leave room for radiation shielding. Any radiation shielding placed between the required clear bore and the magnets increases costs because of greater power dissipation in the winding and the increased cost of the surrounding superconducting coil. The radiation environment degrades the mechanical and electrical properties of the components and reduces their service life.

The requirements for the axicell resistive magnets are summarized in Table 3.1-6, and include central fields of 21 and $24 \mathrm{~T}$, clear bores of 54 and $58 \mathrm{~cm}$, and an operational life of more than $1 \mathrm{yr}$, which is about an order of magnitude longer than that of existing high-field coils.

\subsubsection{Engineering Trade-0ff Studies}

Coil Alternatives. Several types of coils have been considered: (1) Bitter coils; (2) modified Kelvin distribution coils as exemplified by the TASKA design ${ }^{7}$; (3) internally cooled uniform current density coils; and (4) externally cooled uniform current density costs. Our initial choice was the externally cooled, uniform-current density coil shown in Fig. 3.1-10 because of its high achievable current density which results in a much smaller encircling superconducting coil. We did not choose Bitter coils because they must use organic insulation, which does not meet our radiation requirements. The modified Kelvin design, while using less power, requires much larger diameters and axial space.

Most laboratory high-field coils are externally cooled with water. We evaluated several of these, including the 31- $T$ coil at the Francis Bitter National Magnet Laboratory, the 30-T magnet at the Austrailian National University, a 16-T coil at Clarendon, and the Duplex I and II magnets at Grenoble. These magnets have an average service life (without radiation) of about $200 \mathrm{hr}$, which is unacceptable for MARS. We evaluated the causes of 


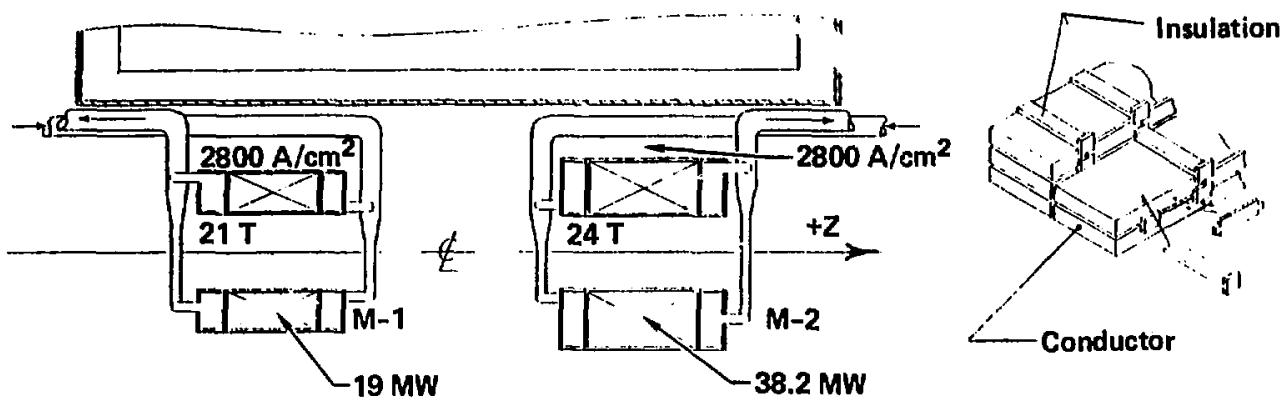

Fig. 3.1-10. Externally cooled resistive coil. 
failures in externally cooled soils. 8,9 Some of the failure mechanisms that we observed for Bitter magnets, their applicability to MARS, and potential solutions are given in Table 3.1-7.

As our investigation continued, it became apparent that separating the coolant and the insulation is necessary to achieve reliable operation for more than. FPY using current or foreseeable technology. Figure 3.1-11 shows our latest concept of an internally coolec coil, our current baseline. The main disadvantages of the internally cooled conductor coil are its lower allowable current density (larger build required) and separate powering for each layer (needed to keep water passages short).

Choice of Conductor and Insulation. The inductor for the resistive magnet has to satisfy two requiraments: high yield strength to withstand hoop stresses and high electrical conductivity for low power dissipation. Silver is the best electrical conductor, copper is next, and gold and aluminum follow. Precipitation-strenythened alloys of copper have high electrical conductivity, high yield strength, and a high recrystallization temperature. We considered and compared several alloys of copper and one alloy of silver. Figure 3.1-12 shows the values for mechanical yield strength and electrical conductivity of these alloys.

The composition of the alloys is:

1. Be - Cu (Be 0.4 to $0.75 \%$, Co 2.4 to $2.7 \%$, balance $\mathrm{Cu}$ ).

2. $\mathrm{Cu}-\mathrm{Ni}-\mathrm{Ti}(5 \% \mathrm{Ni}, 2.5 \% \mathrm{Ti}$, balance $\mathrm{Cu})$.

3. Glidcop Al-20 (0.4\% $\mathrm{Al}_{2} \mathrm{O}_{3}$, balance $\left.\mathrm{Cu}\right)$.

4. Narloy $\mathrm{Z}(2.75$ to $3.25 \% \mathrm{Ag}, 0.3$ to $0.5 \% \mathrm{Zr}$, balance $\mathrm{Cu})$.

5. Consil 900 ; $10 \%, \mathrm{Ag} 90 \%)$.

6. ZAC-1 $(0.1 \% \mathrm{Zr}, 0.3 \% \mathrm{Cr}$, balance $\mathrm{Cu})$.

7. MZC (0.03 to $0.06 \% \mathrm{Mg}, 0.08$ to $0.015 \% \mathrm{Zr}, 0.4$ to $0.8 \mathrm{Cr}$, balance $\mathrm{Cu})$.

8. AMZIRC $(0.13$ to $0.2 \% \mathrm{Zr}$, balance $\mathrm{Cu})$.

Consil 900 is desirable because of its lower induced radioactivity, but it does not have sufficient yield strength. We chose MZC for the conductor material because it has sufficient conductivity and yield strength and is therefore appropriate for the design. 

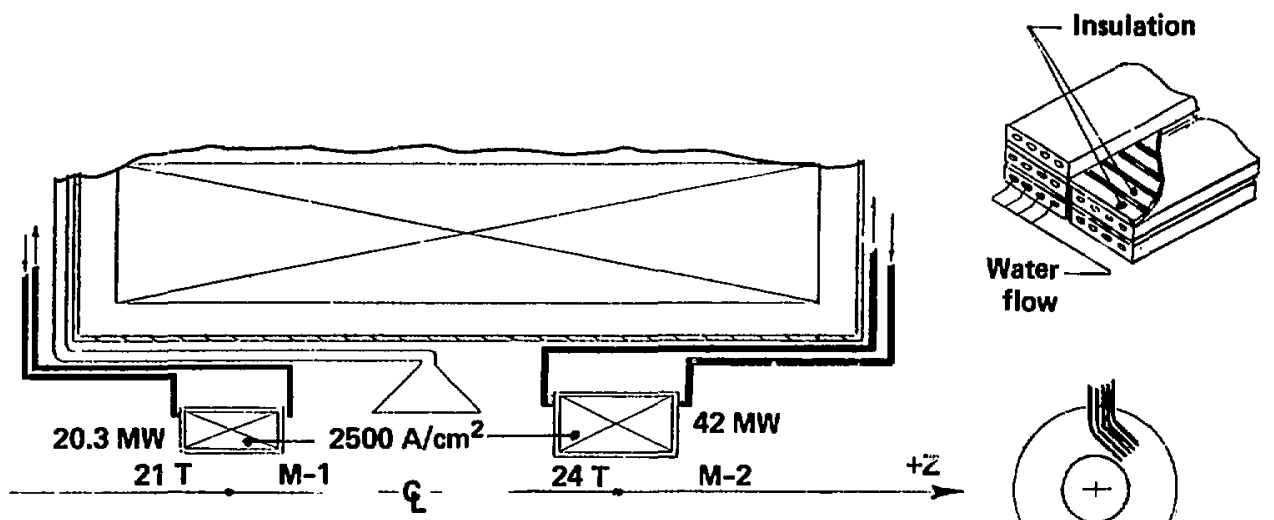

Fig. 3.1-11. Internally cooled resistive coil. 


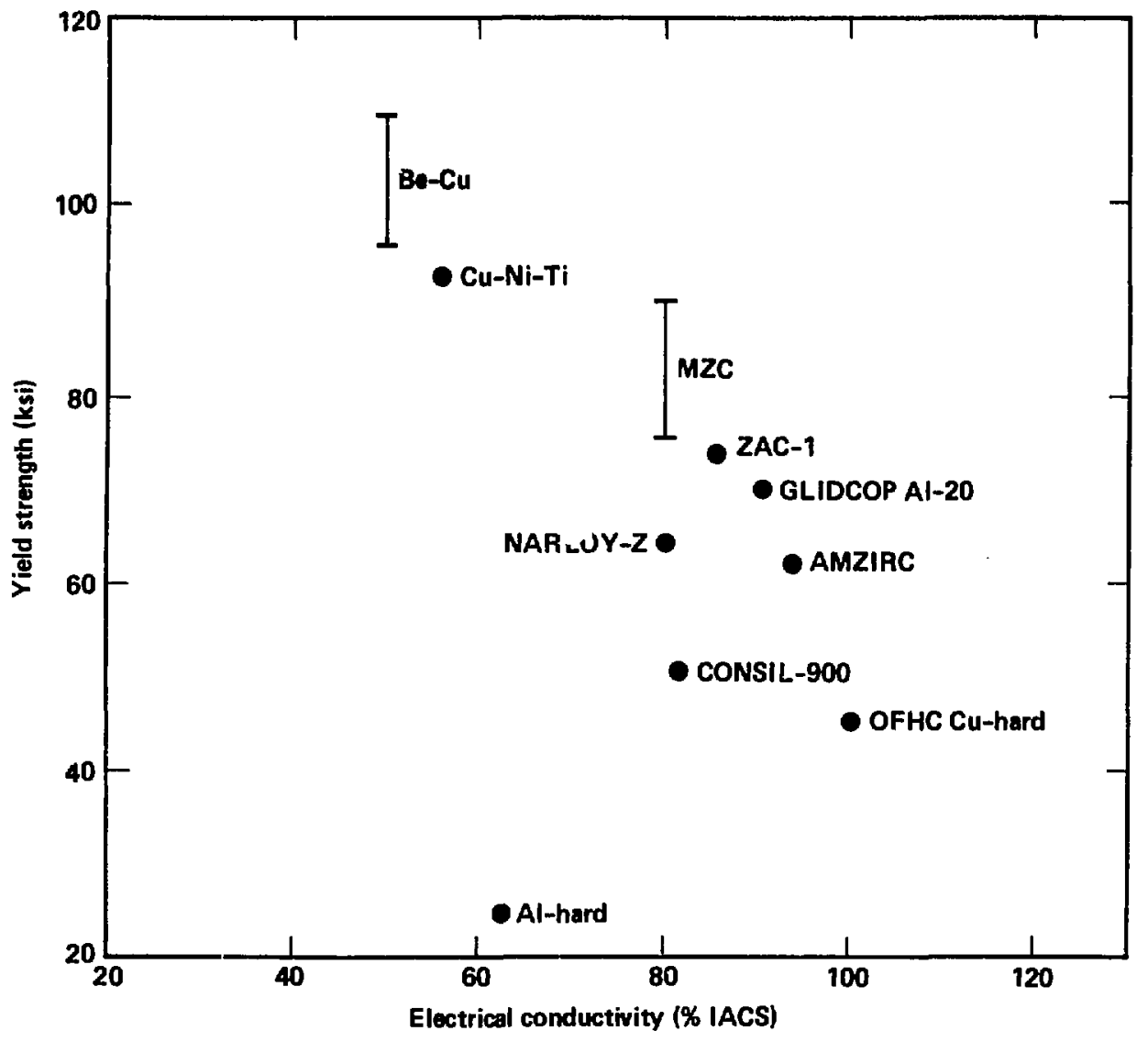

Fig. 3.1-12. Alloy properties for resistive coils. 
Table 3.1-6. Requirements for axicell high-field resistive insert coils.

\begin{tabular}{|c|c|}
\hline Central field & $\begin{array}{l}21.0 \mathrm{~T}\left(M_{1}\right)^{\mathrm{a}} \\
24.0 \mathrm{~T}\left(\mathrm{M}_{2}\right)^{\mathrm{a}}\end{array}$ \\
\hline Operational life & More than $1 \mathrm{yr}$ \\
\hline Clear bore radius & $\begin{array}{l}29 \mathrm{~cm}\left(\mathrm{Ml}_{1}\right) \\
27 \mathrm{~cm}(\mathrm{M2})\end{array}$ \\
\hline
\end{tabular}

Structural case design ASME pressure vessel code

a Combined with superconducting background coll.

Table 3.1-7. Failure mechanisms for Bitter magnets.

Applicability

\begin{tabular}{|c|c|c|}
\hline $\begin{array}{l}\text { Water absorption in } \\
\text { compressed insulation }\end{array}$ & Ho & Copper-clad polyimide \\
\hline $\begin{array}{l}\text { Water attack on copper/ } \\
\text { insulation board }\end{array}$ & No & $\begin{array}{l}\text { Bond epoxy to phenol formal - } \\
\text { dehyde coating }\end{array}$ \\
\hline $\begin{array}{l}\text { Radiolytic corrosion of } \\
\text { copper }\end{array}$ & Yes & Suppresson by coating/plating \\
\hline $\begin{array}{l}\text { Water oxidation of mech- } \\
\text { anical joints }\end{array}$ & Yes & Eliminate or gold-plate joints \\
\hline $\begin{array}{l}\text { Growth of CuO whiskers } \\
\text { in water }\end{array}$ & Yes & Suppression by coating/plating \\
\hline
\end{tabular}

a Ceramics resist water penetration. 
Spinel $\left(\mathrm{Al}_{2} \mathrm{MgO}_{4}\right)$ has low swelling and no microcracking, and it strengthens in a neutron radiation environment. A stress analysis shows that 3 vol \% swelling in spinel can be accomodated in the magnet structure without causing stress problems. This amount of swelling is expected in spinel after 2 FPY of magnet operation. Under the same conditions, alumina $\left(\mathrm{Al}_{2} \mathrm{O}_{3}\right)$ would swell 16\% by volume, develop microcracks, and aeteriorate in mechanical strength. Based on these observations, we selected spinel as the insulator material for the resistive magnets.

Choice of Cooling. He considered water, liquid nitrogen, and gaseous helium to cool the coil. The three coolants were evaluated in an externally cooled coil having a current density of $3700 \mathrm{~A} / \mathrm{cm}^{2}$ (the baseline at that time). The figure of merit was the total power requirement of the coil (resistive losses plus pumping or refrigeration power). We considered water because of its excellent thermodynamic properties; considered the two other coolants because they are chemically inert and could lead to longer lifetimes for externally cooled coils in a radiation environment.

water-Cooled Option. The water-cooled option has a negligible pressure drop and pumping power requirement. The maximum resistive loss in the 24-T coil at end of the magnet lifetime is only 25.9 MN (based on an increase in resistivity of $20 \%$ because of radiation). The conductor temperature is lower than $100^{\circ} \mathrm{C}$. These characteristics are favorable and we tentatively selected water for the baseline coolant. The externally water-cooled case was also used as a point of comparison to evaluate the two other coolants.

Liquid-Nitrogen-Cooled Option. If we assume that the magnet configuration remains the same as the baseline, the resistive power consumption is reduced with $L N_{2}$ coolant by the fraction of copper resistivity that remains at $80 \mathrm{k}$. For pure OFHC copper, resistivity decreases linearly with temperature, from room temperature to $\mathrm{LN}_{2}$ temperature at a rate of about $6.9 \times 10^{-9} \mathrm{ohm}-\mathrm{cm} / \mathrm{K}$, so that the resistivity ratio is approximately 8 . In a neutron environment, this ratio is smaller because the increase in resistivity due to transmutations is independent of temperature. After 1 FPY this resistivity increase is $20 \%$ at room temperature, or $160 \%$ at $80 \mathrm{~K}$. For damaged copper, the ratio of resistivity from 300 to $80 \mathrm{~K}$ is about 3.7 after $1 \mathrm{FPY}$. Thus, for an $\mathrm{LN}_{2}-$ cooled coil, the resistive potver is initially reduced by a factor of 8 , but only by a factor of 3.7 at the end of 1 FPY. 
Conversely, the refrigeration power is high. Theoretically about $3.8 \mathrm{~W}$ of compressor work is required to produce $1 \mathrm{~W}$ of cooling at $\mathrm{LN}_{2}$ temperature. Most real compressors have efficiencies of less than 0.5 , so at best an $\mathrm{LN}_{2}-$ cooled refrigerator requires $7.6 \mathrm{~W}$ input per watt of cooling. Thus, the total power consumed by an $\mathrm{LN}_{2}$-cooled magnet is 8.6 times the joule heat dissipation. For radiation-damaged copper, the trade between water cooling and $\mathrm{LN}_{2}$ cooling shows that the total power requirements for an $\mathrm{LN}_{2}$-cooled magnet is more than twice that of a water-cooled magnet. Moreover, the addition of a cryostat will increase the coll size. For these reasons we rejected the $\mathrm{LN}_{2}$ option.

Gaseous Helium-Cooled Option. The main advantage of GHe as a coolant is that it is inert. Our hope was that the GHe could be pumped through the coil at high enough velocities (and thus a high heat transfer coefficient) to keep the conductor cool without using excessive pump power. However, a heat transfer analysis showed that, for a maxinum conductor temperature of $125^{\circ} \mathrm{C}$, the total power consumption is more than double that for water cooling. There is a 10-MW increase in resistive heating and a 20-MW increase in pump power. Because the GHe cooling concept is not as efficient as water cooling, we rejected it.

We chose water as the baseline coolant because it minimizes the power requirements of the resistive coils. However, because externally cooled coils have reliability problems, ${ }^{8,9}$ we selected an internally cooled conductor coil for the baseline design. Future developments may show that spinel-insulated, externally cooled coils can operate reliably enough, and if this is the case, we will reexamine our design decision.

\subsubsection{Magnet Design}

The two resistive magnet inserts produce axial magnetic fields of approximately 6.0 and $9.6 \mathrm{~T}$. These fields add to the background field of the axicell superconducting coil to obtain total fields of 21 and $24 \mathrm{~T}$.

The coils have a compact build and an efficient shape for reducing ohmic power loss. They are layer-wound with a relatively wide conductor of highstrength low-resistance MZC with insulators made of sintered spinel $\left(\mathrm{Al}_{2} \mathrm{MgO}_{4}\right)$. Electrical leads are ailchored to the coil flanges and are distributed to 
accommodate the ICRH coaxial leads. The coils have inner radi $i$ of 0.30 and $0.32 \mathrm{~m}$ to provide sufficient clearance for the plasma and are enclosed in a casing with a support structure to react the axial magnet forces.

Conductor and Insulation. For reliability of operation, we chose an internally cooled conductor for the baseline. For high current densities, this type of conductor requires relatively short water paths to prevent overheating, which requires cooling and powering each layer separately. The current density of an internally cooled conductor must be less than that of an externally cooled conductor. These factors combine to give a coil that consumes $10 \%$ more power and has a volume $23.5 \%$ greater than a coil using external cooling. However, these disadvantages are offset by increased coil reliability resulting from the use of dry insulation.

The conductor hoop stress is $300 \mathrm{MPa}$ (43.5 $\mathrm{ksi}$ ), which is too high to be able to use OFHC copper. The peak conductor temperature is $200^{\circ} \mathrm{C}$, so to be conservative we selected $M Z C$ copper for the conductor material, which not only has a high allowable stress, but it also has a low resistivity of $80 \%$ of the International Annealed Copper Standard (IACS). The shape of the conductor is a flat bar with four cooling holes. Figure 3.1-13 shows a cross section of the conductor unit cell.

We have chosen spinel for the conductor insulation. Water passages are not necessary between conductors, so we chose the insulation thickness for good electrical isolation of the conductors. Layer-to-layer insulation consists of short rectangular bars butted against each other and the turn-toturn insulation consists of bars curved to match the radius of the coil.

Conductor Cooling. The results of the thermal analyses for the internally and externally cooled resistive coil designs are listed in Table 3.1-8. The flow rate is the major difference between the two cooling schemes. The externally cooled design has a greater flow area, allowing four times the flow rate of an internally cooled coil with negligible pressure drop, and much lower water velocities. Consequently, an externally cooled coil has a cooler conductor at a higher current density as compared with an internally cooled coil. The total power consumption is $124.6 \mathrm{MW}$ for the internally cooled coils vs $114.4 \mathrm{MW}$ for the externally cooled coils. The greater power consumption is justifiable based on the added electrical reliability of internal cooling. 


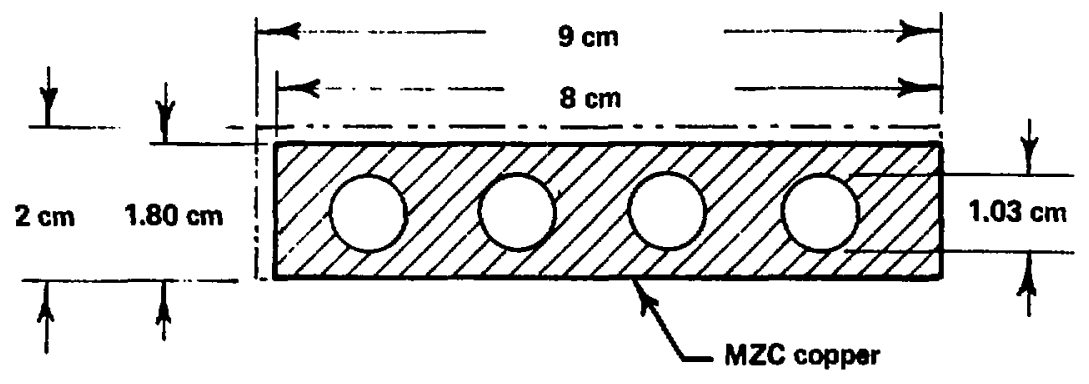

Fig. 3.1-13. Internally cooled conductor design. 
Table 3.1-8. Comparison of operating parameters for internally and externally cooled 24-T resistive coils.

\begin{tabular}{|c|c|c|c|c|}
\hline \multirow[b]{2}{*}{ Operating parameters for $24-T$ coil } & \multicolumn{3}{|c|}{ Internally cooled coil } & \multirow{2}{*}{$\frac{\text { Externally }}{\frac{\text { cooled }}{\text { coil }}}$} \\
\hline & $\begin{array}{l}\text { Inner } \\
\text { layer }\end{array}$ & $\begin{array}{l}\text { Outer } \\
\text { layer }\end{array}$ & $\begin{array}{l}\text { Coil } \\
\text { (average) }\end{array}$ & \\
\hline Overall current density $\left(\mathrm{A} / \mathrm{cm}^{2}\right)$ & 2500 & 2500 & 2500 & 2800 \\
\hline Winding volume $\left(\mathrm{cm}^{3}\right)$ & 38,700 & 88,200 & $1.42 \times 10^{6}$ & $1.15 \times 10^{6}$ \\
\hline Resistivity (ohm-cm $\times 10^{-6}$ ) & 3.6 & 2.8 & 3.4 & 2.9 \\
\hline $\begin{array}{l}\text { Cooling load including } \\
\text { nuclear heat ing (MW) }\end{array}$ & 2.2 & 2.3 & 51.7 & 42.9 \\
\hline Water velocity $(\mathrm{cm} / \mathrm{s})$ & 1000 & 1000 & 1000 & 157 \\
\hline $\begin{array}{l}\text { Heat transfer coefficient } \\
\qquad\left(\mathrm{W} / \mathrm{cm}^{2}-\mathrm{O}^{\mathrm{O}} \mathrm{C}\right)\end{array}$ & 5.1 & 5.1 & 5.1 & 0.657 \\
\hline Flow rate (liter/min) & 200 & 200 & 4600 & 20,000 \\
\hline Water temperature rise $\left({ }^{\circ} \mathrm{C}\right)$ & 147.5 & 162.5 & 16.1 & 27.5 \\
\hline Peak conductor temperature $\left({ }^{\circ} \mathrm{C}\right)$ & 200 & 200 & $N / A$ & 117 \\
\hline Pressure drop (atm) & 21 & 35 & $N / A$ & $w$ \\
\hline Total power consumption (MW) & 1.4 & 2.2 & 42.0 & 38.2 \\
\hline
\end{tabular}




\subsubsection{Engineering Analysis}

We performed the following analyses in support of the design of the axicell resistive coils.

Magnetics. The resistive coils have gone through several changes. The central field strencith, coil inner radius, number of coils, method of conductor cooling, and coll current density have all changed. As we described in Section 3.1.2.4, there are currently two coils at each end of the reactor, surrounded by a large superconducting coil: M-1 has an inner radius of $0.32 \mathrm{~m}$ and a central field of $21 \mathrm{~T}$, and $M-2$ has an inner radius of $0.30 \mathrm{~m}$ and $a$ central field of $24 \mathrm{~T}$. Both coils have a current density of $2500 \mathrm{~A} / \mathrm{cm}^{2}$ and internally cooled conductors (Fig. 3.1-11).

Unlike a superconductor, the current-carrying capacity of a resistive conductor is not limited by field strength, which simplifies the design proach. The power dissipation of a resistive coil in a radiation environment varies with the radius and the type of radiation. We evaluated the resistive losses by determining the radiation damage for each layer of conductor and the resultant increase in resistivity for that layer and then calculating the power.

One disadvantage of the internally cooled conductor coil is that most of the layers must be individually cooled; this calls for separate power supplies. On the other hand, this approach may offer advantages of its own by allowing us to grade the current density to reduce coil size.

Coil shape has a definite effect on the power consumption. For a given field, inner radius, and uniform current density (radiation effects neglected), only one shape will give minimum power consumption. This is the shape that also gives minimum coil volume for the above conditions. He used this concept in sizing the coils. 10 With more study it may be possible to optimize the coil sizes, taking into account the varying resistivity due to radiation.

Another approach to the resistive coil design minimizes ohmic power at the expense of greater coil volume. This is typified by the modified Kelvin distribution described in the TASKA Design Study.7,11 This type of coll uses a series of concentric layers, with each succeeding layer being wider and having a lower current density than its inner neighbor. This type of coil 
substantially reduces the onmic power required, but at the cost of a much larger magnet. In the case of a large encircling superconducting magnet, this approach is uneconomical because the larger resistive coil causes a large increase in the inner radius of the superconducting coil and an even larger increase in the coil volume.

Force and Stress Analysis. For our stress analysis of the baseline resistive coils we evaluated the conductor hoop stresses and the anchoring of the conductor leads, and analyzed the effect of the large axial forces on the coils. The coil cases are of secondary concern because they carry no internal pressure. The hoop stress in the conductors has a maximum value of $300 \mathrm{MPa}$ (43.5 ksi), which is well within the allowable yield for MZC copper. However, developmental work will be required before a fabricator can make the four-hole hollow conductor. Because the individual layers are cooled and powered separately, the conductors must be anchored at each end. Thus, a coil with 24 layers has 48 individual conductor anchorings. The anchoring force is approximately equal to the conductor hoop force. We have taken a preliminary look at the anchoring and determined it is feasible.

Large axial forces also act on the coils. We have not yet evaluated the effect of these forces on the winding, the coil case, or the anchoring of the conductors. The side force of $2.48 \times 10^{7} \mathrm{~N}$ gives an average pressure of $15.23 \mathrm{MPa}(2200 \mathrm{psi})$ on the end of the case. This does not appear to be a problem, but will have to be consider it in the detailed design.

Thermohydraulics. We analyzed the thermohydraulics of the resistive choke coils to determine the water flow rates necessary to keep the coil cooled to below the desired operating temperature and to verify that the capability exists to transfer the heat into the water. The basic principles of heat transfer are identical for either the externally or internally cooled coils, although the details of computation differ.

First, the total heat load is computed by adding the joule heating in the conductor to the heat deposited by neutron radiation. For the internally cooled coil, the heat load is computed for each hydraulic path. For the MARS coils, this corresponds to one layer of conductor. The winding volume, then, is the unit cell areas times the length of conductor required to wind one 
layer. Alternatively, for the externally cooled coil, there is only one equivalent hydraulic path through the magnet and the heat load is computed for the magnet as a whole.

Once the coolant heat load is calculated, the required water flow rate is determined. The heat balances in the conductor and coolant are solved interatively to determine coil operating temperatures, allowing all temperaturedependent properties to vary. The heat transfer coefficient is from the Colburn equation

where

$$
h=\frac{0.23 k v^{0.8}}{0^{0.2} v^{0.8}}
$$

$h=$ heat transfer coefficient $\left(W / \mathrm{cm}^{2} \mathrm{~K}\right)$,

$D=$ flow passage hydraulic diameter $(\mathrm{cm})$,

$k=$ thermal conductivity of water $(\mathrm{W} / \mathrm{cm} \mathrm{K})$,

$v=$ kinematic viscosity of water $\left(\mathrm{cm}^{2} / \mathrm{s}\right)$, and

$V=$ water velocity $(\mathrm{cm} / \mathrm{s})$.

The inlet water temperature is taken to be $32.5^{\circ} \mathrm{C}$, a value expected to be typical of the capability of a cooling tower. For the externally cooled coil, the flow rate is determined by keeping the conductor temperature less than $100^{\circ} \mathrm{C}$. This eliminates the possibility of boiling in a nonpressurized system. For the internally cooled coil, the flow rate is chosen to minimize the total power input to the coil with an additional constraint of a maximum conductor temperature of $200^{\circ} \mathrm{C}$ to avoid recrystallation of the copper and loss of strength of the conductor. The internally cooled coil requires a pressurized system to avoid water vapor formation.

The neutron effects are the most severe at the inner layer of the coil, and they drop off exponentially with radial distance. A volume-averaged value of resistivity was found to be $2.9 \times 10^{-6}$ ohm-cm (this represents a $20 \%$ increase in initial resistivity) for the externally cooled coil. The radial distributions of increased resistivity and neutron heat deposition were used to compute heat transfer for the inner and outer layers of the internally cooled coil.

Radiation Environment. In the resistive magnets, the insulation and conductors near the inner surfaces of the coil see an almost unattenuated 
stream of neutrons and dose rates as high as $10^{6} \mathrm{rad} / \mathrm{s}$. In this radiation environment, organic insulators with a radiation dose limit of $5 \times 10^{9}$ rad are unsuitable and ceramic insulators have to be used. For the reasons given in Section 3.1.3.2, we selected spinel $\mathrm{Al}_{2} \mathrm{MgO}_{4}$ as the material for the insulator.

The neutrons affect both the mechanical and electrical properties of the conductor. The major changes in mechanical properties are caused by radiation-induced void swelling, radiation creep, and radiation embrittlement. Major changes in electrical propertles are caused by defects introduced into the conductor lattice and by transmutations of atoms. The electrical resistivity changes due to defects (sometimes referred to as dpa damage) saturates at any temperature when the recombination rate of defects equals the generation rate of defects. In OFHC copper, the saturation value of induced resistivity is approximately $80 \times 10^{-9} \mathrm{ohm}-\mathrm{cm}$ at $30^{\circ} \mathrm{C}$. The increase in resistivity due to transmutations does not saturate but increases linearly with neutron fluence.

The peak change in conductor resistivity at the inner radius as a result of both transmutations and dpa damage is estimated to be approximately $59 \%$, with an average increase of resistivity throughout the coil of $20 \%$ after 2 FPY.

The corrosion rate of water-cooled copper varies with the resistivity of the water and is minimized for $1 \mathrm{Mohm}-\mathrm{cm}$ water at $0.5 \mathrm{mil} / \mathrm{yr}$. For purer water, 8 to $10 \mathrm{Mohm}-\mathrm{cm}$, it is $7.7 \mathrm{mil} / \mathrm{yr}$, and for $0.004 \mathrm{Mohm}-\mathrm{cm}$ it is $2.8 \mathrm{mil} / \mathrm{yr}$. Irradiation of the coolant (deionized water) affects the rate of corrosion of copper and introduces new mechanisms of corrosion. Radiolysis of the water occurs when radiation is present. Radiation breaks $\mathrm{H}_{2} \mathrm{O}$ molecules down to $\mathrm{H}^{+}$and $\mathrm{OH}^{-}$radicals that interact to produce other radicals and $\mathrm{H}_{2}, \mathrm{O}_{2}$, and $\mathrm{H}_{2} \mathrm{O}_{2}$. It is estimated that the oxygen concentration in the water will be saturated at about $8000 \mathrm{ppb}$. At this concentration and at temperatures of $60^{\circ} \mathrm{C}$, water resistivity cannot be as high as $1 \mathrm{Mohm}-\mathrm{cm}$. Radiolytic corrosion, which is oxidation of copper by $\mathrm{H}_{2} \mathrm{O}_{2}$, may errode $0.65 \mathrm{~mm}$ in $2 \mathrm{FPY}$. Radiolytic corrosion can be lowered by adding reducing agents such as $\mathrm{H}_{2}$ or hydrazine or by using an ion exchanger.

\subsubsection{Transition and Anchor Coils}

These coils are similar enough for us to give a common description. Their basic function is to provide the nonsymmetrical fields necessary to help 
obtain MHD stability. Each coil is a quadrupole. The anchor coils have total sweep angles of $160 \mathrm{deg}$, while the transition coils have maximum sweep angles of 210 and $240 \mathrm{deg}$. They all have a uniform current density and, because they are similar to comparable coils of MFTF-B, they use a copper-stabilized NbTi conductor. The coolant is LHA I.

The coil cases are 304LN stainless steel; insulation is polyimide fiberglass. Each coil has an $\mathrm{LN}_{2}$-cooled radiation shield and vacuum vessel. The forces acting on these coils are not known at this time, but are expected to be large. We assume that each coll will have a structural yoke to contain the spreading forces but we have not determined the location and number of coll supports. Each coll will have a service stack for all current ieids, fluids, and instrumentation leads. Table 3.1-9 compares the major design parameters of the transition and anchor coils of MARS and MFTF-B.

The transition and anchor coils may need some radiation shielding to protect them from neutrons streaming from the central cell and to prevent $D-D$ neutrons born in the anchor from sloshing-ion interactions.

The major areas that we will be investigating in FY 1983 for the transition and anchor coil regions are:

1. Radiation analysis, shielding and neutral beam ccess.

2. Magnet support structure design.

3. Vacuum vessel design.

4. Potential interface problems between transition and anchor magnet cryostats.

5. Design approaches for preventing stagnaticn of helium bubbles. 
Table 3.1-9. Comparison of MARS and MFTF-B transition and anchor coils.

$\frac{\text { Transition coils }}{\text { MARS MFTF-B }} \frac{\text { Anchor coils }}{\text { MARS MFTF-B }}$

Mean major radius (m)

$1.25 / 2.0$

$0.875 / 1.2$

3.0

2.5

Sweep angle (deg)

$210 / 240$

$200 / 220$

160

150

Mean minor radius (m)

1.25

$0.7 / 1.35$

0.80

0.75

Sweep angle (deg)

180

Winding pack height (m)

$0.46 / 0.53$

180

180

180

Winding pack width (m)

$0.46 / 0.53$

$0.45 / 0.34$

1.85

0.82

Current densits $\left(A / \mathrm{cm}^{2}\right)$

$3000 / 2900$

$0.2 / 0.34$

0.32

0.33

Peak field (T)

6.8

$2844 / 3075$

2750

$1977 / 2271$

4.5

8.5

7.0

\section{REFERENCES FOR SECTION 3.1}

1. The Structural Analys is of Non-Homogeneous Soienoids Using the STANSOL Computer Program, Wechanical Research Inc., Report No. MRI-C2754-TR-3 (1975).

2. M. Kohler, Ann. Phsik, 5(32), 211 (1938).

3. R. van-Konynenberg and M. Guinan, Radiation Effects on MFTF-B Magnets, Lawrence Live, riore National Laboratory, Livernore, CA, Interdepartmental Memo (June 16, 1981).

4. Final Design Review for the A-Cell, Transition and Solenoid Coils for the Mirror fusion Test Facility-B (MFTF-B), General Dyramics Convair division, San thego, CA, Report No. GDC-MFTF-B-81-04 (January 1982),

5. J.M. Williams, C.E. Klabunde, J.K. Redman, R.R. Coltman, Jr., R.L. Chaplin, "The Effects of Irradiation on the Copper Normal Metal of a Composite Superconductor," IEEE Transaction on Magnetics, 15(1), 731 (1979).

6. R. Hoard et al., Utilizing Supercooled Superfluid He II in the Design of a 12-Tes la Tandem Mifror Experiment, Lawrence Livermore National Laboratory, Livermore, CA, JCRL 86326 (1981).

7. TASKA University of Wisconsin Report UWFDM-500.

8. J. Schultz, If You Must Run Water Past Your Insulation, MIT Plasma Fusion Center Internal Technical Note, MITN- \$2-06. 
9. J.H. Schultz, Performance Linitations on Highly Irradiated High Performance Solenoids for Use in the Tandem Mirror Demonstration Facility Axicells, MIT (September 14, 1982).

10. D.B. Montgomery, Solenoid Magnet Design, Robert E. Kreigler Publishing Company (1980).

11. G. Logan, A Convenient Analytic Model for Estimating Size and Power Consumption of Constant Stress-Limited, Graded-Current-Density Hybrid Barrier Mirror Coils, Lawrence Livermore National Laboratory, Livermore, CA, private cormunication (January 11, 1982).

12. WITAMIR-I, A University of Wiscons in Tandem Mirror Design, University of Wiscons in Report UWFDM-400 (September 1980). 
Electron cyclotron resonance heating is critical to the operation of the endplugs in a tandem mirror reactor (TMR). As discussed in Section 2.6, ECRH injected at the minimum of the endplug magnetic mirror (Point B) creates a collisionless, mir.or-trapped electron population that helps maintain the thermal barrier. This barrier decouples the central-cell electrons from those in the region of the potential peak. The magnitude of the potential peak is proportional to the electron temperature at that point; the decoupling makes it possible to maintain a high electron temperature with reasonable ECRH power (at point A). Hence, two ECRH systems are included in the MARS baseline. The requirements defined by the TMR physics code are shown in Table 3.2-1.

\subsubsection{ECRH Systems Analysis}

ECRH systems on past and current devices deliver powers in the range of 80 to $200 \mathrm{~kW}$ and have frequencies of 28,35 , or $50 \mathrm{GHz} .1-3$ The basic systems include a pulsed, capacitor-charged power supply, a 200-kW gyrotron, and a 2.5-in. overmoded waveguide transmission system. These systems have been inefficient and expensive, and when scaled to reactor powers they tend to be cumbersome. Hence, we have considered several options that would need development.

The source in an FrRH system has a major effect on both systein cost and eficiciency. In additioii, iffects the power supply and transmission system characteristics. Because experience with other microwave tubes indicates that the use of high power tubes provides a cost benefit, we have considered using 1-MW gyrotrons. In principle, gyrotron efficiencies (30 to 50\%) can be improved by direct recovery of the electron beam. The baseline MARS ECRH source is a 1-MW gyrotron with a depressed collector.

Although this gyrotron is our baseline source, it does not possess some very advantageous features: large bandwidth, frequency variability, and a gaussian wave mode. Free electron lasers (FEL's) contain these features, but they are at an early stage of development and we have no long-term development plan for them. Thus, although the FEL appears to be an ultimately more suitable ECRH source than the gyrotron, we have not selected it as the baseline source. 
Tabie 3.2-1. ECRH system requirements.

\section{At point $A$}

Frequency vacuum (GHz)

$\beta$-corrected

Relativistic

Trapped power (MW)

Trapping fraction

Delivered power (MW)
95

74

44

2

1

2
At point B

64

49

30

54

1

54

Because our experiments have used pulsed systems, and because the actual reactors will need continuous systems, we will have to conitinuously convert the power rather than store it in capacitors for intermittent use. A conventional steady-state power supply includes a fused disconnect, step/start contactor, step controller, transformer/rectifier, capacitor comp crowbar, modulator/regulator, and vacuum disconnect/ground, as well as gyrotron mounts and controls. An induction voltage regulator and cathode crowbar could replace the modulator/regulator tube if the circuit elements are designed to withstand the $10 x$ fault currents for a satisfactory number of crowbars.

To reduce the cost of the ECRH system, we have considered a less costly power supply. The system is based on a Graetz bridge ac/dc converter similar to the type used in a power grid for de transmission. ${ }^{4}$ This option is discussed in Section 3.2.2 as the baseline supply. Note that several key issues are yet to be resolved and the conventional power supply is our backup option.

The transmission system offers two distinct options: the overmoded waveguide system and the quasi-optical transmission system. The efficiency of the overmoded waveguide transmission line is very sensitive to the mode purity requirements of the injected wave, and is usually in the 50 to $80 \%$ range. Even if megawatt gyrotrons are considered, single entry of the guides would be both unwieldy and impractical for high power systems. As a result of the high magnetic fields and high powers, efficient power combination techniques are 
not available. Power density limitations for metallic guides would limit the number of gyrotrons that could be combined in a composite launcher. For high powers, a pressurized guide with a ceramic vacuum window is probably required, but windows capable of handling the high powers in the radiation environment do not currently exist.

In contrast, quasi-optical transmission does not have some of the limitations of waveguide systems, offering low loss power combinations with more relaxed constraints on allowable power density. Because the system is in vacuum, ceramic windows are not required. For these reasons, we chose the quasi-optical transmission system described in Section 3.2.4. It is an evolution of designs previously employed in the WITAMIR ${ }^{5}$ and TASKA 6 tandem mirror fusion devices.

Our baseline ECRH system, which comprises the Graetz bridge power supply, megawatt gyrotrons with depressed collectors, and the quasi-optical launcher, evolved after an extensive trade study. Our analysis compared system capital costs, efficiency, and recirculating power for several ECRH configurations.

\subsubsection{ECRH Power Supply}

The power supply requirement of the ECRH systems is $83 \mathrm{MW}$. One or a number of supplies must provide 150-kV 578-A continuous dc power to the gyrotron cathodes. The choice of the number of supplies necessary to convert to dc power depends on the reliability of the supply, how long the ECRH system will be down if a supply malfunctions, and what impact loss of ECRH power would have on plant operation. The Graetz bridge power supply for dc transmission has proven to be very dependable, and the technology is capable of converting the required power. 7

The Graetz bridge supply includes transformers, thyristor valves, circuit breakers at a number of locations, dc smoothing reactors, dc harmonic filters, lightning arrestors, and overcurrent diverters. The basic power supply configuration is shown in Fig. 3.2-1.7

The bridge is an ac/dc converter consisting of six main thyristor valves connected as a two-way, three-phase circuit with a seventh valve as a bypass valve connected across the $d c$ bus and ground. The group must be rated for $150 \mathrm{kV}$ and $\sim 600 \mathrm{~A} \mathrm{dc}$, and draws from a 230-kV ac line. The MARS requirements 


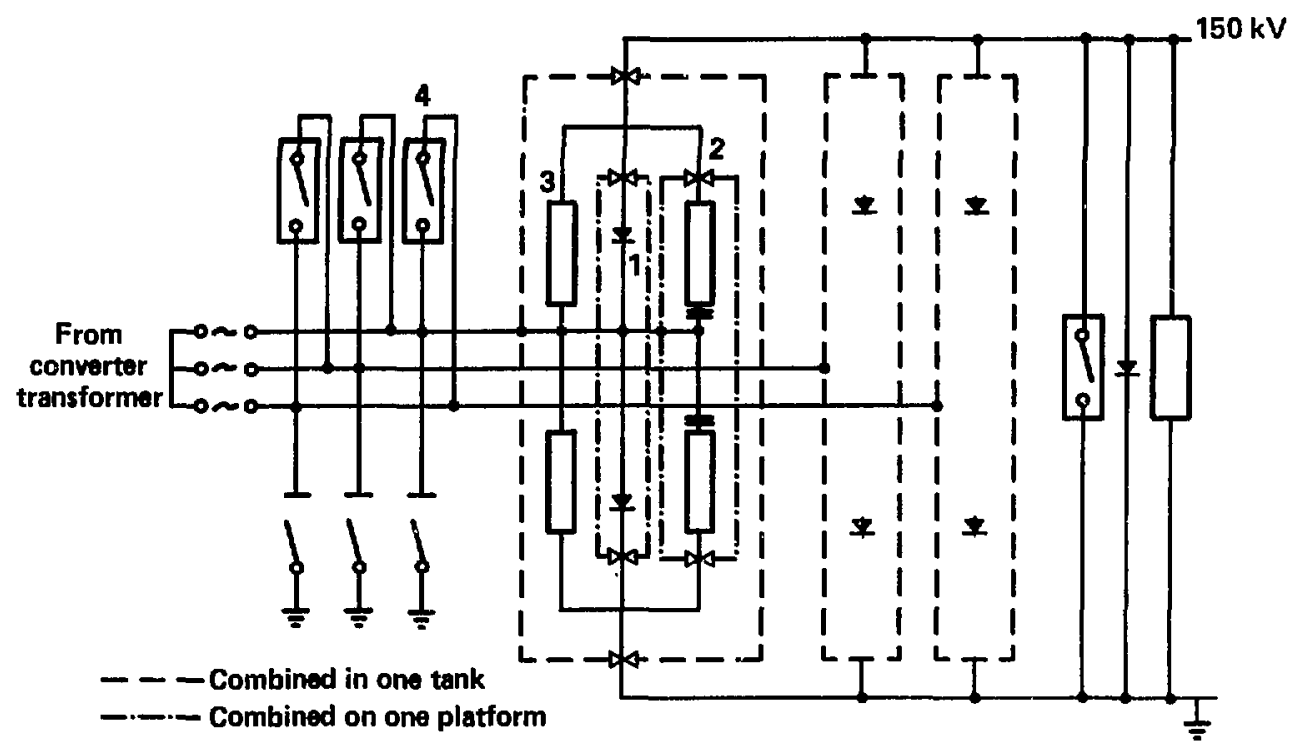

Fig. 3.2-1. Simplified diagram of bridge circuit with valves: (1) externai damping circuits (2), lighting arresters (3), and overcurrent diverters (4). 
are well below state of the art; the typical bridge used in the electrir, power grid is rated at $285 \mathrm{MW}, 133 \mathrm{kV}$, and $2160 \mathrm{~A} .{ }^{8} \mathrm{~A}$ thyristor valve can be designed with a minimum number of thyristor devices in series to reduce costs and power losses and increase reliability. A typical valve is shown in Fig. 3.2-2.

\subsubsection{ECRH Source}

The gyrotron is the microwave source for the frequency range of interest. The development program to achieve the desired operating characteristics for MARS is nearly in place with only the depressed collector to be added. ${ }^{9}$ As described previously, the MARS baseline is a 1-MW gyrotron with a depressed collector. Each tube operates at $150 \mathrm{kV}$ and $8.5 \mathrm{~A}$. The collector voltage is chosen to be $-40 \mathrm{kV}$; the net efficiency is estimated to be $80 \%$.

Current gyrotrons are of the microwave cavity design shown in Fig. 3.2-3. In this configuration, the electron beam propagates in the resonant cavity and also shares the collector cavity with the wave. As the gyrotron power increases, the collector cavity must be enlarged to dissipate the beam power, making it more difficult to couple to the desired cavity mode efficiently. One of the 1-MW gyrotron concepts, the quasi-optical gyrotron, offers a solution to this problem. As shown in Fig. 3.2-4, it is a doublecavity device with the electron ieam traveling through two optical cavities perpendicular to their a:is. 10

\subsubsection{Quasi-Optical Launching System}

The wave mode in a conventicnal metallic waveguide is characterized by a propagation constant and field pattern that is the same at all points along the guide. By contrast, the beam mode in the quasi-optical system consists of a bundle of waves characterized by a spectrum of propagation constants. The field distribution varies along the beam guide but is reset at the conducting mirrors (see Fig. 3.2-5), which act as periodically spaced phase transformers. This permits beam transport with micimal diffraction losses.

The lowest loss-beam mode, designated TEM $_{00}$, is characterized by a Gaussian radial amplitude distribution and linear polarization. Beam modes in general are characterized by the product of a Gaussian and the generalized Laguerre polynomials. ${ }^{11}$ The lowest order Laguerre polynomial, $L_{0}^{0}$, is simply 


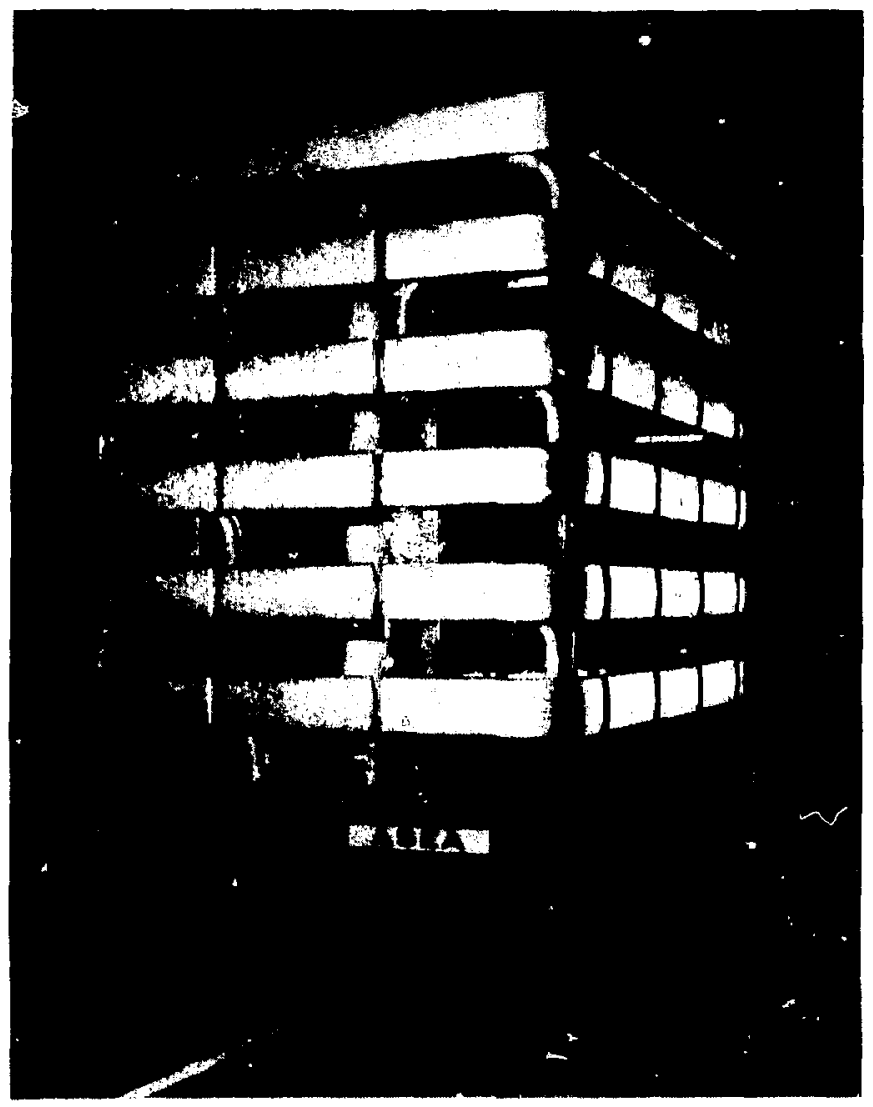

Fig. 3.2-2. A water-cooled thyrister valve with a rating of $133 \mathrm{kV}$ and 2160 $A$, installed at the Sylmar Converter Station of the Pacific Intertie. 


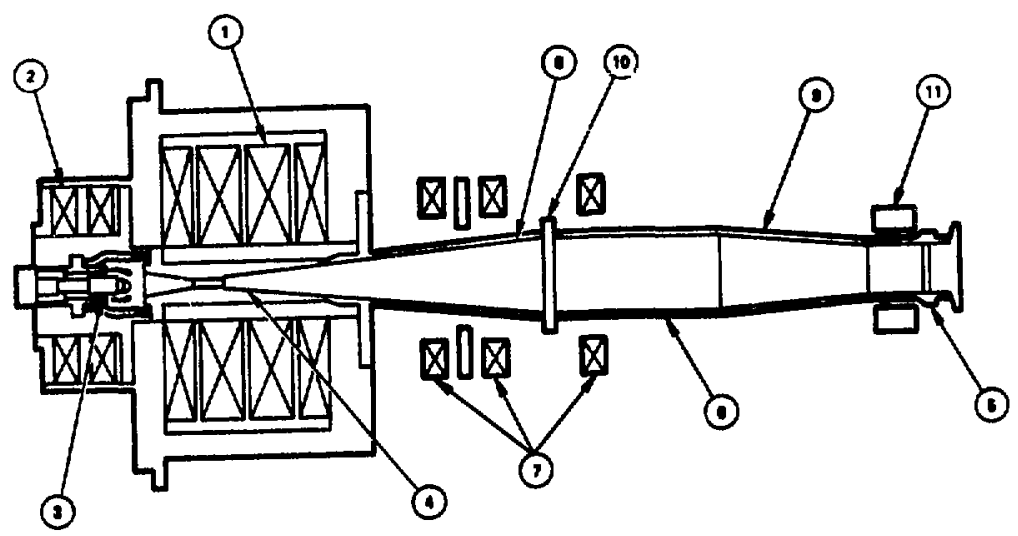
1. Main magnet coils
2. Gun magnet coil
3. Electron gun
4. Cavity
5. Output waveguide and window
6. Beam collector area

7. Collector magnet coils
8. Output guide up-taper
9. Output guide down-taper
10. Lower collector gap
11. Transverse field magnet

Fig. 3.2-3 The conventional gyrotron of a microwave cavity design.

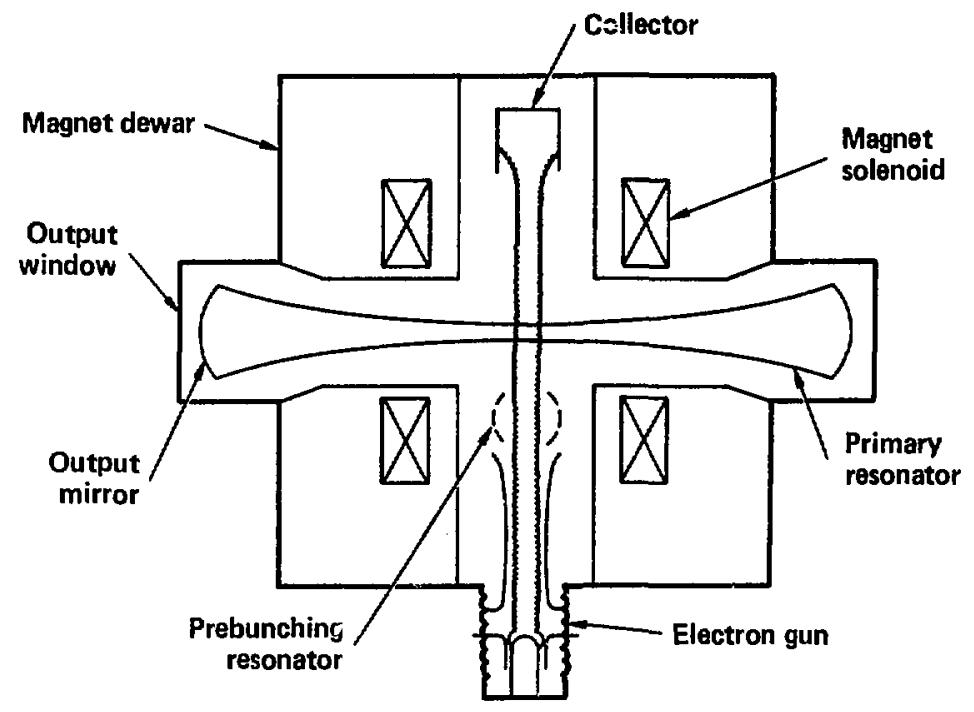

Fig. 3.2-4. Quasi-optical gyrotron. 


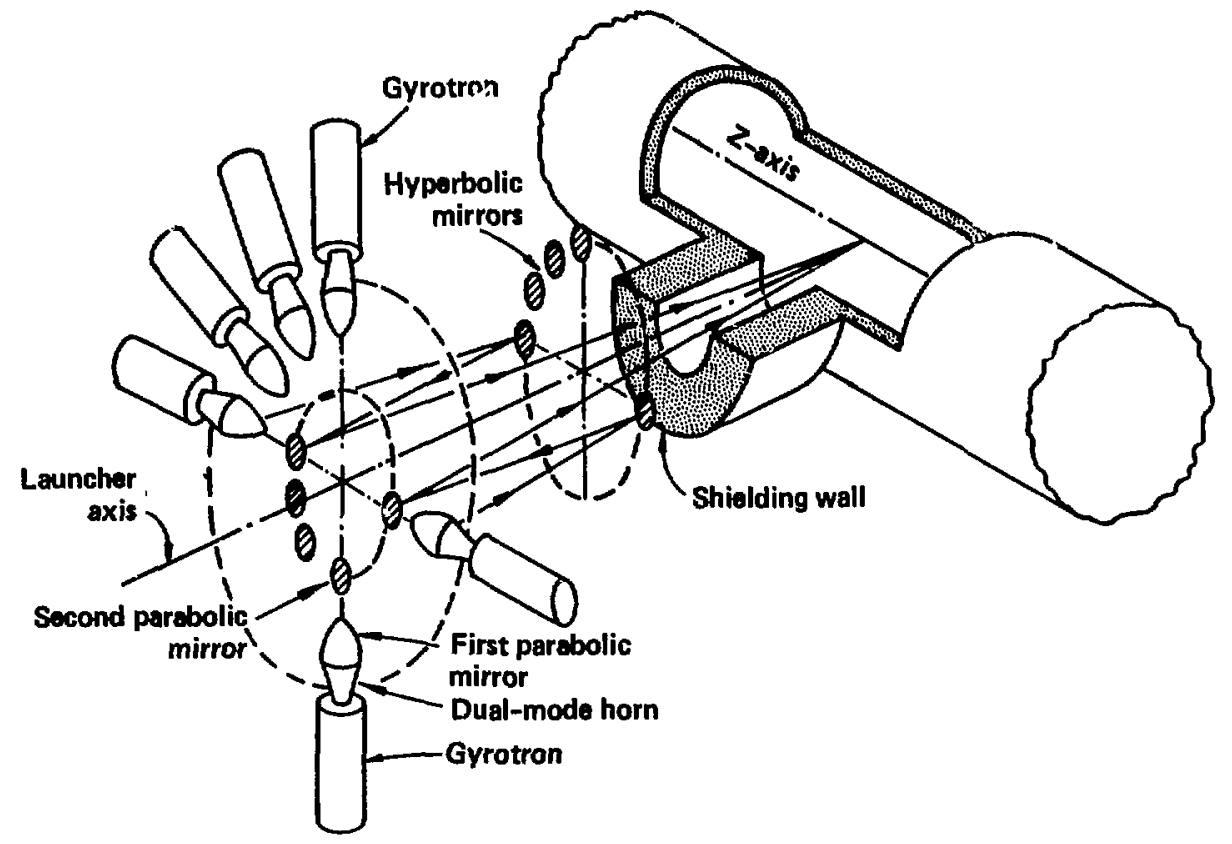

Fig. 3.2-5. Schematic of the MARS quasi-optical launching system. 
equal to one, however, and thus the TEM 00 mode transverse electric fields are given by

$$
E_{r}=E_{00} e^{-a^{2} r^{2} / R^{2}}
$$

where

$$
\begin{aligned}
& R=\text { guide radius (i.e., mirror radius), } \\
& 2 z_{0}=\text { phase transformer spacing or "iteration length", } \\
& a \quad k / 2 z_{0} \text {, and } \\
& k \quad=2 \pi / \lambda_{0} .
\end{aligned}
$$

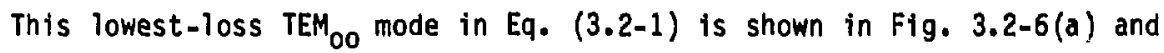
is linearly polarized. Note the Gaussian radial dependence of the electric field. The next lowest-loss mode, TEM $_{01}$, is circular electric (i.e., $\phi-$ independent, not circularly polarized) and is shown in Fig. 3.2-6(b). Because the electromagnetic energy of these beam modes is concentrated near the axis, wall losses and wall breakdown problems associated with metallic waveguides are eliminated.

The parameter "a" in Eq. (3.2-1) is analogous to the Fresnel number of geometrical optics. The diffraction (or iteration) loss, i.e., ioss per mirror spacing, is shown in Fig. 3.2-7 as a function of the parameter "a." It is evident that for a given frequency (i.e., given $k$ ) it is possible to design the mirror spacing and radius for any desired diffraction (iteration) loss.

The function of the mirror configuration in Fig. 3.2-5 is interpreted by Fig. 3.2-8. In the absence of the hyperbolic mirror, excitation at focal point $A^{\prime}$ would result in parallel rays, i.e., a uniform phase front at $B$. This is the conventional Cassegrain system of geometrical optics. In the offset Cassegrain system shown, adding the hyperbolic section merely switches the excilation point to $A$ and aperture blocking is prevented by the offset.

To launch the fundamental beam mode into the mirror system, a conventional dual-mode horn-reflector can be employed. Such an antenna is also an offset paraboloid. This system is shown schematically in Fig. 3.2-9. The apex of the cylindrical horn is placed at the focal point of the paraboloid. The horn has a mode converter in its throat which, if fed with a cylindrical $T E_{11}$ waveguide mode, produces botli $E_{11}$ and $T M_{11}$ modes in the horn. These 

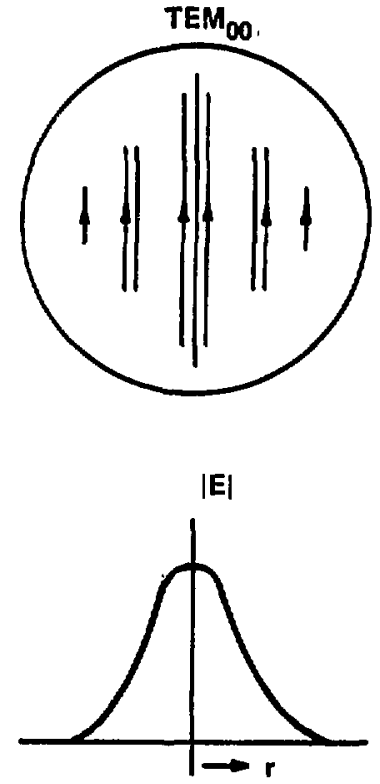

(a)

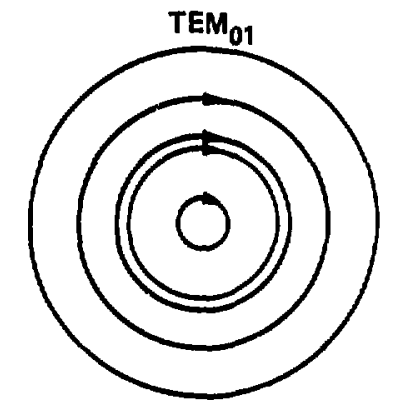

|E

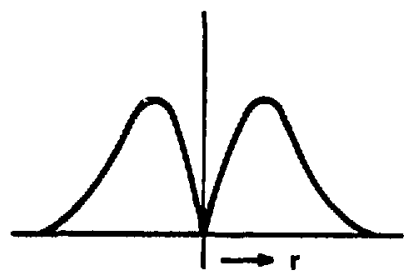

(b)

Fig. 3.2-6. Quasi-optical beam modes illustrating the gaussian radial dependence. 


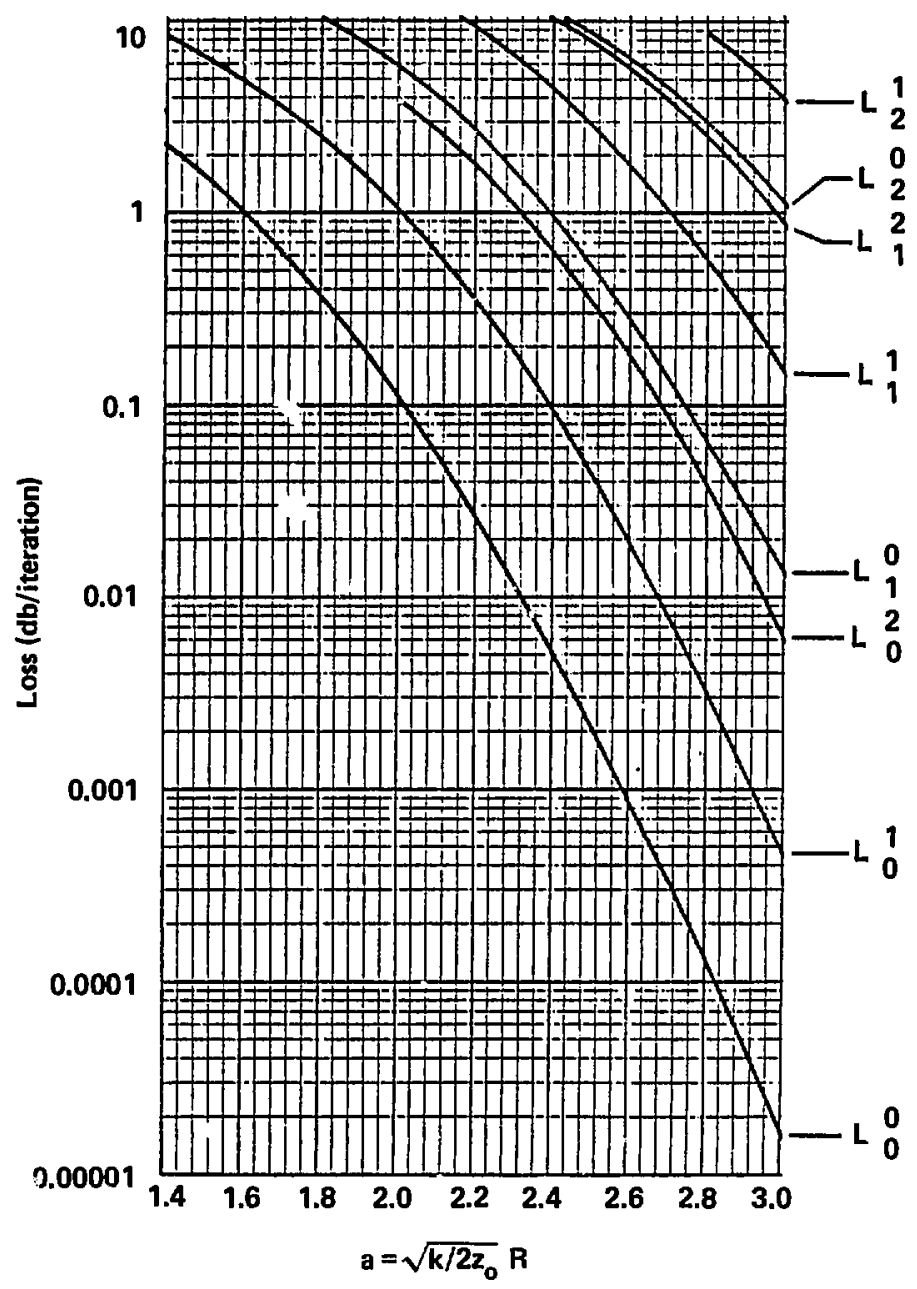

Figure 3.2-7. Diffraction losses for beam mode. 


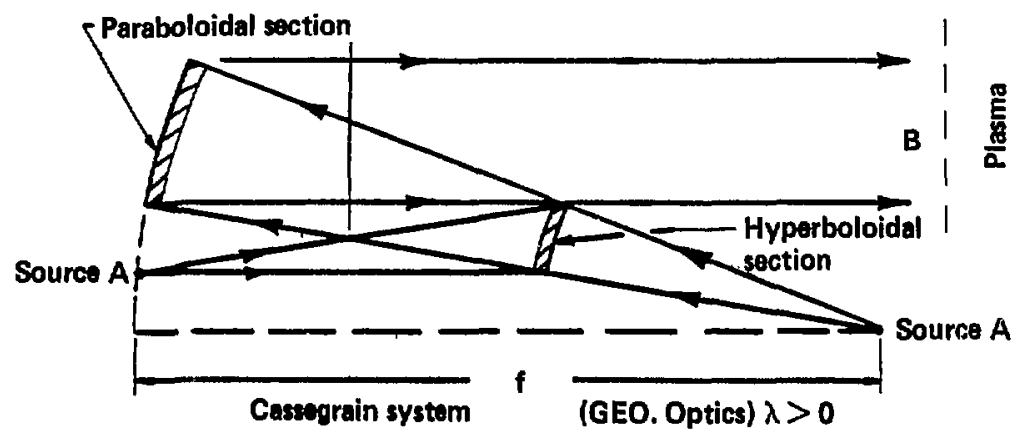

Fig. 3.2-8. Offset Cassegrain mirror system.

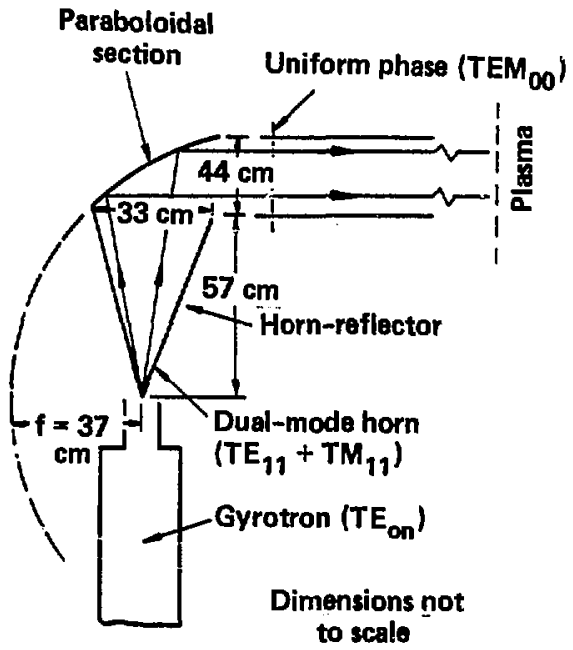

Fig. 3.2-9. The dual mode horn and associated mode conversion. 
modes together in the horn produce, by interference, a near-Gaussian radial amplitude distribution at the horn mouth. ${ }^{12}$ Reflection from the paraboloid creates a uniform phase front and the resulting aperture field is exactly the field required to launch a beam mode. The dimensions shown in Fig. 3.2-9 are for one line of the 64-GHz launching system.

However, gyrotrons operate in the $T E_{\text {on }}$ mode and not in $T E_{11}$. Therefore, an intervening mode converter is required between the gyrotrons and horns. A stepped twist-reflector can te employed here tc provide the necessary conversion to the $\mathrm{TE}_{11}$ mode. A quarter wave grating covers one-half of the reflector and converts the circular symmetric mode electric field into an antisymmetric field. At this point the quarter wave grating of the twistreflector reverses the polarization of only one of the rectangular components of the electric field, with the result that the fields now conform to the $T_{11}$ fields. The desired polarization direction can be obtained by rotating the reflector.

The power ?osses on the system are assumed to be $5 \%$ in each of the mode converters and the dual mode horn and a $0.2 \%$ per mirror. The total transmission loss is then $11 \%$. The assumed efficiencies are those obtained in low power devices (e.g., several watts) that have been and are being tested. ${ }^{13,14}$

\subsubsection{ECRH Systems Layout}

Figure 3.2-10 is a cross-section of the 64-GHz ECRH launcher system. Note that a launcher is defined as one complete circular array of gyrotrons with associated mirrors. The design power of this launcher is $13.5 \mathrm{~m}$. Thus, two launchers will be required at each end of the machine, giving a total ECRH power injection of $54 \mathrm{MW}$ at $64 \mathrm{GHz}$. The dimensions $A$ through $G$ in Fig. 3.2-10, together with other relevant parameters, are given in Table 3.2-2.

The geometrical design of the launcher in Fig. 3.2-10 is taken from the specification of four base requirements, namely, the ECRH frequency (64 GHz), the design power of the launcher $(13.5 \mathrm{NW})$, the diffraction loss per iteration path $(0.01 \mathrm{~dB}$ or $0.2 \%)$ and the angular spread $(2 \alpha)$ around the injection angle $( \pm 5 \mathrm{deg})$. The diffraction loss per iteration path of $0.01 \mathrm{~dB}$ was chosen as a reasonable compromise between the diffraction (scattering) power loss and mirror dimensions. Given these base systen requirements as well as the efficiencies of gyrotrons, mode converters, and mirror reflectivities, the 


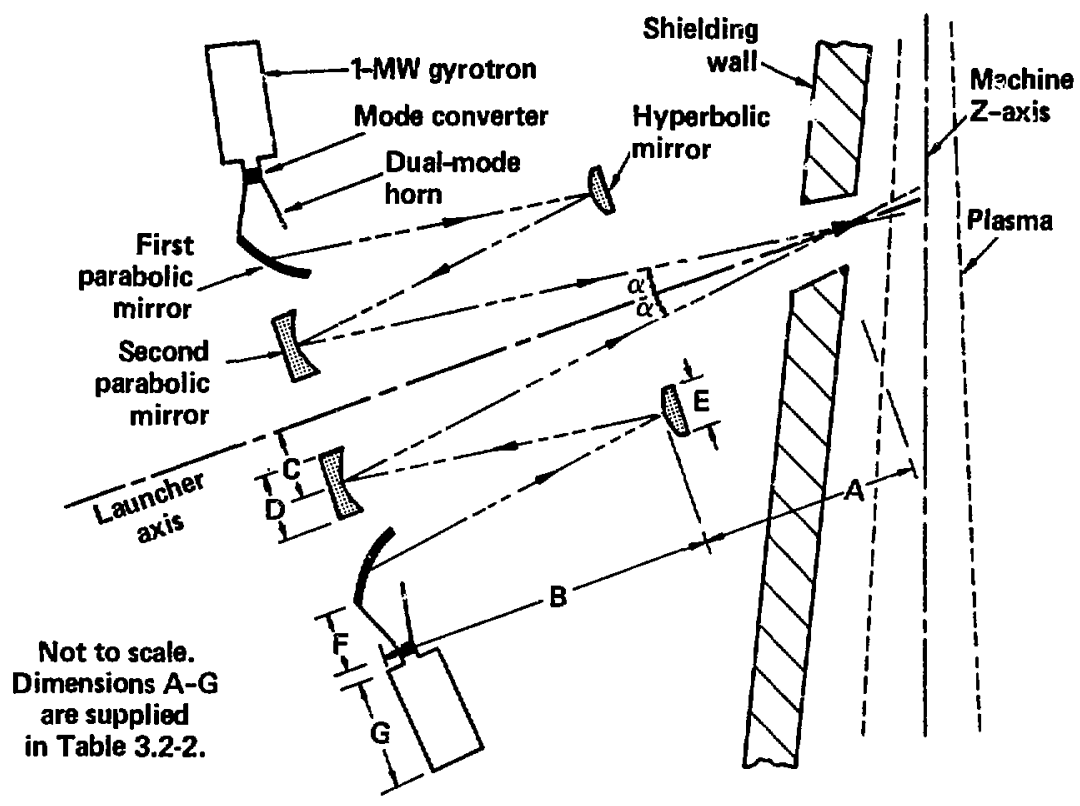

Fig. 3.2-10. Cross section of the quasi-optical 1aunching system. 
minimum dimensions of the launcher in Fig. 3.2-10 can be reduced to a set of coupled algebraic equations. It should be noted that the system in Fig. 3.2-10 and Table 3.2.-2 includes two redundant gyrotrons.

Table 3.2-2 also gives a breakdown of the launching system losses. The design diffraction loss per iteration $(0.01 \mathrm{~dB})$ and the mirror reflectivity losses $(\sim 0.1 \%)$ will be much smaller than the expected power losses in the mode converters.

Table 3.2-2 lists simllar parameters for the 96-GHz ECRH systems. The power requirements here are low compared with those for the 64-GHz system (1.e., $1 \mathrm{~W}$ at each end compared with $27.6 \mathrm{NW}$ ). Therefore, because a maximum of only three gyrotrons is required for each launcher (two for the required power output after losses plus one for redundancy), there is considerably more flexibility in launcher dimensions. Accordingly, with the constraints on diffraction losses per iteration similar to those above, a launcher length of $20 \mathrm{~m}$ was chosen to facilitate ease of location. This resulted in a lower angular spread $(2 \alpha)$ about the injection angle of $\pm 0.7 \mathrm{deg}$.

The consequence of any decrease in frequency is that system dimensions can become very large in order to keep the diffraction losses low. For example, if we use the vacuum field irequency and allow the resonance surface to shift, then a $40 \%$ Doppier shift from the design vaiue of $64 \mathrm{GHz}$ to $38 \mathrm{GHz}$ would require paraboloidal mirror diameters ( $D$ in Fig. 3.2-10) of $\sim 1 \mathrm{~m}$ and launcher lengths (length $A+B$ ) of $\sim 35 \mathrm{~m}$. This would result in a rather unwieldy launcher configuration. Therefore, we are studying the use of second harmonic heating to keep the transmission frequency high.

As the ECRH beam is transported down the launcher to the machine, it encounters a value of the fringing B-field from the magnets that corresponds exactly to the resonance value at the injection point on the z-axis. At this outer resonance point, any residual neutral gas will be efficiently ionized by electron cyclotron resonance, with attendant problems of power absorption and breakdown. It is thus necessary that we specify tre maximum design gas pressure in this region, such that breakdown effects will be minimized. Preliminary results from MFTF-B suggest that the maximum gas pressures of $\sim 10^{-5}$ Tor $r$ will be required. 15 
Table 3.2-2. Parameters for the ECRH launcher systems.

\section{Thermal barrier Potential peak}

ECRH power requirement/endplug (MW)

27.5

1

ECRH frequency $(\mathrm{GHz})$

64

96

No. of launchers (each end)

Design power per launcher (MW)

2

13.5

1

Maximum power per launcher (MW)

15.18

1

No. of gyrotrons/launcher (MW)

$17^{\mathbf{d}}$

$2.68^{\mathrm{a}}$

Gyrotron power/tube (MW)

1

Angular spread at plasma (deg)

$\pm 5$

$3^{b}$

Diffraction loss/iteration (dB)

0.01

0.01

Reflection loss/mirror (\%)

$\sim 0.1$

$\sim 0.07 \%$

Mode converter loss ( $\%$ )

$\sim 5$

$\sim 5$

Horn loss (\%)

$\sim 5$

$\sim 5$

Total loss/launcher ( $(\%)$

$\sim 10.7$

$\sim 10.7$

Launcher length, $A+B(m)$

18.27

20.0

Dia. of second parabolic mirror, 2C, (m)

3.20

0.48

Dia. of second parabolic mirror, $D(m)$

0.54

0.46

Dia. of hyperbolic mirror, $E(m)$

0.38

0.33

Horn length, $F(m)$

0.57

0.43

Dia. of horn mouth $(m)$

0.33

0.26

Gyrotron length, G (m)

$\sim 2$

$\sim 2$

\footnotetext{
a Two redundant gyrotrons per launcher.

b One redundant gyrotron per launcher.
} 
Two remaining issues are tritium contamination and radiation damage. Because no vacuum window is required in the quasi-optical system, no tritium barrier is necessary between the machine vacuum and the launcher. Tritium contamination issues for both the launcher and gyrotrons need to te addressed, especially during gyrotron replacement. The folded-path arrangement of the offset Cassegrain optics in the quasi-optical system offers some unique advantages with respect to radiation damage protection. Figure 3.2-6, with the exception of the second parabolic mirrors, shows that all components can be shielded from direct line-of-sight of the fusion plasma. The key performance parameter for the parabolic mirrors is reflectivity, which is a function of their surface condition and bulk conductivity. However, it is difficult to envisage any radiation effects likely to degrade this reflectivity. Surface damage would have to be comparable to the wavelength $(-0.5 \mathrm{~cm})$ to be appreciable, while conductivity decreases due to neutron-induced transmutations would be negligible given the relatively low neutron flux at the mirrors. It can be concluded, therefore, that radiation effects on the quasioptical system will be minimal. If the neutron flux is low enough to cause minimal damage to the gyrotrons, the folded path could be eliminated, thus simplifying and reducing the size of the ECRH vacuum vessel.

REFERENCES FOR SECTION 3.2

1. R.M. Gilgenback et al., "Heating at the Electron Cyclotron Frequency in the ISX-B Tokamak," Phys. Rev. Lett., 44 (10), 647 (1980).

2. C.P. Moeller, "Electron Cyclotron Resonance Heating on the JFT-2 Tokamak," in Proc. of 4th Topical Conf. on RF Plasma Heating. Austin, Texas (Feb. 910, 1981), 01.

3. J.S. Levine et al., "Electron-Cyclotron heating of the VERSATOR II

Tokamak," in Proc. of 4th Topical Conf. on Plasma Heating, Austin, Texas (Feb. 9-10, 1981), 06.

4. Celilo-Northern Terminal-Pacific HVDC Intertie, U.S. Dept. of the Interior and Bonneville Power Administration (1970).

5. B. Badger et al., WITAMIR-I, a University of Wiscons in Tandem Mirror Reactor Design, University of Wisconsin Fusion Engineering Program Report, UWFON-400 (1980).

6. B. Badger et al., TASKA, A Tandem Mirror Fusion Engineering Facility, University of Wisconsin Fusion Engineering Program Report, UWFDM-500 (1982). 
7. F.C. Beriger et al., "Cabora Bassa HVDC Transmission: 0il Cooled Outdoor Thyristor Valves," in Proc. of IEEE PES Summer Meeting of Energy Resources Conf., Anaheim, CA (JuTy 14-19, 1974), T 74 459-4.

8. HVDC and ASEA Specialty, ASEA Pamphlet, KS10-100E (1979).

9. M. Loring "The OFE/ORNL Kational Gyrotron Development Program," in Proc. of U.S. DOE Office of Fuston Energy-Gyrotron User/Developer Meeting, Germantown, To (April 13-14, 1982) p. 8.

10. M. Read, "NRL Multimegawatt Program," in Proc. U.S. DOE Office of Fusion Energy-Gyrotron User/Developer Meeting, Germantown, MD (April 13-14, 1982). P. 170 .

11. G. Goubau and F. Schwering, IRE Trans. on Antennas and Propagation AP-9, p. 248 (1961).

12. R.J. Chaffin and J.B. Beyer, IEEE Trans. on Microwave Theory and Techniques, Vol. MTT-112, p. 555 (1964).

13. J.B Beyer and E.H. Schreibe, "Loss Measurements of the Beam Waveguide," IEEE Transactions on Microwave Theory and Techniques, MTT-11(1), p. 18 (1963).

14. C.P. Moeller "Status of Components for the Doublett III and the PDX ECH Experiments," in Proc. of U.S. DOE Office of Fusion Energy-Gryrotron User/Developer Meeting, Germantown, MD (April 13-14, 1982), p. 85.

15. G.W. Hamilton, Lawrence Livermore National Laboratory, private comunication (1982). 
In the high-field magnetic well in the axicell region, ions are heated so that the trapping rate in the well balances the loss rate due to collisons into the loss cone, and a potential bump is created. The magnitude of this bump controls the passsing ion density into the anchor; and the density is minimized within stability constraints. Until recently, the potential bump was created and maintained by neutral beam injection; the high energy beam was injected through a split hybrid coil set at 90 deg to the plasma axis. Because of access limitations between the axicell coll set, we have proposed ICRH.

ICRH has proved effective in heating Tokamak and mirror plasmas. Systems with powers up to $\sim 4$ have operated at frequencies from 25 to $46 \mathrm{MHz}, 1-5$ Although the axicell plasma, geometry, and field are distinctly different than what we have encountered up to now, a near proof-of-principle experiment in similar conditions is planned next spring on Alcator $-\mathrm{C} .6$

\subsubsection{ICRH Systems Analysis}

Power balance circulations indicate that $\sim 40$ of ICRH power must be absorbed by the axicell ions to maintain the mirror-trapped distribution. An 85\% ion absorption efficiency has been estimated; therefore, $47 \mathrm{WW}$ of ICRH power must be delivered.

The vacuum field ; the magnetic well is $\sim 18 \mathrm{~T}$. For a fixed frequency corresponding to this field, as beta increases, the resonance surface will move off the midplane. As discussed in Section 2.7, tritium ions need to be heated. The fundamental tritium ion cyclotron resonance fréquency is $f_{c T}=$ $91.2 \mathrm{M} / \mathrm{z}$. Coupling to the plasma is improved by the use of the second harmonic because the evanescent region at the plasma edge, including the halo, decreases. ${ }^{7}$ In addition, second harmonic heating experiments have proven to be quite successful in Tokamaks. ${ }^{2,8}$ Hence, we use a frequency of $f_{2 c T}=182.4 \mathrm{MHz}$.

A schematic of the axicell region is shown in Fig. 3.3-1. When we consider magnets, shields, structure, coolant feeds, etc., the ICRH launcher must fit into a very restricted space. Present ICRH systems have basically consisted of the power supply, coaxial transmission system, and onehalf loop antenna launcher. However, because of radiation damage, lifetime, 


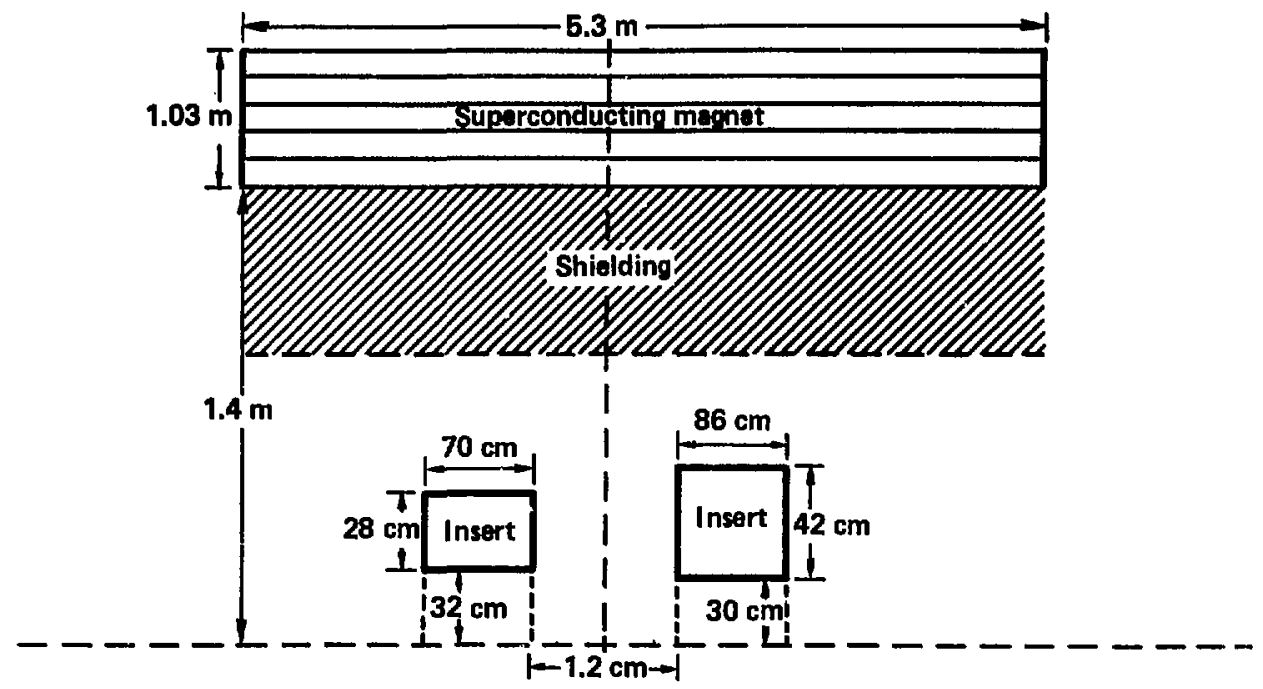

Fig. 3.3-1. MARS axicell geometry. 
and maintenance, several reactor studies have chosen waveguide launchers ${ }^{9-11}$ over antennas, even though the physics of waveguide-plasma coupling is poorly known. For these reasons, we feel that the launcher is the most critical component of the MARS ICRH system.

We initially considered both waveguides and antennas for the MARS axicell. We eliminated waveguides for two important reasons. To match the load, waveguides are generally some multiple of one fourth of a guide wavelength, i.e., $\lambda g / 4$. For $f_{c T} / f_{c}=1.2$, where $f_{c}$ is the waveguide cutoff frequency, the guide length must be greater than $75 \mathrm{~cm}$ with an additional length for matching. This length would reduce the shielding available for the superconducting coll or would force an increase in the bore, neither of which are acceptable. The second reason is that, for a reasonable peak electric field, too many guides would be required for the available surface area (for a $60-\mathrm{cm}$ bore), and the number of feeds to the guides would be too unwieldy.

The MARS ICRH system will thus have an antenna launcher. The issues of radiation damage, lifetime, and maintenance are critical, but since the resistive magnei inserts are faced with the same issues, the antennas will be designed within the same constraints.

\subsubsection{ICRH Amplifier and Power Supply}

The ICRH system must deliver 23.5 MW at $182.4 \mathrm{MHz}$ to each axicell. The power will be launched from an as yet undetermined number of antennas and trainsmission lines. Because of the restricted space in the axicell region, the number of antennas and coax feeds should be minimized. Although we have not yet determined the power of an individual power supply we can calculate the basic amplifier configuration.

Each amplifier will probably consist of IPA/driver units (including local control and monitoring, medium voltage power supplies, and ac distribution), low-power 90-deg hybrid splitters, final power amplifier (FPA) cavities, FPA filament power supplies, and high-power 90-deg hybrid combiners.

The most practical amplifier approach, using current technology, is a cavity-type amplifier with triode or tetrode vacuum tubes. High power tubes rated above $100 \mathrm{MHz}$ do not exist. The RCA 6950 shielded-grid double-anode triode has been tested to $750-\mathrm{kW}$ output, just below the desired frequency. The 
Eimac 4CX50000E tetrode can be operated in a derated mode at 180 to $190 \mathrm{MHz}$ but for only $\sim 100 \mathrm{~kW}$. Because multimegawatt powers per line and antenna are desired, high-power tube development is needed. Otherwise, the power from several tubes will have to be combined. An Eimac 4CX15000A used as a cavity amplifier is capable of driving several FPAs; the $600 \mathrm{~W}$ required to drive the tetrode will be provided by a solid state amplifier.

The quadrative hybrid combiner will be a coaxial type using a 9-3/16-in. or 12-in. pressurized coax. The power supply and cooling requirements should be met with good design practices and presently available components. Figure 3.3-2 shows a schematic of a simflar system at $154 \mathrm{MHz}$ to deliver $1.3 \mathrm{MH} .12$

\subsubsection{ICRH Power Feed}

The transmission system will transport the power from the amplifier to the antenna, whose input impedance is not accurately known and may change with time. A schematic of the line and matching network is shown in Fig. 3.3-3; it is similar in concept to the TEXTOR system. 13

The size of the coax for a majority of the system will be 9-3/16 in. $0 . d$. with 50-ohm impedance, but because of the shield between the superconductor and the resistive insert, the final coax can be no larger than 6-1/8 in. o.d. Active, pressurized cooling inside the inner conductor will be required in $\mathrm{L}_{4}$ and the third stub because of the expected high power (more than several megawatts), and may be needed in the entire line. The volume between the coaxial conductors in $L_{4}, s t_{3}$, and part of $L_{3}$ will be evacuated, while the remainder of the system will contain a controlled atmosphere. The pressure and type of gas used will be determined by the desired safety factor.

Several voltage probes throughout the system will continuously monitor the match; the stubs are controlled by an on-line computer. The two-stub system will hold the voltage standing-wave ratio on $L_{1}$ at unity.

We will also have to include dc breaks to insulate the amplifiers and power supplies from the tandem mirror and coaxial vacuum windows.

\subsubsection{ICRH Antenna}

The Princeton Large Torus (PLT) experiments use end-fed half loop antennas that have an average power density on the antenna surface of 


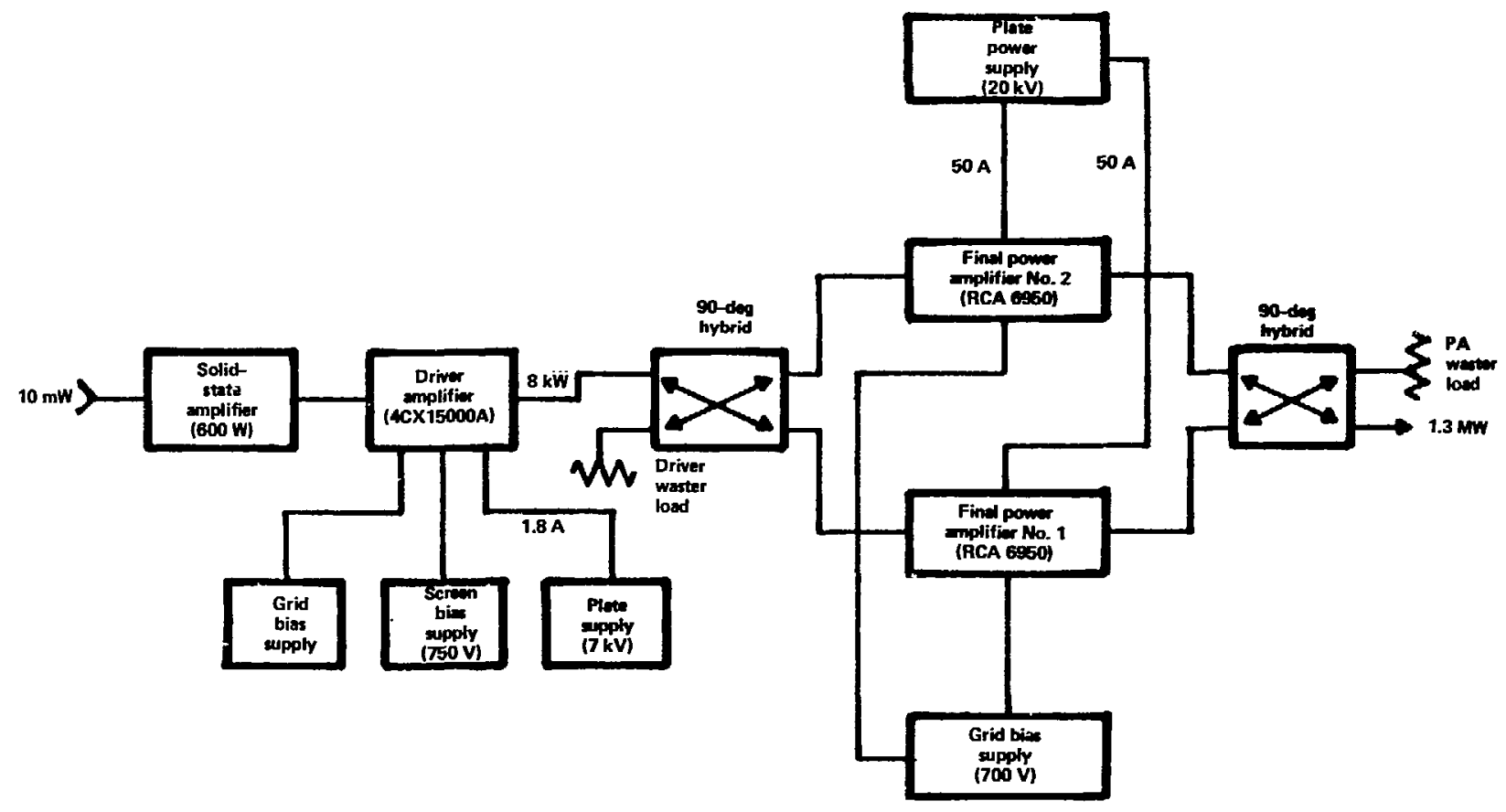

Fig. 3.3-2. A power amplifier chain of an ICRH system similar to that required for MARS. 


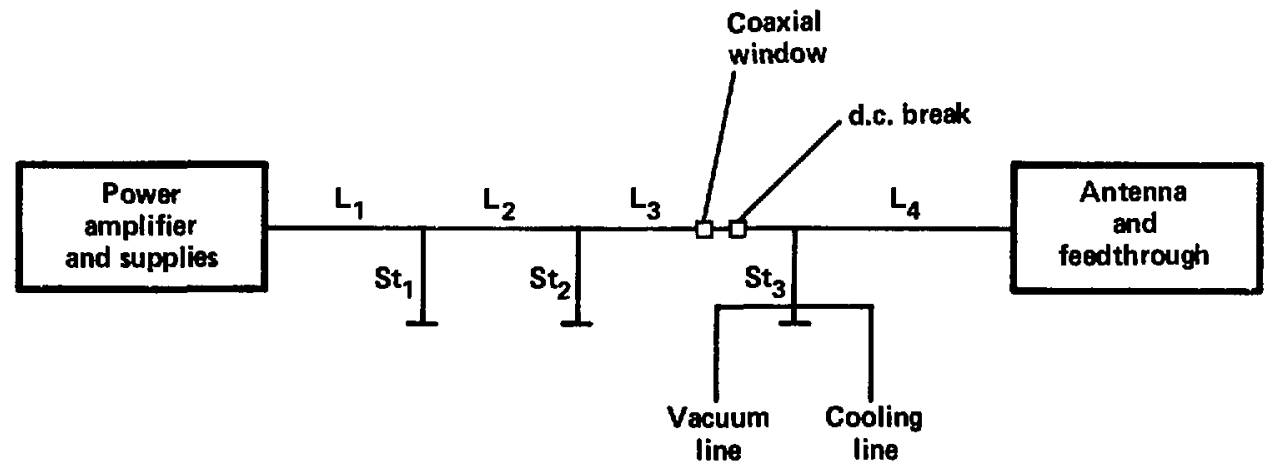

Fig. 3.3-3. Schematic of an ICRH transmission and matching system. 
$\sim 0.5 \mathrm{~kW} / \mathrm{cm}^{2}$. The power has been limited by breakdown at the feedthrough, and not by antenna surface effects. A center-fed antenna with an improved feedthrough is being built for which calculations predict a four-fold increase in power capability. 8 Power densities in tilis range have been achleved on JFT-2, 1.e., $1.4 \mathrm{~kW} ; \mathrm{cm}^{2}$ on the antenna surface. 14

The basic antenna configuration is a short-circuited section of stripline electrostatically shielded by an array of metal strips. The shield prevents the low density plasma from short-circuiting the ICRH voltage and suppresses the excitation of waves with the wrong polarization. The task during the next few months is to determine the antenna parameters necessary to optimize the power handling capability of the total system without exceeding the voltage standoff capabilities of the antenna feedthrough or transmission line components.

An antenna based on a previously developed model should have the following characteristics: ${ }^{13}$

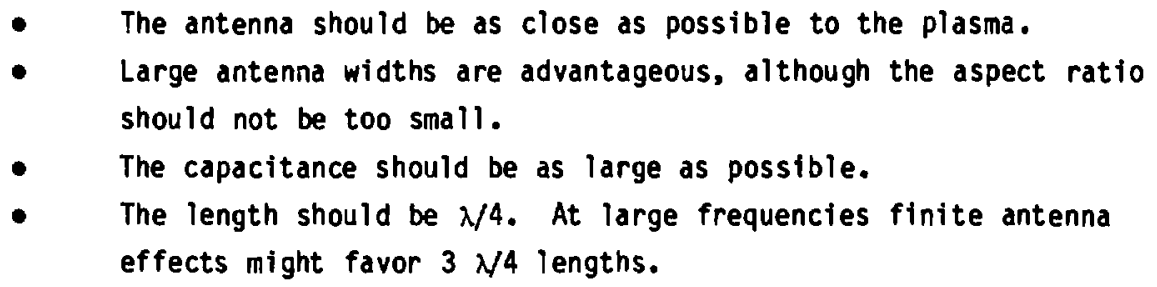

The vacuum feedthroughs are the weakest element in experiments. A large line reduces the field, but the space restriction between the conductor shield and resistive insert limits the conductor size to $6-1 / 8$ in.

\subsubsection{System Layout}

In summary, the ICRH system will consist of a two-stage amplifier system with associated power supplies, protective gear, and controls, as well as a coaxial transmission and multi-stub system and a shielded strip-line antenna.

Because the neutron flux drops off several orders of magnitude on the outboard edge of the axicell, there is an advantage to bringing the coax and feedthrough to the antenna from this side, as shown in Fig. 3.3-4. 


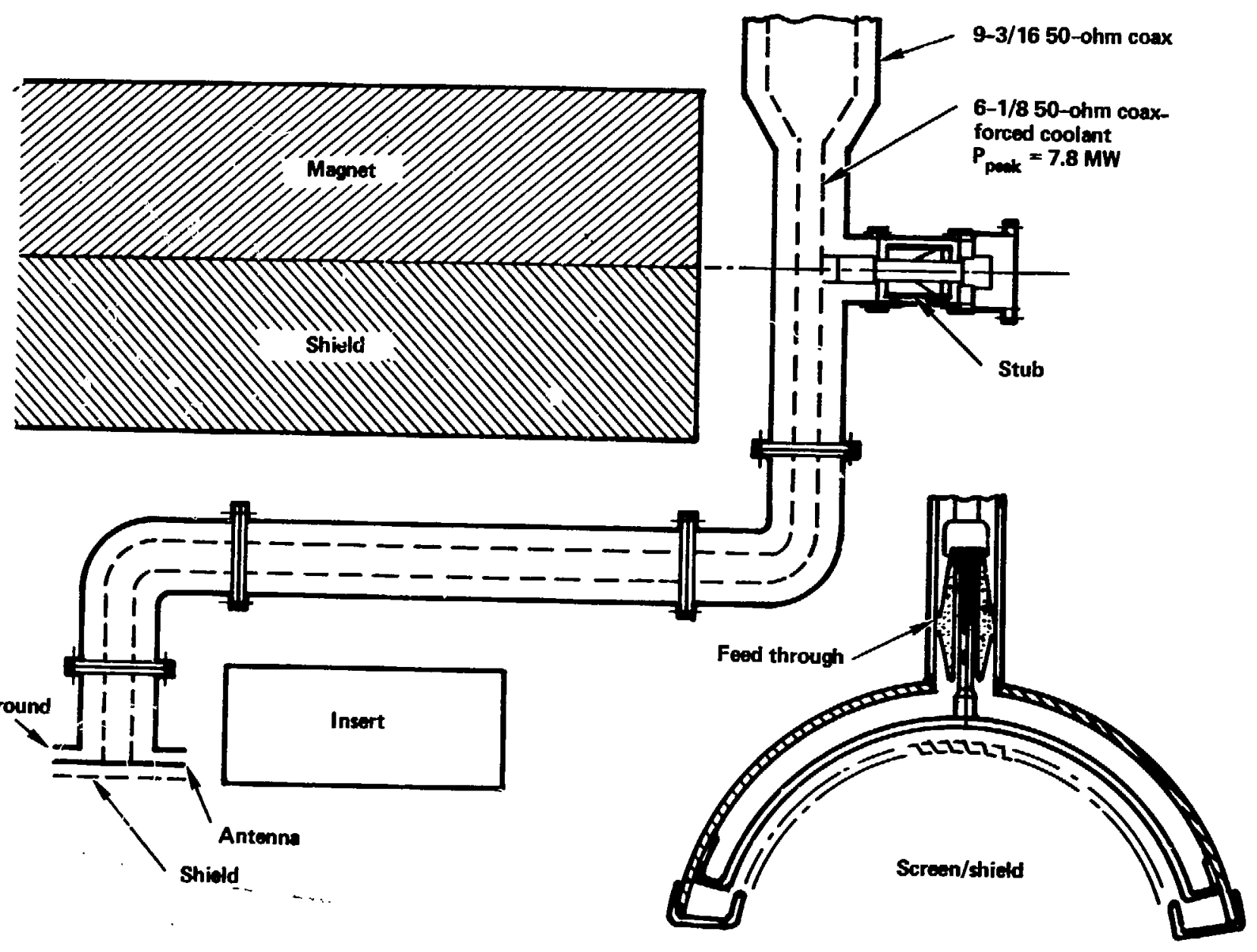

Fig. 3.3-4. ICRH system layout. 


\section{REFERENCES FOR SECTION 3.3}

1. P.L. Colestock, "High Power Ion Cyclotron Heating Experiments on PLT," in

Proc. of the 4th Topical Conference on RF Heating in Plasmas, Austin, TX (Feb. 9-10, 1982), A2.

2. J. Hosea et al., "Fast Wave Ion Cyclotron Heating in the Princeton Large Torus," in Proc. of 8th Int. Conf. on Plasma Physics and Controlled Nuclear Fuston Research, Brussels (July 1-20, 1980), p. 100.

3. TFR Group, "ICRF Heating in TFR 600," in Proc. of 8th Int. Conf. on Plasma Physics and Controlled Nuclear Fuston Research, Brussels (July $1-10,1980), \mathrm{p} 98$.

4. D.K. Snith et al., Bull. Amer. Phys. Soc., 25, 859 (1980).

5. S.N. Golovato et al., Bull. Amer. Phys. Soc., 25, 859 (1980).

6. M. Porkalab, MARS discussions.

7. J.E. Scharer et al., Nuclear Fuston, 17, 297 (1977).

8. D. Hwang, "ICRF Heating Experiments on PLT," in Proc. of INTOR ICRF Workshop, Oak Ridge Natlonal Laboratory, Oak Ridge, TN, (May 6-7, 1982).

9. B. Badger et al ., MUWAK, A Tokamak Reactor Design Study, "University of Wisconsin, UWFDi-330 (march 1979).

1u. C.A. Flanagan et al., Inttial Trade and Design Studies for the Fusion Engineering Device," Oak Ridge kational Laboratory, Oak Ridge, Ti, ORTL7TH-77Th, (1981).

11. S.A. Freije, "FED and STARFIRE ICH Systems," in Proc. of 9th Sym. on Engineering Problems of Fusion Research, Chicago, IL Toct. 26-29, 1981), pp 859.

12. A Design Analysis of Supplemental Heating System, TRW, Redondo Beach, CA, prepared for U.S. Dept. of Energy/0rfice of Magnetic Fusion Energy, (Sept. 1981) D-149.

13. Y.P. Thatnagar et al., Description of the Ion Cylcotron Resonance Heating System to be Applied on the TEXTOR Tokamak, Laboratoire de Physique des Plasma - Laboratoriom voor Plasma fysica, Report $\mathrm{nr}$ 78, (September 1981).

14. H. Kimura et al., "ICRF Heating Experiment in the JFT-2," in Proc. of the IAEA 9th Int. Conf. on Plasma Physics and Control led Nuclear Fuston Research, Baltimore (Sept. 1-8, 1982), IAEA-CN-417J-3. 


\subsection{PUMP EEAM}

The MARS reactor baseline requires that $1480 \mathrm{~A}$ of neutral beam current ( $740 \mathrm{~A}$ per end) at $94 \mathrm{kV}$ be injected from $30 \mathrm{deg}$ off the axis at the 9.3-T magnetic field location. The beam profile spans the range from 7.8 to $10 \mathrm{~T}$. This injection point is $85 \mathrm{~m}$ from the device midplane, between the axicell and transition coils. The beam line location is shown in Fig. 3.4-1. The energy, angle, and magnetic field choices are determined by the physics requirement that the charge-exchanged ions from the beam are in the local loss cone and can pass into the central cell. Because of the high power (140 MW) injected by this system, the pump beam lines must be as efficient as possible and yet they must represent reasonable technology advances in the MARS time frame. We have not yet analyzed either the beam dumps or the heat flux on the walls and other structures from charge-exchange neutrals.

\subsubsection{Pump Beam System Analysis}

We have investigated several options investigated for this very-highpower injection system. Positive ion source beam lines based on the existing technology can be used for the pump beam. These would require an energy recovery system for the unneutralized ions and some advances in positive ion source technology. Negative ion sources can also be used with laser photodetachment neutralization to produce a highly efficient system. These options will be discussed in further detail in the following section.

Positive ion sources produce some fraction of the accelerated current as molecular ions. These particles dissociate during neutralization and form half- and third-energy products that can charge exchange in the plasma. The fractional energy products are then either trapped in the transition region and must be removed by additional pumping current or separated and collected before injection into the reactor. If the partial energy products are allowed to enter the plasma, then roughly $50 \%$ more current must be supplied by $90 \% \mathrm{D}^{+}$ sources to pump the transition region. This represents a large increase in the required injected power to $206 \mathrm{MW}$, and necessitates a much larger neutral beam injection system. System electrical efficiencies of up to $50 \%$ are possible if energy recovery is used.

The molecular species can be removed by magnetic mass filtering before neutralization. A large sweep magnet located behind the accelerator system 


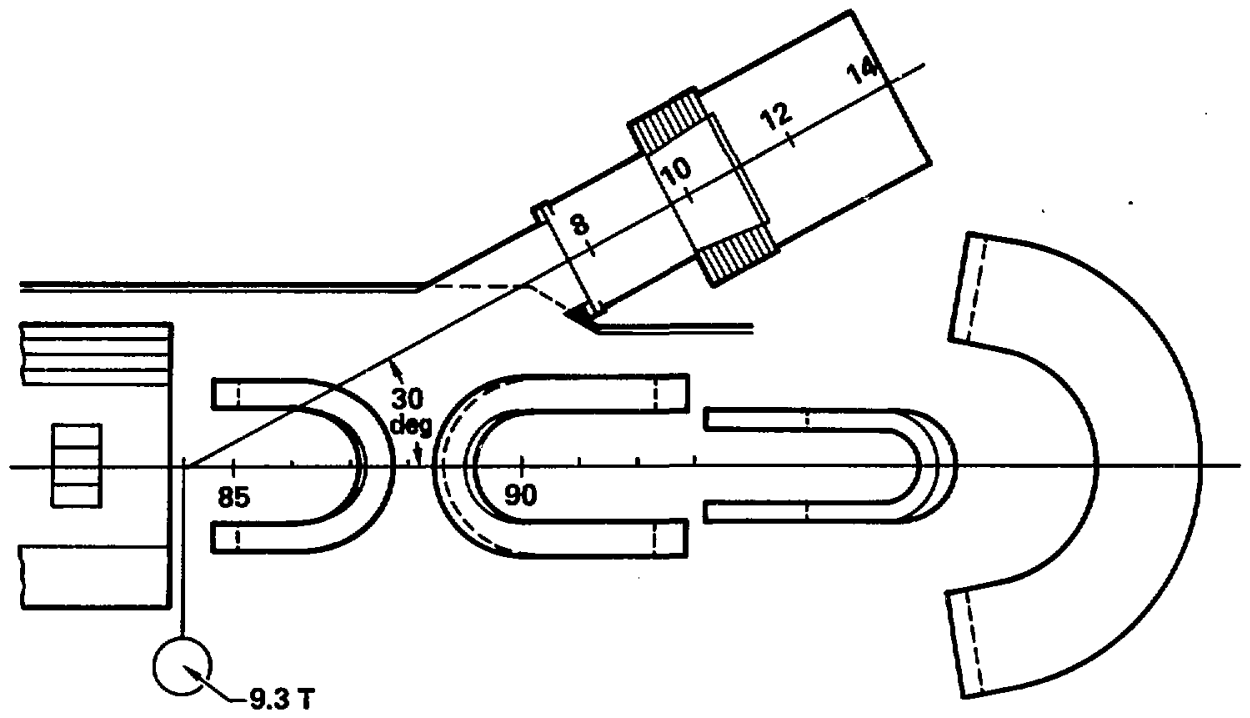

Fig. 3.4-1. MARS pump beam location. 
deflects the atomic ions toward the target and allows the molecular species to be collected in the bean line. The neutralizer would have to be decoupled from the accelerator by a distance of about 0.5 to $1.0 \mathrm{~m}$ and additional gas provided in the duct. The beam losses in the magnet region as a result of charge exchange with the gas from the source are excessively large (20 to 30\%) and cause low system efficiencies of less than $40 \%$. The additional neutral izer gas increases the pumping requirements and enlarges the beam lines.

A new mass filtering technique recently reported by Ken Ehlers of Lawrence Berkeley Laboratory (LBL) would decrease the losses in the magnet region and has revived interest in a positive ion pump beam. The LBL mass filter basically consists of very thin rows of magnets aligned in the decel region of the grids to produce a transverse magnetic field. Each magnet row is shielded from the beam by the accelerator electrodes and produces a relatively short magnetic field region $(4$ to $6 \mathrm{~cm}$ ) between the source and neutralizer. The use of closely spaced $\mathrm{SmCO}_{5}$ magnets produces adequate mass separation if the beam lines are long enough. The mass filter effectively removes the partial energy beam components before injection. Ions that charge exchange with the gas from the source in the magnet region are lost. Calculated values for this beam loss range from only 2.5 to $5 \%$, jepending on the extent of the fringing fields assumed. The deflected atomic ions from the source are then neutralized in a standard gas cell of near equilibrium density. Unneutralized ions from this gas cell can be subjected to energy recovery at high efficiency because the partial energy ions have been removed and a fairly monoenergetic beam results. A schematic representation of this system is shown in Fig. 3.4-2, where the beam has been rotated 90 deg with respect to the accelerator to illustrate the mass separation. A typical positive ion beam line based on these sources is illustrated in Fig. 3.4-3.

A negative ion system with photodetachment is 11lustrated in Fig. 3.4-4. Electrical efficiencies of 55 to almost 7 。 are possible with this system because photodetachment provides 95\% stripl:..5 efficiency. Electrical efficiency vs beam energy is illustrated in Fig. 3,4-5. Since laser efficiencies of more than $10 \%$ are projected, beam line electrical efficiencies of $60 \%$ are achieved at the cost of the laser hardware.

Table 3.4-1 illustrates the comparison between these four options. The negative ion systems are divided into a one-source-per-laser neutralizer and a 


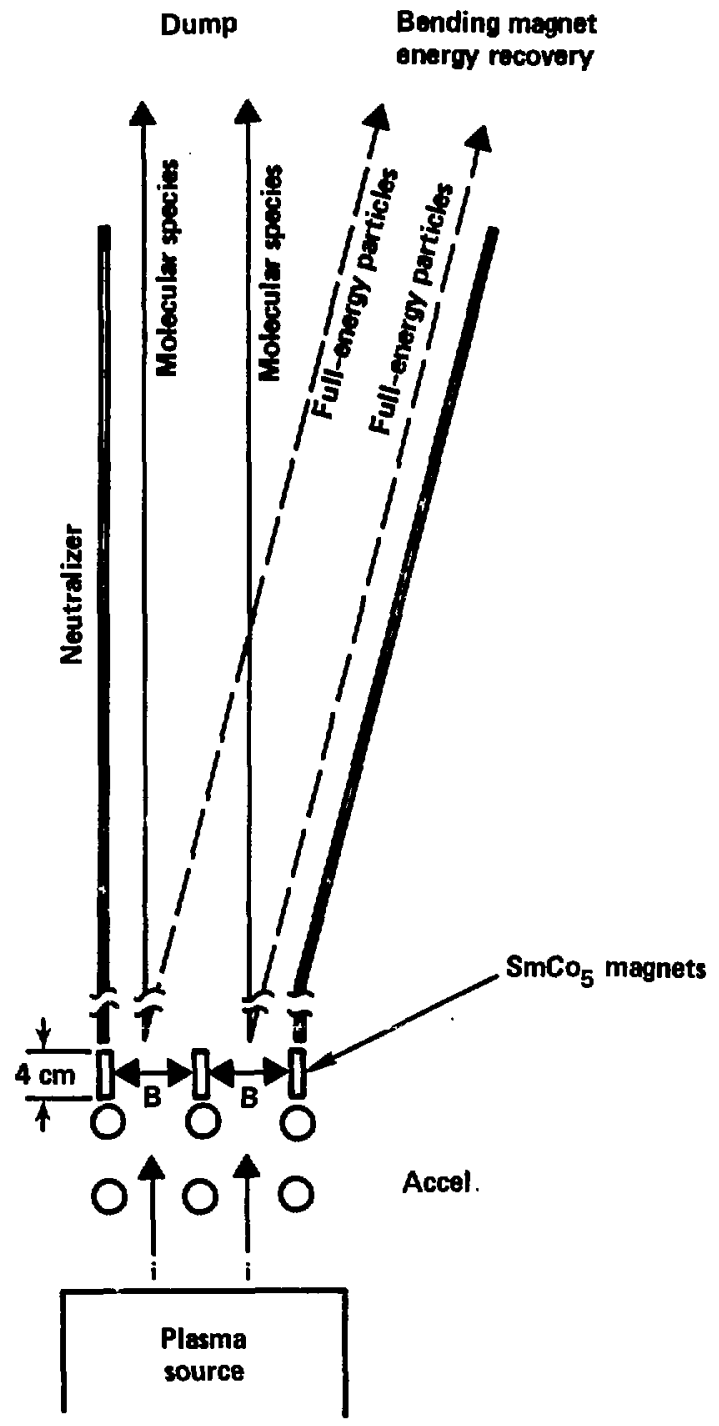

Fig. 3.4-2. Schematic of LBL positive ion mass filter. 


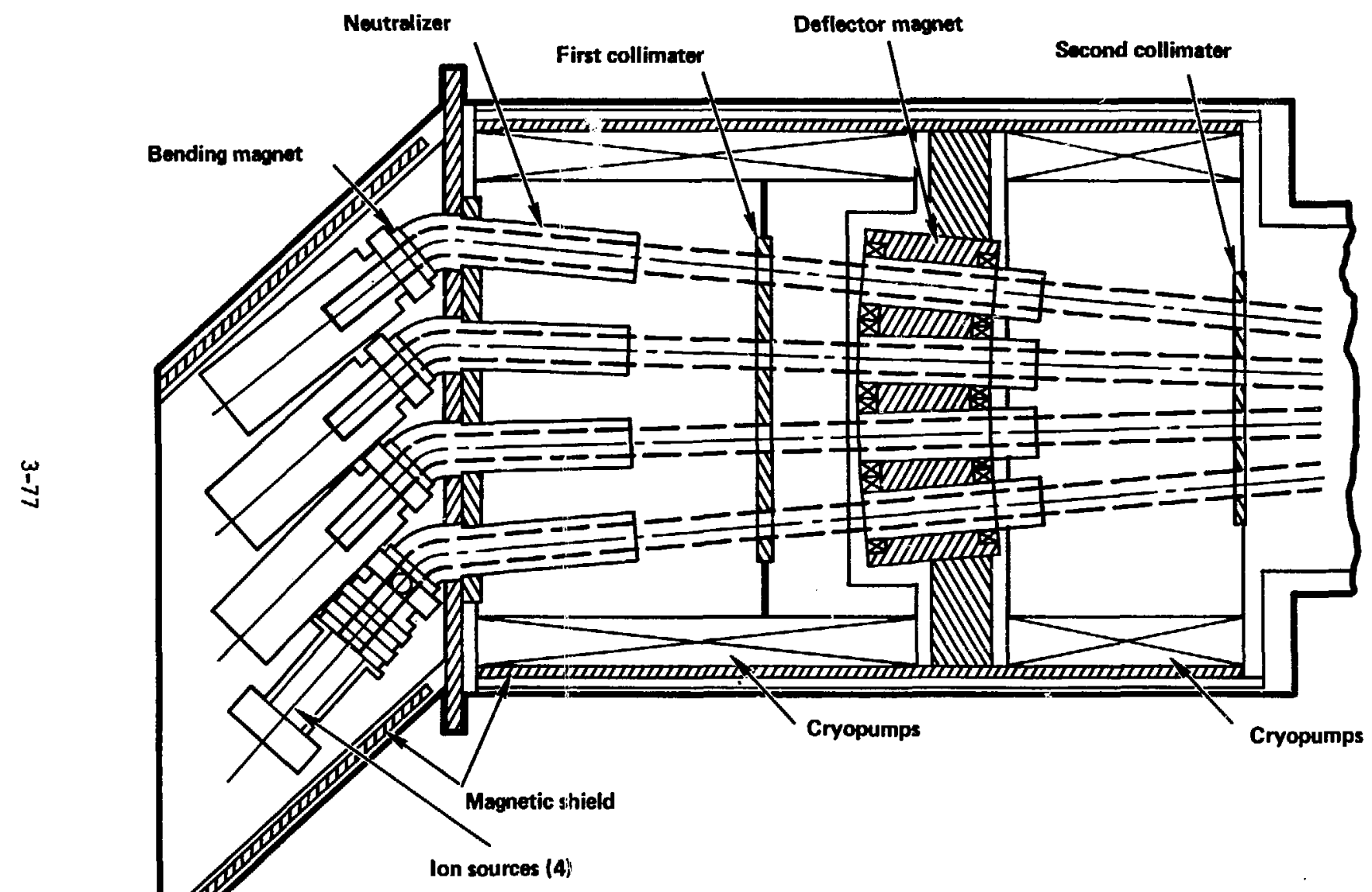

Fig. 3.4-3. MARS pump beam line. 


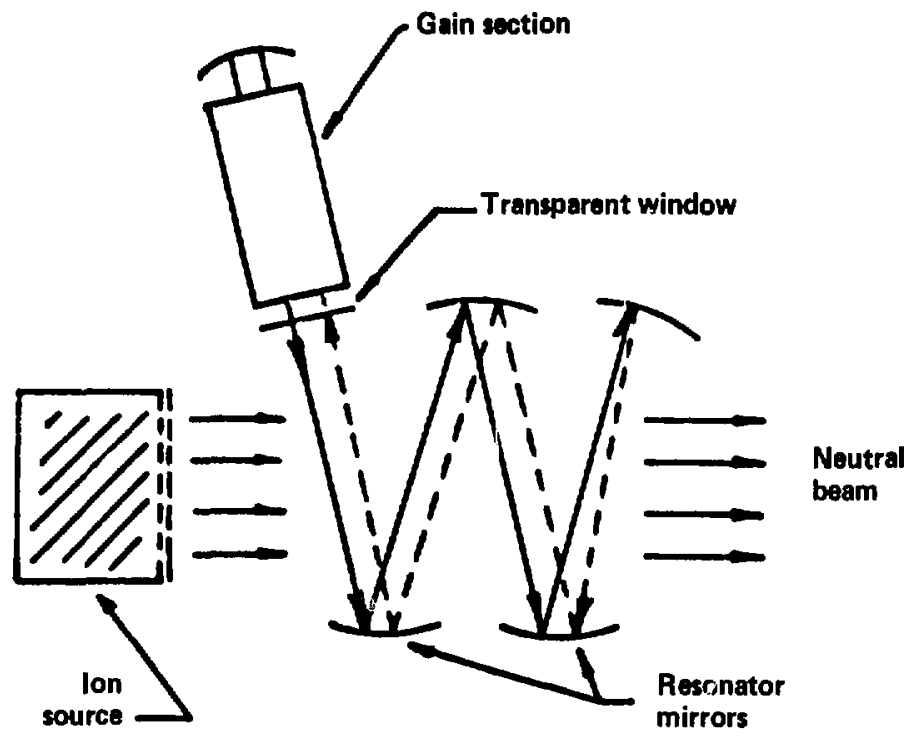

Fig. 3.4-4. Folded iaser resonator with muitiple mirrors. 


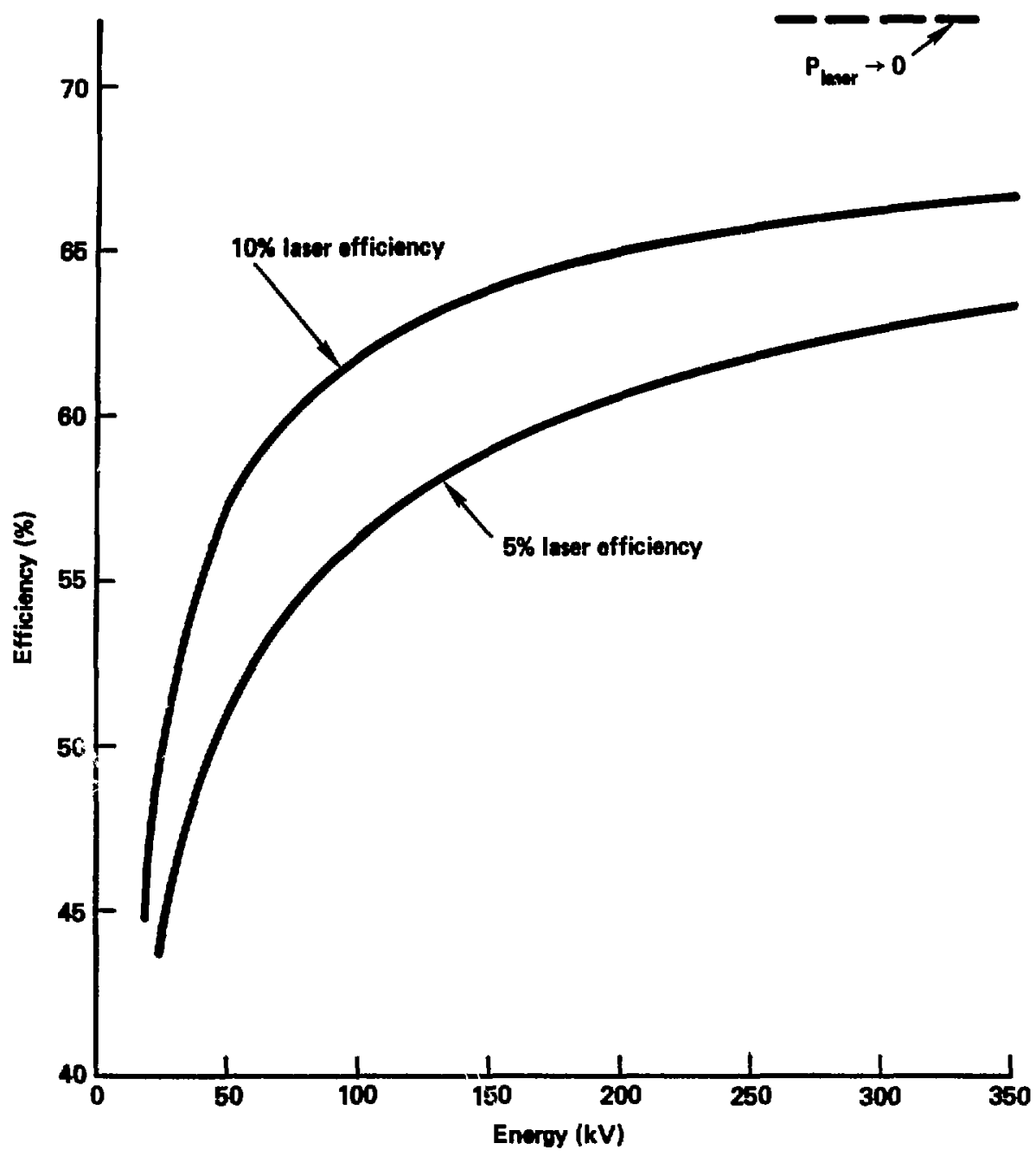

Fig. 3.4-5. Sensitivity of the negative ion beam system efficiency to photodetachment laser efficiency. 
Table 3.4-1. Comparison of pump beam options.

\begin{tabular}{|c|c|c|c|c|c|c|}
\hline $\begin{array}{l}\text { Ion } \\
\text { type }\end{array}$ & $\begin{array}{l}\text { System } \\
\text { electrical } \\
\text { efficiency } \\
(x)\end{array}$ & $\begin{array}{l}\text { Total } \\
\text { injected } \\
\text { power } \\
\text { (MN) }\end{array}$ & $\begin{array}{l}\text { Wal1 } \\
\text { power } \\
\text { (MN) }\end{array}$ & $\begin{array}{l}\text { Minimum } \\
\text { number of } \\
\text { sources }\end{array}$ & $\begin{array}{c}\text { Total } \\
\text { aas } \\
\text { flow } \\
\text { (Torr-1iter/s) }\end{array}$ & Requirements \\
\hline Positive & 50.5 & 206 & 404 & 42 & 945 & Energy recovery \\
\hline $\begin{array}{l}\text { Positive sweep } \\
\text { magnet }\end{array}$ & 38 & 144 & 380 & 40 & 900 & $\begin{array}{l}\text { Energy recovery } \\
\text { Good beam quality }\end{array}$ \\
\hline $\begin{array}{l}\text { Positive LBL } \\
\text { mass filter }\end{array}$ & 47 & 144 & 310 & 35 & 765 & $\begin{array}{l}\text { Energy recovery } \\
\text { Good beam quality }\end{array}$ \\
\hline $\begin{array}{l}\text { Negative } \\
l \text { source/ Taser }\end{array}$ & 55 to 60 & 144 & 240 & 35 & 546 & High current sources \\
\hline $\begin{array}{l}\text { Negative } \\
4 \text { sources/1aser }\end{array}$ & 65 to 70 & 144 & 215 & 35 & 546 & Multiple sources per laser \\
\hline
\end{tabular}


hopeful four-sources-per-laser neutralizer. A multiple-sources-per-laser neutralizer would be difficult for the pump beam because of the injection angle geometry constraints on stacking of the sources. We are examining this problem in further detail. The negative ion and LBL mass-filtered positiveion systems use similar numbers of sources and comparable gas flows. The positive ion system requires an additional 70 to $95 \mathrm{MW}$, the development of energy recovery, and perhaps some beam focusing by the magnetic field.

\subsubsection{Ion Sources}

For positive ton source systems, four beam lines (illustrated in Fig. 3.4-3) are necessary. Each source has an extraction area of $15 \times 70 \mathrm{~cm}$ and produces an average current density of $0.12 \mathrm{~A} / \mathrm{cm}^{2}$ of deuterium ions over that area. The source configuration for continuous plasma production and confinement has not been developed, but will probably contain magnetic multipole confinement and either hollow cathoce or rf plasma generation. The gas flow required for each $50 \%$ utilization efficiency source is 25.2 Torr -1 iter $/ \mathrm{s}$. The $126 \mathrm{~A}$ of accelerated ions consist of $90 \%$ atomic ions, of which $5 \%$ are lost because of charge exchange in the LBL magnetic mass filter region.

The negative ion sources are difficult to project at this time because this is a new technology. A system designed by Fink and Neef at LLNL would use 12 LBL self-extraction negative ion sources per beam line operating at a $50-\mathrm{mA} / \mathrm{cm}^{2}$ current density in a slot $2.5 \mathrm{~cm} \times 3 \mathrm{~m}$. The long dimension would be broken into six equal segments. A system based on Brookhaven National Laboratory's (BNL) sources would consist of four source units per beam line. Each unit would use multiple slots with an accelerator area of $10 \times 150 \mathrm{~cm}$ and an average current density of $50 \mathrm{~mA} / \mathrm{cm}^{2}$.

\subsubsection{Accelerator}

The positive ion source accelerator uses multiple cooled rails with an effective transparency of $40 \%$. The accelerator produces a beam with $1.0-x$ 0.5-deg optics and operates continuously. The LBL filter system would incorporate magnets in the decel regions and has yet to be developed. The negative ion system accelerators are specified to produce the same optics as the positive ion sources, but we have not yet determined the configuration. 


\subsubsection{Neutralizer}

The positive ion source systems use a conventional close-coupled gas neutralizer. The negative ion source system uses a laser resonator photodetachment cell. Competing schemes consist of either multiple lasers spaced along the beam line axis, or a single large laser with multiple mirrors to fold the light flux down along the beam axis. Because the total flux and interaction length is specified by the 95\% stripping requirements, the total power required by both systems is approximately the same. Estimates of the laser wall power produce 2 to 5 of input power per beam line, depending on the laser efficiency and resonator configuration assumed.

\subsubsection{System Layout}

The pump beam layout is illustrated in Fig. 3.4-3 and consists of four beam lines per end of the machine. The choice of positive vs negative ion sources will be made in the next few months after we complete our analyses of the geometry, efficiency, and approximate cost of each system. The higher electrical efficiency of the negative ion beam system (60\%) compared to the 45 to $48 \%$ calculated for the positive ion source system is balanced by the expected higher capital cost of negative ion systems. It appears that the size of both systems is about the same because ine lower negative-ion current density offsets the lower positive-ion neutralization efficiency. Table 3.4-1 compares system characteristics for various pump beam options; technology advances are required in both proposed systems and are briefly described in Table 3.4-2. 
Table 3.4-2. Required technology advances.

LBL positive-ion mass filter system

1. High-current (110-A) continuously operating 100-kV positive-ion sources.

2. Very thin, cooled $\mathrm{SmCO}_{5}$ magnets incorforated into the tecel stages of the ion source.

3. Magnetic focusing of the ion beam to maintain optical quality of the hesm (perhaps).

4. Energy recovery of the unneutralized atomic ions at high efficlency ( 70 to $80 \%$ ).

Negative ion system with laser photodetachment

1. High current (40- to 50-A) continuously operating, $100-\mathrm{kV}$ negative-ion sources.

2. High power (30- to $80-\mathrm{kW}$ ) continuously operating COIL laser resonator.

3. Laser photodetachment system. 
The sloshing ion beam lines must deliver $39.2 \mathrm{~A}$ (19.6 A per end) at $475 \mathrm{kV}$. The injection angle is $90 \mathrm{deg}$ from the MARS axis and the injection point is $100 \mathrm{~m}$ from the device midplane. Because the neutralization effiliency is less than $5 \%$ for positive ions at this energy, negative ions must be used for this beam. Laser photodetachment is then the preferable method of achieving 95\% stripping of the ion beam. The accelerator must produce this beam with a high electrical efficiency and must also contain a system for electron rejection.

\subsubsection{Ion Source}

The ion source uses a cesiated surface to convert into negative ions those positive ions formed by an rf plasma generator in a magnetic multipole bucket containment geometry. Because the acceleratur (discussed in Section 3.5.2) uses circular beamlets, the cesiated surface is machined to focus the ions into two rows of 34 circular apertures located $5 \mathrm{~cm}$ apart. This twodimensional focusing of the ions produces $50 \mathrm{~mA} / \mathrm{cm}^{2}$ of negative ions at each 1.5-cm-radius aperture on the first grid. This geometry produces a total current of $0.35 \mathrm{~A}$ per beamlet. The area of the beam is roughly $10 \times 170 \mathrm{~cm}$, with a majority of the space occupied by the accelerating and focusing electrodes.

The source configuration is based on the LBL self-extraction negative-ion source with some anticipated improvements in current density and gas efficiency. The cesiated surface is located roughly $15 \mathrm{~cm}$ from the accelerator and the electron current from the plasma is suppressed $b y$ a transverse magnetic field produced by permanent $\mathrm{SmCO}_{5}$ magnets. The plasma generacor operates continuously with an electrical efficiency of $5 \mathrm{~kW} / \mathrm{A}$ of negative ion beams and a $30 \%$ gas utilization efficiency.

\subsubsection{Accelerator}

Several accelerator options are possibilities for the sloshing ion beam. Systems that use rf acceleration, such as the LANL-RFQ and the BNLMEQALAC, provide high energy acceleration without using very high voltages. These systems are conceptually safer and easier to protect and milintain than d.c. systems. However, the time-averaged current density is low in the rf 
system, and the electrical efficiency is lower in the rf system than in a d.c. system because of inefficiencies in joule heating and power supply modulators. The standard d.c. multiple grid accelerators used today in positive in systems could possibly be reconfigured to produce a high-voltage regative ion beam. These very high voltages, however, can crntribute to stray electronand radiation-induced breakdowns in the accelerator colum that cause unreliable operation. The sicored energy in each grid must also be minimized to eliminate meltdown problems associated with the arc breakdowns. A d.c. electro-static quadrupnle (ESO) system, in which the beam is focused by electrostatic quadrupoles between accelerator stages, reduces the probability that a breakdown will occur over the entire accelerator column. Accelerator length is also adjustable by the quadrupole parameters so that reasonable voltage gradients can be achieyed.

The d.c.-ESQ accelerator concept is basically a d.c.-MEQA! iC where the acceluration occurs between focusing stages over an extended accelerator length. If a conservative voltage gradient of $10 \mathrm{kV} / \mathrm{cm}$ is selected, the accelerator colum length will range from $65 \mathrm{tc} 80 \mathrm{~cm}$, depending on the space requires by the quad support structures. The pre? iminary accelerator design consists of two rows of 34 beamlets eacii. Each beani? et is $1.5 \mathrm{~cm}$ in radius and carries $350 \mathrm{~mA}$ of negative ion current. The ions from the source are preaccelerated by a standard d.c. multiple grid accelerator to 50 to $100 \mathrm{kV}$ and then injected into the quad system. We estimate that losses of the negative ions as a result of charge exchange in the accelerator column are below $10 \%$. We assume the accelerator electrical efficiency to be $85 \%$.

\subsection{3 leutralizer}

We have chosen a laser photodetachment neutraijizer for this beaml ine because of this neutralizer's very high stripping fractions. A folded resonator with ihe ion beam passing through the peak laser flux produces reasonable requiremer 's for the laser power to achieve $95 \%$ stripping. The initial design uses two resonators (one for each row of beamlets), with a diameter mirrors. Each system requires laser power of $70 \mathrm{~kW}$ and six mirrors to fold the laser resonator along the path of the ions. Assuliing a $10 x$ laser electrical efficiency ( 5 to $16 \%$ projected efficiency), the neutraitizers 
require a total of 1.4 of wall plug power. Focusing of the beam to a smaller size $(<3 \mathrm{~cm})$ by the d.c.-ESQ accelerator will decrease the requirements on mirror size and laser power.

\subsubsection{System}

Figure 3.5-1 shows a schematic representation of the sloshing ion beam line. We are currently working on a detalled design of each component. Some of the parameters chosen for each beamline are indicated in Table 3.5-1. Based on these values, the total system electrical efficlency, defined as beam power on-target divided by the required electrical power, is $69.5 \%$. 


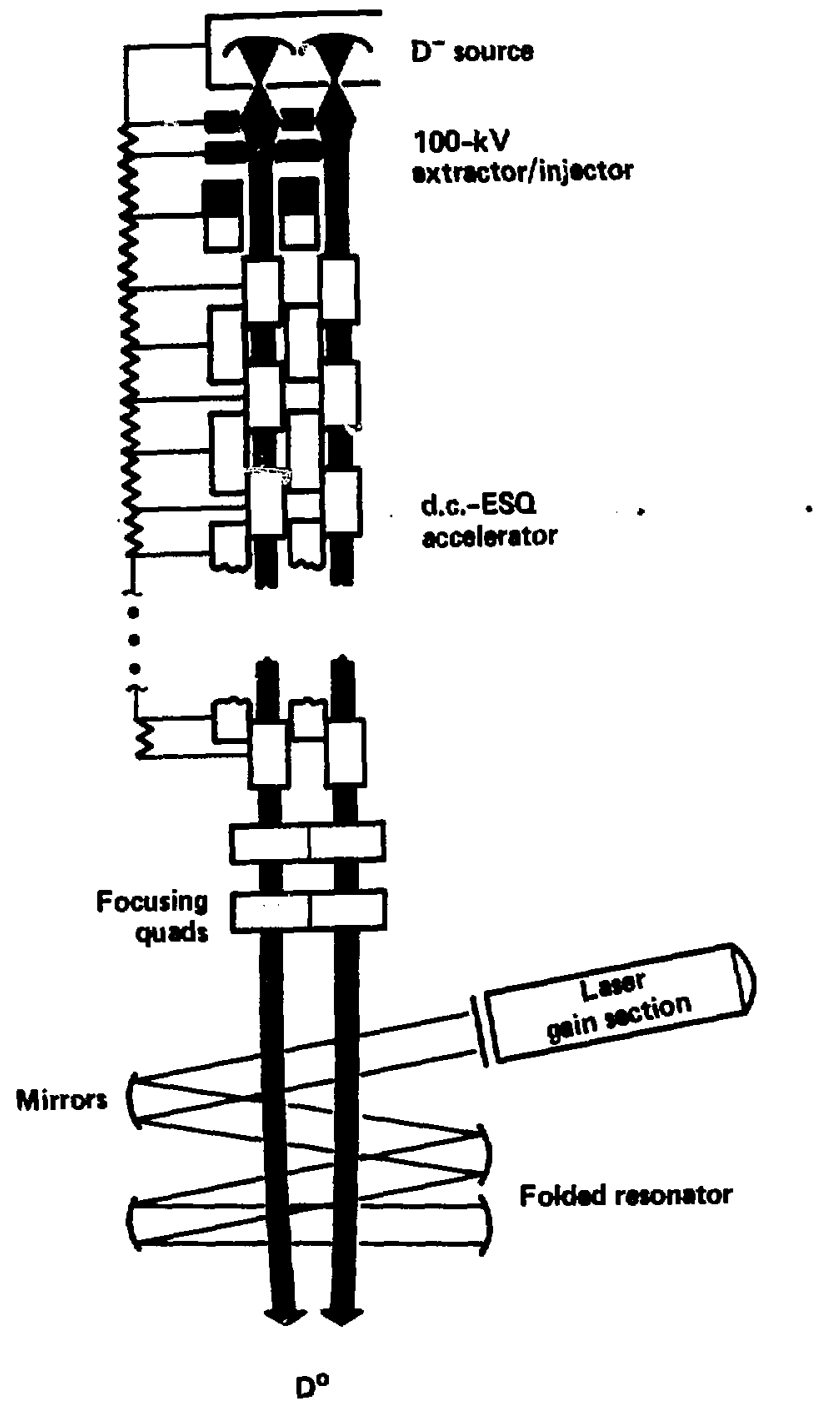

Fig. 3.5-1. Schematic of the sloshing ion beamline. 
Table 3.5-1. Sloshing ion beamline parameters.

Ion source

Modified LBL self-extraction source

Electrical efficiency

Gas efficiency

Total current

Current density

Beam area

Number of apertures

Gas flow
$5 \mathrm{~kW} / \mathrm{A}$

$30 \%$

$24 \mathrm{~A}$

$50 \mathrm{~mA} / \mathrm{cm}^{2}$

$10 \mathrm{~cm} \times 170 \mathrm{~cm}$

68 (1.5-cm radius)

6.75 Torr-liter/s

Neutralizer

Laser photodetachment

Stripping fraction

Number of lasers

Laser efficiency

Mirror reflectivity

Scatter/defraction losses

Window transmission

Number of folds

Mirror di ameter

Laser power

Total wall power
95\%

2

$10 \%$

0.999

$0.2 \%$

0.998

5

$4 \mathrm{~cm}$

$70 \mathrm{~kW}$

$1.4 \mathrm{MW}$

System

Accelerator efficiency

$85 \%$

Ion loss in acceleratior

$6 \%$

Total collimator scrape off

$5 \%$

Duei reionization loss

$5 \%$ 


\subsection{END-PLASMA TECHNOLOGY}

The end-plasma technology area consists of a group of related but separate systems. They are grouped together because they all reside in the end vacuum tanks and are involved in handling charged-particle leakage from the central cell. The end plasma technology consists of the following systems:
- Ion converter end;
- Electron converter end;
- Halo scraper;
- Continuous cryopumps;
- Vacuum tanks and support siructure.

In addition, we discuss the tritium containment and processing problems that occur in these systems.

\subsubsection{Direct Converter}

The direct converter consists of the ion end collector, the electron end collector, and their associated grids. The principal issue is to determine whether it is cost effective to directly convert the approximately $800 \mathrm{~W}$ of charged particle energy that escapes from the central cell. It is obvious that recovering some or all of the charged particle energy is desirable. It is not as obvious, however, that direct conversion is a cost effective method of recovering the additional energy. Physics considerations (see Section 2.2-4) dictate that a small central-cell plasma potential is necessary for stability. One way to create this condition is to depress the end cell potentials several hundred keV below ground. As a result, it is obvious that we can achieve little or no cost savings by eliminating direct conversion, and so our design objectives have been to maximize the energy recovered.

The halo scrapers, although used as plasma limiters, are in fact a part of the direct conversion system. The charged particles that are removed from the plasma edge, flowing against the bias potential of the electron collector, contribute a significant portion of the total recovered energy. 
The present configuration has the electron collector and entrance grid at $-170 \mathrm{kV}$, the suppressor grid at $-270 \mathrm{kV}$, and the ion collector at $156 \mathrm{kV}$. This configuration is expected to recover 426 of electrical energy and dump 408 of thermal energy. The baseline is designed around water-cooled AMAXMZC copper grids and plates. The maximum water temperature obtainable with this design is about $215^{\circ} \mathrm{C}$. This is not high enough quality heat to make thermal recovery efficient. We recognize that recovering a portion of this energy would be useful, and we are conducting studies of the use of higher temperature water or gas cooling. Figure $3.6-1$ is a conception of the ion collector end of the reactor.

The entrance grid (1), suppressor grid (2), and ion collector plate are the major components of the ion converter which, by selective leakage, receives most of the ion flow. The overall layout of these components is shown in Fig. 3.6-1. Electrostatic shields to prevent high voltage-breakdowns are not shown. Each of the components must be segmented to limit the capacitively stored energy in any one segment.

The location of these components is determined by setting the average heat load on grid 2 at $2.5 \mathrm{~mW} / \mathrm{m}^{2}$. Each grid has $5 \%$ opacity and receives the heat and electrical power loads given in Section 2.9. Grid 2 is the most highly loaded, with $30.8 \mathrm{~W}$ giving an area of $246.4 \mathrm{~m}^{2}$. From conservation of magnetic flux, the field strength in the plasma fan when it has expanded to $246.4 \mathrm{~m}^{2}$ is $0.0079 \mathrm{~T}$. EFFI calculations provide coordinates, where the field is $79 \mathrm{G}$, which define the location and shape of grid 2 .

The relative geometry of grids 1 and 2 and the ion collector plate are shown in Fig. 3.6-2. Intergrid spacing is $50 \mathrm{~cm}$ and the distance from grid 2 to the collector plate is $100 \mathrm{~cm}$. The tube-to tube spacing of $20 \mathrm{~cm}$ is fixed by the 1-cm outer diameter of the tubes and the requirement for $5 \%$ opacity in the grid array. We chose the tube size $(1 \mathrm{~cm})$ as the largest practical size before the tube-to-tube spacing becomes too large to create a uniform field. Large-diameter tubes are more desirable because the heated length of the longest tubes is more than $4 \mathrm{~m}$. 


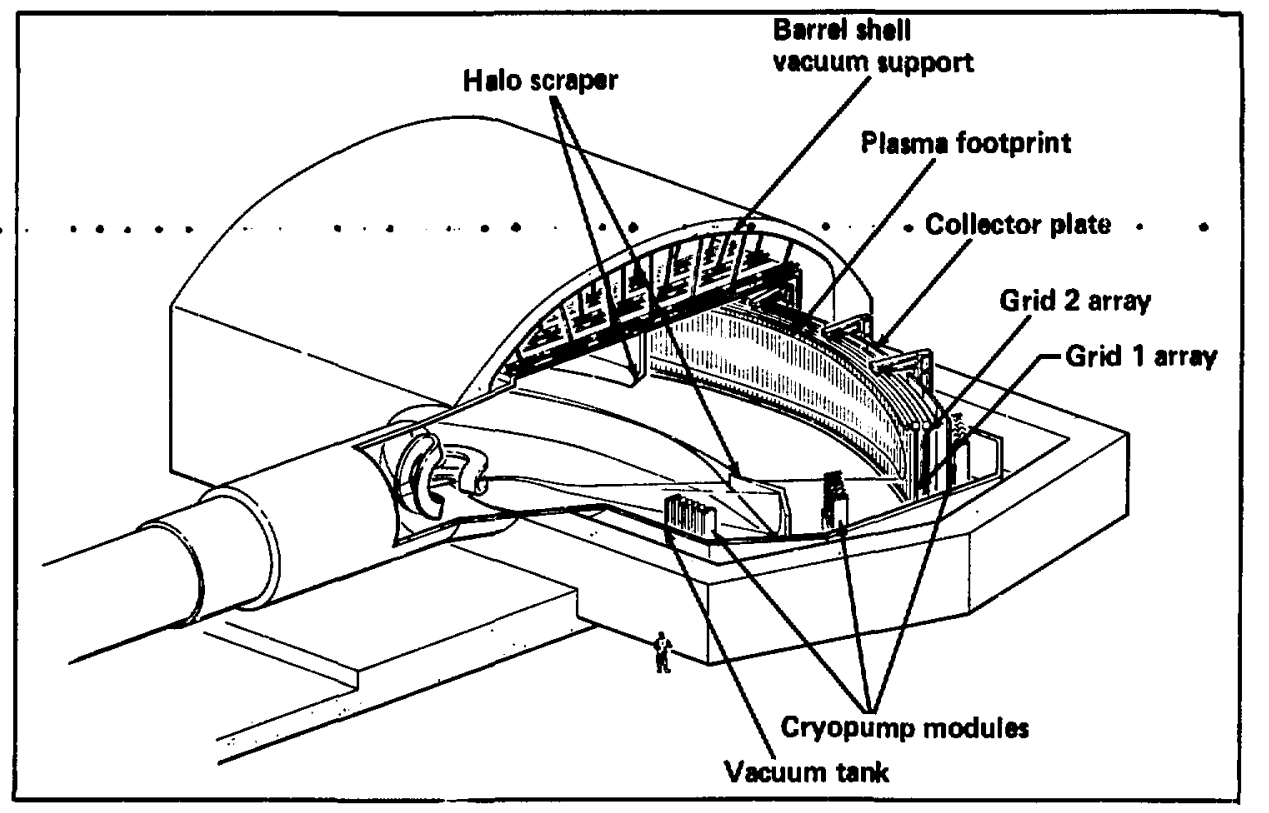

Fig. 3.6-1. MARS ion-end configuration. 


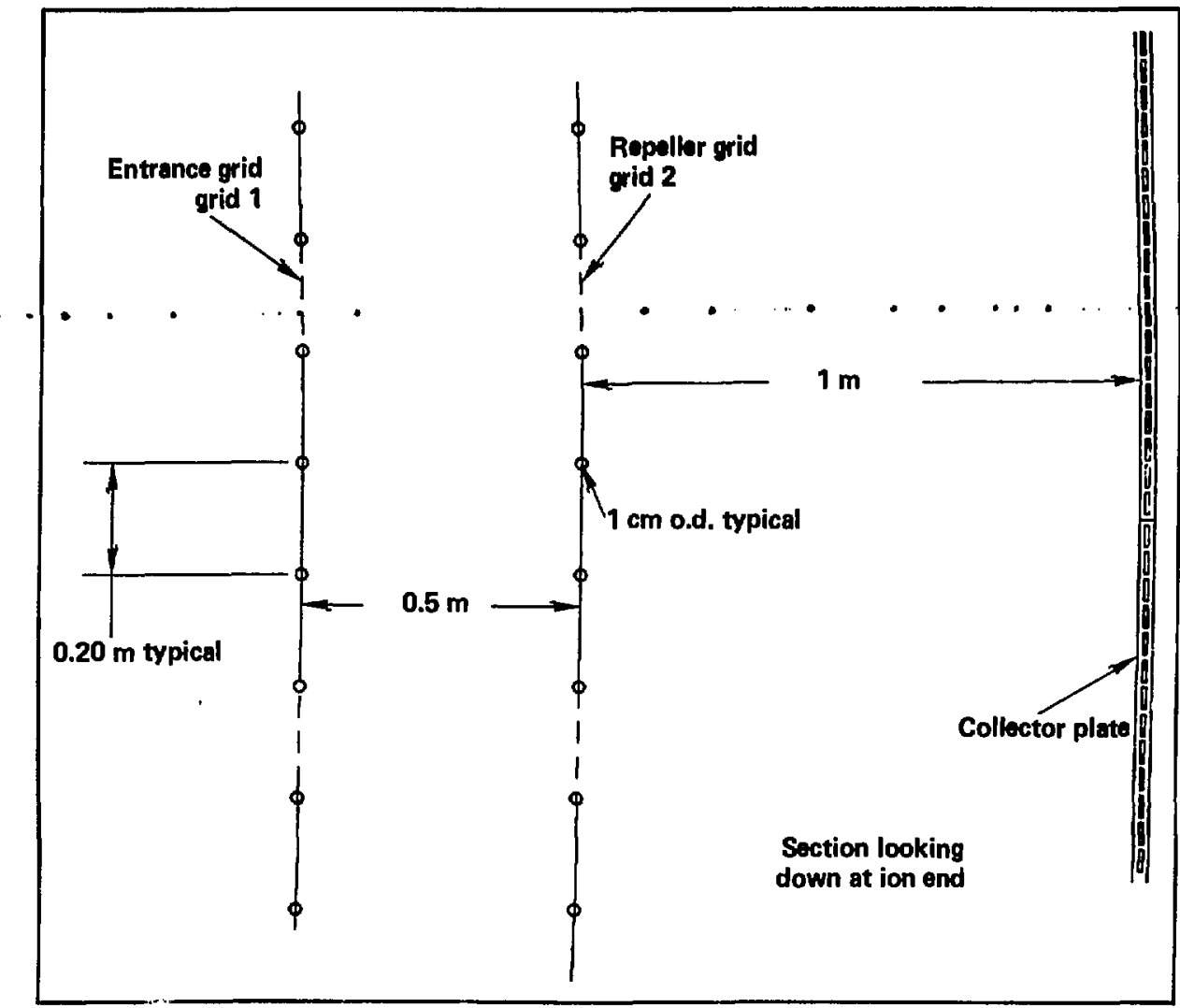

Fig. 3.6-2. Grid geometry. 


\subsubsection{Grid System}

Requirements. The grid tube electrical requirements were presented in Section 2.9.7. In addition, they must perform satisfactorily in the specified thermal, mechanical, and particle environments and maintain their thermalhydraulic and mechanical integrity under all operating conditions.

The thermal load for the grid tubes is defined by the power density distribution contours shown in Fig. 3.6-3. The actual plasma footprint is not exactly an ellipse. The range of power density contours shown is between 0.625 and $7.6 \mathrm{~m} / \mathrm{m}^{2}$. We obtained the direct converter power density contours by assuming that powerflow along a flux line is proportional to the square of the density in the central cell, and that the density falls off radially in accordance with the relationship

$$
n(r): \dot{n}_{c}\left(1: r^{3}\right)
$$

where $n_{c}$ is the density at the center of the cell and $r$ is the fraction of radial distance in the central cell. By assuming flux conservation and by mapping circular cross sections in the central cell into eliiptical cross sections at the direct converter, we can show that the power density $\phi$ is

$$
\Phi=\frac{20}{9} \Phi_{a v} f\left(\beta_{c}, 1\right) \frac{\left(1-r^{3}\right)^{2}}{\sqrt{1-\beta_{c}\left(1-r^{3}\right)}},
$$

where

$$
f\left(\beta_{c}, t\right) \equiv 2 \int_{0}^{t} \sqrt{1-\beta_{c}\left(1-x^{3}\right)} x d x,
$$

$\phi_{a v}$ is the average direct converter power density, and $\beta_{c}$ is the central cell beta. Figure 3.6-3 shows the power density distribution for a $\beta_{c}$ of 0.7 and a $\Phi_{\text {av }}$ of $2.5 \mathrm{mw} / \mathrm{m}^{2}$.

Design. The present grid design evolved from a study of grid cooling methods, configuration optimization, and material suitability considerations. We had to resolve the question of passive (radiation) vs active cooling at an early stage because of the considerable differences in system complexities for 


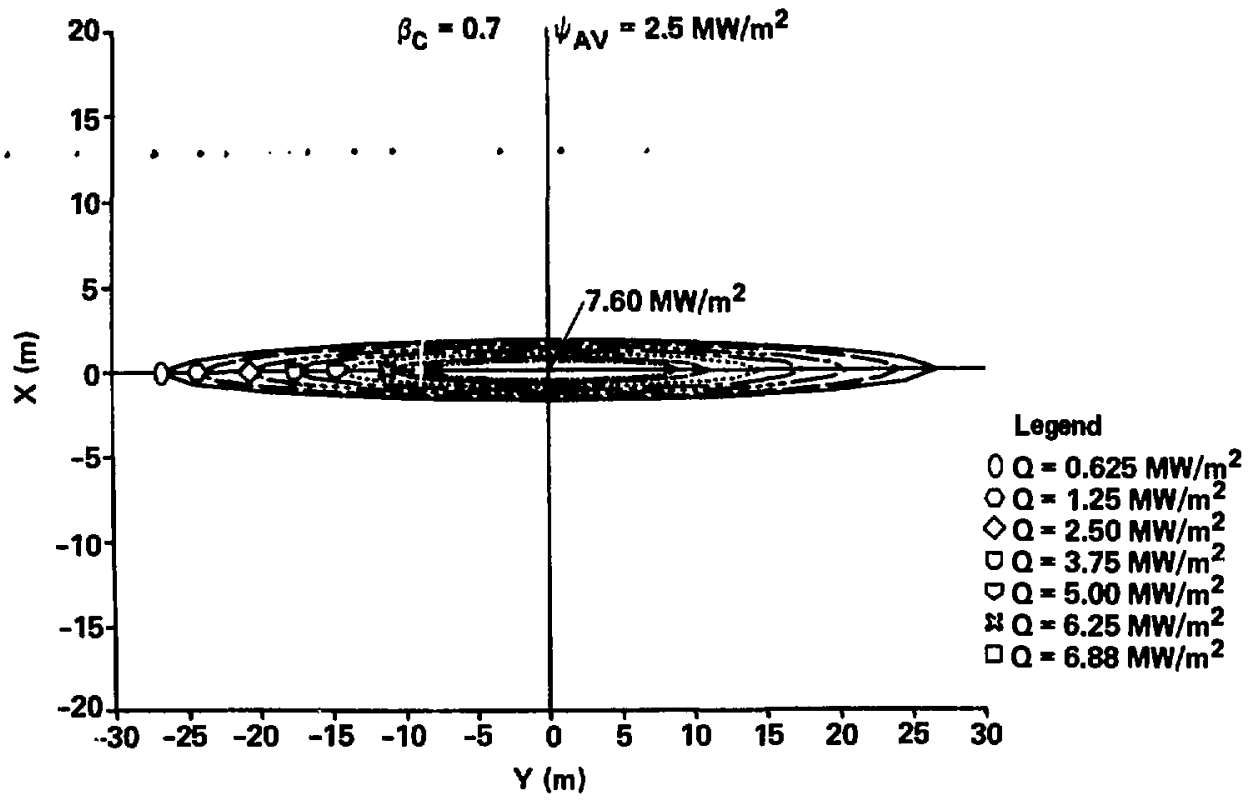

Fig. 3.6-3. Power density distribution. 
the two modes of cooling. Our study was conducted to determine the feasibility of a radiatively cooled grid system. We selected tungsten as the grid tube material because of its high temperature capability.

Figure 3.6-4 shows grid temperature as a function of power density. The two solid curves bracket the grid temperatures obtained when only half of the cylindrical tube is assumed to be radiating and when the full cylinder is radiating. Superimposed on the figure are a few limiting temperature lines, ranging between the temperature at which the material strength goes below $10 \%$ and the melting temperature. Also shown are two thermionic emission lines of $3 \mathrm{~A} / \mathrm{cm}^{2}$ and $80 \mathrm{~A} / \mathrm{cm}^{2}$. He concluded that at a peak power density of $7.6 \mathrm{~mW} / \mathrm{m}^{2}$, a radiatively cooled grid will be unacceptable.

He then performed a thermostructural analysis for active cooling. The assumptions, conditions, and design requirements are listed in Table 3.6-1. Figures 3.6-5 and 3.6-6 show grid performance as a function of grid tube outside diameter. The optimum tube diameter is about $1 \mathrm{~cm}$.

With the tube outside diameter fixed at $1 \mathrm{~cm}$, we investigated the following three water-cooled MZC copper grid-tube point designs:

- Design 1--Maximum grid tube temperature should not exceed $400^{\circ} \mathrm{C}$. Operation at higher than $400^{\circ} \mathrm{C}$ is considered marginal for the expected stress levels.

- Design 2--Coolant should not operate in boiling regime at maximum pressure of 3206 psi.

- Design 3--Coolant should have same exit temperature as collector plate coolant $\left(215^{\circ} \mathrm{C}\right)$.

The objective of the analysis was to discover the maximum coolant outlet temperature and thermal recovery efficiency.

The analysis showed that, within the stated constraints, no significant increase in coolant outlet temperature above $215^{\circ} \mathrm{C}$ can be realized using a water-cooled grid system. He will have to investigate either materials other than copper alloys or gas cooling. 


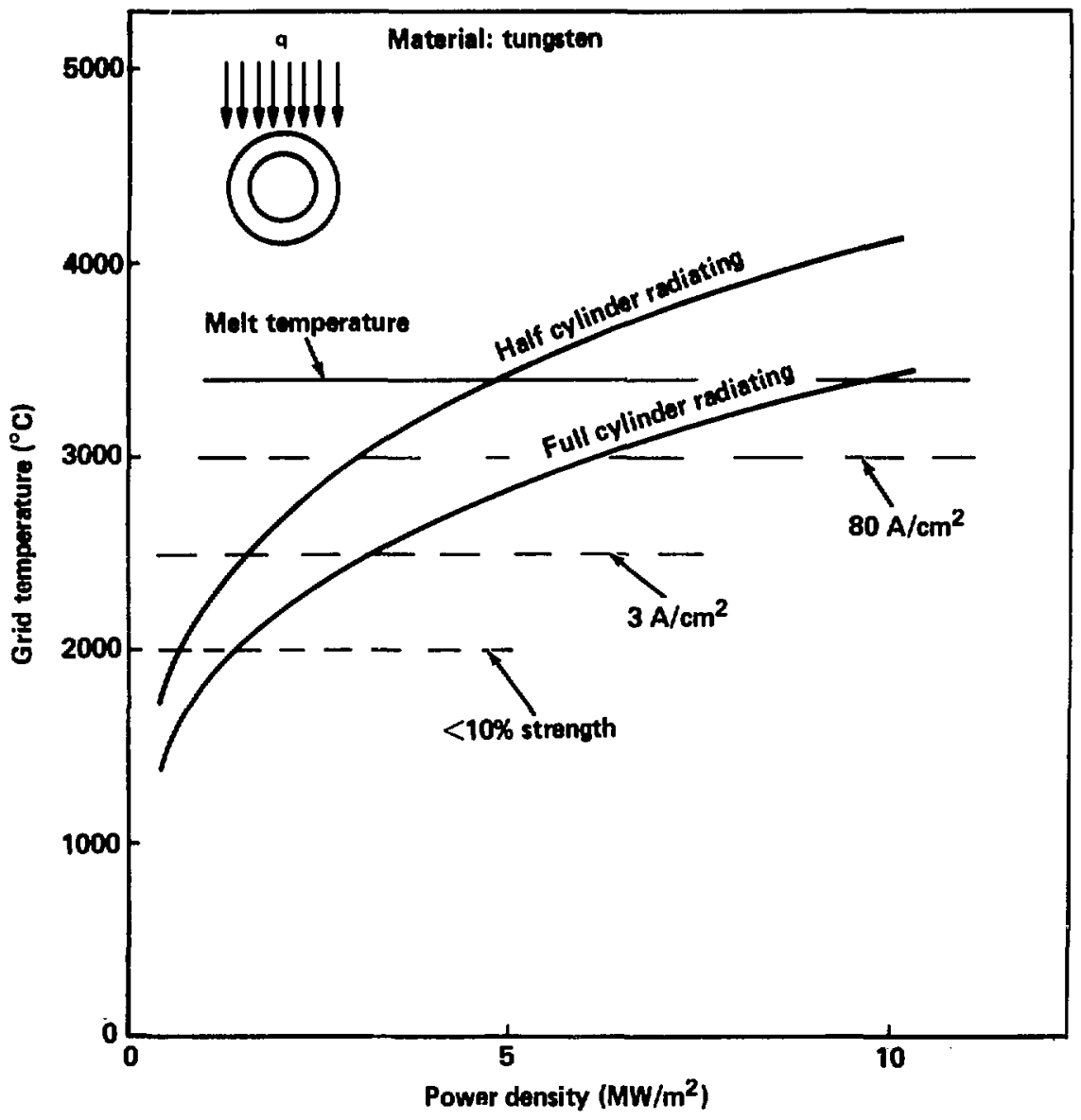

Fig. 3.6-4. Radiation-cooled grid performance. 


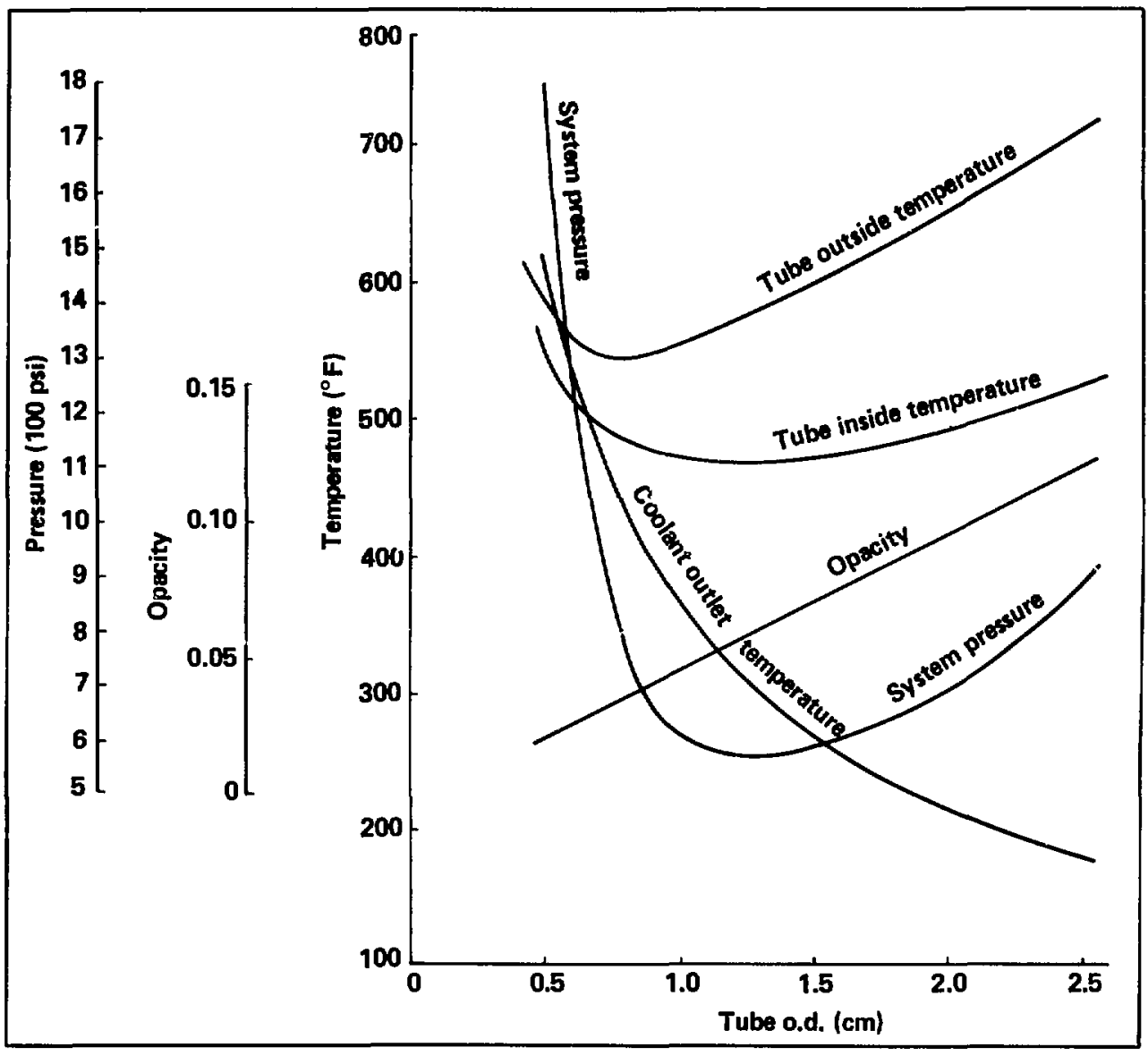

Fig. 3.6-5. Grid thermostructural analysis. 1 


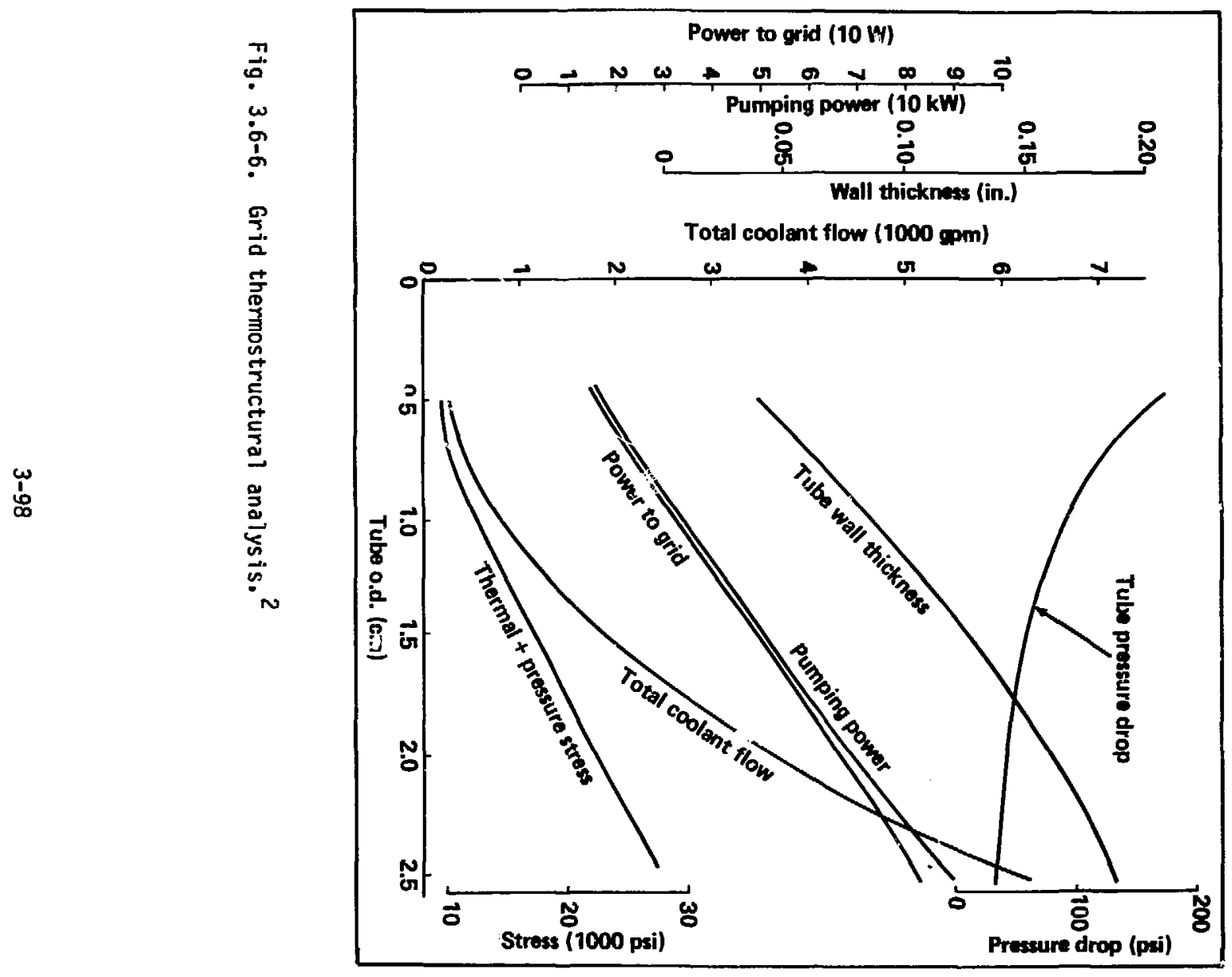


Table 3.6-1. Grid structural aralysis.

Assumptions and conditions

Poak powor dinsity $=7.6 \mathrm{MW} / \mathrm{m}^{2}$ (mid plane)

$\mathrm{d}=\mathbf{2 0} \mathrm{cm}$

$D=65 \mathrm{~m}$

Coolant velocity $=30 \mathrm{fps}$

Coolent inlet: tmp $=30^{\circ} \mathrm{C}$

Tube material: copper alloy

Tube leagth $=5 \mathrm{~m}$

Numiser of tubes $=\mathbf{3 2 5}$

Cooling medium: water

Design requirements

$\overline{d / L} \approx 1 / \Omega(L=50 \mathrm{~cm})$

$T / d<0.05$

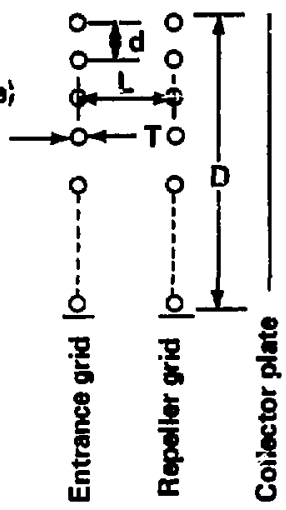


The baseline grid design consists of two arrays, each with 325 tubes spaced at $20-\mathrm{cm}$ intervals. The suppressor grid array is located along the intersection of the 79-G surface over a length of $64.94 \mathrm{~m}$. The longitudinal axes of the individual tubes are located parallel to the MARS X-axis. The entrance grid array is located $50 \mathrm{~cm}$ inboard of the suppressor grid array. Each grid tube is AMAX-MZC copper $5 \mathrm{~m}$ long, $1 \mathrm{~cm}$ outside diameter, and watercooled.

Performance. We performed a thermal analysis on the baseline design to determine the grid temperature distribution and the thermal gradients through the tube walls. Using the power density distribution given in Fig. 3.6-3 and a maximum water outlet temperature of $215^{\circ} \mathrm{C}$, calculated the maximum inner and outer tube temperatures at the grid midplane. The results are shown in Fig. 3.6-7.

We conducted a similar analysis to obtain the distribution of the through-the-tube thickness thermal gradients $(\Delta T)$ shown in Fig. 3.6-8.

To determine the erosion of the grids by energetic plasma particles, we conducted a physical sputtering analys is of the entrance and suppressor grids, focusing on copper and beryllium as the possible materials. He obtained physical sputtering yields as a function of the atomic and mass numbers of the incidence and target atoms, the surface binding energy of the target materials, and the incidence angle and energy of the particles.

The peak particle fluxes and energy levels are given in Table 3.6-2. The erosion rates are given in Fig. 3.6-9 for the entrance grid, suppressor grid, and collector plate. For the baseline tube thickness of $0.159 \mathrm{~cm}$, the lifetime for total erosion of a grid tube is approximately $9 \mathrm{yr}$. Further analysis will be necessary to predict erosion from other sources, such as coolant flow, and to determine minimum wall thickness before replacement is necessary.

Deformation. Because the purpose of the grids is to create potential planes, it is important that they remain in the correct place during operation. A non-planar potential field tends to turn the charged particles, which decreases their axial velocity component and reduces the collection efficiency. 
Table 3.6-2. Peak particle fluxes and energies.

\begin{tabular}{cccc}
\hline Fluxa & $\begin{array}{c}\text { Entrance grid } \\
\text { energy } \\
(\mathrm{keV})\end{array}$ & $\begin{array}{c}\text { Repeller } \\
\text { grid energy } \\
(\mathrm{keV})\end{array}$ & $\begin{array}{c}\text { Collector } \\
\text { plate energy } \\
(\mathrm{keV})\end{array}$ \\
\hline $3.74 \times 10^{15} \mathrm{D}^{+}$ & 319 & 429 & 43.5 \\
$3.74 \times 10^{15} \mathrm{~T}^{+}$ & 319 & 429 & 43.5 \\
$9.48 \times 10^{13} \mathrm{D}^{+}$ & 584 & 694 & 308.5 \\
$7.99 \times 10^{13} \mathrm{D}^{+}$ & 236 & 346 & $-\ldots$ \\
$1.28 \times 10^{14} \mathrm{\alpha}^{++}$ & 739 & 849 & 463.5 \\
\hline
\end{tabular}

afluxes in ions $/ \mathrm{cm}^{2} / \mathrm{s}$. 


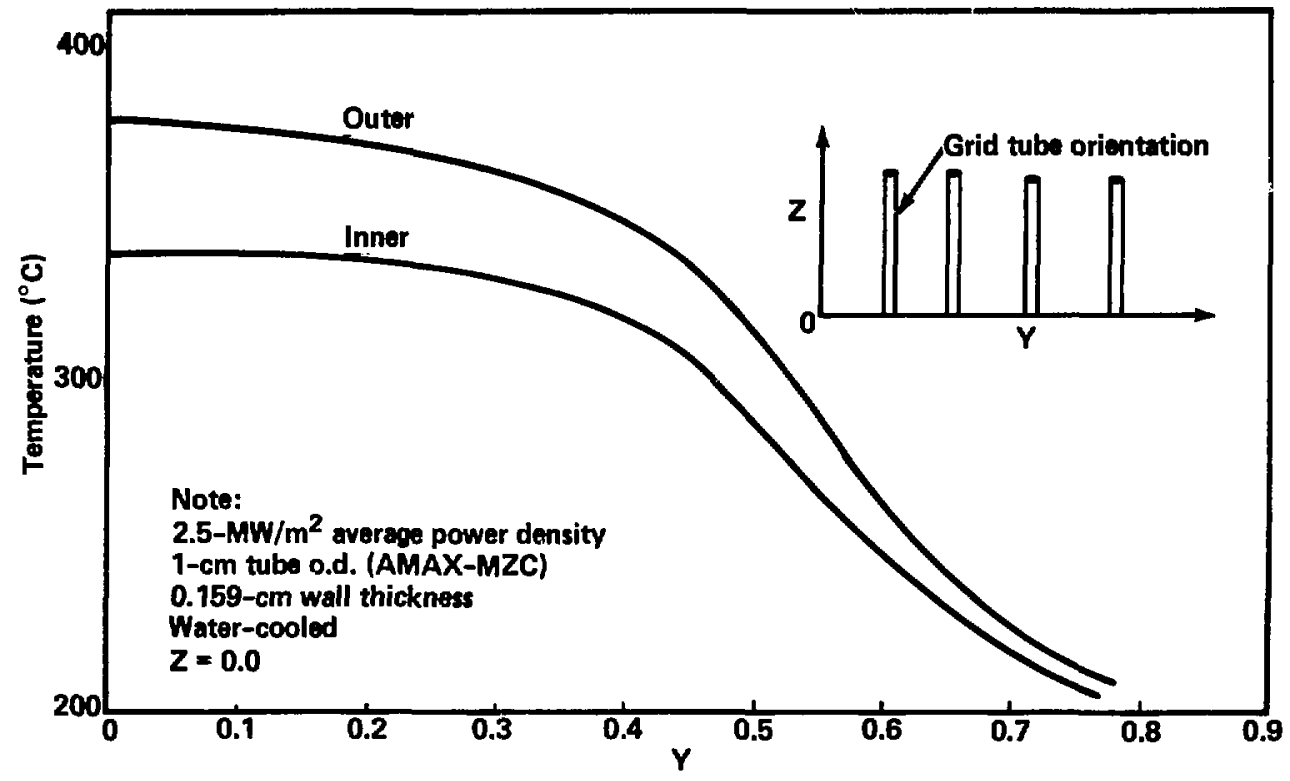

Fig. 3.6-7. Grid tube temperature distribution. 


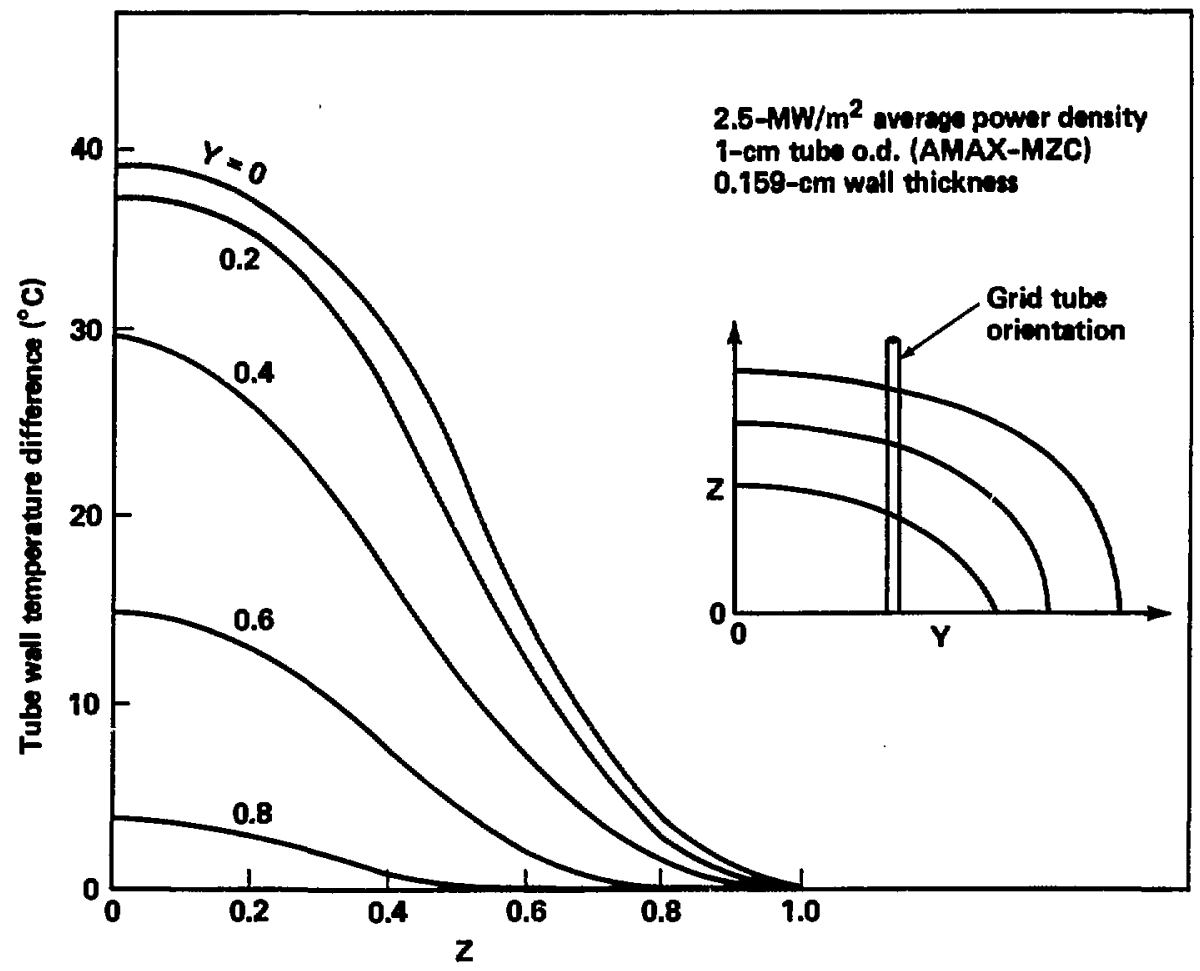

Fig. 3.6-8. Grid tube-wall temperature differences. 


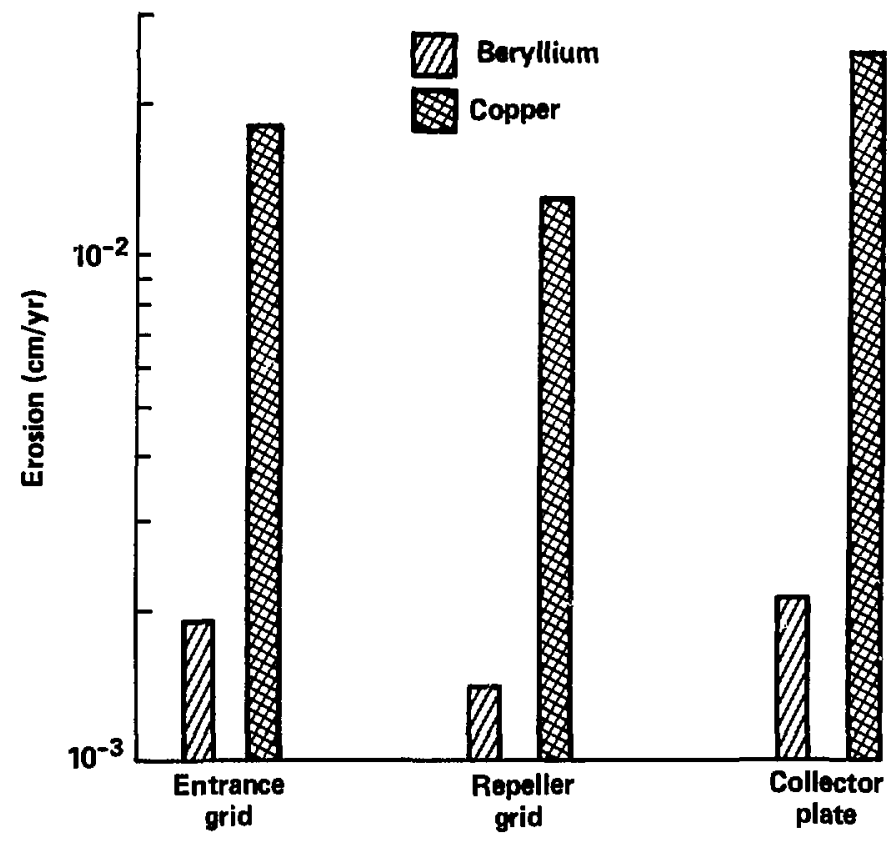

Fig. 3.6-9. Physical sputtering rates. 
Two phenomena can lead to deformation. The first is column buckling due to thermal expansion; each tube is a long column capable of carrying only $76 \mathrm{~N}$ before buckling. The second is bending due to a temperature difference between the front (heated) and back sides of the tube. Any difference in temperature induces a moment in the tube, which then results in deformation of the tube and adds to the column buckling load.

Figure 3.6-10 illustrates tube-end supports that can be added to deal with buckling and bending. Linear expansion of the tube can be handled by allowing the end to slide in a fixture, by spring loading the end, or by pretensioning the tube. Solving the problem of deformation due to differential heating is more difficult. Pretensioning can reduce but never eliminate bending in the tube. For a tube with aniform temperature difference along its length, a simple end moment equal to the induced moment will prevent bending. However, the actual temperature difference varies along the tube length as a function of flux density and coolant temperature. For such a temperature difference variation, a simple end moment cannot eliminate ihe bending.

We performed a detailed analysis of this problem using a 300-segment model for the tube and a realistic heating profile for our baseline watercooled copper design. Our baseline design maintains very high cooling rates which, when coupled with the high thermal conductivity of copper, keep the maximum $\Delta \mathrm{T}$ to $50^{\circ} \mathrm{C}$. This results in the small deformations shown in Fig. 3.6-11 (0.5 cm maximum). With no pretensioning of the tube and an applied end moment, the deflection is maintained at less than $1 \mathrm{~cm}$.

Gas Cooled Grids. The temperature of the grids is 1 imited to $215^{\circ} \mathrm{C}$ in the baseline design. To increase the temperature to a more useful level, other materials or coolants are required. We selected helfum as an alternate coolant to be studied because it has several advantages over water. As with most gaseous coolants, helium can sustain higher temperatures than water and grid tube leaks in a helium cooled system will be less catastrophic than leaks in a water cooled system.

An important consideration in the grid system design is the power required to pump the coolant. A parameter that assesses the effectiveness of a given system is the pumping power ratio (PPR), defined as the ratio of the pumping power to the power absorbed by the coolant. For the baseline grid 


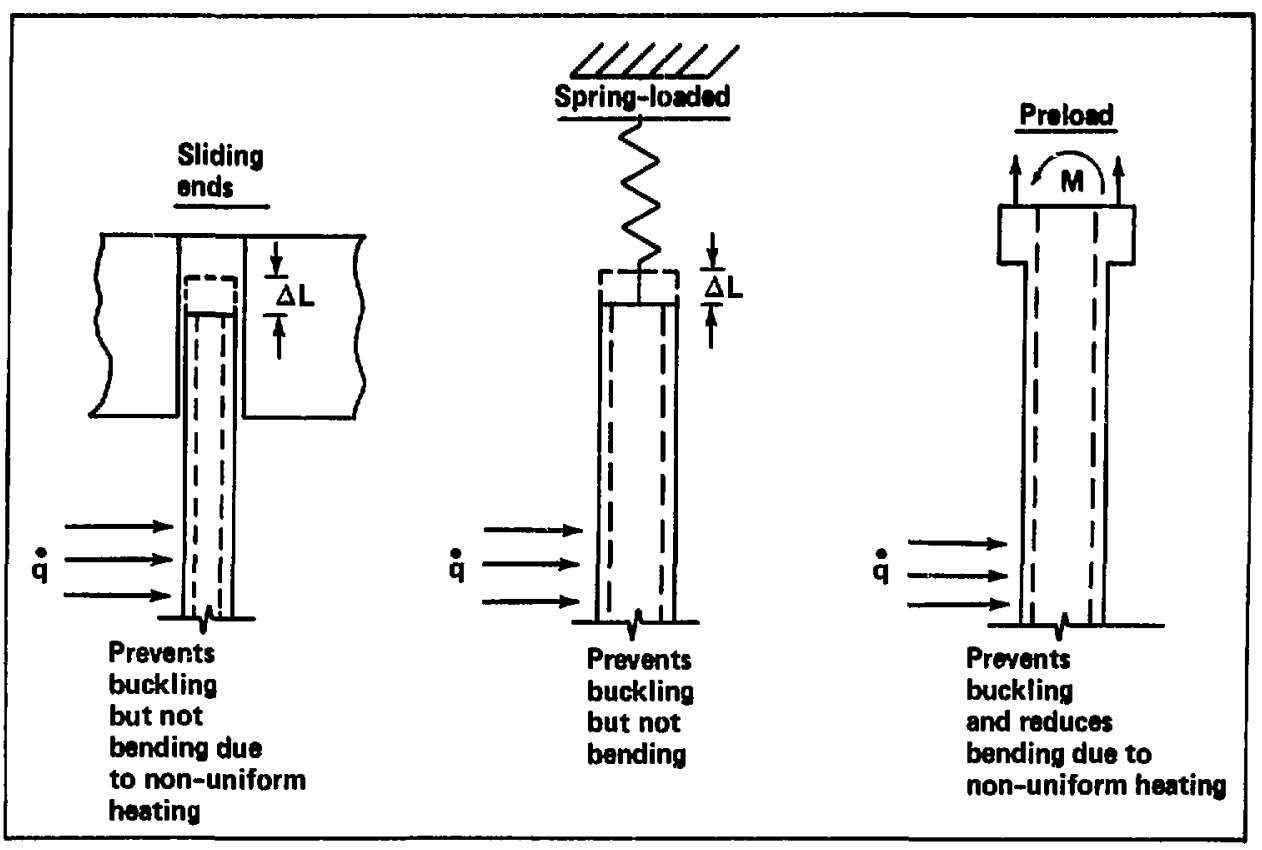

Fig. 3.6-10. Grid tube supports. 


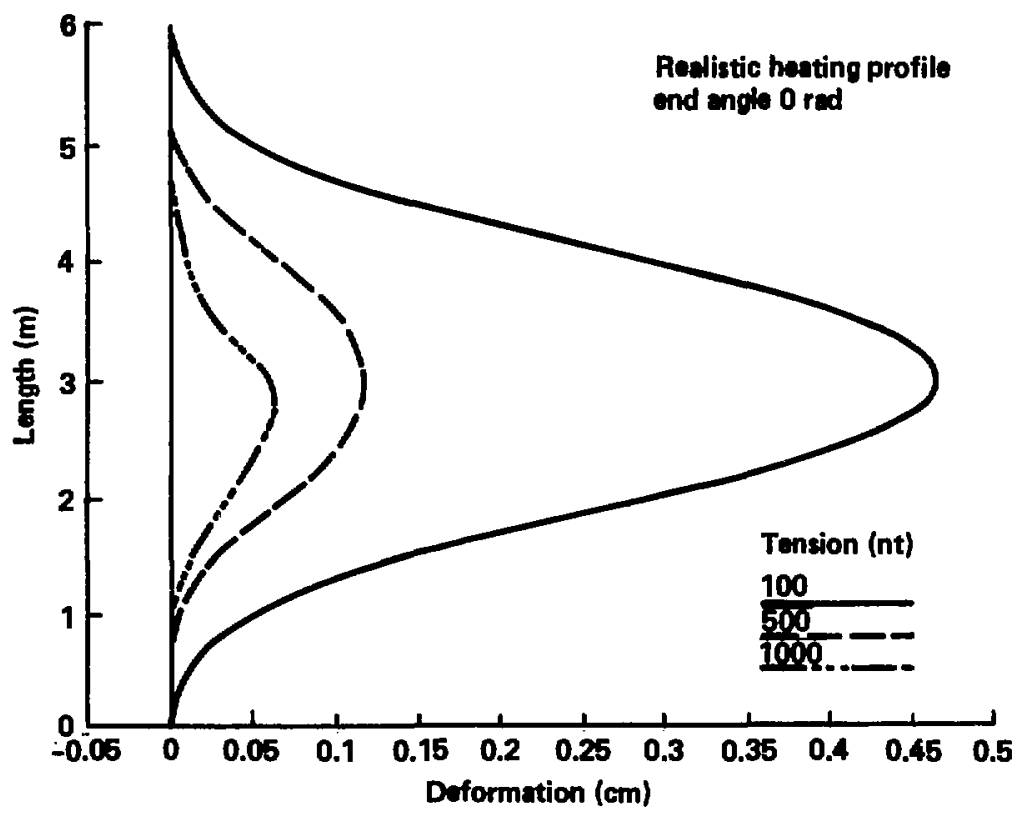

Fig. 3.6-11. Grid tube deformation. 
tube array, the PPR has been calculated as a function of temperature and pressure. The results are shown in Fig. 3.6-12 and indicate the possibility of high frictional losses.

Alternate Grid Materials. As previously stated, the water-cooled copper grid system is temperature-limited. To remove the temperature constraint, several alternate materials were investigated for helium-cooled grids. The selection of a material must include satisfying design constraints imposed by:

- Temperature,

- Erosion (physical and chemical).

- Stress,

- Tritium permeability,

- Vaporization,

- Thermionic emission,

- Grid thermal deflection, and

- Material activation.

We must distinguish between erosion and vaporization for the following reason: The erosion constraint is associated with a limit dictated by the material, e.g. a certain minimum life expeciancy of the grid. The vaporization constraint, on the other hand, is a limit dictated by the plasma.

The materials that we investigated are: copper alloy (AMAX-MZC), molydenum alloy (TZM), niobium alloy (FS-85), and ferritic steel (HT-9). The maximum predicted temperatures for these materials are shown in Fig. 3.6-13. We concluded, on the bas is of temperature alone, that both AMAX-MZC and HT-9 can be eliminated. Temperatures exceeding $400^{\circ} \mathrm{C}$ are considered marginal for AMAX-MZC. Similarly, the strength of HT-9 at $1165^{\circ} \mathrm{C}$ is rapidly approaching zero.

The next step was a stress analysis of the materials. We computed the combined (thermal plus pressure) stress levels as a function of position along the grid midplane $(z=0)$. Our results are shown in Fig. 3.6-14, including the tensile yield strength (FTY) at the appropriate maximum temperature, except for HT-9. For HT-9, the 10,000-hr creep-rupture strength is plotted in 


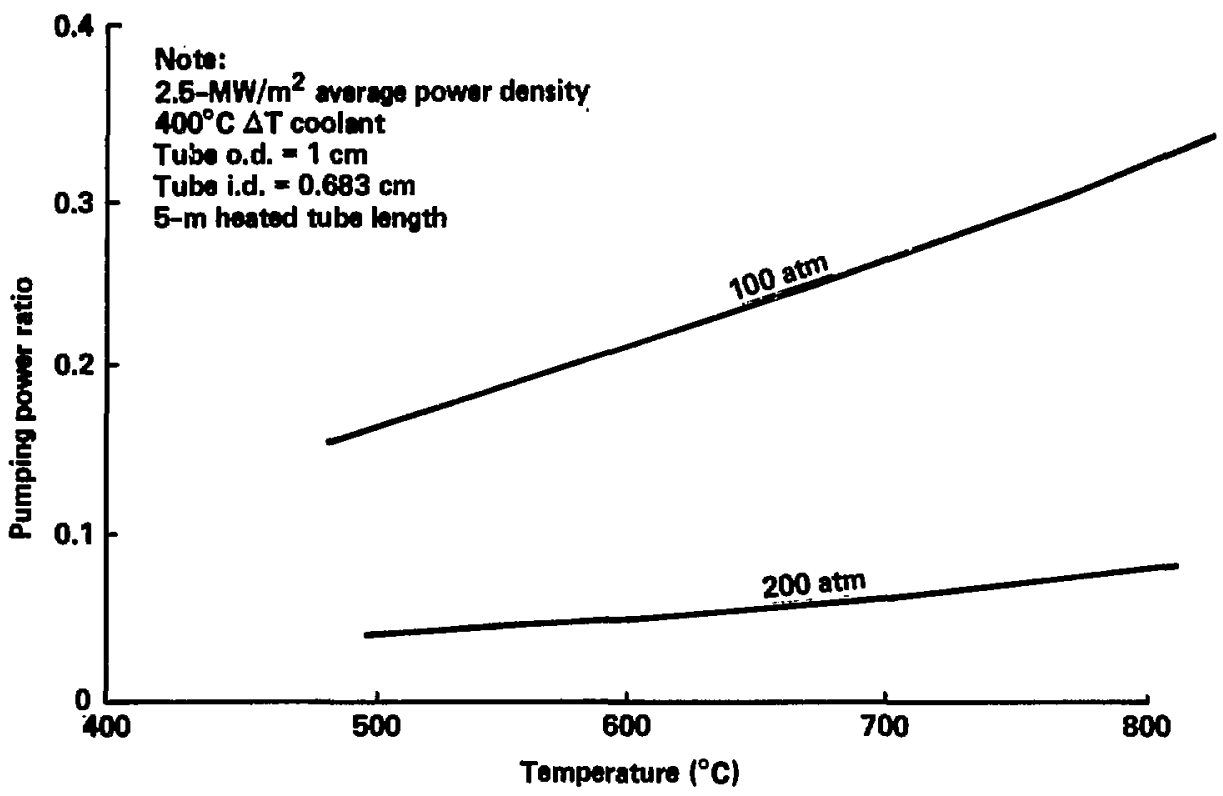

Fig. 3.6-12. Grid pumping power ratio. 


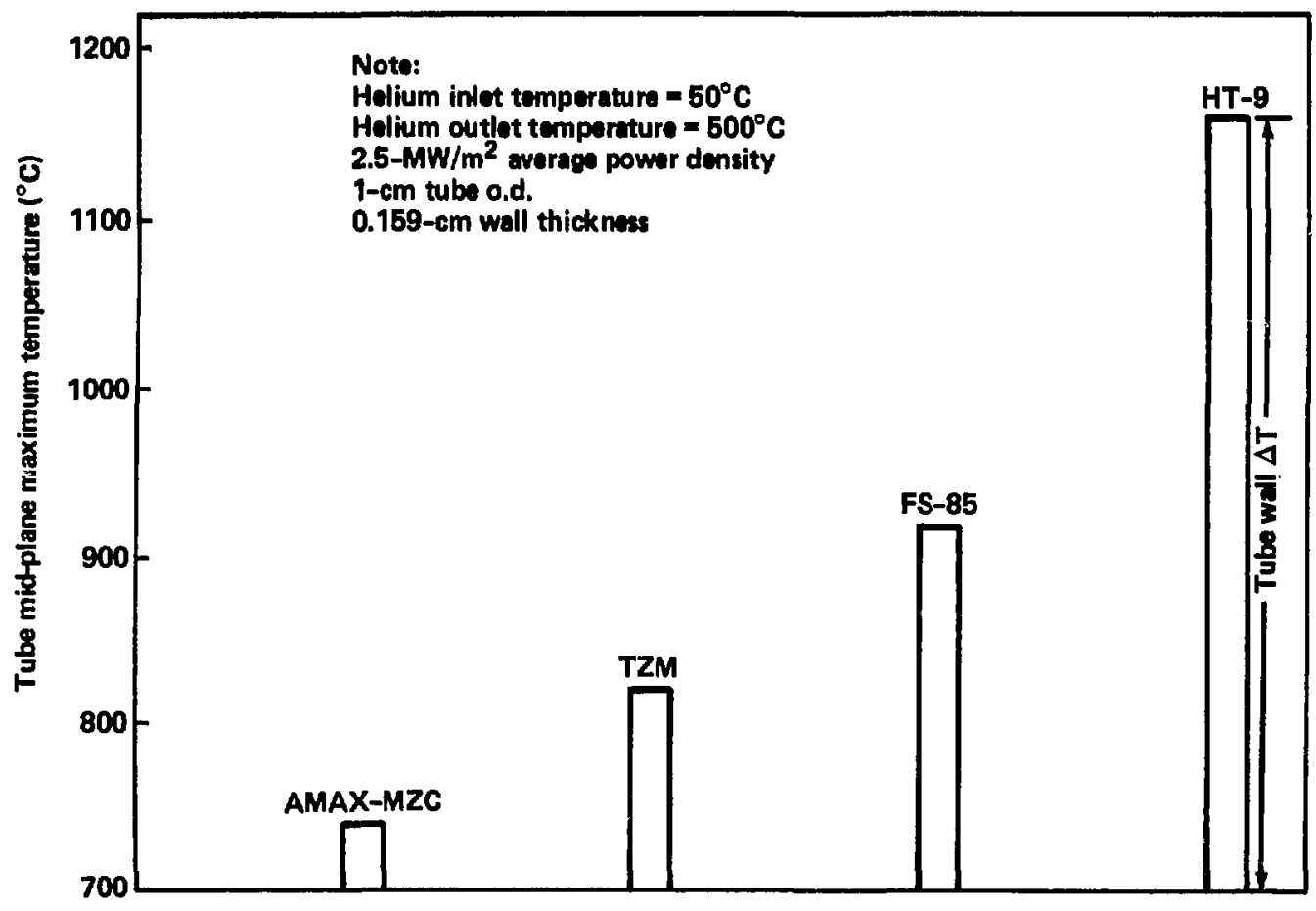

Fig. 3.6-13. Effect of material on maximum grid tube temperature. 


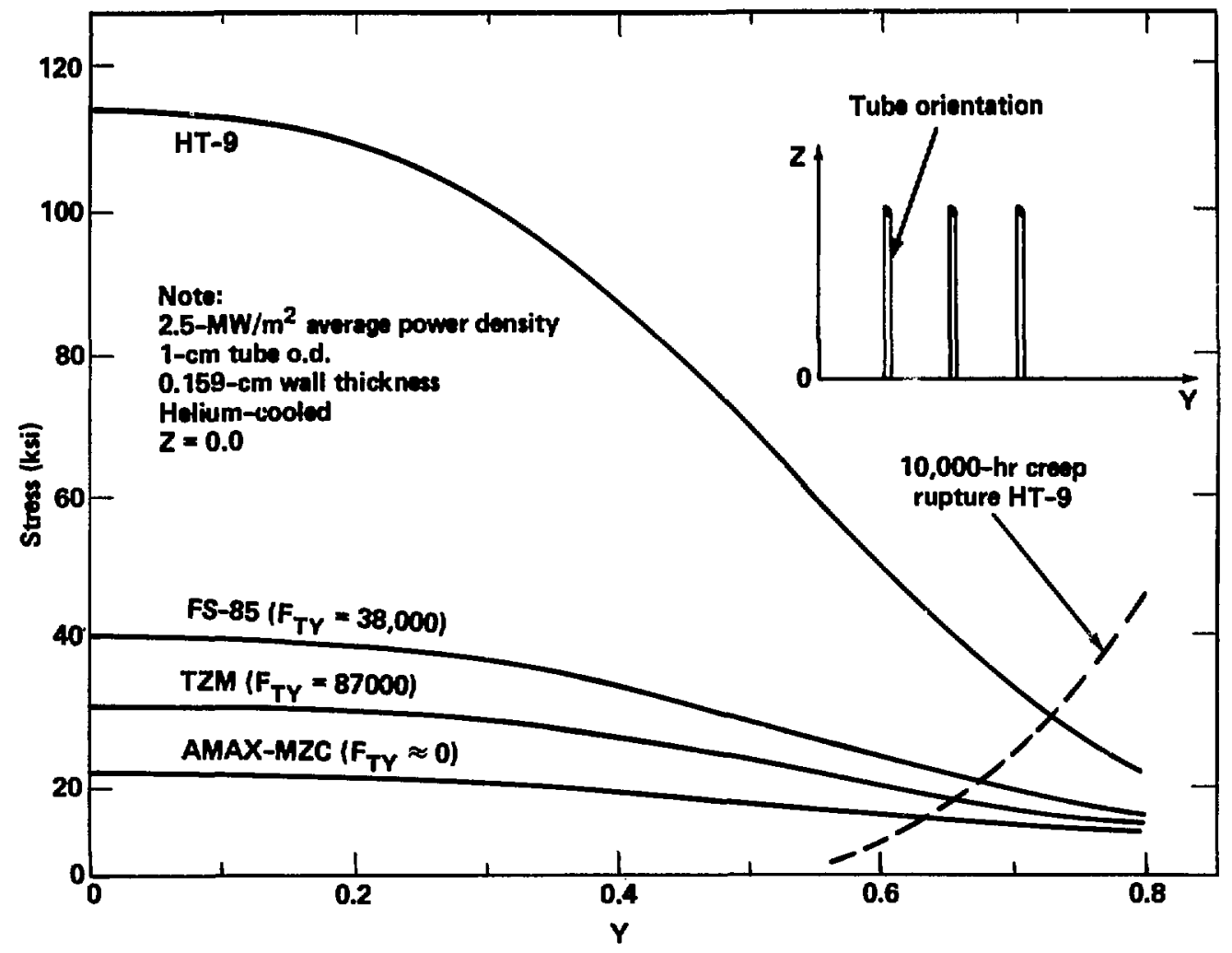

Fig. 3.6-14. Grid tube stress distribution. 
addition to the applied stress. We observed that the applied stresses for HT-9 are higher than the allowable creep rupture except at the very end of the footprint, and that the strength of AMAX-MZC is very low. At the grid midpoint $(y=0, z=0)$ FS-85 is also marginal. However, we would have to do a more detailed andysis to definitely rule out FS-85 as a grid material candidate. The molybdenum alloy, TZM, appears to meet the stress and thermal requirements.

Additional Issues. Several important design issues have not yet been addressec. One of the more important is chemical sputtering. For some materials, chemical sputtering may be a bigger problem than physical sputtering. We did not address the problems of sputtered material transport and redeposition and material activation. Because the basic mode of grid operation is high temperature and long duration, the issue of thermal creep needs further study.

\subsubsection{Collector Plate}

Requirements. The total thermal power to the direct converter collector plate is about $170 \mathrm{MN}$, as specified in Section 2.9.2. The plate is located according to heat load on the grids, which in this case is at an axial $z$ position of $124 \mathrm{~m}$. At this location, the area is $240 \mathrm{~m}^{2}$ for an average power density of $0.7 \mathrm{~m} / \mathrm{m}^{2}$.

Design. We considered two types of cooled surfaces: (1) a fully cooled back and (2) a back with spaced coolant channels. ie used a one-dimensional analysis to scope the maximum temperatures at the front surface of the plate.

The choice of the location for the direct converter collector plate is a compromise between a requirement for a low therma' power density to simplify the thermal design, the need for useful coolant temperature, in the physical size of the converter. The choice for the reference ciesign was to place the converter plate at the $124-m$ station where the thermal fower density is $69.6 \mathrm{~W} / \mathrm{cr}^{2}$ and the impingement area is $240 \mathrm{~m}^{2}$. The magnetic field is $79 \mathrm{G}$ at the 124-m station on the direct converter axis. For this value of constant magnetic field, we have determined an intersection within the horizontal center $\mathrm{plane}$ of the direct converter. This line, the semi-major axis of the elliptical impingement area, is an arc $30 \mathrm{~m}$ long. For convenient installation and maintenance, we designed the collector plates as individual sector panels, 
each $1.5 \mathrm{~m}$ wide and $6 \mathrm{~m}$ high. There are 40 collector sectors, 20 on each side of the center line (Fig. 3.6-15). The sections are numbered 1 through 20, stalting with the center line.

We considered five coolant channel configurations, shown in Fig. 3.6-16, for the ion collector plate. Configuration $A$ has a partially cooled back where the vertically oriented coolant channels are separated from one another. All 40 sectors ha 'e the same configuration. However, the thermal analysis showed that the maximum material iemperature on sector 1 , the sector closest to the axis, is greater than the melting temperature of the MZC copper. Therefore, we rejected this configuration. In configuration $B$ the back is completely cooled by horizontal channels in a switchback arrangement. Again, all 40 sectors have the same configuration. The maximum material temperature is $245^{\circ} \mathrm{C}$, which is below the maximum allowable of $400^{\circ} \mathrm{C}$. However, in the single-channcl horizontal switchback design, the fluid velocity causes a high fluid pressure drop and sufficiently high pumping power (>800 kW) to reject the concept.

We have considered two other horizontal switchback arrangements. Both have maximum material temperatures below $300^{\circ} \mathrm{C}$ and fully cooled backs. In configuration $C$, sectors 1 through 10 are double-channel horizontal switchbacks and sectors 11 through 20 are single-channel horizontal switchbacks. The pumping power is $267 \mathrm{~kW}$. In configuration D, sectors 1 through 12 are triple channel horizontal switchbacks and sectors 13 through 20 are single channel horizontal switchbarks. The pumping power is $118 \mathrm{~kW}$. The pumping power for configuration $D$ is acceptable but the power for configuration $C$ is not. However, we rejected both configurations because the sectors in each configuration, with a mixture of multiple and single channels, lack a common design. Configuration $E$ has a fully cooled back with three channels in series to form a channel path and 13 channel paths per sector. This vertical switchback arrangement has a maximum material termperature of $376^{\circ} \mathrm{C}$ and a pumping power of $31 \mathrm{~kW}$. We have selected it as the baseline.

Certain design objectives have evolved from the coolant configuration study. We selected a modular design consisting of reasonably small individual sectors because it simplifies the installation and liaintenance procedure. Only one type of sector need be kept in inyentory for replacement after a failure or during normal maintenance. The sector coolant configuration is 


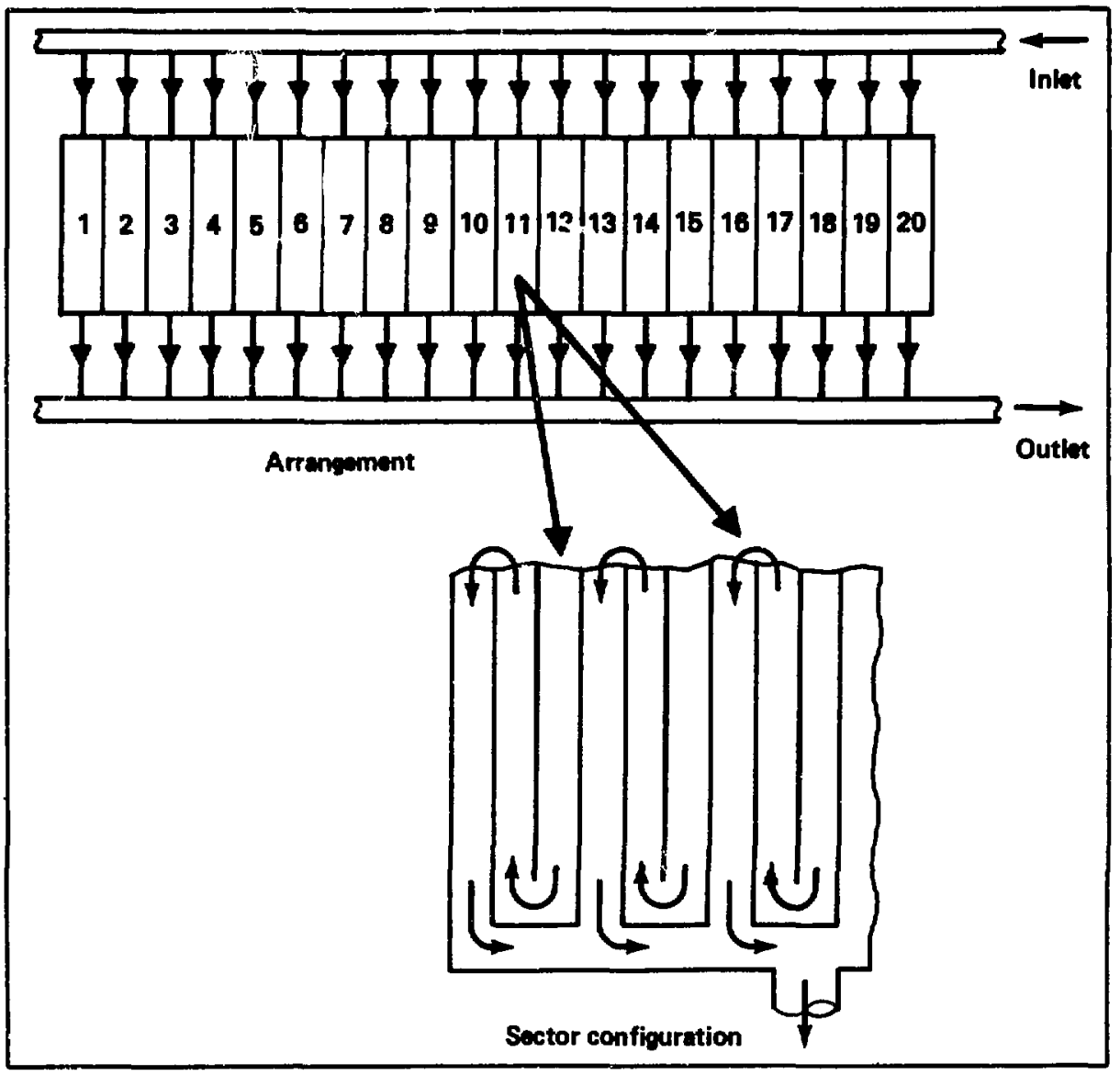

Fig. 3.6-15. Collector plate design. 


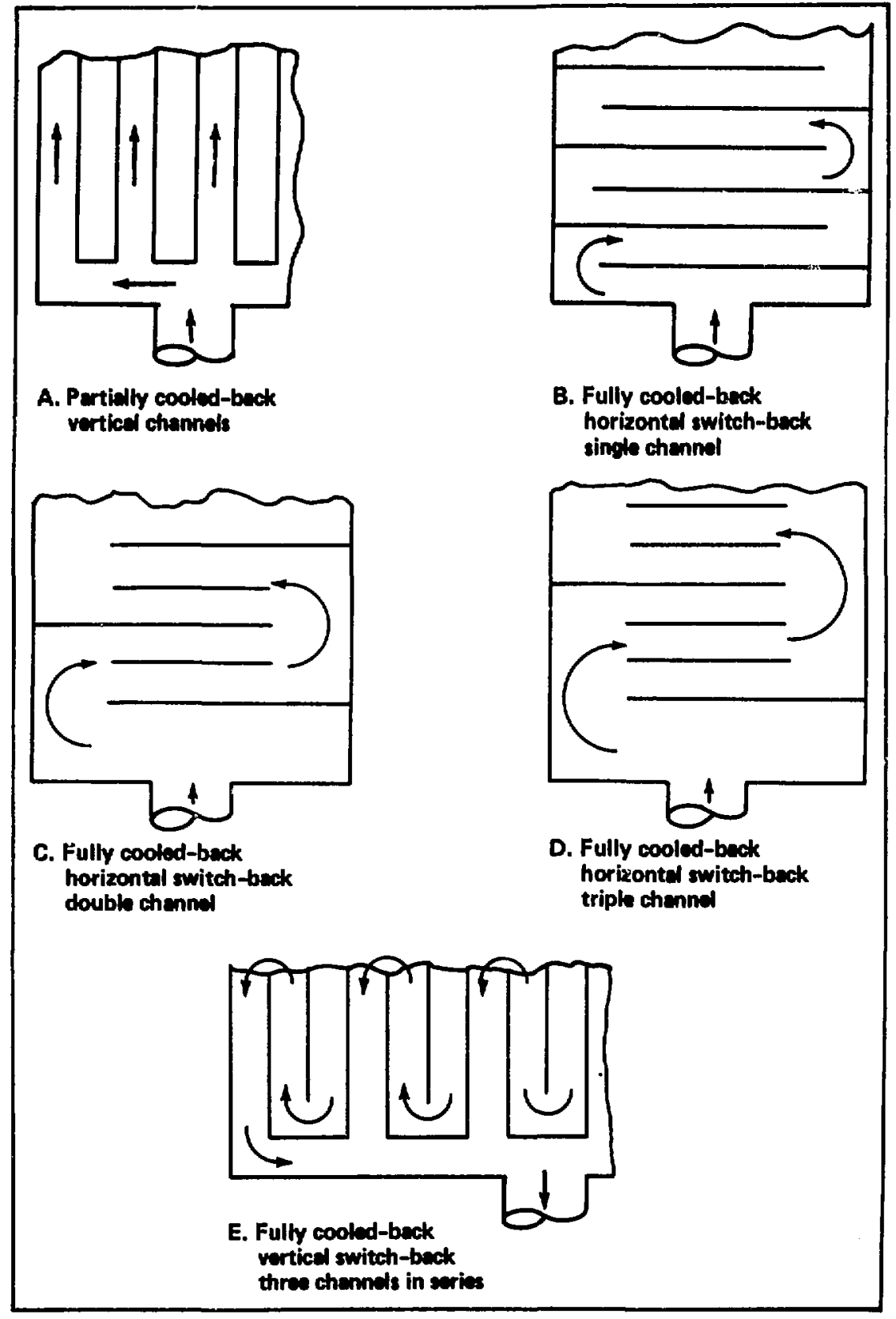

Fig. 3.6-16. Coolant channel configuration. 
designed with straight vertical channels and straight channel walls for ease of milling and brazing sector parts. Each sector has a single inlet and outlet, and all sectors are connected in parallel to a single inlet and outiet header. This simplifies maintenance because only two cuts and welds have to be made for sector replacement. The sector is designed with a fully cooled back to maintain the lowest material temperature. The coolant outlet temperature of all sectors is the same.

The reference design for the total collector is summarized in Table 3.6-3. There is a total of 39 vertical channels per sector. The channel cross section of $3.84 \times 0.72 \mathrm{~cm}$ maintains a relatively high coolant velocity, ensuring that the temperature across the coolant boundary layer is low enough to keep the maximum material temperature below the allowable $400^{\circ} \mathrm{C}$. Because of material degradation by high-energy particle impingement, the front wall thickness is $1 \mathrm{~cm}$ to allow for a reasonable material life. The coolant scheme is turbulent nonboiling flow for all sectors except 19 and 20 which have laminar flow. The sectors are in parallel between the 16-in. inlet and outlet headers, and pressure balance valving is required on each sector inlet to maintain a constant coolant outlet temperature. The sector material is AMAX MZC copper.

Performance. The overall collector plate performance can be characterized by a few key parameters. The maximum inside surface temperature at the wall occurs in section 14 and is $312^{\circ} \mathrm{C}$. The pressure required to prevent boiling at this temperature is $106 \mathrm{~atm}$. We calculated the pressure drop of $1.4 \mathrm{~atm}$ using the Fanning relation. 1 The channel length in the sector for calculating pressure drop is based on the straight length of channel path and the equivalent length of two " $U$ " bends per channel path. The total coolant flow rate is based on a coolant temperature rise of $185^{\circ} \mathrm{C}$ from an inlet temperature of $30^{\circ} \mathrm{C}$. The coolant pumping power of $31 \mathrm{~kW}$ is based on the coolant flow rate and a system pressure drop that is assumed to be double that of the sector to account for the external piping system.

The thermal performance for each of the 20 sectors is given in Table 3.6-4. The maximum material temperatures and coolant velocities are those required to maintain the outlet temperatues of all sectors at $215^{\circ} \mathrm{C}$. Based on the Reynolds number, turbulent flow exists in sectors 1 through 18 and laminar flow in sectors 19 and 20. 
Table 3.6-3. Collector plate design parameters.

- Power input: 167 MW

- Surface area: $240 \mathrm{~m}^{2}$

- Number of sectors: $\mathbf{4 0}$

- Sector height: $6 \mathrm{~m}$

- Sector width: $1.5 \mathrm{~m}$

- Coolant channel arrangement

- Back fully cooled

- 39 vertical channels/sector

- 3 channels in series/channel path

- 13 channe 1 paths/sector

- Channel width: $3.84 \mathrm{~cm}$

- Channel depth: $0.72 \mathrm{~cm}$

- Sector front-surface plate thickness: $1 \mathrm{~cm}$

- Material: AMAX-MZC copper

- Coolant: water

- Coolant scheme: turbulent flow/nonboiling

- Sectors in parallel between inlet/outlet headers

- Inlet header: 16 in. across top

- Outlet header: 16 in. across bottom

- Pressure balance valving on sector inlet piping 
Table 3.6-4. Collector plate sector thermal parameters.

\begin{tabular}{|c|c|c|c|c|c|}
\hline $\begin{array}{l}\text { Sector } \\
\text { number }\end{array}$ & $\begin{array}{l}\text { Center } \\
\text { heat flux } \\
\left(\mathrm{k} / \mathrm{cm}^{2}\right)\end{array}$ & $\begin{array}{l}\text { Heat } \\
\text { input } \\
\text { (MW) }\end{array}$ & $\begin{array}{c}\text { Maximum } \\
\text { material } \\
\text { temperature } \\
\left({ }^{\circ} \mathrm{C}\right)\end{array}$ & $\begin{array}{l}\text { Coolant } \\
\text { velocity } \\
(\mathrm{cm} / \mathrm{s})\end{array}$ & $\begin{array}{c}\text { Reynolds } \\
\text { number }\end{array}$ \\
\hline 1 & 211 & 8.18 & 374 & 294 & $1.3 \times 10^{5}$ \\
\hline 2 & 211 & 8.10 & 375 & 290 & \\
\hline 3 & 211 & 7.97 & 376 & 286 & \\
\hline 4 & 207 & 7.77 & 376 & 276 & \\
\hline 5 & 203 & 7.43 & 376 & 267 & \\
\hline 6 & 194 & 7.10 & 373 & 250 & \\
\hline 7 & 184 & 6.55 & 370 & 235 & $1.0 \times 10^{5}$ \\
\hline 8 & 170 & 6.00 & 364 & 216 & \\
\hline 9 & 156 & 5.42 & 359 & 195 & \\
\hline 10 & 139 & 4.68 & 354 & 168 & \\
\hline 11 & 122 & 3.91 & 349 & 141 & \\
\hline 12 & 101 & 3.14 & 341 & 113 & $4.9 \times 10^{4}$ \\
\hline 13 & 80 & 2.40 & 333 & 86 & \\
\hline 14 & 65 & 1.75 & 333 & 63 & \\
\hline 15 & 49 & 1.25 & 326 & 45 & \\
\hline 16 & 35 & 0.86 & 316 & 31 & \\
\hline 17 & 20 & 0.48 & 302 & 17 & \\
\hline 18 & 12 & 0.33 & 280 & 12 & $5.2 \times 10^{3}$ \\
\hline 19 & 4.7 & 0.11 & 228 & 4 & $1.7 \times 10^{3}$ \\
\hline 20 & 2.4 & 0.03 & 215 & 1.1 & $4.8 \times 10^{2}$ \\
\hline
\end{tabular}

a Laminar flow. 
A parametric study of collector plate operating conditions is shown in Fig. 3.6-17 for a water inlet temperature of $30^{\circ} \mathrm{C}$. The four performance variables of surface temperature, system pressure, flow rate, and pumping power are plotted against the coolant out let temperature. By increasing coolant outlet temperature, the flow rate and pumping power decrease. The maximum matertal surface temperature is influenced by the combined rise in the coolant outlet temperature and the increase in the temperature drop across the film caused by decreasing the flutd velocity. Tha required system pressure is the sum of the pressure required to prevent boiling at the maximum fluid temperature and the pressure drop due to flowing coolant. The combined effects result in a minimum system pressure at a coolar.t outlet temperature of $105^{\circ} \mathrm{C}$.

Design Alternatives. Preliminary work has begun on an investigation of an alternate reference design for the collector plate. A helium-cooled plate with an outlet temperature of $500^{\circ} \mathrm{C}$ is the objective of the alternate design. There are still 40 sectors placed on the 79-G arc at the 124-m axial station. In the alternate design, the coolant is center-fed, splits into two paths, and then flows to the coolant outlets at the top and bottom of the sector. There are 117 parallel channels per sector.

The materials that we considered for the alternate design were AMAX MZC copper, HT-9, V15Cr5Ti, FS-85 niobium, and TZM. Thermal and stress analyses showed that MZC copper and HT-9 are not suitable materials for the same reasons that caused us to reject them for use in grid cooling (discussed in Section 3.6.1.1). Figures $3.6-18,-19$, and -20 present thermal stress results for V15Cr5Tt, FS-85, and TZM ion collector plates, respectively. The data are for the first channel of sector one, wich is at the center of the direct converter. For these three materials, the combined stress is lower than the yield strength over the entire length of the channel, and the maximum outside metal temperature is below the allowable temperature. Therefore, any of these materials could be. used, based on thermal and stress data.

\subsubsection{Electron Collector}

The electron collector plate is heated by 112 of charged-particle power in a plasma fan oriented vertically in the long dimension. To minimize the size of the vacuum tank that encloses this tall plasma fan, the heat flux should be made as large as possible, with a long life design that allows for 


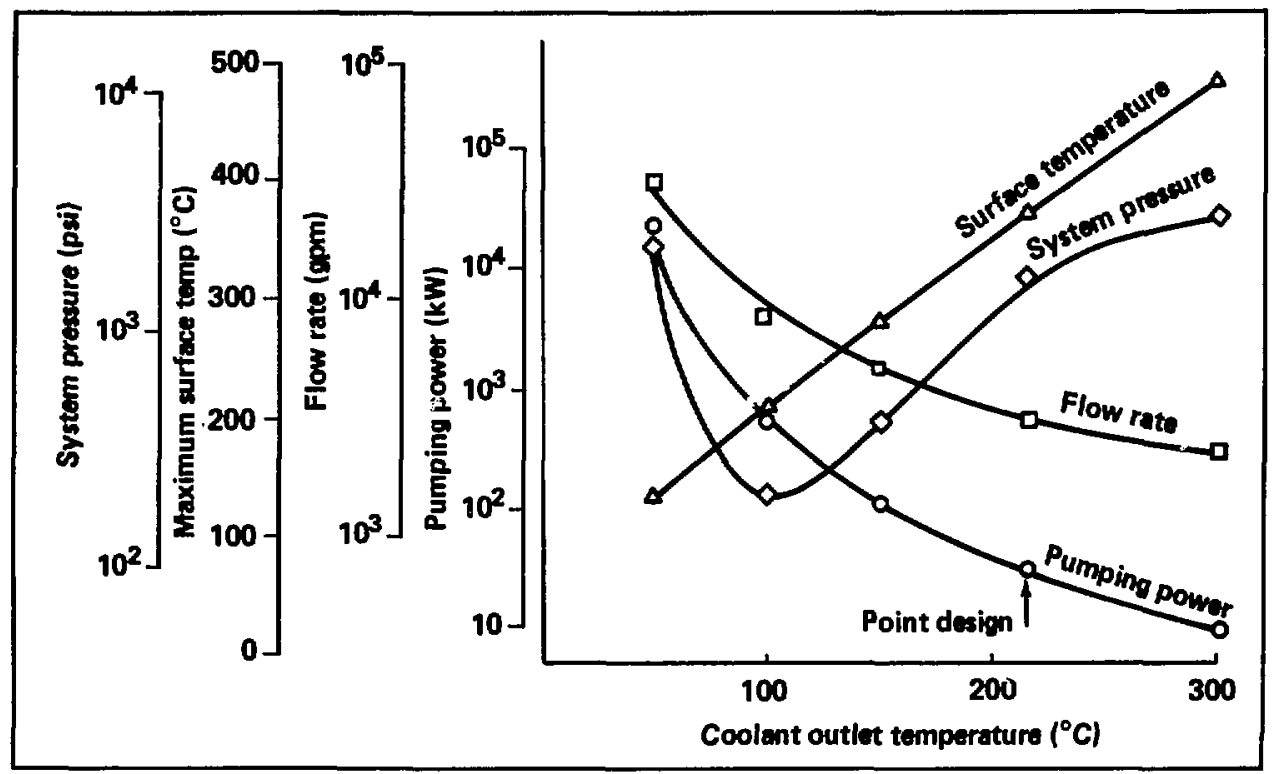

Fig. 3.6-17. Collector plate system study. 


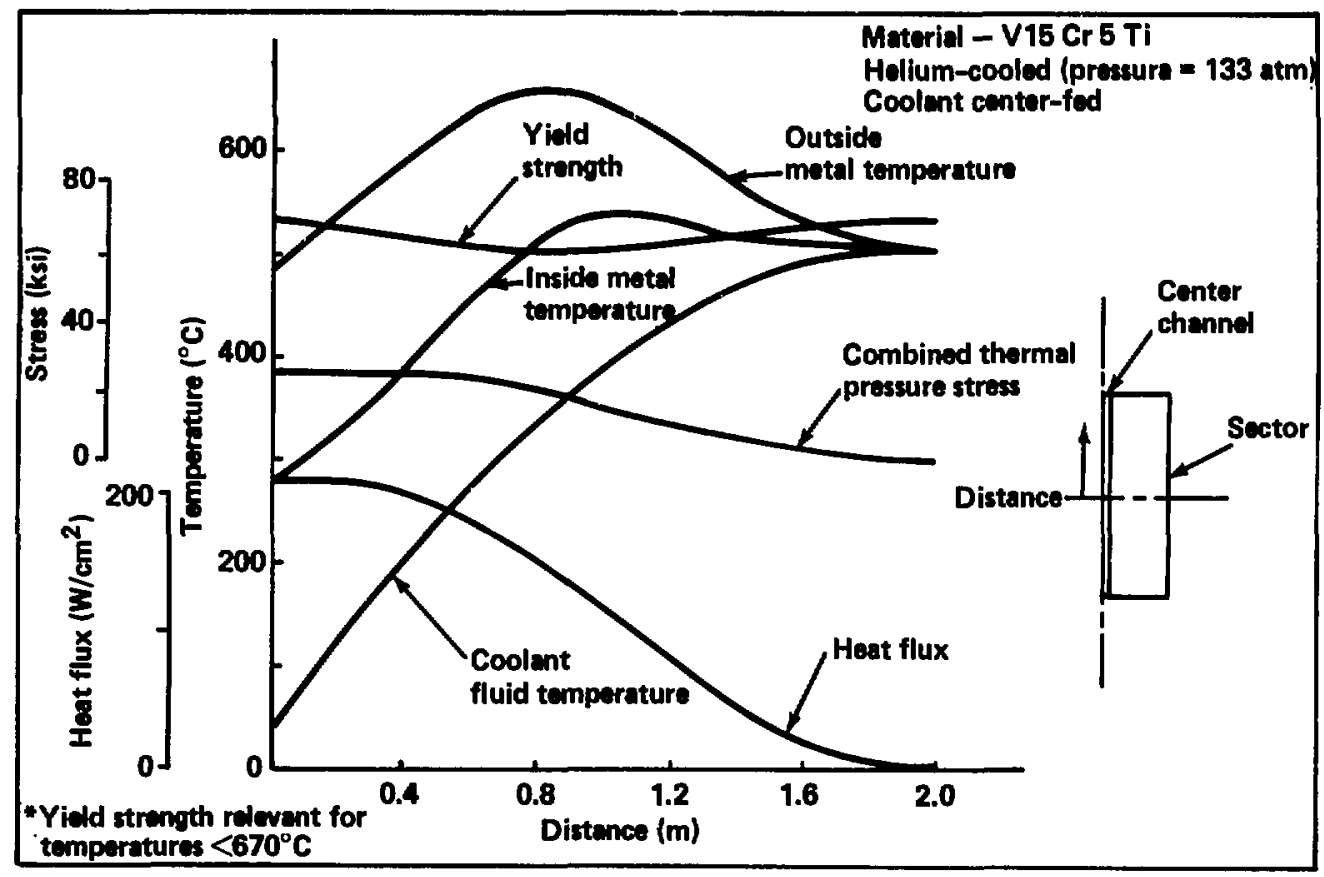

Fig. 3.6-18. Center coolant channel performance--vanadium alloy. 


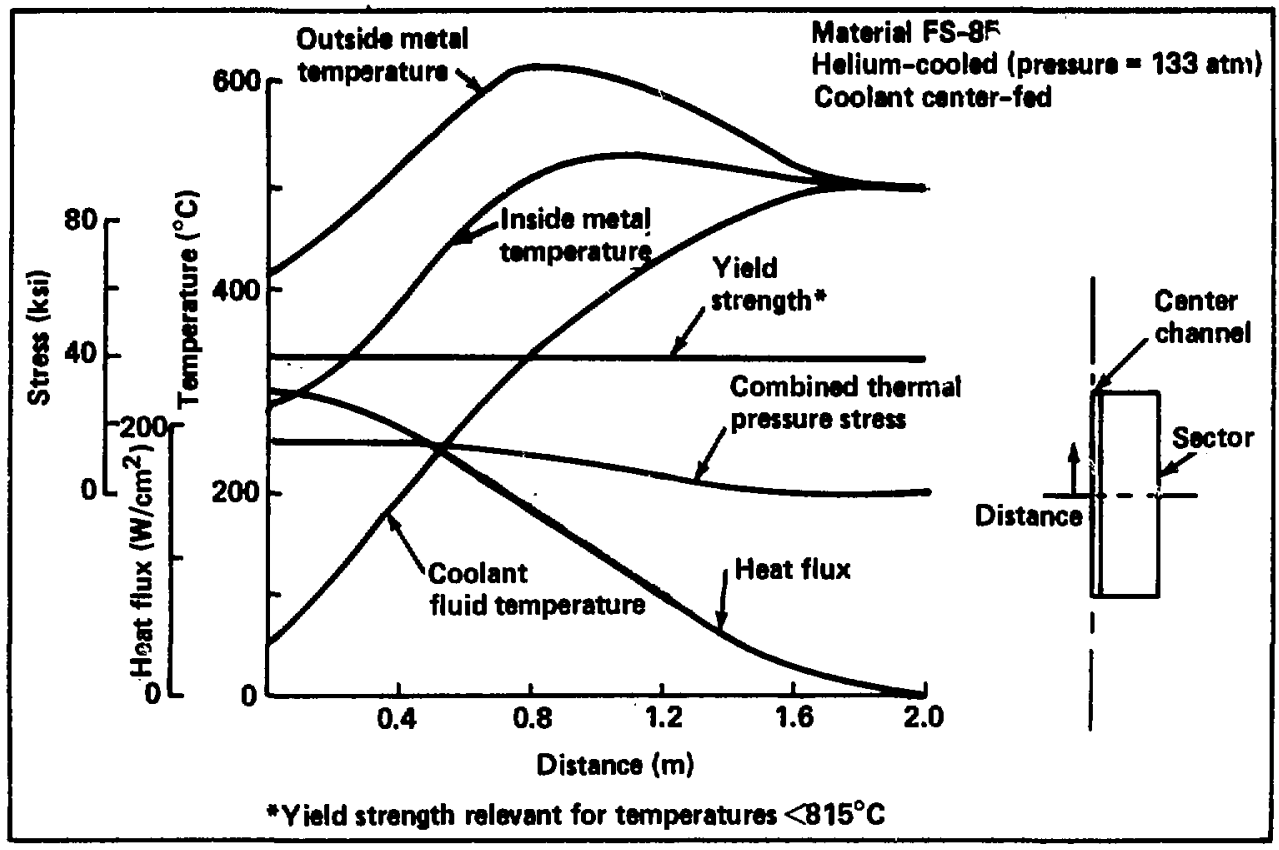

Fig. 3.6-19. Center coolant channel performance--niobium alloy. 


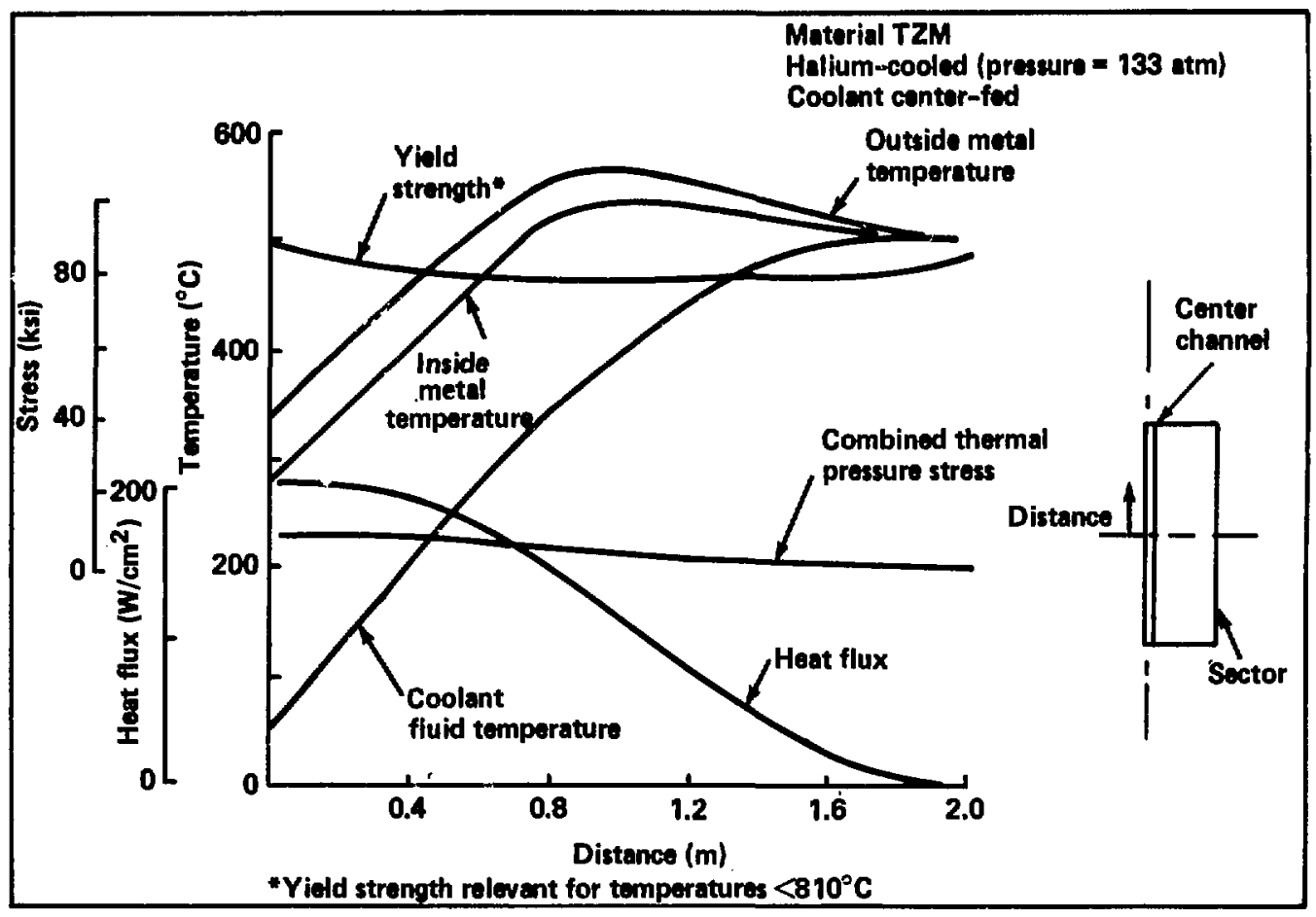

Fig. 3.6-20. Center coolant channel performance--molybdenum alloy. 
thermal recovery. We determined the plate location by assuming $2.5 \mathrm{w} / \mathrm{m}^{2}$ average over an area of $43.8 \mathrm{~m}^{2}$. The flux bundle has the profile shown in Fig. 3.6-21 with the collector at a field of $445 \mathrm{G}$. The variation of flux density across the plate is assumed to be the same as that for the ion collector. We have not yet performed a detailed analysis of the cooling for this plate.

A single-grid array is located in front of the electron collector plate to suppress secondary electron flow from the surface. This will be maintained at about $20 \vee$ negative in relation to the plate.

The halo scraper for the electron end has a different design than the scraper at the ion end even though the heat and particle loads are the same. To reduce the size of the electron end tank, the plate has been placed closer to the yin-yang, which required a modified design. The configuration shown in Fig. 3.6-21 is significantly smaller than the ion-end halo scraper; its leading edge is exposed to a heat flux of $2.5 \mathrm{mw} / \mathrm{m}^{2}$. Our preliminary estimates indicate the design is feasible; the actual analysis will be performed in FY 1983.

\subsubsection{Halo Scraper}

\subsubsection{Requirements}

The halo scraper removes low energy DT and alpha-particles from the unconfined region of the plasma. The halo contains about 80 of power that must be dissipated by the scrapers in the ion and electron ends. We have assumed the flux density in the halo plasma to be uniform so that the load at any location is the total power in the halo divided by the area of the flux bundle. We have designed the baseline halo scraper located at the ion end with the leading edge at a heat flux $1.0 \mathrm{mw} / \mathrm{m}^{2}$ and at a field of $633 \mathrm{G}$. The length of the leading edge is $52.9 \mathrm{~m}$ while the thickness of the halo plasma varies from 0.37 to $2.39 \mathrm{~m}$. As the halo thickness varies, the length of the scraper inlet duct varies, as discussed in Section 3.6.3.

\subsubsection{Design}

We analyzed the halo scraper for a water-cooled plate with both a fully cooled back and a partially cooled back with spaced coolant channels. The 


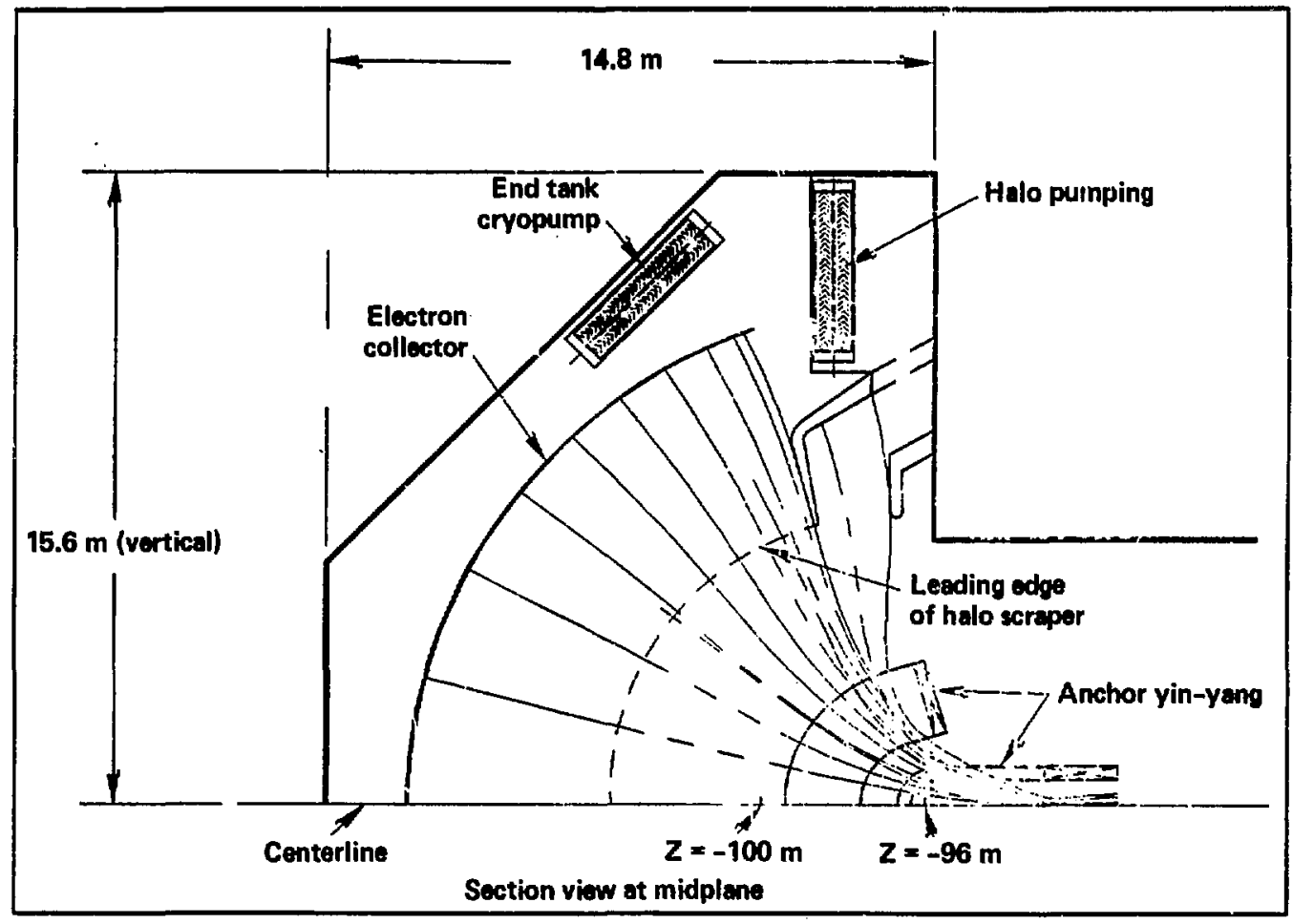

Fig. 3.6-21. Electron collector end tank. 
halo scraper, like the collector plate, is ciesigned for recovery of thermal power. To facilitate this, the water-coolent outlet temperature of all charinel paths is the same and is set at the sari: temperature as the ion collector plate coolant outlet. From the scraper z-plane projection (Fig. 3.6-22) we can see that the coolant channel length will vary with position on

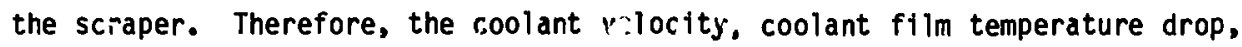
and outside material surface temperature will vary with position. For the partially cooled back at the minimum channel length, the outside material surface temperature is greater than the allowable material temperature of $400^{\circ} \mathrm{C}$ for MZC copper. Therefore, we rejected the partially cooled back design.

The cross section of the halo scraper reference destijn in Fig. 3.6-23 shows the copper channel wall, channel, and center beain. The halo scraper is placed at $45 \mathrm{deg}$ to facilitate the impinging thermal flux to facilitate vacuum pumping of the ipinging particles. The channel dimensions are $4 \times 0.3 \mathrm{~cm}$. An internal web provides increased structural strangth. The web splits the 4-cm channel width in half. Other dimensions are given in Fig. 3.6-22. Tílere are 1320 channels in the scraper connected in series in groups of four; therefore, there are 330 channel paths between the inlet and outlet headers. We used this design scheme to raise the channel velocity so that the film drop is minimized, keeping the material temperature below the allowable $400^{\circ} \mathrm{C}$. The coo'ant will flow down the back face in the first and third channels in series and down the front face in the second and fourth. Both the inlet and outlet coolant headers will be adjacent to the back face. The coolant header is $20 \mathrm{~cm}$.

\subsubsection{Performance}

The halo scaper performance parameters are summarized in Fig. 3.6-22. The power density at the tip, which is normal to the impinging flux, is $100 \mathrm{~W} / \mathrm{cm}^{2}$. Over the majority of the scraper area, which is at $45 \mathrm{deg}$ to the flux, the power density is $71 \mathrm{~W} / \mathrm{cm}^{2}$. The coolant pumping power is $1730 \mathrm{~W}$ and the flo. rate is $820 \mathrm{GPM}$. The coolant temperature rise is $185^{\circ} \mathrm{C}$ from the inlet temperature of $30^{\circ} \mathrm{C}$, with a pressure of $167 \mathrm{~atm}$. The coolant velocity varies between 56.6 and $161.3 \mathrm{~cm} / \mathrm{s}$ with an average of $130 \mathrm{~cm} / \mathrm{s}$. Figure 3.6-22 shows the temperature of the root and tip for botin the maximum and minimum 


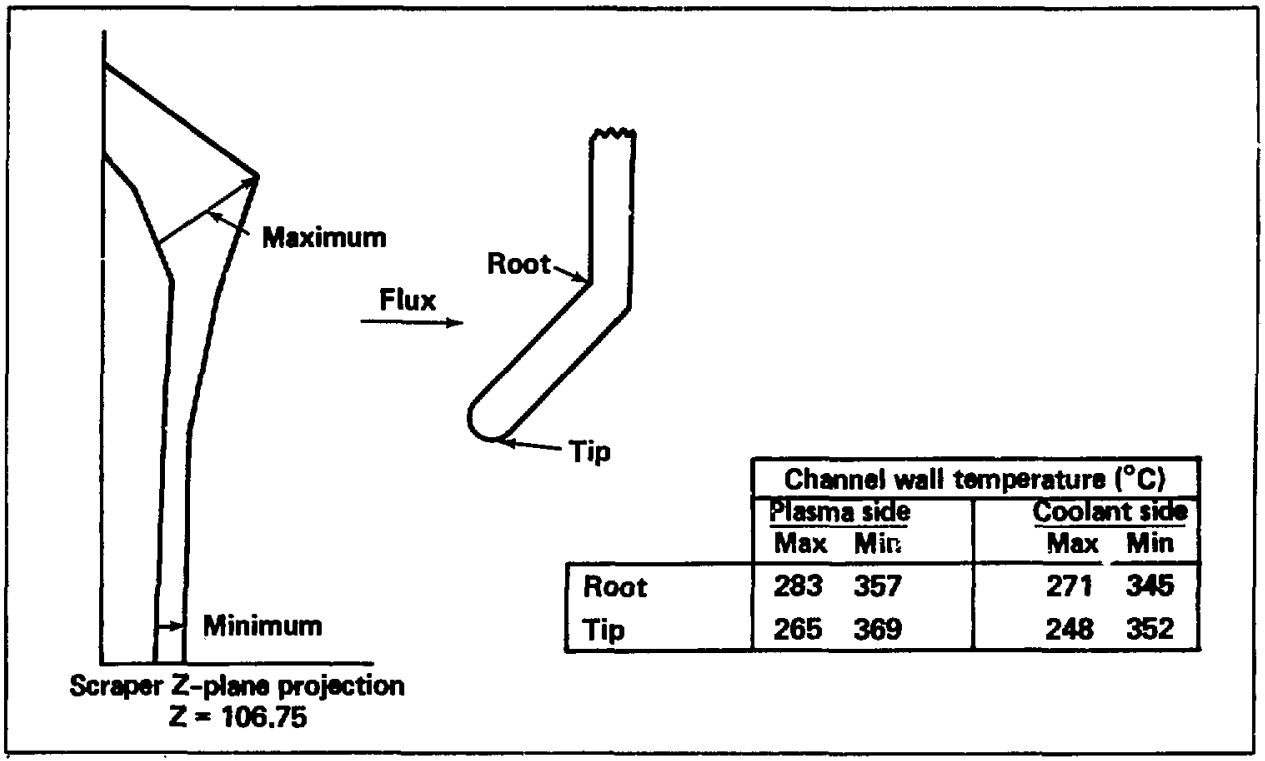

Fig. 3.6-22. Halo scraper performance. 
Channel width

Dopth

Internal wob for structural-strength copper channel wall

Conter beam (CU)

Total scraper thickness

Channel-length

Maximum

Minimum

Dimensions in $\mathrm{cm}$

Material AMAX coppor

Number of chenmels

Number of channeli/channel path

Number of chernel paths

Coolent heeder size

Halo screper station

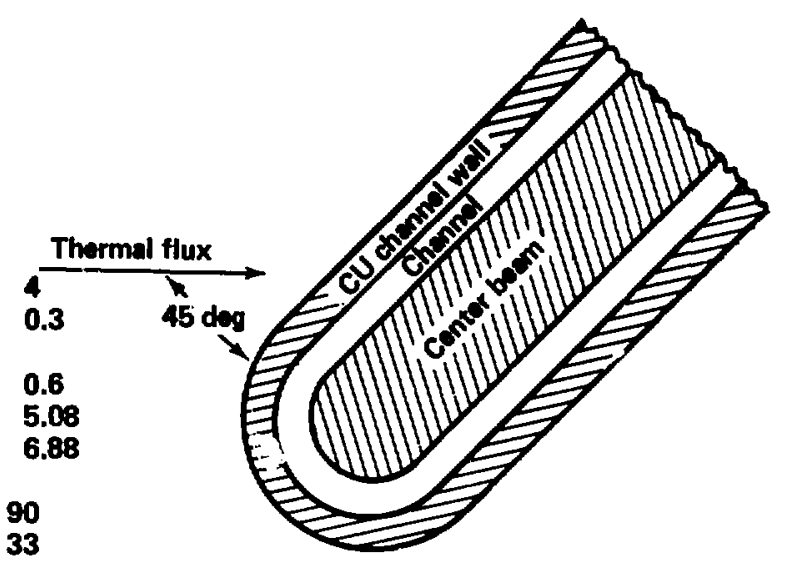

1320

4

330

20

$Z=106.75 \mathrm{~m}$

Fig. 3.6-23. Halo scraper design. 
channel lengths. Both plasma side and coolant side temperatures are given for the downstrean channel in a channel path of four channels. This channel would have the highest root and tip temperatures of all the four channels.

Figure 3.6-24 contains a halo scaper parametric analysis for an inlet temperature of $30^{\circ} \mathrm{C}$. Five performance parameters--maximum surface temperature, coolant pressure, flow rate, pumping power, and combined stress--are plotted against the coolant outlet temperature. The flow rate and pumping power decrease with increasing coolant outlet temperature. As the coolant outlet temperature rises, the temperature drop across the coolant film also rises. Both effects cause the maximum material surface temperature to risa. The required coolant pressure is the sum of the pressure required to prevent the coolant from boiling at the maximum fluid temperature and the pressure drop of the flowing coolant. The pressure drop falls with increasing coolant out let temperature because the flow rate decreases. The result is that the coolant pressure is flat out to about $120^{\circ} \mathrm{C}$ and then rises steeply. The shape of the combined stress curve follows the shape of the pressure.

\subsubsection{Continuous Cryopumping Systems}

The vacuum pumping system is designed to continuously remove the gas load that passes from the reactor central cell to the end tanks. The gases are helium, deuterium, and tritium. Additional vacuum systems that are not discussed are those needed to maintain vacuum beam lines, the pumps used to bring the vessel to a pressure where the cryopumps take over, and the system used to de-gas the continuous cryopumping system.

\subsubsection{Requirements}

The continuous cryopumping system must maintain a total pressure in the reactor end tanks of $2 \times 10^{-5}$ Torr during plasma burn by pumping the particle loads specified in Table 3.6-5. The charged particles pass from the central cell into the fon-end tank and the electron-end tank either into the main tank chambers or into the halo region at each tank perimeter.

\subsubsection{Performance}

We have calculated the performance of a single-stage continuous cryopumping system for the ion-end tank, electron-end tank, and halo scrapers; the 


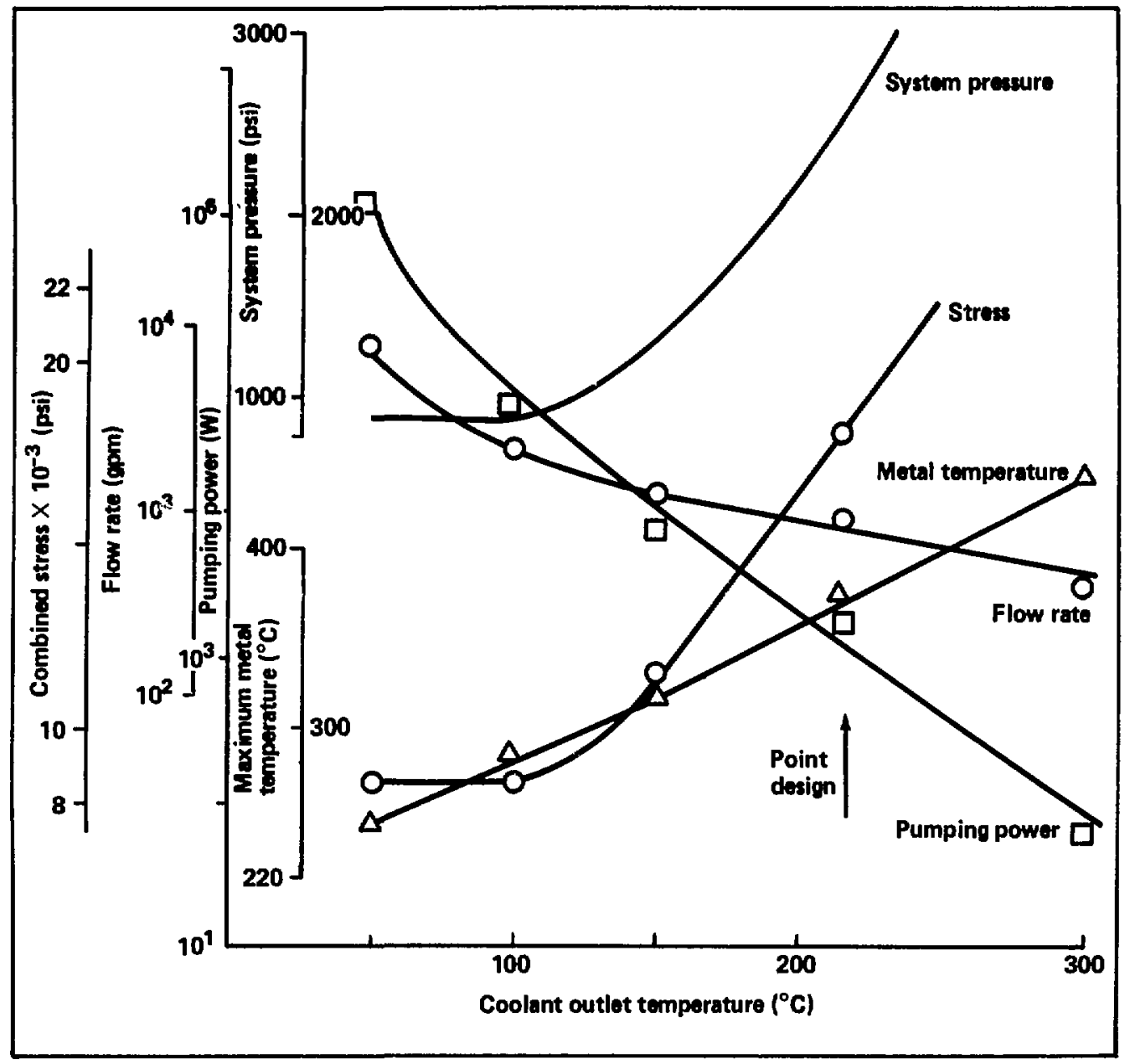

Fig. 3.6-24. Halo scraper system study. 
Table 3.6-5. MARS pumping requirements.

Throughput (Torr-liter/s at $300 \mathrm{~K}$ ) Electron Total

Total current Ion end end both ends

(A)

Core plasma

Deuterium fuel

432.4

42.2

$--$

42.2

Tritium fue 1

432.4

42.2

$--$

42.2

Deuterium sloshing

12.6

0.62

0.62

1.24

Alphas

119.3

5.8

5.8

11.6

Total

-- 90.8

6.4

Halo plasma

Deuterium fuel

696.0

33.7

33.7

67.4

Tritium fuel

696.0

33.7

33.7

67.4

Alphas

278.4

13.5

13.5

27.0

Total

--

80.9

80.9

Overall total

$--\quad 171.7$

87.3

259.0 
results are presented in Table 3.6-6. The pump speeds are $4.5 \times 10^{6} 1$ iter $/ \mathrm{s}$ in the ion end tank, $3.2 \times 10^{5}$. liter/s in the electron end tank, and $1.62 \times 10^{6}$ liter/s in each of the halo scrapers.

The end tank pumps are located within the tanks to maximize conductance. Therefore, the total speed is assumed to equal the speed of the pumps. The halo pumping system is placed in a plenum downstream of the halo scraper. To account for the intervening ducting, the conductance of the duct and plenum is presumed to equal the pump conductance, so that the required pump speed is double the effective speed required at the halo scraper. The halo effective speed is based on a total pressure of $10^{-4}$ Torr maintained in the scraper. We selected this pressure for the given plasma conditions as a compromise between the required effective speed, which varies inversely with the pressure, and the size of the scraper, which increases with the pressure (see Section 3.6.3.4).

\subsubsection{System Description}

The continuous cryopumping system is located in the ion end tank, in the electron end tank, and in plenums downstream of the halo scrapers in each of these end tanks. The system removes the helium and fuel constituents continuously while maintaining a total pressure in the end tanks of $2 \times 10^{-5}$ Torr. The pumping units are sized as shown in Table 3.6-6 to meet the requirements specified for each cavity.

We chose cryopumps because of their ability to be configured for the specified high pumping speeds. Because cryopumps are by nature batch-type devices, it is necessary to include the capability for periodic regeneration of each pump while other pumps in the system are on-line. This excess capacity is included in each pumping module.

The pumping system is made up of individual modules (shown in Fig. 3.6-25) that can be removed for maintenance. The number of pumps (cryopumping units) in each module is derived in Table $3.6-7$, with a $5-m^{2}$ pumping panel (two sides at $2.0 \times 1.25 \mathrm{~m}$ each) selected for commonality. The system has a $20 \%$ excess pump capacity, which, when combined with a short regeneration time, results in a maximum of only $67 \mathrm{~g}$ of tritium being held up in the pumps. The tritium throughput is $3.04 \mathrm{~kg} /$ day, based on $15 \%$ burnup; deuterium throughput is $2.05 \mathrm{~kg} /$ day. In addition, $712 \mathrm{~g} /$ day of helium must be pumped. 
Table 3.6-6. Cryopump sizing.

\begin{tabular}{|c|c|c|c|c|c|c|c|c|}
\hline & & $\begin{array}{c}\text { Throughput } \\
\text { (Torr-liter/s) }\end{array}$ & $\begin{array}{c}\text { Total } \\
\text { pressure } \\
\text { (Torr) }\end{array}$ & $\begin{array}{c}\text { Partial } \\
\text { pressure } \\
\text { (Torr) }\end{array}$ & $\begin{array}{c}\text { Total } \\
\text { speed } \\
(1 \text { iter } / \mathrm{s})\end{array}$ & $\begin{array}{c}\text { Specific } \\
\text { pumping } \\
\text { speed } \\
\left(1 \text { iter } / \mathrm{s}-\mathrm{cm}^{2}\right)\end{array}$ & $\begin{array}{l}\text { Pumping } \\
\text { surface } \\
\text { area } \\
\left(10^{4} \mathrm{~cm}^{2}\right)\end{array}$ & $\begin{array}{c}\text { Pump } \\
\text { speed } \\
(1 \text { iter } / 5)\end{array}$ \\
\hline $\begin{array}{l}\text { Direct } \\
\text { converter }\end{array}$ & DT & 85.0 & $2 \times 10^{-5}$ & $1.87 \times 10^{-5}$ & $4.5 \times 10^{6}$ & 7.9 & 57 & \\
\hline Tank pumps & Helium & 5.8 & $2 \times 10^{-5}$ & $1.28 \times 10^{-6}$ & $4.5 \times 10^{6}$ & 6.5 & 69 & \\
\hline $\begin{array}{l}\text { Electron } \\
\text { end }\end{array}$ & Deuterium & 0.6 & $2 \times 10^{-5}$ & $1.88 \times 10^{-6}$ & $3.2 \times 10^{5}$ & 7.9 & 4.1 & \\
\hline Tank pumps & Helium & 5.8 & $2 \times 10^{-5}$ & $1.81 \times 10^{-5}$ & $3.2 \times 10^{5}$ & 6.5 & 4.9 & \\
\hline \multirow[t]{2}{*}{$\begin{array}{l}\text { Halo pumps } \\
\text { (each end) }\end{array}$} & $\mathbf{D T}$ & 67.4 & $10^{-4}$ & $8.33 \times 10^{-5}$ & $8.1 \times 10^{5}$ & 7.9 & 21 & $1.62 \times 10^{6}$ \\
\hline & Helium & 13.5 & $10^{-4}$ & $1.66 \times 10^{-5}$ & $8.1 \times 10^{5}$ & 6.5 & 25 & $1.62 \times 10^{6}$ \\
\hline
\end{tabular}

aAssume pump speed equails conductance of pump duct system. 
Table 3.6-7. Cryopump installation.

\begin{tabular}{|c|c|c|c|c|c|c|c|c|c|c|}
\hline Location & $\begin{array}{l}\text { Required } \\
\text { pump } \\
\text { area } \\
\left(m^{2}\right)\end{array}$ & $\begin{array}{l}\text { Area per } \\
\text { unit } \\
\left(m^{2}\right)^{a}\end{array}$ & $\begin{array}{l}\text { Actual } \\
\text { unit } \\
\text { area } \\
\left(\mathrm{m}^{2}\right)\end{array}$ & $\begin{array}{l}\text { Number } \\
\text { of } \\
\text { pumping } \\
\text { units }\end{array}$ & $\begin{array}{l}\text { Units } \\
\text { per } \\
\text { moduleb }\end{array}$ & $\begin{array}{c}\text { Number } \\
\text { of } \\
\text { modules }\end{array}$ & $\begin{array}{c}\text { Excess } \\
\text { capacity } \\
(x)\end{array}$ & $\begin{array}{l}\text { Total } \\
\text { pump } \\
\text { area } \\
\left(m^{2}\right)\end{array}$ & $\begin{array}{l}\text { Tritium } \\
\text { through- } \\
\text { put } \\
\text { (g/day) }\end{array}$ & $\begin{array}{c}\text { Tritium } \\
\text { holdup } \\
\text { (g) }\end{array}$ \\
\hline D.C. tank & 69 & - & 75 & 15 & 6 & 3 & 20 & 90 & 1170 & 25.8 \\
\hline $\begin{array}{l}\text { Electron } \\
\text { end } \operatorname{tank}\end{array}$ & 5 & 5 & 5 & 1 & 2 & 1 & 100 & 10 & 0 & 0 \\
\hline $\begin{array}{l}\text { Halo } \\
\text { (D.C. end) }\end{array}$ & 25 & - & 25 & 5 & 6 & 1 & 20 & 30 & 934 & 20.6 \\
\hline $\begin{array}{l}\text { Halo } \\
\text { (electron } \\
\text { end) }\end{array}$ & 25 & - & 25 & 5 & 6 & 1 & 20 & 30 & 934 & 20.6 \\
\hline
\end{tabular}

aEach unit is double-sided, $2 \mathrm{~m} \times 1.25 \mathrm{~m}$ per side.

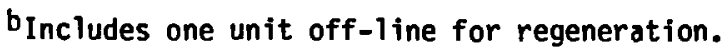




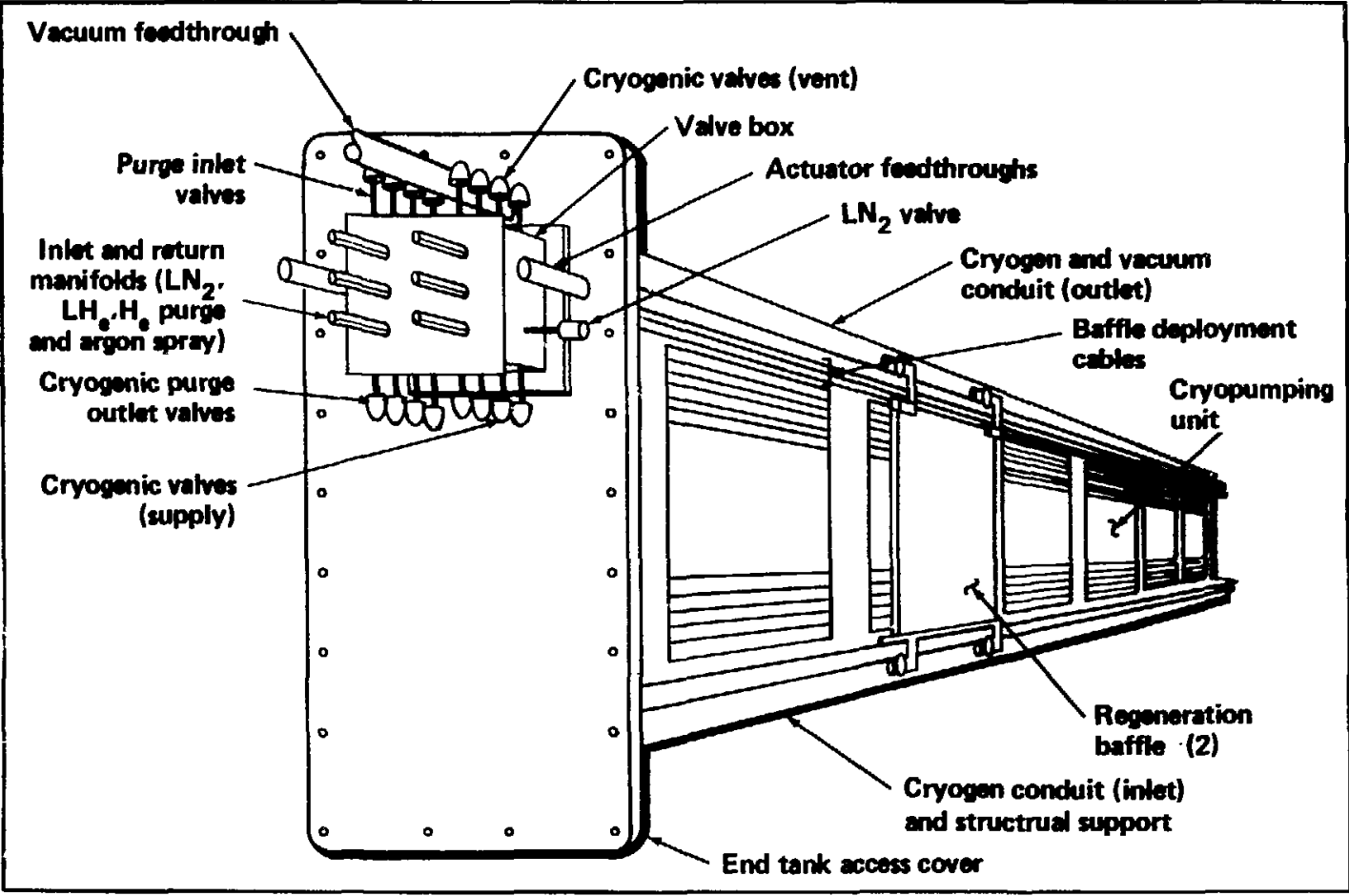

Fig. 3.6-25. Continuous cryopump module. 
The system consists of a total of 32 cryopumps; 26 are pumping during plasma burn while six (one per module) are being regenerated. Helium gas is cryotrapped on a double-sided, liquid-helium-cooled panel using an argon gas spray; the hydrogen isotopes are condensed on the same surfaces. The panel sides are shielded thermally by liquid-nitrogen-cooled chevrons, wich allow passage of the pumped gases from the reactor to the pumping panels. The cryopumps are arranged in an in-line housing so that one pair of baffles can be deployed to de-gas each pump sequentially as is shown in Fig. 3.6-25. The entire assembly is mounted on a vacuum tank cover for removal during maintenance. Valves for cryogen supply and vent, as well as for purge gas control during regeneration, are mounted in an evacuated, shtelded valve box on the tank cover.

An individual pump is regenerated by covering it with the regeneration baffles and purging the liquid helium from the pumping panel. The degassed particles are captured by a collector pump for subsequent recycling.

\subsubsection{Design Options}

We evaluated alternative cryopump and halo scraper configurations before selecting the baseline cryopumping system.

Specific Pumping Speed of Alternative Cryopump Concepts. Sizing of MARS cryopumps for the continuous flow of helium, deuterium, and tritium requires that the specific pumping speed $S_{p}$ be determined for each gas and each configuration of interest. The $S_{p}$ for an ideal pump is

$$
\left.S_{p(\text { idea })}=3.638\left(\frac{T}{M}\right)^{1 / 2} \text { (Torr-liter } / \mathrm{s}-\mathrm{cm}^{2}\right) \text {, }
$$

where $T$ is the temperature $(K)$ of the pumped particles with molecular weight M. For a real installation, $S_{p}=k S_{p}$ (ideal), where $k$ is a complex function of the pump geometry.

A helium and hydrogen cryopump basically consists of a pumping surface or surfaces separated from the source gases by intervening impedences such as chevrons and louvers, that intercept radiated energy and condensible gases. The expression for a single-impedence pump ${ }^{2}$ is

$$
\mathrm{S}_{\mathrm{p} 1}=3.638\left(\frac{\mathrm{T}}{\mathrm{M}}\right)^{1 / 2}\left(\frac{1}{\mathrm{t}}+\frac{1}{\mathrm{c}}-1\right)^{-1} \text {, }
$$


where $t$ is the transmissivity of the impedence and $c$ is the capture coefficient of the pumping surface. Expanding the expression for multiple impedences and that assuming the gas temperature becomes that of the impedence as it passes through, the expression for specific pumping speed of a twoimpedence unit is

$$
s_{p 2} \times 3.638\left(\frac{T}{N}\right)^{1 / 2}\left[\frac{1}{t_{1}}+\left(\frac{T}{T_{1}}\right)^{1 / 2}\left(\frac{1}{t_{2}}-1\right)+\left(\frac{T}{T_{2}}\right)^{1 / 2}\left(\frac{1}{c}-1\right)^{-1}\right. \text {. }
$$

where the subscripts 1 and 2 refer to the first and second impedence, respectively.

Alternative Cryopump Concepts. We evaluated three pump concepts (Table 3.6-8) to determine the best design for MARS. Two of these are compound pumps, each with two impedences; the triird is a single-stage single-impedence pump. Compound pumps have helium and hydrogen isotopes pumped on separate surfaces. The hydrogen is condensed on the second impedence and the helium passes through to be pumped on the final surface. In the single-stage pump, helium and hydrogen are pumped on one surface. In both the compound-and single-stage pumps, the first impedence is an opaque thermal shield.

The compound pump concept has the advantage of separating the helium and hydrogen isotopes, thus avoiding the need for a helium separator in the fuel cleanup system. However, this is gained at the expense of helium pumping speed, which is diminished by the extra surface needed for pumping hydrogen, and at the expense of a more complex regeneration and gas system. The reduced pumping speed can be recovered somewhat by making the first impedence less restrictive, for example by using a louver instead of a chevron, but the pumping speed gain is offset by increased heat load to the helium refrigeration system as is indicated in Table 3.6-9.

The single-stage pump concept features improved helium pumping speed and consequently smaller pumps. However, helium must be separated in the fuel cleanup system; Los Alamos is currently developing this capability as an alternative for TSTA (Tritium System Test Assembly).

We evaluated the effect of the three alternative pump configurations on pumping speed using Eq. (3.6-1). For hydrogen and helfum pumping speeds in the single-stage concept, $t_{2}=1$ because there is no second impedence. Trans- 
Table 3.6-8. Pump configuration.

\begin{tabular}{|c|c|c|c|c|c|c|c|c|c|c|c|c|c|}
\hline \multirow[b]{3}{*}{ Type } & \multicolumn{9}{|c|}{ Conf iguration } & \multirow{2}{*}{\multicolumn{3}{|c|}{$\begin{array}{l}\text { Specific pumping speed } \\
\left(1 \text { iter } / \mathrm{s}^{-1} \mathrm{~cm}^{-2}\right) \\
\end{array}$}} & \multirow[b]{3}{*}{ Remarks } \\
\hline & \multicolumn{3}{|c|}{ First panel } & \multicolumn{3}{|c|}{ Second panel } & \multicolumn{3}{|c|}{ Last panel } & & & & \\
\hline & Function & Style & $\begin{array}{l}\text { Temp } \\
(K)\end{array}$ & Function & Style & $\begin{array}{l}\text { Temp } \\
(K)\end{array}$ & Function & Style & $\operatorname{Temp}_{(\mathrm{k})}$ & Helium & $\begin{array}{l}\text { Hydrogen } \\
\text { isotopes }\end{array}$ & $k^{2}$ & \\
\hline \multirow[t]{3}{*}{ Compound A } & $\begin{array}{l}\text { Thermal } \\
\text { shield }\end{array}$ & Chevron & 77 & $\begin{array}{l}\text { Hydrogen } \\
\text { isotope } \\
\text { pumping }\end{array}$ & Chevron & 4.2 & $\begin{array}{l}\text { Helium } \\
\text { pumping }\end{array}$ & $\begin{array}{l}\text { Trapping } \\
\text { surface }\end{array}$ & 4.2 & 3.5 & 7.9 & 0.11 & $\begin{array}{l}\text { Separation of hydrogen } \\
\text { isotopes and helium }\end{array}$ \\
\hline & $\begin{array}{l}\text { Thermal } \\
\text { shield }\end{array}$ & Louver & 77 & $\begin{array}{l}\text { Hydrogen } \\
\text { isotope }\end{array}$ & Chevron & 4.2 & $\begin{array}{l}\text { He11um } \\
\text { pumping }\end{array}$ & $\begin{array}{l}\text { Trapping } \\
\text { surface }\end{array}$ & 4.2 & 4.3 & 12.7 & 0.14 & $\begin{array}{l}\text { Complex regeneration } \\
\text { scheme }\end{array}$ \\
\hline & & & & pumping & & & & & & & & & $\begin{array}{l}\text { No helium in fue } 1 \\
\text { cleanup system }\end{array}$ \\
\hline \multirow[t]{3}{*}{$\begin{array}{l}\text { Single } \quad C \\
\text { stage } \\
\text { (selected } \\
\text { baseline) }\end{array}$} & $\begin{array}{l}\text { Thermal } \\
\text { shield }\end{array}$ & Chevron & 77 & & Mone & & $\begin{array}{l}\text { Helium } \\
\text { ond } \\
\text { hydrogen } \\
\text { isotope } \\
\text { pumping }\end{array}$ & $\begin{array}{l}\text { Trapping } \\
\text { surface }\end{array}$ & 4.2 & 6.5 & 7.9 & 0.21 & $\begin{array}{l}\text { Improved helium } \\
\text { pumping speed } \\
\text { Simplified pump } \\
\text { design }\end{array}$ \\
\hline & & & & & & & & & & & & & $\begin{array}{l}\text { Helium separator in } \\
\text { fuel cleanup system }\end{array}$ \\
\hline & & & & & & & & & & & & & $\begin{array}{l}\text { Trapping-gas separator } \\
\text { in fuel cleanup systen }\end{array}$ \\
\hline \multicolumn{14}{|c|}{ ' $K=\frac{\text { Helium pumping speed }}{\text { Helfum ideat pumping speed }}$} \\
\hline Configurat & & R & & & & & & & & & & & \\
\hline
\end{tabular}


Table 3.6-9. Electric power requirements for pump options.

\begin{tabular}{|c|c|c|c|c|c|c|c|c|c|c|}
\hline Conftguration & $\begin{array}{l}\text { Heat to } \\
4.2-k \\
\text { surface } \\
\left(\mathrm{H} / \mathrm{cm}^{-2}\right)\end{array}$ & $\begin{array}{l}\text { Pumpling } \\
\text { system }\end{array}$ & $\begin{array}{c}\text { Total } \\
\text { pump } \\
\text { speed } \\
\left(11 \text { ter } / s^{-1}\right)\end{array}$ & $\begin{array}{c}\text { Specific He } \\
\text { pumping } \\
\text { speed } \\
\left(1 \text { titer } / \mathrm{s}^{-1} \mathrm{~cm}^{-2}\right)\end{array}$ & $\begin{array}{l}\text { Pumping } \\
\text { surface } \\
\text { ared } \\
\left(10^{4} \mathrm{~cm}^{2}\right)\end{array}$ & $\begin{array}{c}\text { Heat } \\
\text { load } \\
(\mathrm{H} \text { at } 4.2 \mathrm{~K})\end{array}$ & $\begin{array}{l}\text { Thernil } \\
\text { load } \\
\text { (1 at } 4.2 \mathrm{~K} \text { ) }\end{array}$ & $\begin{array}{l}\text { Gas } \\
\text { enthalphy } \\
\text { (U at } 4.2 \mathrm{~K} \text { ) }\end{array}$ & $\begin{array}{c}\text { Total } \\
\text { load } \\
\text { (H at } 4.2 \mathrm{~K})\end{array}$ & $\begin{array}{l}\text { Electric } \\
\text { power } \\
\text { at } 500: 1 \\
\text { (kH) }\end{array}$ \\
\hline \multirow{3}{*}{$\begin{array}{l}\text { A. Compound } \\
\text { (chevron- } \\
\text { chevron) }\end{array}$} & $2.0 \times 10^{-5}$ & $\begin{array}{l}\text { Direct } \\
\text { converter }\end{array}$ & $4.5 \times 10^{6}$ & 3.5 & 129 & 25.8 & 46 & 3 & 49 & 25 \\
\hline & $2.0 \times 10^{-5}$ & $\begin{array}{l}\text { Electron } \\
\text { end }\end{array}$ & $3.2 \times 10^{5}$ & 3.5 & 9.1 & 1.8 & 46 & 3 & 49 & 25 \\
\hline & $2.0 \times 10^{-5}$ & $\begin{array}{l}\text { Halo } \\
\text { (each) }\end{array}$ & $1.6 \times 10^{6}$ & 3.5 & 46 & 9.2 & 46 & 3 & 49 & 25 \\
\hline \multirow{3}{*}{$\begin{array}{l}\text { B. Compound } \\
\text { (1ouver- } \\
\text { chevron) }\end{array}$} & $1.8 \times 10^{-3}$ & $\begin{array}{l}\text { Direct } \\
\text { coiverter }\end{array}$ & $4.5 \times 10^{6}$ & 4.3 & 105 & 1890 & 3355 & 3 & 3358 & 1679 \\
\hline & $1.8 \times 10^{-3}$ & $\begin{array}{l}\text { Electron } \\
\text { end }\end{array}$ & $3.2 \times 10^{5}$ & 4.3 & 7.4 & 133 & 3355 & 3 & 3358 & 1679 \\
\hline & $1.8 \times 10^{-3}$ & $\begin{array}{l}\text { Halo } \\
\text { (each) }\end{array}$ & $1.6 \times 10^{6}$ & 4.3 & 37 & 666 & 3355 & 3 & 3358 & 1679 \\
\hline \multirow{3}{*}{$\begin{array}{l}\text { C. Single } \\
\text { stage }\end{array}$} & $2.0 \times 10^{-5}$ & $\begin{array}{l}\text { Direct } \\
\text { converter }\end{array}$ & $4.5 \times 10^{6}$ & 6.5 & 69 & 13.8 & 24 & 34 & 58 & 29 \\
\hline & $2.0 \times 10^{-5}$ & $\begin{array}{l}\text { Electron } \\
\text { end }\end{array}$ & $3.2 \times 10^{5}$ & 6.5 & 4.9 & 1.0 & 24 & 34 & 58 & 29 \\
\hline & $2.0 \times 10^{-5}$ & $\begin{array}{l}\text { Halo } \\
\text { (each) }\end{array}$ & $1.6 \times 10^{6}$ & 6.5 & 25 & 5.0 & 24 & 34 & 58 & 29 \\
\hline
\end{tabular}


missivities are 0.28 for a chevron and 0.45 for a louver. To compare alternatives, the capture coefficient $c$ for helium in the compound pump and for hydrogen is taken as unity. However, a mare conservative value of $c=0.6$ is assumed for helium pumping in the single-stage pump where the gas inixture is pumped on a single surface.

We selected the single-stage cryopump design because of its simplified design, higher pumping speed, and, thus, smaller-sized units.

Halo Scraper Configurations. The halo scraper system, shown schematically in Fig. 3.6-26, neutralizes particles in the halo plasma so they can be removed by the continuous cryopumping system. Ionized particles entering the scraper are neutralized at the elbow formed by the scraper and the vacuum pumping duct. Their path depends on the conductance of these two passages. Neutral particles reentering the scraper can be reionized or can return to the plasma. Reionization probabilities have been based on STARFIRE data. The fraction escaping from the scraper, because of its symmetry, is based on an assumption that $50 \%$ of the neutrals reentering the scraper escape if the scraper length is zero. This fercentage rapidly decreases as the scraper length-to-height ratio is increased.

The performance and design paraneters for the hato scraper in the ion-end tank are tabulated in iable 3.6-10. The inlet area and plasma filux are based on the halo plasma $F$.jfile defined in Section 3.6.2. The results show that. the halo scraper has to be $8.8 \mathrm{~m}$ long for the required throughput.

We evaluated the practicality of reducing pumping speed to a range where turbomolecular (TM) pumps could be used. For this study, we held the inlet width constant and allowed the height to vary as a function of the plasma flux density. Pump speed reduces inversely with halo pressure. However, for a fixed-plasma particle and energy flux, the halo inlet conductance must be reduced (increased length) to maintain the particle balance within the halo scraper as the halo pressure is increased. To reduce pump speed while mainraining a scraner size comparable to the baseline, the plasma density and heat flux must ir rease. We selected two plasma conditions for comparing TM and cryopumps. The results are shown in Fig. 3.6-27 and Table 3.6-11, which present the changes required in plasma conditions to reduce halo pump speed while maintaining the scraper length at $8.8 \mathrm{~m}$. He will evaluate the consequences of the low pump speed option in FY 1983. 
Table 3.6-10. Ion-end halo scraper parameters.
Helium-pumped (Torr-liter/s)
$\$ 3.5$
DT-puriped (Torr-1iter/s)
67.4
Halo pressure (Torr)
$10^{-4}$
Halo plasma inlet molecular flux $\left(\mathrm{cm}^{-2}-\mathrm{s}^{-1}\right)$
$1.2 \times 10^{100}$
Halo plasma power (MW)
40.15
Heat flux $\left(M W / \mathrm{m}^{2}\right)$
1
Halo inlet area $\left(\mathrm{m}^{2}\right)$
40.2
Halo inlet height, averaged $(\mathrm{cm})$
100
Halo inlet iength for $1-m$ height $(\mathrm{m})$
8.9
Pump speed (1iter/s)
$1.62 \times 10^{6}$

Table 3.6-11. Alternative pump parameters.

Baseline

Scraper length (m)

Inlet height $(\mathrm{cm})$

Molecular flux $\left(\mathrm{cm}^{-2}-\mathrm{s}^{-1}\right) 1.2 \times 10^{16}$

Plasma power $\left(\mathrm{W} / \mathrm{cm}^{2}\right)$

Halo pressure (Torr)

Pump speed (1 iter/s)
8.8

100

100

$10^{-4}$

$1.6 \times 10^{6}$
Low pump speed option

8.8

27

$4.5 \times 10^{16}$

375

$3.3 \times 10^{-3}$

$4.9 \times 10^{4}$ 


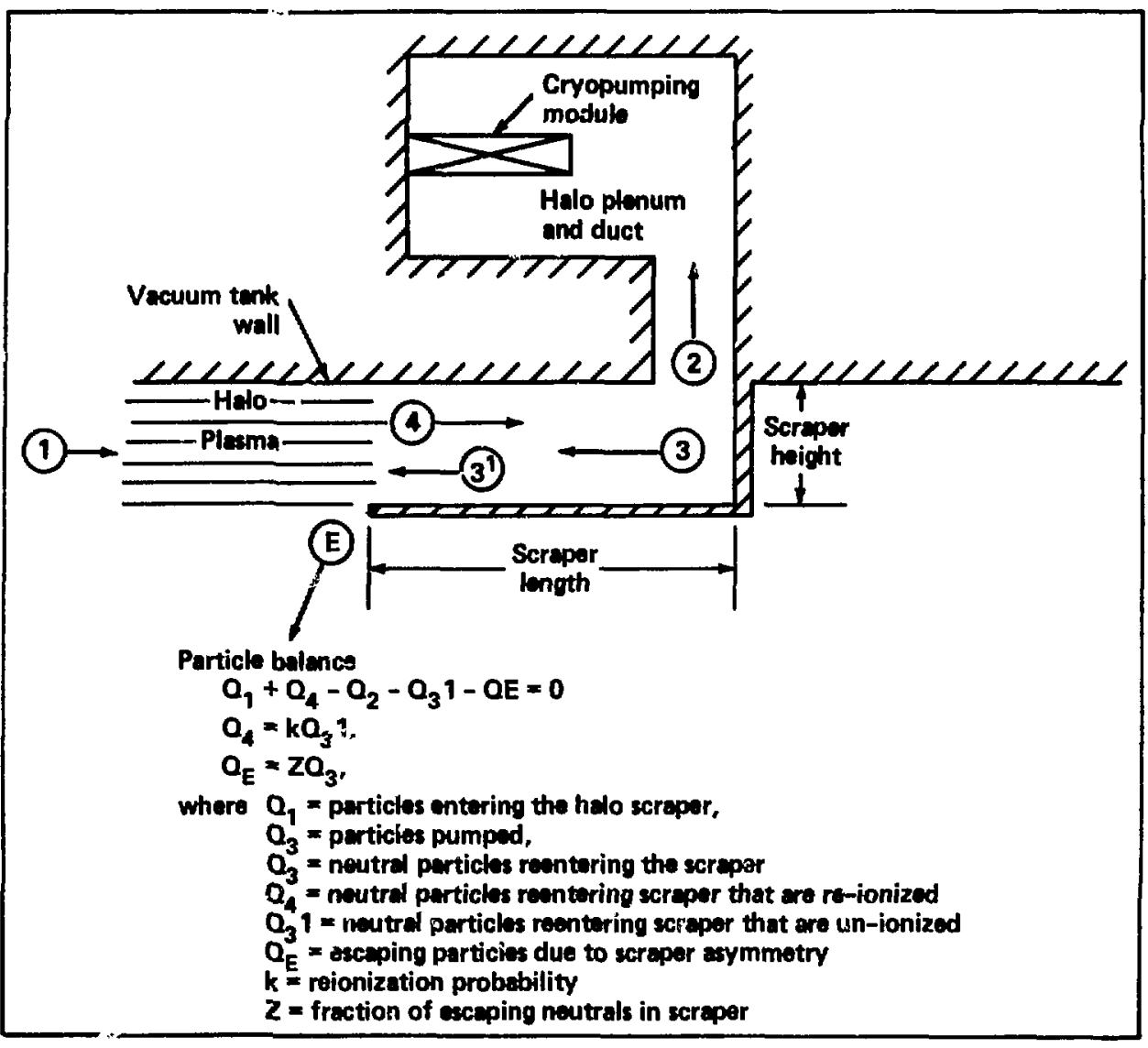

Fig. 3.6-26. Halo scraper model. 


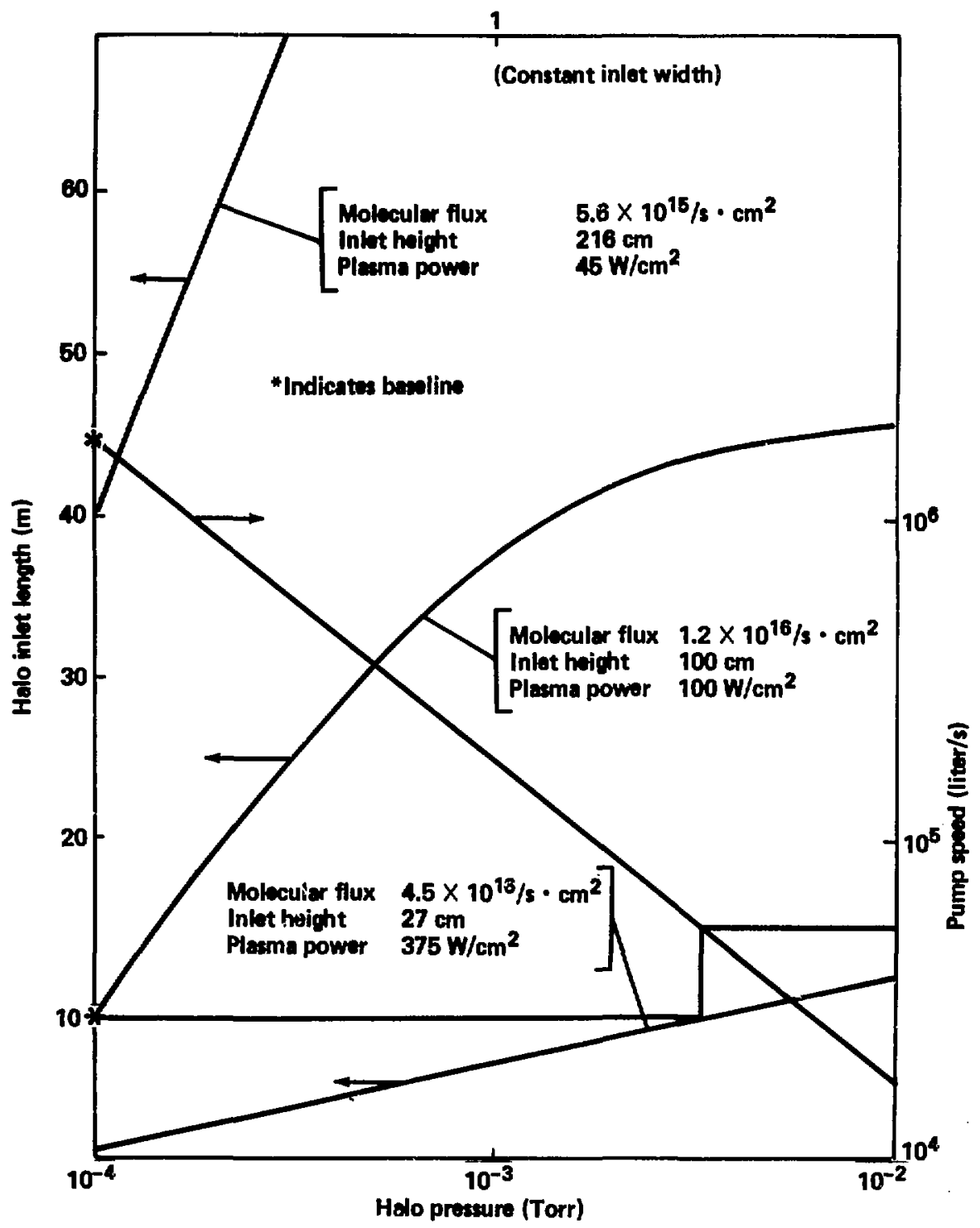

Fig. 3.6-27. Halo pump parameters. 
The particle flow to the electron-end halo is identical to the ion-end halo flow; however, the profile is not yet defined. Therefore, its parameters are to be determined.

\subsubsection{Helium Refrigeration Load}

We have completed a preliminary estimate of the helium refrigeration load for the continuous cryopumping system. The load is principally that required to support the regeneration function and to of fset heat leak from the distribution system.

Each of the 32 cryopumps is regenerated every $48 \mathrm{~min}$. During regeneration the Lhe-cooled pumping surface is allowed to warm to $18 \mathrm{~K}$ by purging the coolant with warm helium gas. The helium refrigeration recools the panel to $4.2 \mathrm{~K}$ for operation. The refrigeration load for regeneration depends mainly on the control of purge temperatures. Use of ambient helium purge gas requires $24.4 \mathrm{~W}$ of cooling per pump ( $778 \mathrm{~W}$ total). If a lower purge gas temperature is used--125 $\mathrm{K}$ assumed--this requirement drops to $9.2 \mathrm{~W}$ per pump (295 $\mathrm{W}$ total), which is the value used in the baseline. The additional load for cooling the metal panel after regeneration is small (49 $\mathrm{W}$ total) because of the panel's low specific heat at cryogenic temperatures. The heat loss from the helium distribution system is given in Table 3.6-12.

The total helium refrigeration load, which includes energy extracted from the pumped gas and energy radiated to the pumping surfaces, is summarized in Table 3.6-13.

\subsubsection{Vacuum Tanks and Support Structure}

The end tanks for MARS will be different from each other in many ways. The two biggest differences are in size and orientation. The end tank for the ion end of the machine is significantly larger than the end tank for the electron end because the high heat load on srid 2 dictates its large area. The plasma fans are rotated $90 \mathrm{deg}$ with respect to one another for plasma stability regions. Thus, while the large ion-end tank lies flat, the electron-end tank fans out vertically. In both cases, the tanks must be designed considering remote maintenance of the plasma direct converter components. 
Table 3.6-12. Helium distribution system heat loss.

\begin{tabular}{|c|c|c|}
\hline Item & Quantity & $\begin{array}{l}\text { Heat loss } \\
\text { (W) }\end{array}$ \\
\hline Supply valve & 38 & 67 \\
\hline Return valves & 38 & 76 \\
\hline Ffll bayonets & 6 & 12 \\
\hline Return bayonets & 6 & 12 \\
\hline Fteld joints & 12 & 28 \\
\hline Fill lines & 250 & 53 \\
\hline \multirow[t]{2}{*}{ Return lines } & 250 & 75 \\
\hline & & 323 \\
\hline
\end{tabular}

Table 3.6-13. Total helium heat load (W).

Pumped gas energy 34

Radfation to pumping surfaces 32

Distribution system 323

Regeneration $\quad 344$

Contingency

Total 805 W 


\subsubsection{Ion-End Tank}

The halo scraper, cryopump modules, and ion converter components requite a vacuum tank with overall dimensions of $32.5 \times 60 \times 10 \mathrm{~m}$, as shown in fig. 3.6-1. Several possible schemes were considered for supporting the vacuum loads introduced on such a tank before declding upon the configuration shown in Figs. 3.6-28 and 3.6-29.

The concepts considered but rejected were: a stiffened flat plate design (sinflar to the TFTR neutral bean boxes); a flat plate stiffened by large reinforced concrete beams; and suspension-type construction. The Idea of bridging through the plasma fan with compression members to most directly react the vacuum loads was not considered because of the plasma-wall interaction problems and the resulting loss in effictency from blocking particle flow.

A reinforced concrete barrel shell enclosure is the design we chose to support the vacuun vessel. As Figs. 3.6-28 and 3.6-29 show, the end tank vacuum boundary is determined by flat plates that approximate the shape of the direct converter components. The flat plates are stiffened and supported by beams on 3-m centers that are in turn supported from the barrel shell. On the upper surface, this support is via cables, while on the lower side tile rods are used since both gravity and vacuum loads must be reacted.

The total estimated cost for this structure is $\$ 55$ million, which includes $\$ 50$ million for the stainless steel vacuum tank and $\$ 5$ million for the concrete barrel shell. A less expensive option may be to use a magnetic material for the vacuum vessel, bat this will require additional study of the effect on the plasma fan.

The components in the end tank are remotely maintained through access ports in the top of the yacuum vessel. These ports are located between the stiffening bearis, witich are spaced at $3-m$ intervals. Each cryopump module is the size of one port, ant the grid structures and ion collector plate can be reduced to $1.5-\mathrm{m}$ segments for replacement. Service cranes run in the direction of the axis of the barrel sheli between the service ports and the service bay area adjacent to the end tank. 


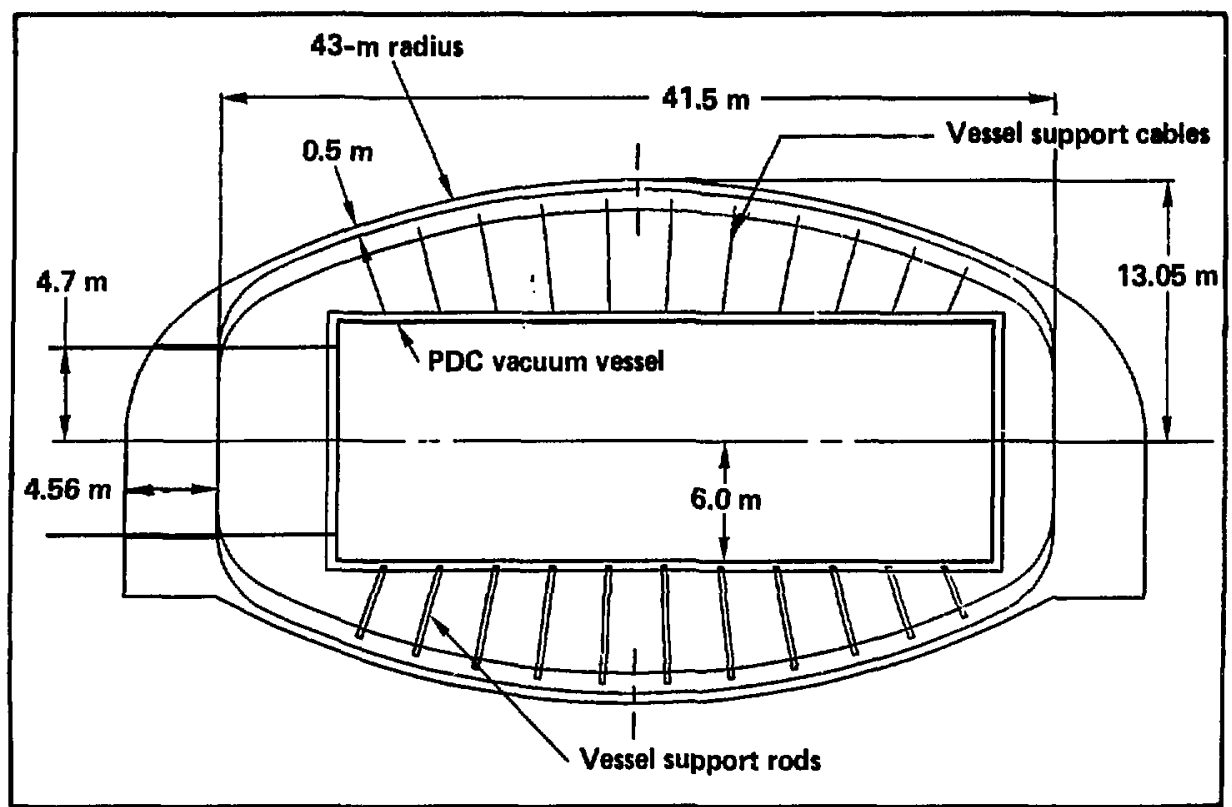

Fig. 3.6-29 Barrell shell support structure. 


\subsubsection{Electron-End Tank}

The electron-end tank and supporting structure are shown in Fig. 3.6-30. The vacuum tank is supported by a concrete barrel shell structure simflar to the structure on the fon end, except that the plasma fan expands vertically. Access to the tank for remote maintenance must be through the top because the clearance between the barrel shell and tank wall is not sufficient. The tank itself has overall dimenstons of $15 \times 31 \times 6 \mathrm{~m}$, approximately half the size of the lon-end tank. It is made from 1.9-cm-thick stainless steel plates stiffened by back-to-back channels at 3-m spacing. The vacuim loads are transferred to the barrel shell structure through tie-rods connected to the channels.

\subsubsection{Tritium Considerations}

\subsubsection{Tritium Permeation into Cooling Water}

In the ion converter, high-energy trittum fons (40 to $400 \mathrm{keV}$ ) strike the collector plate and grid array assemblies to enable the plasma energy to be recovered in the form of electrical and thermal energy. In addition, the halo plasma containing tritium impinges on the halo scraper. The energetic impingement of tritium ions on these surfaces will result in a steady-state tritium loss to the water coolant as well as in a tritfum inventory in the walls. This steady-state tritlum loss to the coolant poses a potential environmental hazard because of subsequent tritlated water leakage from the cooling system. In addition, the tritium inventory must be kept low enough to prevent material fallure due to hydrogen embrittlenent. To estimate the extent of these problens, we applied a simple model of tritium diffusion and holdup, which takes into account the recombinative properties of the sur.ace and the depth of the plasma implant, to the surfaces of interest. The model is based on the recommended approach of Baskes, 3 which has been successfully used to investigate reemission of implanted deuterium from stainless steel walls, thermal absorption, and nuciear reactor profiling of deuterium near surface concentrations. The analysis, however, has not been verified experimentally for permeation implantation problems. Furthermore, in our analys is, we had to rely solely upon analytic estimates of the recombination rate coefficients and implant depth proftles for energetic tritfum/copnor interaction because we could not find any experimental data. 


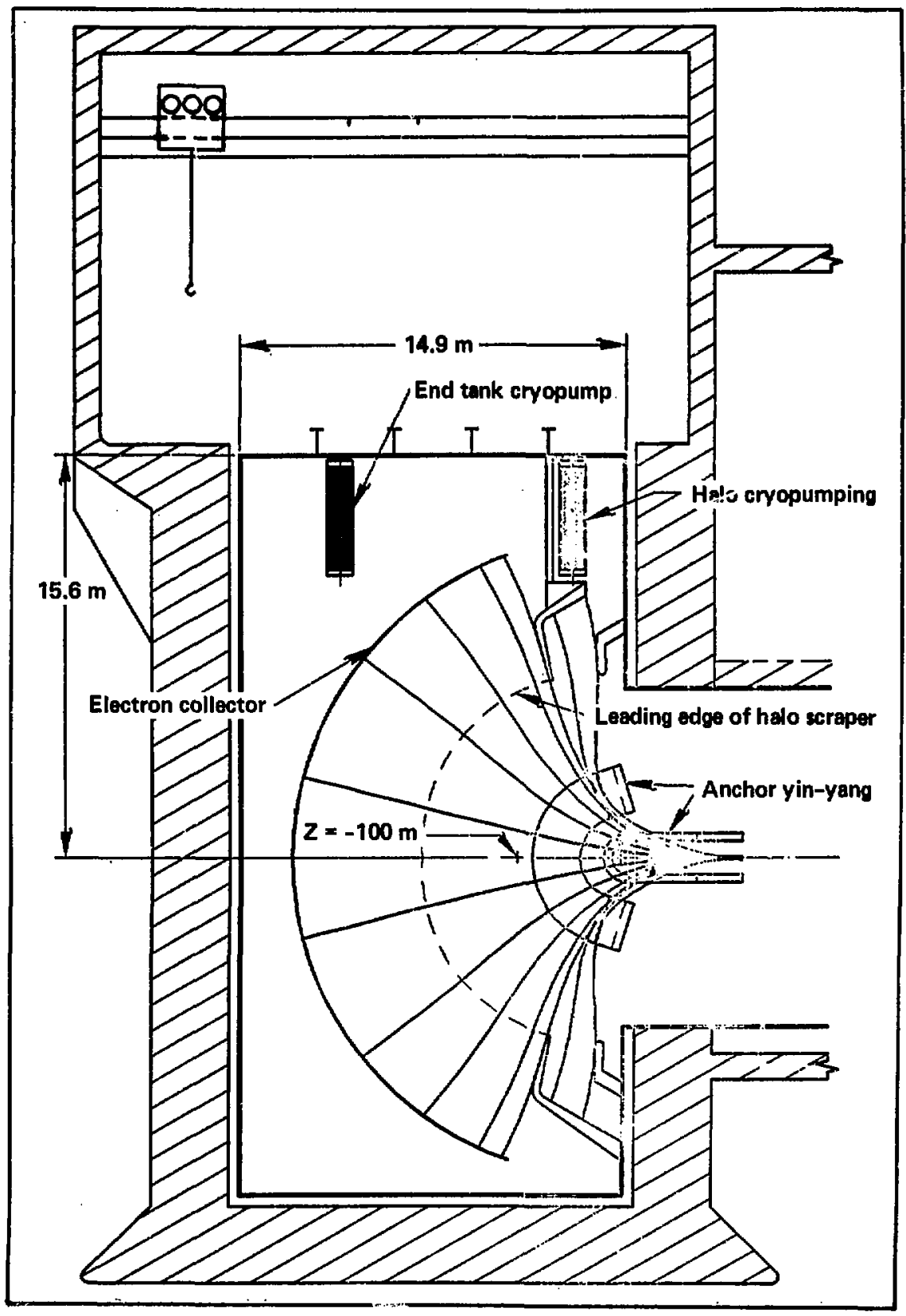

Fig. 3.6-30 Electron-end tank anci support structure. 


\subsubsection{Tritium Diffusion Model}

The analytic model for tritiun diffusion is briefly described, sample calculated results are presented, and methods for reducing the permeation loss are suggested for further investigation. More detalls of the analysis, along with a sensitivity analysis of predicted loss and holdup to uncertainties in the recombination rate coefficient and implant depth parameters, may be found in Ref. 4.

We estimated the permeation and inventory in the fon collector plate, entrance and suppressor grid arrays, and halo scraper using solutions of the steady-state one-dimensional Fick's law, with the following assumptions:

1. A monoenergetic DT plasma interacting with a clean, semi-infinite one-dimensional, homogenous $\mathrm{Cu}$ slab with a linear temperature profile across the slab.

2. Source term due to implanted tritium only.

3. The release of tritium from the external surfaces requires recombination of the tritium atoms; f.e., second-order desorption is the dominant mechanism.

4. No trapping, i.e., no hydrides are formed.

Figure 3.6-31 illustrates the salfent features of the analytic model that can be expressed with the following four equations:

$$
\begin{aligned}
& D(T) \frac{x}{\partial x}=\text { CONST }=J, \\
& \phi=D\left(T_{1}\right)\left[c^{*}-c(-\delta)\right] / \delta \text { at } x=0 \text { for } u_{B} \ll v_{\delta^{\prime}} \\
& K_{r}\left(T_{1}\right) c^{2}(-\delta)=D\left(T_{1}\right)\left[c^{*}-c(-\delta)\right] / \delta \text { at } x=-\delta, \text { and } \\
& K_{r}\left(T_{2}\right) c^{2}(L)=\left.D\left(T_{2}\right) \frac{x}{\partial x}\right|_{x=L},
\end{aligned}
$$

where the recombination rate coefficfent $K_{r}(T)$ was estimated using the model developed in Ref. 5 assuming a sticking probabllity of one; the implant depth for tritium in copper was obtained from Ref. 6. 


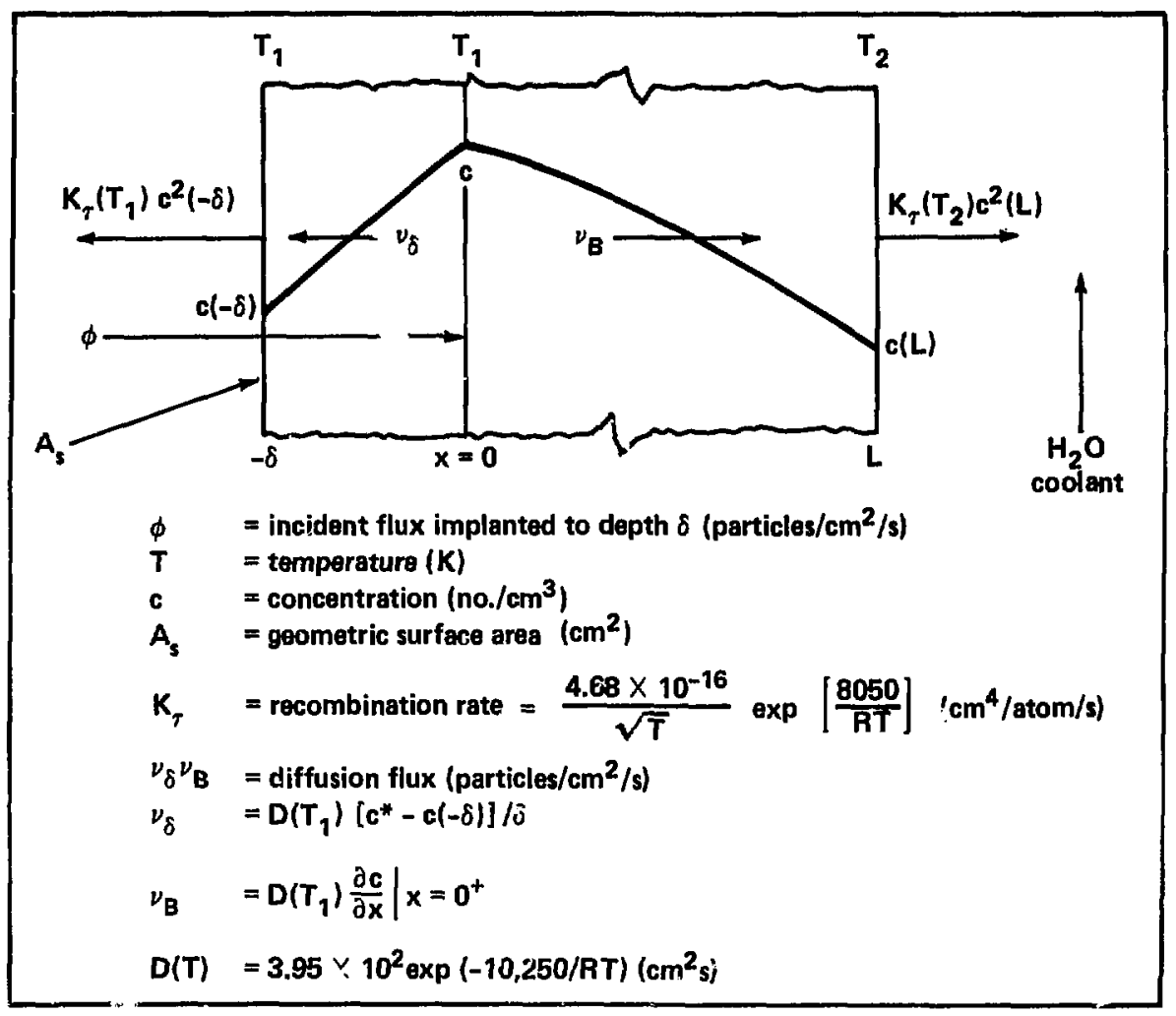

Fig. 3.6-31. Tritium diversion mode1. 
From the solution of Eq. (3.6-2) we obtain the concentration profile in the wall

$$
\begin{aligned}
\tilde{c}(\xi) & =1+J^{\prime} \int_{0}^{\xi} \frac{d \xi^{\prime}}{\left(3.95 \times 10^{-2}\right) \exp \left(\frac{-10,250}{R\left[T_{1}+\left(T_{2}-T_{1}\right) \xi\right]}\right)}, 0<\xi<1 \\
& =1+J^{\prime} I(\xi),
\end{aligned}
$$

and the wall inventory

$$
I N V=A_{s} L c^{\star} \int_{0}^{1} c\left(\xi^{\prime}\right) d \xi^{\prime},
$$

where a linear temperature profile is assumed in the wall, $T=T_{1}+\left(T_{2}-T_{1}\right) \xi$, $\xi=x / L, c=c(x) / C^{*}$, and $J^{\prime}=J L / C^{*}$.

From Eqs. (3.6-3) and (3.6-4), we obtain expressions for the implant concentration $c *$ and the upstream wall concentration $c(-\delta)$, such that

$$
c^{*}=c(-\delta)+\phi \delta / D\left(T_{1}\right)=\sqrt{\phi / K\left(T_{1}\right)+\phi \delta / D\left(T_{1}\right)} \text {. }
$$

From Eq. (3.6-6) and the boundary condition at $x=L$ given by Eq. 3.6-5,

$$
J^{\prime}=-1 / I(1)
$$

The permeation to the coolant is then

$$
P=-\left.A_{s} D\left(T_{2}\right) \frac{\partial C}{\partial x}\right|_{x=L}=-A_{s} J=A_{s} C^{*} / L I(1) .
$$

The integral I(1) was solved numerically for the cases investigated with the assumed linear temperature gradient. For $T_{1}=T_{2}=$ const $=T, I(1)=1 / D(T)$ and $P=A_{s} D c^{*} / L$, the correct result for $c(L)=0$. For all the cases we have inyestigated to date, $c(-) \sim 0$ because the ratio of the recombination rate flux to the diffusion rate flux is very large and the permeation is diffusionrate limited; i.e., Eq. $(3.6-7)$ is only correct for $K\left(T_{2}\right) C * L / D\left(T_{2}\right) \gg 1$. If the rate of tritium removal at the coolant-wall interface is limiting (e.g., by an oxide coating formed by $\mathrm{H}_{2} \mathrm{O} / \mathrm{Cu}$ reaction), the wall loading will increase 
and the permeation will decrease. For the cases we have considered, we obtained conservative estimates of the permeation and underestimates of the wall inventory.

\subsubsection{Results}

Table 3.6-1.4 summarizes our estimates of the tritium permeation to the coolant and the wall inventory for the ion collector plate, the entrance and suppressor grid arrays, and the halo scraper. Th: principal input parameters specified in the calculations are also shown in the table. The total tritium loss and wall inventory obtained represent roughly $0.013 \%$ of the incident flux. The peak implant concentration observed was 1.4 appm (centerline suppressor grid tu.e) and does not present material failure problems from hydrogen embrittlement.

In the analysis, we assumed that a constant tritium flux and surface temperature was appropriate for the current halo scraper design. For the ion collector plate, the tritium flux variation along the major axis of the elongated ellipitical plasma footprint was approximated by average values over each of the $6-\mathrm{m}^{2}$ sectors (Table 3.6-15). Note that there are approximately seven grid tubes per sector for each grid array. We did not include detailed wall temperature gradients in the present analysis of these designs because the temperature gradients are too small to significantly reduce permeation and holdup. For example, two cases are shown in Table 3.6-14 for the grid arrays: (1) a constant temperature for all tubes in the array and (2) a case where each tube has a different constant temperature corresponding to its local heat 1oad. These results differ by only $20 \%$ in the permeation and by 5 to $8 \%$ in the inventory. We obtained similar results for the collector plate with and without temperature gradients.

Temperatu.e gradients can significantly reduce the permeation and holdup. 4 However, for copper walls with the coolant wall temperature maintained at a minimum of $200^{\circ} \mathrm{C}$ and a maximum allowable surface temperature of $500^{\circ} \mathrm{C}$, a factor of 10 reduction in permeation and inventory would be the most optimistic resuit. The effectiveness of the temperature gradient in reducing permeation and holdup is primarily caused by the decreased implant concentration level with increasing temperature in the implant region. On the other hand, the increased uncertainity of the uredictions due to the uncertainty in the 
Table 3.6-14. Tritium permeation (al1 surfaces constructed of AMAX-MZC materia1).

\begin{tabular}{|c|c|c|c|c|c|c|c|c|c|}
\hline Surface & $\begin{array}{c}\text { Thickness } \\
\text { (m) }\end{array}$ & $\begin{array}{l}\text { Total } \\
\text { area } \\
\left(m^{2}\right)\end{array}$ & $\begin{array}{c}\text { Tritium } \\
\text { energy } \\
\text { (keV) }\end{array}$ & $\begin{array}{l}\text { Tritium } \\
\text { flux } \\
\left(\mathrm{cm}^{2}-\mathrm{s}\right)\end{array}$ & $\begin{array}{l}\text { Implant } \\
\text { depth } \\
\text { (Im) }\end{array}$ & $\begin{array}{c}\text { P1 asma-wal1 } \\
\text { temperature } \\
\left({ }^{\circ} \mathrm{C}\right)\end{array}$ & $\begin{array}{c}\text { Coolont-wall } \\
\text { temperature } \\
\left({ }^{\circ} \mathrm{C}\right)\end{array}$ & $\begin{array}{c}\text { Permeation } \\
\text { (mg/day) }\end{array}$ & $\begin{array}{c}\text { Inventory } \\
\text { (mg) }\end{array}$ \\
\hline Converter collector plate & 1 & 240 & 45 & Voried & 0.3 & 225 & 225 & 33 & 142 \\
\hline Entrance grid array & 0.16 & 11.2 & 371 & Vorieda & 2.04 & $\left\{\begin{array}{c}276 \\
\text { Varieda }\end{array}\right.$ & $\left\{\begin{array}{c}276 \\
\text { Varieda }\end{array}\right.$ & $\left\{\begin{array}{l}65 \\
54\end{array}\right.$ & $\left\{\begin{array}{l}2.0 \\
1.9\end{array}\right.$ \\
\hline Repeller grid array & 0.16 & 11.2 & 471 & Varieda & 2.6 & $\left\{\begin{array}{l}276 \\
\text { varfeda }\end{array}\right.$ & $\left\{\begin{array}{c}276 \\
\text { Yaried }\end{array}\right.$ & $\left\{\begin{array}{l}83 \\
68\end{array}\right.$ & $\left\{\begin{array}{l}2.6 \\
2.4\end{array}\right.$ \\
\hline Halo scraper & 0.6 & 103 & 52.5 & $1.06 \times 10^{15}$ & $0.24^{b}$ & 250 & 250 & 19 & 18 \\
\hline
\end{tabular}

asee Table 3.6-15.

bSurface is inclined at 45 deg relative to the inconing tritium stream. 
Table 3.6-15. Tritium flux and temperature profiles.

\begin{tabular}{|c|c|c|c|}
\hline $\begin{array}{l}\text { Sector } \\
\text { number }\end{array}$ & $\begin{array}{l}\text { Flux } \times 10^{15} \\
\left.\text { (no./ } \mathrm{cm}^{2}-5\right)\end{array}$ & $\begin{array}{c}\text { Plasma-wal? } \\
\text { temperature } \\
\left({ }^{\circ} \mathrm{C}\right)\end{array}$ & $\begin{array}{c}\text { Coolant-wall } \\
\text { temperature } \\
\left({ }^{\circ} \mathrm{C}\right)\end{array}$ \\
\hline 1 & 1.86 & 378 & 340 \\
\hline 2 & 1.86 & 376 & 338 \\
\hline 3 & 1.86 & 374 & 337 \\
\hline 4 & 1.82 & 370 & 336 \\
\hline 5 & 1.79 & 367 & 334 \\
\hline 6 & 1.71 & 362 & 330 \\
\hline 7 & 1.62 & 356 & 326 \\
\hline 8 & 1.50 & 348 & 320 \\
\hline 9 & 1.38 & 334 & 306 \\
\hline 10 & 1.23 & 314 & 287 \\
\hline 11 & 1.08 & 288 & 266 \\
\hline 12 & 0.89 & 262 & 246 \\
\hline 13 & 0.71 & 242 & 232 \\
\hline 14 & 0.57 & 226 & 218 \\
\hline 15 & $0.43^{\circ}$ & 214 & 208 \\
\hline 16 & 0.31 . & 206 & 200 \\
\hline 17 & 0.18 & 200 & 200 \\
\hline 18 & 0.11 & 200 & 200 \\
\hline 19 & 0.042 & 200 & 200 \\
\hline 20 & 0.021 & 200 & 200 \\
\hline
\end{tabular}


recombination rate coefficient (dirty surface effects) with increasing plasmawall surface temperature could easily offset the anticipated reduction in permeation. 4

\subsubsection{Permeation Barriers}

The permeation rate can be significantly lowered by using a barrier layer, cladding, or coating on the coolant wall surface. Oxide ceramics such as $\mathrm{Al}_{2} \mathrm{O}_{3}, \mathrm{BeO}$, and $\mathrm{MgO}$ would prove very effective in reducing triti $n$ permeation by several orders of magnitude and can be formed for this application.

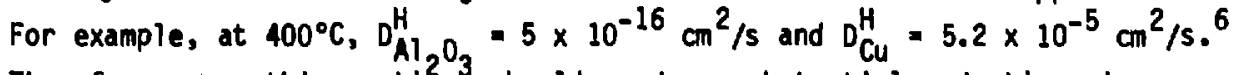
Therefore, very thin coatifigs should produce substantial reductions ir permeation. To apply this approach, a detalled analysis of the tritium build-up at the ceramic-metal interface would be needed in addition to a reevaluation of the thermal design. Ceramic liners have the advantage of reducing tritium contamination to the coolant to such a low level that iritium recovery mo not be required. However, the increased costs of such a procedure would neer to be balanced against the actual costs of tritium recovery without a barriei. A tradeoff study is recomended before any final engineering decisions are made on the need for tritium barriers.

The tritum permeation rate could also be reduced by employing low $Z$ coatings such as $C$ and TiC on the plasma-wall surface. Coatings of this type have been investigated in a number of implantation studies which have shown that the implanted hydrogen concentration saturates at a relatively low level. 3

The next phase of our analysis will consider these possibilities for permeation in the current design. We will also investigate the tritium problems associated with higher-temperature helium-cooled direct converter designs.

\subsubsection{Tritium Extraction From Cooling Water}

We investigated tritium permeation into the reactor water coolants. The direct-converter ion-collector coolant water will receive the largest tritium contamination of $\sim 750 \mathrm{Ci} / \mathrm{day}$. The water supply to this area will be kept separate and tritium will be recovered from the water when the tritiun concentration reaches a specified level, at wich time a slip stream will be removed 
and the tritium recovered at the permeation rate.

The parameters anticipated for the MARS water recovery unit (WRU) are a tritium permeation rate and subsequent recovery rate of $1000 \mathrm{Ci} /$ day, a concentration limit in water of 1 Ci/liter, a $1 \%$ slip stream, and a slip stream flow rate of $10^{3}$ liter/day.

He anticipate that the concentration level of $1 \mathrm{Ci} /$ day can be handled safely with respect to personnel exposure and environmental release. The CANOU reactor systems presently handle concentrations of $24 \mathrm{Ci} / \mathrm{liter}$ in moderator $\mathrm{D}_{2} \mathrm{O}$ and $0.65 \mathrm{Ci} / \mathrm{liter}$ in the reactor coolarit $\mathrm{D}_{2} \mathrm{O}$. The maximum recommended concentration level for the FED/INTOR water coolant is also 1 Cilliter.

Several methods can be used to recover tritfum from water. Many of these methods are in developmental stages, and choosing an optimum WRU for a future reactor is difficult. Following are several options considered for the MARS WRU.

LPCE. Liquid phase catalytic exchange is favored for its simplicity and operation at low temperature and pressure, and also because the tritium concentration as HTO is kept below the feed level. In addition, tritium can be removed at low concentrations and $T_{2}$ is produced in the exchange reaction. This process is presently in developmental stages, but it appears promising for future reactors.

VPCE. Vapor phase catalytic exchange is more complex than LPCE because it requires repeated evaporation and condensation cycles and higher operation temperatures. However, the technology for the process is available now. A $\$ 58$ million VPCE process is being installed for operation in 1985 at the Ontario Hydro Pickering site. Considerable industrial experience will exist for this process.

CECE. Combined electrolysis and catalytic exchange consists of a front end exchange column using a hydrophobic catalyst that enriches the HTO concentration. The enriched HrO is electrolyzed and hydrogen species are cryogenically distilled. This process is in development and should be available for future reactors.

Water Distillation Front End. We have investigated low pressure distillation of $\mathrm{H}_{2} \mathrm{O} / \mathrm{HTO}$ as a possible front end process. The distillation is 
based on the relative volatilities of the $\mathrm{H}_{2} \mathrm{O} / \mathrm{HTO}$ system presented in Table 3.6-16. 7 The design of the distillation system is summarized in Table 3.6-17. From the parameters listed, water distillation appears to be a simple and economical front end process for enrichment of hTO.

Table 3.6-16. Relative volatility for $\mathrm{H}_{2} \mathrm{O} / \mathrm{HTO}$.

Temperature

( $\left.{ }^{\circ} \mathrm{C}\right)$

40

55

76
Relative volatility

(a)

1.19

1.14

1.12
System pressure $(\mathrm{mHHg})$

118

296 
Table 3.6-17. Distillation coium design for $H_{2} /$ HTO separation.

Spectfied quantities

Feed HTO (CI/liter)

1

Overhead HTO

0.1

Botton HTO

100

Feed rate (1iter $/ \mathrm{m} / \mathrm{n})$

0.77

Overhead rate (1iter/min)

0.763

Bottom rate (1iter/hr)

0.415

Relative volatility

1.14

Tower operating pressure (mbar)

150

Reflux ratio

$2 \times$ minimum

Calculated results

Theoretical trays in rectifying section

27

Theoretical trays in stripping section

39

Reboiler power (MW)

0.4

Reflux ratio

12.3

Tower dimensions: 8

Diameter (m)

$0.5 \mathrm{~m}$

Height (m)

$17 \mathrm{~m}$

Cost of distillation system ${ }^{9}$

$\$ 600,000$ 
1. W. McAdams, Heat Transmission, McGraw Hill Book Co., Mew York (1954).

2. L.C. Pittenger, Vacuum Engineering for Fusion Research and Fusion Reactors, Lawrence Livermore Laboratory. AVS Symposium 1976.

3. M.I. Baskes, W. Bauer, and K.L. Wilson, Tritium Permeation in Fusion Reactor First Walls, Sandia Report SAND 82-8698, Unlimited (June 1982).

4. V. Calia and A. Tobin, Estimate of Tritium Permeation and Inventory in Copper Grid Tubes Immersed in A High Energy Plasma, Research Department Note, RN-423 (June 1982).

5. M.I. Baskes, "A Calculation of the Surface Recombination Rate Constant for Hydrogen Isotopes on Metals," Jour. of Nucl. Mat'15, 92, 318-324 (1980).

6. H. H. Anderson and J.F. Ziegler, "Hydrogen Stopping Powers and Ranges in all Elements", Vol. 3 of the Stopping and Ranges of Ions in Matter, organized by J.F. Ziegler, Permagon Press (1977).

7. A.H. Price, "Vapour, Pressure Tritiated Water," Nature, 181, 262 (1958).

8. M. Huber and W. Meier, "SULZER Columns for Vacuum Rectification and Mass Transfer," Sulzer Technical Review, 57, 3-15 (1975).

9. R.S Hal1, J. Matley, and J. McNaughton, "Current Costs of Process Equipment," Chem. Eng., pp 80-110, April 5, 1982. 


\subsection{CRYOGENICS}

We have performed a preliminary analysis of MARS cryogenic requirements. The subsystems needing refrigeration are: the superconducting magnets, vacuum system cryopanels, beamlines, and tritium processing equipment. These requirements are summarized in Table 3.7-1. Boiloff gas from refrigeration is returned to the cryoplant at a low temperature $(25 \mathrm{~K})$. The liquid requirement, given in liter/hr, refers to helium that will be returned to the cryoplant at approximately room temperature. Thus, the two requirements are very different. The MARS magnet cryogenic requirements were obtained through analysis of baseline design. Each of the systems that contribute to the cryoplant load will be discussed separately.

Table 3.7-1. MARS cryoplant heat load summary.

\begin{tabular}{|c|c|c|c|c|}
\hline System & $\begin{array}{c}\text { LHe I } \\
(\mathrm{kW} \odot 4.4 \mathrm{~K})\end{array}$ & $\begin{array}{c}\text { LHe I } \\
\text { (1iter/hr) }\end{array}$ & $\begin{array}{l}\text { LHe II } \\
(W \circ 1.8 \mathrm{~K})\end{array}$ & $\begin{array}{c}\mathrm{LN}_{2} \\
(\mathrm{~kW} \odot 80 \mathrm{~K})\end{array}$ \\
\hline \multicolumn{5}{|l|}{ Magnets } \\
\hline Central cell (48) & 22.4 & 1,344 & & 468 \\
\hline Axicells & & 108 & 3,700 & 48 \\
\hline Anchor cell pair (2) & 0.48 & 64 & & 61 \\
\hline Transition pair (2) & 0.26 & 42 & & 30 \\
\hline Magnet totals & 23.2 & 1,558 & 3,700 & 608 \\
\hline Cryopanels & 0.82 & & & 12 \\
\hline Miscellaneous & 7.85 & & & 17 \\
\hline$\frac{\text { MARS total cryogenic }}{\text { requirements }}$ & 30.8 & 1,600 & 3,700 & 638 \\
\hline
\end{tabular}




\subsubsection{Central Cell Magnets}

The central-cell magnet requirements are comprised of six components listed in Table 3.7-2.

Table 3.7-2. Central cell magnet requirements with LHe cryogen.

\begin{tabular}{|c|c|c|}
\hline Component & $\begin{array}{c}\text { Load } \\
(\mathrm{kW} @ 4.4 \mathrm{~K}) \\
\end{array}$ & Basis \\
\hline Conduction & 210 & Analysis \\
\hline Radiation & 58 & Scaled from MFTF-B \\
\hline Instrumentation & 25 & Scaled from MFTF-B \\
\hline Fluid lines & 12 & Scaled from MFTF-B \\
\hline Splice heating & 12 & Scaled from MFTF-B \\
\hline Neutronic & 150 & Scaled from axicell loads \\
\hline Total & 467 & \\
\hline
\end{tabular}

This results in $22.4 \mathrm{KW}$ of refrigeration at $4.4 \mathrm{~K}$ for the 48 central cell coils. The liquid helium (LHe) requirement for the power leads is estimated at $1344 \mathrm{liter} / \mathrm{hr}$ based on $0.35 \mathrm{liter} / \mathrm{hr}$ per $100 \mathrm{~A}$. The liquid nitrogen heat load $\left(\mathrm{LN}_{2}\right)$ required by the thermal radiation shields is calculated to be $468 \mathrm{kH}$.

\subsubsection{Axicell Magnets}

The axicell magnet requirenents were scaled from central cell loads (since no support/shield designs are complete), except for neutron heating loads, which were obtained from neutronics analyses presented in Section 3.1. The LHe II requirements for each end are given in Table 3.7-3. 
Table 3.7-3. Requirements for end-cell magnets with LHe II cryogen.

\begin{tabular}{lcl}
\hline \multicolumn{1}{c}{ Component } & Load & \\
\hline & (W $1.8 \mathrm{~K})$ & \multicolumn{1}{c}{ Basis } \\
Conduction & 870 & Scaled from central cells \\
Radiation & 111 & Scaled from MFTF-B \\
Instrumentation & 69 & Scaled from MFTF-B \\
Fluid lines & 138 & Scaled from MFTF-B \\
Splice heating & 163 & Scaled from MFTF-B \\
Neutronic & 500 & Analysis \\
\multicolumn{1}{c}{ Total } & 1850 & \\
\hline
\end{tabular}

\subsubsection{Yin-Yang and Transition Magnets}

The yin-yang (anchor cel1) and transition coil cryogenic requirements were obtained from MFTF-B requirements ${ }^{1,2}$ because the MARS anchor cells are similar. These requirements are given in Table 3.7-4.

Table 3.7-4. Yin-yang and transition coil requirements with LHe cryogen.

\begin{tabular}{|c|c|c|c|}
\hline \multicolumn{2}{|c|}{ Anchor cell pair } & \multicolumn{2}{|l|}{ Transition coils } \\
\hline Component & $\begin{array}{c}\text { Load } \\
(W \circ 4.4 K) \\
\end{array}$ & Component & $\begin{array}{l}\text { Load } \\
(W \in 4.4 K) \\
\end{array}$ \\
\hline Conduction & 87 & Conduction & 143 \\
\hline Radiation & 22 & Instrumentation and radiation & 43 \\
\hline Instrumentation & 63 & Fluid lines & 2 \\
\hline Fluid lines & 25 & Splice heating & 28 \\
\hline Splice heating & 42 & Neutronic & 46 \\
\hline Totals & 239 & & 262 \\
\hline
\end{tabular}




\subsubsection{Vacuum System}

Cryogenic requirements for the vacuum cryopanel system (without allowance for neutron heating) are given in Table 3.7-5.

Table 3.7-5. Cryogenic requirements for the vacuum cryopanel system.

\begin{tabular}{|c|c|c|c|}
\hline \multicolumn{2}{|l|}{ LHe } & \multicolumn{2}{|l|}{$\mathrm{LN}_{2}$} \\
\hline Component & 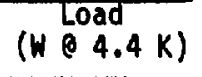 & Component & (W $4.4 \mathrm{~K})$ \\
\hline Sensible heat & 34 & Radiation on chevrons & 5.6 \\
\hline Radịation/panels & 32 & Radiation on piping & 1.4 \\
\hline Radiation/piping & 5 & Distribution & 4.3 \\
\hline Distribution lines & 323 & 10\% margin & 1.1 \\
\hline Regenerative operation & 350 & & \\
\hline 10\% margin & 74 & & 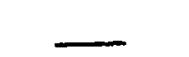 \\
\hline Totals & 818 & & 12.4 \\
\hline
\end{tabular}

\subsubsection{Additional Systems}

Other subsystems that will require cryogenic refrigeration include: tritium processing, main beamlines, and additional distribution. However, we have not assessed the requirements and loads for these subsystems to date. We have estimated these loads based on a use fraction rate obtained from the TDF cryoplant study. The miscellaneous loads were found to account for approximately 29 and $2.8 \%$ of major $L$ He and $L_{2}$ heat loads, respectively. Thus, the estimated loads for LHe and $L_{2}$ systems are $7850 \mathrm{~W}$ at $4.4 \mathrm{~K}$ and $17.5 \mathrm{KW}$ at $80 \mathrm{~K}$, respectively.

\subsubsection{Cost Estimate}

We estimated the capital and operating costs for a large cryoplant and have established incremental capital and oprating costs in $\$ / W$ or $\$ / k W-h r$. 
For an LHe system, the incremental capital cost ia $\$ 1330 / \mathrm{W}$ of heat removed at $4.4 \mathrm{~K}$, and the incremental operating cost is $\$ 37.50 / \mathrm{kW}-\mathrm{hr}$ of heat removed per hour. For an $\mathrm{LN}_{2}$ system, the incremental capital cost is $\$ 15.90 / \mathrm{W}$ of heat removed at $80 \mathrm{~K}$, and the incremental operating cost is $\$ 0.75 / \mathrm{kW}-\mathrm{hr}$ of heat removed per hour.

\section{REFERENCES FOR SECTION 3.7}

1. R. F. O'Neill and D. H. Riemer, Thermodynamic Analys is of the Magnet System for Mirror Fusion Test Facility (MFTF-B), CASD-LLL-78-002, pp. 4-11.

2. J. F. Bartkowski, Thermal Design Analys is of Supports and Heat Load Analysis of MFTF-B Supports for Solenoid, A-Cell, Transition and Yin-Yang Magnets, TM-696-82-186, p. 28 (May 18, 1982). 


\section{POWER SYSTEMS}

\subsection{WASTE MANAGEMENT CONSIDERATIONS}

To date we have devoted only a modest effort to the generic issue of waste management in fustion power systems. Now that we have developed several blanket concepts, we wili be sncentrating on more spectfic problems of waste management. The lithium-lead and high-temperature blankets use nonflammable coolants and have low tritfum inventories, which greatly reduces the. severity of possible accidents and mitigates handing of tritium in waste disposal. During Fy 1982 we also assessed the problem of radioactive waste generated by neutron activition of structural materials. We found that by carefully selecting structural materials we will be able to make reactors with radioactive components that can be disposed of by near-surface burlal. This would greatly simplify waste processing and handling, including disposal both during life of plant and after decomissioning. To realize near-surface disposal, it may be necessary to modify current structural steels to meet both the waste performance goal and other performance requirements.

In our assessment we ascumed that fusion waste will be disposed of under the same institutional conditions required for fission waste disposal. Currently, no regulations are prepared specifically for fusion waste. However, future regulations can be expected to depend on waste form, isotoplc composition, and levels of activity, without rejard to the primary source of the waste (1.e., fission or fusion). Therefore, we examined the problem of assuming that it will regulated by KRC Rule 10CFR61, "Licensing Requirenents for Land Disposal of Radioactive Waste." This regulation assumes that the waste will be under administrative control for $100 \mathrm{yr}$. In this time period, the activities of isotopes with half lives less than $5 \mathrm{yr}$ will have decayed to insignificant levels from any possible initial activity. Therefore, isotopes with half lives less than 5 yr pose ne hazards for long-term waste disposal.

However, isotopes with half lives greater than 5 yr can be hazardous after $100 \mathrm{yr}$, depending on the wastc form, quantity, and half life. The isotopes of direct concern to fustion raste treated specifically in WRC Rule 10CFR61 are tritium, C-14, Mi-59, Ni-63, Co-60, Nb-94 and Tc-99. Several other long-lived iscitopes may be subjected to direct regulation. Table 4.1-1 contains a 11st of long-lived isotopes that may be produced in a fuston reactor. 
Table 4.1-1. Long half-life isotopes (> $5 \mathrm{yr}$ ) potentially important for near-surface disposai of fusion activation wastes.

\begin{tabular}{|c|c|c|c|}
\hline Element & $\begin{array}{l}\text { Natural isotope } \\
\text { mass number } \\
\text { ( } \chi \text { abundance) }\end{array}$ & $\begin{array}{l}\text { Long } \\
\text { half-ifife } \\
\text { isotope }\end{array}$ & $\begin{array}{l}\text { Half-life } \\
(y r)\end{array}$ \\
\hline Beryllium & $9(100)$ & $B e-10$ & $2.7 \times 10^{6}$ \\
\hline Carbon & $\begin{array}{l}12 \\
13\end{array}\left(\begin{array}{l}98.892) \\
1.108)\end{array}\right.$ & - $\quad$-14 & 5730 \\
\hline Aluminum & $27(100)$ & $A 1-26$ & $7.4 \times 10^{5}$ \\
\hline stitcon & $\begin{array}{l}28(92.18) \\
29 \\
30(4.71) \\
3.12)\end{array}$ & $51-32$ & $\sim 700$ \\
\hline Carcium & $\begin{array}{l}40(96.97) \\
42(0.64) \\
43(0.145)\end{array}$ & $\mathrm{Ca}-41$ & $8 \times 10^{4}$ \\
\hline Titantum & $\begin{array}{l}46(7.99) \\
47(7.32) \\
48(73.99) \\
49(5.46) \\
50(5.25)\end{array}$ & $\mathrm{TI}-44$ & 47 \\
\hline Manganese & $55(100)$ & $m-53$ & $2 \times 10^{6}$ \\
\hline Iron & $\begin{array}{ll}54 & (5.84) \\
56 & (91.68) \\
57 & (2.17) \\
58 & (0.31)\end{array}$ & $\mathrm{Fe}-60$ & $3 \times 10^{5}$ \\
\hline Nickel & $\begin{array}{ll}58 & (67.76) \\
60 & (26.16) \\
61 & (1.25) \\
64 & (1.16)\end{array}$ & $\begin{array}{l}N 1-59 \\
N 1-63\end{array}$ & $\begin{array}{c}8 \times 10^{4} \\
92\end{array}$ \\
\hline Strontium & $\begin{array}{l}84(0.56) \\
86(9.86) \\
87(7.02) \\
88(82.56)\end{array}$ & $S r-90$ & 28 \\
\hline Zirconium & 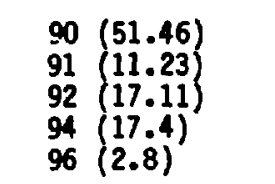 & $2 r-93$ & $1.5 \times 10^{6}$ \\
\hline
\end{tabular}


Table 4.1-1. Continued.

\begin{tabular}{|c|c|c|c|}
\hline Element & $\begin{array}{l}\text { Matural isotope } \\
\text { mass number } \\
\text { ( } \mathrm{x} \text { abundance) }\end{array}$ & $\begin{array}{l}\text { Long } \\
\text { half-life } \\
\text { Isotope }\end{array}$ & $\begin{array}{c}\text { Half-life } \\
(y r)\end{array}$ \\
\hline IHobium & $93(100)$ & $\begin{array}{l}N b-92 \\
N b-93 \text { in } \\
N b-94\end{array}$ & $\begin{array}{l}3.2 \times 10^{7} \\
13.60 \\
2 \times 10^{4}\end{array}$ \\
\hline Molybdenum & $\begin{aligned} 92 & (15.86) \\
94 & (9.12) \\
95 & (15.7 \\
96 & (16.5 \\
97 & (9.45) \\
98 & (23.75) \\
100 & (9.62)\end{aligned}$ & No-93 & 3500 \\
\hline Technicium & None & Tc-97 & $\begin{array}{l}2.6 \times 10^{6} \\
1.5 \times 10^{6} \\
2.12 \times 10^{5}\end{array}$ \\
\hline Palladiun & $\begin{array}{l}102(0.96) \\
104(10.97) \\
105(22.2) \\
106(27.3) \\
108(26.7) \\
110(11.8)\end{array}$ & $P d-107$ & $7 \times 10^{6}$ \\
\hline Silver & $\begin{array}{l}107(51.35 \\
109\end{array}(48.65)$ & Ag-108 & $\stackrel{>5}{(127} \mathrm{yr})$ \\
\hline Cadatium & 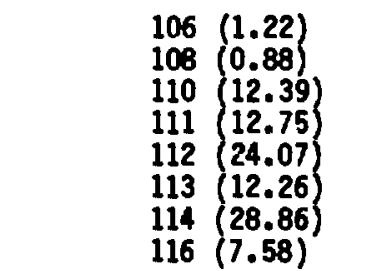 & $\mathrm{Cd}-113 \mathrm{~m}$ & 13.6 \\
\hline
\end{tabular}


Because reactor materials will be composed of a very wide range of elements, an index system, called the Waste Disposal Rating (WOR), serves as a guide to designers in evaluating activated alloys and other materials with respect to their classification for disposal as waste. The WDR assigns an index number to each material, which is given by

$$
\text { WOR }=\sum \frac{A(i)}{L(i)}
$$

where $A(1)$ is the activity in $\mathrm{Cl} / \mathrm{m}^{3}$ of isotope $\mathrm{I}$ and $L(1)$ is the maximum allowable activity of isotope 1 as specificied by whatever regulation is in force. This definition was suggested by the rule in 10CFR61 that governs how allowable concentrations of isotopes in mixtures are determined. Presumably, if WOR > 1, the material cannot be disposed of by near-surface burial.

The WDR gives designers quantitative guides as to the actions needed to meet the goal of near-surface disposal. For example, if materials with WDR > 1 are used to enhance performance, then the procedures necessary to process this waste to put it in a form suitable for near-surface burial must be examined. The magnitude of the WDR directly gives the volume to which a unit volume of waste must be diluted with inert material to make WDR $<1$. The individual terms in the WOR can guide the evaluation of possible chemical processing to partition the waste into batches so that most of the bulk volume could be disposed directly by near-surface burial and only a small volume would have to be disposed of by other methods (e.g., deep geologic burial) or by dilution for near-surface burial.

If new materfals with WDR $<1$ are selected to produce a reactor that inherently meets the performance goal of near-surface disposal for long-term waste, then the properties of the new materials that affect rellability, lifetime of components, etc., must be critically examined. These new materials might be developed to meet WDR $<1$ by elemental substitutions in existing alloys to eliminate the elements causing long-lived isotopes (e.g. substituting vanadium for molybdenum). This would undoubtedly shift significant burdens to developers of materials and system designers to make trade-offs related to the mechanical, chemical, and magnetic properties of the new materials. In particular, the system designer should make certain that optimizing long-term waste disposal does not create severe short-term 
activation problems in the case of accidents or cause a significant increase in maintenance difficulty.

Another approach to producing a reactor that inherently meets the performance goal for long-term waste would be to use existing materials modified by tafloring the isotopic composition but retaining the original elemental composition. Presumably this would not change the knotm mechanical, chemical, and magnetic properties of the alloys. The absence of natural isotopes that result in long-1ived 1sotopes through activation/dacay chains would ensure KDR < 1. For example, if the molybdenum content of an alloy was only Mo-96, the long-1 lyed activation products (Mo-93, Mb-92, Mb-94, Tc-97, Tc-98, and TC-99) would be generated in such quantities that the waste disposal rating for molybdenum alone would be small enough to allow near-surface disposal. This approach would require little change in present systems designs based on existing materials. However, research would be required to develop isotopic tafloring processes for new or modifled materials.

From a systems design view, it must be remembered that during reactor operation and for a time after shutdown, the radiation hazards are mainly produced by Fe-55 $\left(t_{1 / 2}=2.7 \mathrm{yr}\right), \mathrm{m}-54\left(\mathrm{t}_{1 / 2}=303\right.$ days $), \mathrm{m}_{\mathrm{r}}-56\left(\mathrm{t}_{1 / 2}=\right.$ $2.8 \mathrm{hrs}), \mathrm{Co}_{-60}\left(\mathrm{t}_{1 / 2}=5.3 \mathrm{yr}\right)$, and a few other short half-lived isotopes in iron alloys. Therefore, the in-plant radiation hazards governing waste handling and processing procedures for a fusion reactor using tron alloys will be essentially the same regardless of the stracegy for long-term waste performance of the iron alloys. However, the waste performance strategy and the resulting WDR will have significant effects on long-term disposal. For example, if WOR $<1$, one could consider using designs with throw-away components that might be safely stored for the life of the plant and then permanently disposed of by decomissioning rules. These decomissioning rules may be considerably different from ordinary waste disposal rules if MDR $<1$.

We have prepared two examples of $k$ applied to conceptual first-wall and first-shield region designs. 1 These designs are for WITAMIR ${ }^{2}$ and the MARS high temperature blaket discussed in Section 4.3. The relatively more compact structure of the MARS central cell uses a thicker breeder section with less reflector and shield. Designs for MITAMIR and MARS also differ in the postulated fast neutron loading on the first wall. WITAMIR is designed to 
have a neutron loading of $2.4 \mathrm{~m} / \mathrm{m}^{2}$ wth a planned first willolanket replacement time of $5 \mathrm{yr}$. The comparable numbers for MaRS are $4.8 \mathrm{~mm} / \mathrm{m}^{2}$ and $3 \mathrm{yr}$. For this study, we assumed that in both cases the shield would last for $30 \mathrm{yr}$. Based on neutronic calculations, these wall loadings correspond to the neutron fluxes presented in Table 4.1-2. The liniting activities used in the examples are shown in Table 4.1-3.

Table 4.1-2. Meutron flux assumptions.

\begin{tabular}{|c|c|c|}
\hline \multirow[b]{2}{*}{ Design } & \multicolumn{2}{|c|}{$\begin{array}{l}\text { Neutfon F }\left\{u x^{a}\right. \\
\left(10^{18} n / m^{2}-5\right)\end{array}$} \\
\hline & First wall & Shield \\
\hline \multicolumn{3}{|l|}{ WITAMIR } \\
\hline 0 to 1 HeV & 26.6 & 1.9 \\
\hline 1 to $15 \mathrm{MeV}$ & 3.5 & 0.005 \\
\hline \multicolumn{3}{|c|}{ MARS high temperature } \\
\hline 0 to $1 \mathrm{MeV}$ & 30.9 & 0.2 \\
\hline 1 to $15 \mathrm{MeV}$ & 9.9 & 0.0023 \\
\hline
\end{tabular}

\footnotetext{
a Neutron fluxes are taken in the first calculational zone of the first wall and in the first calculatioilal zone of the shield.
} 
Table 4.1-3. Concentrations of radioactive wastes allowable for near-surface disposal.

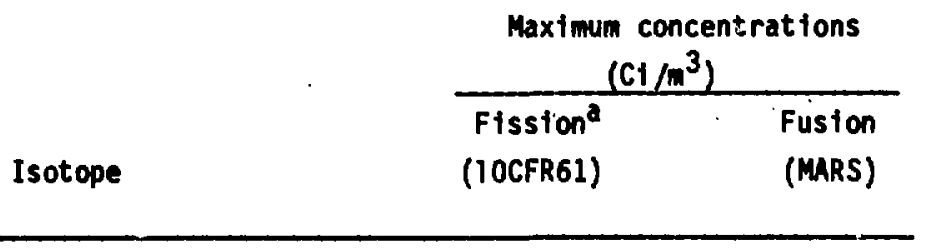

$\begin{array}{lll}\text { Any with half life }<5 \text { yr } & \text { TMSA } & \text { TMSA } \\ H-3 & \text { TMSA } & \text { TMSA } \\ C-14 & 0.8 & 8 \\ \text { Mr-53C } & -- & 22 \\ \text { Ni-59 } & 2.2 & 22 \\ \text { Co-60 } & \text { TMSA } & \text { TMSA } \\ \text { Ni-63 } & 70 & 700 \\ \text { Zr-93C } & -- & 0.02 \\ \text { Mo-935 } & -- & 22 \\ \text { No-94 } & 0.002 & 0.02 \\ \text { Sr-90 } & 700 & 7000 \\ \text { Tc-99 } & 0.3 & 3\end{array}$

abased on Table 1 , Federal Register, Voi. 46, No. 142, p. 38097. For 1sotopes contained in metals, metal alloys, or permanently fixed on metals as contanination, the values given in this column may be increased by a factor of 10 .

bTheoretical maximum specific activity (TMSA): the activity that would result If all the elements were converted to this radioactive daughter.

Cirot explicitly covered in the proposed 10CRF61. The limit given is an estimate based on the closest analogy in terms of isotope haif life, type of radiation, and decay energy to an isotope explicitly covered in locrf61. 
Table 4.1-4 contains the natural elements comonly found in steels that generate long-lifed radfoactive daughters. Although we have exanined all of these radioactive daughters only a few of them appear to occur at levels which significantly affect long-term waste disposal. These are $\mathrm{Ni}-59, \mathrm{Ni}-63, \mathrm{Nb}-94$, Mo-93, Tc-99, and $\mathrm{m}-53$.

Table 4.1-5 gives the composition of several steels that may be candfdates for structural materials in fusion reactors. The weight percentages of the five elements that affect WOR are specified. These few alloying elements produce radioactive daughters that exceed the 1 inits proposed in IOCFR61 for class $C$ near-surface disposal. The other elements in these alloys have daughters that contribute to high radioactivity imnediately after shutdown of a fusion reactor; however, the half lives are short enough for the radioactivity to decay after 20 to $30 \mathrm{yr}$ to levels not significant for longterm waste disposal.

Table 4.1-4. Long-lived radioactive daughters from natural elements in steel al loys.

\begin{tabular}{|c|c|c|}
\hline Parent element & Daughter isotope & $\begin{array}{c}\text { Daughter half-life } \\
(y r)\end{array}$ \\
\hline $\begin{array}{l}\mathrm{C} \\
\mathrm{N} \\
\mathrm{Al} \\
\mathrm{Cr} \\
\mathrm{Mn} \\
\mathrm{Fe} \\
\mathrm{Co} \\
\mathrm{Ni} \\
\mathrm{Cu} \\
\mathrm{Zr} \\
\mathrm{Nb} \\
\mathrm{Mo}\end{array}$ & $\begin{array}{l}\mathrm{C}-14 \\
\mathrm{C}-14 \\
\mathrm{Al}-26 \\
\mathrm{Mn}-53 \\
\mathrm{Mn}-53 \\
\mathrm{Mn}-53 \\
\mathrm{Ni}-59 \\
\mathrm{Ni}-59 \\
\mathrm{Ni}-63 \\
\mathrm{Ni}-63 \\
\mathrm{Sr}-90 \\
\mathrm{Zr}-93 \\
\mathrm{Zr}-93 \\
\mathrm{Nb}-94 \\
\mathrm{Nb}-94 \\
\mathrm{Mo}-93 \\
\mathrm{Tc}-99\end{array}$ & $\begin{array}{r}5,700 \\
5,700 \\
740,000 \\
1,900,000 \\
1,900,000 \\
1,900,000 \\
80,000 \\
80,000 \\
92 \\
92 \\
28 \\
1,500,000 \\
1,500,000 \\
20,000 \\
20,000 \\
3,600 \\
210,000\end{array}$ \\
\hline
\end{tabular}


Table 4.1-5. Crittcal natural elements in various steels.

$$
\text { Alloya }
$$

We1ght Percent

Fe In N1 Nb Ho Otherb

\begin{tabular}{lllllll} 
SS316 & 64.44 & 1.43 & 14 & -- & 2.8 & 17.33 \\
PCA & 64.68 & 1.8 & 16 & 0.03 & 2.0 & 15.49 \\
HT-9 & 85.1 & 0.55 & 0.5 & -- & 1.0 & 12.85 \\
$2.25-1$ & 94.58 & 0.45 & -- & -- & 1.0 & 3.97 \\
Tenelon & 68.0 & 14.5 & -- & -- & -- & 17.5 \\
Nonagne & 79.35 & 15.0 & 2.2 & -- & -- & 3.45 \\
Nir-1 & 76.92 & 20.0 & -- & -- & -- & 3.08 \\
$25-5-1$ & 66.22 & 26.0 & 1.2 & 0.07 & -- & 6.51 \\
$32-7$ & 60.24 & 31.6 & 0.23 & -- &.- & 7.93 \\
JUS289-N & 72.45 & 20.0 & -- & -- & -- & 7.55 \\
JUS289-Y & 72.15 & 20.0 & -- & -- & -- & 7.85 \\
\hline
\end{tabular}

a Nomagne is made by Kobe Steel, M-1 by Mippon Korean, 25-5-1 by Nippon Steel Ltd., and 32-7, JUS289- $M$, and JUS289-V by Japan Steel Horks. Tenelon is a steel developed by the U.S. Steel Corp. We have no information on these steels regarding radiation danage in high-flux neutron fields.

bot critical for iong-term waste disposa?. 
Tables 4.1-6 through 4.1-9 show the calculated specific waste disposal ratings for the steels in Table 4.1-5. Both the WITAMIR and MARS first walls are nominally HT-9; the shields are austenitic manganese steels. These tables show the effect of material replacement on WDR. We have not included changes in the design to accomodate these materials.

of the steels examined, only Tenelon, MM-1, JUS289-N, and JUS289-V can be used in the first walls and shields of HITAMIR or MaRS with the resulting induced radioactivity low enough so that the waste can be disposed of directly by near-surface burial. The $2.25 \mathrm{Cr} / 1 \mathrm{mo}$ steel can be dfsposed of directly when used in the shields but not in the first walls. The elements that cause problems in the vartous steels are nickel, molybdenum, and ntoblum.

Thus, the above results suggest that only two major natural elements-nickel and molybdenum--prevent near-surface disposal of the activated steels according to the HRC's proposed 10CFR61. Cobalt, copper, niobium, and zirconium could also prevent near-surface disposal, but they can generally be avoided completely in the structural alloys. The natural isotopes of nickel and molybdenum that are particularly troublesome are $\mathrm{Ni}-58, \mathrm{Ni}-60, \mathrm{Ni}-62, \mathrm{Ni}-$ 64, M0-92, Mo-94, Mo-95, Mo-98 and Mo-100.

There are three alternatives for selecting structural materials that produce radioactive wastes that can be disposed of solely by near-surface burial. Two of these alternatives are elemental tafloring (el/minating offending elements in the steels) and isotopic talloring (reducing the relative amounts of the specific natural isotopes that cause unacceptable activities for nearsurface burial). The third alternative is to use nonferrous alloys with short-lived activation products that are acceptable for near-surface burial. We have not considered this last alternative; however, the methods of analysis could be extended to nonferrous materials without modification to determine their waste disposal ratings. 
Table 4.1-6. WITAMIR first wall (parent elements in parentheses).

\begin{tabular}{|c|c|c|c|c|c|c|c|}
\hline \multirow[b]{2}{*}{ Alloy } & \multicolumn{6}{|c|}{ MDR } & \multirow{2}{*}{$\begin{array}{l}\text { Alloy } \\
\text { total }\end{array}$} \\
\hline & $\mathrm{Ni}-59$ & $\mathrm{Ni}-63$ & $\mathrm{Nb}-94$ & Mo-93 & Tc-99 & $M n-53$ & \\
\hline S\$316 & $26(N i)$ & $69(\mathrm{Ni})$ & $0.5\left(M_{0}\right)$ & $16(\mathrm{Mo})$ & $2.7(\mathrm{Ho})$ & $0.21(\mathrm{Fe})$ & 114 \\
\hline PCA & 29 (N1) & $77(\mathrm{Ni})$ & $1800(\mathrm{Nb})$ & $12\left(\mathrm{Mo}_{0}\right)$ & $2.0\left(M_{0}\right)$ & $0.21(\mathrm{Fe})$ & 1920 \\
\hline HT-9 & $0.91(\mathrm{Ni})$ & $2.4(N t)$ & $0.15(M 0)$ & $5.9(\mathrm{Mo})$ & $1.0\left(\mathrm{Mo}_{0}\right)$ & $0.27(\mathrm{Fe})$ & 11 \\
\hline $2.25-1$ & -. & -- & 0.15 (Mo) & $5.9\left(\mathrm{Mo}_{0}\right)$ & $1.0\left(M_{0}\right)$ & 0.31 (Fe) & 7.4 \\
\hline Tene ion & -- & -- & - & -- & -- & $0.23(\mathrm{Fe} / \mathrm{mm})$ & 0.23 \\
\hline Momagne & $3.6(\mathrm{NI})$ & $11(\mathrm{Ni})$ & -- & -- & -- & $0.26(\mathrm{Fe} / \mathrm{Mn})$ & 15 \\
\hline MM-1 & -- & -- & -- & -- & -- & $0.26(\mathrm{Fe} / \mathrm{Mn})$ & 0.26 \\
\hline $25-5-1$ & $2\left(\mathrm{Ni}_{i}\right)$ & $5.7(\mathrm{N1})$ & $4200(N b)$ & -- & -- & $0.23(\mathrm{Fe} / \mathrm{Mn})$ & 4210 \\
\hline $32-7$ & 0.39 (NA) & $1.1(N 1)$ & -- & -- & - & $0.21(\mathrm{Fe} / \mathrm{Mn})$ & 1.7 \\
\hline JUS289-N & -- & -- & -- & $\cdots$ & -- & $0.24(\mathrm{Fe} / \mathrm{Mn})$ & 0.24 \\
\hline JUS289-V & -- & -- & - & -- & -- & $0.24(\mathrm{Fe} / \mathrm{Mn})$ & 0.24 \\
\hline
\end{tabular}


Table 4.1-7. MARS first wall MOR (parent elements in parentheses).

\begin{tabular}{|c|c|c|c|c|c|c|c|}
\hline \multirow[b]{2}{*}{ Alloy } & \multicolumn{6}{|c|}{ MDR } & \multirow{2}{*}{$\begin{array}{l}\text { Alloy } \\
\text { total }\end{array}$} \\
\hline & Ni-59 & $N 1-63$ & $M b-94$ & Ha-93 & TC-99 & $m-53$ & \\
\hline SS316 & $23.0(\mathrm{Ni})$ & $71.0(N i)$ & $1.5\left(M_{0}\right)$ & $26.0(M 0)$ & $3.7(\mathrm{Mo})$ & $0.33(\mathrm{Fe})$ & 130 \\
\hline PCA & $26.0(\mathrm{Ni})$ & $83.0(\mathrm{Ni})$ & $1400(N b)$ & $18.0\left(\mathrm{Mo}_{0}\right)$ & $2.7(\mathrm{Mo})$ & $0.33(\mathrm{Fe})$ & 1500 \\
\hline HT-9 & $0.82(\mathrm{Ni})$ & $2.6(\mathrm{Ni})$ & $0.5\left(M_{0}\right)$ & $9.1(\mathrm{MO})$ & $1.3(\mathrm{Mo})$ & $0.42(\mathrm{Fe})$ & 15 \\
\hline $2.25-1$ & -- & -- & $0.5\left(M_{0}\right)$ & $9.1\left(M_{0}\right)$ & $1.3(\mathrm{Mo})$ & $0.45(\mathrm{Fe})$ & 11 \\
\hline Tenel on & -- & -- & -- & -- & - & $0.37(\mathrm{Fe} / \mathrm{Mn})$ & 0.37 \\
\hline Momagne & $3.6(\mathrm{Ni})$ & $11.0(\mathrm{Ni})$ & - & -- & - & $0.42(\mathrm{Fe} / \mathrm{Mn})$ & 15 \\
\hline m-1 & -- & - & -- & - & - & $0.42(\mathrm{Fe} / \mathrm{Mm})$ & 0.42 \\
\hline $25-5-1$ & $2.0(\mathrm{Ni})$ & $6.1(N i)$ & $3200(N b)$ & $9.1 E-5$ & - & $0.37(\mathrm{Fe} / \mathrm{Mn})$ & 3200 \\
\hline 32.7 & $0.36(N i)$ & $1.2(\mathrm{Ni})$ & - & -- & - & $0.35(\mathrm{Fe} / \mathrm{Mn})$ & 1.9 \\
\hline JUS289-N & -- & - & - & -- & - & $0.43(\mathrm{Fe} / \mathrm{Mn})$ & 0.43 \\
\hline JUS289-V & -- & -- & - & -- & -- & $0.43(\mathrm{Fe} / \mathrm{Mm})$ & 0.43 \\
\hline
\end{tabular}


Table 4.1-8. WITMIR shield WOR (parent elements in parentheses).

\begin{tabular}{|c|c|c|c|c|c|c|c|}
\hline \multirow[b]{2}{*}{ Alloy } & \multicolumn{6}{|c|}{ MOR } & \multirow{2}{*}{$\begin{array}{l}\text { Alloy } \\
\text { total }\end{array}$} \\
\hline & $M i-59$ & $N i-63$ & $\mathrm{Nb}-94$ & Mo-93 & TC -99 & $M n-53$ & \\
\hline SS316 & $18.0(N i)$ & $100.0(\mathrm{Hi})$ & $5 E-5\left(M_{0}\right)$ & 4.6E-2(Mo) & $1 E-2\left(H_{0}\right)$ & $2 \mathrm{E}-3(\mathrm{Fe})$ & 1.8 \\
\hline PCA & $21.0(\mathrm{Ni})$ & $114.0(\mathrm{Ni})$ & $1200(\mathrm{Nb})$ & $3.2 E-2\left(\mathrm{Ho}_{0}\right)$ & $6.7 \mathrm{E}-3\left(\mathrm{Mo}_{0}\right)$ & $2 E-3(F e)$ & 1300 \\
\hline HT-9 & $0.64(\mathrm{Ni})$ & $3.6(\mathrm{Ni})$ & $2 E-5(M 0)$ & $1.8 E-2\left(M_{0}\right)$ & $3.3 \mathrm{E}-3\left(M_{0}\right)$ & $3 \mathrm{E}-3(\mathrm{Fe})$ & 4.7 \\
\hline $2.25-1$ & -- & - & $2 E-5\left(\mathrm{Mo}_{0}\right)$ & $1.8 E-2\left(M_{0}\right)$ & $3.3 E-3(\% 0)$ & $3 E-3(\mathrm{Fe})$ & $\sim 0$ \\
\hline Tenelon & -- & -- & -- & - & $-\infty$ & $2 \mathrm{E}-3(\mathrm{Fe})$ & $\sim 0$ \\
\hline Momagne & $2.7(\mathrm{Ni})$ & $14.0(\mathrm{Ni})$ & - & -- & -- & $3 E-3(F e)$ & 17 \\
\hline$m-1$ & -- & -- & -- & -- & -- & $2 \mathrm{E}-3(\mathrm{Fe})$ & $\sim 0$ \\
\hline $25-5-1$ & 1.5 (Ni) & $8.6(\mathrm{Ki})$ & $2800(\mathrm{HB})$ & - & - & $2 \mathrm{E}-3(\mathrm{Fe})$ & 2800 \\
\hline $32-7$ & $0.27(\mathrm{Ni})$ & 1.7 (Ni) & -- & -- & - & $2 E-3(F e)$ & 2 \\
\hline JUS289-N & -- & - & -- & -- & - & $2 E-3(\mathrm{Fe})$ & $\sim 0$ \\
\hline JUS289-Y & -- & -- & -- & -- & - & $2 \mathrm{E}-3(\mathrm{Fe})$ & $\sim 0$ \\
\hline
\end{tabular}


Table 4.1-9. MARS shield WDR (parent elements in parentheses).

\begin{tabular}{|c|c|c|c|c|c|c|c|}
\hline \multirow[b]{2}{*}{ Alloy } & \multicolumn{6}{|c|}{ WDR } & \multirow{2}{*}{$\begin{array}{l}\text { Allo: } \\
\text { total }\end{array}$} \\
\hline & $N i-59$ & $\mathrm{Ni}-63$ & $N b-94$ & Mo-93 & Tc-99 & $M n-53$ & \\
\hline SS316 & $6.36(\mathrm{Ni})$ & 26.0 (Nf) & $1 E-4(M 0)$ & $1.4 \mathrm{E}-2(\mathrm{Mo})$ & $2 E-3\left(M_{0}\right)$ & $7.3 \mathrm{E}-\mathrm{E}(\mathrm{Fe})$ & 32 \\
\hline $\mathrm{PCA}$ & $7.3(\mathrm{Ni})$ & 29.0 (Ni) & $300(\mathrm{Nb})$ & $9.1 E-3\left(M_{0}\right)$ & $1.32-3\left(M_{0}\right)$ & $7.3 \mathrm{E}-5(\mathrm{Fe})$ & $34 c$ \\
\hline HT-9 & $0.23(\mathrm{Ni})$ & $0.91(\mathrm{Ni})$ & $3 E-5\left(M_{0}\right)$ & $4.6 \mathrm{E}-3(\mathrm{Mo})$ & $6.7 E-4(m o)$ & $9.6 \mathrm{E}-5(\mathrm{Fe})$ & 1.1 \\
\hline $2.25-1$ & -- & -- & $3 E-5\left(M_{0}\right)$ & $4.6 \mathrm{E}-3$ (Mo) & $6.7 \mathrm{E}-4(\mathrm{Mo})$ & $7.3 \mathrm{E}-5(\mathrm{Fe})$ & $\sim 0$ \\
\hline Tenelon & -- & - & - & -- & -- & 8.6E-5(Fe) & $\sim 0$ \\
\hline Momagne & 0.91 (Ni) & 4.0 (Ni) & - & - & - & $8.6 \mathrm{E}-5(\mathrm{Fe})$ & 5 \\
\hline MM-1 & -- & -- & -- & -- & -- & $7.3 \mathrm{E}-5(\mathrm{Fe})$ & $\sim 0$ \\
\hline $25-5-1$ & 0.46 (Ni) & $2.1(N i)$ & $650(\mathrm{Nb})$ & $\cdots$ & -- & $7.3 \mathrm{E}-5(\mathrm{Fe})$ & 652 \\
\hline $32-7$ & 9.1E-2(NI) & $0.43(\mathrm{Ni})$ & -- & -- & -- & $6.3 \mathrm{E}-5(\mathrm{Fe})$ & 0.5 \\
\hline JUS289-N & -- & - & -- & -- & -- & 8.2E-5(Fe) & $\sim 0$ \\
\hline JUS289-V & - & -- & -- & -- & - & 8.2E-5(Fe) & $\sim 0$ \\
\hline
\end{tabular}




\section{REFERENCES FOR SECTION 4.1}

1. R. C. Maninger and D. W. Dorn, Issues in Radioactive Waste Management for Fusion Power, Lawrence Livermore Mational Laboratory, Livermore, CA, UCRL-87786, in Proc. of IEEE 1982 Nuclear Sctence Symposjum, Nashington, D.C. (October 20-22, 1982).

2. 8. Badger, et.al., MITAMIR-I, A University of Wisconsin Tandem Mirror :eactor Design, University of Wisconsin Rept. IMFDM-400, (1980). 


\subsection{LITHIUM-LEAD BLANKET}

\subsubsection{Blanket Design \\ 4.2.1.1 Design Sumary}

objectives. The main objective of the lithium-lead blanket is to produce electricity in the most cost effective manner. (This design differs from the high temperature blanket described in Section 4.3, which has a dual application to provide high temperature heat consistent with synthetic fuel production or cost effective electricity.) We established the following design criteria at the start of this study to gutde our work.

Capital costs. One of our primary objectives was to minimize cost of the product. We found that we could do this best by maximizing the blanket multiplication factor $M$, while at the same time achieving an adequate tritium breeding ratio (e.g., > 1.1). Costs are also minimized by having a high thermal conversion efficiency $\eta_{\text {. }}$ In fact, the final figure of merit $\left(\$ / \mathrm{kW}_{e}\right)$ strongly depends on the product of $M_{\eta_{0}}$ which maximized.

Ease of Fabrication. Many of the past designs for fusion power reactors required such precise manufacturing tolerances and so many complicated welds that the credibility of the blanket design could be legitimately questioned. Our objective in the MARS lithium-lead blanket design was to work closely with manufacturing experts to ensure that the blanket could be fabricated in present facilities with current, well-understood techniques. The present design satisfies these requirements.

Maintenance. The MARS lithium-lead blanket design should take maxinum advantage of the linear geometry of the mirror. To that end, we determined that the following criteria should be met:

1. Small, light blanket modules.

2. Minfmum number of coolant connections.

3. Maximum use of remote decoupling of coolant connections.

4. No vacuum seals between modules to be replaced.

5. No movement of superconducting colls to remove damaged modules.

6. "Hands on" maintenance at the bulk of the shield within 2 days.

The present design incorporates all of the above features. 
Radianctive Waste. Our objecicive was to reduce both the level of long term activity and the mass of radioactive waste to be handled. Because MARS has such a high neutron wall loading (hence, short-lived blanket components), we neded to choose aterial that is highly resistant to radiation danage and also contains the fewest possible elements that yield long-lived isotopes. We feel that HT-9 satisfies this requirement.

It is epparent that we could not meet all of the objectives simultaneous1y, but we have made considerable progress in this area and expect even more progress in FY 1983.

Eeneral Features of MRS Lithium-Lead Blanket. Figure 4.2-1 shows a vertical cross section of the central cell containing the $\mathrm{LI}_{17} \mathrm{~Pb}_{83}$ blanket moduies; Fig. 4.2-2 shows the horizontal cross section. Table 4.2-1 lists the main features of the blanket design.

The basic design of the bianket is quite simple. The $\mathrm{Li}_{17} \mathrm{~Pb}_{83}$ alloy (LiPb) is fed at $350^{\circ} \mathrm{C}$ to the HT-9 blanket module through a large coolant header at the top. From there, it flows through (in parallel) the rectangular HT-9 bean structure and the 10-Cin-diameter circular HT-9 tube section. The tubes in the front ensure uniform flow in the regions where the neutron heating is the highest, and the rectangular sections are better sulted to attain a low void fraction to incraase $M$. At $500^{\circ} \mathrm{C}$, the LiPb exits through a large header at the bottom of the blanket and finally through a large-diameter coolant pipe to a doublewalled heat exchanger. The large pipes are necessary to reduce the MOD pumping power losses.

The central-cell neutron-produced power is $3892 \mathrm{MH}_{\mathrm{th}}$. This energy is deposited in a 38-cm-thick blanket module and a 43-cm-thick reflector. It is important to note that the blanket modules are separated from the reflector region by a 2-cin gap so that they can be rewoved without interference from the reflector and shield region. The latter two water-cooled regions are made from water-cooled austenitic steel because of the lower temperature and because the neutron damge rate is lower in those areas.

The tritium breeding ratio is 1.13 from the LiPb which is enriched to $90 \%$ L1-6. An important feature of the LIPb system is its low tritium solubility, only $5 \mathrm{~g}$ in the entire blanket and heat transport system. There is actually only $1 \mathrm{~g}$ of tritium in the blanket modules at any one time, and the rest is in the heat transport system. 


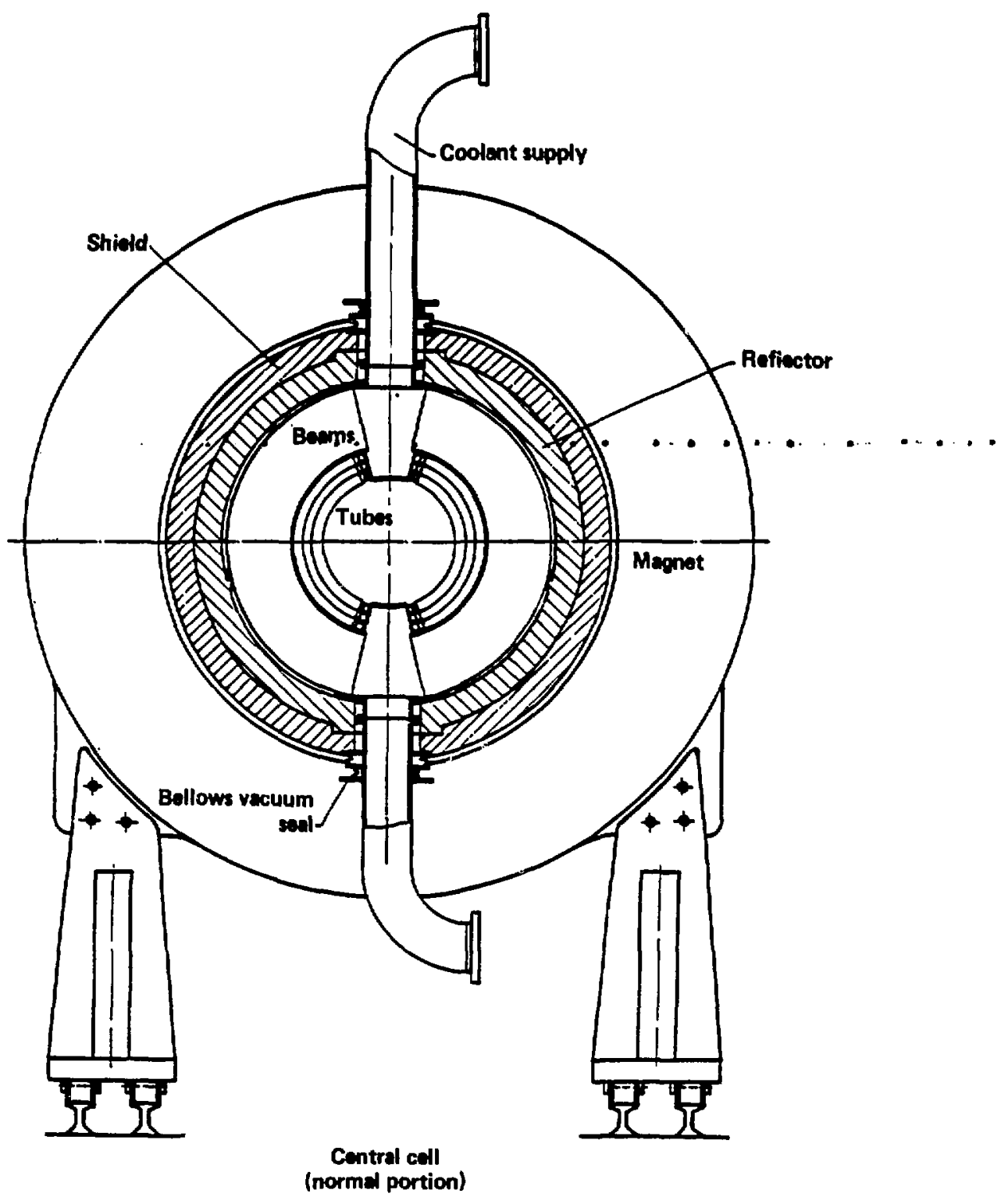

Fig. 4.2-1. Vertical cross section of the central cell for the LiPb blanket. 4- 18 

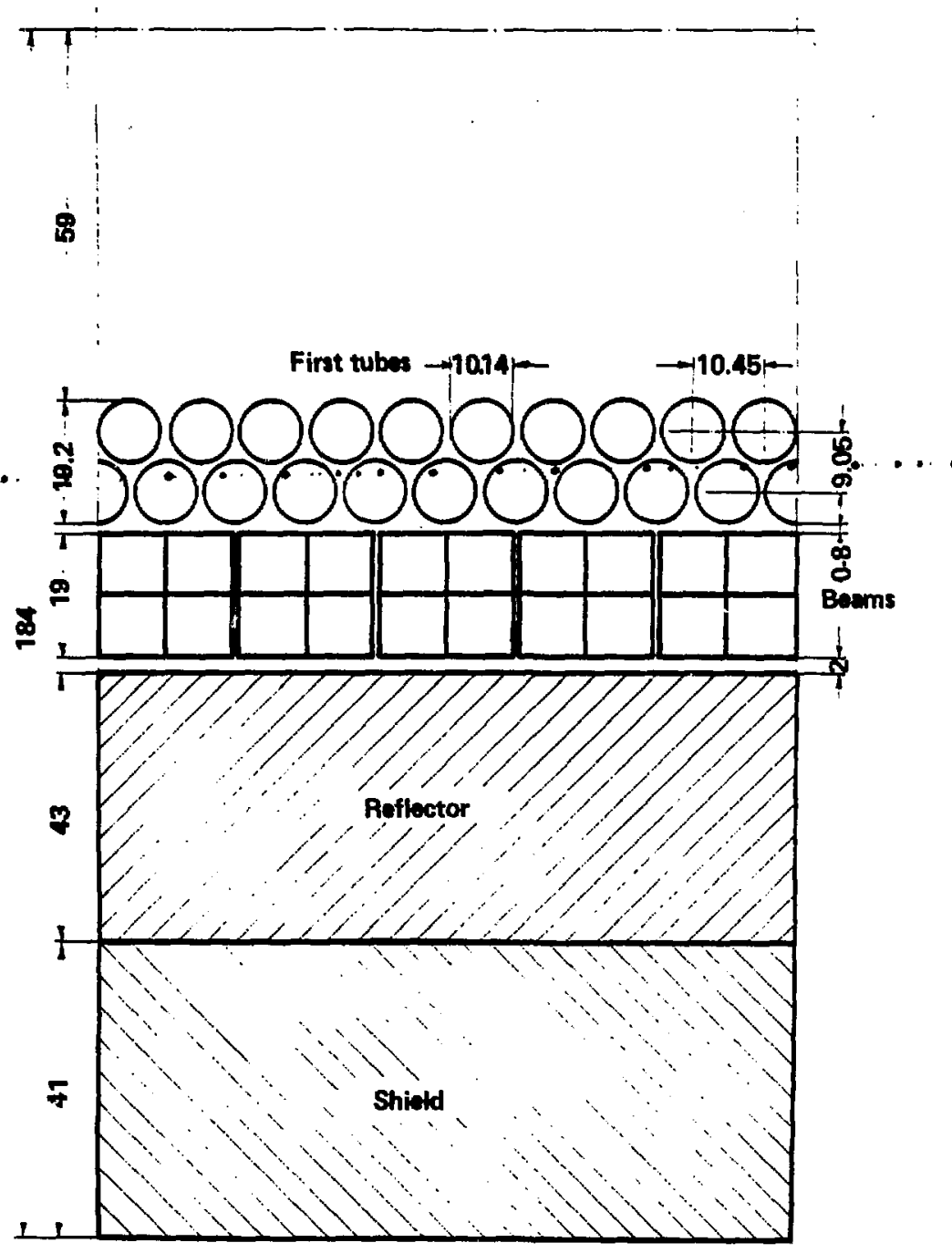

Fig. 4.2-2. Horizontal cross section of the LiPb blanket. 
Table 4.2-1. Major LiPb blanket paraneters.

$\begin{array}{ll}\text { Central cell power }\left(\mathrm{m}_{\mathrm{th}}\right) & 3892 \\ \text { Structural material } & \mathrm{HT}-9 \\ \text { Blanket coolant and breeder } & \mathrm{Li}_{17^{\mathrm{Pb}} 83} \\ \text { First wall radius (cm) } & 59 \\ \text { Neutron wall loading (mw/m }{ }^{2} \text { ) } & 5 \\ \text { Blanket/reflector multiplication (M) } & 1.39 \\ & \\ \text { Tritium breeding ratio } & 1.13 \\ \text { Blanket } \mathrm{T}_{2} \text { inventory (kg) } & 0.005 \\ \text { Net conversion efficiency (x) } & 38.6\end{array}$

Finally, the net conversion efficiency, including the pumping power for the LiPb, is $38.6 \%$. This is possible because of the high outlet temperature $\left(500^{\circ} \mathrm{C}\right)$ and the use of a double-walled heat exchanger to eliminate an intermediate loop.

\subsubsection{Structural Design}

The LiPb blanket consists of two distinct zones:

1. The front zone, which is composed of two rows of closely packed tubes.

2. The back zone, which consists of a single row of hollow rectangular beans that provide the structural support for the blanket.

Figure 4.2-1 shows that both zones are attached to top and bottom manifolds. The plasma is surrounded by two rows of tubes that are bent semicircularly. The end of each tube is attached to a smaller dianeter tube by means of a conical transition section. A small diameter tube is then attached to the manifold. The tubes are arranged so that the structural support in the back is concealed from the direct line of sight of plasma neutrons. The 
rectangular beans behtnd the tube banks are $19 \mathrm{~cm}$ deep. Each beam has four square passages. Table $4.2-2$ contains the pertinent mechanical parameters of the blanket.

Each blanket module has one coolant inlet pipe header at the top and one outlet pipe header at the bottom. The molten breeding materlal, $\mathrm{Li}_{17} \mathrm{~Pb}_{83}$, comes in through the top header, atstributes axially along the top manifold, flows through tubes and bearis perpendicularly to the magnetic fleld lines, converges in the bottom munifold, and finally exits through the bottom pipe header.

The cylindrical blanket modules are enclosed in a 43-cm-thick reflector covered by a 41-cm-thick shield. The total length of the central cell, which contains 84 blanket modules of identical axial width, is $151.68 \mathrm{~m}$. The blanket module is supported on ralis attached to the reflector structure. A 2-cm-thick gap is located between the outer surface of the blanket and the inner surface of the reflector to alleviate the thermal stress problems. The vacuun boundary is defined as the Inner surface of the reflector. Bellows and 0-rings guarantee the vacuum for the penetrations in the shield and reflector, which are required for coolant supply pipes.

The attractive features of this design are:

1. Both the blanket module configuration and fabriction are simple.

2. The coolant channel design for the blanket modui: and the connected pipe headers constitute an excellent drainable system.

3. Each blanket module has only two coolant connections.

4. The field perturbation is minimized by the uniform distribution of the ferromagnetic structural material HT-9. 
Table 4.2-2. Mechanical parameters for the blanket module.

Structural material

Axtal length, room temperature $(\mathrm{cm})$

Axtal length, operating temperature $(\mathrm{cm})$

Inner radius $(\mathrm{cm})$

Outer radius $(\mathrm{cm})$

Tube zone thickness ( $\mathrm{Cm}$ )

Bean zone thtckness (cm)

Tube outer diameter ( $\mathrm{cm}$ )

Tube wall thickness $(\mathrm{cm})$

Axial tube spacing (cm)

Radial tube spacing ( $\mathrm{cm}$ )

$\mathrm{Li}_{17}{ }^{\mathrm{Pb}} 83$ weight contained in a module (ton)

Structural material weight of a module, drained (ton)
HT -9

178.6

180.57

59

98

19.2

19

10.14

0.227

10.45

9.05

26

2.7

\subsubsection{Neutronics}

The primary goal of our neutronics analysis is to choose a blanket design that yields a large energy multiplication $M$ with an adequate tritium breeding ratto (TBR > 1.10). We performed calculations to determine the appropriate composition and thickness of the blanket and reflector. We used the coupled neutron-gama continuous-energy Monte Carlo code MC:1 with cross section data based on the ExidF/B- $Y$ evaluation. In addition, the continuous energy treatment of MCNP avoids any energy group structure effects. Because we are interested only in integral quantities such as TBR and $M_{2}$ low statistical uncertainties can be achieved with a reasonable number of histories. He used 4,000 histories in the calculations resulting in fractional standard deviations less than $1 \%$ for the quantities of interest.

The liquid metal eutectic $\mathrm{Li}_{17}{ }^{\mathrm{Pb}}{ }_{83}$ is the breeder and coolant in the blanket and the ferritic steel alloy HT-9 is the structural material. As we stated before, the blanket includes a front tube zone composed of several rows of closely packed tubes $10.14 \mathrm{~cm}$ in diameter that provide for good heat 
rewoval where the energy deposition rates are highest. The back zone of the blanket consists of a single row of hollow rectangular beams that provide structural support for the blanket. The volumetric fraction in the blanket tube zone was selected as a result of structural and thermal hydraulics considerations.

Our results show that neutron multiplication through the $P b(n, 2 n)$ reaction saturates to a value of 1.75 at a blanket thickness of $\sim 30 \mathrm{~cm}$. This implies that, from the point of view of neutron multiplication, the lead in the back region of the blanket is not effective. Hence, we chose a thin blanket design. However, 1 ithium enrichment is required to achieve adequate tritium breeding in such a design. We performed neutronics calculations for a thin blanket with the lithium enriched to $90 \%{ }^{6}$ LI and a thick blanket with natural lithium. We saved $49 \mathrm{~cm}(38.2$ vs $87.2 \mathrm{~cm})$ in the blanket thickness with the thin blanket design' for the' same TBR. "This savings in turn reduces the central cell cost because the magnets are smaller. Furthermore, the blanket weighs less and can be easily replaced at end of life. However, the thinner blanket with the HT-9 reflector produces a smaller $M$ than the thick blanket. $M$ is defined as the ratio of the total recoverable energy deposited in the blanket and reflector to the fusion neutron energy of $14.1 \mathrm{MeV}$.

We have made several attempts to enhance energy multiplication while preserving the advantages of the thin blanket design. Increasing the lithiumlead content in the beam zone of the blanket was found to increase energy multiplication, and we chose a beam zone to achieve high $M$ with adequate structural support. The tube and bean zones of the blanket are 19.2 and $19 \mathrm{~cm}$ thick, respectively.

Energy multiplication can be further increased by using appropriate structural material in the reflector. The reflector is water-cooled and the energy deposited in it is a part of the recoverable energy. For a thin blanket, a relatively large fraction of energy is deposited in the reflector and the overall energy multiplication is sensitive to the choice of reflector material and thickness. In our analysis we included HT-9, 316 SS, graphite, and Fe-1422. The 10w, long-term activation steel, fe-1422 (14 wt\% $\mathrm{m}, 2$ wt\% $\mathrm{N1}, 2 \mathrm{wtx} \mathrm{Cr}$ and 82 wt\% Fe), yielded the largest energy multiplication. This is primarily because of the large gama production in $\mathrm{m}$. Furthermore, $\beta$ decay of $56 \mathrm{wn}_{\mathrm{m}}$, which is a product of the ${ }^{55} \mathrm{~m}(\mathrm{n}, \gamma)$ reaction, 
contributes to local nuclear heating. Increasing the reflector thickness from 28 to $43 \mathrm{~cm}$ increased $M$ from 1.35 to 1.39 . The fraction of energy deposited in the reflector increased from 24.5 to 27.6\%; the fraction of energy deposited as low grade heat in the shield decreased from 3.85 to $0.76 \%$.

Figure 4.2-3 shows the effect of reflector thickness and material on the energy multiplicaton for a thin blanket that uses $90 \%$ enriched lithium and a thick blanket with natural lithium. Both blanket designs have similar TBRs. The improvement in $M$ is clearly more pronounced for the thin blanket. Using a thick Fe-1422 reflector with the thin blanket results in $M$ greater than that for the thick blanket. Table 4.2-3 gives the compositions and thicknesses for the different zones of the blanket and reflector chosen for the MARS LIPb blanket design. The main disadvantage of the thin blanket design is the large fraction of heat deposited in the water-cooled reflector (27.55\%), which reduces overall therial efficfency. 'This was taken' into account in the deisign' optimization analysis, presented in Section 4.2 .3 , which reveals that the thin blanket design has a better overall economic figure of merit than the thick blanket design.

Table 4.2-3. Dimensions and composition of the MARS LIPb blanket and reflector.

\begin{tabular}{|c|c|c|}
\hline & Thickness $(\mathrm{cm})$ & Composition \\
\hline $\begin{array}{l}\text { Blanket tube zone } \\
\text { HT-9 }\end{array}$ & 10.2 & 73.7 vol $\% \mathrm{LI}_{17} \mathrm{~Pb}_{83}, 7.1$ vol $\%$ \\
\hline $\begin{array}{l}\text { Blanket beam zone } \\
\text { Reflector }\end{array}$ & $\begin{array}{l}19 \\
43\end{array}$ & $\begin{array}{l}85 \text { vol } \& \mathrm{Lf}_{17} \mathrm{~Pb}_{83}, 15 \text { vol } \% \mathrm{HT}-9 \\
95 \text { vol } \times \mathrm{Fe}-1422,5 \text { vol } \& \mathrm{H}_{2} \mathrm{O}\end{array}$ \\
\hline
\end{tabular}




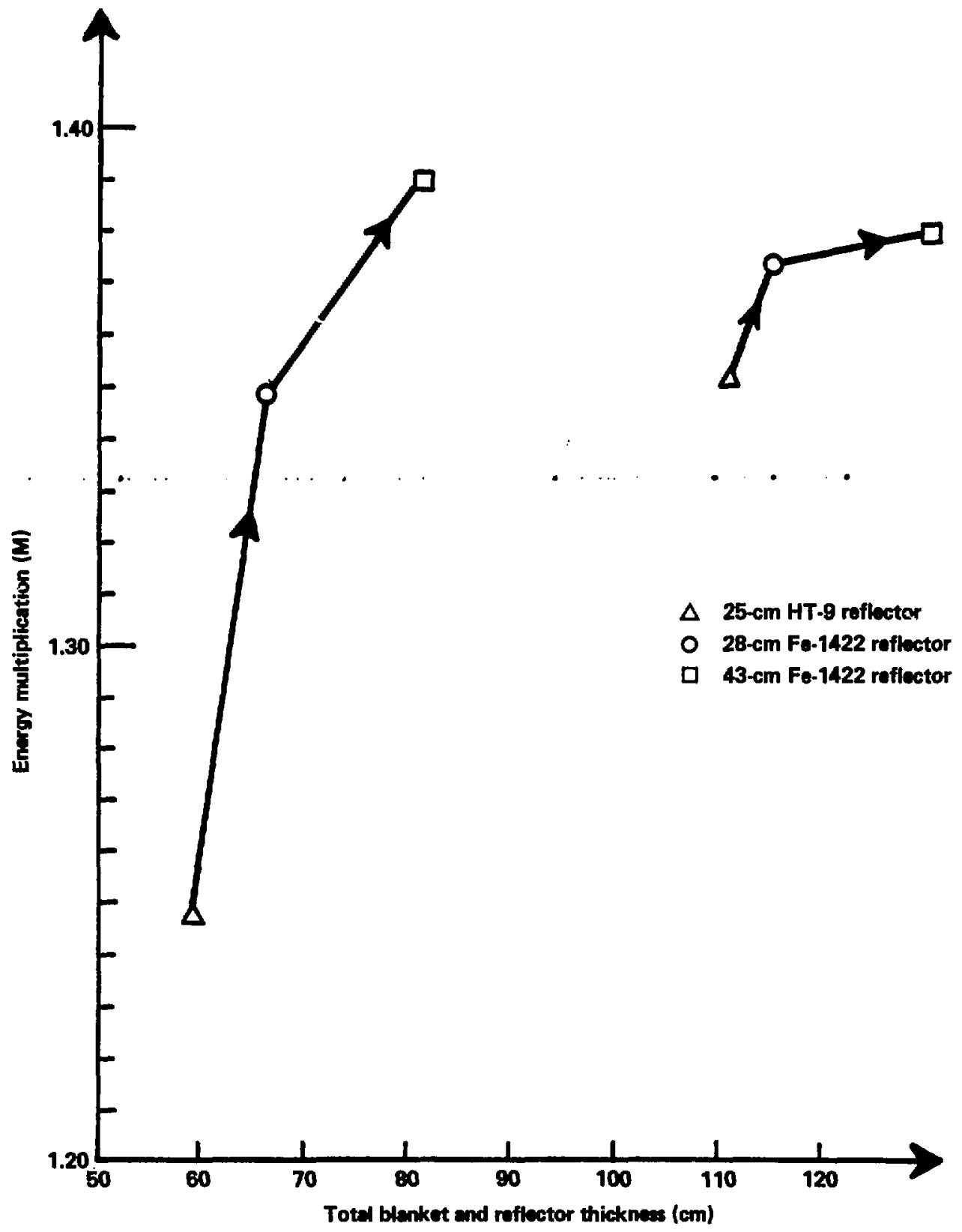

Fig. 4.2-3. Effect of reflector composition and thickness on energy multiplication. 
Table 4.2-4 gives a sumary of the tritium production results for the MARS LiPb blanket design. The overall tritium breeding ratio is 1.134 . Almost all tritium is produced in the ${ }^{6} \mathrm{LI}(n, \alpha) t$ reaction. The nuclear heating results are summarized in Table 4.2-5. The total recoverable energy is 19.604 MeV/fusion. About $60 \%$ of the total heating comes from garmas. This is a direct result of using a metallic reflector. Based on a neutron wall loading of $5 \mathrm{mw} / \mathrm{m}^{2}$, the peak power densities in the blanket, reflector, and shield are $36.0,3.62$, and $0.10 \mathrm{~W} / \mathrm{cm}^{3}$, respectively.

Table 4.2-4. Tritium production in the MARS LIPb blanket.
$6_{L I}(n, \alpha) t$
$T_{\operatorname{Li}\left(n, n^{\prime} \alpha\right) t}$
Total

Tube zone

0.565

0.0022

0.567

Bearn zone

0.567

0.0004

0.567

Total blanket

1.132

0.0026

1.134

Table 4.2-5. Nuclear heating (MeV/fusion) in the MARS LIPB blanket.

\begin{tabular}{llrr} 
& Neutron & $\frac{\text { Gamma }}{4}$ & $\frac{\text { Total }}{9.145}$ \\
\cline { 2 - 2 } & 4.488 & 4.657 & 5.016 \\
Blanket beam zone & 3.296 & 1.720 & 5.442 \\
Reflector & 0.339 & 5.103 & 0.151 \\
Shield & 0.016 & 0.135 & 19.754 \\
Total & 8.139 & 11.615 &
\end{tabular}




\subsubsection{Thermal Hydraulics}

The flow of a liquid metal perpendicular to a strong magnetic field, called Hartman flow, is laminar with a constant veloctty profile and a very thin boundary layer. The heat transfer in such a system is dominated by conduction and, under such conditions, is poor because of the lack of turbulence. For a TMR, the surface heating load in the central cell is small enough that a poor heat transfer coefficient is not a major drawback. For this reason, a tube flow provides enough cooling to the first 11 .

We cannot obtain an exact solution for conduction heat transfer in a coolant channel with both surface heating and nonuniform volumetric heating. However, a numerical solution is easily ubtained using fintte-difference techniques. The energy is derived from neutronic calculations and the coolant velocity is calculated from energy balance. With this input, the temperature profile in a coolant tube can be calculated step-by-step from an initlal temperature distribution. The heat transfer calculations are summarized in Table 4.2-6.

Table 4.2-6. Thermal hydraulic paraneters of the LIPb blanket.

Structure/coolant

Neutron wall loading $\left(m / m^{2}\right)$

Surface heat flux $\left(\mathrm{H} / \mathrm{Cm}^{2}\right)$

Blanket thermal power (NW)

Reflector thermal power (MW)

Blanket coolant inlet/out let temperature $\left({ }^{\circ} \mathrm{C}\right)$

Structural temperature $\left({ }^{\circ} \mathrm{C}\right)$

Maximum blanket coolant velocity $(\mathrm{m} / \mathrm{s})$

Coolant flow rate $(\mathrm{kg} / \mathrm{hr})$

Reflector coolant

Reflector coolant inlet/outlet temperature $\left({ }^{\circ} \mathrm{C}\right)$

Reflector coolant velocity $(\mathrm{m} / \mathrm{s})$

Reflector coolant flow rate $(\mathrm{kg} / \mathrm{hr})$
$H T-9 / \mathrm{LI}_{17} \mathrm{~Pb}_{83}$

5

6

2880

1012

$330 / 500$

$330 / 550$

0.19

$3.9 \times 10^{8}$

$\mathrm{H}_{2} \mathrm{O}$

$200 / 300$

3

$5.1 \times 10^{6}$ 


\subsubsection{MAD Considerations}

The dominant force on a conducting fluid flowing across magnetic field ines is the MDD force. The effect of the MHD force is to increase the pressure drop and retard heat transfer by suppressing turbulence. The MHD pressure drop will increase the stresses in the blanket and will increase the pumping power. Therefore, we have to calculate the effect of the MD forces on both heat transfer and pressure drop.

The Hartman pressure gradient arises in fully developed laminar flow across a uniform transverse magnetic field. For such a flow in a cylindrical tube, in a uniform magnetic field normal to the tube, the pressure gradient is given by ${ }^{2}$

$$
\text { - . } \quad-\frac{d P}{d x}=\frac{\mu}{a^{2}}\left(\frac{H^{2} \tanh H}{H-\tanh H}+\frac{H^{2} C}{1+C}\right) \text {, }
$$

in which $C=$ wall conductance $=q_{w} t_{w} / a \sigma$,

$a=$ radius of the tube,

$H=B_{1} V \sigma \mu_{,}$

$v=$ bulk velocity,

$\mu=v i s \cos i t y$,

$B_{\perp}=$ component of the magnetic field perpendicuiar to the bulk velocity,

$\sigma=$ electrical conductivity of the coolant,

$q_{w}=$ electrical conductivity of the wall, and

$t_{w}=$ wall thickness.

When the fluid is in a channel with a conducting will and under large magnetic fields $(H \gg 1+1 / C)$, the second term of the equation dominates, giving

$$
-\frac{d P}{d x} \approx v B_{1}^{2} q_{w} t_{w} /[a(1+c)]
$$

For a thin-walled tube, $c$ is on the order of $10^{-2}$, or

$$
-\frac{d P}{d x} \simeq \frac{v B_{1}^{2} q t_{w}}{d} \text {. }
$$


This equation contains an interesting conclusion. The WHD pressure drop for sully developed laminar flow across a unfform magnetic fieli is independert of the properties of che flowing material. The wall thickness $t_{w}$ of the feed and discharge tubes is kept small by using a 1-mm-thick insert inside the structural tube. The insert is kept separate from the main struciural tube by an electrical insulation layer. The eddy current generated by the $\checkmark \times B$ effects is theroby reduced.

The calculaton of pressure drop is summarized in Table 4.2-7. In this table, $\Delta P_{E}$ is the pressure drop associated with turns and end-of-100F effects and $\Delta P_{H}$ is the Hartman pressure drop. The pressure distribution in the blanket is shown in Fig. 4.2-4. The total pressure drop is $1.32 \mathrm{MPa}$, which corresponds to the pumping nower requirement of 40 with an assumed pump efficiency of $50 \%$.

\subsubsection{Lipb Corroston}

There is a scarctiy of corrosion data between $\mathrm{Li}_{17} \mathrm{~Pb}_{83}$ and structural materials. The only avatlable data are for a static system, ${ }^{3}$ as shom in Figure 4.2-5. By comparing 1000- and 3000-hr tests, we see significant uncertainties in tha dependence of the corrosion rate on temperature. The presence of themal gradients in the capsules increases the corrosion rate above what it would be $i n$ an isothermal capsule. Tise allowable limits on corrosion are not well established; however, the levels indicated in Fig. 4.2-5 are small enough so that material thinning is not a problem. Redtposition of transported corrosion products is the more important effect. The MARS system is a dynanic system. The corrosion rate in MARS is expected to be higher than the rate shown in Fig. 4.2-5. However, there is virtually no turbulence in flow perpendicular to strong magnetic field with very low velocity. He expect the fiisss transfer in the liquid side to be dominated by molecular diffusion and, therefore, will resemble a static system more than a dynamic system.

Although there are no dynamic tesis of steel with $\mathrm{Li}_{17} \mathrm{~Pb}_{83}$, we expect that $\mathrm{Li}_{17}{ }^{\mathrm{Pb}} 83$ will reseable pure $\mathrm{Pb}$. Observation of corrosion of steel by lead will provide some indication of $\mathrm{Li}_{17} \mathrm{~Pb}_{83}$ compatibility. The corrosion is primarily caused by straightforward dissolution by lead. In commenting on such corrosion. it is essential to be specific not only with respect to 
Table 4.2-7. Sumary of blanket MHD calculations.

\begin{tabular}{|c|c|c|c|c|c|c|c|c|}
\hline & $\begin{array}{c}V \\
(m / 5) \\
\end{array}$ & $\begin{array}{c}a \\
(m)\end{array}$ & $t_{w}$ & $\begin{array}{l}L \\
(m)\end{array}$ & $\begin{array}{c}\text { B } \\
(\mathrm{T}) \\
\end{array}$ & $\begin{array}{c}\Delta \mathbf{P}_{\mathbf{E}} \\
(\mathbf{M P a})\end{array}$ & $\begin{array}{c}\Delta \mathbf{P}_{\mathbf{H}} \\
(\mathbf{M P a})\end{array}$ & $\begin{array}{c}\operatorname{IAP} \\
(\mathrm{MPa})^{\mathbf{a}}\end{array}$ \\
\hline Feed pipe & 0.50 & 0.30 & 1 & 3 & 4.7 & 0.2 & 0.1 & 0.3 \\
\hline $\begin{array}{l}\text { Feed pipe turn to supply } \\
\text { header }\end{array}$ & 0.5 & 0.3 & 1 & - & 4.7 & 0.2 & - & 0.2 \\
\hline $\begin{array}{l}\text { Supply header turn to } \\
\text { blanket tube }\end{array}$ & 0.19 & 0.05 & 2 & - & 4.7 & 0.01 & - & $\dot{0.01}$ \\
\hline Blanket tube & 0.19 & 0.05 & 2 & 1.88 & 4.7 & - & 0.3 & 0.3 \\
\hline $\begin{array}{l}\text { Blanket tube turn to } \\
\text { return header }\end{array}$ & 0.19 & 0.05 & 2 & - & 4.7 & 0.01 & - & 0.01 \\
\hline $\begin{array}{l}\text { Return header turn to } \\
\text { discharge pipe }\end{array}$ & 0.5 & 0.3 & 1 & - & 4.7 & 0.2 & - & 0.2 \\
\hline Discharge pipe & 0.5 & 0.3 & 1 & 3 & 4.7 & 0.2 & 0.1 & 0,3 \\
\hline
\end{tabular}

\footnotetext{
a Total pressure drop is $1.32 \mathrm{Ma}$.
} 


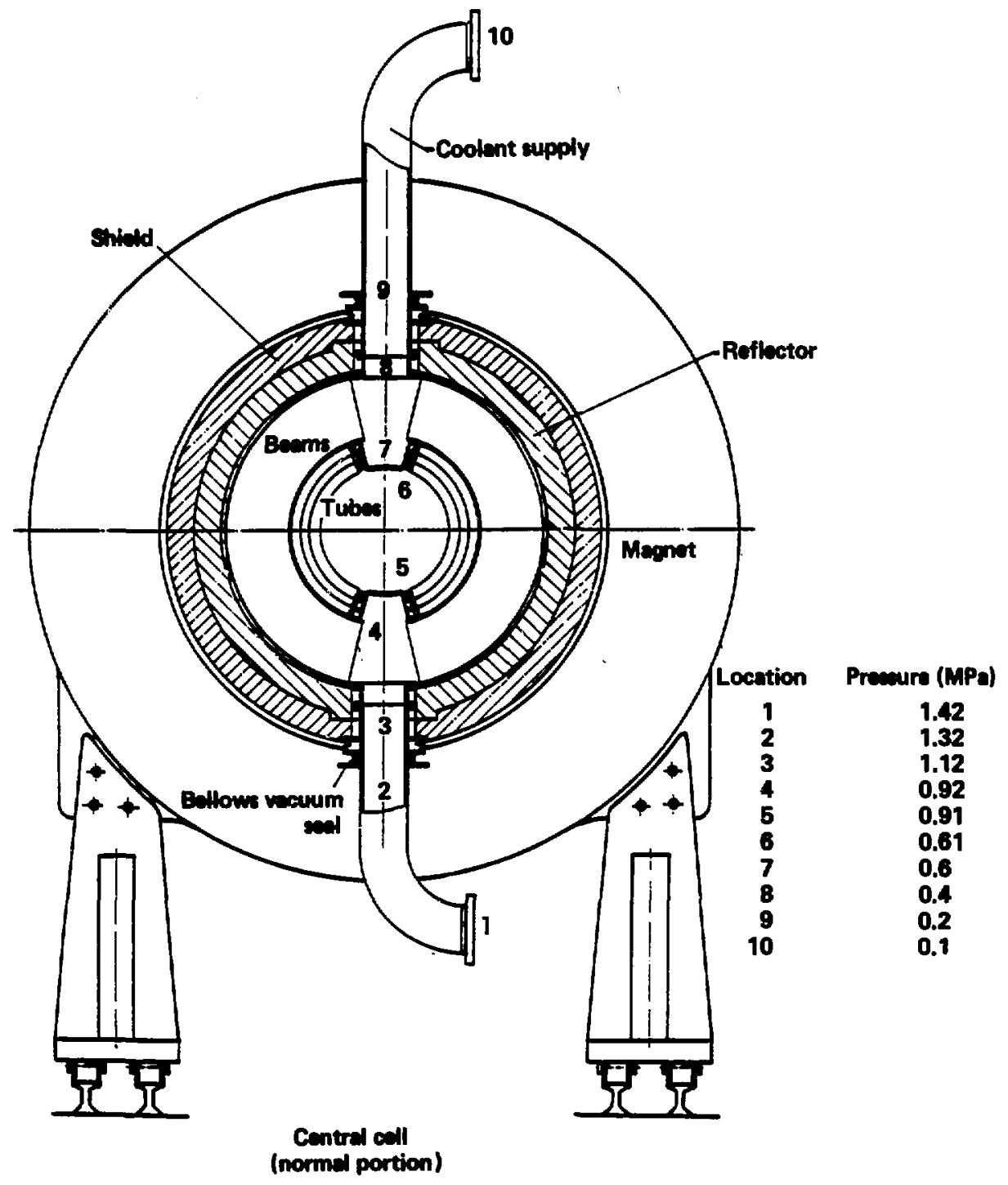

Fig. 4.2-4. Pressure in MaRS blanket (static head is not included). 


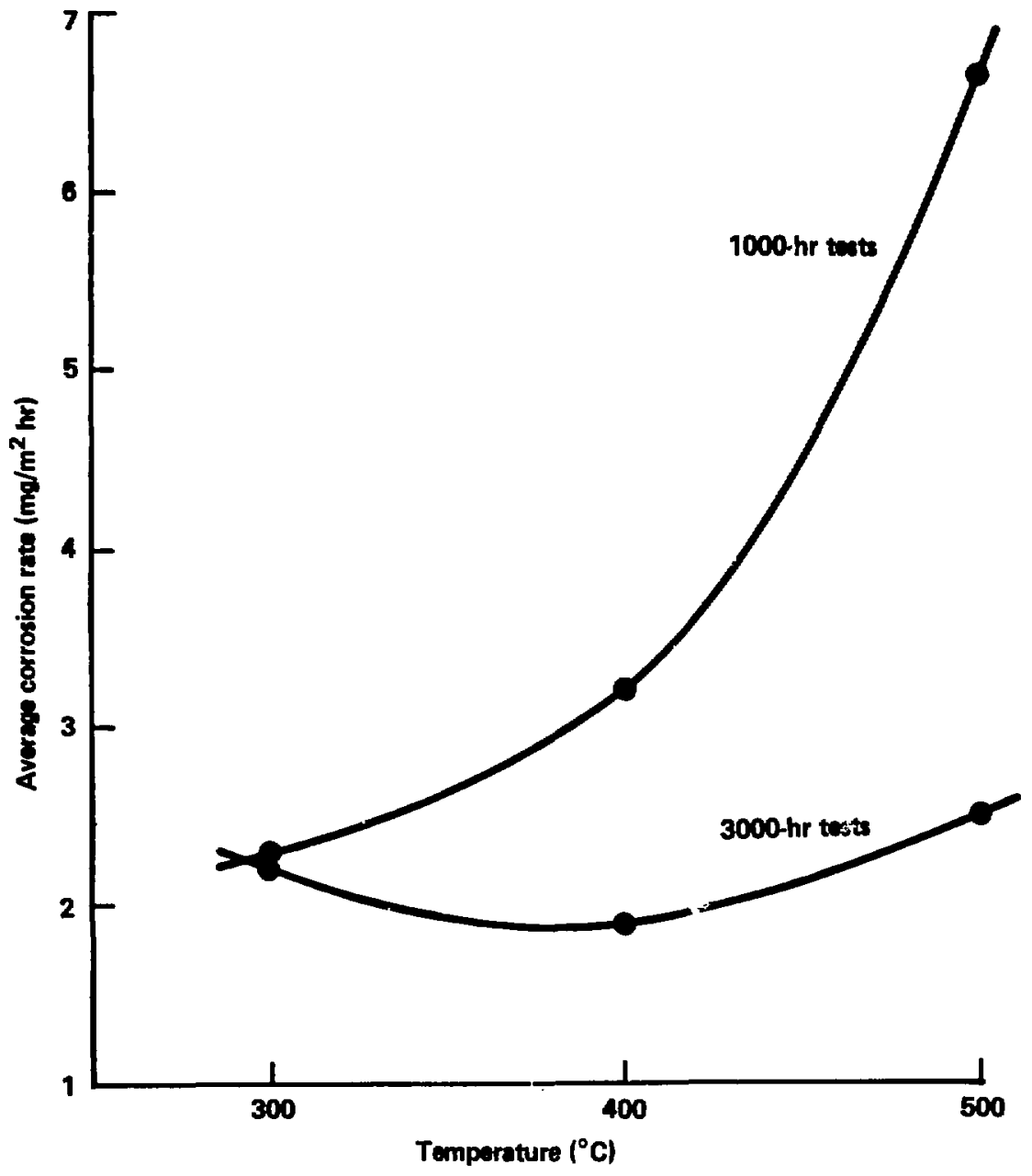

Fig. 4.2-5. LIPb/HT-9 corrosion data. 
temperature, but also with respect to temperature gradient, coolant velocity, and coolant impurity level. At $1000^{\circ} \mathrm{C}$, one source 4 reports a rapid intergranular attack of various steels, but another source ${ }^{5}$ cites only a slight attack on 304 SS.

At temperatures around $600^{\circ} \mathrm{C}$, however, the alloys are far more corrosionresistant, as is shown in Table 4.2-8.6 For compartson, corroston of 316 SS by lithium at $600^{\circ} \mathrm{C}$ is estimated to be $23 \mathrm{mil} / \mathrm{yr} .^{7}$ If the temperature is reduced to $400^{\circ} \mathrm{C}, 304$ ss shows no attack after $500 \mathrm{hr} .8$ On the basis of these data, we conclude that at temperatures below $500^{\circ} \mathrm{C}$, dissolution attack by lead is reasonably slow even for stainless steels.

Because nickel and nickel-based alloys are much more soiuble than iron, It is not surprising that the austenitic stainless steels with their higher nickel content are somewhat more easily corroded. Chromium also has a higher solubility than iron, but lower than nickel. At $600^{\circ} \mathrm{C}$ the solubility of iron was reported 9 as only $2.3 \times 10^{-4}$ wt; at $635^{\circ} \mathrm{C}$ the solubility of nickel was reported to be $0.85 \mathrm{wt} \%$. For this reason we favor the use of ferritic or martensitic steels rather than the higher nickel austenitic alloys.

Finally, we found that little eiffort has yet been extended to further reduce the corrosion rate by the use of inhibiting techniques. The addition of trace amounts of zirconiun and magnesium has been shown 11,12 to provide complete protection of Croloy 2-1/4 over three years with temperatures up to $550^{\circ} \mathrm{C}$ in spite of strong thermal gradients in the test loop.

We have concluded that we can be reasonably optimistic about the use of steels as structural materials in liquid lead (and hence LiPb) up to $500^{\circ} \mathrm{C}$. This initial conciusion, however, should not be used as an argument to reglect corrosion studies for these systems. The data we have used, while encouraging, are far from definitive and we heartily endorse the proposal for simple coupon tests to validate the alloys proposed for use in LiPb. 
Table 4.2-8. $\mathrm{Li}_{17} \mathrm{~Pb}_{83}$ corrosion.

\begin{tabular}{lcccc}
\hline Alloy & $\begin{array}{c}\text { Maximum } T \\
\left({ }^{\circ} \mathrm{C}\right)\end{array}$ & $\begin{array}{c}\Delta \mathrm{T} \\
\left({ }^{\circ} \mathrm{C}\right)\end{array}$ & $\begin{array}{c}\text { Exposure } \\
(\mathrm{hr})\end{array}$ & $\begin{array}{c}\text { Calculated } \\
\text { corrosion rate } \\
\text { (mil/yr) }\end{array}$ \\
\hline ASTM A106 & 573 & & & \\
Croloy 2-1/4 & 593 & 111 & 5064 & 12 \\
410 SS & 655 & 111 & 5156 & 10 \\
& & 167 & 1346 & 15 \\
\hline
\end{tabular}

\subsubsection{Lithium-Lead/Water Interactions}

We recognize that a molten metal can interact with water to produce hydrogen and an accompanying release of mechanical energy. However, the magnitude of the interaction depends strongly on the metal composition, temperature, and mass as well as on the mode of contact between the metal and water. He have quantified the magnitude of the lithium-lead/water interaction that might occur in the blanket of the fusion power reactor, in the accompanying prinary coolant loop piping, or in the metal-water steam generator. However, because some of the physical processes involved with molten metalwater interactions are not fuily understood, our analysis is only preliminary and should be vertffed by experiments and more detailed analysis.

Some preliminary experiments have been performed at Argonne Mational Laboratory 13-15 using lithium and lithium-lead alloys as the molten metal, which is poured into a waterpool. The results of the experiments, shown in Table 4.2-9, indicate that molten lithium-lead undergoes a qualitatively more benign reaction than lithium. Two physical processes are involved in a matalwater interaction:

1. Physical exploston (also called vapor explosion or fuel-coolant interaction), where the hotter liquid (molten metal -." fuel ") mixes with the colder liquid (water--"coolant"), rapidly producing high pressure steam as the fuel is finally fragmented. 
2. Chemical reaction and burning when the molten metal that wixes with the water undergoes exothermic oxidation, produces hydrogen gas, and the hydrogen gas burns (deflagration or detonation).

The results of the All experiments suggest that the lithium-lead alloys (in particular $\mathrm{Li}_{17} \mathrm{~Pb}_{83}$ ) do not undergo as vigorous or damaging a reaction as molten lithium.

Although the reasons for such a difference are not completely known at this time, we propose three possible explanations:

1. The relative temperature rise for the lithium-lead alloy $\left(\mathrm{Li}_{17} \mathrm{~Pb}_{83}\right)$ is smaller than for molten lithium during oxidation.

2. The lower reaction temperature coupled with realistic chemical kinetics produces slower reaction rates.

3. The surface area available for reaction is probably smaller for the lithlum-lead alloys.

The relative temperature rise for a $\mathrm{Li}_{17} \mathrm{~Pb}_{83}$-water chemical reaction is lower than the relative temperature rise for lithium-water. The thermodynamic maximum reaction zone temperature is $\sim 4350 \mathrm{~K}$ for lithium and $\sim 1750 \mathrm{~K}$ for $\mathrm{LI}_{17}{ }^{\mathrm{Pb}} 83$. The major reason is the large thermal inertia of the lithlum-lead alloy.

This difference in the reaction zone temperature manifests itself in the chemical kinetics of the reaction. As the reaction zone temperature increases, the process of vapor-phase oxidation becomes more dominant. Vapor-phase oxidation occurs when the molten metal temperature increases to such an extent that the metal vapor pressure rises, the metal vapor diffuses toward the water vapor, and oxidation occurs. The kinetics of the vapor-phase reaction are much more rapid than the kinetics of liquid metal-water reaction because the protective oxide layer on the metal surface does not inhibit the vapor phase reaction. For lithium at $\sim 4350 \mathrm{~K}$ the partial pressure is $\sim 10^{5}$ Torr, whereas for $\mathrm{LI}_{17} \mathrm{~Pb}_{83}$ at $1750 \mathrm{~K}$ the lithium partial pressure is $\sim 1$ Torr. This suggests that vapor phase oxidation would be much less of a concern for $\mathrm{Lf}_{17}{ }^{\mathrm{Pb}}{ }_{83}$. In fact, If we use the data from scdium-water reactions, vapor 
phase oxidation becomes dominant when the partial pressure of metal vapor rises above 100 Torr. This analogy suggests that the vapor phase combination is not a concern with $\mathrm{Li}_{17} \mathrm{~Pb}_{83}$.

In the absence of vapor phase oxidation, the kinetic rate of reaction per unit area of a molten metal in water is controlled by the rate of stean diffusion to the metal surface and the rate of oxygen diffusion through the oxide surface layer into the liquid fuel phase. Although the kinetics of the 11thium-lead alloy reaction have not been measured, we belleve that the rate of reaction would be lower than the rate of reaction with pure lithium for two reasons. First, the chemlcal activity of $\mathrm{LA}_{17} \mathrm{~Pb}_{83}$ is much lower than 1ithium. Second, the rate of reaction would be reduced because the lithium would be more quickly depleted near the surface of the metal for lithium-lead because of its low mole fraction.

Finally, the total rate of oxidation is the product of the kinetic rate per unit area and the total molten metal surface area. The rate of mixing and, therefore, the rate of fuel surface area generation is proportional to the square root of the ratio of coolant density to fuel density. Thus, the higher density of $\mathrm{Li}_{17}{ }^{\mathrm{Pb}_{83}}$ causes slower mixing and slower area generation. This implies that less surface area is avallable for chemical reactions. Experiments should be performed to verify these results.

\subsubsection{Emergency Cooling}

Loss of Flow and Loss of Coolant Accidents. The temperature responses of the first wall due to loss of flow accident (LOFA) and loss of coolant accident (LOCA) are calculated and shown in Fig. 4.2-6. The rates of the first wall temperature rises are very similar in the two cases. This unexpected result is caused by the much higher volumetric heat capacity for steel $\left(\rho c_{p}=3.6 \mathrm{~J} / \mathrm{cm}^{3}-{ }^{\circ} \mathrm{C}\right)$ than for the $\mathrm{Li}_{17}{ }^{\mathrm{Pb}} 83\left(\rho c_{p}=1.5 \mathrm{~J} / \mathrm{cm}^{3}-{ }^{\circ} \mathrm{C}\right)$. Therefore, in the event of a LOFA, the temperature rise of the $\mathrm{Li}_{17}{ }^{\mathrm{Pb}} 83$ is actually faster than that of the structure, although the volumetric heating rate is less. The coolant will heat up the first wall and, consequently, the first wall is heated up faster than in the event of a LOCA. As the time reaches $\sim 20 \mathrm{~s}$, the $\mathrm{Lf}_{17} \mathrm{~Pb}_{83}$ farther away from the first wall (and, consequently, with a much lower volumetric heating) starts to cool the first wall by conduction. This explains the cross-over point of the two temperatureresponse curves. 


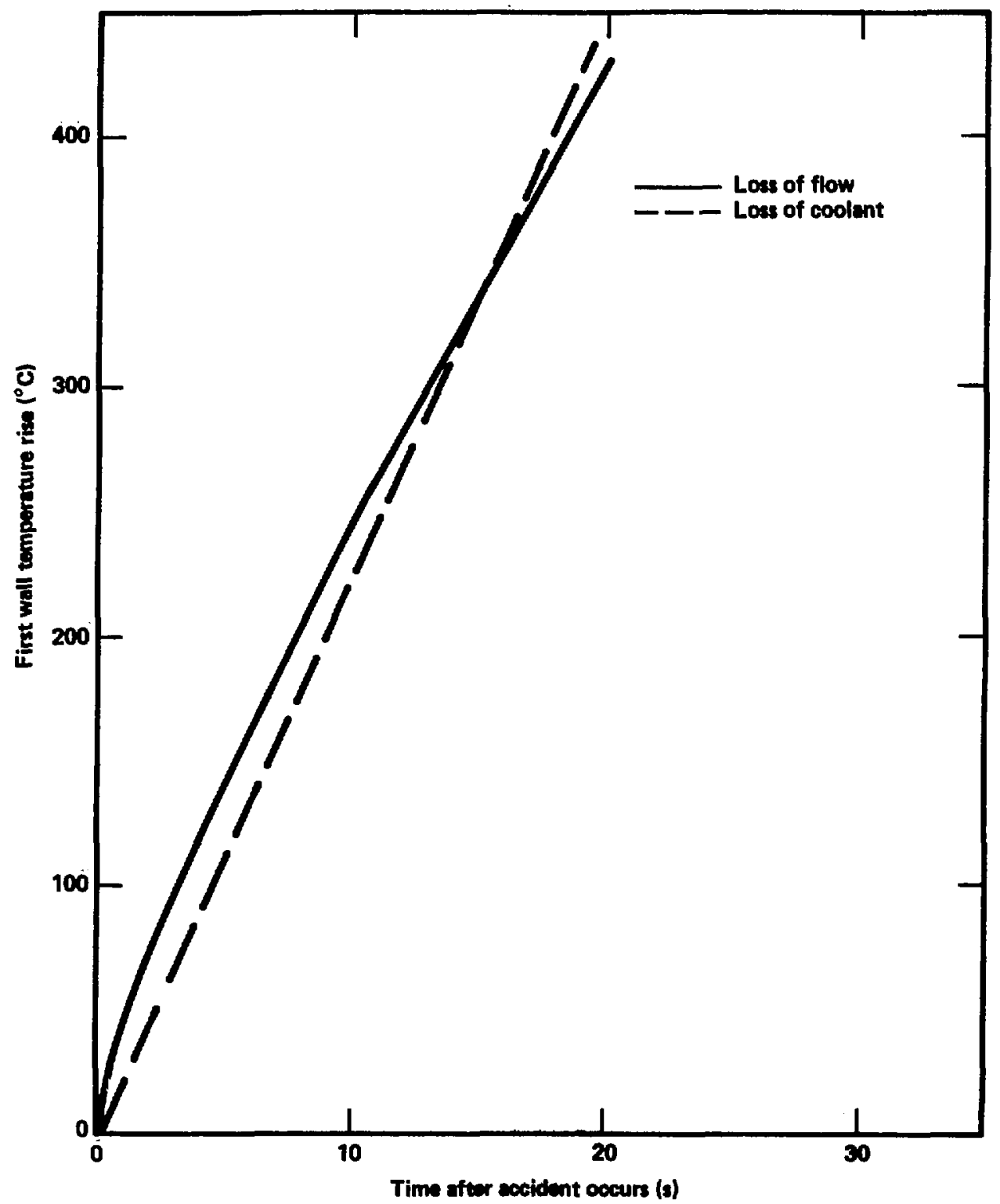

Fig. 4.2-6. Temperature response of the first wall due to loss of flow and loss of coolant accidents. 
If a LOFA or LOCA occurs, the blanket will suffer permanent damage if the plasma is on for $10 \mathrm{~s}$. If the plasma is not stopped in $\sim 35 \mathrm{~s}$, the first wall will melt.

In a liquid-meta? flow system, a surge tank is always provided to maintain a free surface. The $\mathrm{LI}_{17} \mathrm{~Pb}_{83}$ in the surge tank can be used as the energency coolant by using gravitational force to pump. A coolant velocity of $7 \mathrm{~cm} / \mathrm{s}$ can be reached by using the gravitational head. The temperature response of the first wall, shown in Fig. 4.2-7, will reach a maximum of $800^{\circ} \mathrm{C}$ in $\sim 30 \mathrm{~s}$. The volumetric flow rate required for the coolant is $5 \mathrm{~m}^{3} / \mathrm{s}$ for the entire reactor. The total energency cooling time provided has to be optimized between the surge tank cost and accident detection time.

Table 4.2-9. Reactions of LiPb alloys and lithium with water.

Case Composition $\begin{aligned} & \text { Sample } \\ & \text { state }\end{aligned}$

$\begin{array}{ll}1 & \mathrm{Li}_{7} \mathrm{~Pb}_{2} \\ 2 & \mathrm{Li}_{7} \mathrm{~Pb}_{2} \\ 3 & \mathrm{Li}_{7} \mathrm{~Pb}_{2} \\ 4 & \mathrm{Li}_{7} \mathrm{~Pb}_{2} \\ 5 & \mathrm{Li}_{62} \mathrm{~Pb}_{38} \\ 6 & \mathrm{Li}_{17}{ }^{\mathrm{Pb}} 83 \\ 7 & \mathrm{Li}_{83} \\ 8 & \mathrm{Li}^{\mathrm{b}}\end{array}$

s

s

$\ell$

$\ell$

$\ell$

$\ell$

$\ell$

$\ell$
$\underset{(K)}{\operatorname{Temp}}$

773

773

873

1103

773

773

773

773
298

369

368

368

368

368

368

368
Reaction

Modest

Vigorous

Vigorous

Very vigorous

Vigorous

Very modest

$\mathrm{H}_{2}$ Detonation

Detonation

a Where $s=$ solid, $l=1$ iquid.

$b$ Injected under water. 


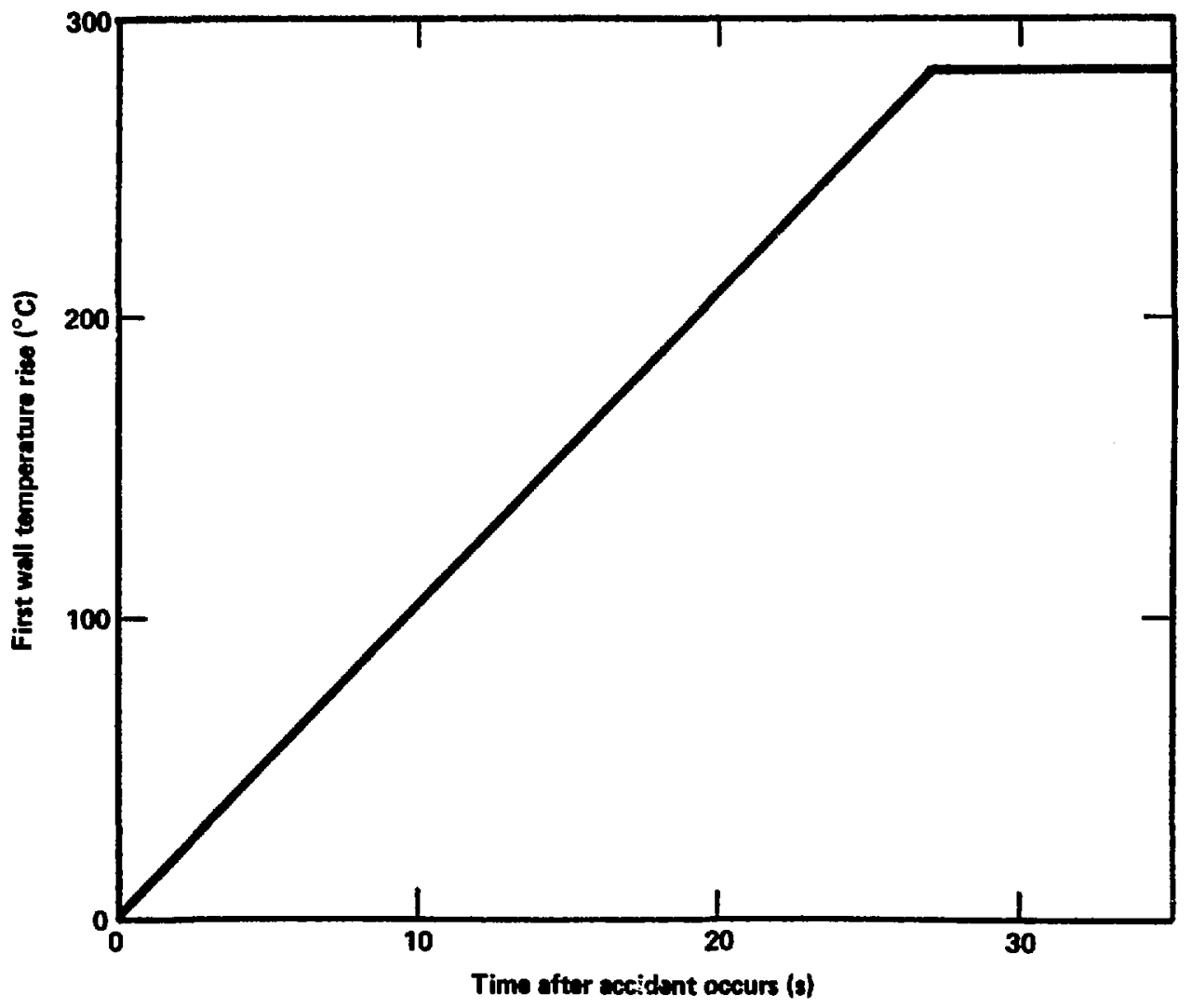
Fig. 4.2-7. Temperature response of the first wall with gravitational coolant
flow. 
Afterheat Calculations. The afterheat levels in the blanket and shield are shown in Figs. 4.2-8 and 4.2-9. Figure 4.2-8 shows the afterheat with the blanket filled with $\mathrm{Li}_{17} \mathrm{~Pb}_{83}$ and Fig. 4.2-9 shows the afterheat with the $\mathrm{Li}_{17} \mathrm{~Pb}_{83}$ drained. Table 4.2-10 shows the adiabatic temperature rise rate in different regions of the blanket and shield. Because of the manganese in Fe1422, the afterheat in the reflector is very $\mathrm{high} l \mathrm{hr}$ after the reactor is shutdown. The half-life of $56 \mathrm{Mn}$ is $2.6 \mathrm{hr}$; therefore, the afterheat in the reflector decays rapidiy in the first day. After one day, the reflector can be used as the sink to radiation-cool the blanket.

Table 4.2-11 sumnarizes the thermal radiation calculation for the blanket one day after the reactor is shut down. We see that the blanket can be cooled effectively by thermal radiation. The best estimate of emissivity of the steel tube is 0.4 . The maximum temperature that can be reached is $390^{\circ} \mathrm{C}$ at $\varepsilon=0.4$.

Table 4.2-10. Rate of adiabatic temperature rise (in ${ }^{\circ} \mathrm{C}$ ).

\begin{tabular}{llll}
\multicolumn{2}{c}{$\begin{array}{l}\text { With } \mathrm{Li}_{1} 7^{\mathrm{Pb}} 83 \\
\text { hr after }\end{array} \begin{array}{c}1 \text { day after } \\
\text { shutdown }\end{array}$} & $\begin{array}{c}\text { Without } \mathrm{Li}_{17} \mathrm{~Pb}_{83} \text { hr after } \\
\text { shutdown }\end{array}$ & $\begin{array}{c}1 \text { day after } \\
\text { shutdown }\end{array}$ \\
\hline & & & \\
$7.7 \times 10^{-2}$ & $7.6 \times 10^{-3}$ & $1.0 \times 10^{-1}$ & $1.6 \times 10^{-2}$ \\
$1.7 \times 10^{-2}$ & $2.5 \times 10^{-3}$ & $6.2 \times 10^{-2}$ & $9.3 \times 10^{-3}$
\end{tabular}

Second blanket $\quad 9.1 \times 10^{-3} \quad 6.9 \times 10^{-4} \quad 3.5 \times 10^{-2} \quad 2.7 \times 10^{-3}$

Reflector $\quad 3.3 \times 10^{-2} \quad 9.7 \times 10^{-5} \quad 3.3 \times 10^{-2} \quad 1.4 \times 10^{-4}$

Shield $\quad 1.0 \times 10^{-3} \quad 3.3 \times 10^{-6} \quad 1.0 \times 10^{-3} \quad 3.3 \times 10^{-6}$ 


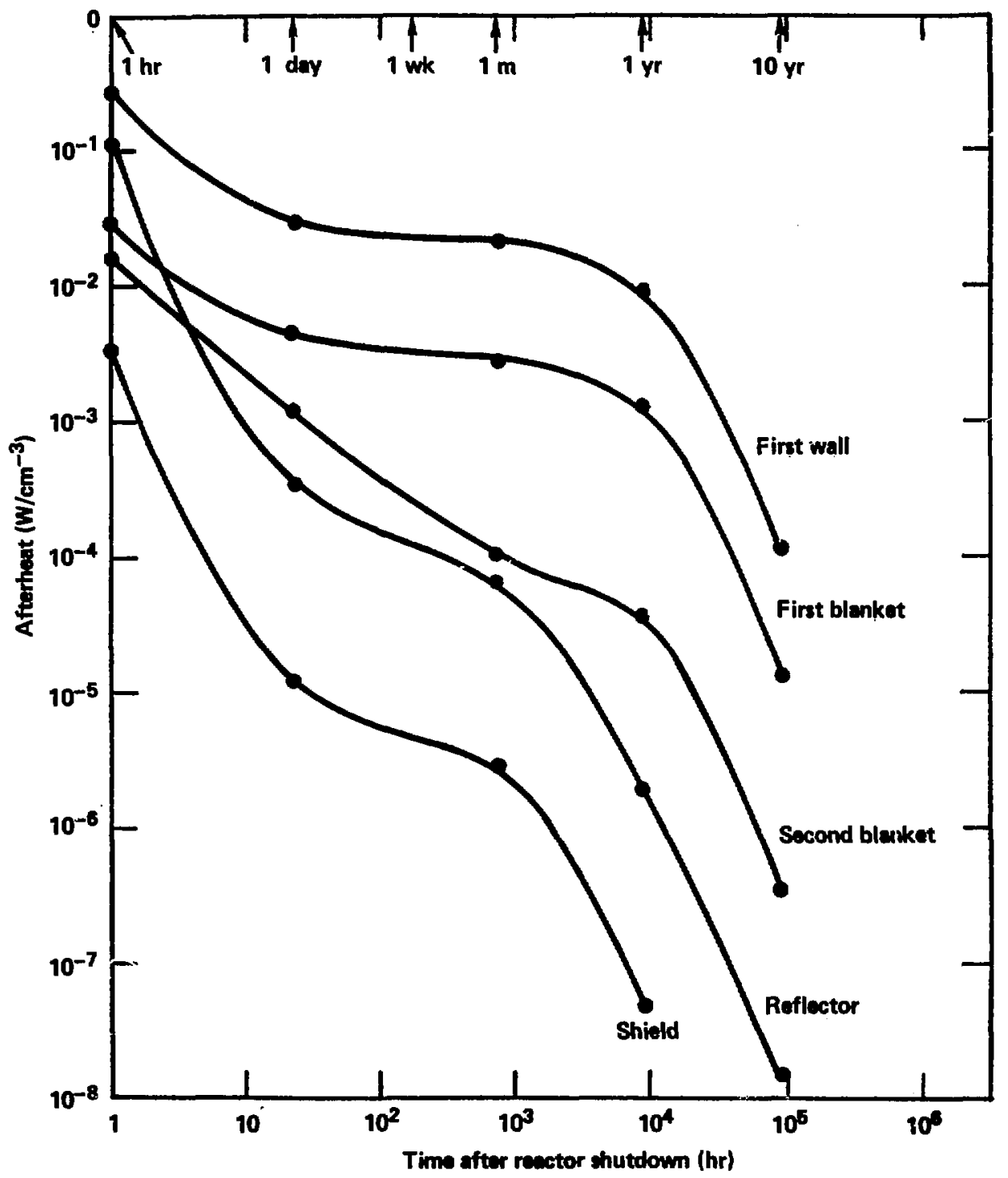

Fig. 4.2-8. Afterheat in different region with $L_{17} \mathrm{~Pb}_{83}$. 


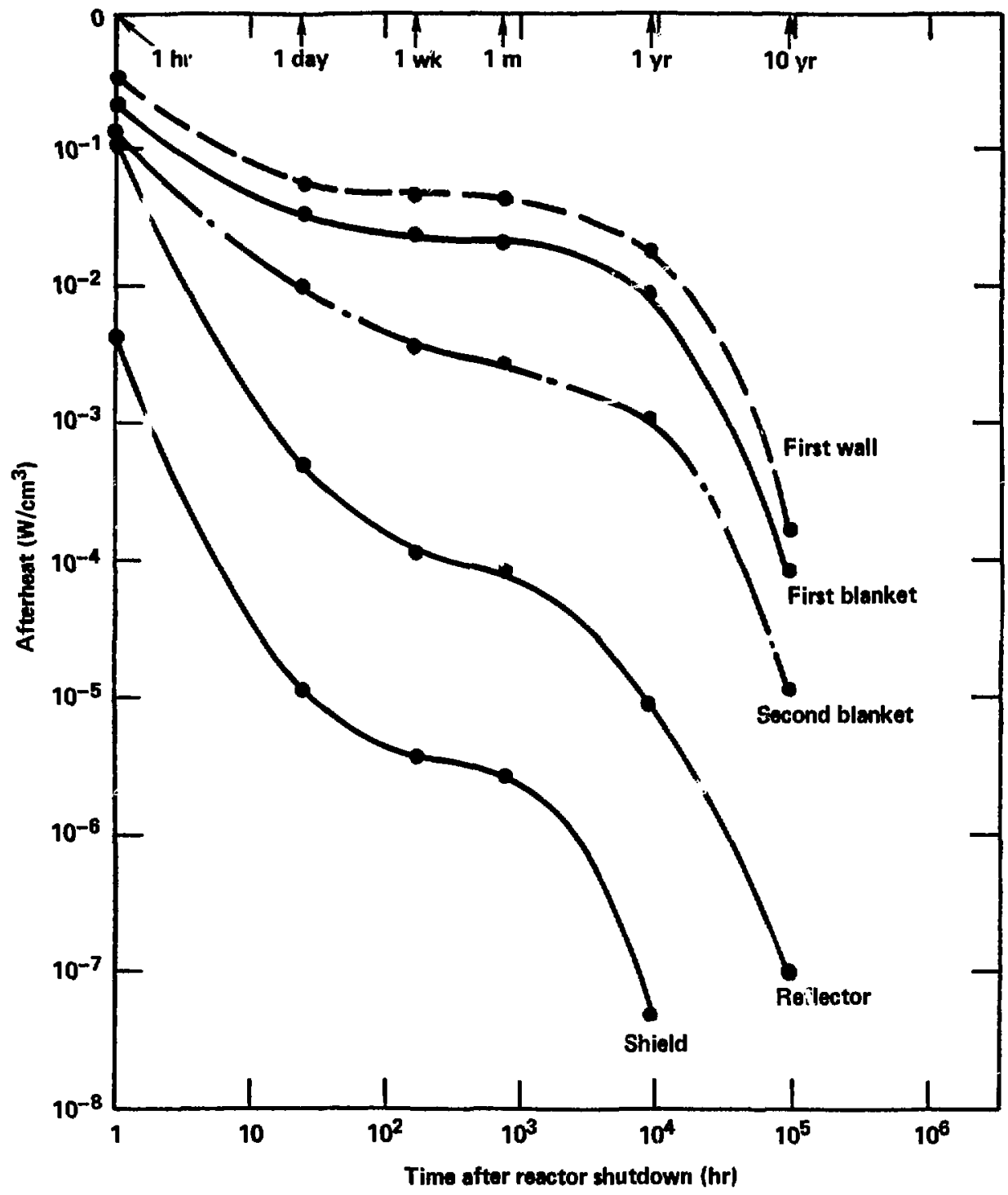

Fig. 4.2-9. Afterheat in different regions after $\mathrm{Li}_{17} \mathrm{~Pb}_{83}$ is drained. 4- 42 
Table 4.2-11. Radiation-cooled blanket temperature. (Forced cooling was stopped one day after shutdown.)

Maximum blanket temperature $\left({ }^{\circ} \mathrm{C}\right)$

Steel tube emissitivity

\begin{tabular}{l}
0.5 \\
0.4 \\
\hline
\end{tabular}

First wall

Second wall

Third wall

Fourth w? ll

Second blanket

Reflector
357

347

331

307

272

200
390

379

360

332

290

200
508

493

467

426

363

200

\subsubsection{Radiation Effects on Central Cell Materials}

Five inportant materials in the central cell need to be analyzed:

- HT-9 (blanket material);

- Fe-1422 (reflector and shield structure);

- Copper (stabilizer in the indgnets);

- MbTf (superconductor in the magnets); and

- Polyimide (magnet electrical insulation).

Figure 4.2-10 shows the spatial variation of dpa and helfum production in the blanket and shield. Figure 4.2-11 shows the spatial :arlation in the dpa rate for the Cu stabilizer.

An analysis of these figures reveals that the maximum damage rate in the HT-9 is 70.6 dpa/FPY, while helium is produced at a rate of 561 appm per Fry. The present assessment of the useful life of HT-9 at $500^{\circ} \mathrm{C}$ is 10 to 20 Mu-yr/m (100 to $200 \mathrm{dpa}$ ) so that we expect a first wall life of 1.5 to 3 FPY. 


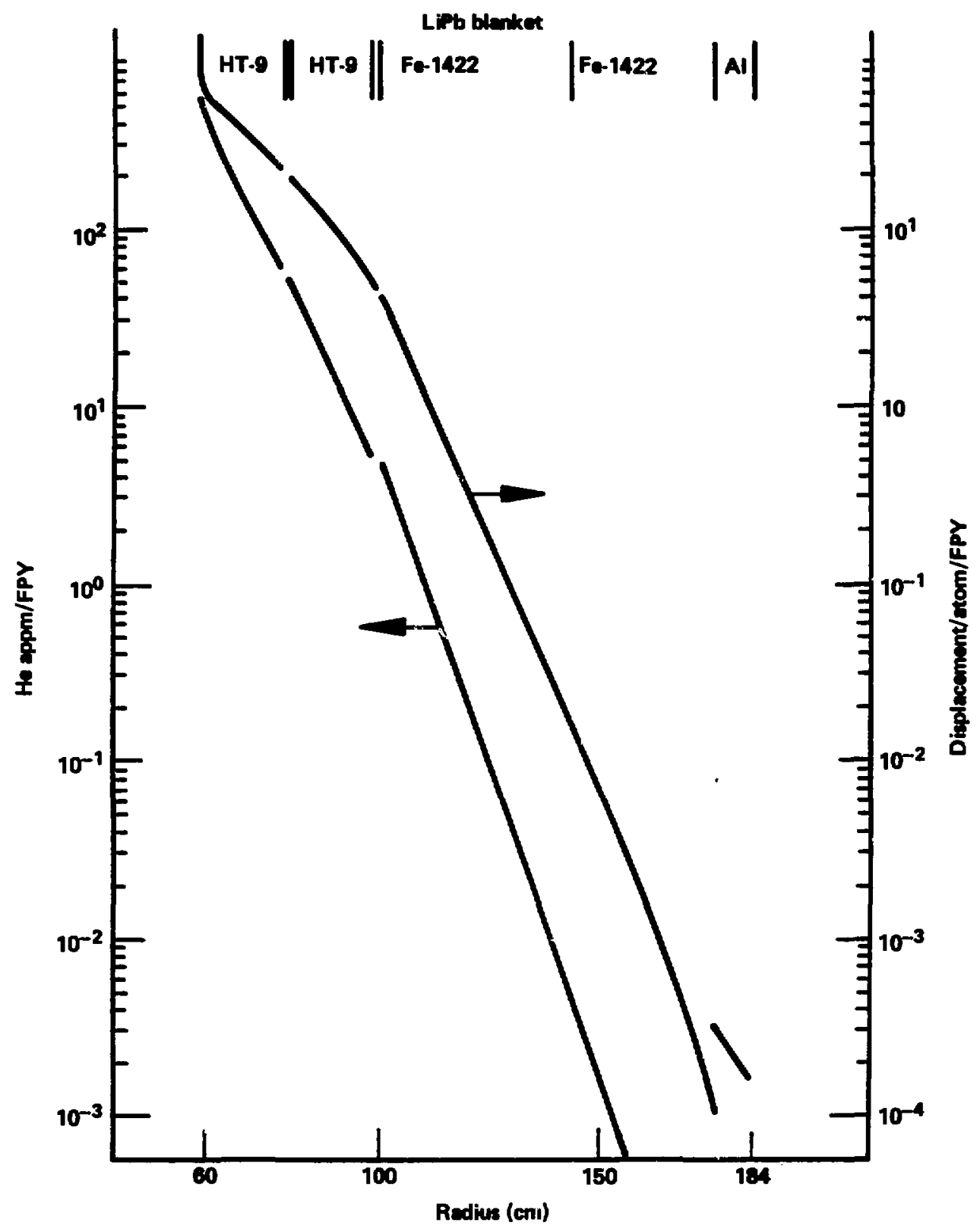

Fig. 4.2-10. Spatial variation of displacement damage and helfum production in MARS. 


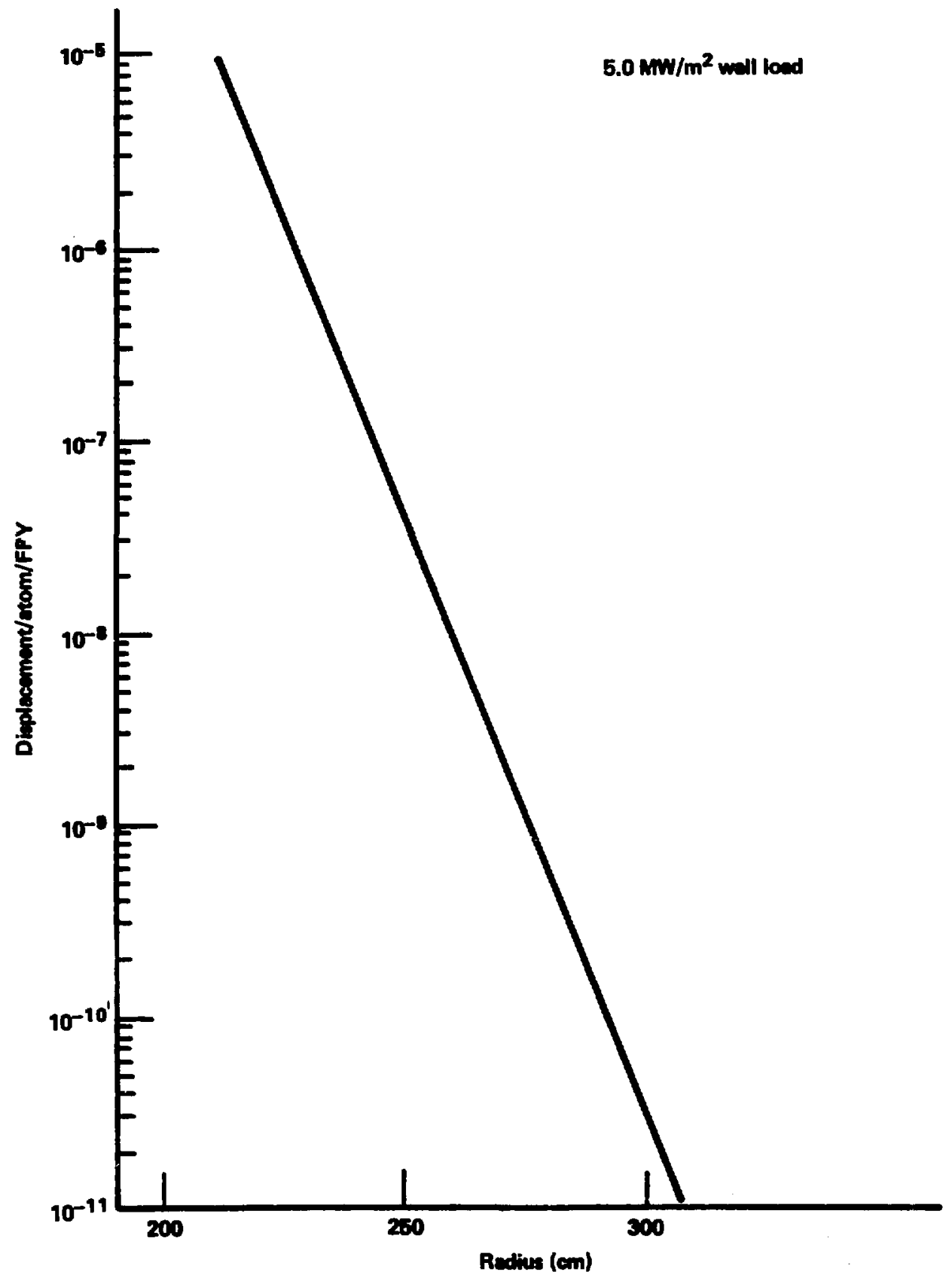

Fig. 4.2-11. Spatial variation of displacement damage in copper stabilizer in MARS central-cell solenoid magnets. 
The maximum damage rate in the Fe-1422 is 3.8 dpa/FPY and 4.9 appm He/FPY at $300^{\circ} \mathrm{C}$. Without high surface heat fluxes or cycling there is ro apparent reason why this steel cannot achieve useful lifetimes of $\sim 100$ dpa at such a low temperature. This means that it will be reasonable to expect a full reactor lifetime ( 24 FPY) for the reflector and shield.

The damage rate of $9 \times 10^{-6} \mathrm{dpa} / \mathrm{FPY}$ in the copper stabilizer is sufficient to achieve 12 FPY of operation before annealing is required (at $1.1 \times 10^{-4} \mathrm{dpa}$ ). Even assuming $80 \%$ recovery for each anneal means that only three anneals are required in the 24 FPY of reactor lifetime.

The low damage level in the NbTi superconductor $\left(\sim 8.9 \times 10^{-6} \mathrm{dpa} / 24 \mathrm{FPY}\right)$ indicates that we should expect full reactor lifetime from the magnet. The threshold for property degradation in $\mathrm{NbTi}$ is $\sim 10^{-3} \mathrm{dpa}$.

Finally, the maximum dose in the polyimide magnet insulation is $4.3 x$ $10^{7} \mathrm{rad} / \mathrm{FPY}$. The cumulative damage is only $1 \times 10^{9}$ rad in $24 \mathrm{FPY}$ which is well below the design limit of $5 \times 10^{9} \mathrm{rad}$.

In sumary, there appear to be no major lifetime problems in the central cell blanket, reflector, shield or magnet structures. Scheduled replacement of the blanket moduies on a 2 to 3 year cycle does not appear to be too restrictive from an avallability standpoint.

\subsubsection{Magnetic Effects on Structural Materials}

The use of HT-9, wich has a high resistance to radiation damage, should greatly increase the operating life of the MARS blanket. However, because this alloy is ferromagnetic, one must be concerned about the effect of the ferritic structure on the magnetic field and the effect of the magnetic field on the ferritic structure. It is well known that the presence of a ferromagnetic material in a magnetic field will change the shape of the magnetic fleld in a way that depends on the geonetry and the magnetization of the structure. Relatively small changes in the field ripple can cause enhanced particle leakage and possibly reduce the stability of the plasma. It is also knom that the ferromagnetic structure will be subjected to magnetic forces and moments if placed in a magnetic field. Such forces can require a substantial amount of support material. Therefore, an accurate description of the magnetic loading is required from both safety and economic points of view. 
Magnetic Forces. The forces on a magnetic body depend on the magnetization of the body and the gradient of the field within the bodyc In regions where the field is above $1.5 \mathrm{~T}$, the HT-9 structure will be magnetically saturated independent of its orientation with respect to the field. Therefore, in these regions, the magnitude of the magnetization will be almost constant and the force variations will depend mainly on the gradient of the magnetic field and the direction of the magnetization. The latter quantity depends on the direction of the field.

Two cases fllustrate the magnitude of the magnet force variations. The inlet coolant pipe for the liquid metal blanket was placed in two different locations. It has an inner diameter of $43 \mathrm{~cm}$ and thickness of $0.6 \mathrm{~cm}$, and is simulated by a solid bean of square cross section $9.1 \times 9.1 \mathrm{~cm}, 1 . e$. , an equivalent volume of the iron. The beam is $460 \mathrm{~cm}$ long. The bean and the field along its center are shown in Fig. 4.2-12, and the calculated magnetic forces per unit length are shown in Fig. 4.2-13. In this figure the forces are relative to the magnitude of the weight of HT-9 per unit length. The large arrow in Fig. 4.2-13 represents the magnitude of the weight per unit length of the LiPb coolant that would fill the original pipe (in the same units). In this case, the magnetic force could have a maximum value silghtly larger than the weight of the coolant.

When the position of the pipe is raised to that shown in Fig. 4.2-14, the magnetic forces on the corresponding beam are much smaller as show in Fig. 4.2-15.

The general conclusions drawn from these preliminary calculations are: the forces on such a beam could vary according to its location; the force distribution must be known to design the appropriate support structure; the magnitudes of these forces are not very high compared to those al ready associated with the liquid-metal-filled pipe itself.

Field Perturbation. As stated previously, the magnetic field that confines the plasma will be perturbed by the presence of the ferromanetic steel. These perturbations can be categorized as either azimuthal or axial. The azimuthal perturbation will most probably be caused by a nonuniform azimuthal distribution of the HT-9; the axial perturbation is merely an end 


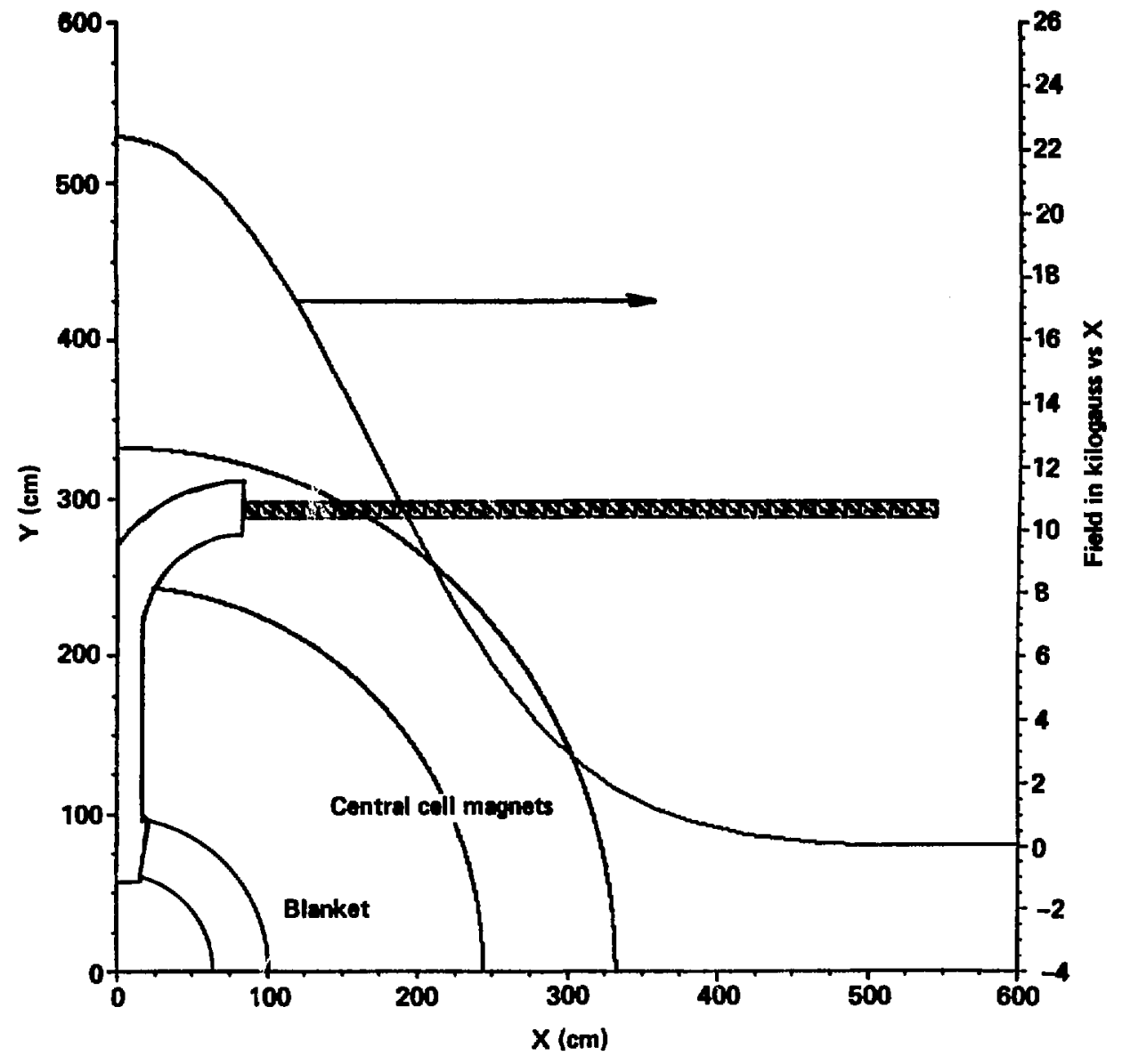

Fig. 4.2-12. The first position of the beam and the magnetic field along its axis. 


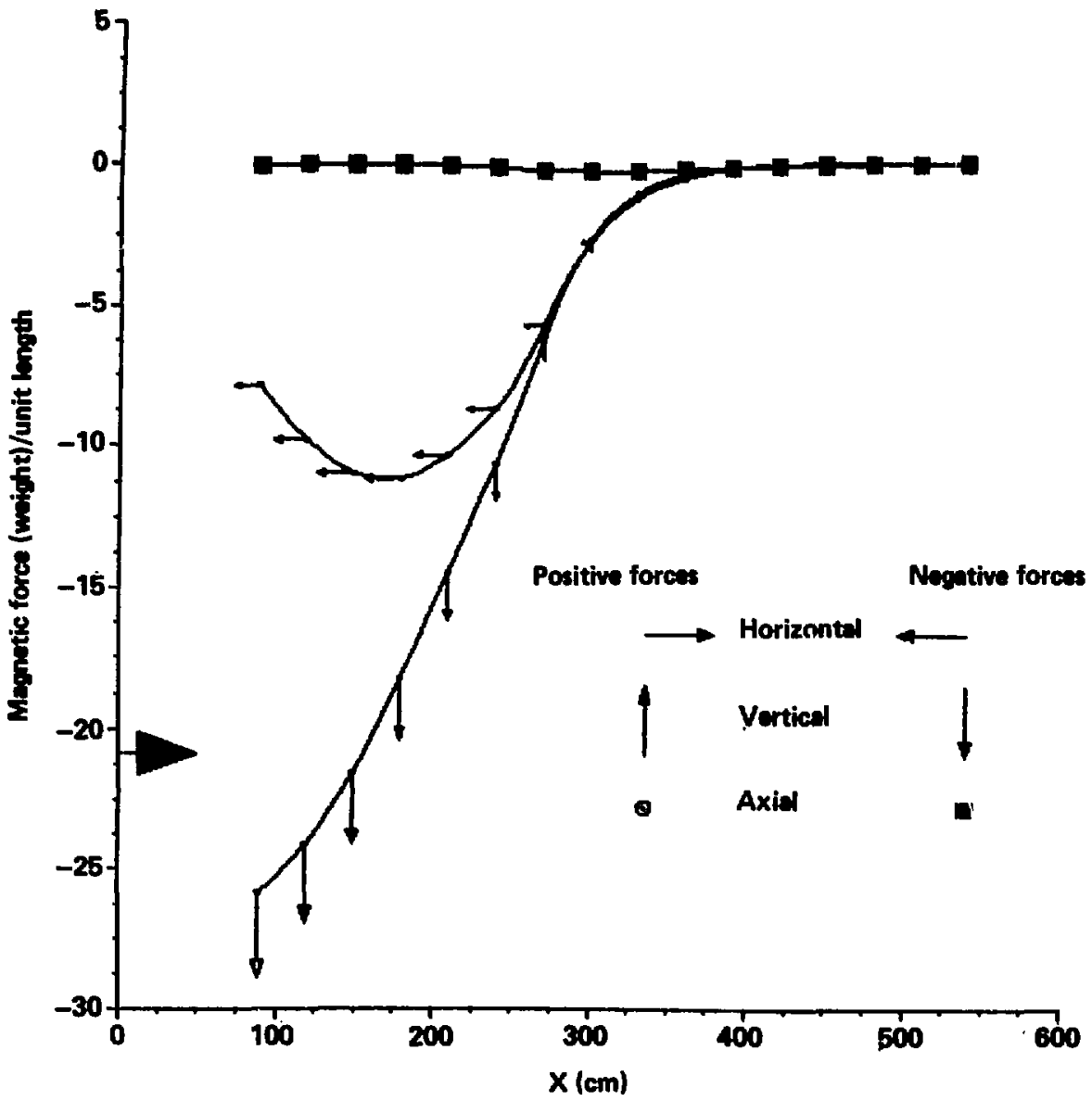

Fig. 4.2-13. The force distribution on the beam shown in Fig. 4.2-12. The large arrow represents the magnitude of the weight per unit length of $L i P b$ that would fill the original pipe.

$4-49$ 


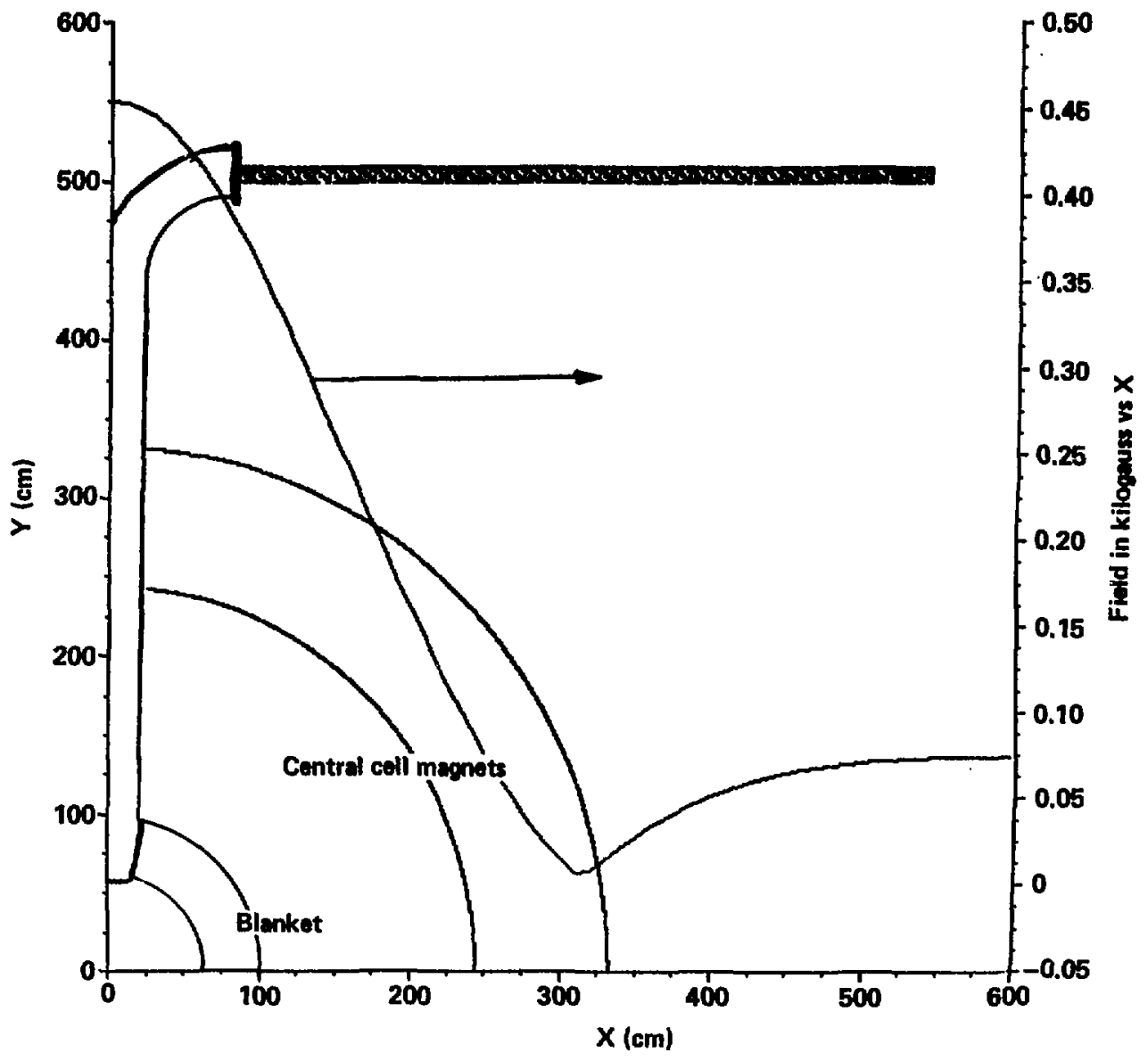

Fig. 4.2-14. The second position of the bean and the magnetic field along its axis. 


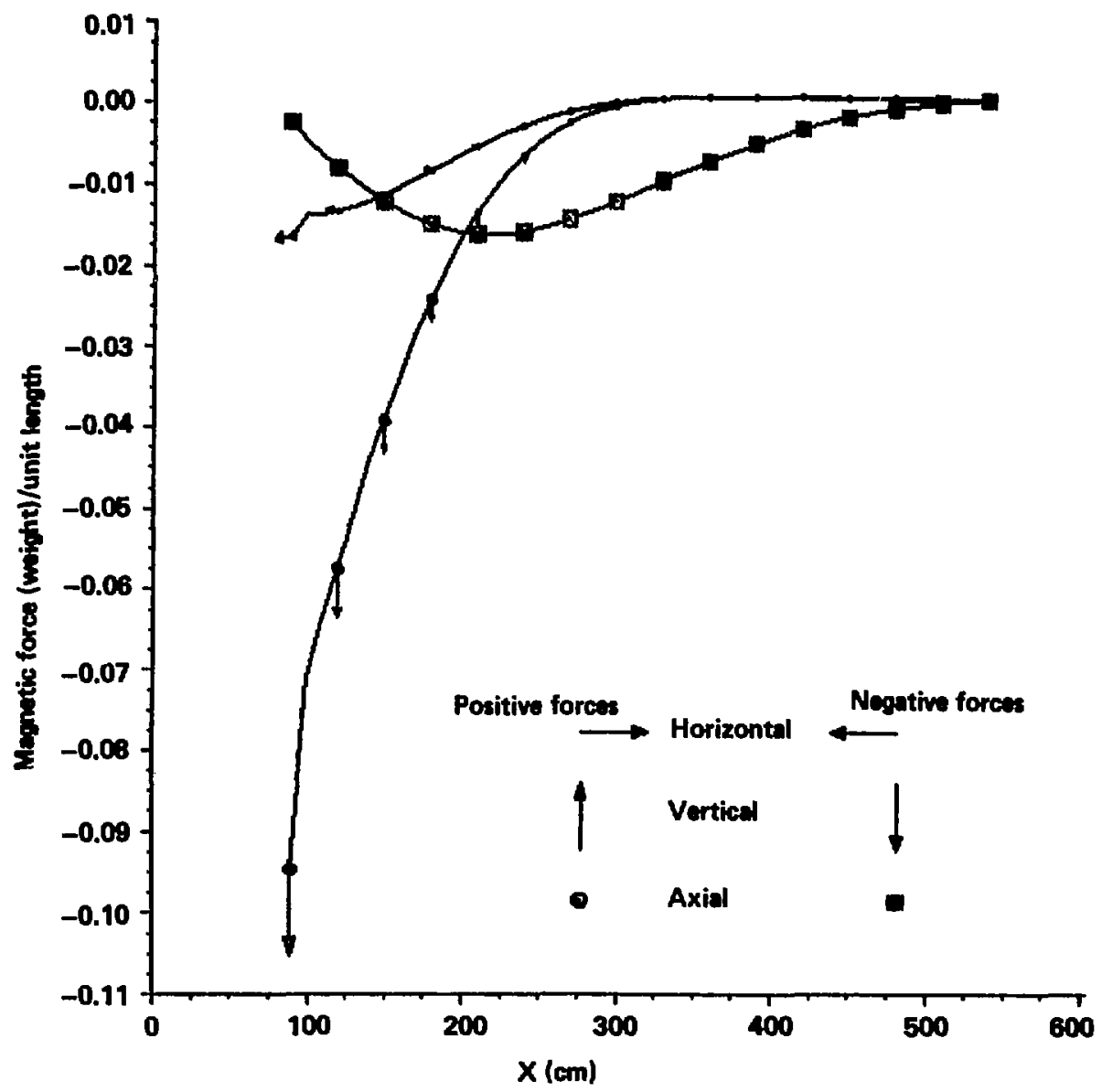

Fig. 4.2-15. The force distribution on the bean shown in Fig. 4.2-14. 
effect that depends on the length of the blanket (see Ref. 16 for a detailed description). In FY 1983 we will calculate the field ripple associated with the MRS blanket, but at this time we expect it to be less than $4 \%$. 


\subsubsection{Shield and Radioactivity Considerations}

\subsubsection{Design Criterla}

Shielding is required to protect the central cell solenoids from neutron and gama radiation. The blanket is a poor attenuator of low energy neutrons because of the large volume fraction of lead, and a separate shield is needed.

The shielding requirements for the central cell magnets are determined by a number of radiation linits. The damage to the copper stabilizer should not exceed $1.1 \times 10^{-4}$ dpa before the first anneal. This corresponds to a $20 \%$ increase in resistivity. Annealing at roon temperature results in about $80 \%$ recovery of radiation induced defects. 17 For an estimated reactor 1 ife of 24 FPY and 5 FPY before the first anneal, at least 1 FPY is avallable between the final two anneals. Therefore, the damage rate in $\mathrm{Cu}$ should not exceed $2.2 \times$ $10^{-5} \mathrm{dpa} / \mathrm{FPY}$.

Mechanical strength tests of irradiated magnet insulacors have shown that polyimides are 5 to 10 times more radiation-resistant than comparably prepared epoxies. 18 polyimide is used as an electrical insulator in the MaRS central-cell coils. The radiation effects on the organic insulators used in the magnets are not reversible. Hence, it is essential that they last for the whole reactor life. The design criterion used for polyinide is that the radiation dose should not exceed $5 \times 10^{9}$ rad after 24 FPY. The 11 mit on the peak heat load in the magnet winding pack is determined by the refrigeration requil rements and is taken to be $0.06 \mathrm{~nm} / \mathrm{cm}^{3}$.

Although these constraints adequately protect the radiation-sensitive components of the magnets, other considerations influence the blanket and shield design. One of these is the desire to minimize long-term radioactivity in the system. Previous experience has shown that nickel in structural materials produces significant long-term radioactivity. Consequently, we have selected Fe-1422 as the basic structural material for the blanket and shield because it is a low-nickel high-manganese steel.

The final design constraint is the requirement for hands-on maintenance after shutdown. The selection of an allowable dose at the back of the shielu is based on the limits set in part 20 of chapter 10 of the Code of Federal 
Regulations (10CFR20). Stated very briefiy, these regulations require that the dose to workers in a restricted area be kept as low as possible and no greater than $1.25 \mathrm{rem} / \mathrm{calendar}$ quarter. This dose rate is equivalent to a continuous exposure of $100 \mathrm{mrem}$ for a $40-\mathrm{hr}$ work week, or $2.5 \mathrm{mrem} / \mathrm{hr}$. In interpreting these requirenents, considered several aiternative criteria both with regard to dose and to the time after a shutdown at which the exposure would start. The alternative criterla considered were (1) 2.5 $\mathrm{mrem} / \mathrm{hr}$ after 1 day, (2) $2.5 \mathrm{mrem} / \mathrm{hr}$ after 2 days, (3) $10 \mathrm{mrem} / \mathrm{hr}$ after 1 day, (4) $10 \mathrm{mrem} / \mathrm{hr}$ after 2 days, and (5) $100 \mathrm{mrem}$ for 5 working days after 1 to 2 days. Although the first criterion is preferabie because it permits relatively quick access and low dose, it is overly restrictive. Both 10-mrem criteria were rejected since they could result in excessive doses. We adopted the last criterion (100 mrem for 5 working days after 1 to 2 days) for this design because it satisfyies the limits set in 10CFR20 for the first week, results in lower doses for subsequent pertods, and still permits reasonably prompt access for servicing.

\subsubsection{Neutronics}

We performed one-dimensional neutronics calculations to determine the thickness of the shield required to protect the magnets. We considered a water-cooled shield consisting of layers of Fe-1422, $B_{4} C$, and lead. The calculations were performed with $A$ ANISN $^{19}$ using a $\mathrm{P}_{3}-\mathrm{S}_{8}$ approximation. A coupled 46-neutron 21-gamma-group cross section set collapsed from the 'RSIC DLC-41B/VITAMIN-C 11 brary, 20 and the DLC-60B/MACKLIB-IV-82 response '1 ibrary 21 was used. These data libraries are based on the ENDF/B-IV evaluation.

The model used for the magnet shielding calculations is show in Fig. 4.2-16. The magnet case, cryostat, and vacuum dewar were included in the model as $6.3 \mathrm{~cm}$ of stainless steel. The shield consists of four zones. Shield $A$ is used primarily to attenuate and moderate the high-energy neutrons that penetrate the blanket and reflector. The lead in shield $B$ effectively attenuates gammas produced in the stetl structure of the blanket, reflector, and shield. The boron carbide in shield $C$ thermalizes neutrons and subsequent neutron absorption in boron produces only a soft $0.5-\mathrm{MeV}$ gama and an easily absorbed alpha particle. The Fe-1422 structure used in shields $B$ and $C$ will 

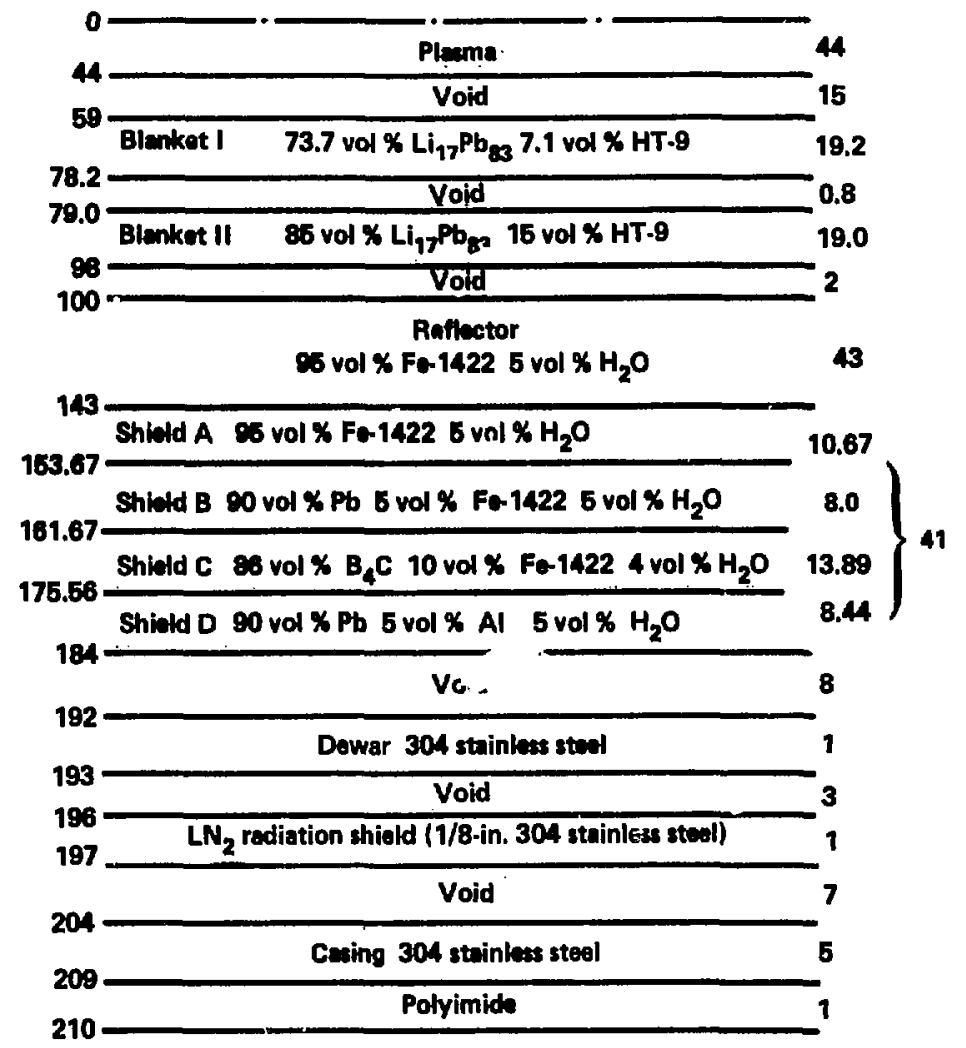

Winding peck

35 vol \% 304 stainleses sted 31.5 vol \% Cu

15 vol $\%$ polyimide

117.3

15 vol \% LHe

3.6 vol \% NbTi

327.3

Redius (cm)

Thickness (cm)

Fig. 4.2-16. Geonetrical model for magnet shielding calculations. 
help attenuate the high-energy neutrons that succeed in penetrating that deep in the shield. Shield D is required primarily i,o attenuate the gammas resulting from radioactive decay of activated materials in the reactor. A1 6061 is used as a structural material in shield $D$ to reduce the activation and to yield acceptable biological doses at the back of the shield after shutdown, as discussed in Secton 4.2.2.5. Aithough a smaller thickness of this shield zone can provide adequate protection for the magnets, a zone thickness of 8.44 $\mathrm{cm}$ is used based on radioactivity considerations.

Using the shield configuration shown in Fig. 4.2-16, we obtained a peak damage rate of $8.94 \times 10^{-6}$ dpa/FPy in the copper stablitizer. This implies that the first magnet anneal is required aicer 12 FPY. its pes:s radiation dose in the polyimide insulator after 24 FPY is $1.0 \times 10^{9}$ rad, which is well below the design limit of $5 \times 10^{9} \mathrm{rad}$. The peak heat load in the magnet is $0.05 \mathrm{mH} / \mathrm{cm}^{3}$, whici. is also lower than the specified limit. Therefore, the shield configuration used provides adequate magnet protection with a comfortable margin that allows for possible data uncertalnties and calculational deficiencies.

\subsubsection{Structural Design}

One possible design for the 41-cm-thick shield is shown in Fig. 4.2-17. The jockets in the shield are filled with canned slabs of $B_{4} C$ and lead, with spaces in between for cooling water. The pockets are then capped off and welded shut.

Nuclear heating in the shield will be low enough to allow low-velocity and low-pressure water cooling. Thus, water traversing one shleld pocket from one end to the other will be routed onto the next, snaking its way around the shield. In this way a minimum number of coolant connections will be necessary.

Critical areas in the design are:

1. The penetrations in the shield required for coolant supply pipes.

2. Shield cutting positions for unexpected component replacement (see Section 4.2.6). 


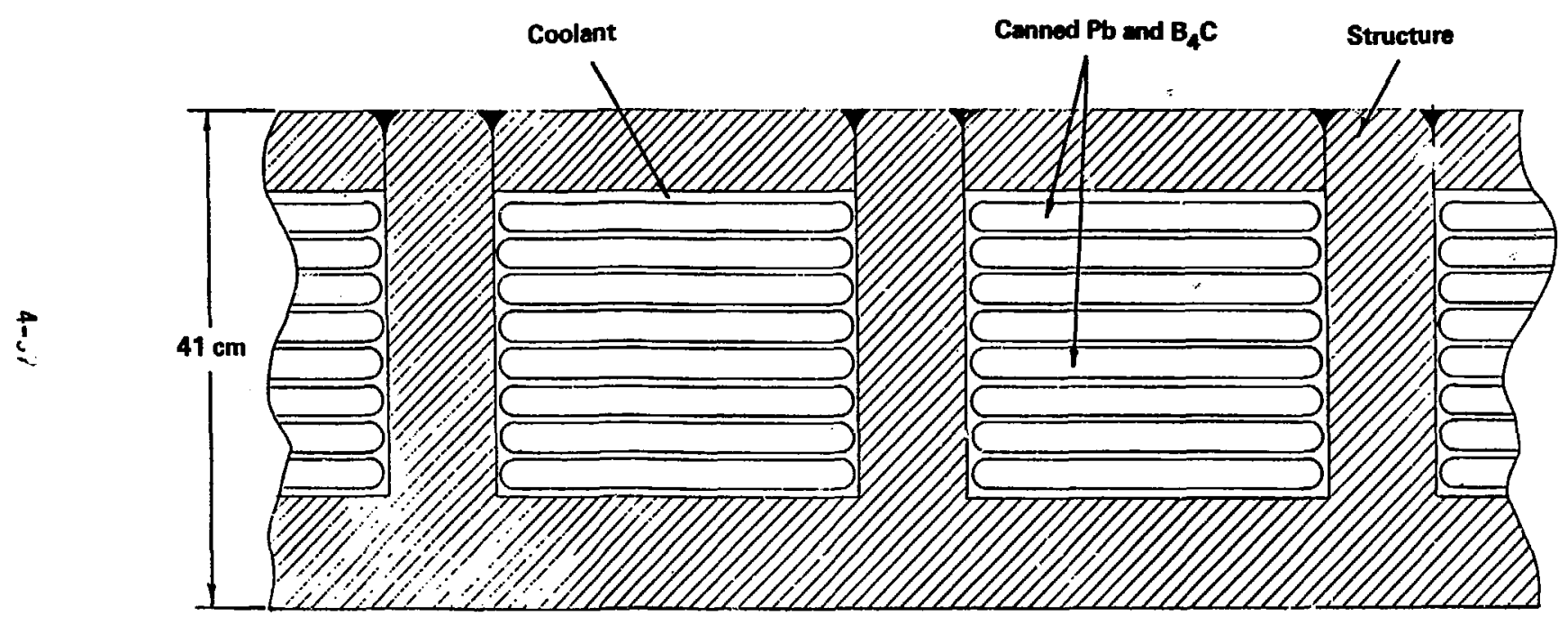

Fig. 4.2-17: A possible design option for the shield structure. 
3. The bulk shield and built-in reflector in the servising stations (see Section 4.2.6).

The shield should be firmly supported, since it also carries the weight of the blanket modules and reflector structure. We expect that the support structure will resemble the magnet support structure. Both of them should be able to move either in the axial direction or in the transverse direction for installation and maintenance.

\subsubsection{Thermal/Hydraulics}

The shield is cooled by water entering at $50^{\circ} \mathrm{C}$ and exiting at $80^{\circ} \mathrm{C}$. The maximum water temperature is kept below $100^{\circ} \mathrm{C}$ to avoid pressurized containment. The total thermal energy deposited in the shield is $150 \mathrm{~m}$. Shield parameters are sumarized in Table 4.2-12.

\subsubsection{Radioactivity}

The neutron fluxes from Sections 4.2.1.3 and 4.2.2.2 were used with the DKR code to calculate the activity of the blanket and shield after 2 FPY. The results of these calculations are summarized in Fig. 4.2-18. There is a fairly rapid initial decrease corresponding to the decay of $56_{\mathrm{mm}}\left(t_{1 / 2}=i=38\right.$ $h r)$. Subsequently, the activity is dominated : $y 4_{\mathrm{Mn}}\left(t_{1 / 2}=312 \mathrm{~d}\right)$ followed by ${ }^{55} \mathrm{Fe}\left(t_{1 / 2}=2.7 \mathrm{yr}\right)$. In terms of activicy per unit volume of material, the average activity after $10 \mathrm{yr}$ is $1.7 \mathrm{ci} / \mathrm{cm}^{3}$ but is reduced to $2.8 \times 10^{-3} \mathrm{ci} / \mathrm{cm}^{3}$ after $100 \mathrm{yr}$. The activity at long times is doninated by activities from molybdenum, specifically $\left.93_{\mathrm{Mo}}\left(\mathrm{t}_{1 / 2}=3 \times 10^{3} \mathrm{yr}\right),{ }^{92_{\mathrm{Hb}}\left(t_{1 / 2}\right.}=2 \times 10^{7} \mathrm{yr}\right)$, and ${ }^{99} \mathrm{Tc}\left(t_{1 / 2}=2 \times 10^{5} \mathrm{yr}\right)$.

With the activity levels of the various isotopes calculated as a function of position in the bianket and shield, we used ANISN to calculate the gamma dose rate at the outer edge of the shield. As mentioned in Section 4.2.2.2, al though the original shleid design provides adequate protection for the magnets, it does not give adequate biological protection. The calculated dose rates were $250 \mathrm{mrem} / \mathrm{hr}$ after 1 day, $70 \mathrm{mrem} / \mathrm{hr}$ after 2 days, and $60 \mathrm{mren} / \mathrm{hr}$ after 1 meek. To reduce this dose rate, we added $10 \mathrm{~cm}$ of shielding (95\% Pb, 5\% Fe-1422), which reduced the dose rates to $18 \mathrm{mrem} / \mathrm{hr}$ after 1 day, $10.5 \mathrm{mrem} / \mathrm{hr}$ after 2 days, and $10.3 \mathrm{mrem} / \mathrm{hr}$ after 1 week. While this was a significant improvement, it was still not adequate to meet the design criter- 


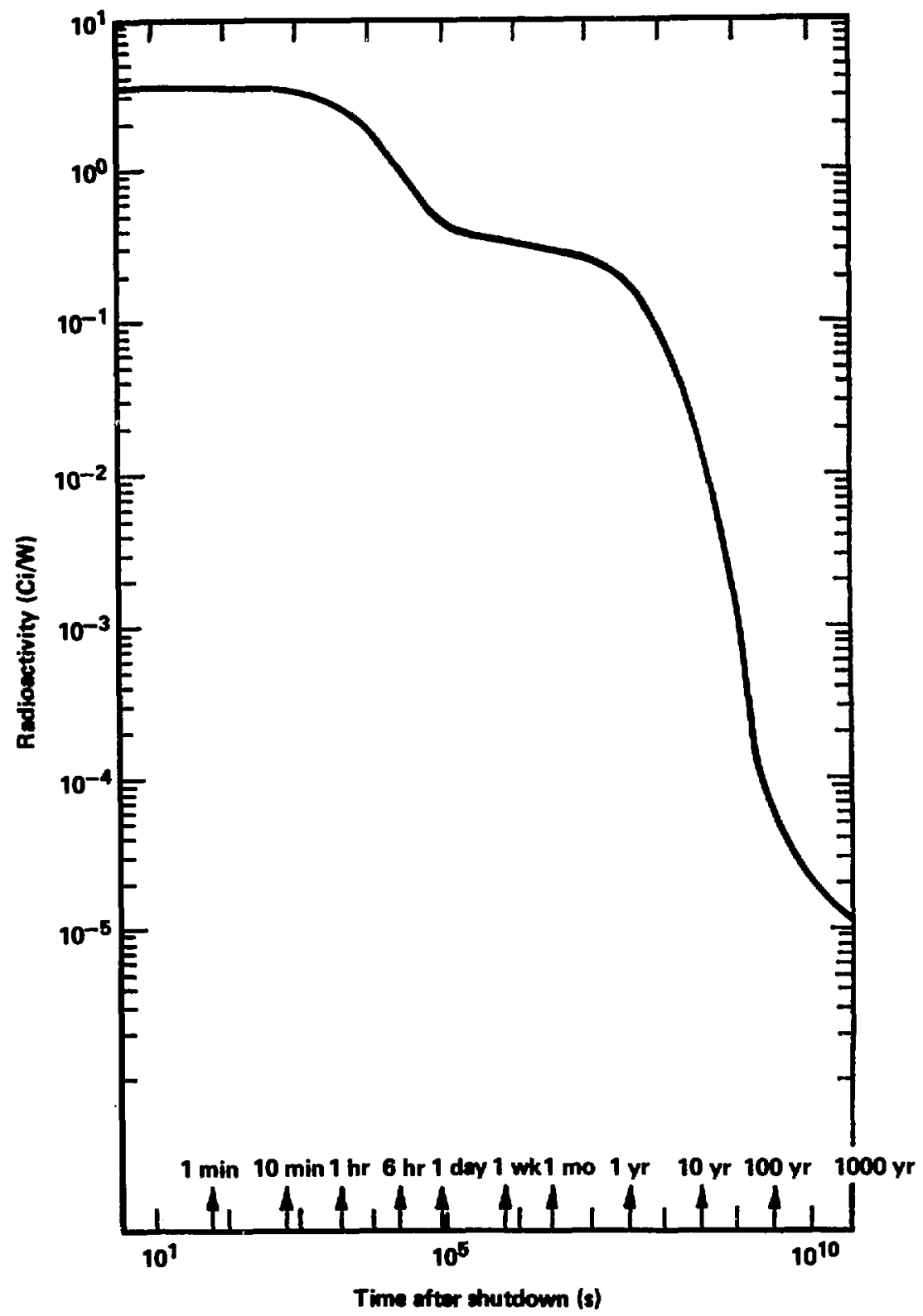

Fig. 4.2-18. Radioactivity vs time after shutdown following two years of operation. 
Table 4.2-12. Thermal hydraulic paraneters for the shield.

Thermal power (IN)

Coolant

Coolant temperature $\left({ }^{\circ} \mathrm{C}\right)$

Coolant flow rate $(\mathrm{kg} / \mathrm{hr})$

Maximum water temperature $\left({ }^{\circ} \mathrm{C}\right)$

Maximum shield temperature $\left({ }^{\circ} \mathrm{C}\right)$

Maximum coolant velocity $(\mathrm{m} / \mathrm{s})$

Maximum coolant pressure drop ( $\mathrm{kPa})$

Coolant pumping requirenent (kW)
150

$\mathrm{H}_{2} \mathrm{O}$

$50 / 80$

$4.3 \times 10^{6}$

90

140

1

20

10 
Ion. Previous studies have shown that in many cases the activated material in the outer part of the shield contributes significantly to the radiation dose. We confirmed this in our study by running a case in wich the structural material in the outer region of the shield was concentrated on the inner side of that region. The resulting dose was further reduced to $6.8 \mathrm{mrem} / \mathrm{hr}$ after 1 day and only $0.5 \mathrm{mrem} / \mathrm{hr}$ after 2 days. Because this arrangement of the structural material would not be satisfactory from a mechanical design point of view, we replaced the Fe-1422 structure with Al 6061 in the outer region of the shield. Subsequent calculations shawed that the dose rate for this arrangement would be $34 \mathrm{mrem} / \mathrm{hr}$ after 1 day, $11.6 \mathrm{mrem} / \mathrm{hr}$ after 2 diys, and $0.6 \mathrm{mrem} / \mathrm{hr}$ after 1 week. The major contributor to the dose during this tim period is ${ }^{24} \mathrm{Ma}\left(\mathrm{t}_{1 / 2}=15 \mathrm{hr}\right)$. Calculating the integrated dose for a 40-hr work week showed that a 100-mrem dose would be obtained if entrance was delayed to 2.3 days after shutdown. Thus, by adding $10 \mathrm{~cm}$ of shielding and replacing Fe-1422 with $A 16061$ in the outer layer, the criterion of $10 \mathrm{mrem} / \mathrm{hr}$ could be met after silightly more than 2 days. More importantly, the linit of 100 mrem in 1 working week could be met after 2.3 days and any subsequent doses received would be significantly smailer.

It must be noted that the activation of materials near the edge of the shield plays an important role in determining radiation levels. Consequently, we expect that activating the components outside the shield will also contribute to the radiation level. Chief among these components are the magnets as well as their assoctated dewars and support structures. This problen is complex because of the geometry of the systen and we cannot attempt to analyze it until these components are more completely designed. Thus, their contribution of materiais near the edge of the shield to the radiation level is undetermined but must be considered before the shieid design is finished. 


\subsubsection{Blanket Design Optinization}

\subsubsection{Design Philosophy}

The MRS lithlum-lead blanket has a flowing LiPb coolant/breeder contained in a ferritic steel structure. It is simple in concept, easy to fabricate, and provides for convenient maintenance. Given these characteristics, how should the blanket be designed with regard to neutronic and economic performance? For example, what value of tritium breeding ratio is required? How high should the blanket multiplication factor $M$ be? What maximum fraction of the neutron energy should be deposited in the reflector?

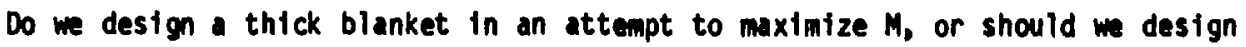
a thin blanket in an attempt to minimize central cell costs?. Would a thin blanket and corresponding thick shield be more expensive than thick blanket with a corresponding thin shield? How do superconducting magnet radiation constraints affect tire overall design, etc?

We examined in detall design questions such as these during the early design phase of the LIPD blanket; we concluded that all constraints and requirements should be integrated into a systematic trade study. Accordingly, we developed a blanket design optimization procedure to assess LiPb blanket designs in terms of their relative economic performance. This procedure is necessarlly iterative and requires that an economic figure of merit be minimized for the overall reactor system.

\subsubsection{Design Optimization Procedure}

We developed the following procedure for blanket design optimization:

1. For the desired tritium breeding ratio (TBR), estabijsh a LiPb blanket and reflector point design.

2. Perform neutronics analyses to obtain the blanket multiplication factor $M$ and energy deposition in the blanket and reflector.

3. Given the blanket and reflector thermal powers, determine the gross efficiency $\eta$ and LiPb pumping power. 
4. Determine both the neutronics design constraints for the central cell magnets (i.e., maximum dpa in the stabilizer, maximum dose limit for the organic insulator, maximum nuclear heating rate) and the required radioactivity conditions at the back of the shield.

5. Given these magnet and radioactivity constraints, design the blanket shielding so that all conditions are satisfied.

6. Given the outer radius of the shield from above, determine the minimum. Inside radius of the central cell magnet winding pack.

7. Specify the magnitude and on-axis ripple (1.e, $\Delta B / B$ ) of the centralcell magnetic field and, given the inside radius of the winding pack from above, determine the magnet winding pack dimensions and centercenter spacing.

8. For these magnet dimensions, ensure that the magnet configuration is consistent with blanket maintenance requirements.

9. Determine the cost of the blanket, reflector, shield, magnets, thermal hydraulic system, turbine plant equipment, and electrical plant equipment (i.e., those items whose costs depend on the blanket multiplication factor and, therefore, the blanket design).

10. Establish a self-consistent economic figure of merit $F$ for overall system optimization.

11. Repeat the above procedure and, by varying the blanket/reflector design, minimize $F$ through iteration.

With regard to this procedure, the following points should be noted:

1. When varying the blanket design in this iterative manner, it is very difficult (if not impossible) to precisely achieve a predetermined tritium breeding ratio (TBR). Accordingly, we specified a desired range for TBR of 1.05 to 1.15 at the outset and taflored subsequent design iterations to lie somewhere in this range.

2. Only the radiation dose limit to the organic magnet insulation is an absolute design constraint. The other radiation limits are tradeoffs of shield cost vs cryogenic systen cost, magnet cost, or cost of lower avallability. 
3. For the LiPb blanket, the limiting constraint on the shield design was imposed by the required radianctivity conditions at the back of the shield.

\subsubsection{Figure-of-Merit Parameter}

It is now necessary to select an appropriate economic figure of merit $F$ for the blanket system so that minimization of $F$ leads to an optimized design. From a cursory view of this problen, it would appear expedient to mininize the capital cost of the central cell. However, a low-cost blanket may yield only modest values of recoverable power. Therefore, a better figure of merit would be to normalize the central cell costs by the recoverable central cell thermal power, i.e.,

where

$$
F_{1}=\frac{C_{c c}}{P_{c c}^{t}}=\frac{C_{c c}}{P_{N}},
$$

$C_{c c}=$ total central cell cosis (blanket, reflector, shield, and magnets),

$P_{c c}^{t}=$ central-cell thermal power, and

$P_{M}=$ fusion neutron power.

Therefore, $F_{1}$ is minimized by minimizing central cell costs while maximizing M.

However, Eq. 4.2-2 is deficient in that it does not account for the thermal conversion efficiency, the costs of the thermal hydraulic system, or the LiPb pumping power. Therefore, it $\mathrm{might}$ be expected that an improved figure of merit would be

$$
F_{2}=\frac{C_{C C}+C_{T H}}{P_{C C}^{e}-P_{P}^{e}}=\frac{C_{C C}+C_{T H}}{P_{N}+H-P_{P}^{e}},
$$

where $M=$ neutron energy multiplication factor,

$$
C_{T H}=\text { cost of thermal hydraulic system, }
$$


$P_{c c}^{e}=$ gross central-cell electric power,

$P_{p}^{e}=$ electric power requitrements of LiPb pumps, and

$\eta=$ thermal cycle effictency.

Again, however, use of $F_{2}$ leads to spurtous conclusions. In particular, the blanket optinization process should seak to minimize the overall capital cost of the reactor through blanket design. This requires that the figure of merit explicitly contain the cost of other reactor items such as the plasma heating systems, inscellaneous plant equipment, direct converter, etc., even though they are not dependent on the blanket design. Accordingly, the figure of merlt chosen for this study is

$$
F=\frac{C_{C C}+C_{T H}+C_{T P}+C_{E P}+C_{M P}+C_{P M}+C_{H}+C_{D C}+C_{B}}{P_{C C n}^{e}+P_{D C}^{e}-P_{R}^{e}},
$$

where

$C_{c c}=$ total central cell costs,

$C_{T H}=$ cost of thermal hydraulic system,

$C_{T P}=$ cost of turbine plant equipment,

$C_{E P}=$ cost of electrical plant equipment,

$C_{\text {MP }}=$ cost of miscellaneous plant equiprent,

$C_{P M}=$ cost of plug magnets,

$C_{D C}=$ cost of di rect convertor,

$C_{H}=$ cost of supplemental heating systems,

$C_{B}=$ cost of bulldings and site factlities,

$P_{c c n}^{e}=$ net electric power from central cell $\left(P_{c c}^{e}-P_{p}^{e}\right)$.

$P_{D C}^{e}=$ electric power from direct convertor and halo convertors, and

$p_{R}^{e}=$ recirculating electrical power requirements for reactor (except LiPb pumps).

Here we define $F$ as the overall capital cost of the reactor normalized to the net electrical output. It is commonly expressed in units of $\$ / k \mathrm{k}_{\text {. }}$. 
Note the following interesting properties of $F$ from Eq. 4.2-3. The first four costs $\left(C_{C C}, C_{T H}, C_{T P}\right.$, and $\left.C_{E P}\right)$ are dependent on the blanket and thermal cycle properties, wile the last five terms $\left(C_{M P}, C_{P M}, C_{H}, C_{D C}\right.$ and $\left.C_{B}\right)$ are independent of the blanket properties. In the denominator, $P_{C C n}^{e}$ is blanketdependent while $P_{D C}^{e}$ and $P_{R}^{e}$ are blanket-independent. However, for a tandem mirror reactor such as MRS, the electrical output from the direct convertor $P_{D C}^{e}$ and the recirculating electric power requirements $P_{R}^{e}$ are comparable in magnitude and approximately cancel each other in Eq. 4.2-3. It is therefore possible to represent this figure of merit by

$$
F=\frac{C+C^{\prime}}{P_{c c n}^{e}}
$$

where

$$
\begin{aligned}
C & =\text { cost of all blanket-dependent items, } \\
C^{\prime} & =\text { cost of all non-blanket-dependent items, and } \\
P_{C C n}^{e} & =\text { net electric power from central cell. }
\end{aligned}
$$

Note that now there is a large term $C$ that is independent of the blanket multiplication factor $M$ and thermal cycle efficiency $n$, while the denominator $P_{c c n}^{e}$ is directly proportional to $M_{n}$. The figure of merit parameter $F$ is, therefore, quite sensitive to changes in $p_{c c n}^{e}$ due to changes in $M_{n}$. It is not as sensitive to changes in the blanket-dependent costs $C$ because of the large constant term C'. Basically, we wish to amortize the end cell costs over a central cell that produces as much electricity as possible. Additional details of this methodology are given in Ref. 22.

\subsubsection{Figure-of-Merit Application}

We studied the performances of two near-optinum designs for the LIPb blanket and reached two main conclusions. First, maximum values of the product of $M_{n}$ (and thus maximum values of $p_{c C}^{e}$ ) are obtained for a blanket with a large radial thickness and a natural isotopic composition of lithium in the LiPb coolant/breeder ("thick blanket"). Second, the cost of the blanketdependent items is minimized for a blanket with small radial thickness and highly enriched lithium ("thin blanket"). However, the thin blanket has a 
lower $M$ than the thick blanket and, in addition, has a higher fraction of the total neutron energy deposition in the reflector, thus resulting in a lower value of $n_{0}$ Further details of these neutronics analyses are found in Section 4.2.1.3. The optimum blanket is, therefore, that which minimizes the figureof-merit parameter $F$ in terms of overall $\$ / \mathrm{kW}_{\mathrm{e}}$.

Table 4.2-13 lists the central cell parameters for the thick and thin blankets. Table 4.2-14 itemizes approximate costs for those items that depend on the blanket design and, therefore, on the parameters $M$ and $\eta_{0}$. Note that the central cell costs are dominated by the magnets as is the difference between the total blanket-dependent costs. Table 4.2-15 shows an approximate cost breakdown for the blanket-independent itens in the reactor system. These figures are based on rough estimates of direct capital costs and should not be taken as final or definitive cost figures for MARS.

Table 4.2-16 sumarizes the economic tradeoff using the figure-of-merit paraneter $F$. Table 4.2-15 shows that $F$ for the thin blanket is $\sim 2.4 \%$ smaller than $F$ for the thick blanket: This is a small but significant difference and indicates that the thin blanket is an improved blanket design. It might be argued that, because of the uncertain costs of the blanket-independent items in Table 4.2-16, a 2.4\% difference in $F$ is not significant. However, Fig. 4.2-19 shows the ratio of $F$ for the thin blanket to that of the thick blanket plotted as a function of the cost of the blanket-independent items ( $C^{\prime}$ ). Although this ratio remains less than unity, the thin blanket is economically preferable. The present design point of $C^{\prime}=1610$ with an F-parameter ratio of 0.976 is also indicated. To change the conclusion as to which blanket is preferred, the magnitude of $C^{\prime}$ would have to change by a factor of about 2.7 to $\sim 4375 \$ M$. The uncertainty in the ratio of the $F$ parameter is, therefore, very insensitive to the uncertainty in $\mathrm{C}^{\prime}$. 
Table 4.2-13. LiPb blanket paraneters.

\begin{tabular}{|c|c|c|}
\hline & $\begin{array}{c}\text { Thin } \\
\text { blanket }\end{array}$ & $\begin{array}{c}\text { Thick } \\
\text { blanket }\end{array}$ \\
\hline Blanket thickness (m) & 0.382 & 0.872 \\
\hline Reflector thickness (m) & 0.43 & 0.28 \\
\hline Shield thickness (m) & 0.41 & 0.44 \\
\hline Instde radius of magnet winding pack (m) & 2.10 & 2.48 \\
\hline Winding pack dimenstons $\left(m^{3}\right)$ & $0.78 \times 0.78$ & $0.90 \times 0.90$ \\
\hline CC magnet spacing (m) & 2.75 & 3.225 \\
\hline $6_{\text {LI }}$ enrichment $(x)$ & 90 & Nat. \\
\hline Fraction of eneray in reflector (x) & 27.6 & 13.4 \\
\hline Blanket multiplication $M$ & 1.390 & 1.374 \\
\hline Tritium breeding ratio & 1.135 & 1.078 \\
\hline Gross cycle efficiency $\eta(x)$ & 40.0 & 41.7 \\
\hline \multirow[t]{2}{*}{$M \times \eta$} & & 0.556 \\
\hline & & 0.572 \\
\hline LiPt pumping power ( $\left(W_{e}\right)$ & 54.7 & 63.6 \\
\hline Net cycle effictency (x) & 38.6 & 40.0 \\
\hline CC thermal power $\left(w_{t}\right)$ & 3892 & 3847 \\
\hline CC gross electrical power $\left(\mathrm{NW}_{\mathrm{e}}\right)$ & 1556 & 1603 \\
\hline CC net electrical power $\left(M W_{e}\right)$ & 1501 & 1539 \\
\hline
\end{tabular}


Table 4.2-14. Costs for blanket-dependent items.

\begin{tabular}{|c|c|c|}
\hline & $\begin{array}{c}\text { Thin } \\
\text { blanket } \\
\end{array}$ & $\begin{array}{c}\text { Thick } \\
\text { blanket }\end{array}$ \\
\hline Blanket cost $/ m(\$ M / m)^{a}$ & 0.241 & 0.425 \\
\hline Reflector cost $/ m(\$ M / m)^{a}$ & 0.356 & 0.312 \\
\hline Shield cost/m $(\$ M / m)^{a}$ & 0.207 & 0.272 \\
\hline Magnet cost $/ m(S M / m)^{a}$ & 2.060 & 2.602 \\
\hline Total CC cost/m $(\$ M / M)^{a}$ & 2.863 & 3.611 \\
\hline Total $C C$ cost $(\$ N)^{a}$ & 429.5 & 541.7 \\
\hline Thermal hydraulic system $(\$ M)^{b}$ & 262.5 & 279.1 \\
\hline Turbine plant equipment $(\$ M)^{c}$ & 262.7 & 266.8 \\
\hline Electric plant equipment $(\$ M)^{c}$ & 158.1 & 161.2 \\
\hline Total (\$M) & 1113 & 1249 \\
\hline
\end{tabular}
a Costed on a consistent \$/unit-mass basis.
b Costed on a \$/unit-mass basis with suitable representative costs for values, pumps, double-walled $H X$, etc.
c Costed via algorithms from STARFIRE 23 
Table 4.2-15. Costs for blanket-independent items. (All costs are in $\$ 4)$.

\begin{tabular}{llc}
\hline & Subsystem & System \\
\cline { 2 - 3 } & & \\
Miscel laneous plant equipment & & 150 \\
Piug magnets & & 250 \\
Direct convertor & & 142 \\
Bufldings and site :acflfties & $30 n$ \\
Heating systems & 240 & 768 \\
ECRH & 528 & \\
Neutral beams & & 1610 \\
Total blanket-independent items & & \\
\hline
\end{tabular}


Table 4.2-16. Sumary of blanket optimization.

\begin{tabular}{cc}
$\begin{array}{c}\text { Thin } \\
\text { blanket }\end{array}$ & $\begin{array}{c}\text { Thick } \\
\text { blanket }\end{array}$ \\
\hline
\end{tabular}

Blanket-dependent items (SM)

1113

1249

Blanket-independent items (\$M)

1610

1610

Total direct costs (\$M)

2722

2858

Blanket net electrical power $\left(\mathrm{MH}_{\mathrm{e}}\right)$

1501

1539

Direct converter electric power $\left(M_{e}\right)$

565

565

Recirculating electric power $\left(\mathrm{MH}_{\mathrm{e}}\right)$

527

527

System net electric power $\left(m_{e}\right)$

1529

1577

Figure of merit $\left(\$ / \mathrm{kW}_{\mathrm{e}}\right)$

1769

1812

Normalized figure-of-merit

1.0

1.024 


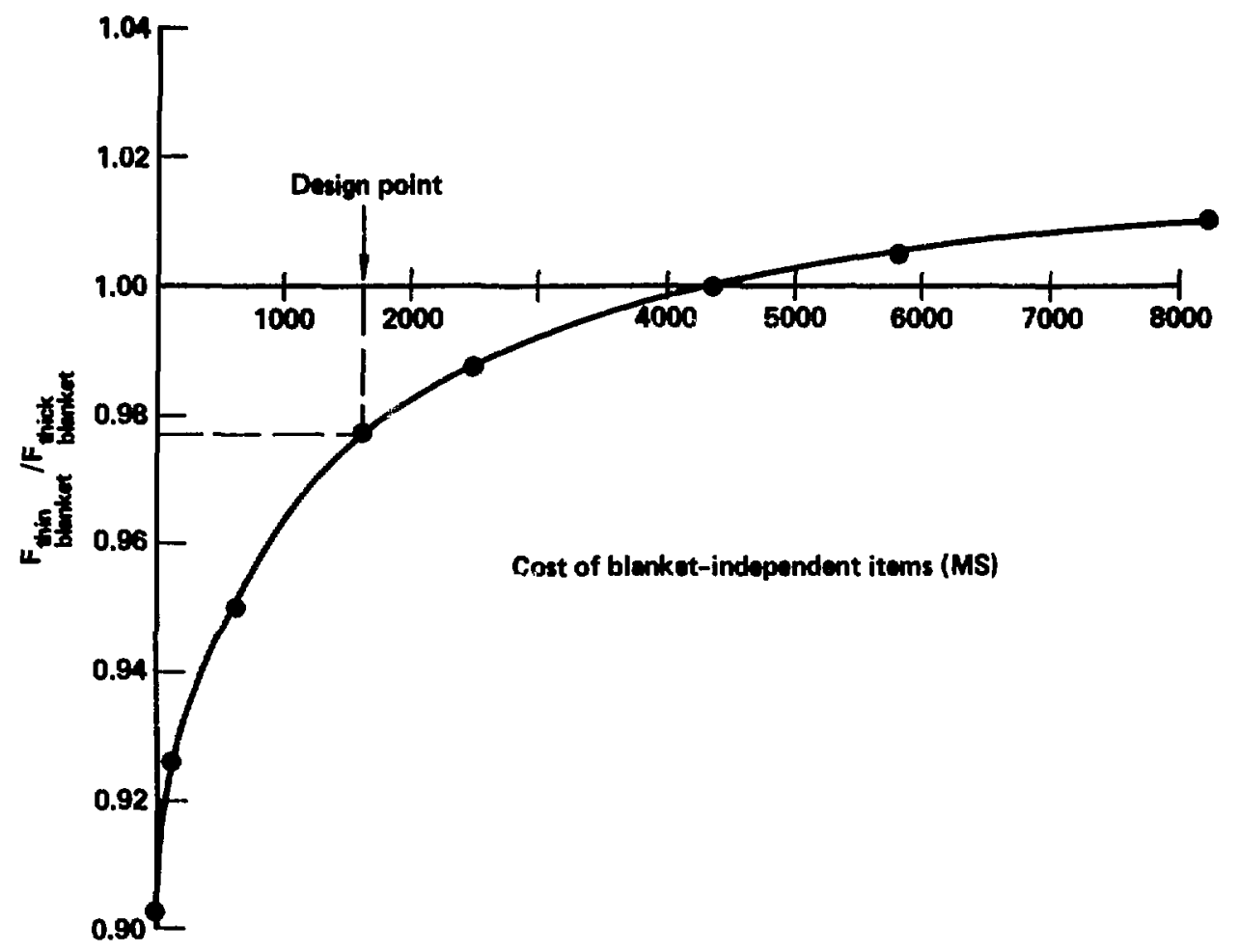

Fig. 4.2-19. Ratio of the thin blanket/thick blanket F-parameters as a function of the cost of the blanket-independent items. 


\subsubsection{Tritium Removal}

\subsubsection{Trittuin Extraction from LiPb}

Tritium is bred in the $\mathrm{LA}_{17}{ }^{\mathrm{Pb}} \mathrm{B3}_{\mathrm{B}}$ eutectic at a rate of $0.59 \mathrm{~g} /$ day and must be extracted at this rate. Tritium can be removed from 11thium-lead alloys 24,25 by vacuum pumping, inert gas sparging, and molten salt extraction. We chose vacuum pumping for MARS as the primary trittum recovery scheme. The 1ithiumlead system is a unique liquid breeder because of its extremely low. trit lum solubility. The Steverts' constant for tritium in $\mathrm{LI}_{17} \mathrm{~Pb}_{83}$ in the temperature range from 400 to $600^{\circ} \mathrm{C}$ is $0.051 \mathrm{kppm} T / T_{0} \mathrm{rr}^{1 / 2.26}$ Even at very low concentration levels (wppb), tritiun will establish a reasonably high partial pressure above the alloy. Vacuum pumping at or below this partial pressure should provide an effective means of removing the tritiun in a relatively simple and economical fashion. Although no experiments are presently being conducted on vacuum removal of gases from $\mathrm{LIPb}$, considerable experfence exists in the degassing of liquid steels to remove hydrogen and other volatile impurities. 27 He chose vacuun pumping without a helfum purge gas in the base design to avold possible large hellum flow rates and hellum/tritium separation at large He: $T_{2}$ ratios. This option, however, is still being investigated. Molten salt extraction from lithium-lead alloys appears feasible and has been suggested in other designs. $24,25,28$ Vacuum removal is a simpler alternative and it avoids the possible transport of molten salt residues through the coolant loop.

To deteraine the optimum steady-state concentration level in the breeding material, both the extraction and the containment systems must be considered. High concentrations place a burden on the containment systems whereas low concentrations make extraction more difficult. The trade-off between the partial pressure and the pumping requiments is 111 ustrated in Fig. 4.2-20. The major containment problen is in the transport of tritium through the heat exchanger. For single-walled tube with an oxide coating giving a resistance factor of 100 , the tritium partial pressure must be $2.5 \times 10^{-9}$ Torr to keep losses through the heat exchanger to $10 \mathrm{cl} / \mathrm{d}$. The pumping rate of $2.1 \times$ $10^{10}$ ifter $/ \mathrm{s}$ at $500^{\circ} \mathrm{C}$ prohibits extraction at this low pressure. 


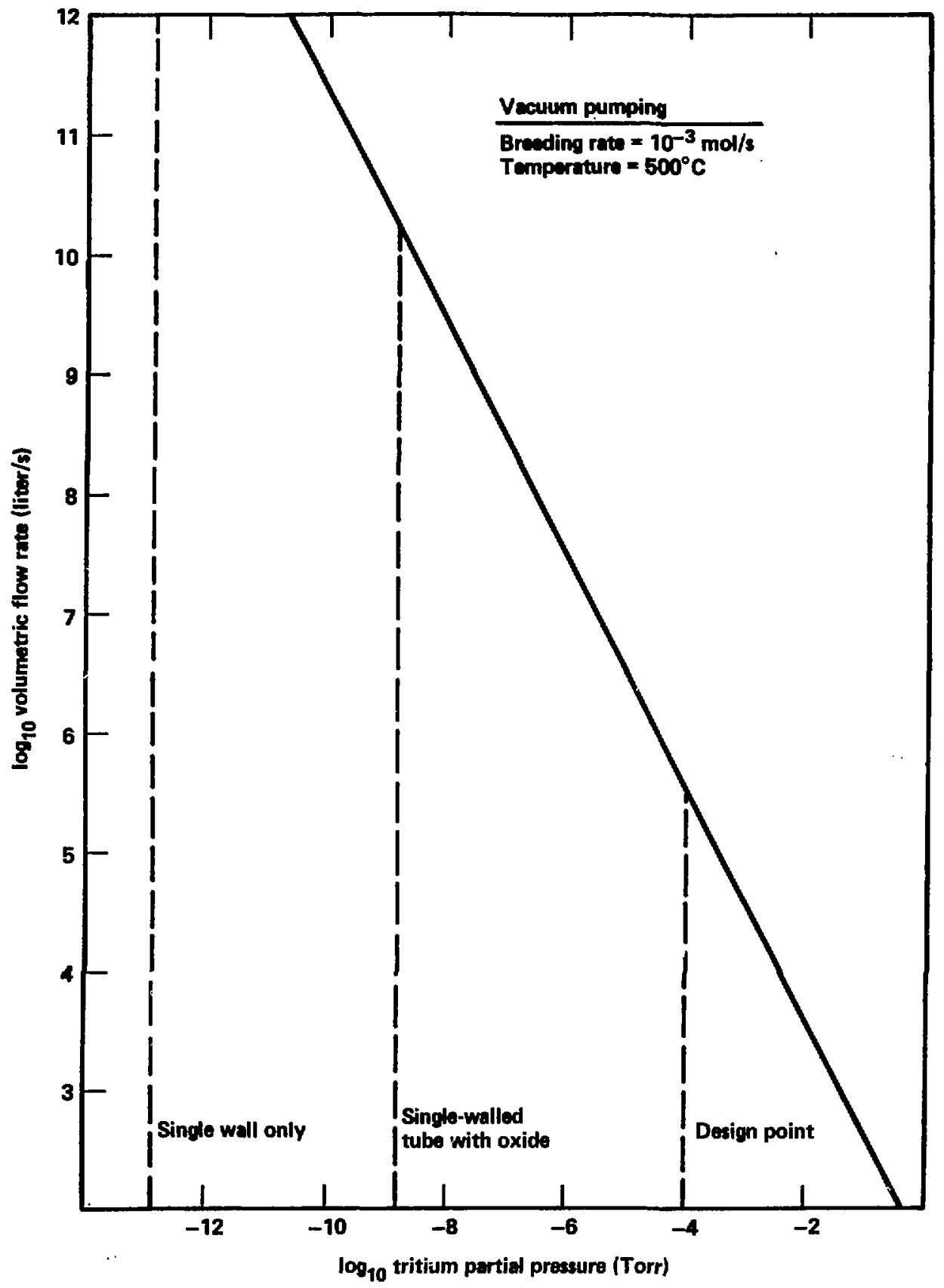

Fig. 4.2-20. Volumetric flow rate for tritium extraction by vacuum punping at various tritium partial pressures. 
Table 4.2-17. Point design parameters for tritium removal system by vacuum pumping.

Tritiun removal rate ( $\mathrm{kg} /$ day)

Vacuum pumping speed at $10^{-4}$ Torr and $500^{\circ} \mathrm{C}(11$ ter/s)

Tritius removed ( $(x)$

LIPb sent to extractor $(x)$

Lipb flow rate into extractor $(\mathrm{kg} / \mathrm{s})$

Extraction temperature

Tritium inventory in pumps $(g)^{a}$

Inlet tritium conditions

Pressure (Torr)

Concentration (wppm)

Outlet tritlum conditions

Pressure (Torr)

Concentration (wppm)
0.59

$5.3 \times 10^{5}$

44

22

$3.1 \times 10^{4}$

$500^{\circ} \mathrm{C}$

49

$1 \times 10^{-4}$

$5.1 \times 10^{-4}$

$3.0 \times 10^{-5}$

$2.8 \times 10^{-4}$

2 hr on-line.

We selected a tritium pressure of $10^{-4}$ Torr for the point design to ease the burden on the extraction system. At this pressure and $500^{\circ} \mathrm{C}$, the punping rate is $5.3 \times 10^{5} 1$ iter/s and the vacuum system cost is low, about $\$ 100,000$. This pressure will require a confinenent system that reduces the tritium permeation through the heat exchanger by a factor of $\sim 10^{5}$. The double-walled tubes in the heat exchanger are expected to be able to provide a barrier of this magnitude. (See Section 4.2.5.3.)

The parameters for the preliminary point design of the tritium recovery system (TRS)--using vacuum pumping at a tritium pressure of $10^{-4}$ Torr--are given in Tabie 4.2-17. With a LIPb flow rate about $1.4 \times 10^{5} \mathrm{~kg} / \mathrm{s}$, the tritium flow rate is $7.1 \times 10^{-5} \mathrm{~kg} / \mathrm{s}$. To extract tritium at the breeding rate of $6.8 \times 10^{-6} \mathrm{~kg} / \mathrm{s}, 9.6 \%$ of the tritium must be renoved. The extractor is assumed to remove $44 \%$ of the tritiuin. Therefore, $22 \%$ of the LIPb must be continually diverted to the TRS. 
At $500^{\circ} \mathrm{C}$ the vapor pressure above the eutectic corresponds to $\mathrm{P}_{\mathrm{Pb}}=1.4$ $\times 10^{-5}$ Torr and $P_{L i}=3.7 \times 10^{-7}$ Torr: In the vacuum system, both 1 fthium and lead will be transported from the alloy in the ratio of their vapor-phase composition, at transport rates of $2.9 \times 10^{-8} \mathrm{~kg} / \mathrm{s}$ and $3.3 \times 10^{-5} \mathrm{~kg} / \mathrm{s}$, respectively. Cooled surfaces will be used to trap the lithium and lead vapors before they can reach the cryopumps.

There are several possible ways of creating favorable transport conditions in the liquid metal to allow for tritium removal by vacuum: largesurface-area pools, pools with agitation or gas sparging, or a thin stream or droplet spray introduced into the vacuum vessel. We are currently comparing these methods for extraction capability, heat loss from the liquid metal, and simplicity of design. The following are some preliminary assessments and calculations for various extraction schemes:

Degassing From Liquid Metal Pools. The steps for transporting tritium gas from a liquid metal pool are: ${ }^{27}$

1. Transport from the interior to the interface of the metal. This occurs in liquids by convective transport processes in the interior to a zone near the surface and from there by diffusion to the interface.

2. Transition from the dissolved state to the adsorbed state at the surface.

3. Assoctation of gas atoms in the adsorbed layer with each other to form molecules.

4. Desorption of molecules from the surface.

5. Diffusion of gas molecules into the gas space and removal by the pumping system.

Step 1, transport in the liquid phase, determines the rate of the entire process. Because mass transport in the liquid intertor is by convection, generating additional convection in the pool with a stirring coll or an inert gas purge improves performance. The key parameter is the mass transfer coefficient $\beta$, which is not known for hydrogen in $\mathrm{Li}_{17} \mathrm{~Pb}_{83}$. Known values of 3.9 $\times 10^{-2} \mathrm{~cm} / \mathrm{s}$ for hydrogen in 1 tquitd aluminum at $700^{\circ} \mathrm{c}^{29}$ and $1 \mathrm{~cm} / \mathrm{s}$ for hydrogen in liquid steel at $1600^{\circ} \mathrm{C}^{30}$ give an idea of the order of magnitude. The mass 
transfer coeffictent can also be determined from the diffusion coefficient and boundary layer thickness, which are themselves unknown. An estimate gives $\beta=1$. Experiments and further calculations are necessary to determine the value for the mass transfer coefficient.

Figure 4.2-21 shows the dependence of $\beta$ on the extraction system parameters. For $\beta \leq 10^{-1}$, the extraction systen requires high surface-areato-volume ratios for residence times of $\lesssim 100 \mathrm{~s}$. An extraction system that creates a large surface area, such as a droplet spray, would be an alternative to creating large thin pools. If $\beta \sim 1$, then the ratio of area to volume is low enough to create a reasonably sized pool for residence times $\lesssim 100 \mathrm{~s}$. Table 4.2-18 gives an example of the parameters for a pool degassing system with $\beta \sim 1$.

Gas Sparging. The use of a scavenging gas has two important effects on mass transfer. First, it provides an increased surface area available for release; second, it causes agitation of the melt. Tritium is removed from the bulk of the liquid by diffusion into the gas bubbles and from the surface by the mechanism described in Section 4.2.4.1. Surface losses may be increased because of the increased surface area caused by the bolling action of the bubbles.

We developed a simplified model to give a preliminary assessment of the purge gas requirements for tritium removal from the base design. The total tritium lost from the system is the tritium removed by transport into the gas bubbles and the tritium that diffuses from the surface. The tritium that enters the bubbles is calculated by assuming that the mass transfer into the bubbles is perfect and, therefore, the bubble is always saturated at the equilibrium tritium pressire. This assumption should give the most optimistic result.

Table 4.2-19 shows the inert-gas fiow rate required to remove various amounts of $T_{2}$. The amount of tritium removed from the bulk by the inert gas is small unless relatively large purge-gas flows are used; tritfum losses from the surface dominate at low flow rates. Tritium can be separated from helium at a molar ratio of $10^{-4}$; however, to avoid this separation process we did not use the helium purge in the baseline design. 


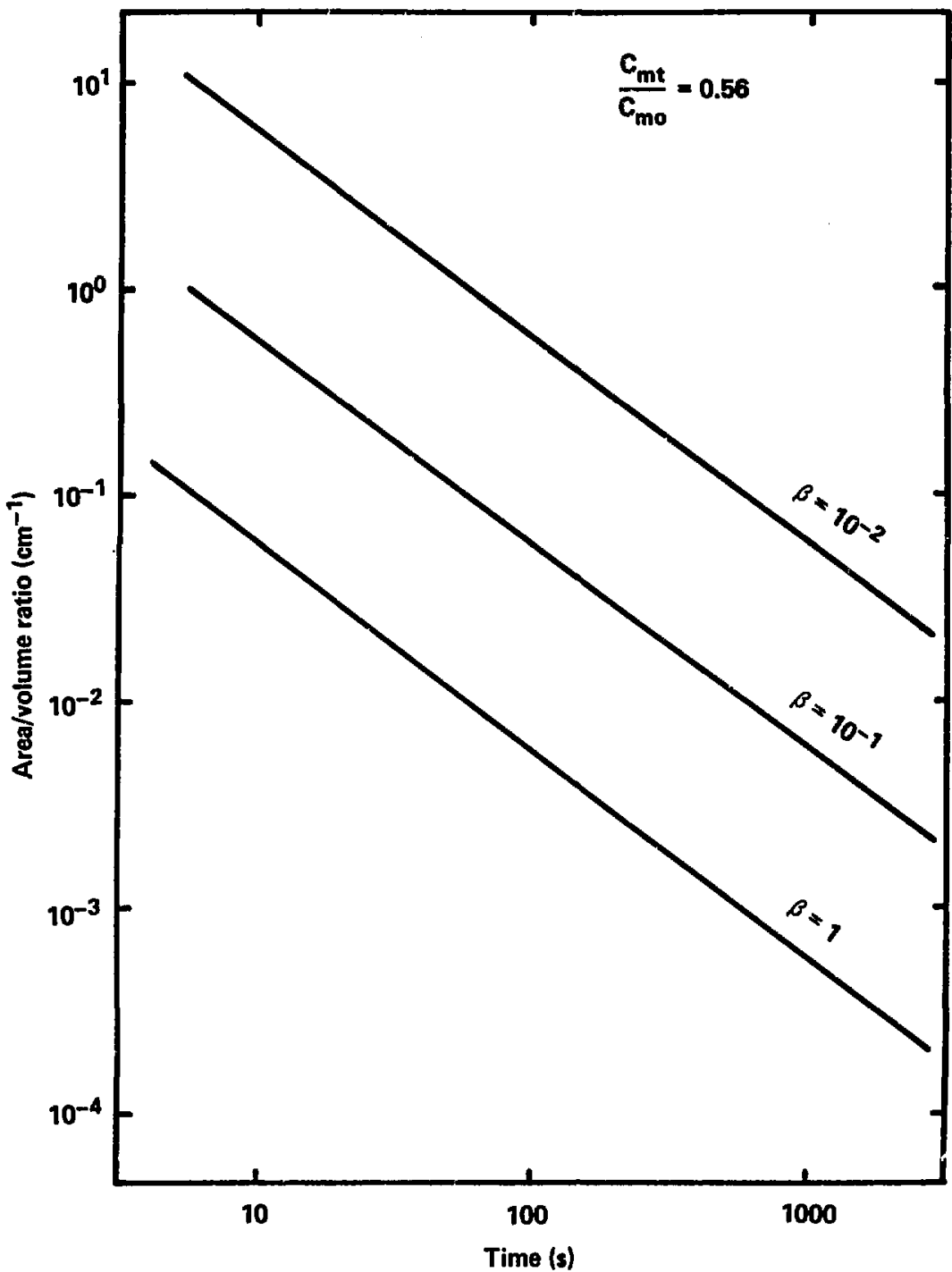

Fig. 4.2-21. Effect of the mass tronsfer coefficient, $B$, on area-to-volume ratio and the residence time for liquid metal degassing. 
Table 4.2-18. Vacuum extraction from a liquid-metal-pool by vacuum pumping with stirring.

\begin{tabular}{ll}
\hline Mass transfer coefficient $(\mathrm{cm} / \mathrm{s})$ & 1 \\
Residence time (s) & 100 \\
Mass of LiPb in extractor $(\mathrm{kg})$ & $3.1 \times 10^{6}$ \\
Tritium inventory in LiPb $(\mathrm{g})$ & 1.6 \\
Pool dinensions & \\
$\quad$ Radius $(\mathrm{m})$ & 7.8 \\
$\quad$ Height $(\mathrm{m})$ & 1.7 \\
Surface area $\left(\mathrm{m}^{2}\right)$ & $1.9 \times 10^{2}$ \\
Volume $\left(\mathrm{m}^{3}\right)$ & $3.3 \times 10^{2}$ \\
\hline
\end{tabular}

Table 4.2-19. Removal of tritium from the bulk of the melt with an inert gas purge. a

$\begin{array}{cccc}\begin{array}{c}\mathrm{T}_{2} \\ \text { removed }\end{array} & \begin{array}{c}\text { Mol } \mathrm{r}_{2} / \mathrm{s} \\ \text { removed }\end{array} & \begin{array}{c}\text { Volumetric flow } \\ \text { rate }(1 \mathrm{ter} / \mathrm{s})^{\mathrm{b}}\end{array} & \begin{array}{c}\text { Purge rate } \\ (\mathrm{g} \mathrm{He} / \mathrm{s})\end{array} \\ 100 & 1.1 \times 10^{-3} & \frac{2.2 \times 10^{8}}{2.2 \times 10^{7}} & 190 \\ 10 & 1.1 \times 10^{-4} & 2.2 \times 10^{6} & 19 \\ 1 & 1.1 \times 10^{-5} & 2.2 \times 10^{5} & 0.19 \\ 0.1 & 1.1 \times 10^{-6} & & 9\end{array}$

a Does not include surface losses, which may be dominant.

b The volunetric flow rate of helium is calculated at $450^{\circ} \mathrm{C}$ and $10^{-2}$ Torr. 
Strean Degassing. In stream degassing, the liquid metal is forced through a nozzle to form a strean of droplets that fall from a predeterained height into a pool. The height of fall and dwell time are minimized to reduce heat losses. We have studied the rate of diffusion from a spherical droplet in a vacuum and the time of fall. 31

Table 4.2-20 shows the anount of gas liberated from falling drops with radil of 1 to $3 \mathrm{~mm}$ for fall times of 0.5 to $1 \mathrm{~s}$. The desired value of $44 \%$ removal can be achieved for fall times slightly greater than $0.2 \mathrm{~s}$ with radti of $1 \mathrm{~mm}$. Some degassing will also occur in the poor at the bottom of the chamber. This process can achieve a high degree of degasification in a short time period and, therefore, it appears to be the superior method. We are continuing to study the design of this system.

\subsubsection{Iritfum Isolation}

The secondary and tertiary containnent systems minimize personnel exposure to tritium and environmental effects of tritium by preventing tritium from escaping by permeation, leakage, or system malfunction. Containment systems have been developed for TSTA and other tritium facilities and should be well-established on the time scale for a comercial fusion reactor.

We are particularly concerned about tritium losses from systems containing LiPb at high-tritium partial pressures of $10^{-4}$ Torr. This problem is especially critical in the heat exchanger; an analysis of tritium isolation by the use of double-walled tubes is given in section 4.2.5.3. In the central cell tritium that permeates through the blanket tubing is pumped by the reactor yacuum system along with the exhaust gases. The tritium extraction unit ant liquid metal holding tanks will have thick, well-insulated walls to prevent neat loss and reduce tritium permeation. Large-diameter thick-walled well-insulated piping is used to transport the LiPb. In addition, aluminum sleeves can be used to provide a secondary containment barrier around the pipes. Additional jacketing or glove boxes can be used to enclose valves or other components where leakage may occur. 
Table 4.2-20. Gas 1iberation from droplets during strean degassing.

\begin{tabular}{clll}
\hline & \multicolumn{3}{c}{ \% gas 1 lberated } \\
Drop radius (m) of fall (s) \\
\cline { 2 - 4 } & 0.2 & 0.5 & 1.0 \\
\hline 1 & 0.42 & 0.60 & 0.77 \\
3 & 0.15 & 0.24 & 0.32 \\
\hline
\end{tabular}




\subsubsection{Energy Conversion System}

\subsubsection{Heat Exchanger Arrangement and Power Cycle}

Figure 4.2-22 shows the power cycle for the LIPb blanket. The 2940-M $W_{t}$ heat input is split in two places to increase the effectiveness of heat transfer. The stean system is $2400 \mathrm{ps} 1 \mathrm{~g} / 482^{\circ} \mathrm{C}\left(900^{\circ} \mathrm{F}\right)$ with $482^{\circ} \mathrm{C}\left(900^{\circ} \mathrm{F}\right)$ reheat. The heat transfer temperature diagram in Fig. 4.2-23 11lustrates the effectiveness of the cycle in a system where the first-stage reheat and economizer do not share the blanket heat. The evaporator pinch point increases from 23.5 to $30 \%$ of the total heat transferred, and the $\Delta T$ is raised from 15 to $25^{\circ} \mathrm{C}$. This causes larger log-mean-temperature differences (LMTD) in the evaporator, first-stage reheater, and economizer, which in turn reduces heating surface requirements and LiPb inventory. A larger pinch-point $\Delta T$ may make it possible to use a highen steam-cycle pressure, which would increase steam-cycle efficiency over the efficiency possible at 2400-psig.

Similar procedures can be used advantageously to integrate power recovery from water used to cool the reflector. Cooling water at $300^{\circ} \mathrm{C}$ with a $27.8^{\circ} \mathrm{C}$ $\Delta T$ can use PWR-type steam generators to produce 640-psig saturated steam. A reflector power of 980 MWt will generate $4.04 \times 10^{6} \mathrm{lb}$-steam/hr from $204^{\circ} \mathrm{C}$ feedwater.

To improve the thermal effictency of this steam cycle, the 600-psi steam (at turbine throttle) can be superheated to $357^{\circ} \mathrm{C}$ from the blanket coolant (152.5 wt) by adding a superheater in parallel with the first stage reheater and economizer shown in Fig. 4.2-22. This will obviously improve the 600-psi reflector power cycle. In addition, it will shift the pinch point in Fig. 4.2-23 frail 30 to $35 \%$ to further improve component LMTDS and provide greater flexibility in going to a higher blanket-power-cycle pressure.

Similarly, cooling water heated to $250^{\circ} \mathrm{C}$ can be used to produce 250-psia saturated steam (at turbine throttle) for a third power cycle. This can be superheated by reflector cooling water at $300^{\circ} \mathrm{C}$ and possibly reheated by blanket-derived-steam at 600-psi. 


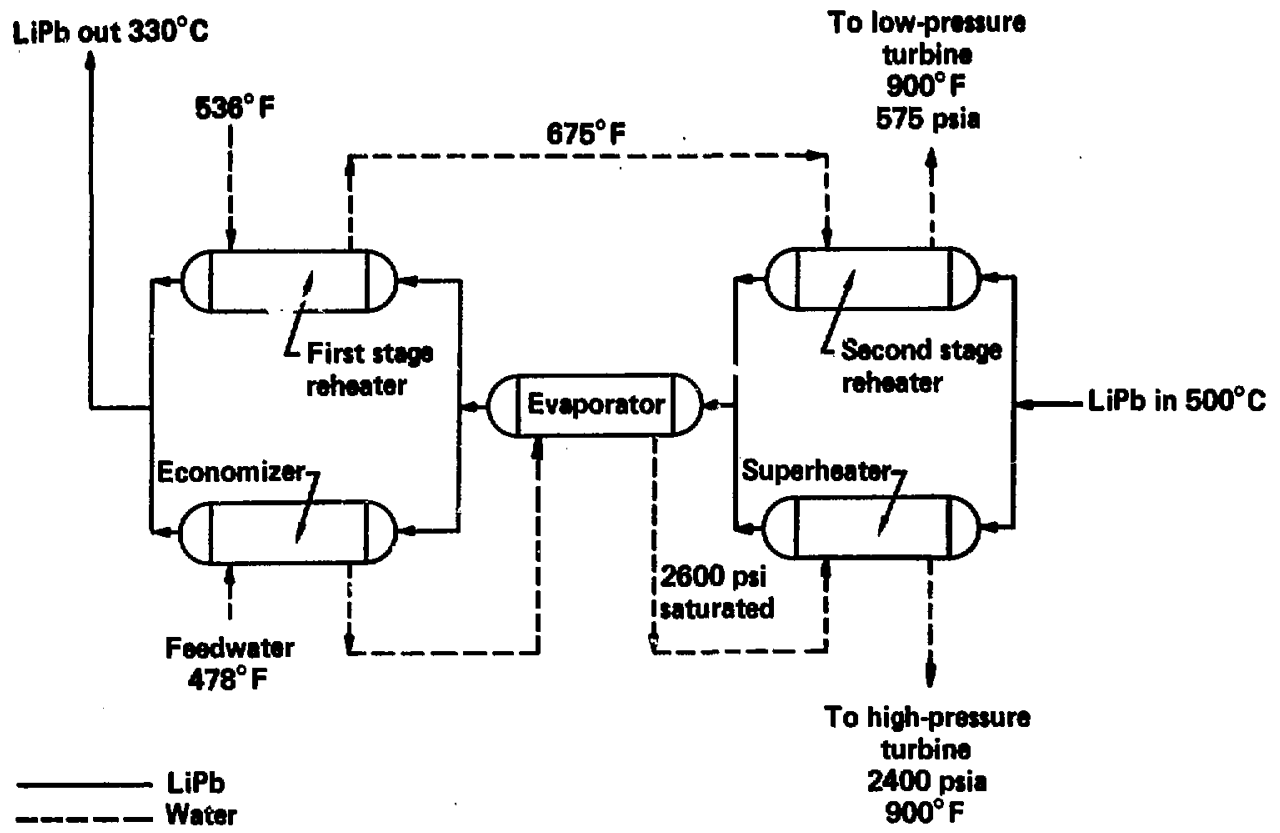

Fig. 4.2-22. Heat exchanger arrangement. 


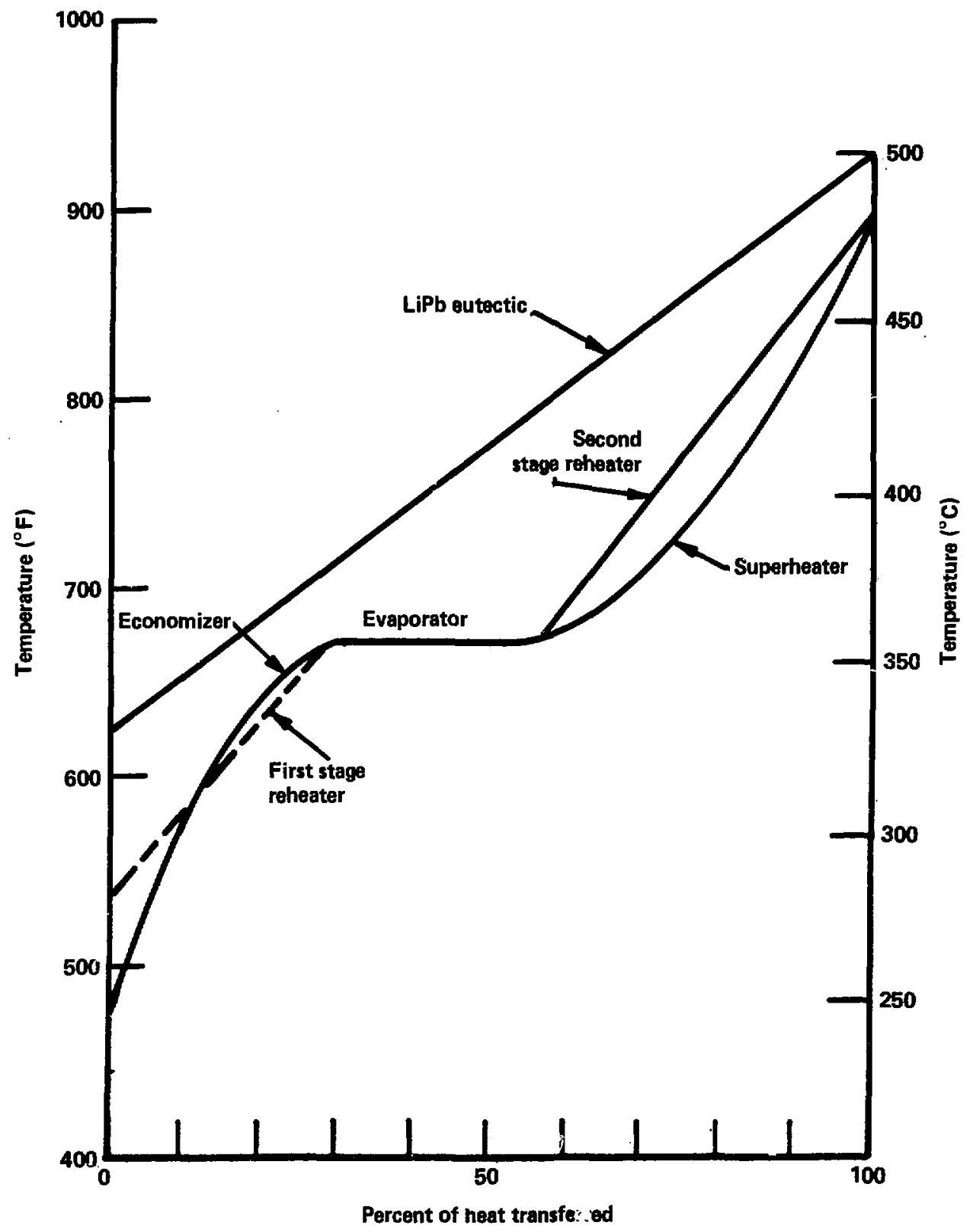

Fig. 4.2-23. MARS temperature diagram for $2400-p s i a 900^{\circ} \mathrm{F} / 900^{\circ} \mathrm{F}$ steam cycle. 


\subsubsection{Double-Walled Steam Generator}

Heat exchange equipment for the MARS power cycle must fulfill exacting requirements while at the same time retaining design features acceptable to the utility industry. The design requirements of 1iquid-metal-to-water heat exchangers are complicated by two factors:

- It must provide a tritium diffusion barrier between blanket coolant and steam cycle.

- It must permit practical inspection and mat-tenance procedures in spite of radiation levels produced by activated corrosion products present in the blanket coolant.

The double-walled steam generator described in Figs. 4.2-24 through 4.2-27 was created to meet the above requirements. It provides the following features:

1. An adequate tritium diffusion barrier.

2. Detection of potential tube defects that could lead tc metal/water reactions.

3. Avoidance of size-limitation problems typical of double-walled heat exchangers.

4. Detection, location, and repair of leaks by conventional methods (eddy current).

5. More compact construction.

6. More conventional, less difficui: component assembly.

The space between the inner and outer shells and between the inner and outer walls if each tube assembly is filled with helium that contains oxygen at a partial pressure of 1 Torr. A pumping system (not shown) is used to circulate this gas longitudinally through each tube wall gap as a sweep (or monitor) gas to detect water vapor.

The hamispherical heads shown in Fig. 4.2-24 are similar in configuration to those used for "once-through" PWR steam generators. Thus, the tube inspection and repair equipment developed for this steam generator is applicable to 


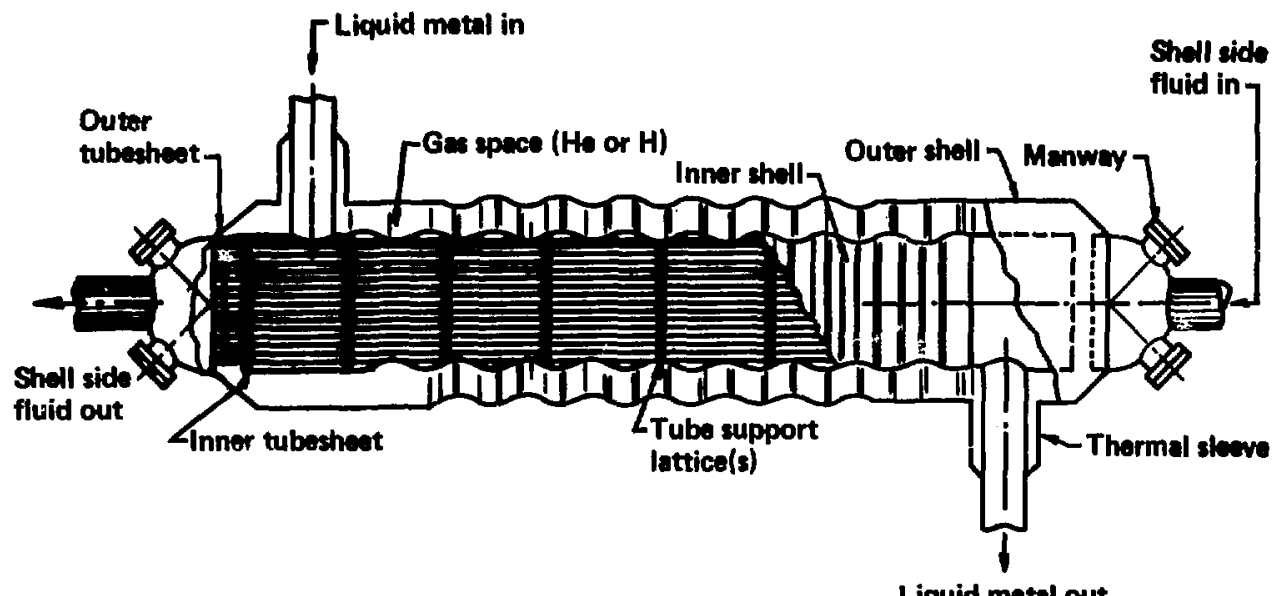

Liquid motal out

Fig. 4.2-24. General arrangement. 


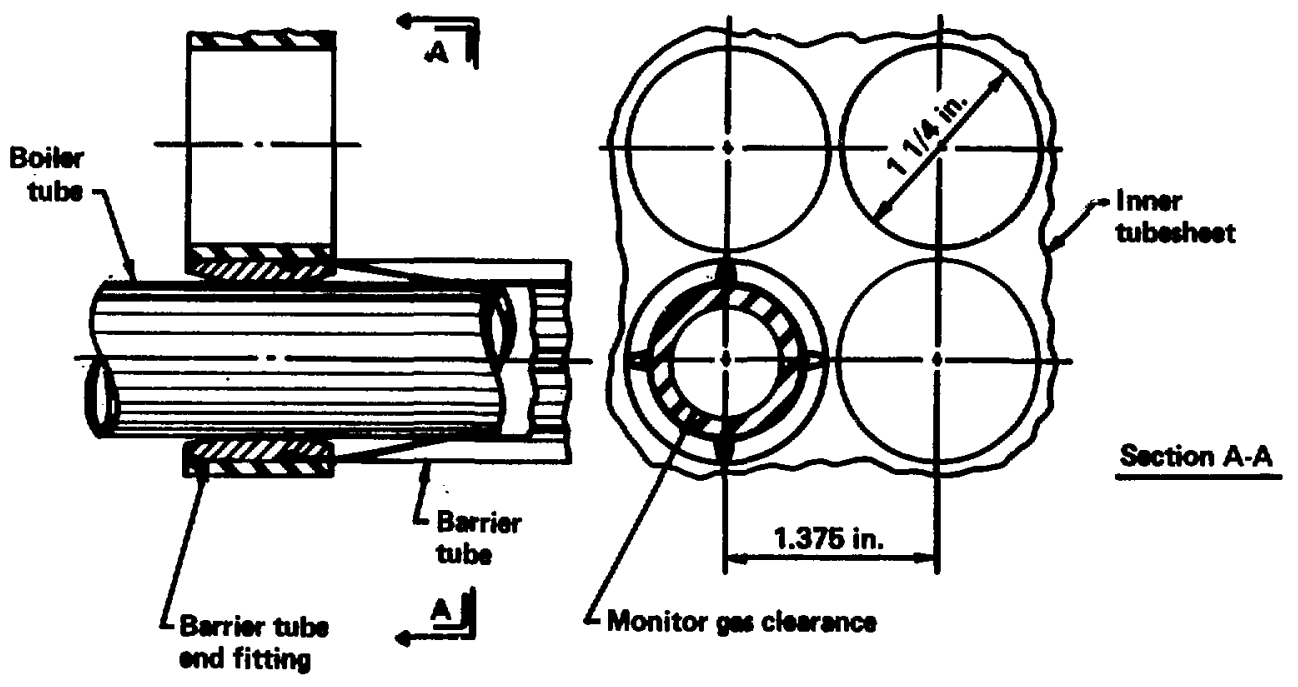

Fig. 4,2-25. Barrier tube to inner tubesheet detail. 

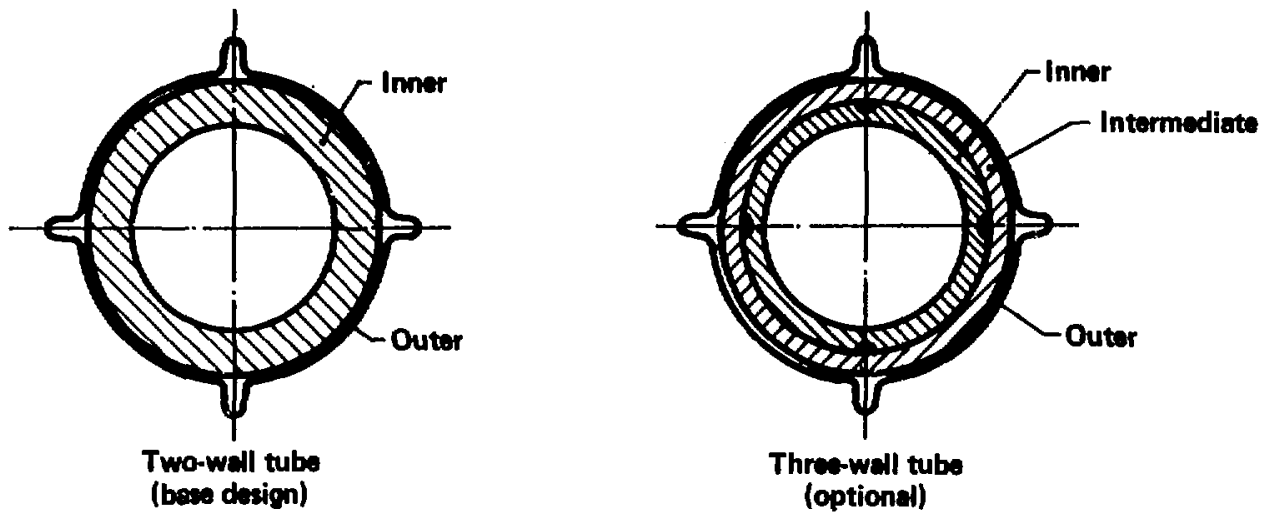

Fig. 4.2-26. Double wailed tube structure. 


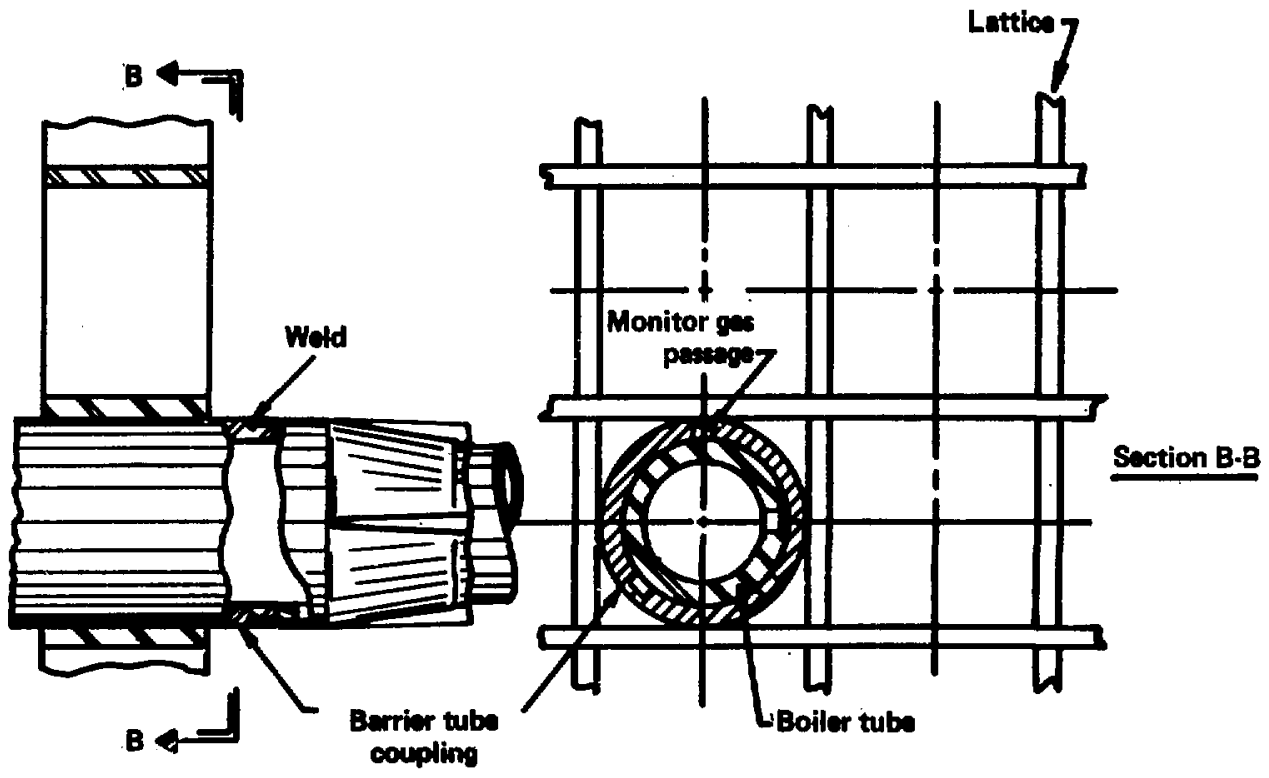

Fig. 4.2-27. Tube support detail. 
the design shown in Fig. 4.2-24. This equipment has been used satisfactorily to perform inspection and maintenance work in radiation levels up to about 15 rem at the henispherical head tube face.

To cope with higher radiation levels, the manways can be replaced by full opening closures, wich in turn will permit use of rotating plugs and other equipment developed in the past for hot maintenance. The effect of activated corrosion-product radiation on inspection and maintenance tends to be minimized by the following:

1. Vertical (instead of horizontal) tube sheet faces that reduce collection of corrosion particles on the surfaces.

2. Inner and outer tube sheets with a space between that attenuates streaming of ganna rays through tube inside diameters.

3. Corrosion products will tend to collect at the top of the inner shell. Radiation from this location will not be in line with tube sheet holes.

\subsubsection{Tritium Isolation}

Tritium confinement is one of the most difficult problems steming from the use of $\mathrm{Li}_{17} \mathrm{Fb}_{83}$. The very low tritiun solubility in $\mathrm{LI}_{17} \mathrm{~Pb}_{83}$ results in a very high tritium partial pressure. The most vulnerable region in the primary loop for tritiun ?aakage is the primary heat exchanger. To improve heat transfer, the su"race of the heat exchanger is large, with thin-walled tube construction. Therefore, an effective tritiun diffusion barrier is required between the primary loop and the steam cycle to reduce the tritium leakage to an acceptable level.

Hydrogen permeability through different ferritic steels is summarized in Fig. 4.2-28. The best estimation of tritium permeability through HT-9 is

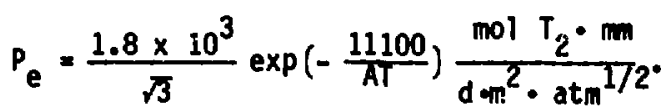

With this permeability and a steam generator area of $3.46 \times 10^{4} \mathrm{~m}^{2}$, the iritium leakage rate $1 \mathrm{~s} 3.5 \times 10^{5} \mathrm{ci} /$ day. Therefore, a tritiun diffusion barrier of $10^{5}$ is needed. 


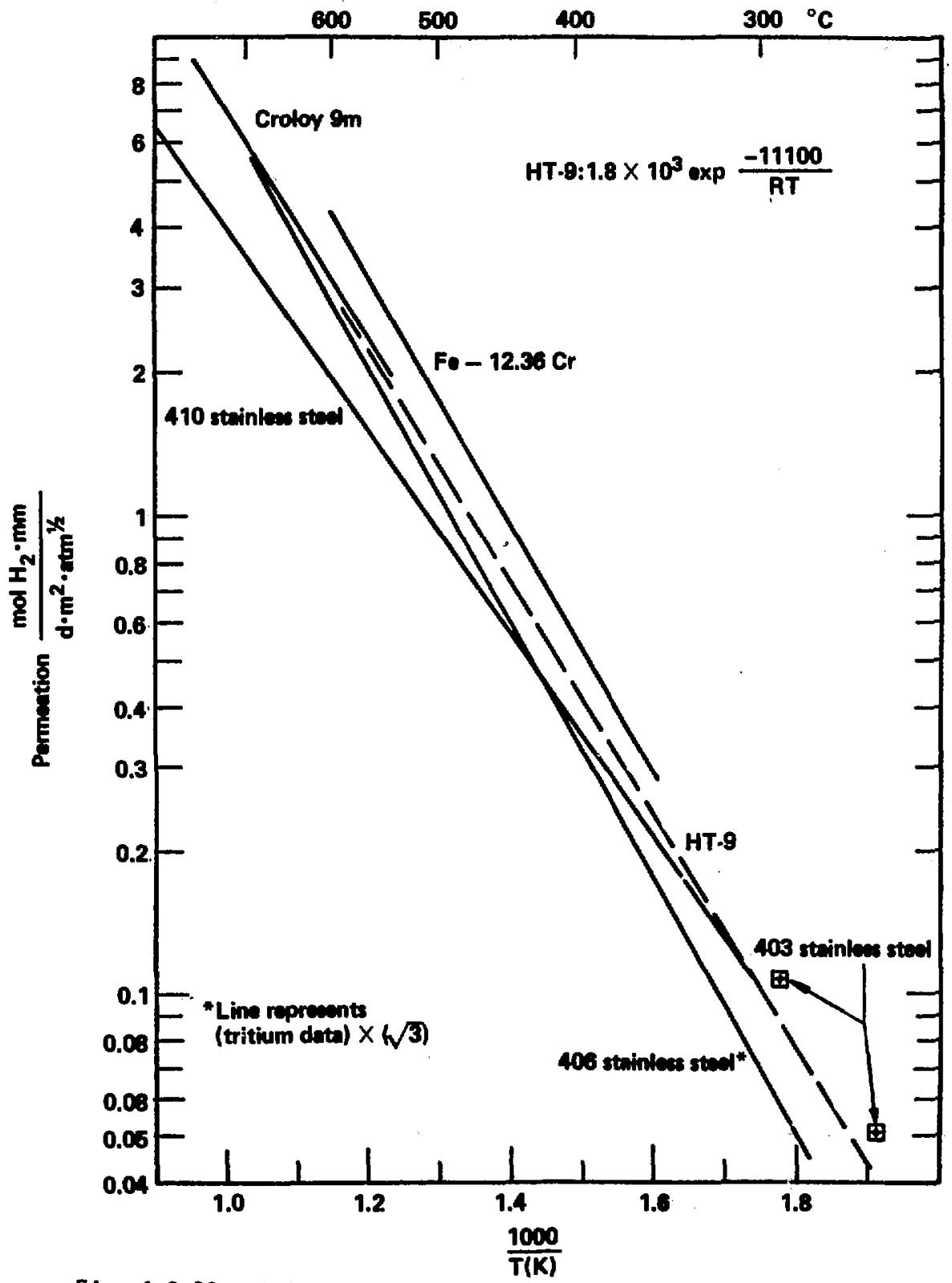

Fig. 4.2-28. Hydrogen permeation through chromiun ferritic steels. 
In MRS, a double-walled heat exchanger provides the required tritium diffusion barrier. The construction, with two tubes in close contact, is shown in Fig. 4.2-26. A helium purge with 1 Torr of oxygen pressure passes through the channels to provide an oxidizing atmosphere between the two tubes. The oxide flims formed between the two tubes, as well as the oxide film on the water side, provide an effective diffusion barrier.

Figure 4.2-29 shows two tritiun diffusion paths through the double-walled stean generator. This is an enlarged view around one contact point between the tubes.

The resistance to the diffusion across the gap is the product of the resistance of the oxide films and the resistance of the gap. Figure 4.2-30 shows the attenuation factor caused by the gap as a function of the gap width; Fig. 4.2-31 shows the attenuation factor resulting from the combined effect of three oxide coatings as a function of single oxide coating layer factor. If the gap width is $10^{-3} \mathrm{~cm}$, the attenuation factor from the gap is $3 \times 10^{-3}$. To obtain a total attenuation factor of $10^{-5}$, an attenuation factor of $3 \times 10^{-3}$ is needed from the oxide coatings, which corresponds to a single layer factor of 20. Experiments have indicated that a single-layer factor of a few hundred is available for ferritic steel. 32 It can be concluded, therefore, that the attenuation factor of $\ll 10^{-5}$ is available for tritium leakage across the gap.

He performed a two-dimensional finite-difference tritiun diffusion calculation for tritium leakage along path 2 (through the contact point). The result shows that an attenuation of $10^{-5}$ is also available along this path. The total attenuation is the sum of the attenuation along those two paths and is, therefore, $<10^{-5}$.

\subsubsection{Removal of Corrosion Products}

The corrosion in the blanket causes two problens. The first is thinning of the blanket structural material, which can weaken the structure. This effect appears to be acceptable. The second problem is caused by corrosion products transported to the primary heat exchanger and deposited there. This may cause problems such as channel blockage, which will require the heat exchanger to be remotely maintained. 


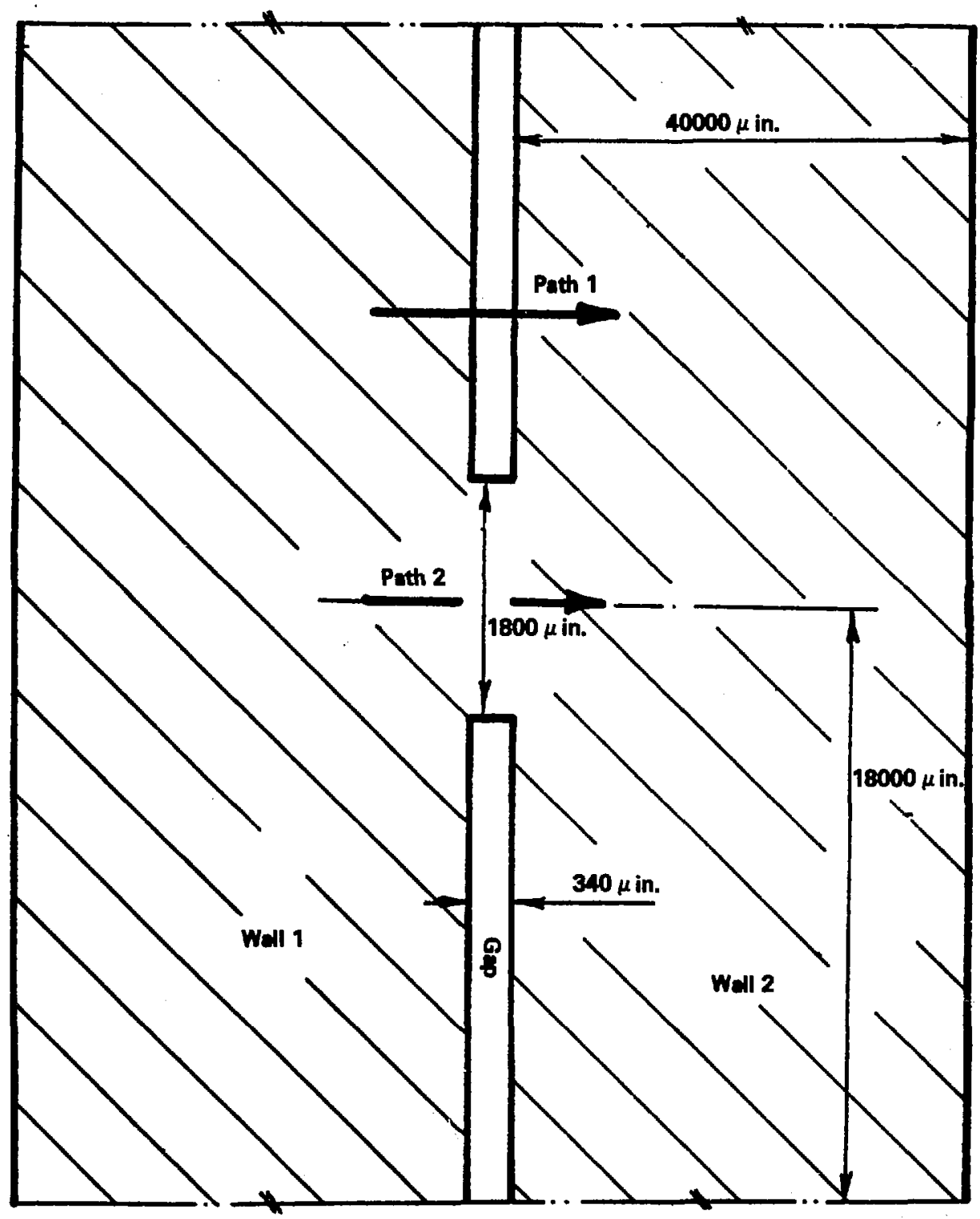

Fig. 4.2-29. Tritiun diffusion path. 


$$
10
$$




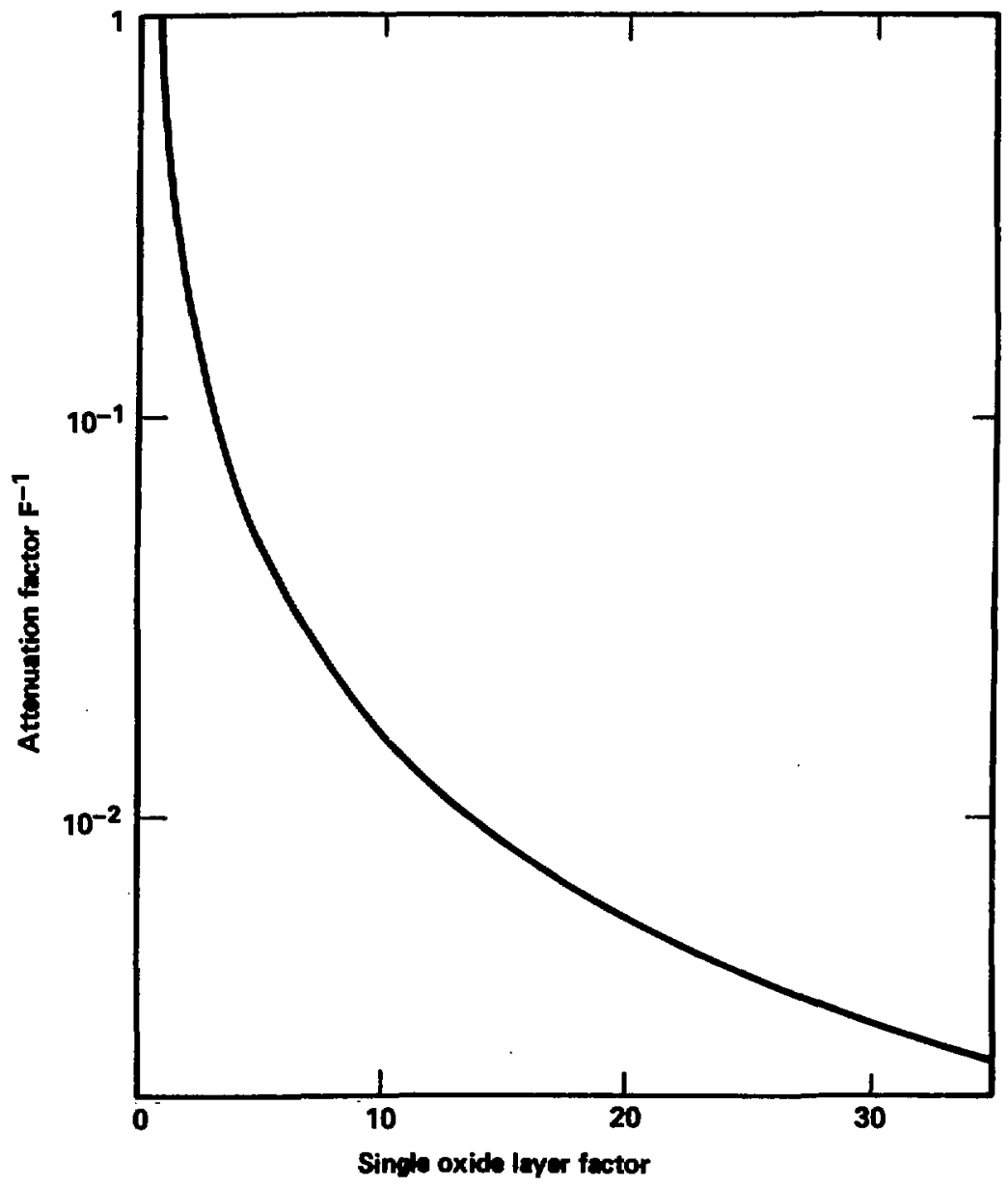

Fig. 4.2-31. Attenuation factor due to oxide coatings. 
The LIPb blanket has a surface area of $10^{4} \mathrm{~m}^{2}$. The corrosion rate in a static system is estimated to be $2 \times 10^{-7} \mathrm{~g} / \mathrm{cm}^{2}-\mathrm{hr} \cdot{ }^{33}$ If the MD effects dominate the mass transfer in the liquid phase, a dynamic system in a strong MHD environment will resemble a static system. For sucil a system, the total corrosion rate from the blanket is $175 \mathrm{~kg} / \mathrm{yr}$. This appears to be an acceptable amount.

Corrosion product transport in a liquid-metal system is significantly different from transport in a non-liquid-metal system. For a liquid-metal system, the Prandtl number is much smaller than the Schmidt number, or the heat transfer is much faster than mass transfer. Therefore, the liquid metal in the center of the flow region will be cooled to supersaturation before the mass in the center of the flow region is about to diffuse out. This is the reason for the well-known fact that dissolved material in a liquid metal will form particulates and be suspended in the flow when the flow is being cooled. In contrast, in a regular coolant, the dissolved material will be deposited on the wall.

As the coolant passes the heat exchanger, the majority of the corrosion products will be suspended in the flow and pass through the heat exchanger. Various methods can be used to partially separate the suspended particulates and the coolant. The methods tried in other 11quid-metal systems are filtration processes and magnetic separation. We are still working to determine which is the perferable process and its separation efficiency.

\subsubsection{Blanket Maintenance}

\subsubsection{Maintenance Philosophy}

The components of the central cell must be replaceable. By synthesizing and comparing several design options, we have developed a straightforward maintenance approach that emphasizes high avallability and totally remote handling in two distinct maintenance categorles:

1. Routine blanket module changeout. Maintenance operations are performed only on the blankets themselves, so the shield is not cut and the magnets are not moved. Spares are provided and pretested; as one section of the blanket is rewoved, an immediate replacement is avatlable. 
2. Unscheduled component replacement. Provision for maintaining the long-term components, such as the colis, the ralis on the reflector, and a section of shield, etc., must be included. These components are designed for maintainability in all contingencles. Spares for superconducting magnets, standard reflector, and shield structure are also provided.

Remote maintenance is mecessary because of the radiation hazard to plant personnel. Also, the use of remote equipment permits maintenance with a mintmal cool-down pertod. Personnel access is not allowed once the shield is removed.

The design philosophy results in the following maintenance design features:

1. The cylindrical blanket module lying on the rafls attached to the reflector structure is capable of being translated axially within the reflector tunnel by a spectally designed machine.

2. The coolant supply plpe headers--patterned after the WITAMIR-1 design--are designed for rewote disconnection and reconnection, which takes advantage of the fact that the $\mathrm{Lf}_{1} \mathrm{~Pb}_{83}$ coolant/breeder is an excellent solder. A trace mater and a coolant line are located on the flange edge to control the temperature so that the headers can be separated or frozen together with the help of a mechanical, hinged bracket as shom in Fig. 4.2-32.

3. Accessibility to the blanket module is provided by 12 servicing stations, or ports for blanket module ramoval. The vacuum seals for the ports are flat flanges. In addition, a vacuum barrier is designed for redundancy. Flgure 4.2-33 shows the cross section of the servicing stations, ach of witch services the seven marest blankets.

4. The axial dimensions are defined in Figs. 4.2-34 and 4,2-35. Because the magnets remain in position during routine maintenance, space is provided to transiate the blanket module and manifold to the service station. The inlet and exit pipes pass through the shield in the vertical direction. The attachment position to the blanket varies, wich necessitates four different blanket patterns. 


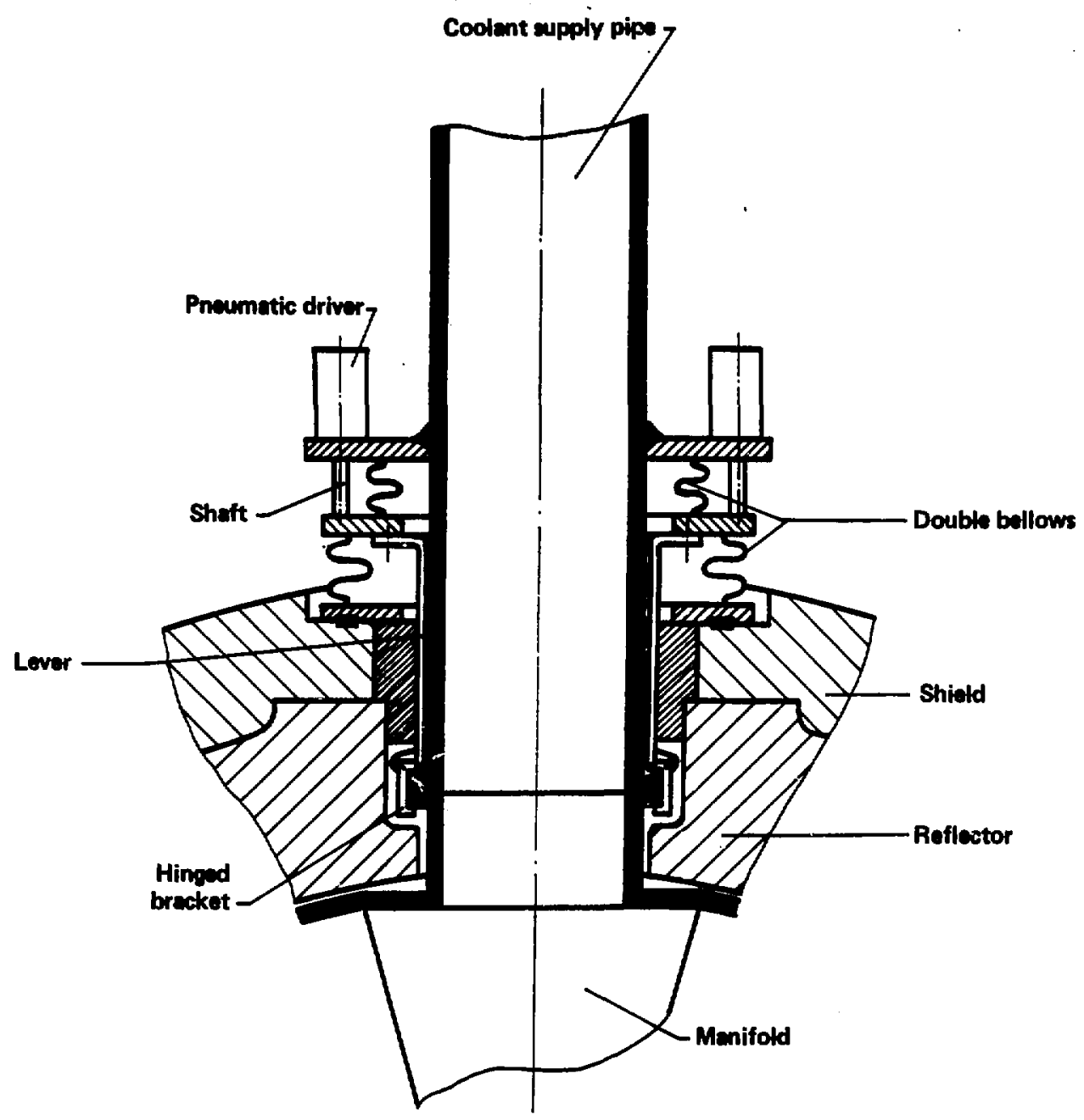

Fig. 4.2-32. Pipe connection and vacuum seals. 


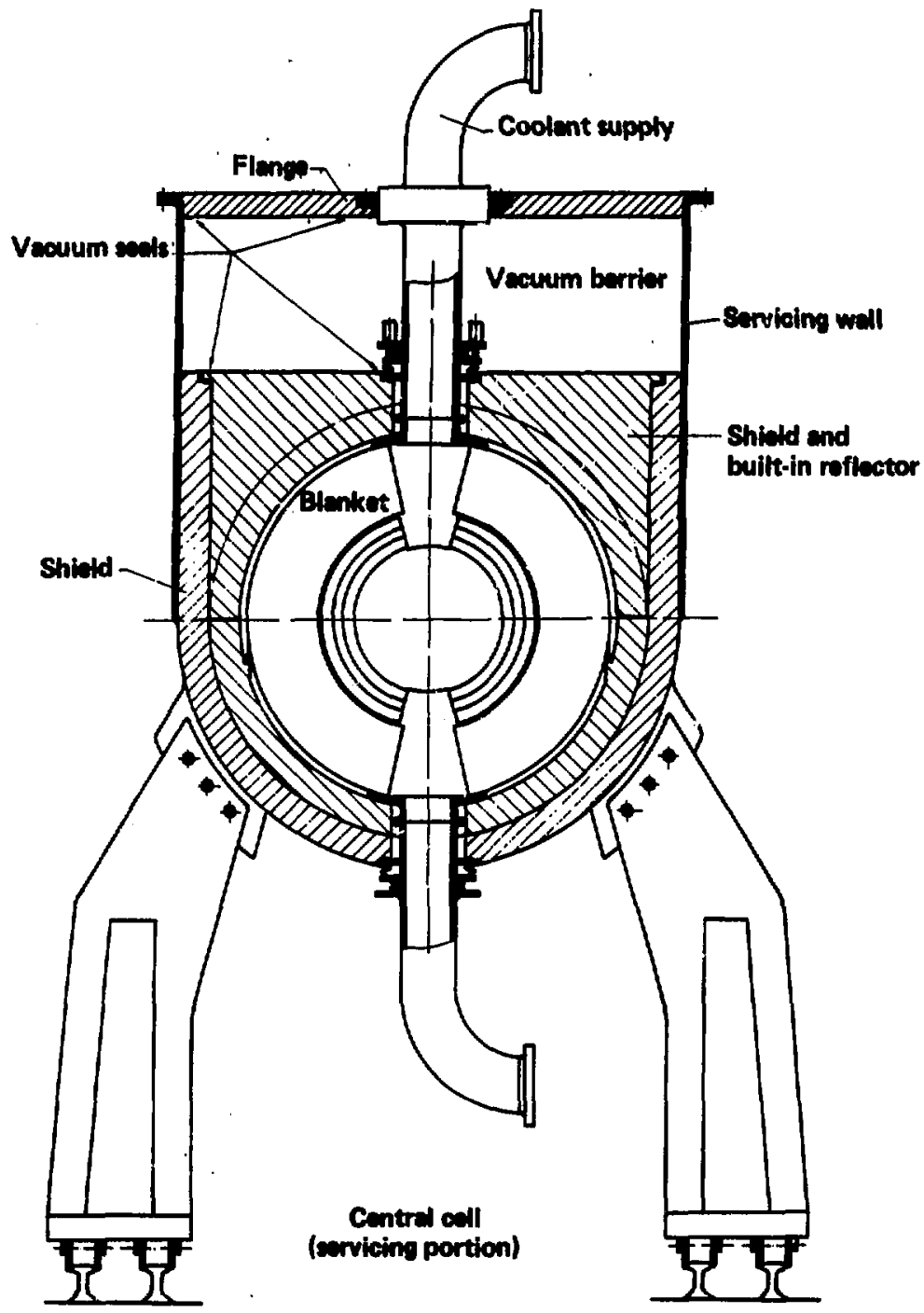

Fig. 4.2-33. Vertical cross section of the central cell showing the blanket servicing region. 


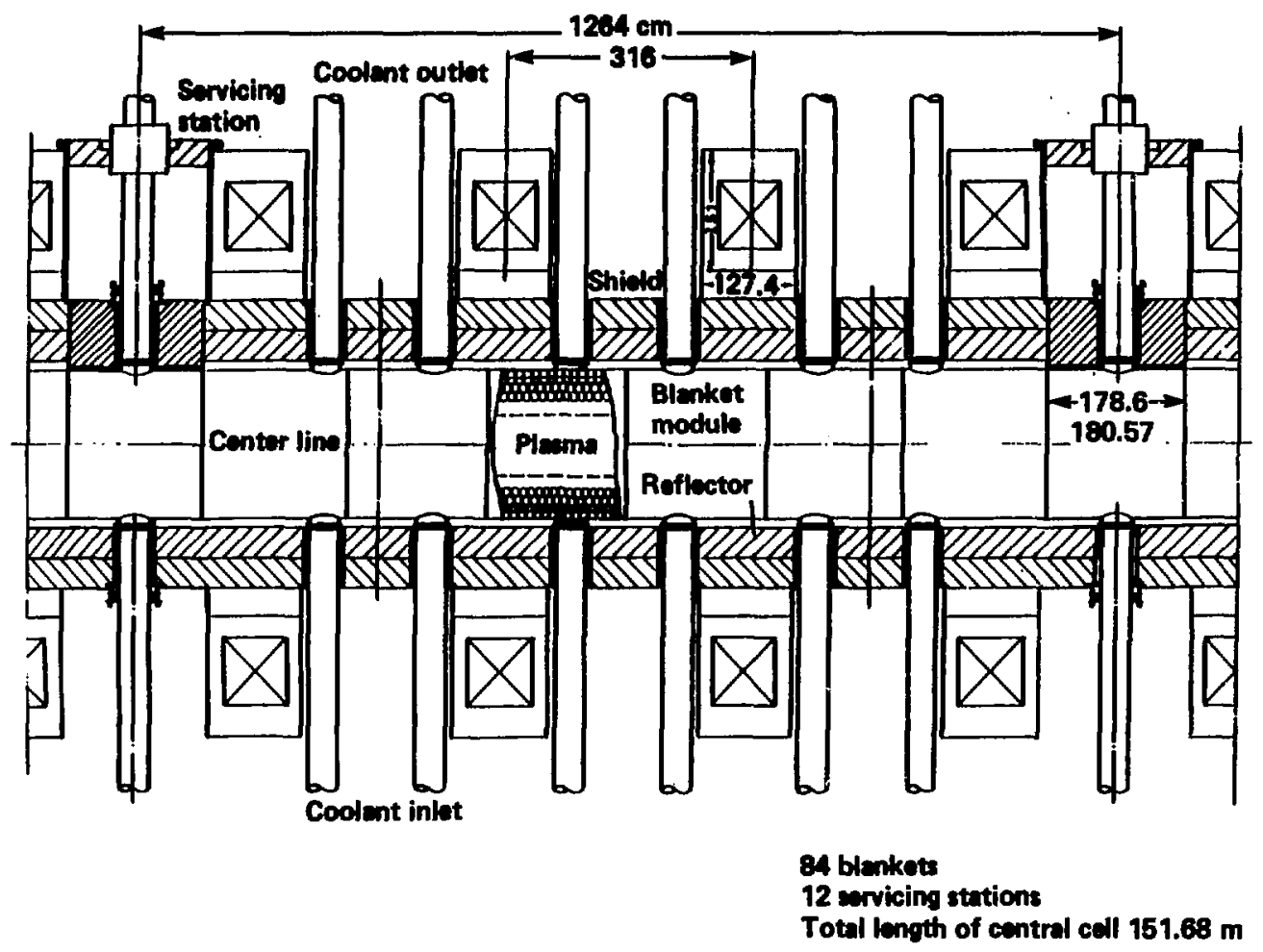

Fig. 4.2-34. Cross section of the central cell showing details of the blanket module. 


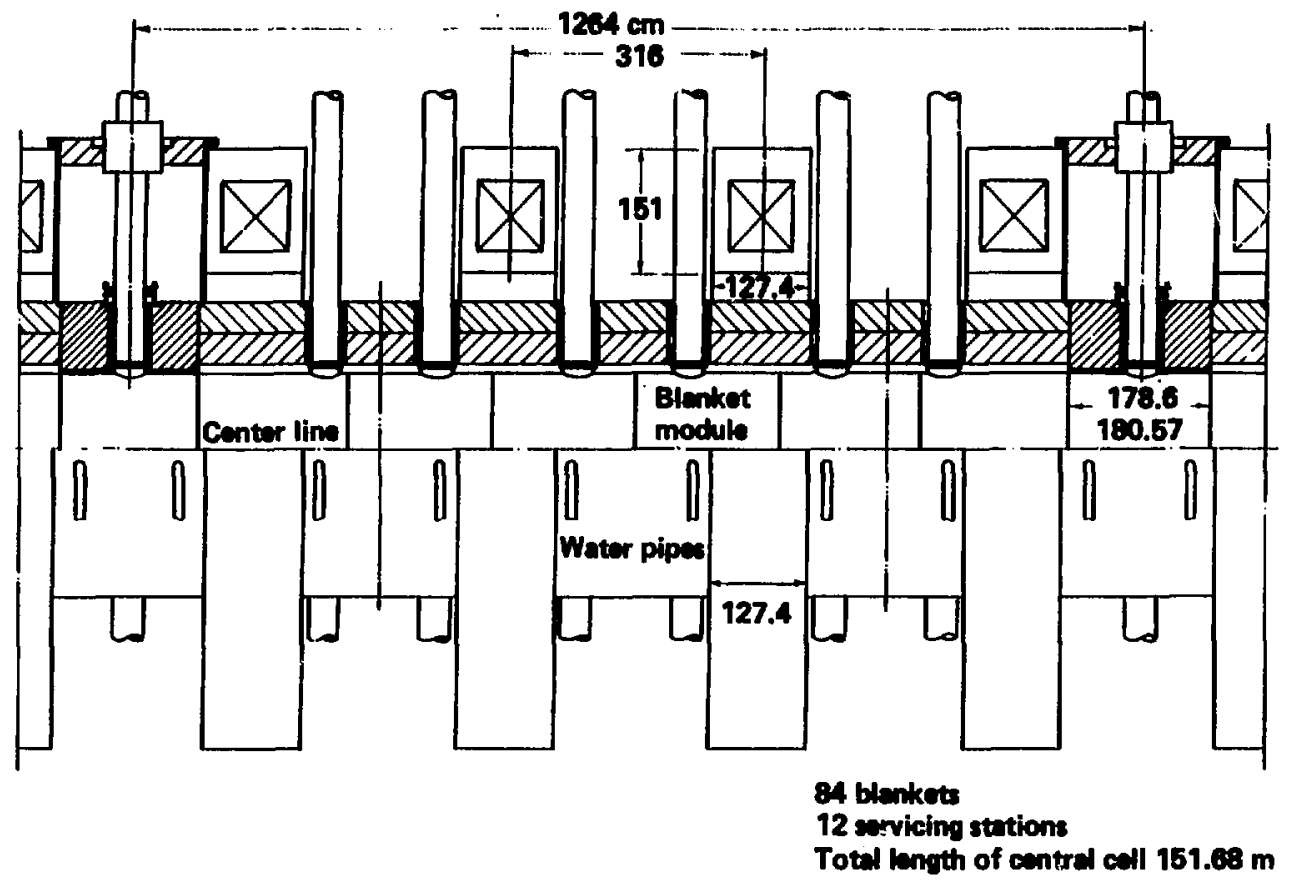

Fig. 4.2-35. Cross section of the central cell showing the reflected water pipes. 
5. The shield and reflector can be cut for replacing a failed component. The cutting positions are shown in Figs. 4.2-34 and 4.2-35 by dashed lines perpendicular to the reactor axis; the details of the structure are shown in Fig. 4.2-36. The upper and lower parts of the piece of the shield surrounding the vacuum seal can be removed separately. Each section of the central cell, including the associated magnets, is capable of being translated radially through the side of the reactor. The magnets can then be translated axially to the right or left and ramoved.

\subsubsection{Maintenance Procedure}

The routine mintenance sequence would be as follows:

1. Drain the coolant into dump tanks.

2. Heat solid LiPb on pipe connections until molten.

3. Release the mechanical, hinged brackets.

4. Lift all top pipe headers up 1 to $2 \mathrm{~cm}$.

5. Pull out the pipe headers in the servicing stations.

6. Open flanges at servicing stations.

7. Withdraw the bulk shield and butlt-in reflector in the servicing stations.

8. Extract the blankets immediately below.

9. Translate the six adjacent blankets to each servicing station.

10. Repeat step 8.

11. Reverse steps 3 to 10 for inserting new modules.

12. Bake-out the whole reactor system except the flange of the coolant pipe headers.

13. Evacuate.

14. Reverse step 1.

15. Restart.

F1g. 4.2-37 shows the exploded view of the maintenance procedure.

We are investigating a timetable for each of the above operations. We anticipate that the most time-consuming aspects are steps $6,9,12$, and 13 , including their reverse operations (where applicable). 


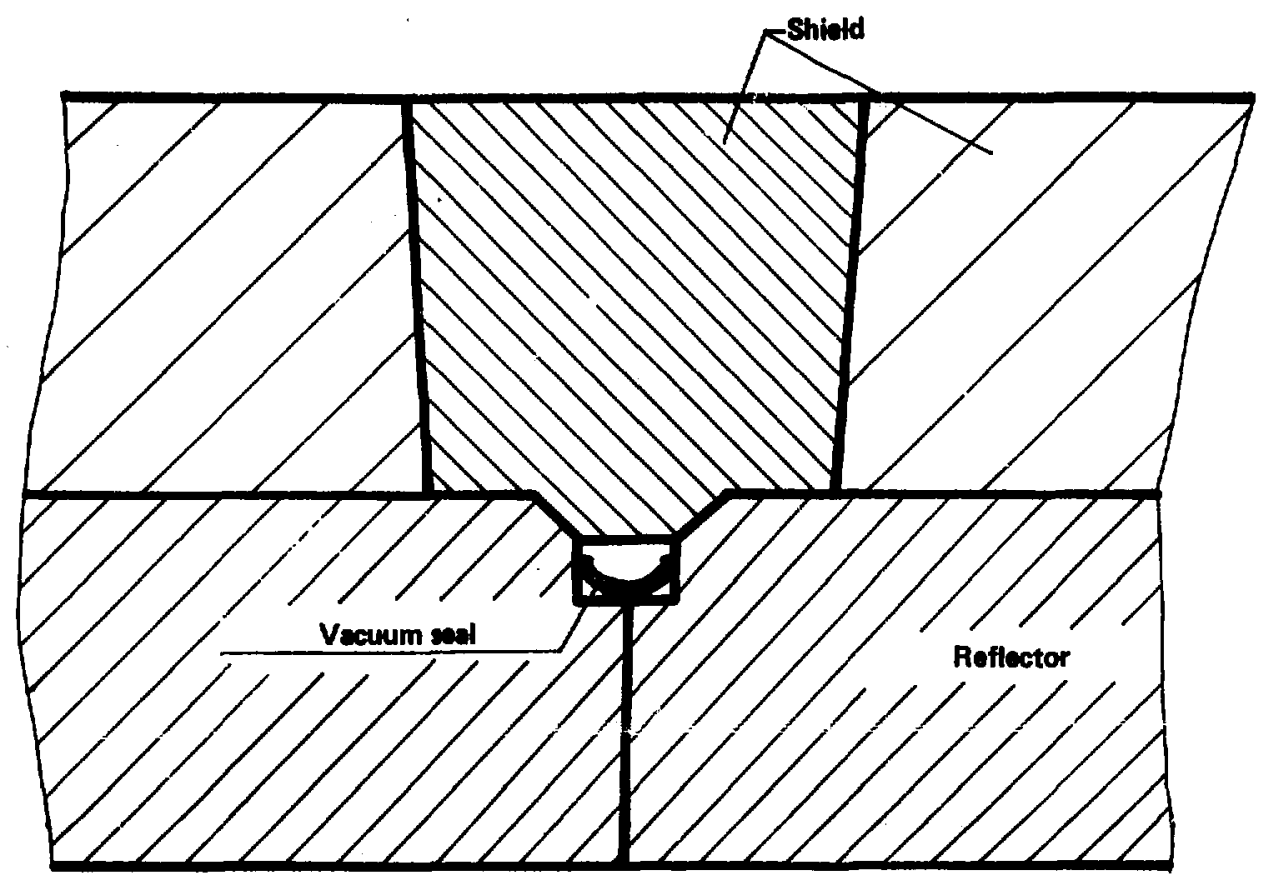

Fig. 4.2-36. Details of the vacuum seal weld for reflector disassembly (schematic). 


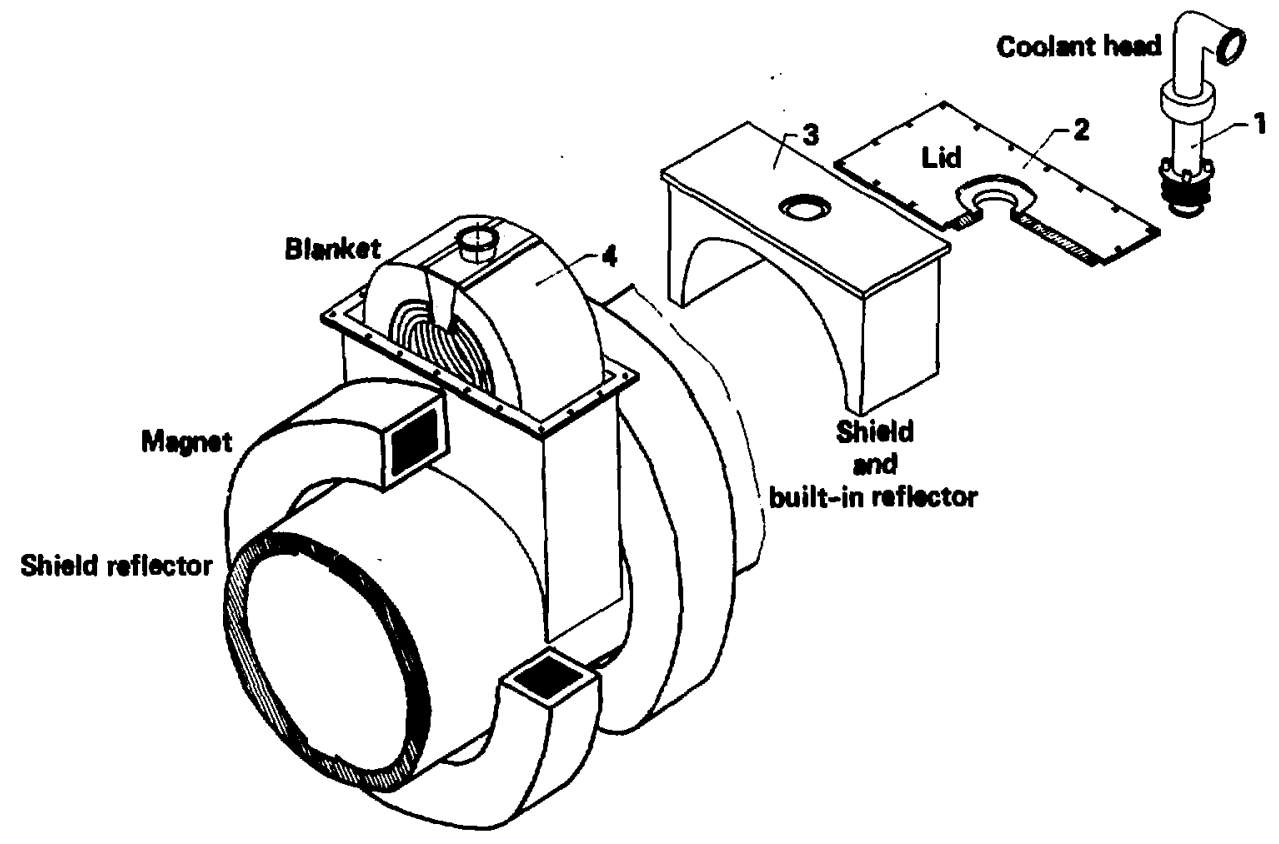

Fig. 4.2-37. Blanket maintenance procedure. 
1. MCMP - A General Monte Carlo Code for Meutron and Photon Transport, Los dTamos hational Laboratory, Los Alanos, Kew Mexico, LR-7396-N, (1981).

2. M.A. Hoffman and G.A. Carlson, Calculation Techniques for Estimating the Pressure Losses for Conducting Fluld Flows in magnetic FeTds, Lawrence ITrermore hational Laboratory, LIVermore, CA, UShEC Report UCRL-50110 (1971).

3. J. DeVan, Compatibility of Structural Materials with Fusion Reactor Coolant and orecer Flutd.

4. W.D. Wilkinson, E.W. Hoyt, and H.V. Rhude, Attack on Materials by Lead at $1000^{\circ} \mathrm{C}$, USAEC Report AML 5449 (Oct. 1955).

5. R. Parkman and 0.C. Cutler, Investigation of Materials for Use in a Heat Transfer Loop Containing Liquid Lead Alloys, Report XII, USAEC Report ORO-45 (Juine 1951).

6. G.M. Tolson and A. Taboda, A Study of Lead and Lead Salt Corrosion in Thermal-Convect ion Loops, Oak Ridge Rational Laboratory, Oak RTdge, IN, USAEC Report ORILLTII-1437 (April 1966).

7. W.N. Gill et al., Mass Transfer in Liquid-Lithium Systens," AICHE $\underline{J} \underline{6}$, $139(1960)$.

8. J.H. Frye, H.D. Manly, and J.E. Cunningham, Metaliurgy Division Annual Progress Report for Period Ending September 1, 1959, Oak Ridge Rational Laboratory, oak Ridge, Th, USAEC Report ORRL-2839 (12/16, 1959).

9. J.R. Weeks, MASA Spectal Publ. SP-41 (1963).

10. M. Hanson, Constitution of Binary Alloys, McGraw H1ll (1958).

11. R.C. Asher, D. Davids, and S.A. Beetham, "Some Observation on the Compatibility of Structural Materials with Molten Lead, "Corrosion Science 17, 545 (1977).

12. A.J. Romano, C.J. Klamut, and D.H. Gurinsky, The Investigation of Container Materials for Bi and Pb Alloys, Part 1, Thermal Convect ion Loops, Brookhaven attonal Laboratory, Brookhaven, WY, USAEC Report BiL-811 (JuTy 1963).

13. R.G. Clemerer and D.R. Aristrong, "An Experimental Study of the Reaction of $\mathrm{Li}_{7} \mathrm{~Pb}_{2}$ with Water," IRSAW, 32, p. 71 (1979).

14. R.G. Clemmer and D.R. Armstrong, "The Reaction of Lead-Lithium Alloys with Nater," TRSANO, 34, P. 55 (1980).

15. A.G. Rogers at al., Miguid Lithium-Leid-Bismuth, A New Tritium Breeder," in Proc. of gth Symposium on Engimering Problems in Fuston Research, Chicago (1981). 
16. B. Badger et al., TASKA, UAFDM-500/FPA-82-1/KfK-3311, Sec. V.9 (1982).

17. B. Brown, T. Blewitt, T. Scott, and A. Klank, J. Mucl. Mat.., 52, 215 (1974).

18. R. Coltman, Jr., and C. Klabunde, J. Mucl. Mat., 103 \& 104, 717 (1981).

19. "ANISH-ORM," RSIC Code Package CCC-254, Radiation Shielding Information Center, Oak Ridge Mational Laboratory, Oak Ridge, TK, (1979).

20. VITAMIN-C, 171 Neutron, 36 Gama-Ray Group Cross Section Library in AMPX Interface format for Fuston lieutronles Studies, DLC-41-B, RSTC bata Itbrarym Oak sidge Kational Laboratory, Odk RTdge, TH, (1977).

21. MCKLIB-IE, 171 Neutron, 36 Gama-Ray Group Kerma Factor Library, DLC-60B, RSTC Data Library, Oak RTdge National Laboratory, Oak Ridge, TN, (1982).

22. L.J. Perkins, Optimization of Fusion Blanket Design by Minimization of the Economic Figure of herlt for the Overall Reactor System, University of Wisconsin Fuston Engineering Program Report (to be published).

23. C.C. Baker et al., STARFIRE - A Commercial Tokamak Fusion Power Plant Study, Argonne Mational Laboratory, AIL/FPP-80-1 (1981).

24. B. Badger et al., WITAMIR-1 - A Tanden Mirror Reactor Study, University of Wisconsin Fusion Englneering Program Report UWFDH-400 (Dec. 1977), Ch. XI.

25. B. Badger et al., MUWuK - A Tokamak Reactor Design Study, University of Wisconsin Fusion Engineering Program Report UWFDH-330 (1979), Ch. XI.

26. E. Veleckis, Argonne National Laboratory, private communication (June 1981). (A $B$ isotope effect is assumed in converting hydrogen data to tritium data).

27. K. Ruttinger, "Vacuum Degassing in the Liquid State", Vacuum Metallurgy, 0 . Winkler and R. Bakish (Eds.), Elsevier Pub. Co., New York, (1971) Pp. 337-463 and references therein.

28. C. Baker et al.. STARFIRE/DEMO - A Demonstration Tokamak Power Plant Study, Argonne National Laboratory, ANL/FPP/IM-154 (1982), Ch. 4.

29. R.D. Pehlke and A.L. Bemen, Jr., Mass Transfer of Hydrogen Between Liquid Aluminum and Bubbles of Argon Gas," Trans. AIME 224, 1239-1242 (1962).

30. C.B Alcock, Principles of Pyrometal lurgy, Academic Press, New York (1976) 305-312.

31. J.D. Sharp, "Physical Considerations in the Stream Degassing of Steel" and references therein in Proc. of the Iron and Steel Institute Annual General Meeting, London, England (hay 5-6, 1965), Special Report 92, Perry Lund, Humphries and Co. Ltd., London, 50-54. 
32. J.T. Bell et al., "Tritium Permeability of Structural Materials and Surface Effects on Permeation Rates", in Proc. on the Tritiun Technology in Fission, Fusion and Isotopic Applications, Dayton, of (1980).

33. P. Tortorelli and J. DeVan, "Compatibility of Stainless Steel with Pb-17 at. $\mathrm{LI}$ ", in Proc. of The International Corrosion Forum," Houston, TX, (March 1978). 


\subsection{HIGH TEMPERATURE BLANKET}

The principal goal of the high temperature blanket design is to produce high temperature heat for synthetic fuel or electricity production. This year we have concentrated on electricity production; the blanket design has been optinized in conjunction with the energy conversion cycle to maximize thermalto-electrical efficiency. Additional goals that have had a significant effect on the design are manufacturability, low tritium inventory, no reactive liquid metals, no exotic materials, and a maintenance concept that al lows all central cell components to be replaced as part of nomal operations.

\subsubsection{Blanket Destgn}

The MARS high temperature blanket concept is pictured in Fig. 4.3-1. All structural steel (HT-9) is th direct contact with $\mathrm{Lf}_{17}{ }^{\mathrm{Pb}} 83$ for cooling and to minimize neutron capture in the steel. The high temperature zone consists of pods containing silicon carbide pebbles doped with $2 \%$ lithium aluminate for breeding tritium and cooled with 80 atm helfum exiting at $900^{\circ} \mathrm{C}$.

The major goal of the high temperature blanket design is to meet the dual purpose of high effictency electrictty or synfuels production. Performance goals include a high temperature $\left(900\right.$ to $\left.1000^{\circ} \mathrm{C}\right)$ heat fraction greater than 40\%, tritium self sufficiency (TBR > 1.05), and a 3-to-4 yr first wall/blanket lifetime. Major safety goals are low tritium inventory (less than $1 \mathrm{~kg}$ ), no reactive liquid metals, minimum long-term activation, and a passive loss of coolant flow safety system. Other design goals are the elimination of exotic materials (because of 1 imited resources and costs), a blanket and shield thickness less than 1.85 m to reduce central-cell magnet costs, and easily renovable magnets to simplify module change out and magnet replacement.

\subsubsection{Performance Summary}

Meutronics analyses show that the high temperature blanket has an energy multiplication $M$ of 1.15, a TBR of 1.13 and a deposited fraction of high temperature heat FHT of 0.48. First wall damage is $69 \mathrm{dpa} / \mathrm{FPY}$. Thermal losses from the high temperature to low temperature zones reduce FHT to 0.46 . A maximum structural steel temperature of $520^{\circ} \mathrm{C}$ is expected, with a $24^{\circ} \mathrm{C}$ maximum $\Delta T$ through the first wail. Silicon carbide temperatures are expected to remain below $1000^{\circ} \mathrm{C}$ with the $900^{\circ} \mathrm{C}$ helium exit temperature. The helfum 


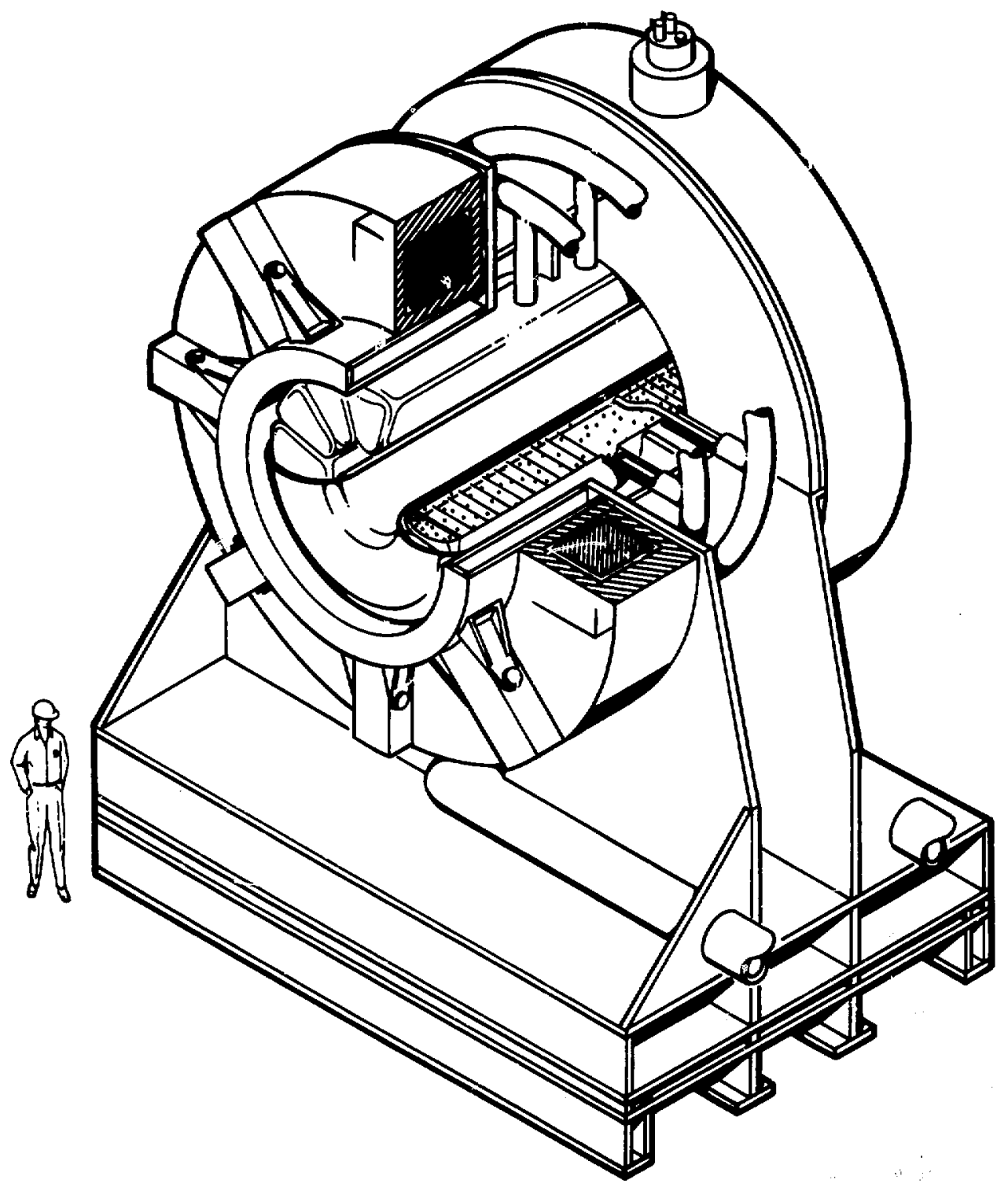

Fig. 4.3-1. High temperature blanket module. 
pumping power in the blanket is calculated to be $1.6 \mathrm{~m}$. Hellum inlet/outlet temperatures are 450 and $900^{\circ} \mathrm{C}$, and lithium-lead inlet/outlet temperatures are 372 and $482^{\circ} \mathrm{C}$. The 3-to-4-calendar year lifetime at $80 \%$ availability corresponds to 12 to $16 \mathrm{yr} / \mathrm{m}^{2}$ at the first wall. Fusion blanket 11 fetime studies remiln speculative; however, we consider 3-to-t calendar years reasonable at $5 \mathrm{~m} / \mathrm{m}^{2}$ wall loading.

The high temperature blanket and energy conversion cycle have a gross thermal to electrical conversion effictency of 46\%. Tritium breeding in the high temperature zone is mecessary to simultaneousiy achieve tritium self sufficiency and a high FHT. Using a small amount $\{2 x$ by volume) of lithium aluminate reduces the problems associated with sintering wile exceeding the tritiun and heat fraction goils. Tritium inventory in the solid breeder is predicted to be about $100 \mathrm{~g}$.

We are currently studying blanket lifetime. At this time we can only estimate the effects of swelling, embrittlement, and creep of structural steels at the fusion neutron fluences and energies on lifetime because we lave only recently begun studies of the combined effects of these phenomena. The blanket has been designed to tolerate swelling by allowing room for expansion. Predicted swelling and creep rates and neutron effects on ductile-tobrittle transition temperature are given in Section 4.3.1.8.

Tritium inventory in the solid breeder is predicted to be about $100 \mathrm{~g}$. Activation is minimized by materials selection. The most activated materials in the blanket are elements added to the steel such as molybdenum, nickel, and manganese. HT-9 with isotopically tallored molybdenum may be a feastble low activation structural material. Minimizing the steel in the blanket also reduces the amount of activated material. We have not yet analyzed LOCA and LOFA. The two coolant blanket design should prove advantageous if one of the coolants is lost.

We have successfully avolded exotic materials in this blanket concept. The blanket and shield thickness of $1.6 \mathrm{~m}$ has not yet been fully optimized; further work is expected to reduce this value. The current magnet mounting system is designed for simple removal. Work is continuing on the magnet mounts to reduce heat leak. 
The major issues associated with the high temperature blanket design are: Lipb corrosion, structural IIfetint, WD effects on liquid metal cooling, and tritium inventory in the solid breeder. LiPb corrosion of HT-S is currently being investigated is the fusion coimurity. New experimental results are not likely to be avallable in time to affect the design. We can only roughly estimate the lifetime of structures in the fusion environment, and this will remain an open issue for all fusion reactors until better analytical tools and experimental results are available. Tritiun hold-up in the porous silicon carbide and the lithium aluminuie solid breeder is expected to be small. Howei, $r$, neutronic effects on sintering, radiochemical effects on solubility, and precise rates of diffusion and removal are not well known.

\subsubsection{Module Description}

The MRS high temperature blanket is a two temperature zone blanket: a helfum-cooled high-temperature $\left(900^{\circ} \mathrm{C}\right)$ zone and LtPb-cooled iow-temperature $\left(500^{\circ} \mathrm{C}\right)$ zane. Tia blanket structural material is HT-9 ferritic steel kept below $520^{\circ} \mathrm{C}$ by the LiPb coolant. Each blanket module is composed of a cylindrical first wall structure and two LiPb end-closure plena; a set of 12 pods (submodules) that are susperided with in the LiPb form the high temperature zone. The encire mass plus the nagnets are supporied by the shfeld structure (see Fig. 4.3-1).

Low-Temperature Zone Structure. The low temperature structure is r.0oled by axtally flowing LiPb surrounding the pods. All steel components are kept below $520^{\circ} \mathrm{C}$. This region consists of: (1) the first wall structure, (2) interface panels between pods, (3) an edge support panel between the shield and back of the pods, (4) the pod support rails, and (5) the end plenum closures. These are shown in Figs. 4.3-2 and 4.3-3.

The first wall consists of a cylindrical, corrugated sandwich wall of HT-9. The composite wall is $5 \mathrm{~cm}$ thick, with a $0.5-\mathrm{cm}$ first wall and a $1.5-\mathrm{cm}$ second wall. The corrugation stabilizes the structure and provides flow direction for the LiPb [see Fig. 4.3-3(a)]. Corrugated steel interface panels are placed between adjacent pods to provide LiPb flow passages for cooling and structural support for the pod sidewalls [see Fig. 4.3-3(b)]. The edge support panels are sintlar to the interface panei, consisting of corrugated, sandwich steel panels placed between the back wall of the posts ard the shield to pro- 

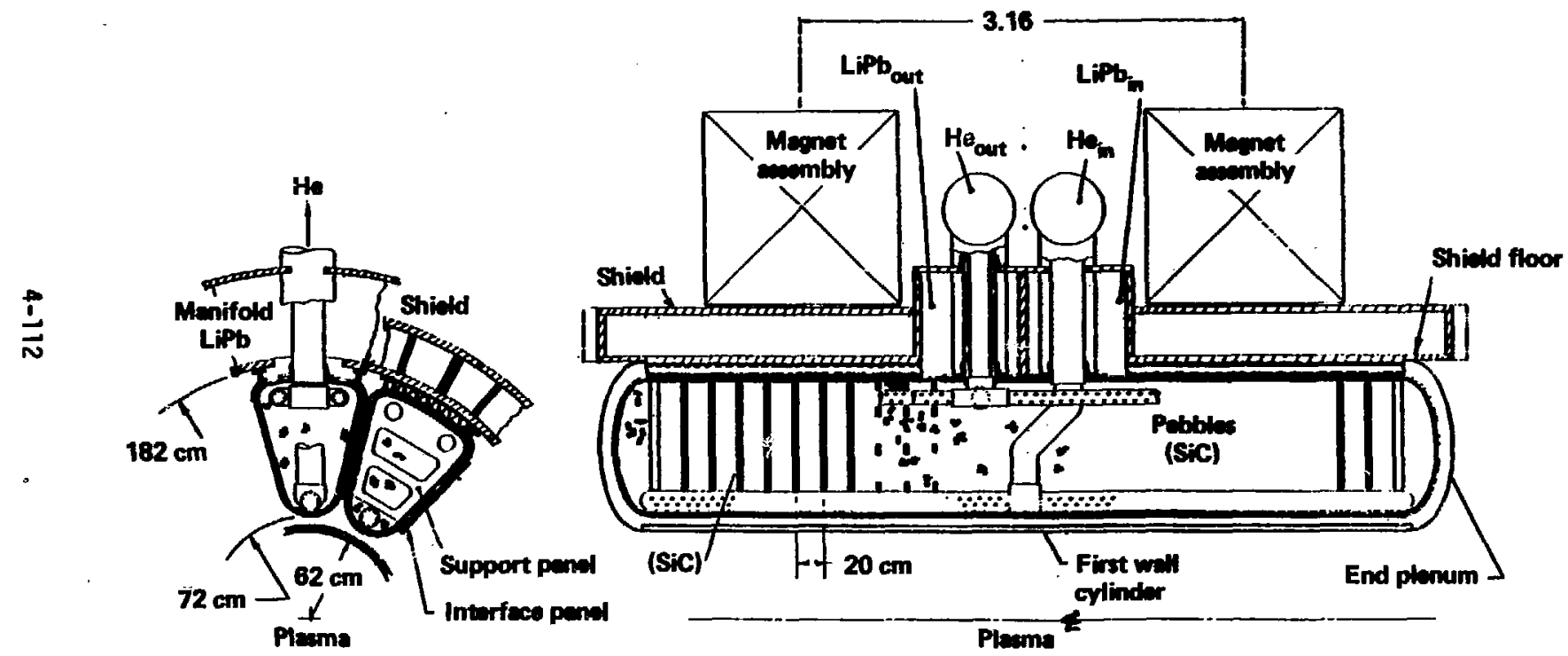

Fig. 4.3-2. High temperature blanket structure. 


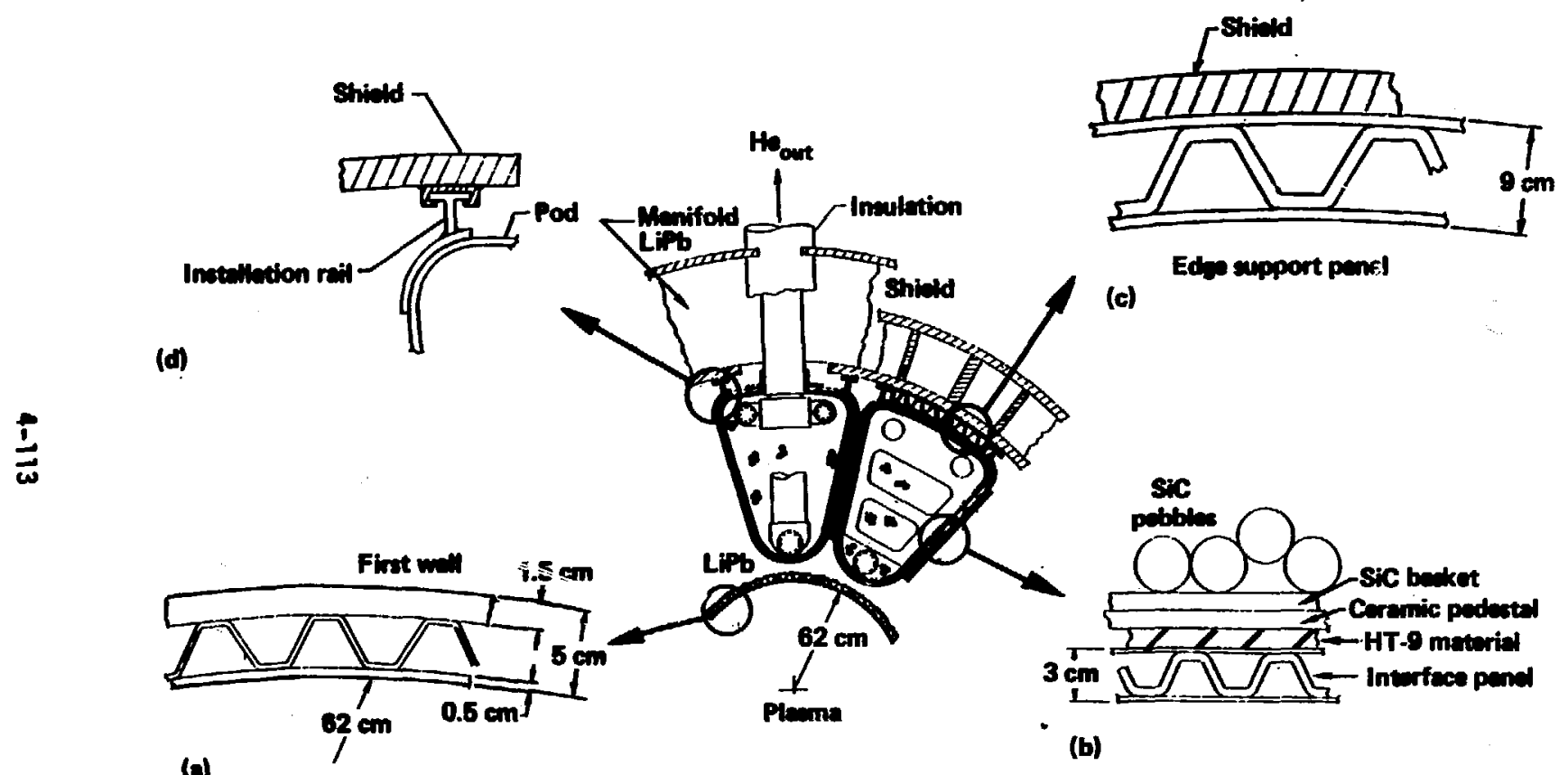

(a)

Fig. 4.3-3. Blanket first wall and interface structure. 
vide Lipb flow passages for cooling, and to transfer the hellum pressure load from the pods into the shield structure [see fig. 4.3-3(c)]. The pod support system is a T-section support rail of HT-9 attached to each back corner of the pods. The support rails are mounted to the shield structure for support during assembly (see Fig. 4.3-3). Thie end plena consist of an annular end closure cap (5 min thick) welded to each end of the first wall and the blanket shleld (see Fig. 4.3-2).

High-Temperature Zone Structure. The high temperature pod is a 30-deg circular-sector-shaped pressure vessel constructed of HT-9. The vessel is $592 \mathrm{~cm}$ long and $100 \mathrm{~cm}$ high with a nose radius of $23 \mathrm{~cm}$. The vessel wall is $1.1 \mathrm{~cm}$ thick with $2.1-\mathrm{cm}$-thick semi-elliptical done welded to each end, as shown in Fig. 4.3-4. The steel vessel wall is maintained at or below $520^{\circ} \mathrm{C}$ by Lipb circulating on the outside of the pod.

Silicon carbide (SiC) spherical pebbles $(3.4 \mathrm{~cm}$ in diameter) and support panels ( $3 \mathrm{~cm}$ thick) are supported on a $1 \mathrm{~cm}$ thick SiC basket. The pebbles contain 2 atomic \& lithium aluminate. The basket supports the pebbles and panels and separates then from the steel vessel. Thermal insulation (lowdensity alumina-silica mat) fills a 1-cm gap between the steel vessel wall and SiC basket. The gap is provided by $1-\mathrm{cm}-$ high ceramic spacing pedestals placed between the steel vessel wall and the basket in allgnment with the support panels (see Fig. 4.3-4). There are 23 support panels in each pod, spaced at 20-cm intervals, except in the central helium inlet/outlet area. The support panels preclude a domino fallure in which a sudden loss of pressure in one pod causes adjacent pods to burst in series.

Module Structures. The shield is the main structural skeleton of the high temperature blanket. The end plena and high temperature pods are attached to and supported by the shieid. Because the shield, magnet supports, piping structures, and module support have been integrally designed with the blanket, these structures are described here.

Magnet Support. The shield structure acts as an integrated box beam and as such is designed to transmit loads resulting from the magnet weight (200 tons) and critical fallure condition (13,000 tons axially). The magnet loads are transmitted to the shield via the efght-pin joint-attach fittings shown in Fig. 4.3-5, wich are secured to a continuous circumferentialstructural ring that attaches through bolt patterns to the shield. 


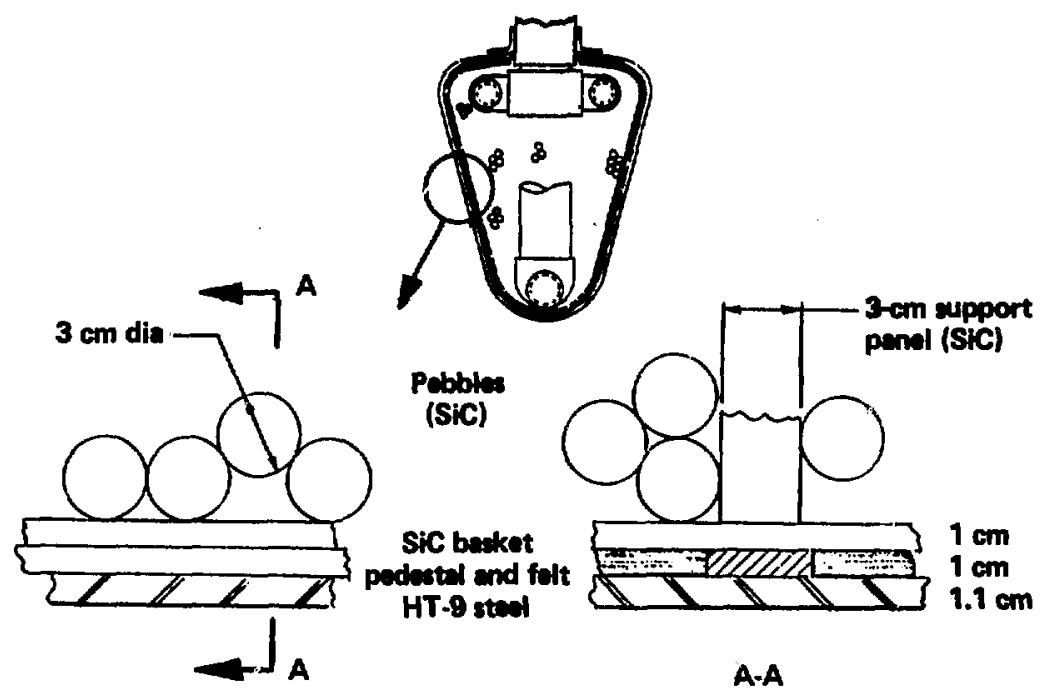

Fig. 4,3-4. Helium pod. 


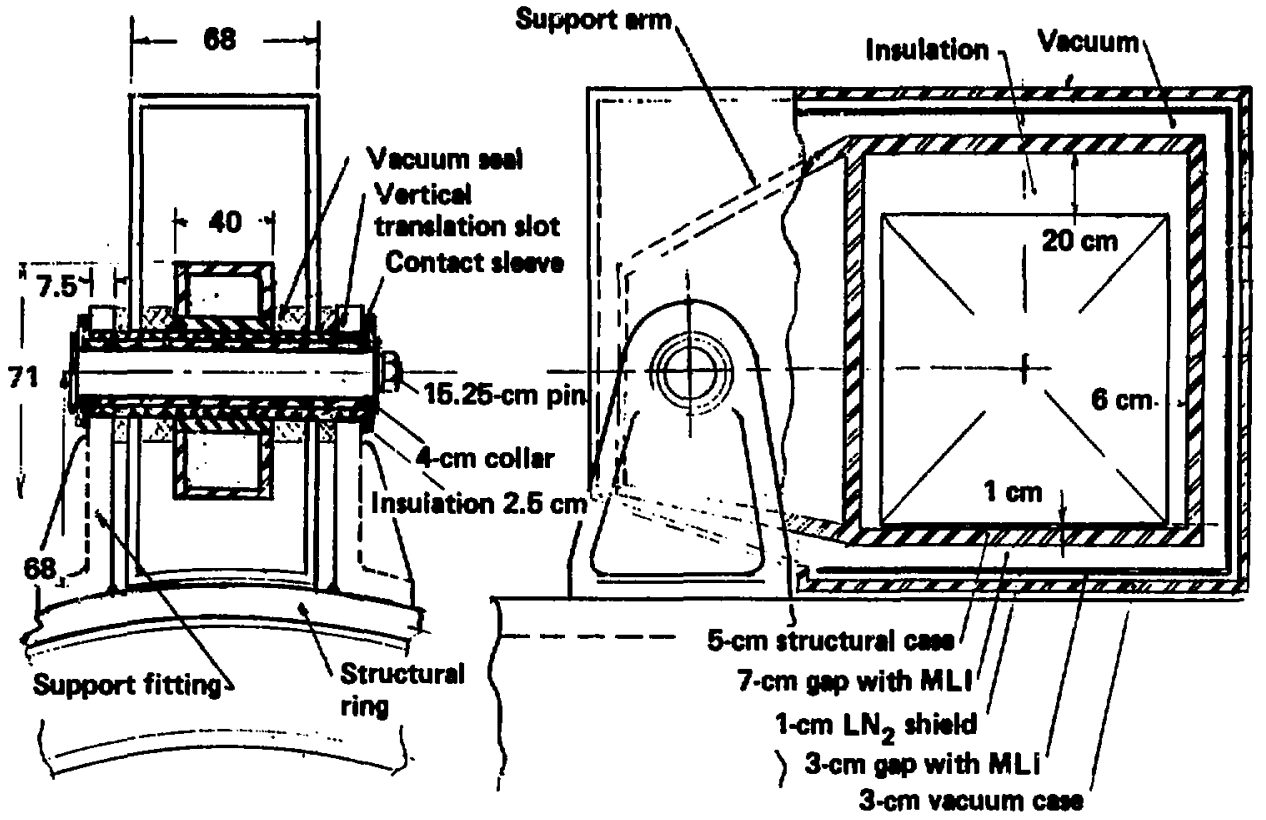

Fig. 4.3-5. Magnet support structure. 
This type of support has several unique design advantages that permit minimu effort for removal of the magnets, restrict paths for minimizing magnet heat loss, and can accommodate magnet fallure loads. Magnets can be separated from the module by renoving the shear bolts used to secure the structural ring to the shield. Enough stiffeners are provided to ensure minimum out-of-round deflection of the ring. Conduction heat paths are intercepted at the pin joint by $2.5-\mathrm{cm}$ polyimide insulating collar. Active liquid-nitrogen cooling can be introduced within the pin (by providing a 1.5-1n.-diamerer hole) to increase the collar effectiveness. Additional intercepts can be provided by isolating the magnet support arms with a pin/truss type structure.

The superconductor will contract during chilldown by approximately $2 \mathrm{~cm}$. This deflection, as well as vertical allgnment tolerances, can be accomodated by incorporatjing, vertical translation slots into.the support . . . . fitting. Because of the geometry of the magnet support structure, this also defines a single predictable load path for the magnet wight; the magnet is essentially hung from the top pin. Because the support pins are sized for the fallure load, they provide a simple method of supporting the weight wille relfering tolerance requirements.

The ilquid-nitrogen magnet heat shield is attached directly to the magnet vacuum case by spacers, while the vacuum case itself is directly supported on the pin and is therefore free from physical contact with the superconductor. The vacuum. is isolated at the pin foint by insulating vacuun seals.

Lithiun-Lead and Helium Piping Structures. Figures 4.3-6 and 4.3-7 show the flow paths for the LiPb and helium coolants. The relatively low temperature and pressure requirements for the $L \mathrm{LP}$ allows the use of rectangular flow paths with conventional stcel plate construction. The shield construction geometry allows the water and LIPb flow paths to be separated from each other by double wall construction, and also allows the separation of any interface weld joints. The high-temperature, high-pressure hellum pipes are insulation-lined.

Assembly Structural support. The module weight and magnet loads are carried to the floor with conventional plate steel construction. This support structure uses box-type construction to maximize rigidity and redundancy. Loads can be reacted in the floor through grips and/or bolt tie downs. 

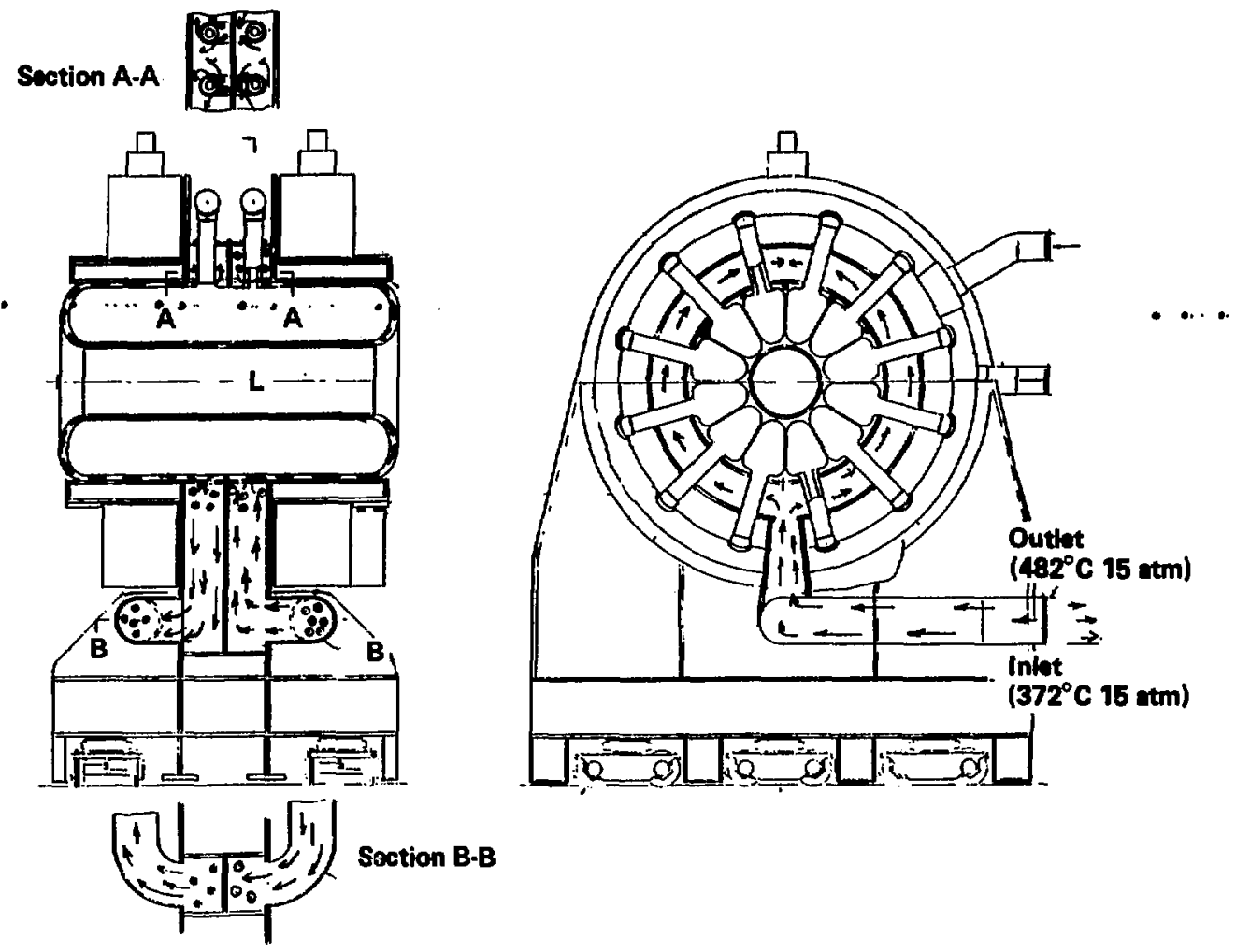

Fig. 4.3-6. Lipb coolant flow paths. 

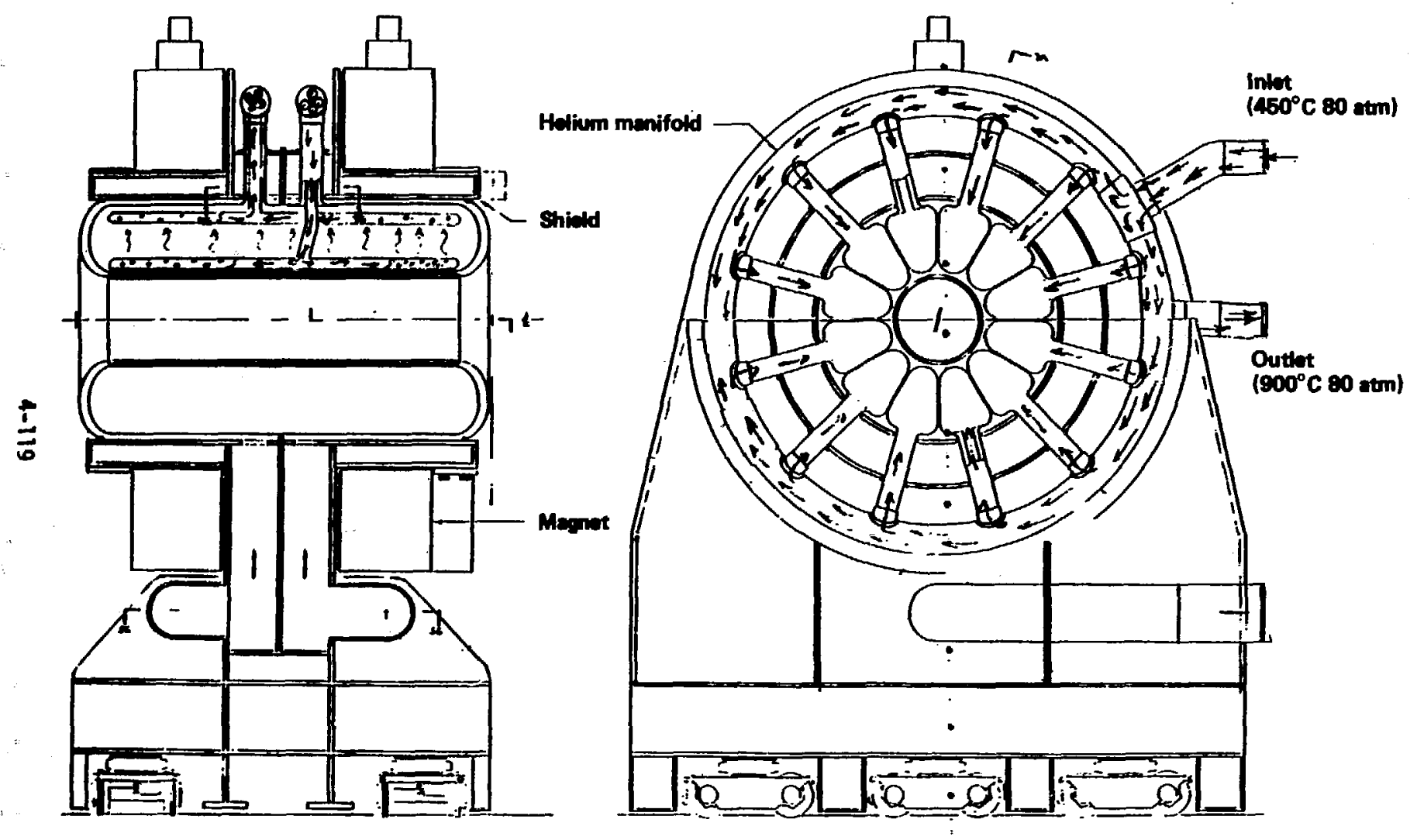

Fig. 4.3-7. Heilum coolant flow paths. 
Reactor alfgniment is provided by module-mounted jacks. Transhaulers are used to move the modules into the reactor for positioning and alignment. Six of these rail-type movers are required to move a module. They are remotely operated, permit 360-deg module movement and incorporate a vertfcal ifft stroke of $19 \mathrm{~cm}$.

\subsubsection{Structural Analysis}

First Mall. The first ill is a cylindrical shell of corrugated sandwich construction with the corrugation orfented in the axial direction. This shell is subjected to an external pressure load from the LiPb. The shell first-wall structure is classified as an orthotropic shell. Reference 1 gives the procedures used to analyze sandwich cylinders. However, if the structure has to be designed for a specific lifetime at an elevated temperature, an isochrqnous stress-strain, diagcan for the material. is required. The ASME Botler Code ${ }^{2}$ only contains procedures for analyzing monocoque cylindrical shells under external pressure. To use the design procedures of the boller code, we derived an equivalent cylinder thickness based on the sandwich shell having equal stiffness in both the hoop and axial directions for a sandwich construction of a total thickness of $5 \mathrm{~cm}$, with the outer facing $1.5 \mathrm{~cm}$ thick and inner facing $0.5 \mathrm{~cm}$ thick; the equivalent thickness is computed to be $4.05 \mathrm{~cm}$. The allowable pressure is not currently avaflable in the boiler code for HT-9. We used the allowable for nickel-iron-chromium alloy 800 because the mechanical properties for this alloy are close to those of HT-9. The design based on this analysis is rated for 200-psi allowable external pressure, which produces a hoop stress of $5.9 \mathrm{ksi}$. Boller code section 4.2.12.1 lists the 100,000-ini creep rupture stress of $16 \mathrm{ksi}$ for HT-9. Hence we have concluded that the chasen geometry satisfies the design requirements.

Pod Analysis. The pods are basically single-wall HT-9 construction under internal pressure of $80 \mathrm{~atm}$. A corrugated sandwich panel is inserted between the side walls of the adjacent pods to reduce the bending stress on the flat portion of the pods. The nose section of the pods is of cylindrical construction. The minimum thickness of the nose section was obtained using VG-27(C) (1). ${ }^{2}$ Using an al lowable stress of $28 \mathrm{ksi}$ (1.e., $2 / 3$ yield stress), we computed the minimum thickness of the pod nose section to be $1.4 \mathrm{~cm}$. 
The design has $23 \mathrm{SiC}$ panels $3 \mathrm{~cm}$ thick. These panels are of nonuniform width to accomodate the pod section and have cutouts. If a fault condition artses where the pressure in a pod drops to zero, the pressure from the pods on either side has to be resisted to avoid a propagating failure. The structures that resist these forces are the side wall acting as a sandwich panel under bending and the SIC panels acting as plates under compression. A preliminary analysis showed that these parels do not have a problem resisting the bending and compressive loads.

The side walls of the pods can be treated as flat rectangular sandwich plates supported by SIC panels. Other considerations dictate maximum spacing of $100 \mathrm{~cm}$ between the stlicon carbide panels near the helfun entrance piping. Treating this $100-x-70-\mathrm{cm}$ panel as a simply supported sandwich plate and using the procedure outlined in Ref. 3, we computed that a pressure of 850 pst produces a maxtmon bending stress , of $.42 . \mathrm{ks1}$. . In bending.. the botler code allows the stress level to reach as high as the yield stress. This means that the sidewalls of the pods can withstand a pressure drop of 850 psi as a fault condition. Analysis has shown that an 8-in.-diameter hole in a pod will cause a maximum pressure drop of 350 psi between adjacent pads.

End Closure Analysis. There are two types of end closures: Individual pod end closures of approximately semi-ellfptical dome shape under an internal pressure of $80 \mathrm{~atm}$, and a torotdal ring end plenum under an internal pressure of 200 psi. For preliminary analysis purposes, both end closures may be approximated as ellipsotal heads with a ratio of 2:1. Boiler code ${ }^{2}$ equation VG-32d is used to determine the minimu thickness. This results in minimum thickness of $2.1 \mathrm{~cm}$ for the pod end closure and of $0.5 \mathrm{~cm}$ for the end plenum.

Magnet Support Analysis. The magnet support consists of elght doubleshear-pin assemblies that transfer magnetic and gravitational forces from the magnet into the support structure. The support structure then transfers these forces to the ground. The gravitational forces are from the weight of the magnet and the structure. The magnetic forces are caused by a fault condition whers one manet denergizes but the others keep functioning. This results in a 2.7-x-107-lb laad axially applied on the manet center line.

Materials and dimenstons of some of the magnet support components muve not yet been specified. We have analyzed the key areas of high stress (the support pins and the magnet mounting bolts). A von Mises faflure criterion is 
assumed for a biaxial state of stress with a safety factor of 1.5. The pin was analyzed as two short cantllevers, rigidly fixed at the center section. Because of the shear and bending, the 10-1n.-diameter pin material meeds to have a yield stress of $\sim 140 \mathrm{ks1}$ (e.g., SS 304LK). When the material is selected, a fracture analysis will be performed to avold catastropic brittle faflure. The bolts mounting the magnet assembly to the module support structure require a shear yfeld strength of $80 \mathrm{ksi}$ (e.g., heat-treated SS 316). A total of 20 2-in.-diameter bolts is required for each pin support assembly (10 per side).

Module Structural Support Analysis. Module structural supports are also in the preliminary stages of design. The critical section for the support occurs midway down where simple box structure resists the bending moment and shear. At this section, assuming 2-in. thickness on inner walls and.4in. thickness on outer walls, material yield stress should be on the . order of $64 \mathrm{kst}$. The base section for transfer of the loads to the ground should have a yield stress of $78 \mathrm{ksi}$. Because high-strength heat-treated materlals are impractical for the large support structures, these areas will be reinforced. We have reported the strength requirements for this first cut design because they show that the stresses are within reason, requiring less than a factor of two reduction to allow the use of any of several steels.

Main Areas for Study. He have presented the results of our preliminary analysis for the first wall, pods, and end closures. A detalled analysis is still necessary, including thermal stresses based on realistic values of thermal gradients and stresses due to swelling of the materials, If any. A detalled analysis of the pod support system and the discontinulty areas of the structure is also necessary. The structural integrity requires that fracturemechanics-based crack growth analysis be made to show that there is no fatigue crack growth problem.

\subsubsection{Meutronics}

Our neutronics analyses have concentrated on the analysis of module lifetime, tritium self-sufficiency, and the fraction of energy deposition in the form of high iemperature heat. We have covered the following areas:

1. Blanket ceramics (other than graphite) to overcone swelling 
problems (11fetime constraint).

2. Addition of lithlum-bearing compounds to the solid breeder (tritium production constraint).

3. Addition of a thick, frontal LiPb zone to enhance breeding and reduce parasitics in the solid breeder (tritiun production constraint).

4. Dimensional adjustment to balance tritium production, energy multiplication, and FHT.

5. Three-dimensional calculations to fine-tune the design.

6. Burn-up calculation to verify adequate module lifetime and demonstrate adequate module performance over that lifetine.

One-Dimenstonal Meutronics Analyses. Table 4.3-1 presents selected results from series of one-dimensional (1-D) calculations to evaluate the thickness of the front LiPb zone and thickness of the silicon carbide "breeder" region. The LiPb has natural lithium, while the breeder has 1 vol $x$ $\mathrm{LIAlO}_{2}$, 90\% enriched in $6 \mathrm{LI}$, in SiC. Earlier neutronics results indicated tritium production in the breeder was clearly dominated by the ${ }^{6}{ }_{L}(n, T)$ reaction; to resuce sintering, the volume fraction of lithium-bearing compound was minimized by $6_{L I}$ enrichment. We selected LiPb as the 11 thium-bearing compound for reasons of its chemical compatibility with SiC, as is discussed in Section 4.3.1.9.

Tables 4.3-1 and 4.3-2 present results of selected calculations of PbL1 lithium enrichment and volume fraction of $\mathrm{LAAlO}_{2}$ for fixed blanket dimension corresponding to a front $\mathrm{LiPb}$ zone of $5 \mathrm{~cm}$ and a breeder zone of $1 \mathrm{~m}$.

Table 4.3-3 shows spatial energy deposition for the one-dimenstonal model shown in Fig. 4.3-8: FMT is equal to the higher of the bracketing values given in Table 4.3-2. The FHT bracketing calculations are done by first assuming that the LiPb at the pod edge is to be ignored (generating the lower limit) and second by assuming the LiPb a. the edge is uniformly distributed throughout the pod (generating the upper 1imit). As input to thermal analysis, the upper linit on FHT is the most conservative.

Three Dimensional Neutronics. Figure 4.3-8 shows the cross-sectional equivalence between 1- and 3-D neutronics models; not shown are the axial zones corresponding to LIPb plena and module ends. The 3-D model was deemed 


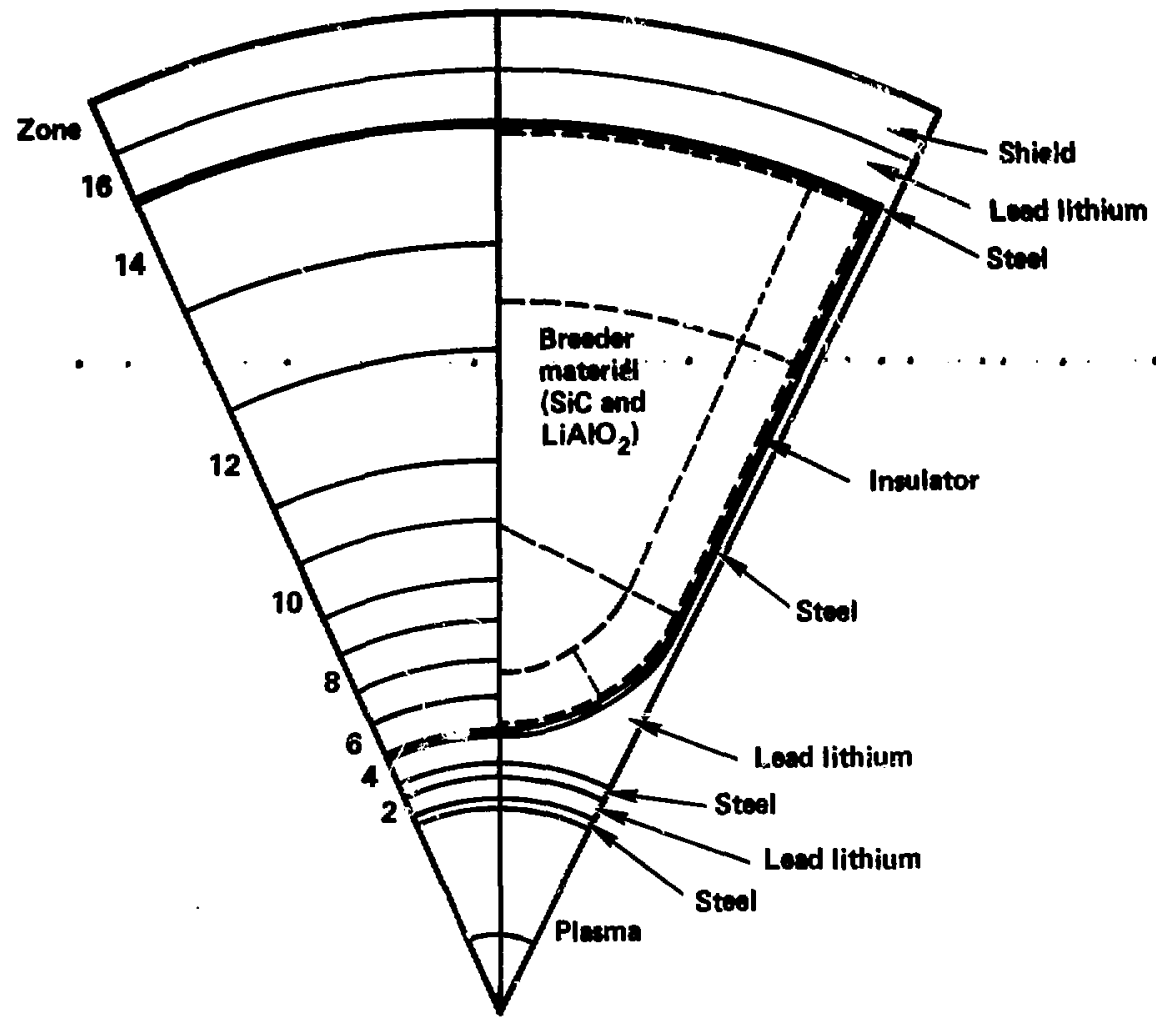

Fig. 4.3-8. One- and three-dimensional neuironics models (axtal details not depicted). 
Table 4.3-1. Blanket thickness snaiysis.

\begin{tabular}{|c|c|}
\hline $\begin{array}{c}\text { Thickness } \\
\text { of } \\
\text { front } \\
\text { PbLI } \\
\text { (cm) }\end{array}$ & $\begin{array}{l}\text { Thickness } \\
\text { of } \\
\text { breeder } \\
\text { region } \\
\text { (cm) }\end{array}$ \\
\hline
\end{tabular}

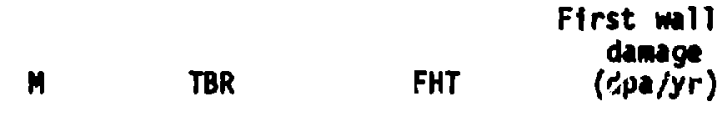

Leakage

$\begin{array}{rrrrllll}10 & 100 & 1.25 & 1.31 & 0.54 & 71 & 9.3 \mathrm{E}-4 \\ 14 & 148 & 1.26 & 1.38 & 0.62 & 74 & 4.5 \mathrm{E}-5 \\ 14 & 60 & 1.29 & 1.23 & 0.45 & 73 & 6.6 \mathrm{E}-3 \\ 5 & 148 & 1.22 & 1.25 & 0.74 & 68 & 8.6 \mathrm{E}-5 \\ 5 & . & 60 & 1.25 & 1.04 & 0.53 & 66 & 1.1 \mathrm{E}-2\end{array}$

Table 4.3-2. Lithium enrichment analysis.

\begin{tabular}{|c|c|c|c|c|c|c|}
\hline $\begin{array}{l}6_{\text {Li }} \\
\text { Enrich- } \\
\text { ment in } \\
\text { PbLt } \\
\text { ( } x_{\text {; }}\end{array}$ & $\begin{array}{l}\text { Fraction } \\
\mathrm{LAIIO}_{2} \text { in } \\
\text { SiC }^{-} \\
\text {breeder }\end{array}$ & $M$ & TBR & FHT & $\begin{array}{c}\text { First wit } \\
\text { damage } \\
\text { (dpa/yr) }\end{array}$ & $\begin{array}{l}\text { Energ } \\
\text { depositif } \\
(\mathrm{H} / \mathrm{cm}\end{array}$ \\
\hline $7.6^{\mathrm{a}}$ & 0.01 & 1.24 & 1.21 & 0.35 to 0.53 & 69.0 & 38.0 \\
\hline 7.6 & 0.02 & 1.23 & 1.24 & 0.39 to 0.54 & 69.0 & 37.8 \\
\hline 1 & 0.01 & 1.26 & 1.13 & 0.41 to 0.54 & 69.4 & 39.2 \\
\hline 1 & 0.02 & 1.24 & 1.19 & 0.44 to 0.56 & 69.4 & 38.7 \\
\hline
\end{tabular}

a Matural. 
Table 4.3-3. Blanket energy deposition.

\begin{tabular}{|c|c|c|c|}
\hline Zone & $\begin{array}{l}\text { Inner radius } \\
(\mathrm{cm})\end{array}$ & Material & $\begin{array}{l}\text { Energy deposition } \\
\text { (MeV/source neutron) }\end{array}$ \\
\hline 1 & 62 & Steel & 0.569 \\
\hline 2 & 62.5 & LIPD & 2.498 \\
\hline 3 & 65.0 & Steel & 1.695 \\
\hline 4 & 67.0 & LIPD & 2.361 \\
\hline 5 & 72.0 & LIPb & 0.267 \\
\hline 6 & 72.75 & Breader+steel+LIPD & 1.345 \\
\hline 7 & 77.5 & Breeder+steel +LIPb & 0.092 \\
\hline 8 & 82.5 & Breeder+steel+LIPD & 0.874 \\
\hline 9 & 87.5 & Breeder+stee $1+L 1 P b$ & 1.462 \\
\hline 10 & 92.5 & Breeder+stee $1+\mathrm{LIPb}$ & 1.143 \\
\hline 11 & 102.5 & Breeder+steel +L1Pb & 1.543 \\
\hline 12 & 112.5 & Breeder+steel $+L$ I $\mathrm{Pb}$ & 0.948 \\
\hline 13 & 132.5 & Breeder+steel +LiPb & 0.449 \\
\hline 14 & 152.5 & Breeder+steel+LIPD & 0.013 \\
\hline 15 & 171 & Steel & 0.107 \\
\hline 16 & 172 & LIPt & 0.107 \\
\hline \multirow[t]{3}{*}{17} & 182 & Shield & 0.241 \\
\hline & & & 17.65 \\
\hline & & & 0.56 \\
\hline
\end{tabular}


necessary to adequately assess the axial competition for neutrons between stlicon carbide regions without LIPb and the LiPb-filled regions between adjacent pods and at the module ends. Because the LIPb reglons are not int 1 mately dispersed within the breeder material, 1-D analysis will tend to overpredict tritium production and underpredict parasitic capture. FHT is also dffficult to treat adequately in 1-D analyses. (Table 4.3-2 shows the bounds.)

The 3-D performance parameters are seen to be $1.13,1.15$, and 0.48 for TBR, $M_{0}$ and FHT, respectively. These compare to the 1-D parameters of 1.24, 1.19 and 0.44 to 0.56 . It is worth noting that the depletion of ${ }^{L} \mathrm{LI}$ in the LiPb dinintshes the disparity between the 1-D homogenized zone descriptions and the 3-D system they model. Were the MRS high-temperature blanket design to use natural or enriched lithium in LIPb, the 1-D results would tend to further overpredict tritium production and energy multiplication.

Burnup Calculations. Using a solid breeder for both tritium breeding and high temperature heat production requires a time-dependent calculation to evaluate the effects of burnup. Lithtum burnup is particularly important since the neutrontcs performance is affected by the stze of the regions and the distribution of $6_{\mathrm{LI}}$ and $7_{\mathrm{LI}}$. The LiPb, which is distributed anong all the modules and mixed, is assumed to be maintained at a constant concentration. The principal effect of meutron interactions with $\mathrm{LAAlO}_{2}$ is a reduction in ifthium concentration. The $7_{L i}$ burnup is almost 3 orders of magnitude less than the ${ }^{6} \mathrm{Lf}$ burnup and $w 11$ be less than 18 over the life of the module. Therefore, only ${ }^{6}$ LI burnup is explicitly calculored. An average burnup rate for each zone is calculated from the 3-D TART run by

$$
K=\frac{R F}{L},
$$

where

$K$ is the burnup per atom of $6 \mathrm{Lt}$ per year,

$R$ is the reactions per source meutron (TART output),

$F$ is the source neutrons per moduie-year (for a mall loading of $\left.4.9 \mathrm{~m}^{2} / \mathrm{m}^{2}\right)$, and

$L$ is the ${ }^{6} L$ atoms per zone. 
The $6 \mathrm{LI}$ is then depleted at this rate for one year by $L I(T+1)=L(T) e^{-K}$, and a new 3-D TART run is performed. One-year time steps can allow burnup of $30 \%$ of the ${ }^{6 f}$ in the front zone, which changes the burnup rate by $15 \%$, but has at most a few percent effect on performance. TART is a Monte Carlo calculation; results for individual zones have standard deviations of about $1 \%$; performance summary results, combining several zones, have correspondingly better statistics.

The module performance for 4 FPY is sumarized in Table 4.3-4 and in Fig. 4.3-9. In the top portion of Fig. 4.3-9, tritium breeding is shom for four regions. Middle, front, and back refer to radial position in the solid breeder, and liquid refers to the LiPb. In the lower portion, low temperature and high-temperature zone energy depositions are shown. Energy multiplication is the sum of the two divided by 14.1, while FHT is their ratio. As $6_{\mathrm{Li}}$ is depleted (particularly in the front), tritium breeding and energy deposition shift back in the breeder and out to the LiPb. The increase in $M$ is primarily caused by increased neutron capture in lead.

These results demonstrate the neutronic viablilty of the solid breeder concept. Tritium breeding, based on average breeding from modules of different ages, is sufficient beyond 4 FPY. In the first 3 FPY, performance changes only slightly. The TBR drops only $4 \%$ while FHT drops $3 \%$ for an individual module. The change in TBR and FHT averaged over the central-cell is a factor of two lower.

First Wall Demage. Damage calculations were performed with the 1-D code ONEDAMT. The neutron flux was normalized to $4.9 \mathrm{~mm} / \mathrm{m}^{2}$ at the first wall. The results presented in Table 4.3-5 show the highest damage in the first wall of about $70 \mathrm{dpa} / \mathrm{yr}$.

Areas Requiring Further Analysts. Foremost anong the blanket analysis needs will be interactive development of a module-to-module interface design and the effect of the interface on neutronic performance and shielding. Activation analysis of major blanket components will be required as an integral part of the development of a mintenance scheme and as a component of the waste disposal contributions to systems economics. 
Table 4.3-4. Burn-up summary.

Tritium breeding ratio

T1me

(FPY)
M

FHT

Total

$\mathrm{LAAlO}_{2}$

LiPb

$\begin{array}{llllll}0 & 1.153 & 0.481 & 1.126 & 1.056 & 0.070 \\ 1 & 1.155 & 0.474 & 1.104 & 1.029 & 0.075 \\ 2 & 1.162 & 0.465 & 1.092 & 1.009 & 0.083 \\ 3 & 1.173 & 0.457 & 1.087 & 0.997 & 0.090 \\ 4 & 1.162 & 0.448 & 1.030 & 0.932 & 0.098\end{array}$


Table 4.3-5. Neutron damage.

First wall

Radius (cm)

\section{dpa/FPY}

62.125

69.3

62.375

66.4

Corrugation

$\begin{array}{ll}62.75 & 63.3 \\ 63.25 & 60.2 \\ 63.75 & 57.3 \\ 64.25 & 54.6 \\ 64.75 & 52.1\end{array}$

Second wall

$\begin{array}{ll}65.5 & 47.8 \\ 66.5 & 42.3\end{array}$

Pod wall

$\begin{array}{ll}72.375 & 27.3 \\ 73.542 & 24.9 \\ 75.125 & 22.3 \\ 76.708 & 19.9 \\ 78.333 & 17.7 \\ 80.0 & 15.9\end{array}$



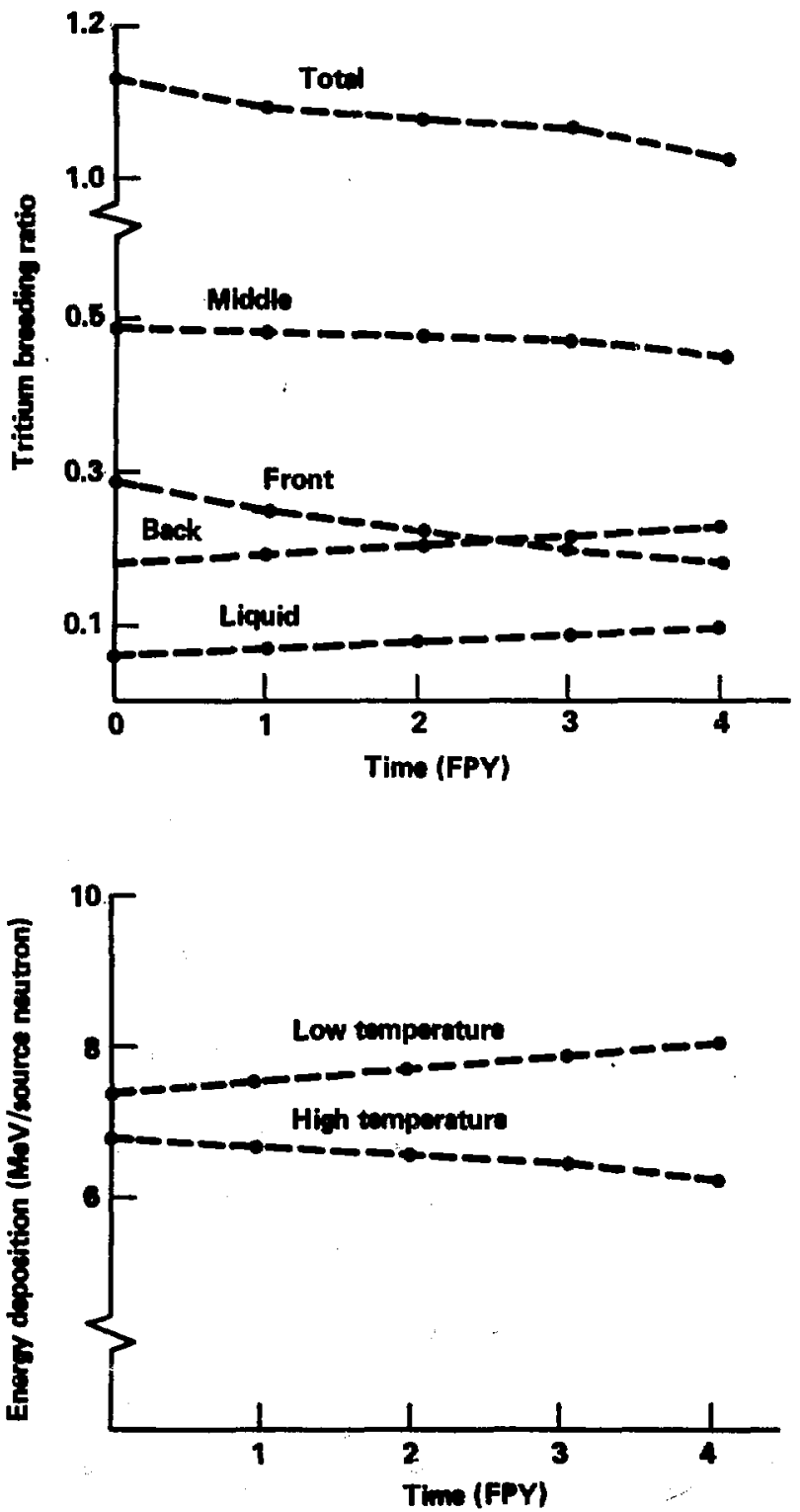

Fig. 4.3-9. Blanket lithium burnup. 


\subsubsection{Thermal Hydraulics}

High Temperature Zone. Hellum is used as the high-temperature working fluld to carry away the heat absorbed in the stlicon carbide and spherical pebbles. Calculation of the heat transfer coefficient in the packed bed is done using a stanton number correlation 4

$$
S t=0.40 \mathrm{Re}^{-0.437} \mathrm{Pr}^{-2 / 3}
$$

where

St Stanton number $=h / G C_{p}$,

Re - Reynolds number.

$\mathrm{Pr}$ - Prandtl number,

$C_{p}=$ Specific heat,

$G=$ fluid mass velocity based on unpacked tube, and

$h$. heat transfer coefficient.

The temperature drop between the surface of the ball and the surrounding helium can then be calculated.

The temperature distribution in the packed bed varies significantly with pebble radius. Figure 4.3-10 deplcts the relationship that exists between the pebble diameter and the film drop proflle through the bed. At small pebble dlameters, the larger surface area keeps the flim drop low, causing a large front-to-back temperature gradient in the surface temperature of the pebbles, side wall, and support blocks. A ball radius exists that produces a nearly flat, pebble-surface temperature profile throughout the bed, with a large film drop in the front of the bed, and a smaller film drop in the pack of the bed. This ball radius is about $1.9 \mathrm{~cm}$ as is shown Fig. 4.3-10. At this radtus, the structural blocks and sidewalls will not be subjected to large front-to-back temperature gradients. The maximum film drop temperature of $455^{\circ} \mathrm{C}$ occurs in the front of the bed and maximum STC ball surface temperature is $910^{\circ} \mathrm{C}$. The maximum ball centerline temperature is $950^{\circ} \mathrm{C}$.

Helium Fluid Hydraultes. One consideration of using helium as the high-temperature working fluid is the pumping power needed to flow large volumes of the gas through the system. The pressure drop through the module has been calculated for the flow paths shown in Figs. 4.3-2 and 4.3-7. The 

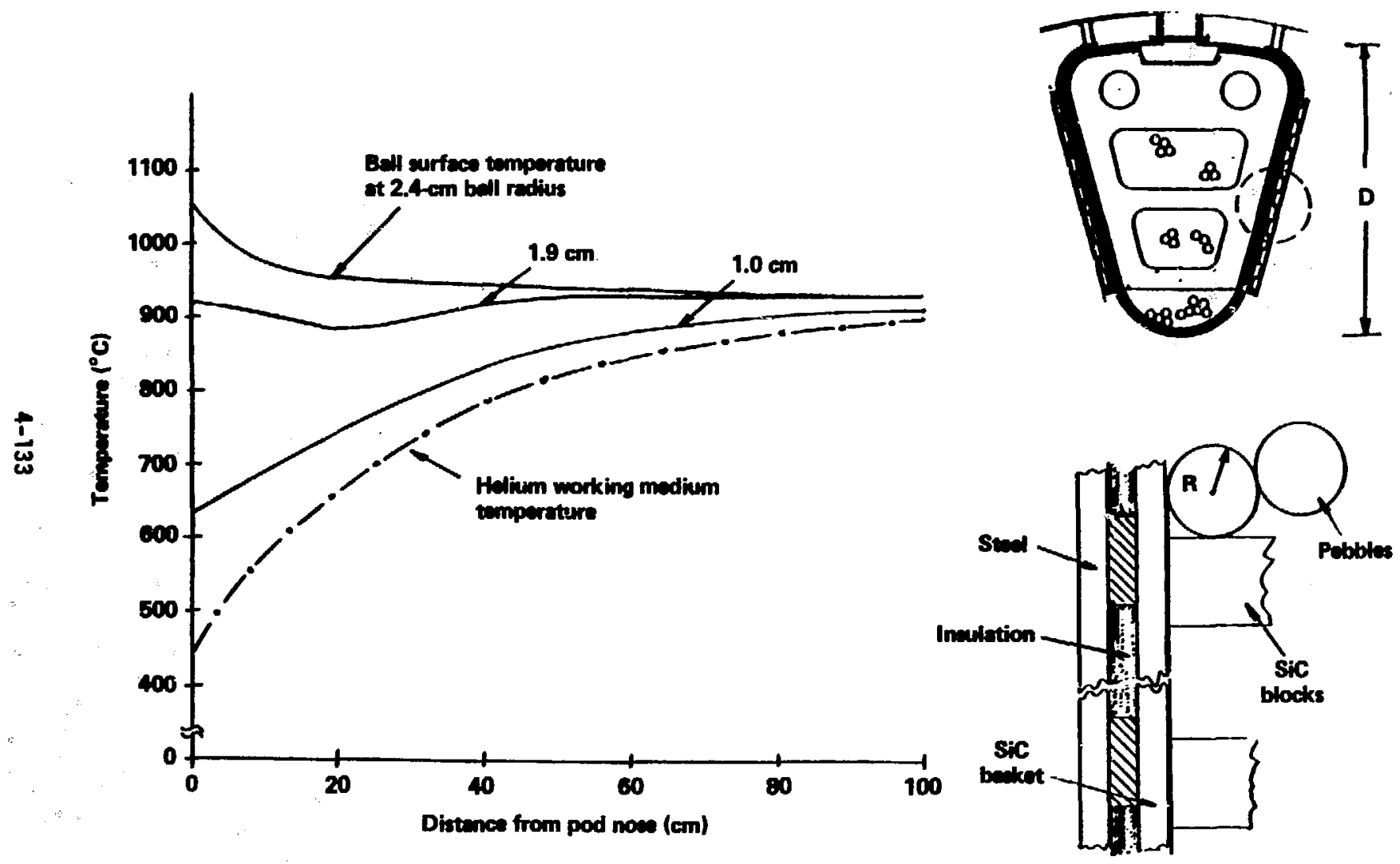

Fig. 4.3-10. Effect of pebble size on bed temperature. 
coolant operates at 80 atm with $T_{\text {in }}$ at $450^{\circ} \mathrm{C}$ and $T_{\text {out }}$ at $900^{\circ} \mathrm{C}$. A simplified flow schematic of the hellum piping system is show in Fig. 4.3-11, 11lustrating the detail of the pressure drop calculation. The total pressure drop is a sumation of the pressure drops through inlet/outlet manifold straight pipes and manifold tees, pod distribution and collector pipes, and packed bed.

To estimate the pressure di op through the pipes, we used a conservative value of friction factor to account for high-temperature piping insulating material. This value may be relatively rough. We calculated the pressure drop in the manifold and pod entrance/exit tees using the equivalent length method outlined in Ref. 4. This method estimates the equivalent length of straight pipe that would cause the same frictional pressure drop as flow through a standard tee.

We estimated the pressure drop in the pod distribution entrance pife and the pod coliector pipe according to the procedure outlined in Ref. 5. The method provides empirical data for calculating the pressure change through holes of 90-deg turns of flow into and from a branch side duct.

We calculated the pressure drop in an incompressible packed bed composed of nearly spherical particles as described and developed in Ref. 4. The continuity equation was our basis for caiculating the bulk velocity of the helium in the packed bed. The total pressure drop through the module helfum piping system is $5.6 \mathrm{psi}$. The pumping power output requirements is $0.067 \mathrm{~m}$ per $6.3+m$ module.

Further work must be done to size the holes in the pod diffuser and collector for proper distribution of the flow through the pod. A1so, flow orifices will be needed in the large feed manifold.

Low Temperature Zone. The first wall coolant, LiPb, is a conducting liquid metal; the effect of the strong magnetic field on the heat transfer of the coolant must be considered. Not only is the Reyr.jld's number (Re) used to describe the fluid flow in the first wall, but an additonal parameter, the Hartmann number $(\mathrm{Ha})$ is used to describe the flow regime:

$$
H a=a \quad \frac{q}{\mu} \text {. }
$$




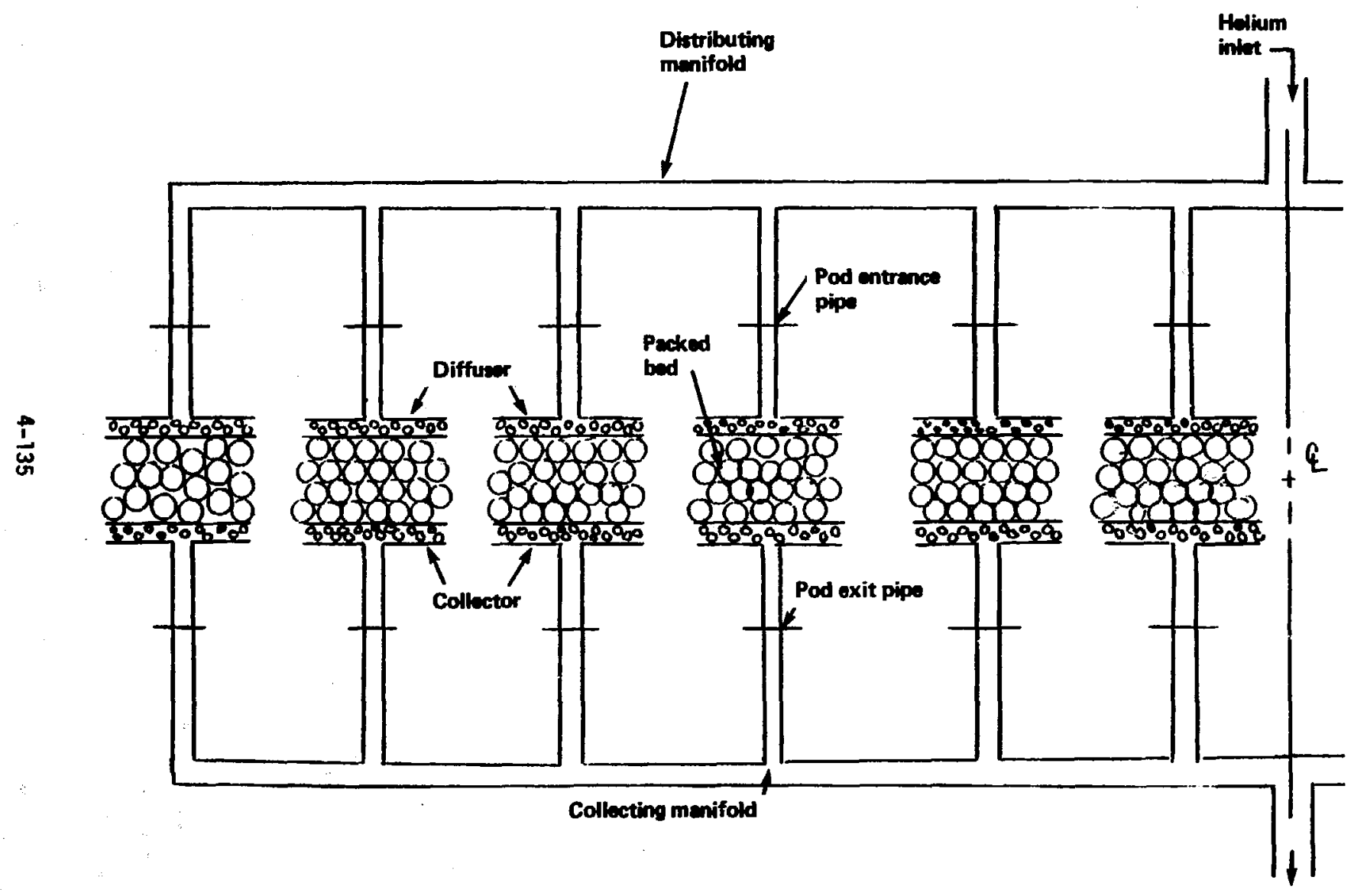

Fig. 4.3-11. Simplified schematic of helium system. 
where

$$
\begin{aligned}
& \text { a channel half-width, } \\
& \text { B } \quad \text { = field strength, } \\
& \text { p } \quad \text { = flutd electrical conductivity, and } \\
&
\end{aligned}
$$

The factor Re/Ha, which represunts the ratio of viscous to electromagnetic forces, is used as a measure of the importance of the magnetic fleld effects on the flow of the fluid. Turbulent flow will be dampened at Re/Ha < 60 for flow parallel to the magnetic field. 6 since Re/Ha for our design is approximately 200, no significant dampening effect is expected to occur. Thus, we used the following turbulent, liquid-metal Husselt-number (Mu) empirical correlation 6

$$
\mathrm{Nu}=6.5+0.025(\operatorname{RePr})^{0.8} \text {, }
$$

where

$\operatorname{Pr} \quad=\quad$ Prandtl number.

The main objective in cooling the first wall is to maintain a maximum temperature in the steel below $520^{\circ} \mathrm{C}$. The LiPb enters the module at $372^{\circ} \mathrm{C}$ and exits at $482^{\circ} \mathrm{C}$; thus, a total LIPb mass flow of of $3600 \mathrm{~kg} / \mathrm{s}$ is needed to cool the low temperature region. The maximum heat deposition of $28 \mathrm{~W} / \mathrm{cm}^{3}$ occurs in the first wall. Figure 4.3-12 shows the temperature profile at the maximum temperature locations of the first and second walls. The film drops for the first and second walls are 1,2 and $8^{\circ} \mathrm{C}$, wile the conduction temperature drops in the first and second steel walls are 24 and $18^{\circ} \mathrm{C}$, respectively. The maximum temperature that the steel reaches in the first wall region is about $515^{\circ} \mathrm{C}$.

Thermal Interface. To maximize FHT, the heat flow between zones must be kept low. As the number of pods increases or the inner radius of the module increases, the heat leak from the high to low temperature zone increases as a result of the increase in surface area between the two regions. This was one reason for selecting a small number of pods and the small inner radius of the module. The operating conditions of the module also affect the performance of the blanket: the larger the temperature difference between the two regions, the more heat that is lost. 


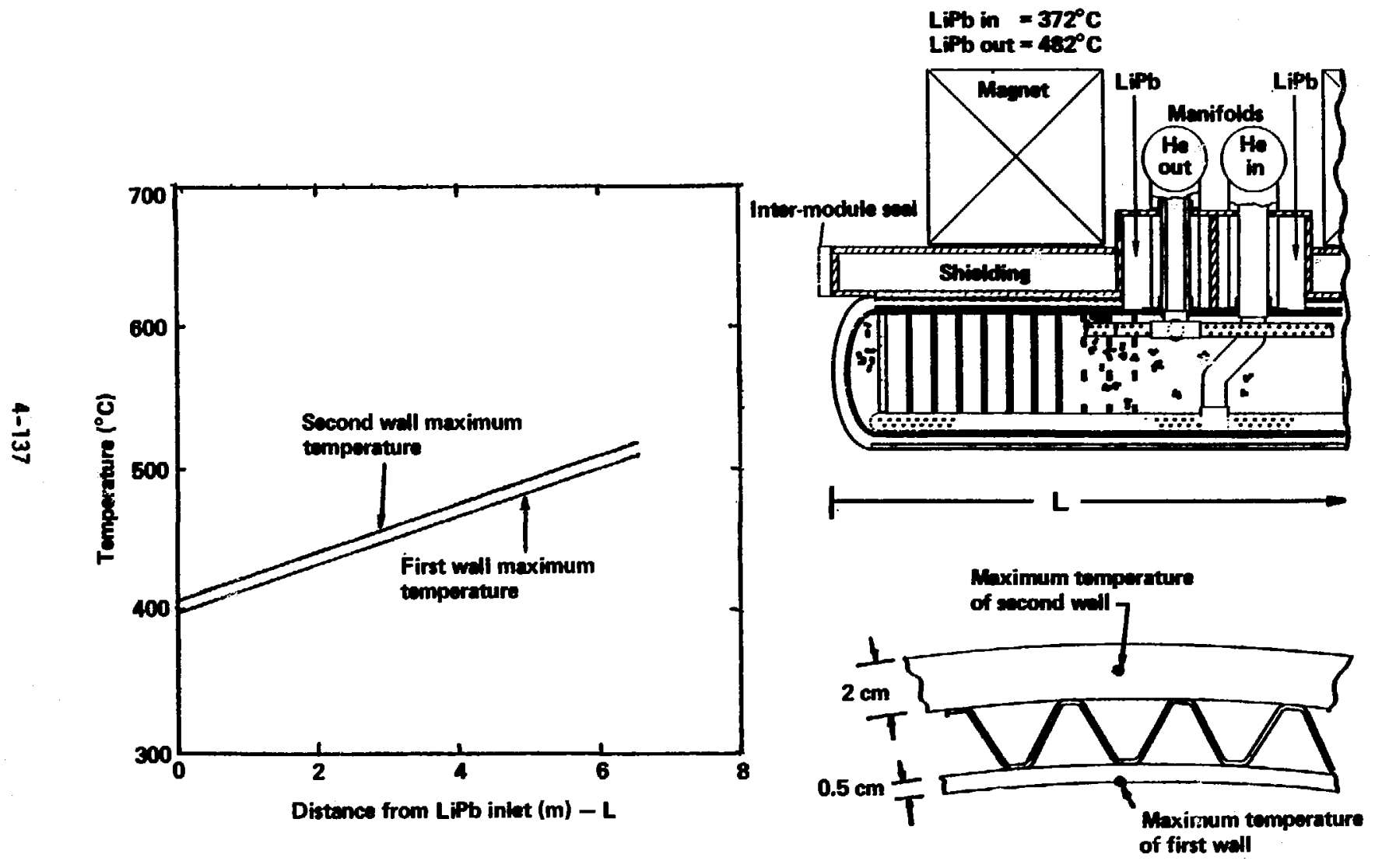

Fig. 4.3-12. First and second wall temperature maxima. 
It is necessary to insulate the two regions from one arwother. The following properties of the insulation are assential: (1) high resistance to radiation damage; (2) the ability to maintain structural integrity of the blanket; (3) low density to minimize internal heat generation; and (4) low effective thermal conductivity. Table 4.3-6 shows the experimental values of thermal conductivity for some low-density fibrous insulators at high temperatures in stagnant gas. ${ }^{7}$ The effective thermal conductivity approaches that of the conductivity of the stagnart gas.

As Fig. 4.3-13 shows, the MRS baseline insulat1i.:. design consists of ceranic (MgO) pedestals separating the pebble basket and the pod wall, fibrous aluminum silica mat between pedestals with stagnant helfum filling the vo:ds. As determined by the structural analysis, the ceramic pedestals are assumed to cover $2 \%$ of the total surface area of the interface between the high and low temperature regions. Also shown in Fig. 4.3-13 is the re?ationship between the amount of heat lost to the low temperature region and the thickness of insulation and the pebble radius. As the insulation thickness increases, the heat leak decreases. At the larger pebble radius $(1.7 \mathrm{~cm})$, the heat leak is larger because SiC surface temperature increases at the front of the blanket. For the baseline design of a $1.9-\mathrm{cm}$ pebble radius and insulation thickness of $2 \mathrm{~cm}$, the heat loss to the low temperature zone is only $3.4 \%$ of the total high temperature heat. 


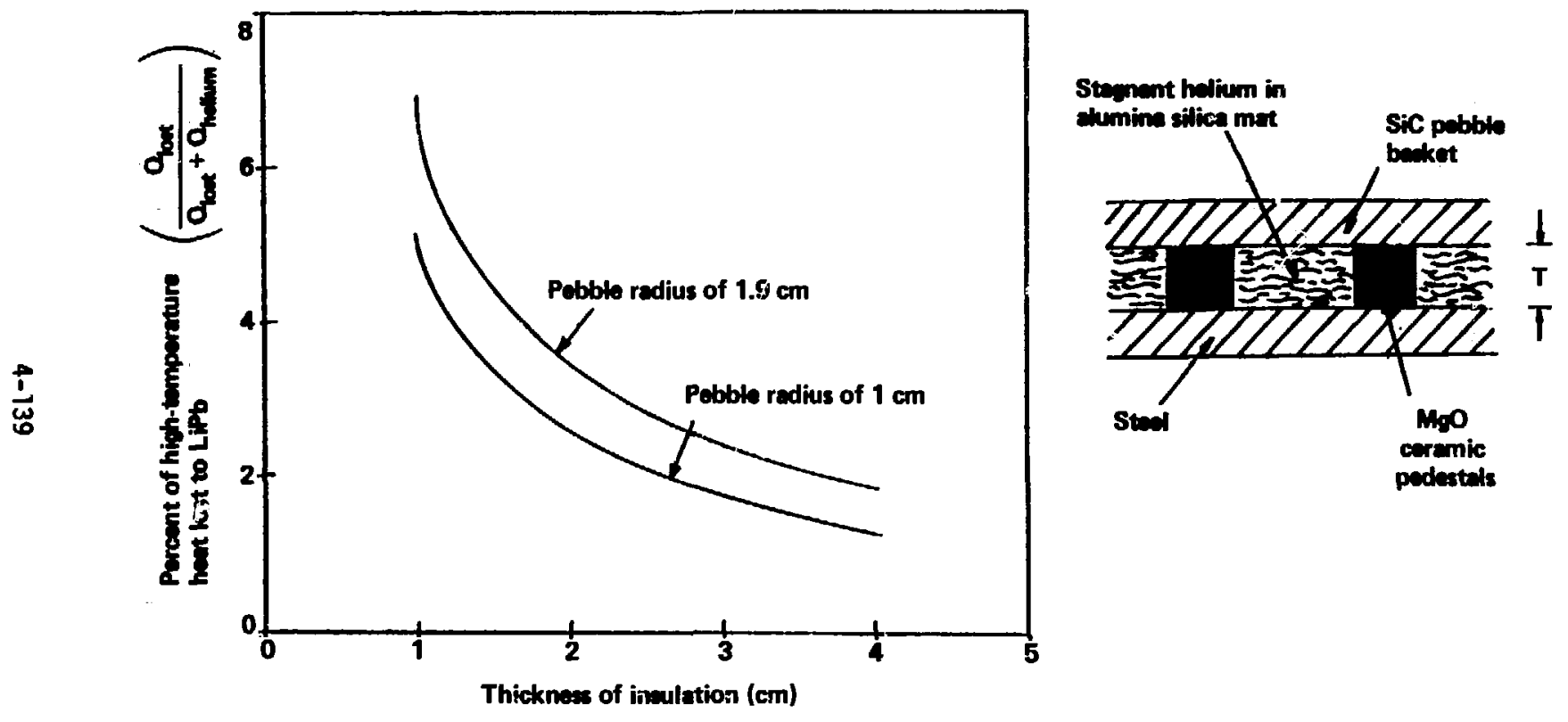

Fig. 4.3-13. Performance of insulation at various pebble radii ârid thicknesses. 


\subsubsection{Mid Pressure Drop}

Magnetohydrodynaaic effects dominate pressure, pumping, and heat transfer in the lithium-laad loop. Wo pumping power is high enough (20 in) to not Icably affect met plant efficiency, and MD pressure strongly affects the structural design and ilfetime of the first wil. High pressures are more easily handled in piping and toward the back of the blanket where thicker structures can be used and where these structures are not under buckling loads.

As discussed in the preceding section, monetic fields affect hait transfer by changing the laminar/turbulent flow transition velocity. ${ }^{8}$ Our knowledge of liquid-matal flow is linfted because there is a lack of experiments in this ares and bacause of the complexity of possible current paths in changing flow situations.9 Experiments have been performed for flow in straight pipes and between conducting plates in uniform magnetic fields. 10-14 However, the flow paths in the MRS high temperature blanket are considerably more complex than those verified experimentally, making the calculational results somethat uncertain.

The Hartman and end-of-loop pressure drops were calculated using

$$
\begin{aligned}
& \Delta P=\frac{V B^{2} q t_{W} L}{a(1+C)} ; c=q t_{w} / 0 \\
& \Delta P=0.062 \mathrm{drB}^{2},
\end{aligned}
$$

where

$$
\begin{aligned}
& y=\text { velocity of fluid, } \\
& B=\text { magnetic field strength, } \\
& q_{w}=\text { electrical conductivity of wll, } \\
& t_{w}=\text { wall thickness, } \\
& ==\text { hydraulic radius, } \\
& \sigma=\text { electrical conductivity of fluid, and } \\
& L=\text { flow path length. }
\end{aligned}
$$

Equation (4.3-1) was used for flow perpendicular to the magnetic field and Eq. (4.3-2) for turns and flow into and out of the field. 15 
Figure 4.3-14 and Table 4.3-7 show the locations and magnitudes of the field strengths used and the calculated mo pressure drops. The maximum first wall pressure is $1.32 \mathrm{ma}$ (194 psi) including the static head.

\subsubsection{Lithlum-Lead Compatibility}

The upper temperature for LiPb will be linited by either the creep rupture strength of the structure or by corrosion. Static tests of HT-9 in LiPb have been performed, 16 but are not directly applicable to the flowing conditions and $110^{\circ} \mathrm{C}$ temperature difference in the high temperature blanket. Dynamic tests of HT-9 in flowing LiPb are being performed at ORNL and A.M. Unt1l these data becom avatlable, the maximum LiPb Interface temperatures cannot be chosen with confidence. The maximum LiPb/HT-9 interface temperature is $490^{\circ} \mathrm{C}$, corresponding to a corrosion rate of $5.9 \mathrm{mg} / \mathrm{m}^{2}-\mathrm{hr}$ based on the 1000-hr static test data presented in Fig. 4.3-15. These data indicate that the Lifo had reached saturation by $3000 \mathrm{hr}$ at all three temperatures. At $500^{\circ} \mathrm{C}$ the corrosion rate has apparently already begun to level off at the 1000-hr test. Flgure 4.3-16 shows the corrosion rates for the 3000- and 1000-hr tests. The points plotted are weight lost at the end of each static test. The data at $1000 \mathrm{hr}$ may be closer to flowing conditions because saturation was not affected the results as much as in the $3000-\mathrm{hr}$ data as indicated by the curved lines in Fig. 4.3-15. On the other hand, short term surface effects my dominate the 1000-hr tests. We belfeve that more experimental results are meeded. As mentioned above, these data are not directly applicable to flowing conditions, but the temperature dependence indicated by the 1000-hr results in Fig. 4.3-15 may be significant.

At the corrosion rates expected in MARS, the major problems will occur on the colc side of the loop where material precipitates out of the coolant, possibly clogging the valves and steam gemerator tubes. Methods of removing these corrosion products fram the syste are discussed in section 4.3.5.5.

\subsubsection{Rediation Effects on Structural Meterials}

As we stated prevfously, whe chosen ferritic steels for the blanket because of their excellent physical and mechanical properties and their resistance to meutron-induced roid swelling and melfum embrittlement. Ferritic steels (HT-9 and 9 (r-2 Mo) have been shown to reach state of 


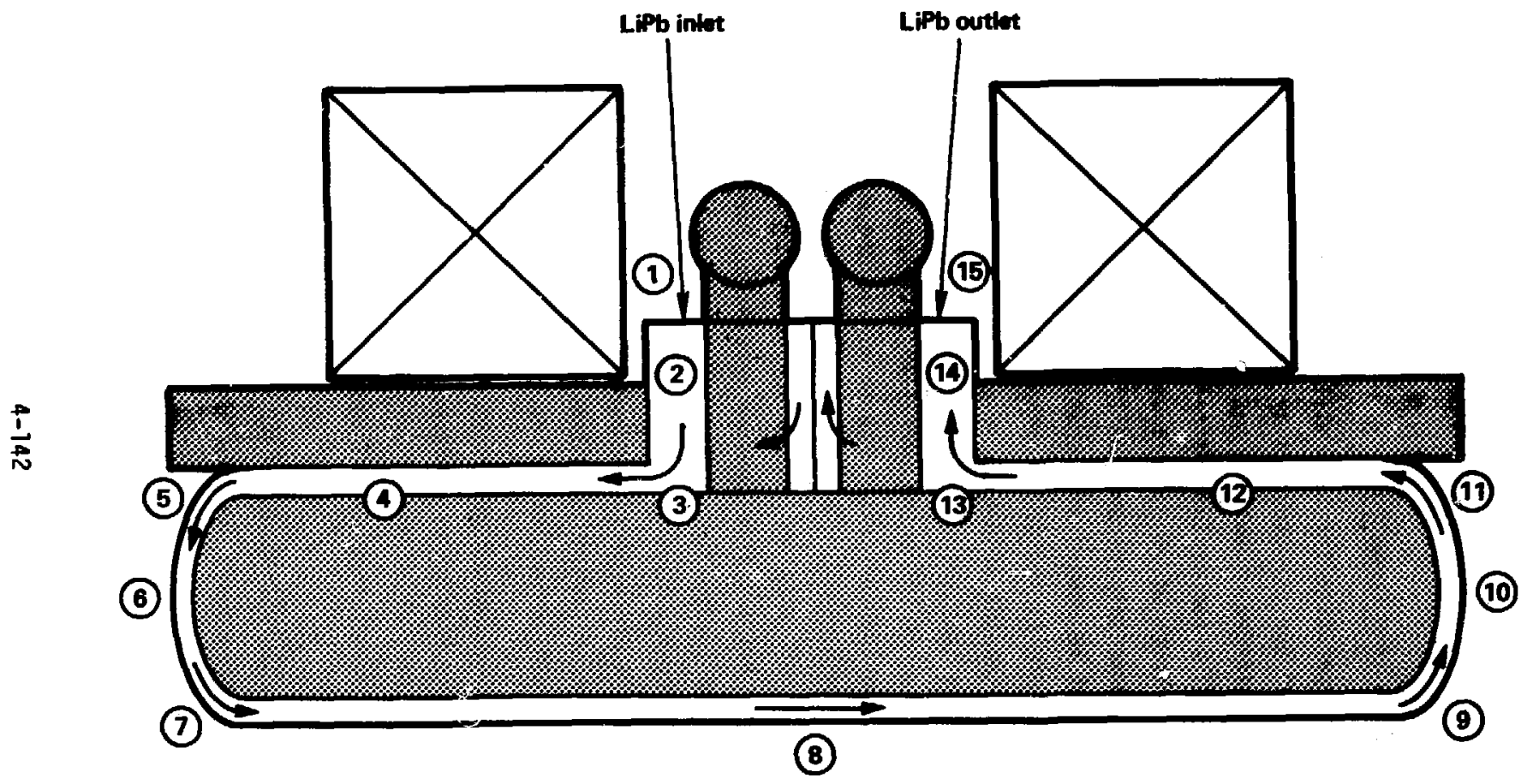

Fig. 4.3-14. LiPb flow. 


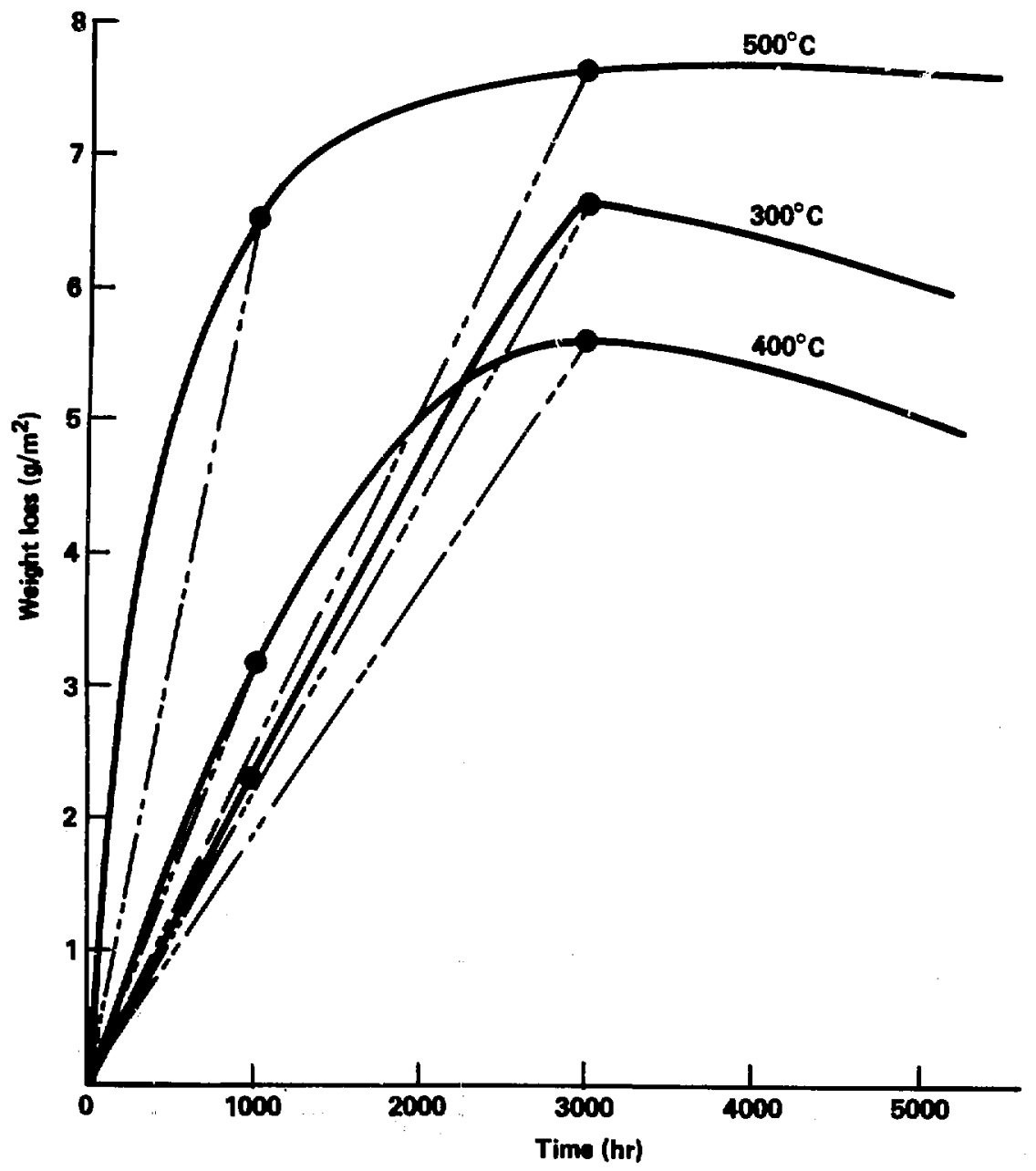

Fig. 4.3-15. Results of static corrosion test of HT-9 in LiPb. 


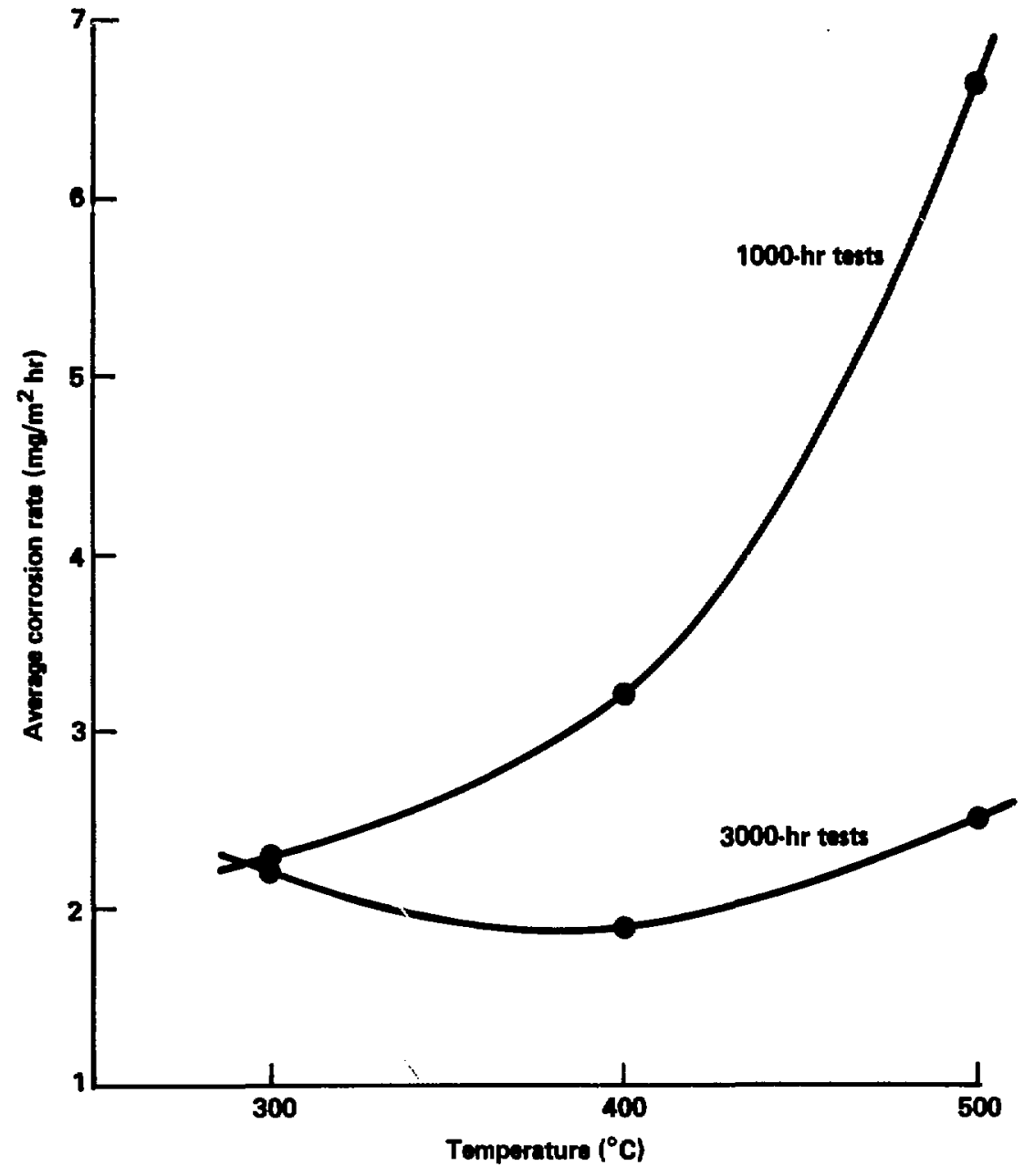

Fig. 4.3-16. LiPb/HT-9 corrosion data. 
Table 4.3-7. WD pressure drop.

\begin{tabular}{|c|c|c|c|c|}
\hline Posftiona & $\begin{array}{c}B \\
(T)\end{array}$ & $\begin{array}{c}V \\
(m / s)\end{array}$ & Flow analyzed & $\begin{array}{c}\Delta P \\
(k P a)\end{array}$ \\
\hline 1 & 3.0 & 0.184 & Inlet/outlet & 68.8 \\
\hline $2^{b}$ & 3.5 & 0.234 & Field & 315 \\
\hline 3 & 4.0 & 0.291 & Turn & 27.7 \\
\hline 4 & 5.4 & 0.291 & Viscous & 0.2 \\
\hline 5 & 4.1 & 0.291 & Turn & 83.7 \\
\hline 6 & 4.3 & 0.239 & Field & 505 \\
\hline 7 & 4.5 & 0.239 & Turn & 36.0 \\
\hline 8 & 4.5 & 1.01 & Viscous & 19.4 \\
\hline 9 & 4.5 & 0.239 & Turn & 36.0 \\
\hline 10 & 4.3 & 0.239 & Fteld & 505 \\
\hline 11 & 4.1 & 0.291 & Turn & 83.7 \\
\hline 12 & 5.4 & 0.291 & Viscous & 0.2 \\
\hline 13 & 4.0 & 0.291 & Turn & 27.7 \\
\hline $14^{b}$ & 3.5 & 0.234 & Field & 315 \\
\hline 15 & 3.0 & 0.184 & Inlet/outlet & 68.8 \\
\hline
\end{tabular}

see Fig. 4.3-14.

b Torotdal flow around module, average velocity. 
magnetic saturation at relatively low field strengths (1 to $2 \mathrm{~T}$ ), indicating that ferromagnetism will not present a problem in the tandem mirror. 17 We have considered using HT-9 (12 $\mathrm{Cr}-1 \mathrm{mo})$ and 2-1/4 $\mathrm{Cr}-1$ mo for the MRS high temperature blanket. These steels are limited in their useful lifetimes by irradiation embrittlement at low temperatures, by swelling, and by creep rupture and liquid-metal corrosion at high temperatures. We have updated and used already-developeci, design equations for the shift in ductile-to-brittle transition temperature ( $\triangle D B T T$ ), swelling, and creep rupture time.

Neutron embrittlement causes crack propagation at low applied stress. Crack growth rates, and hence fallure criteria, are usually based on static fracture toughness masurements $\left(K_{I C}\right)$ for plane strain. On the other hand, the majority of ractor desfigns in the nuclear industry are based on mitigating the shift in the ductile-to-brittle transition temperature. This may be a more conservative approach where crack propagation due to dynanic loading is avoided. We have followed this philosophy in our analysis.

Figure 4.3-17 shows the estimated DBTT for HT-9 during irradiation. The DBTT is calculated for the worst case, which is the minimum structure temperature $\left(388^{\circ} \mathrm{C}\right)$, and the shift shown to saturate after only a few months of operation. After operation for about $1 \mathrm{yr}$, the plant is shutdown for scheduled maintenance and the structure temperature is held constant at $450^{\circ} \mathrm{C}$ for approximately $60 \mathrm{hr}$. This allows an almost full recovery of the $\triangle D B T T$ as shown in Fig. 4.3-17. The procedure is repeated once every scheduled maintenance (approximately every year). The annealing procedure is calculated according to a functional fit to the results of the RANEL annealing computer model. 19

Neutron-induced swelling of ferritic steels depends on dpa, irradiation temperature, and chromiun content. $(18,20)$ Iron-chromium-carbon alloys show a systematic dependence on chromium content, as shown in Fig. 4.3-18. Unfortunately, the peak swelling temperature for HT-9 and 2-1/4 $\mathrm{Cr}-1$ to is approximately $426^{\circ} \mathrm{C}$, occurring within the expected operating temperature range. The predicted first-wall volumetric swelling after three calendar years (80\% availability) is $4.0 \%$ and $1.8 \%(\Delta V / V \%)$ for $H T-9$ and 2-1/4 $\mathrm{Cr}-1 \mathrm{No}$, respectively. This extrapolation is for a similar heat treatment of steel assuming a predominantly bainitic microstructure. 


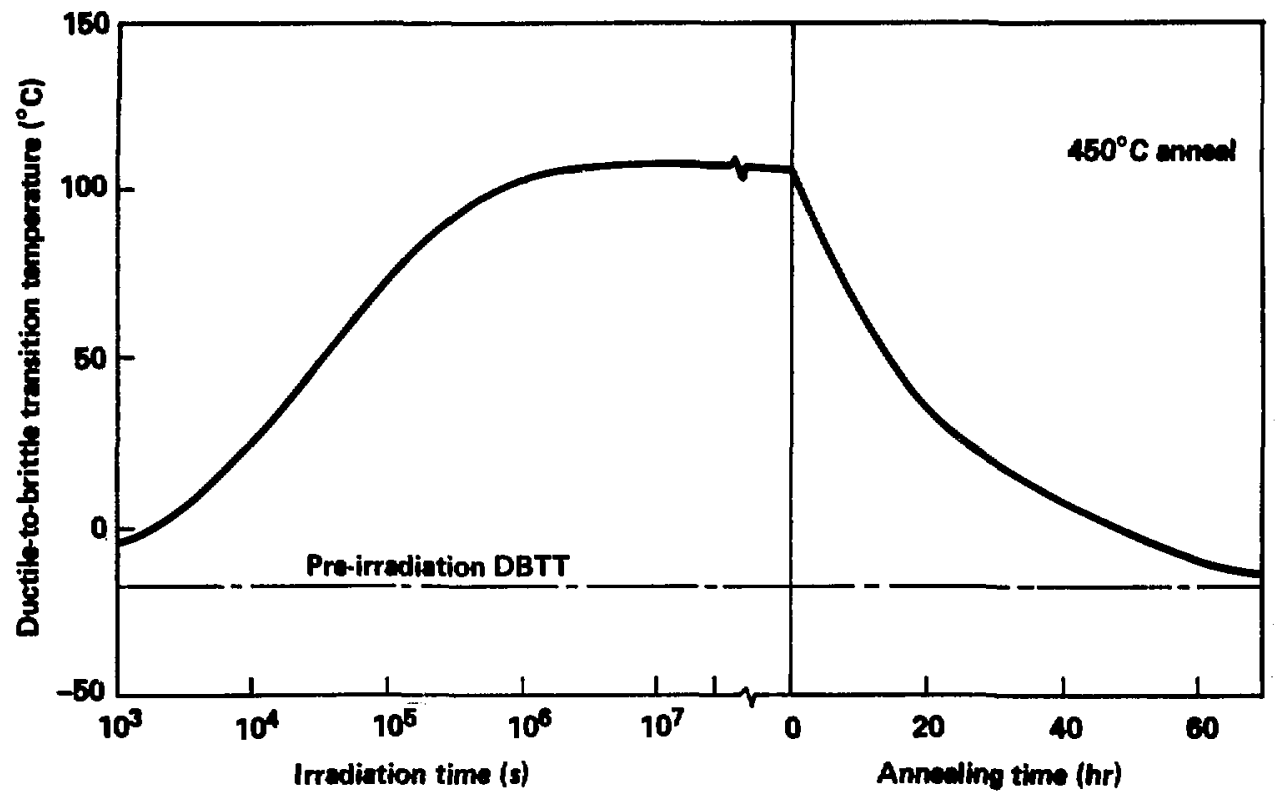

Fig. 4.3-17. Ductile-to-brittle transition temperature vs radiation and annealing $t$ ine. 


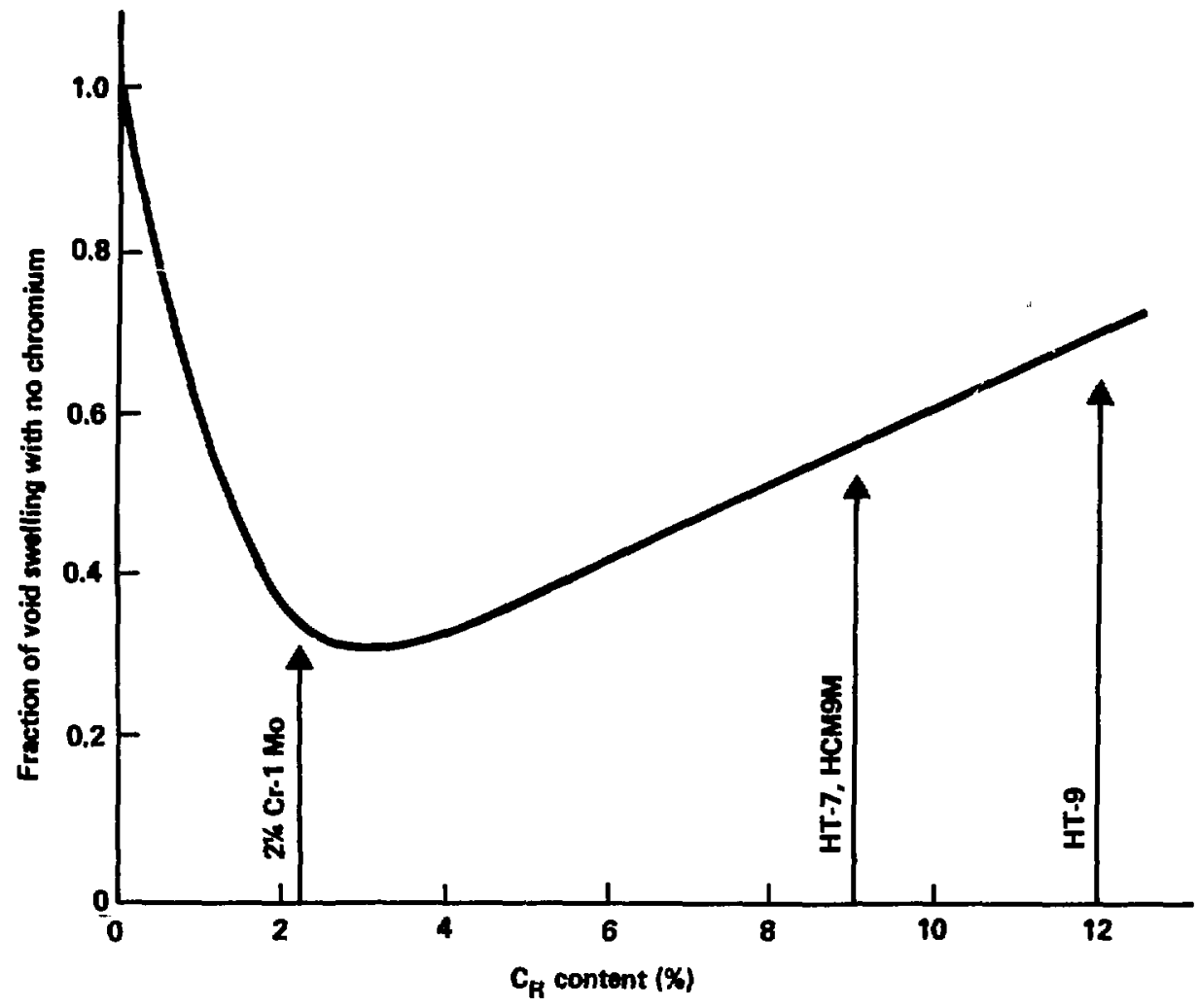

Fig. a.3-18. Effect of chromium content on swelling of ferritic steels. 
The lower swelling of 2-1/4 $\mathrm{Cr}-1 \mathrm{Mo}$, as compared with HT-9, is the primary reason for considering it for interest in the MARS design. However, the high yleld and thermal creep strength of HT-9 mean that less material will be requiled (thimer sections), resulting in lower costs, less activated material to dispose of, and possibly reduced swelling-induced stresses. Swelling-induced stress can be dependent on the thickness of structural menbers when temperature (and therefore swelling) differentlals are dependent on thickness, and when swelling strains are taken up in bending. The temperature drop through the first mall causes a swelling differential across the wall, inducing stress in the section. Table 4.3-8 compares the two alloys in this respect. The first wall thickness is assumed to vary inversely with yield strength, the peak swelling temperature $\left(426^{\circ} \mathrm{C}\right)$ is assumed to occur on the inside surface of the first wall, and the section is assumed to be constrained. Stress rellef due to creep is ignored. The stress in HT-9 is 82\% of its yield st, rangth and the stress in the 2-1/4 Cr-1 to is $86 \%$ of yield.

Swelling strain (elongation) of the first wall parallel to the plasma axis is taken up in bending of the sorodial end caps. Because the stronger Hi-9 will allow thinner end caps than 2-i/4 $\mathrm{Cr}-1$ Mo, lower stress would be induced in the HT-9 by the same anount of bending. The HT-9 will, of course, swell more and therefore bend more, but the thinner section and higher working strength will tend to cancel the higher swelling.

It has been show that irradiation creep significantly relaxes the swelling-induced stresses. 21 As shown above, the stresses generated by swelling differentials will not lead to plastic deformation at the end of life (3 yr) for elther HT-9 or 2-1/4 Cr-1 Mo. The fractional stress is silightly higher in 2-1/4 Cr-1 tho than in HT-9. When irradiation creep is included, the stress in the first wall will be a function of irradiation time. The magnitude of the stress is determined as a balance between swelling and irradiation creep. Hatson 21 has shown that, for a clamped plate, the steadystate residual stress is proportional to the ratio of swelling to creep compliance. Even though the swelling is a factor of two smaller in $2-1 / 4 \mathrm{Cr}-1$ ino than in HT-9, the magnitude of creep compliance is uncertain. It is premature, therefore, to make a final selection before a complets analysis is pizformed. 
Table 4.3-8. First wall temperature-dependent swellng-induced stress. Creep relaxation is neglected.

\begin{tabular}{|c|c|c|c|c|c|}
\hline & \multicolumn{2}{|c|}{ Material } & & \multicolumn{2}{|c|}{ Material } \\
\hline & $H T-9$ & $\mathrm{Cr}^{2-1 / 2} \mathrm{MO}^{2}$ & & $H T-9$ & $\mathrm{Cr}=1 \mathrm{Mo}$ \\
\hline $\begin{array}{l}\text { Yield strength } \\
\left.\text { (MPa at } 400^{\circ} \mathrm{C}\right)\end{array}$ & 290 & 218 & $\begin{array}{l}\text { Swelling } \\
\text { difference }(x)\end{array}$ & 0.61 & 0.46 \\
\hline Thickness $(\mathrm{cm})$ & 0.50 & 0.67 & $\begin{array}{l}\text { Young's modulus } \\
\text { (GPa at } 400^{\circ} \mathrm{C} \text { ) }\end{array}$ & 175 & 183 \\
\hline $\begin{array}{l}\text { Thermal } \\
\text { conductivity } \\
\text { (W/NK) }\end{array}$ & 29 & 34 & $\begin{array}{l}\text { Induced stress } \\
\text { (MPa) }\end{array}$ & \pm 238 & \pm 187 \\
\hline$\Delta T\left({ }^{\circ} \mathrm{C}\right)$ & 24 & 32 & Percent of yield & \pm 82 & \pm 86 \\
\hline
\end{tabular}


The slow deformation of structures at high temperature unat applied stress will eventually lead to faflure. Creep will also tend to relieve swelling and thermal stress. In some areas creep and swelling can combine to cause excessive elongation. For example, swelling and creep will cause the first wall of the MARS high-temperature blanket to grow in axial length because of the tension caused by the LiPb pressure acting on the torodial end caps. Creep can therefore limit lifetime by contributing to excessive deformation or by creep rupture. Thus, total deformation at end of life as well as the time to rupture should be quantified. Ghoniem and Conn 18,20 have developed a method to calculate time to rupture. It appears that HT-9 will not be creep-rupture-limited below about $520^{\circ} \mathrm{C}$. Swelling elongation of the first wall is predicted to be $1.3 \%$ or $8 \mathrm{~cm}$ over the length of the module (3-yr lifetime). We have not yet cal iated creep elongation, but we expect it to be small compared with swelling =longation because the stress in this direction is low.

We have chosen HT-9 over 2-1/4 $\mathrm{Cr}-1$ Mo because its strength advantages were judged to outweigh the problems associated with swelling. The higher yield strength and creep strength of HT-9 allow the use of less structural material. This affects cost both by reducing the amount of material itself and because of easier manufacturing and handling of the thinner sections. Less material also results in lower activation. The HT-9 contains $1 / 2 \%$ nickel, while 2-1/4 $\mathrm{Cr}-1$ to does not, but long-term activation of both steels is dominated by molybdenum. Isotopic separation of molybdenum or substitution of molybdenum with vanadium would greatly reduce structural activation, making it desirable to reconsider using 2-1/4 $\mathrm{Cr}-1$ to to eliminate the nickel in HT -9.20

The study of of fusion blanket lifetimes is in its infancy. Analyses of the combined effects of swelling, creep, embrittiement and thermal stresses have been performed in only a few cases. ${ }^{21}$ A finite-element code that includes radiation effects would be a very useful tool in this type of study. Quantification of the combined stresses and fatlure criterla in complex structures such as the MARS high temperature blanket will require a detalled structural analysis that includes radiation effects on material properties. 


\subsubsection{Solid Breeder Performance}

One of our major concerns is the cherical compatibility of the solid breeder materials, including possible chemical react: ons between a breeding material and the SiC carrier. Siribering of these materials is also a concern. Although many litiniun crimpounds have been considered as breeding materials, only a few are sultable for the maRs blanket because of its relatively high operating temperature $\left(1000^{\circ} \mathrm{C}\right)$. We reviewed the literature on solid breeders and selected potential candidates for the MARS high temperature blanket. Because there is acarcity of data on solid breeders in the temperature range of interest; some preliminary screening tests were performed as a part of a TRW research project.

Potential lithium compounds have been catego:1zed and discusssed by many authors. 22,23,24 These can be put into several groups including liquid metals (e.g., $\mathrm{Li}, \mathrm{LI}_{17} \mathrm{~Pb}_{83}$ ), intermetalics (e.g., LiAl, $\mathrm{LI}_{7} \mathrm{~Pb}_{2}$ ) binary compounds (e.g., $\mathrm{Li}_{2} \mathrm{O}, \mathrm{Li}_{2} \mathrm{C}_{2}, \mathrm{LIF}_{2} \mathrm{Li}_{3} \mathrm{~N}, \mathrm{Li}_{4} \mathrm{SI}$ ) and ternary oxides (e.g., LiAlO $\mathrm{O}_{2}$, $\left.\mathrm{Li}_{2} \mathrm{SiO}_{3}, \mathrm{Li}_{2} \mathrm{TiO}_{3}, \mathrm{LiFeO}_{2}, \mathrm{Li}_{4} \mathrm{SiO}_{4}, \mathrm{Li}_{2} \mathrm{ZrO}_{8}\right)$. All compounds that melted or decomposed at less than $900^{\circ} \mathrm{C}$ were rejected. In addition, any material that was belfeved to react with oxygen or SiC was eliminated. The most promising compounds remaining were $\mathrm{LI}_{2} \mathrm{O}$ and the ternary oxides.

Lithium oxide has a high melting point, high lithfum-atom density, good tritium extraction, and relatively iaw chemical reactivity. However,

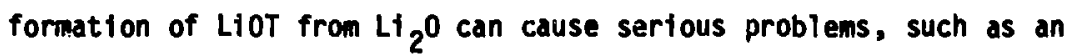
accieration of sintering and corrosion of structural materials. Therefore, we turned our attention to the ternary oxides. One of the disadvantages of the ternary oxides is their low lithium density; thus, a neutron multiplier is required. In the MARS high temperature blanket, however, the $\mathrm{Li}_{17}{ }^{\mathrm{Pb}}{ }_{83}$ coolant provides adequate multiplication. The remaing concem was high temperature operation because of self-sintering or sintering to the SiC carrier. Based on melting point and neutrontcs, we selected $\mathrm{LAAlO}_{2}$ and $\mathrm{LI}_{2} \mathrm{TiO}_{3}$ for testing.

Lithiun Titanate and Stlicon Carbide Heated Separately. He conducted se arate tests for possible self-sintering of $\mathrm{Li}_{2} \mathrm{THO}_{3}$ and $\mathrm{SiC}$ powder. The tests took place in the BREW furnace at $1100^{\circ} \mathrm{C}$ and $1 \times 10^{-5}$ Torr for $5 \mathrm{hr}$. The SiC did not show any sign self-sintering; $\mathrm{Li}_{2} \mathrm{TiO}_{3}$ sintered and its color changed from white to bluish gray. The color change of $\mathrm{Li}_{2} \mathrm{THO}_{3}$ may be caused by trace impurities. Based on the $x$-ray diffriction pattern, no 
chemical reaction occurred. The SEM micrograph (Fig. 4.3-19) shows a selfsintering of $\mathrm{LH}_{2} \mathrm{THO}_{3}$.

Mixtures of Lithium Titanate and Silicon Carbide. The test conditions were identical to those in the previous experiment. Two mixture ratios were tested; both mixtures (1:1 and 1:6) sintered during heating. The shape of the particies, deterinined by SEM, seems to indicate both self-sintering of $\mathrm{LI}_{2} \mathrm{THO}_{3}$ and $\mathrm{Li}_{2} \mathrm{TiO}_{3}-\mathrm{SiC}$ sintering. The $x$-ray diffraction shown in Fig. 4.3-20 gives some evidence of a chemical reaction between $\mathrm{Li}_{2} \mathrm{TiO}_{3}$ and $\mathrm{SiC}$. The $\mathrm{Li}_{2} \mathrm{THO}_{3}$ seaned to disappear and new peaks appeared after heating corresponded to $\mathrm{LI}_{2} \mathrm{SiO}_{3}$. Unfortunately, the x-ray diffraction spectra of TIC is identical to that of SiC, and the formation of TIC cannot be confirmed by $x$-ray diffraction analysis. The thermodynamic calculation predicts the following reaction to occur spontaneousily:

$$
\begin{aligned}
& \mathrm{LI}_{2} \mathrm{THO}_{3}+\mathrm{SIC}+\mathrm{LI}_{2} \mathrm{SiO}_{3}+\mathrm{THC} \\
& \Delta 6_{\text {ranction }}^{\mathrm{O}}=-20.305 \mathrm{kcal} .
\end{aligned}
$$

Although we have not seen definitive evidence, it appears that the above reaction took place during the heating at $1100^{\circ} \mathrm{C}$. From this result and based on the thermodynamics, it is also likely that lithium zirconate $\left(\mathrm{Lt}_{2} \mathrm{ZrO}_{3}\right)$ another solid breeder candidate, will react with silicon cartide to form lithium silicate and zirconium carbide.

\section{Lithium Aluminate and Silicon Carbide. Mixtures of $\mathrm{LIAlO}_{2}$ and SiC} $(1: 1$ and $1: 5)$ were heated to $1100^{\circ} \mathrm{C}$ in a similar manner as in the previous case. When the furnace was opened, we found that nost of the sample powders spilled all over the furnace. An excessive outgassing must have occurred at approximately $300^{\circ} \mathrm{C}$. Because of this problem; we carried out a degassing by laboratory box furnace. The $\mathrm{LiAlO}_{2}$ powder $(100 \mathrm{~m})$ was heated at 750 to $\mathrm{B00}$. $\mathrm{C}$ for $4 \mathrm{hr}$. The weight loss by heating was approximately 25\%. Rdditional heating at $800^{\circ} \mathrm{C}$ for $4 \mathrm{hr}$ did not change the apht apreclably. The $x$-ray diffraction spectra (Fig. 4.3-21) showed that the commerctal $\mathrm{LHAlO}_{2}$ contains a hydrated $\mathrm{LAAlO}_{2}$ and some $\mathrm{Lt}_{2} \mathrm{CO}_{3}$.

$A$ thermal gravimetric analysis of $\mathrm{LHAlO}_{2}$ indicated a weight loss of $2 \mathrm{AX}$ at 200 to $300^{\circ} \mathrm{C}$ and $4 \%$ at 550 to $650^{\circ} \mathrm{C}$. The first weight loss is due to 


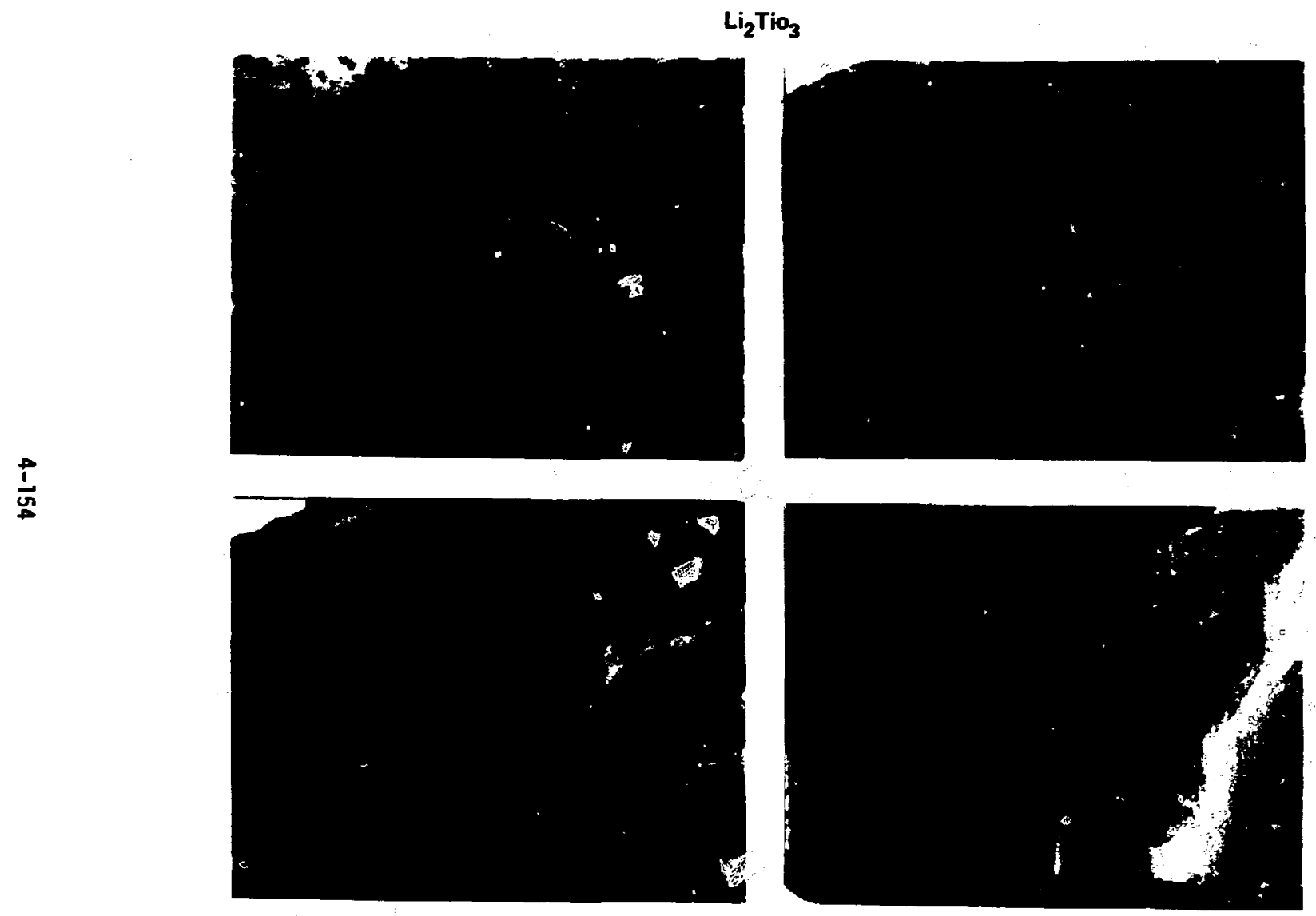

Fig. 4.3-19. $\mathrm{Li}_{2} \mathrm{TiO}_{3}$ after $1100^{\circ} \mathrm{C}$ 4-hr vacuum exposure. 
$\mathrm{Li}_{2} \mathrm{TiO}_{3} / \mathrm{siC}(1: 1)$ Before expowur

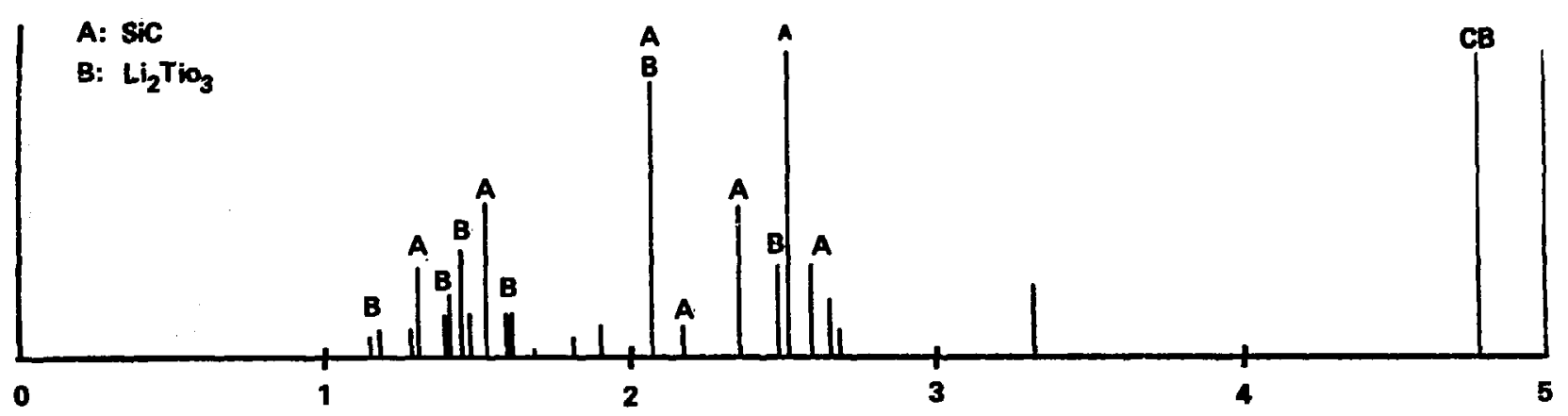

ज़

$\mathrm{Li}_{2} \mathrm{TiO}_{3} / \mathrm{SiC}(1: 1)$ After expocure (at $1100^{\circ} \mathrm{C}$ for $5 \mathrm{hr}$ )

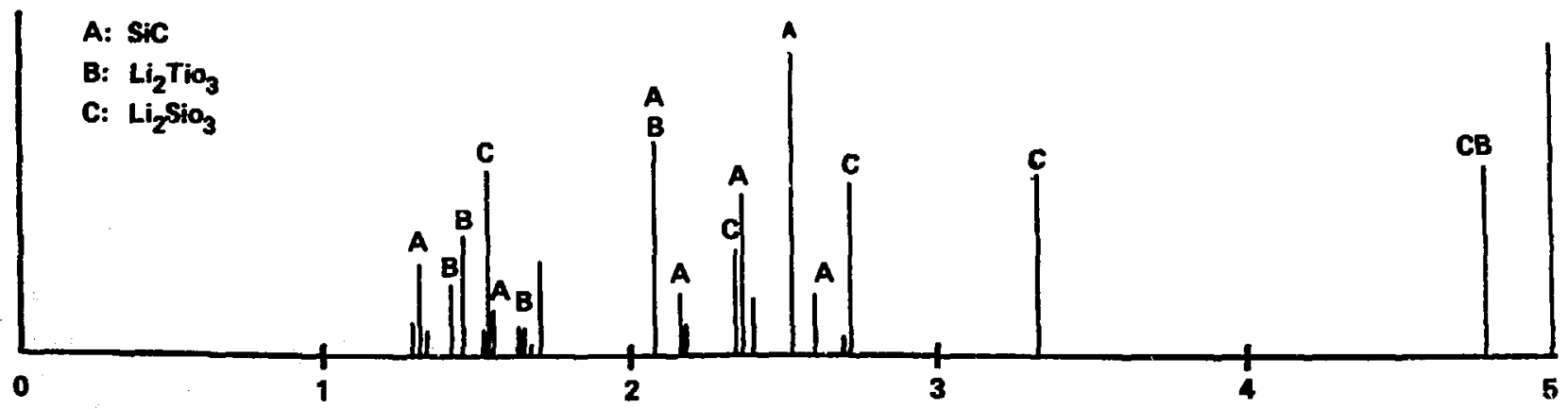

Fig. 4.3-20. X-ray diffraction pattern of $\mathrm{Li}_{2} \mathrm{TiO}_{3} / \mathrm{SiC}(1: 1)$. 


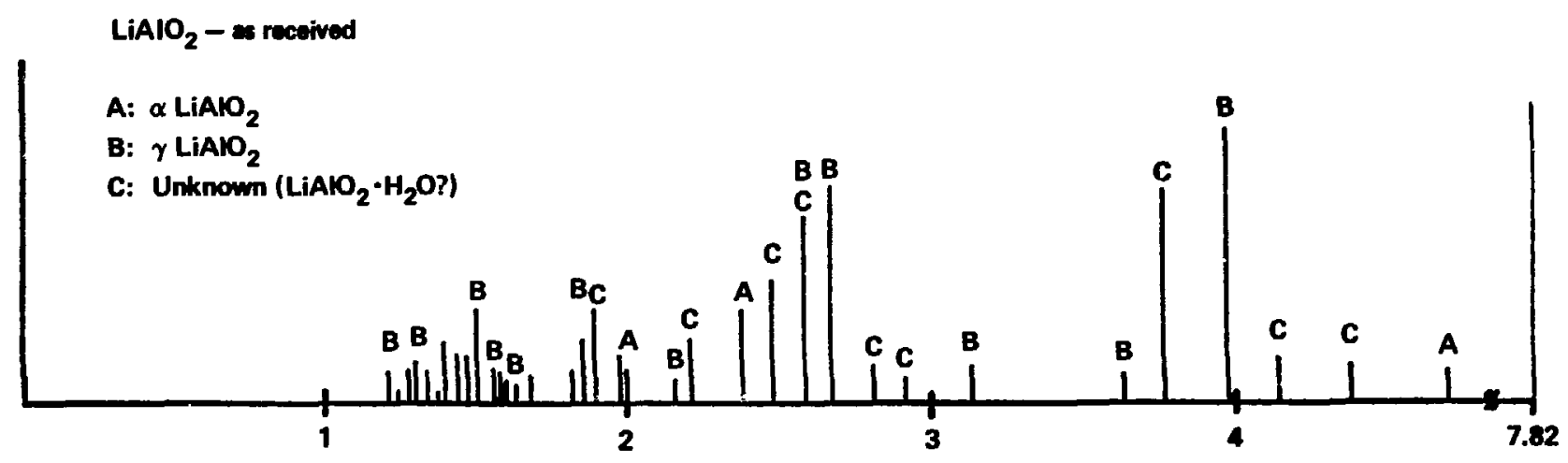

$\frac{1}{\text { ज̆ }}$

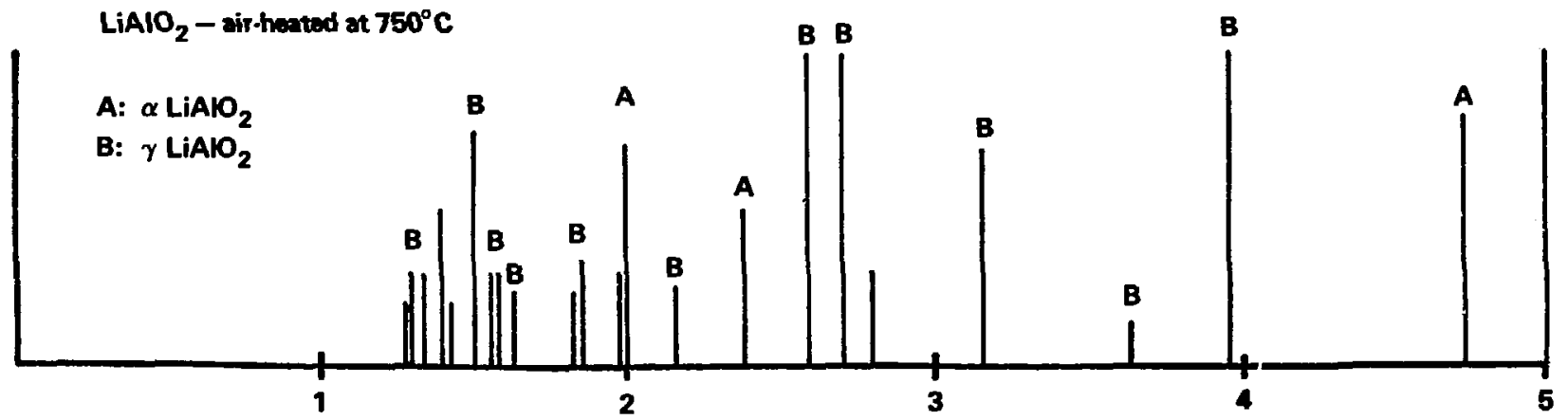

Fig. 4.3-21. X-ray diffraction pattern of $\mathrm{LiAIO}_{2}$. 
dehydration of $\mathrm{LAAlO}_{2} \times \mathrm{H}_{2} \mathrm{O}$ and the second weight loss seems to be due to a decomposition of $\mathrm{LI}_{2} \mathrm{CO}_{3}$. The hydration number is calculated to be 1 .

Whe again blended silicon carbide and the preheated lithium aluminate to make $1: 1$ and $1: 5$ ratios. we then conducted the compatibility test in a sintlar menner as in the previous case. This time, we did not see an excessive pressure rise as the temperature was increased to $500^{\circ} \mathrm{C}$ in the vacuuin. The folght change was only 1.7 and $0.8 \%$ for the $1: 1$ and $1: 5$ mixtures, respectively. The $x$-ray diffraction spectra did not indicate a significant change except for the change of a-LiAlO $\mathrm{L}_{2}$ to $\mathrm{rLAAO}_{2}$ during the hating at $1100^{\circ} \mathrm{C}$, as shom in Fig. 4.3-22. This phase change was also reported by Johnson, at al., 25 The Irreversibility of the phase change is reconfimed by the present work.

Table 4.3-9 sumarizes the chemical compatibility test of a lithium compound with silicon carbide. The following conclusions may be dram from the present study:

1. $\mathrm{Li}_{2} \mathrm{THO}_{3}$ reacted with $\mathrm{SIC}$ and formed $\mathrm{LI}_{2} \mathrm{SHO}_{3}$ at $1100^{\circ} \mathrm{C}$ after $5 \mathrm{hr}$.

2. $\mathrm{Lf}_{2} \mathrm{THO}_{3}$ showed self-sintering during heating at $1100^{\circ} \mathrm{C}$ for $5 \mathrm{hr}$.

3. Based on the preliminary test results, $\mathrm{LAAlO}_{2}$ is compatible with SiC at $1100^{\circ} \mathrm{C}$; no evidence of chemical reaction or sintering was detected.

Suggestions for Future Study. Our preitminary test results indicate that the $\mathrm{LAAlO}_{2}$ and SIC $\mathrm{mlxture}$ is the leading candidate as the breeder materfal. Future tests should include tritfum solubility as a function of temperature. The effects of radiation danage on solid breeder perforannce must also be assesed experimentally. Experimental data must be systematically complled before we can make a conclusion on the $\mathrm{LAAlO}_{2}-\mathrm{SIC}$ system. 
$\mathrm{LiAlO}_{2} / \mathrm{SiC}(1: 1)$ Before exposure

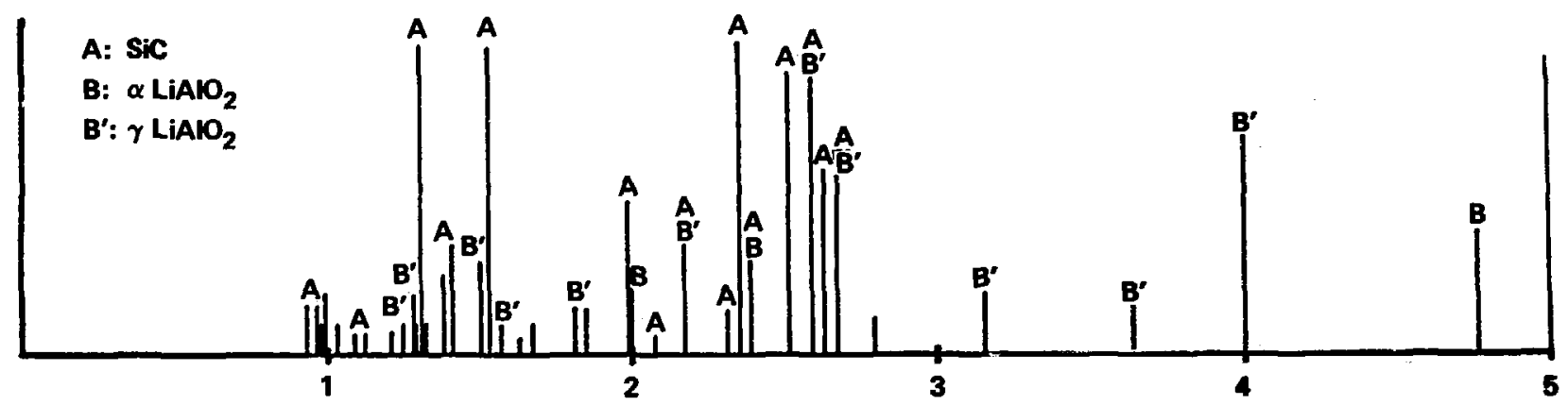

$\frac{1}{\dot{1}}$

$\mathrm{LiAlO}_{2} / \mathrm{SiC}(1: 1)$ After exposure (at $1100^{\circ} \mathrm{C}$ )

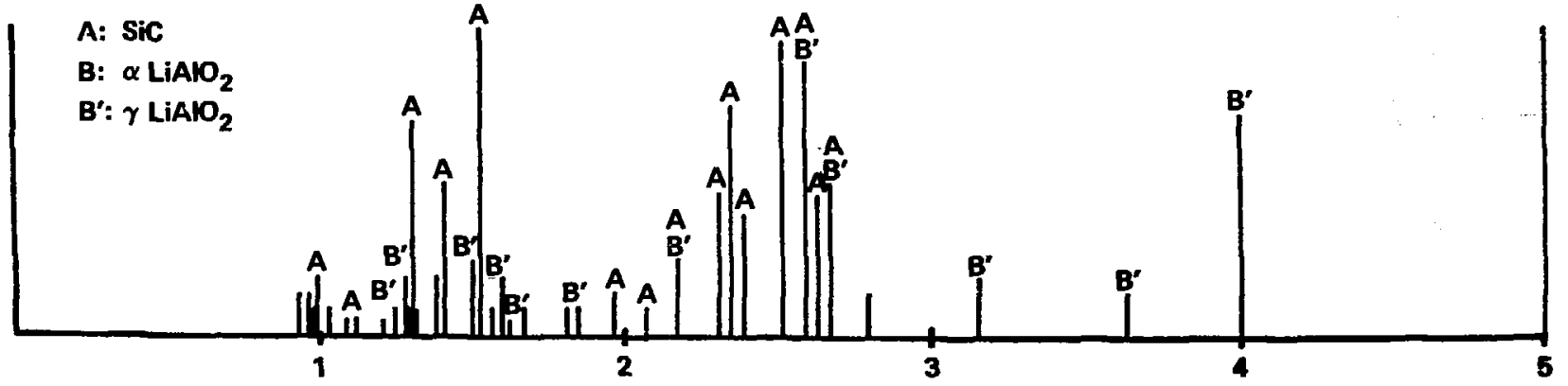

Fig. 4.3-22. X-ray diffraction pattern of $\mathrm{LiAIO}_{2} / \mathrm{SiC}(1: 1)$. 
Table 4.3-9. A sumary of chemical compatibility tests of lithium compounds and silicon carbide.

Wefght change $X$-ray diffraction SEM Observation

(g)

\begin{tabular}{|c|c|c|c|c|}
\hline SiC & $0.472 / 3.682$ & No change in the spectra & - & No sintering \\
\hline $\mathrm{Lt}_{2} \mathrm{TtO}_{3}$ & $0.444 / 2.767$ & No change in the spectra & $\begin{array}{l}\text { Self- } \\
\text { sintering }\end{array}$ & $\begin{array}{l}\text { Color change, } \\
\text { white to bluis } \\
\text { gray, self } \\
\text { sintering }\end{array}$ \\
\hline $\operatorname{LAA1O_{2}}$ & $5.256 / 20.351$ & $\begin{array}{l}\text { Substantial change in } \\
\text { the spectra--clear peaks } \\
\text { of a } \& \mathrm{LtAlO}_{2}\end{array}$ & - & No sintering \\
\hline $\mathrm{Lit}_{2} \mathrm{TiO}_{3}$ & $0.200 / 6.146$ & $\begin{array}{l}\text { Disappearing of } \mathrm{LI}_{2} \mathrm{TiO}_{3} \\
\text { peaks --appearing of } \\
\mathrm{LI}_{2} \mathrm{SiO}_{3} \text { peaks }\end{array}$ & $\begin{array}{l}\text { Shows } \\
\text { sintering }\end{array}$ & $\begin{array}{l}\text { Color change, } \\
\text { gray to black } \\
\text { sintering, }\end{array}$ \\
\hline $\operatorname{LiAlO}_{\text {SiC }}$ & $0.056 / 3.2239$ & $\begin{array}{l}\text { Disappearing of a LiAlO } \\
\text { no new peaks }\end{array}$ & - & No sintering \\
\hline
\end{tabular}

- Atr heated at approximately $750^{\circ} \mathrm{C}$ for $4 \mathrm{hr}$. 


\subsubsection{Pad Aupture malysts}

During steady-state operation, the pressure in all 12 pods should be identical so that there are no unbalanced stresses in the pod walls. We performed a prelintary amalysis to deternine the manitude of the maximum pressure differentials resulting between any two pods in the event of a pod rupture, and the blowdown that follows it.

When a pod ruptures, a transfent blowdown will occur. During this process all module helfum supply and return Ifnes must be closed to prevent addittonai heltum from escaping. All helfum present in the pods and mantfolds will exit to the plasma region through the fallure point. Figure 4.3-23 shows the 12-pod arrangament and also indicates helium flow directions during a fallure blowdown.

A worst case analysts has shown that the maximum pressure differential extsting between pods across the common wall regtons will be 24 atw for an 8-in.-diameter hole and 80-atm operating pressure. Analysis of the pod structure shows that it can withstand a 58-atm different1al. The maximum pressure drop occurs near the inner radius of the power blanket across the common pod wall only at the instant of failure, and decrease very rapidly as the blowdown proceeds. Figure 4.3-24 shows the maximum and average pressure differentials as a function of hole size for 50- and 80-atm operating pressures.

The approach used to determine the pressure differential between adjacent pods involves calculating the pressure drop through a pod for a given rupture hole dlameter and the corresponding mass flow rate. The total pressure drop through a falled pod consists of three parts. The first pressure drop is from flow through the helium outlet ducting. This is the sum of losses in the stralght header section, the perforated mantfold, and numerous perforations in the mantfold. The second major pressure drop is from flow through the StC packed bed. The third major pressure drop is from losses through the inlet header and its perforated manifold.

The largest fallure size investigated, which is not shown in Fig. 4.3-24, was a tear along the length of the pod $0.5 \mathrm{ln}$. wide and $13.0 \mathrm{ft}$ long, simulating a separation or stress crack fallure. The local maximum and average pressure differentials for such a faflure at a 50-atm operating pressure are 30.8 and $15.9 \mathrm{~atm}$, respectively. For an 80-atm operating pressure, these 


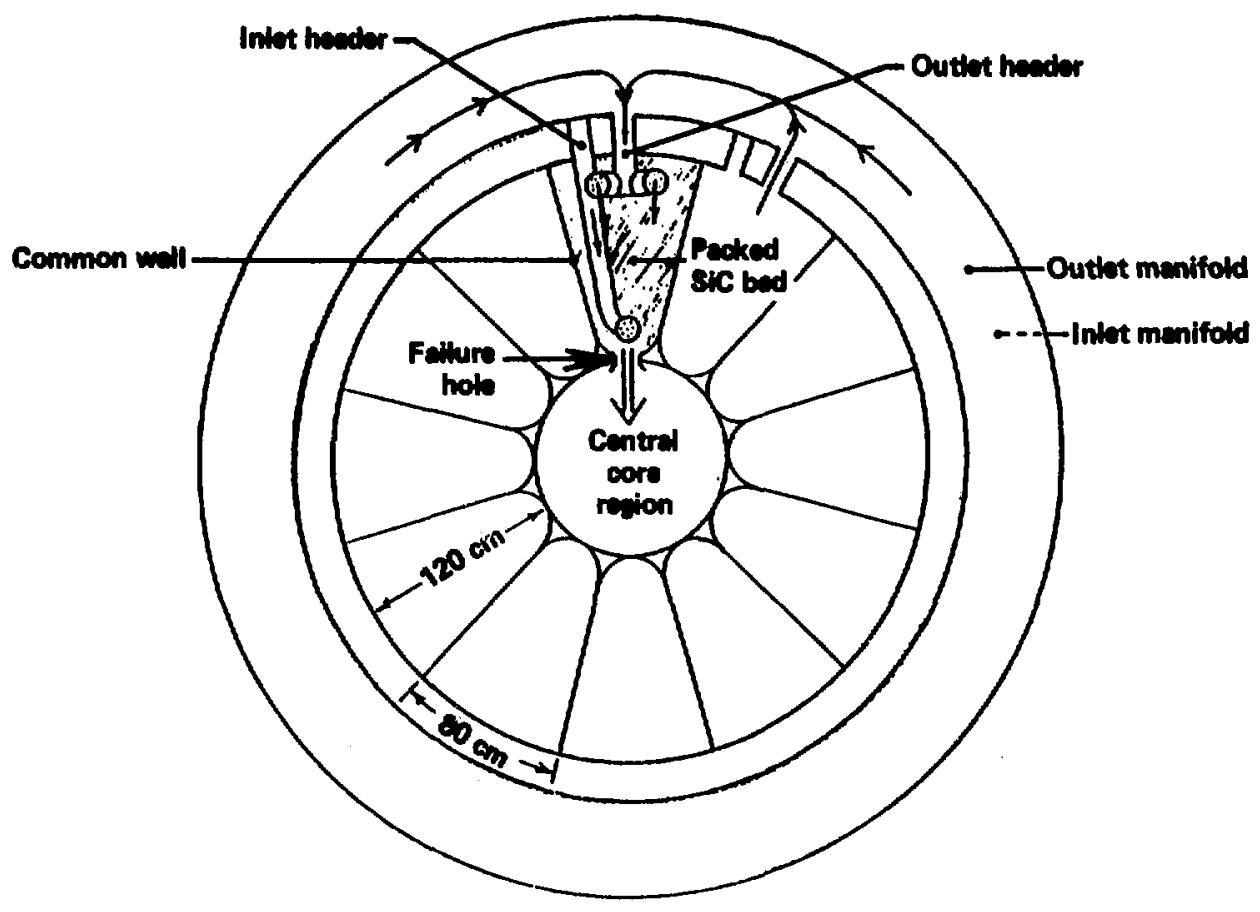

Fig. 4.3-23. Pod rupture flowdown paths. 


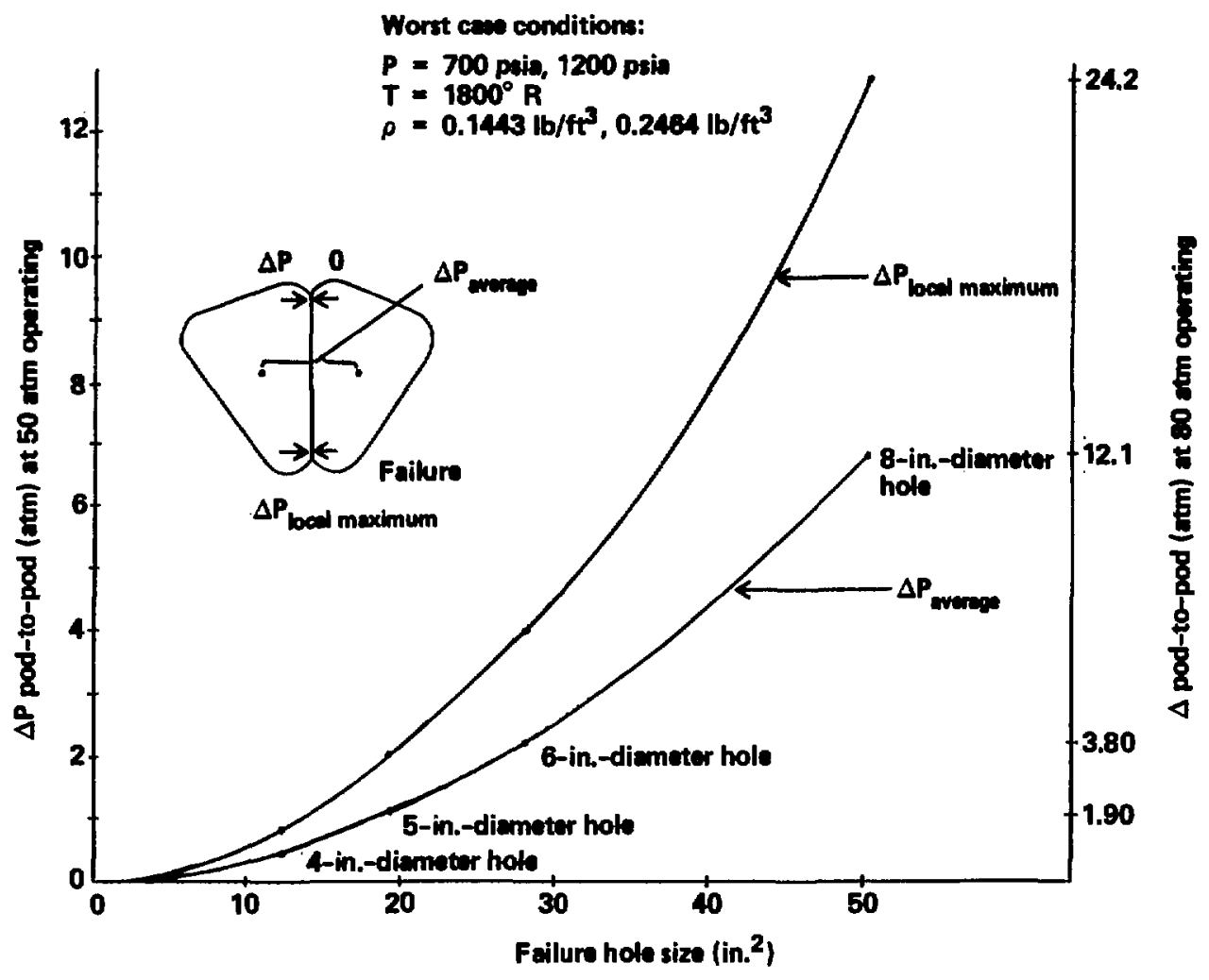

Fig. 4.3-24. Pod-to-pod pressure differentials vs failure hole size. 
values are 50.4 and 29.1 atm, respectively. Thus, even in the case of a split dom two-thirds of the length of a pod, the pressure differential is below the 58-atm linit for the structure. We belleve that the pod design is strong enough to preclude a domino fallure.

\subsubsection{Shield}

The shield design has only been scoped at this time to show that it is feasible to met the requirements for the shield. Further design iterations will refine and optimize the concept. The function of this shield is to protect the superconducting magnets from radiation and to protect workers in the reactor area during mintenance. The MRS high-temperature blanket shield uses steel as the shielding and main structural material. It also provides structural support for the blanket and magnets. Figure 4.3-25 shows the shleld materials and thicknesses used in the neutronics and thermal hydraulics scoping analyses described below. Further optimization is expected to result in a thinner shield.

\subsubsection{Shield Design Criterfa}

The principal criteria for shield design is to protect the superconducting magnets from neutron and garma radiation. The same magnet shielding criterla discussed in Section 4.2.2.1 apply to the LiPb and high temperature blankets. To reiterate, three values characterize the central-cell shield requirements: (1) $2.2 \times 10^{-5}$ dpa/FPY in the Cu stabllizer to allow 5 FPY before the first anneal; (2) $2.0 \times 10^{8}$ rad (insulator)/FPY to ensure a Iffetime dose less than $5 \times 10^{9} \mathrm{rad}$; (3) $0.06 \mathrm{~m} / / \mathrm{cm}^{3}$ peak heat load in the winding to minimize cryogenic costs.

\subsubsection{General Structural Description}

The primary purpose of the cylindrical shield is to provide radiation protection for the central cell magnets during reactor operation. In addition, the shield has been structurally integrated into and forms a part of the module support structure and, therefore, provides support for the magnets and blanket.

The shield is a box structure supporting the blanket and magnet. It encloses circulating borated water for cooling and lead and polyethyleme slabs 


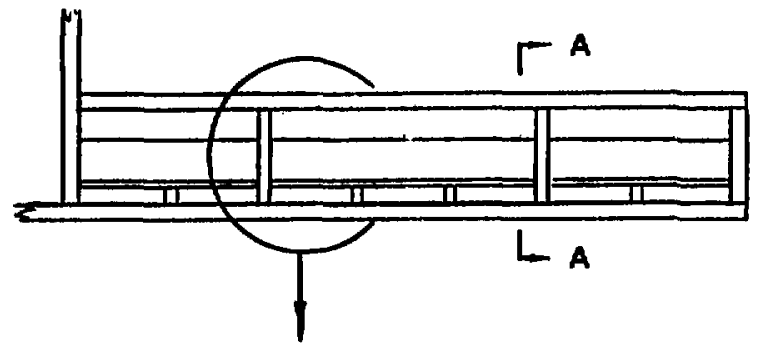

Suction A-A

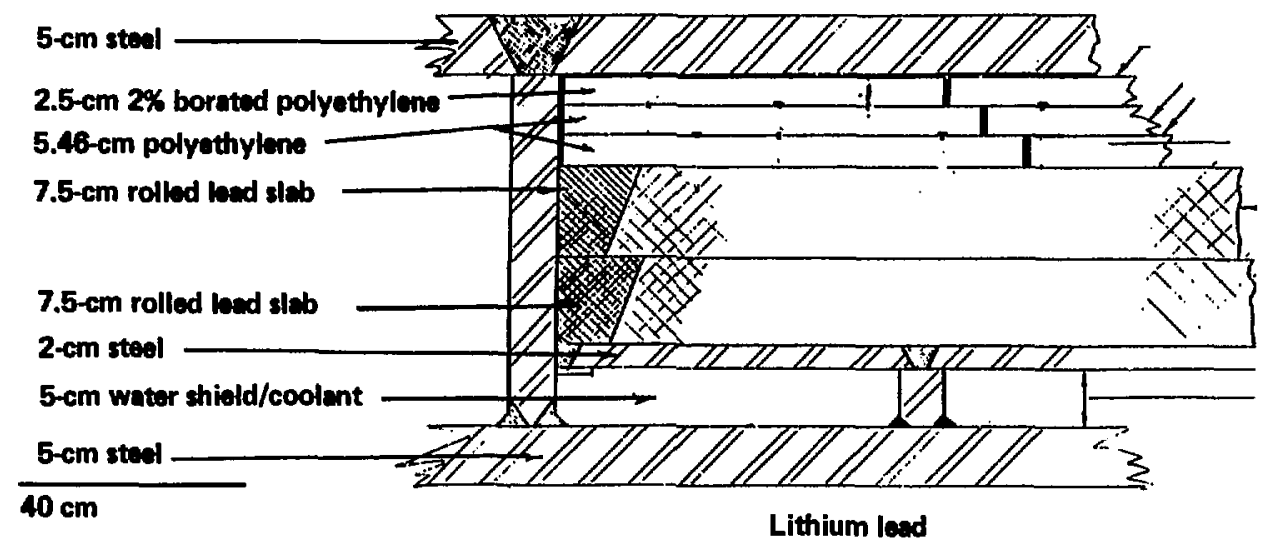

Fig. 4.3-25. Shield. 
to attenuate neutron and gama radiation. The 5-cm steel-box floor (inner radius) interfaces with the blanket LiPB coolant (maximim temperature $\sim 500^{\circ} \mathrm{C}$ ) and pods on one side, and the shield borated mater coolant (maximum temperature $\sim 100^{\circ} \mathrm{C}$ ) on the other. An insulation layer is required in this region. The water coolant is enclosed with $2 \mathrm{~cm}$ of steel, which also acts as a support for the two $7.5 \mathrm{~cm}$ lead slabs. The three polyethylene slabs are capped by a 5-cm steel cover. Channels located on the shield floor at the LiPb interface are designed to accept the pod support rafls.

\subsubsection{Shield Meutronics}

We have demonstrated the feasibility of the shield for the MARS hightemperature bianket. The final design of the shield has lot been completed, but it presents little conceptual difficulty. He still need to address the shield design th the vicinity of the module-to-module interfaces.

We performed one-dimensional neutronics analysis of the shield using the OHEDANT code. The shield geonetry is shown in Fig. 4.3-25. Its performance against the criteria is given in Table 4,3-10, and the energy deposition as a function of position is given th $T a$ le 4.3-11.

Although the proposed shield fails to satisfy the first criterion, it appears that compliance can be achleved by trading some lead for polyethylene. Future work will address optimization and model plumbing penetrations. (e.g., helium and LiPb pipes).

Table 4.3-10. Shield performance.

\begin{tabular}{|c|c|c|}
\hline & Criteria & Performance: \\
\hline $\begin{array}{l}\text { Stabilizer damage (dpa/FPY) } \\
\text { Insulator dose (rad/FPY) }\end{array}$ & $\begin{array}{l}<2.2 \times 10^{-5} \\
<2.0 \times 10^{8}\end{array}$ & $\begin{array}{l}3.5 \times 10^{-5} \\
3.6 \times 10^{6}\end{array}$ \\
\hline $\begin{array}{l}\text { Peak conductor heat deposition } \\
\left(\mathrm{mH}_{\mathrm{H}} / \mathrm{cm}^{3}\right)\end{array}$ & $<0.05$ & 0.053 \\
\hline
\end{tabular}


Table 4.3-11. Energy deposition profile.

\begin{tabular}{|c|c|c|c|}
\hline \multirow[b]{2}{*}{ Material } & \multirow[b]{2}{*}{ Radtus $(\mathrm{cm})$} & \multicolumn{2}{|c|}{ Energy deposition } \\
\hline & & $\begin{array}{l}\text { Heutrons } \\
\left(\mathrm{mW} / \mathrm{Cm}^{3}\right)\end{array}$ & $\begin{array}{l}\text { Ganmas } \\
\left(\mathrm{mW} / \mathrm{cm}^{3}\right)\end{array}$ \\
\hline \multirow[t]{3}{*}{ Steel } & 182.8 & 2.37 & 256 \\
\hline & 184.5 & 1.74 & 301 \\
\hline & 186.2 & 1.19 & 304 \\
\hline \multirow{3}{*}{. Water } & 187.8 & 9.50 & 34.1 \\
\hline & 189.5 & 4.52 & 33.0 \\
\hline & 191.2 & 3.25 & 32.2 \\
\hline Steel & 193.0 & 0.33 & 212 \\
\hline \multirow[t]{5}{*}{ Leád } & 195.5 & 0.039 & 134 \\
\hline & 198.5 & 0.035 & 9.6 \\
\hline & 201.5 & 0.029 & 2.5 \\
\hline & 204.5 & 0.024 & 1.3 \\
\hline & 207.5 & 0.020 & 1.7 \\
\hline \multirow[t]{2}{*}{ Polyethylene } & 210.4 & 0.346 & 0.17 \\
\hline & 213.1 & 0.168 & 0.20 \\
\hline \multirow[t]{2}{*}{ Borated polyethylerie: } & 215.1 & 5.58 & 0.18 \\
\hline & 216.4 & 0.507 & 0.17 \\
\hline \multirow[t]{2}{*}{ Steel } & 218.3 & 0.0115 & 0.87 \\
\hline & 220.8 & 0.0092 & 0.31 \\
\hline
\end{tabular}




\subsubsection{Shield Thermal Hydraulics}

The shield configuration is shown in Fig. 4.3-25. The themal-analyzer finite-element computer model ${ }^{26}$ was used to predict temperature profiles in the shield. One requirement is to maintain a maximum temperature in the polyethylene below $120^{\circ} \mathrm{C} .{ }^{27} \mathrm{Also}$, the heat leak from the LiPb region should be low, because the heat carried out by the water will be low temperature heat. It is necessary to keep thermal gradients in the shield low to maintain structural integrity.

Insulation will be required between the LiPb itl the back wall region and the shield. Without this insulation, two design problems exist: (1) a large temperature differential on thie order of 300 to $400^{\circ} \mathrm{C}$ will cecur through the first $5 \mathrm{~cm}$ of the shifeld, and (2) a large heat leak on the order of $16 \mathrm{w} / \mathrm{cm}^{2}$ will occur between the LiPb region to the water-cooled shield, causing intolerable film drop temperatures in the water cooling system.

To keep the water pressure around 100 to $150 \mathrm{psi}$, the inner shell must be insulated from the LiPb so that the maximum temperature of the inner shell is around 150 to $200^{\circ} \mathrm{C}$. This decreases the heat leak and reduces the film drop on the inner shell side to a reasonable value. If the inner shell of the shield is constrained at $165^{\circ} \mathrm{C}$ and the maximum water temperature is $56^{\circ} \mathrm{C}$, polyethylene reaches a temperature of $120^{\circ} \mathrm{C}$, which just meets the maximum service temperature. Thus trace cooling will not be needed at the outer shell of the shield. The film drop temperatures are $53^{\circ} \mathrm{C}$ on the shield side and $84^{\circ} \mathrm{C}$ on the blanket side.

The 6.32-m-long shield module section must accomodate about 1.9 w of heating, not including the heat leak from the LiPb regiori. Assuming an inlet water temperature of $40^{\circ} \mathrm{C}$ and a temperature rise of $16^{\circ} \mathrm{C}$, a water flow rate of $28 \mathrm{~kg} / \mathrm{s}$ is required. The water :elocity of $0.146 \mathrm{~m} / \mathrm{s}$ will cause a very $10 \mathrm{w}$ pressure drop through the channels, requiring only $0.14 \mathrm{~W}$ of pumping power per module. 


\subsubsection{Seals}

\subsubsection{Vacuum Seals}

The module interface with each other through module-to-module seals designed to maintain the reactor vacuum and yet, for safety, allow and the joint to be sealed and disconnected by remote operation. Studies indicate the seal can use brazing techniques, allow for verification of each joint without requiring a reactor vacuum, and yet be flexible enough to permit deflections resulting from module temperature expansions. Figure 4.3-26 show a schematic of the intermodule seal concept.

The flat seal plate is closed agalnst the grooved seal plate on the adjacent module by pressurizing the bellows. The bellows chamber contains a steel plug for shtelcing. Backing plates gulde and support the movable be?lows, seal plate, and shield plug assembly. The seal is formad by allowing a molten brazing alloy to flow into the grooves in the statfonary seal plate surface. Resistance heating melts the alloy and warms the flow paths. This allows a relatively low pressure in the bellows to position the seal. Two seal grooves allow the seal integrity to be tested through a port between the grooves.

\subsubsection{Renote Actuate. Mining Lithium-Lead Connections}

For protection against potential tritium residue and to minimize ifsengagement time, the Lipb pipe connections are also designed to be operated remotely. Because of the relatively low operating temperatures, the LIPb joint can be brazed with a design similar to that used in the module vacuum seal. The joints use brazing matal from a reservoir that is allowed to flow into twa areas located between the two surfacas to be joined. Heating colls in the joint areas ensure continuous flow of the brazing material. Cooling jackets, if required, are provided to ensure that the brazing compound remains confined whlle the seal hardens. A pressure/vacuum test connection port to a chamber between the two joints allows the integrity of the seal to be verifted. The concept is shown in Fig. 4.3-27. 


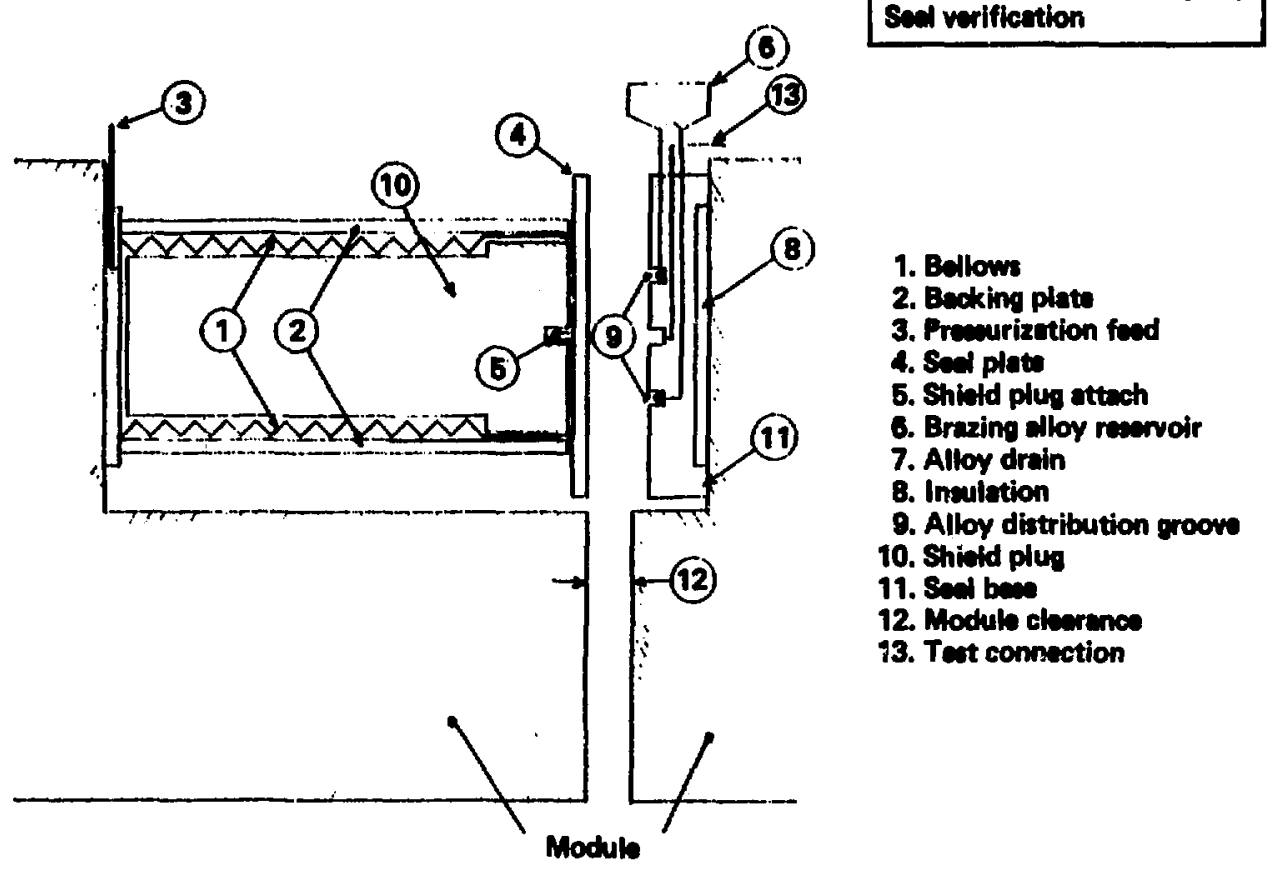

\section{Fentures}

Motel ending

Remote coupling/decoupling

Senl veriflestion

2. Bodking platt

3. Prenurization feed

4. Saed plat

5. Shisld plur attuch

6. Bruzing elloy rewenoir

7. Alloy drain

8. Imvilation

9. Alloy divtribution groow

10. Shisid plu.

11. Sow ben

12. Module clearence

13. Tent connection

Fig. 4.3-26. Module-to-wodule interface seal. 


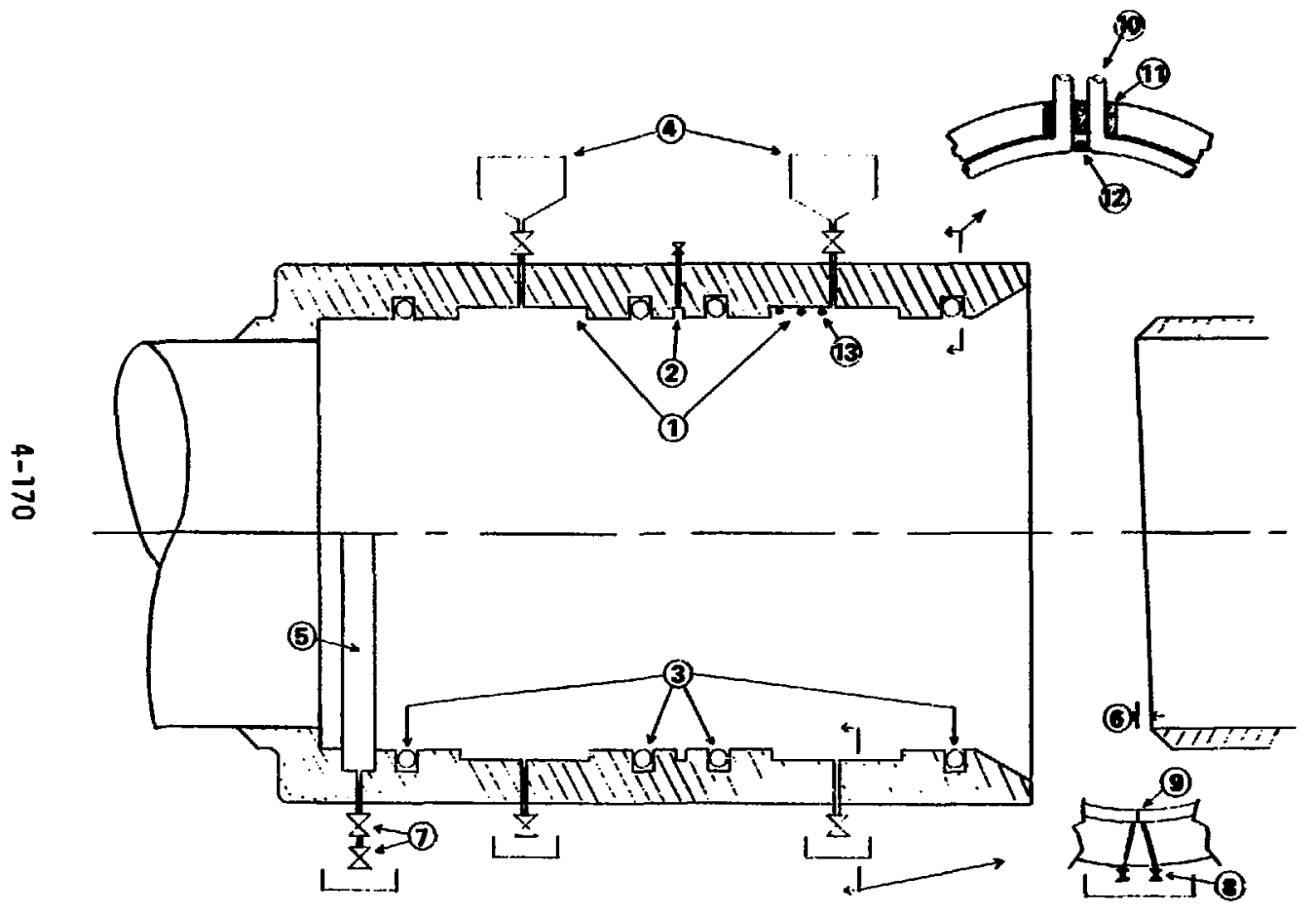

\section{Features:}

Notal sall

Remote operation

Sed verification
1. Joint area
2. Test connection
3. Cooling tubses
4. Breze slloy remervoir
5. Drip grow
6. 1-cm offert
7. Lipb drin
8. Brazedloy drain
9. Baffis
10. Cooling tubss
11. Buthing
12. Thermal biidse
13. Heating coil

Fig. 4.3-27. LiPb pipe coupling. 


\subsubsection{Tritium Removal}

Tritlum is produced in the high temperature blanket at a rate of $450 \mathrm{~g} /$ day. The tritium is bred in a solid lithium-containing material and diffuses into the helium coolant. Tritium inventory in the solid breeder is estimated at $100 \mathrm{~g}$. A partial pressure of oxygen on the order of 1 Torr in the helium maintains the tritium primarily as $\mathrm{T}_{2} \mathrm{O}$. By maintaining the chemical form of tritium as $\mathrm{T}_{2} \mathrm{O}$, the contalment and recovery of tritium is considerably easier thian containment and recovery in system that contains molecular tritium $\left(T_{2}\right)$. This is because $T_{2} 0$ does not permeate materiais as does $T_{2}$, and $T_{2} 0$ can be recovered from hel fum with present-day technology wile the technology for recovering $\mathrm{T}_{2}$ from hellum is less developed. The tritium path through the high-temperature helium loop begins in the breeding blanket, then passes through the recirculating heliun (whtch comprises the power loop), and finally to recovery from the hellum by phystcal adsorption. A small amount of tritfum, on the order of $50 \mathrm{~g} /$ day, is bred in the LIPb. It is recovered by a flash unit operation.

Tritium loss from the process system occurs minly in the form of $\mathrm{T}_{2} \mathrm{O}$ the high-temperature power loop through turbine and compressor leaks. Additional loss is as $T_{2}$ permeation and leaks from the LiPb loop. Accidental release of a fraction of the tritium in these loops is possible, and the fraction released provides a basis for sizing the tritium control equipment for the reactor hall.

\subsubsection{Solld Breeder Tritium Inventory}

We have made estimates of the tritium inventory and the rate of formation of $\mathrm{T}_{2} \mathrm{O}$ in the high-temperature solid breeder. The tritiun inventory depends on the particle size of the breeder compound and is estimated at $100 \mathrm{~g}$ for the reactor if the particle radius is 50 pm (roughly 150 mesh material). This estimate is influenced strongly by the tritium diffusivity value, wich is poorly known and little understood in the lithium compounds of interest. Assuming $1 \mathrm{Ppm}$ oxygen in the helium stream at $1000^{\circ} \mathrm{C}$, calculations indicate that the rate of $\mathrm{T}_{2} \mathrm{O}$ formation is controlled by diffusion of tritium through the solid breeder compound, and that tritium will ract with oxygen as soon as it reaches a solid surface that contains adsorbed oxygen.: 
The tritium inventory in a breeder blanket has been estimated based on the assumptions that:

1. Tritium is swept away by the coolant gas (containing 1 ppm $\mathrm{O}_{2}$ ) as soon as $\mathrm{T}_{2} \mathrm{O}$ is formed.

2. Negligible $\mathrm{T}_{2} \mathrm{O}$ is present in the SIC portion of the solid blanket.

3. All the "stationary Inventory" of tritium is present as tritiun in the lithium compound.

4. The lithium compound particles are spherical.

Under these assumptions, the inventory of tritium in each particle, $S$, is the volume integral of the tritium concentration, 1.e..,

where

$$
S=\int_{0}^{r} C_{T}(r) 4 \pi^{2} d r,
$$

which is

$$
S=\frac{4 R_{T}}{450} r_{0}^{5}
$$
$R_{T} \quad=\quad$ tritiun generation rate in (atoms $/ \mathrm{cm}^{3}-\mathrm{s}$ ),
$D=$ diffusivity of tritium in the breeder compound $\left(\mathrm{cm}^{2} / \mathrm{s}\right)$, and $r_{0}=$ radius of particle sphere $(\mathrm{cm})$.

The total tritlum inventory is obtained by using the total generation rate and assuming uniform particle size, such that

$$
S(\operatorname{total})=\frac{R(\operatorname{total})}{150} r_{0}^{2}
$$

At this point, it is necessary to estimate values for the $D$ because no data are avaflable for the diffusivity of hydrogen (much less tritium) in $\mathrm{LI}_{2} \mathrm{TiO}_{3}$ or LIAIO

We chose a value of $\mathrm{O}$ based on the diffusivity of tritium in $\mathrm{Al}_{2} \mathrm{O}_{3}$ where

$$
D=4 \times 10^{-10} \mathrm{~cm}^{2} / \mathrm{s}
$$

With this value, the total reactor inventory is $25 \mathrm{~g}$. We note that the estimated value depends strongly on the diffusivity of tritium in the breeder, whtch can be altered many orders of magnitude by heat treatment, environmental exposure, and bombardment by ionizing radiation. We recomnend that tritiun 
diffusion in lithium-bearing compounds be further exanined and analyzed.

Finally, the calculated inventory values for the MARS solid breeder scales as the particle radius squared. For a 100-pan particle it is $100 \mathrm{~g}$. This has been used as the baseline. If the diffusion coefficient were an order of magnitude lower, $4 \times 10^{-11} \mathrm{~cm}^{2} / \mathrm{s}$, the trittum inventory would be 260 g. We have not considered $T_{2} 0$ formation in the solid, which could increase the inventory.

\subsubsection{2 $T_{2} 0$ Formation Rate}

A key factor in the blanket design is the formation of $T_{2} 0$ by the oxygenated helium strecu to prevent tritium loss by permeation. How fast this occurs is the critical issue a : to determine this we had to examine the data avallable on hydrogen oxidation. A large amount of data is summarized in Fig. 4.3-28, where different rate-1initing regimes are identifted. 28 The regime of interest is the extrene lower right corner because the total pressure of $\mathrm{H}_{2}$ and $\mathrm{O}_{2}$ is $0.12 \mathrm{~m} \mathrm{Hg}$ at $1000^{\circ} \mathrm{C}$. This is the regime where an explosion will not occur but where the oxidation of hydrogen is i imited by recombination at a wall. The important point is that the gaseous mixture is not in the explosive regime. Thus, we turn to an analysis of the heterogeneous reaction rates.

The rate of oxygen adsorption on a solid surface can be expressed as

$$
r(\mathrm{ads})=\frac{3.51 \times 10^{22}}{(M T)^{1 / 2}} \text { Pf }\left(\frac{\text { molecules }}{\mathrm{cm}^{2}-\mathrm{s}}\right) \text {, }
$$

where

$$
\begin{aligned}
& P=\text { partial pressure of } \mathrm{O}_{2}=(0.06 \text { Torr }), \\
& M=\text { molecular weight of } \mathrm{O}_{2}(32), \\
& T=\text { absolute temperature }(1273 \mathrm{~K}) \text {, and } \\
& f=\text { fraction of the collisions that result in } \\
& \text { chemisorption. }
\end{aligned}
$$

The fraction of collisions $f$ that resuit in chemisorption is

$$
f=e^{\frac{-\Delta E}{R T}}
$$


where $\Delta E$ is the activation energy (for the chen'sorption process).

As a general rule-of-thumb, $E$ is 10 to $15 \mathrm{kcal} / \mathrm{mole}$, and we used these values because no data were avaflable for the activation energy of $\mathrm{O}_{2}$ on $\mathrm{Li}_{2} \mathrm{THO}_{3}$ or $\mathrm{LiAlO}_{2}$.

Thus, $r_{\text {ads }}=2.0 \times 10^{17}$ for $E=10 \mathrm{kcal} / \mathrm{mole}$, and $r_{\text {ads }}=2.8 \times 10^{16}$ for $E=15 \mathrm{kcal} / \mathrm{mole}$.

Later results show that this sensitivity is not important. This is the rate of chemisorption of oxygen on any solid surface in the breeder module; this, of course, includes the surfaces of the solid breeder compounds.

The next task is to determine the rate of arrival of tritiun atoms from within the breeder compound. The flux of tritium at the surface is found from the solution of the diffusion equation assuming a diffusivity of $4 \times 10^{-10}$ $\mathrm{cm}^{2} / \mathrm{s}$ and a rate of tritium generation that is constant throughout the particles. For a particle size of $50 \times 10^{-4} \mathrm{~cm}(50 \mathrm{~m})$, the flux of tritium atoms at the surface is $1.3 \times 10^{11}$ atorn $\mathrm{T} / \mathrm{cm}^{3}-\mathrm{s}$.

This atomic tritfum flux is much lower than the rate of chemisorption of oxygen $\left(6 \times 10^{16}\right)$, and indicates that the rate of tritium oxidation is 1 inited by the rate of diffusion of tritium through the solfd breeder. The surface is effectively covered with oxygen and, based on FIg. 4.3-28, the oxtdation rate at the surface is the same as the diffusion rate. As a rough estimate, the fraction of tritium desorbed as $T_{2}$ is proportional to the ratio of diffuston of $T$ to adsc-ption of $\mathrm{O}_{2}$. This is approximately $2.2 \times 10^{-6}$. 


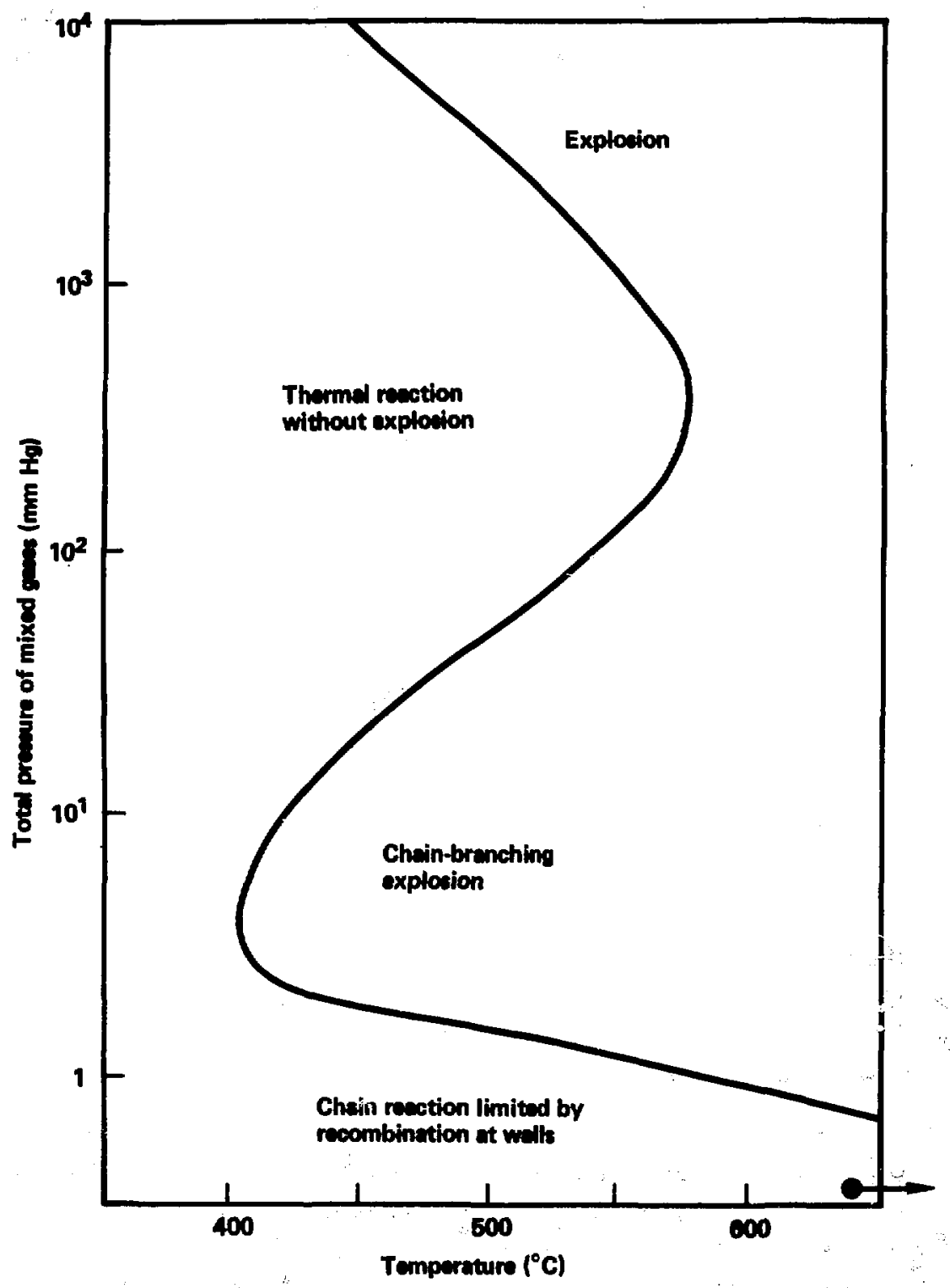

Fig. 4.3-28. Nature of reaction of stoichiometric mixtures of hydrogen and oxygen. 


\subsubsection{Tritium Extraction from Helium}

Tritium is produced in the solid breeding blanket and diffuses into the helium loop as $\mathrm{T}_{2} \mathrm{O}$ at the rate of $450 \mathrm{~g} /$ day. The function of the tritium extraction process is to recover the tritium produced in the blanket from the heliun loop.

A slip-stream recovery process for steady-state tritium extraction is lllustrated in Fig. 4.3-29. The input variables for the process design are the tritium production rate, the chemical form of tritfum, the slip-stream flow rate, and the min helium flow rate. The engineering variables to be specified or calculated are the selection of the therwodynamic sink for $\mathrm{T}_{2} \mathrm{O}$ in the recovery process, the operating temperature for the $\mathrm{T}_{2} \mathrm{O}$ recovery process, and the tritium concentrations in all the process streams. Flgure 4.3-39 and the above independent/dependent variables apply to any tritium extraction process for the helfum process loop, as shown.

The tritiun extraction process chosen for $T_{2} 0$ recovery is physical adsorption using molecular sfeve. The molecular sieve, designated 4 , provides the thermodynamic sink for $T_{2} 0$ and results in a $T_{2} 0$ partial pressure at the exit of the recovery unit on the order of 0.01 Torr at $60^{\circ} \mathrm{C} .29$ The $60^{\circ} \mathrm{C}$ process temperature exists in the main helium loop at the exit of the Ho. 2 precooler and before reaching to the hellum compressor, as discussed in Section 4.3.5. A higher or lower extraction temperature produces a correspondingly higher or lower $\mathrm{T}_{2} \mathrm{O}$ exit partial pressure. Lowering this temperature to $40^{\circ} \mathrm{C}$ would result in a $\mathrm{T}_{2} \mathrm{O}$ exit partial pressure of approximately 0.001 Torr.

The molecular steve bed is regenerated to recover $\mathrm{T}_{2} \mathrm{O}$ by ratsing the bed temperature to 200 to $300^{\circ} \mathrm{C}$ and purging off the $T_{2} \mathrm{O}$, or by vacuum punping. 30 The $T_{2} \mathrm{O}$ is recovered in a cold trap and the 11 quid $T_{2} \mathrm{O}$ delivered for final $T_{2}$ purification by electrolysis and cryogentc distillation.

Table 4.3-12 contains the results of calculations of the pertinent quantitfes of a steady-state $T_{2} 0$ material balance for s1ip-stream fractions of 1 and 5\%. The recirculating tritium radioactive inventory, i.e., that tritium as $T_{2} \mathrm{O}$ in helium, is calculated from $\mathrm{Cl}=8.7 \times 10^{9}(y)\left(t_{r}\right)$, where $t_{r}$ is the helfum residence time (time to complete one loop of the coolant ctrcuit) in seconds and $y$ is the $\mathrm{T}_{2} \mathrm{O}$ concent ation in moles $\mathrm{T}_{2} \mathrm{O} /$ mole He. For the case of 


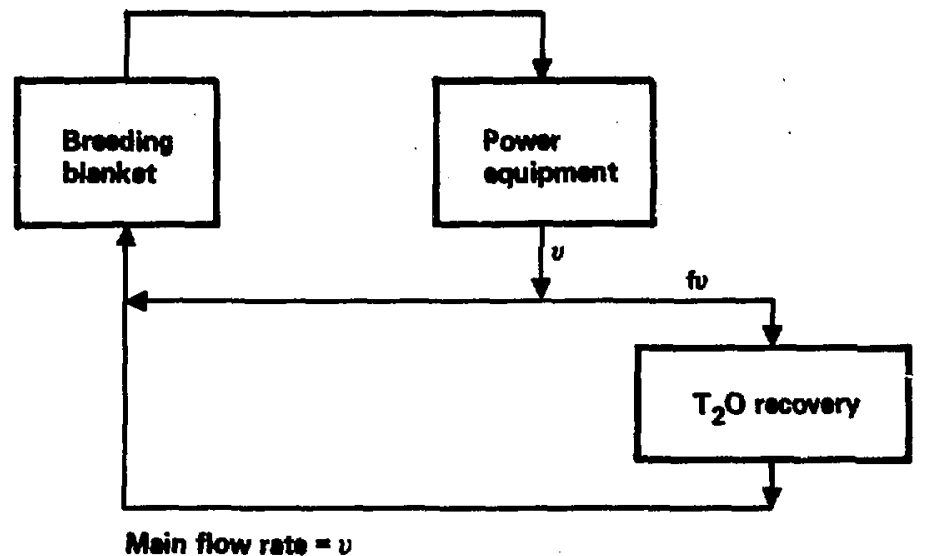

Slip streem flow rate $=$ fo; $f \simeq 0.01$

Fig. 4.3-29. Material balance diagram of slip-strean tritium recovery process. 
Table 4.3-12. Steady state material bulance for a $T_{2} 0$ slip stream recovery.

\section{Input variables}

Tritium production (g/day)

450

Hellum flow 80 atm (1bm/s)

1322

$\mathrm{T}_{2} \mathrm{O}$ recovery exit pressure $60^{\circ} \mathrm{C}$ (Torr)

0.01

Blanket exit' temperature $\left({ }^{\circ} \mathrm{C}\right)$

900

18 silp stream

Si ip stream exit conceniration $T_{2} \mathrm{O}$ (moles/mole)

$1.645 \times 10^{-7}$

Return concentration (moles/mole)

$7.374 \times 10^{-7}$

Blanket exit concentration (noles/mole)

$7.432 \times 10^{-7}$

$\mathrm{T}_{2} \mathrm{O}$ partial pressure entering blanket (Torr)

$4.483 \times 10^{-2}$

$\mathrm{T}_{2} \mathrm{O}$ partial pressure exiting blanket (Torr)

$4.519 \times 10^{-2}$

silp strean flow $\left(m^{3} / 9\right)$

0.512

5x slip stream

Slip stream exit concentration (moles/mole)

$1.645 \times 10^{-7}$

Return concentration (noles/mole)

$2.744 \times 10^{-7}$

Blanket exit concentration (moles/mole)

$2.802 \times 10^{-7}$

$\mathrm{T}_{2} \mathrm{O}$ partial pressure entering blanket (Torr)

$1.669 \times 10^{-2}$

$T_{2} 0$ partial pressure exiting blanket (Torr)

$1.704 \times 10^{-2}$

silp strean flow $\left(m^{3} / 9\right)$

2.56 
a 17 slip stream where the $T_{2} 0$ concentration is approximately $7.4 \times 10^{-7}$ moles $T_{2} 0 /$ mole He, the recirculating activities in curies is $6.4 \times 10^{3} t_{r}$; for a $5 \%$ slip strean the result is $2.4 \times 10^{3} t_{p}$.

To design the hardware for $T_{2} 0$. recovery using a 4 molecular sieve, we had to spectfy the size of the ressels. Vessel sizing is based on 24-hr operation, a $1 \%$ slip stream, a tritium loading of $450 \mathrm{~g}$ (as $T_{2} 0,1650 \mathrm{~g}$ ), a loading of $1 \mathrm{lb}$ of trittated water per $100 \mathrm{lb}$ of sieve, and a superficial velocity of $22.8 \mathrm{~m} / \mathrm{min}$. These specifications produce a process pressure drop of approximately $3.4 \mathrm{kPa}(0.5 \mathrm{psi})$, bed area of $1.33 \mathrm{~m}^{2}$, a vessel diameter of $1.2 \mathrm{~m}$ and bed depth of $33 \mathrm{~cm}$. The cost for a single stainless steel vessel rated at $1.03 \times 10^{4} \mathrm{kPa}(1500 \mathrm{psi})$ is $\$ 66,000^{31}$. Three vessels are requited with one in operation, one in regeneration, and one in standby mode.

The tradeoffs for the process design of the tritium extraction system involve cost rs tritium in the process system. For the base case we selected a slip strean of 18 and cycle time of $24 \mathrm{hr}$. This results in approximately $470 \mathrm{~g}$ of tritium $\left(4.5 \times 10^{6} \mathrm{Cl}\right.$ ) in inventory (450 g on the bed, and $20 \mathrm{~g}$ recirculat: $j$ with a $30-s$ residence time). Clearly, cutting the cycle time in half would result in a 250-g tritium inventory reduction. However, at the present it is not clear how fast a molecular sieve vessel can be heated, purged or vacuum pumped, and cooled for return to the process. The 24-hr cycle time is subjective specification and appears reasonable from an engineering perspective. Thus, the apparent cycle time could be shortened by adding more ressels. Noting that the single stainless-steel ressel cost is $\$ 65,000$ and the installed cost is approximately 5.4 times this cost (including 25\% contingency), increasing the number of vessels costs $\$ 35,5,000$ per installed vessel. Further trade-off studies must involve cost versus the tritiun process inventory.

\subsubsection{Tritiun Extraction fron LiPb}

Tritiun will be extracted from the $L i P b$ iy evacuation at a free surface. The system is identical (except in size) to the tritiun extraction syster for the LiPb blanket discussed in Secicion 4.2.4.1. The process relies on the very lon solubllity of tritium in LIPb. 


\subsubsection{5 : Tritium Control in the Reactor Hall}

Tritium will enter the MRS reactor-hall atmosphere elther as the oxide or as molecular tritium. It can. enter the hall as a result of accidents, normal process leaks such as helfum $\left(\mathrm{T}_{2} \mathrm{O}\right)$ from the turbines and compressors, Lipb leaks containing molecular tritium $\left(T_{2}\right)$, and permeation losses from molecular trituim inventories. In any event, the reactor-hall tritium-control process must recover tritium that exists as $T_{2} 0, H T O$, or $T_{2}$.

The tritium recovery process that accepts $T_{2} 0$ and $T_{2}$ as feed is called catalytic oxidation/adsorption. It is shown in Fig. 4.3-30 with minor modifications. 32 In the process, the reactor-hall atmosphere is accepted and compressed to approximately $405 \mathrm{kPa}$ ( $3 \mathrm{~atm}$ ). The compressed gas is heated and passed through a catalytic recombiner where molecular tritium (or HT) is oxidized to $\mathrm{T}_{2} \mathrm{O}$ (HTO). The gas exiting the catalytic recombiner is cooled and refrigerated to $275 \mathrm{~K}\left(2^{\circ} \mathrm{C}\right)$ to condense water. The condensed water is dratned from the process and this is essentially the only recovered-water exit from the process. The drfed gas from the refrigerated dryer then passes through a molecular-sieve bed, to remove more water. At the exit of this bed, stean is added to the process gas and the gas is passed through a final molecular sieve bed. The stean is added to allow a larger fraction of the tritiated water to be recovered. By increasing the dew point of the process stream before the final molecular sieve bed, a larger percentage of the water entering this bed can be recovered. Because the tritiated water and the added stean w111 be well-mixed, the same percentage of steam and water will be recovered. If the stean were not added, the potentfal for mass transfer of the water would be relatively low and thus its percentage of recovery would be low. The exiting gas from the last molecular sieve bed is vented to the stack or returned to the reactor hall. The molecular steve beds are regenerated with hot process gas and the regeneration exhaust is recycled to the entrance of the process. The unit capital cost of this catalytic oxidation/adsorption process is estimated to be $\$ 690,000$ per $30 \mathrm{~m}^{3} / \mathrm{min} .32$

To estimate the number of catalytic oxidation/adsorption units required to control the tritium concentration in the reactor hall, it is necessary to assume values for certain independent variables. $T$ : reactor-hall volume is assumed to be on the order of $1.2 \times 10^{6} \mathrm{~m}^{3}$. An instantaneous tritium input of 


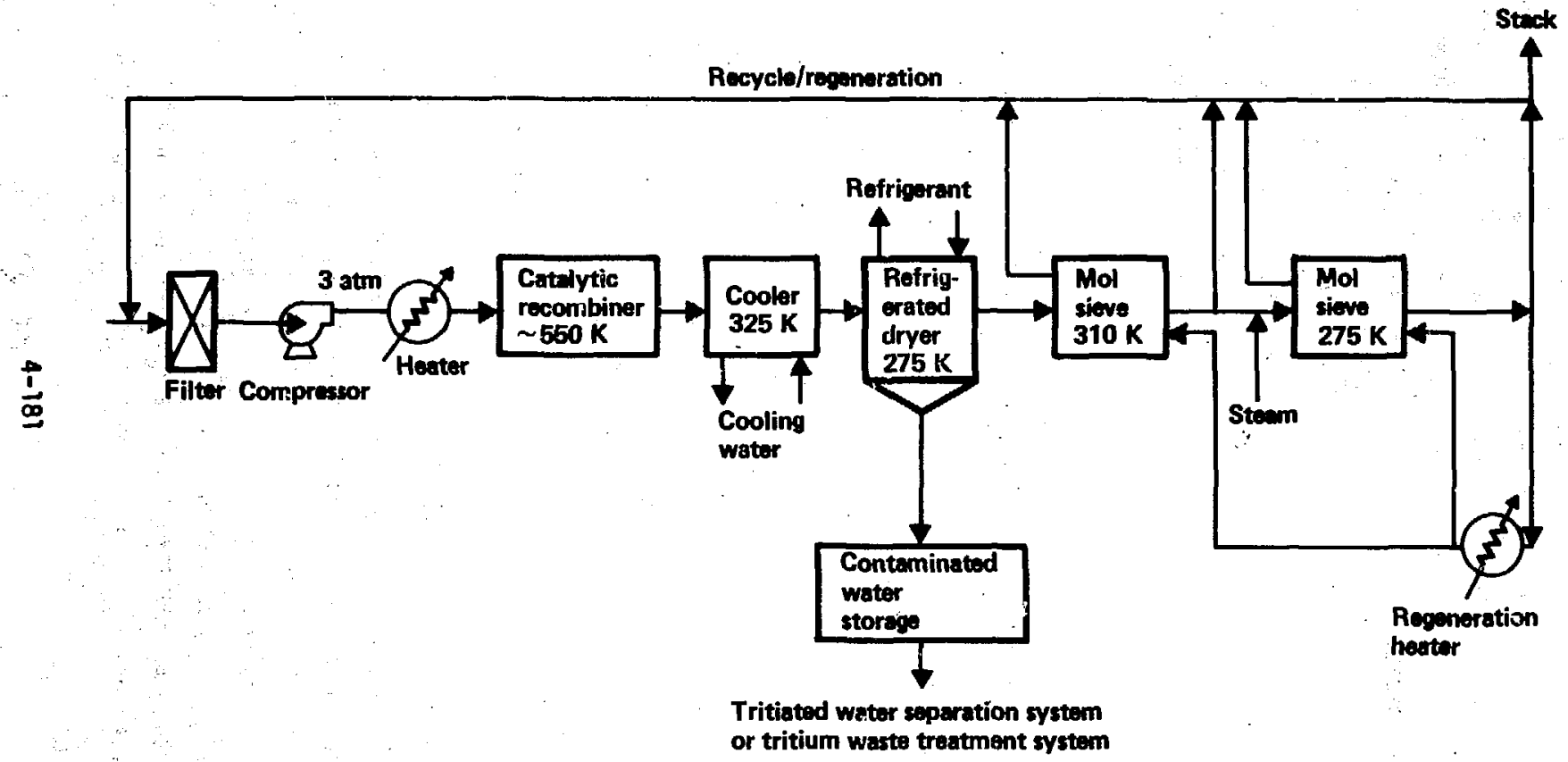

Fig. 4.3-30. MARS reactor-hall tritium recovery by catalytic oxidation/adsorption. 
$6.46 \times 10^{4} \mathrm{Cl}$ is assumed from the helium loop (approximately one-third of the recirculating inventory for the base case $\mathrm{T}_{2} 0 /$ hel fum loop because the total loss of pressure in the helium loop can be prevented by isolation valves). The system cleanup objective is assumad to be a reactor hall tritiun concentration of $500 \times 10^{-6} \mathrm{ci} / \mathrm{m}^{3}$ in 14 days (1.e., bubble suit protection). Assuming the reactor-hall atmosphere to be well mixed, the tritium concentration as a function of time will be $C / C_{0}-\exp [-v t / V]_{3}$, where $C_{0}$ is the time 0 concentration, $v$ is the reactor hall volume, and $v$ is the total feed rate capacity of the catalytic oxidation/adsorption process. Our assumption that the stean and water are mil-mixed ignores tritiun adsorption on the surfaces of the reactor hall. The required catalytic oxidation/adsorption flow rate for our assumptions is $280 \mathrm{~m}^{3} / \mathrm{min}$, or 10 units. Scaling directly at $\$ 690,000$ per unit yields a total cost of $\$ 6.9 \times 10^{6}$. We anticipate that the total cost for the capacity required will scale to some power less than unity. Therefore, because our assumptions are only preliminary and because of the lack of construction experience in this area, the stated cost should be regarded as an upper bound.

We still have to address the issue of the validity of the assumptions used in sizing the process equipment for the reactor hall. This equipnent is well understood and in an advanced state of design and testing ${ }^{32}$. However, to determine equipnent requirements, a range of material balance conditions must be provided. For this particular case, me must quantify tritfum input to the reactor hall atmosphere by way of normal process leaks and potential accidents. We also need to specify the acceptable steady-state tritium concentration in the reactor hall atmosphere and the allowable time to attain a specified tritium concentration after accidental release.

\subsubsection{Energy Conversion System}

We have developed a highly efficient energy conversion system for the high temperature blanket. The two-coolant two-temperature scheme using LiPb and helium offers an exciting opportunity for a hybrid heat transport/energy conversion system designed for maximum conversion efficiency. Within the constraints of blanket neutronic performance, energy multiplication $M, t r i t$ ium breeding ratio TBR and structural requirements, it is possible to vary the high temperature fraction FHT. Such flexibility gives us the opportunity to 
optimize the integrated blanket and energy conversion systems.

The baseline system has both a closed-cycle high-temperature hellum-gas turbine and a conventional stream-turbine cycle fed by the LiPb. The system is a hybrid bacause exhaust heat from the the helium turbine is used as a source of feedwater heating in the steam cycle. A gross thermal efficiency of 45.3\% is achfeved with a FHT of 46\%.

The equipment used for the baseline is efther comerctal y avaflable or state of the art and, therefore, does not require extensive R\& programs for implementation. We have chosen a modular concept to mintinize system cost, 1mprove avaflability, and increase system maintainabilfty.

\subsubsection{System Malysis}

As noted above, the two-fluid two-temperature blanket approacn offers the opportunity for a hybrid systen of maximum thermal conversion efficiency. The LiPb has a blanket outlet temperature of $482^{\circ} \mathrm{C}\left(900^{\circ} \mathrm{F}\right)$ with a retur temperature of $372^{\circ} \mathrm{C}\left(700^{\circ} \mathrm{F}\right)$. When these temperatures are conblned with the helium outlet temperature of $900^{\circ} \mathrm{C}\left(1652^{\circ} \mathrm{F}\right)$ and an allowable blanket helfum $\Delta T$ of $450^{\circ} \mathrm{C}$, atal blanket thermal energy of 3250 mit is provided.

A wide variety of hybrid and energy conversion options are possible. In each of the cases studied, LIPb is used to produce steam for a more-or-less conversion steam-turbine cycle. For the high temperature helfum, a number of options are potentially attractive. The major considerations in evaluating each energy conversion system option include gross electric output, net electric output (gross electric minus pumping power), tritium permeation and leakage, avallabilfty of system components, and overall system cost. The hightemperature blanket design allows a range of FHT, which is defined as that percent of total blanket energy avaflable as $900^{\circ} \mathrm{C}$ melfum. As the FHT is varied, the gross cycle effictency and, thus, the gross and net electric energy generated wary. Bacause $M$ and TBR also vary as a function of FHT, some iteration with the blanket design is necessary to optinize electricity generation. Fortunately, for the cycle chosen, system efficiency is falrly flat in the FHT range of interest.

The baseline cycle is deplcted in the form of an abbreviated hat belance (shom in Fig. 4.3-31). The thermal input is 1750 mit in L1Pb and 1500 mit in 

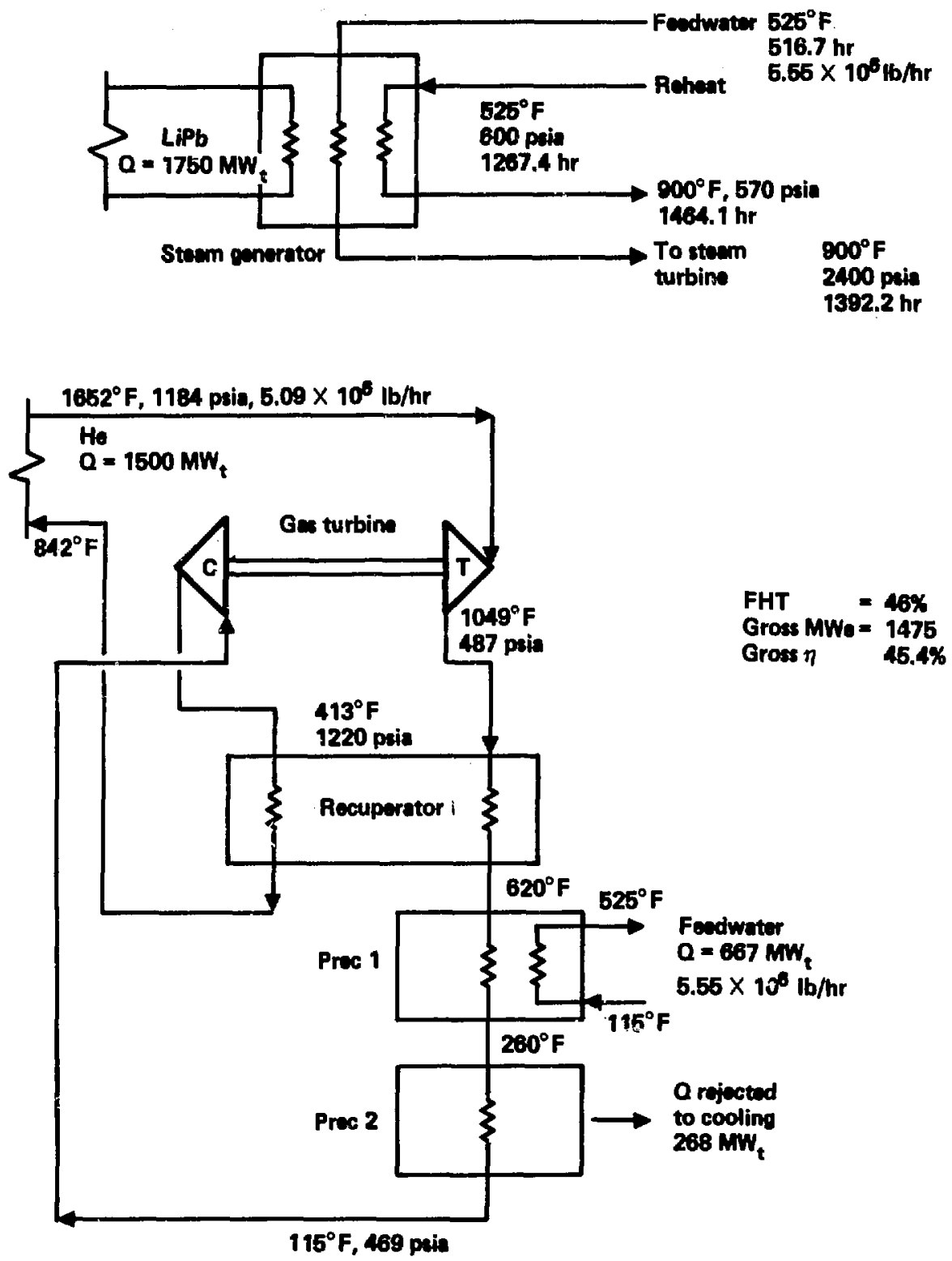

Fig. 4.3-31. MARS heat balance. 
hellum. The $200^{\circ} \mathrm{F} \Delta T$ for LiPb requires a flow of $805 \times 10^{6} \mathrm{lb} / \mathrm{hr}$. This LIPb flow is passed through a number of heat exchangers to transfer the energy to a more-or-less conventional superheater/reheater stean turbine cycle. All LIPb/ $\mathrm{H}_{2} \mathrm{O}$ heat exhangers use the double wall stean generator design developed by $\mathrm{B} \& \mathrm{~W}$ and discussed in Section 4.2.5.3.

Steam conditions are $865^{\circ} \mathrm{F}$ at 2400 psta for the matn stean and $865^{\circ} \mathrm{F}$ at 570 psta for the reheat stean. It should be noted that the stean flow of 5.71 $x 10^{6} \mathrm{lb} / \mathrm{hr}$ is mintalned for both the superheat and reheat cycles. Because helfum turbine exhaust is used for feedwater heating, stean extraction for this purpose is not requitred, thereby increasing the mass flow through the low pressure turbines. The stean turbine generator produces 939 me.

The hellum flow of $5.09 \times 10^{6} \mathrm{lb} / \mathrm{hr}$ passes through a conventional closedloop gas turbine cycle. The gas turbines produce 1115 of shaft power while the helium compressor consumes 551 m. Accounting for some 28 w of gas turbine/compressor losses yields a net gas turbine generation output of 536 we. The precooler normally assoctated with a closed loop gas turbine cycle has been divided in two stages to recover some of the gas turbine exhaust energy. The first stage is, in effect, a feedwater heater for the steam turbine cycle. Approximately 868 mut are transferred to the stean cycle via this precooler/feedwater heater, thus eliminating the need for extraction steam. The remaining $248 w_{t}$ of hellum is rejected to the atmosphere via the second stage and the station cooling towers.

Table 4.3-13 presents a gross energy balance for the heat transport/ energy conversion systen. Gross thermal efficiency is calculated to be $45.4 \%$. Pumping power for the LIPb is 57 Mile, largely attributable to the high MAD pressure-drop through the blanket. Subtracting this pumping power from the gross thermal electric power of 1475 mile yields a net electric output of 1418 Wive for a net thermal efficiency of $43.6 \%$.

The 248 wit of rejected hat from the helium cycle implies that we have room for Improving the overall hybrid cycle efficiency. By decreasing the FHT, the LIPb and steam flows are increased, creating a greater demand for feedwater heating in the nonextraction sted cycle. This greater demand can be satisfled via larger and/or additional helium to feedwater heaters. Preliminary calculations indicate a gain of approximately $1 \%$ in gross thermal 
Tabie 4.3-13. Energy conversion system.

Gross blanket thermal power $\left(m_{t}\right)$

3250

Gross blanket electric power $\left(W_{t}\right)$

1475

Stean turbine

939

Helium turbine

536

Gross thermal effictency (x)

45.4

Lithium-lead punping power $\left(m_{e}\right)$

57

Net thermal output $\left(W_{e}\right)$

1418

Net thermal efficiency (x)

43.6 
effictency if the FHT is lowered to 40\%. The increased LIPb flow will require a slightly greater LiPb pumping power. Therefire, the gain in net thermal efficiency will be somewhat under $1 \%$.

\subsubsection{Component Description}

The heat transport/energy conversion system is shown schematically in Ffg. 4.3-31. There are three main subsystems of this system: the lithium-lead loop, the halium loop, and the feedwater loop.

Lithfum-Lead Loop. To remove the 1750 mit from the central cell in LiPb requires a total flow of $805 \times 10^{6} \mathrm{lb} / \mathrm{hr}$. The high density of LiPb and the length of the central cell (150 m) have anajor influence on component selection. The piping loops for transporting LiPb are a major part of heat transport system costs and, therefore, piping runs must be kept as short as practical. To provide for a reasonable pipe support system under selsmic conditions with the high-density flutd, we restricted the maximum pipe diameter to $1 \mathrm{~m}$ except for short headers and manifolds. With this restriction, 12 primary pipe loops are needed to pass the LIPb flow at an average velocity of $6.4 \mathrm{fps}$. Relatively thin-wall pipe (schedule 30 ) may be used because the system operating pressure is generally low.

We decided to use a modular design for the heat exchanger/steam generator layout because of the number of pipe loops, the length of the central cell, and the desire to minimize the length of pipe runs. Our decision was reinforced by rellability/maintenance considerations, with the need to keep the weight of individual components within reason when full. There are four steam generator/superheater loops, each with one economizer, two evaporators, and a superheater. The reheat cycle has three heat exchangers operating in parallel. All heat exchangers, with the exception of the evaporators were toiling takes place, will be mounted horizontally to factlitate structural support. The five Lipo loops (four superheat and one reheat) each require approximatiely the same flow; therefore, five pumps are baselined. Each pump is driven by a 15000-HP motor.

Hellum Loop. A helium flow of $5.09 \times 10^{6} \mathrm{lb} / \mathrm{hr}$ is neaded to remove the 1500 mit. Again, we chose a modular design because of the length of the central cell and relfabllity/maintenance considerations. In this case, there 
are two identical, helium-gas turbine loops, one at each end of the central cell. A total of six pipe loops, three for each helium turbine, are required to keep helium velocity under 20,000 fps with 30-in. pipe. The high pressure pipe from the blanket modules to the turbine inlet will be refractory-1ined to handle the $900^{\circ} \mathrm{C}\left(1652^{\circ} \mathrm{F}\right)$ helfum.

Each gas turbine will pass one half the helium flow for a combined total shaft power of 1115 m. The shaft-driven heliun compressors, which also satisfy helium pumping requirements, consume 551 w. Each turbine-driven generator then produces 283 we after subtracting turbine and compressor losses.

There are two recuperators per gas turbine, each with a shell and tube design and a surface area of approximately $5000 \mathrm{ft}^{2}$. Precooler 1, which contains the feedwater heaters, is composed of four shell and tube heat exchangers in series for each gas turbine. Heat is rejected by one heat exchanger (Precooler 2) for each gas turbine.

Stean/Feedwater Loop. The remaining major component of the energy conversion system is the steam turbine generator. He have chosen a 3600-rpm unit with a single-flow high-pressure turbine, a double-flow intermediatepressure turbine, and a six-flow low-pressure turbine. Throttle flow is 5.71 $\times 10^{6} \mathrm{lb} / \mathrm{hr}$ and inlet conditions are $865^{\circ} \mathrm{F}$ at slightly under 2400 psia for the high-and intermediate-pressure turbines and $865^{\circ} \mathrm{F}$ at about 570 psia for the low-pressure turbine.

\subsubsection{Double-Walled Steam Generator}

A double-walled steam generator is the baseline for the LiPb loop because it provides for tritium 1solation, inspection and maintenance and can be manufactured in large sizes. The steam generator is discussed in more detall in Section 4.2.5.2.

\subsubsection{Tritium Isolation}

Tritium breeding takes place in both the helfum and LIPb zones of the high temperature blanket. As such, both coolants will contain significant quantities of tritium. The high temperature of the working fluids and, therefore, the high temperature of the metallic containment for these fluids 
facilitates tritium permeation. The major potential tritium leakage paths will be through the high temperature piping, heat exchangers, and helium gas turbines. Permation of the tritium through the steam cycle is adequately. controlled by the double-walled stean generator discussed in Sections 4.2.5.3 and 4.3.5.3.

We have designed at least two lines of defense to guard against tritium leakage. First, the insulation required for all high temperature components to minimize thermal energy losses also provides an adequate tritium barrier. Each high temperature pipe, heat exchanger, turbine, and punp will be sheathed by a metallic skin outside of the thermal insulation. Because these skins will be at room temperature, the tritium barrier nead not be thick, nor is any special coating anticipated. The skin must of course be continuous throughout the heat transport system.

Channels may be carved in the thermal insulation to provide for a purge gas, probably helium doped with oxygen, to collect tritium in the annular space between the pipe or component wall and thin metallic skin. Special bagging provistons may be required around turbine seals, pump shafts, and valve stems.

The second line of defense is to build containment around those areas that could become contaminated. The reactor hall and all heat transport equipment, with the exception of steam turbine generators, must be located within these secondary tritium barriers. All entrances and penetrations must be sealed against tritium permeation and the enclosed areas kept at a negative pressure. The areas may be divided into separate zones, each sealed-off from the other, to minimize the total volume that could potentially be contaninated.

\subsubsection{Corrosion Product Removal}

Corrosion problems in liquid metal loops are usually most severe on the cold side, where corrosion products precipftate and can clog steam generators and valves. This problem can be dealt with in several ways. In the steam generator, heat transfer to the tube walls mill occur faster than mass transfer, causing a super-saturated solution of the corrosion products in LiPb. The addition of particulates will cause most of the corrosion products to 
precipitate out in the flowing strean (rather than on the tube walls), allowing them to be removed by filtering, magnetic separations, and other methods. Alternatively, it may be possible to keep corrosion product concentrations to fairly low levels by removing them on chevrons at the cold side of the loop, The static test data (see Fig. 4.3-15) indicate that it will take about $1000 \mathrm{hr}$ to reach saturation at $500^{\circ} \mathrm{C}$. Because this is a long time compared to a single loop through the system, it may be possible to remove corrosion products in a slip stream, keeping concentrations in the LiPb to acceptable levels. Another method of controlling corrosion is to add a corrosion inhibitor to the LiPb.

Without improved measurements of the corrosion rates it is difficult to choose an appropriate control method or to determine if corrosion control is needed. We are currently, working to quantify and further define some of the concepts mentioned above. At present it appears feasible to use LiPb with HT-9 at $\approx 500^{\circ} \mathrm{C}$.

\subsubsection{Blanket Maintenance}

\subsubsection{Maintenance Philosophy}

The objective of the blanket module maintenance system on MARS is to minimize machine down-time for normal and off-normal events while safely renoving and replacing the blanket modules. Remote, manual, and automated handling methods will be used depending on radiation levels and the task. Because blanket replacenent is a scheduled yearly matintenance operation, we are expending considerable effort to design the modules and module handing systems for rapid removal and replacement. The use of remote techniques increases the time required to perform any task. Although good component design for remote handling can lessen maintenance time, it cannot compare with normal manual servicing. Therefore, it is prudent to maximize the number of operations that can be performed "hands-on." Maintenance time can be reduced by increasing shielding in certain high-maintenance areas, but the cost of additicinal shielding must be compared with the cost of providing for remote maintenance procedures. The availablity of the machine is maximized by moving a small number of large integral units on a fixed-raill or roller system. The number of operations, their complexity, and the cost of equipment is also 
reduced. This technique has been used successfully in shipbuilding prograns to decrease construction time. All central cell components are designed for rapid replacement. Faflure of any component, including a central-cell superconducting magnet, will not result in excessive downtime.

In general, blanket module components weighing several hundred tons or less will be handled with an overhead (gantry) crane. Heavier items will be moved on a track or roller system specifically designed for and incorporated into the MARS facillity. The use of several mobile transporters (see section 4.3.6.3) will eliminate the need to have a transport system built into each of the 24 modules, which produces considerable cost savings and reduced machine complexity. In such a system, access to the modules must be provided. Consequently, services lines, main coolant piping, and bus sys : must approach the machine from one side and from the top of the machine. Jisconnection and reconnection of these connections could be done by hands-on maintenance.

A separate blanket maintenance cell must be provided apart from the machine containment area for module repair work. This cell will be capable of handling sufficient modules simultaneously to allow repair of six blankets within the 1-yr perfod batween changeouts. This area will be sealed from the main reactor containment cell following module replacement to allow replacement work on the blanliet structures to proceed during machine operation. Each of the hot bay areas will be equipped with overhead cranes for handling large components. Manipulator systems will be provided where required for disassembly operations, and spectaltized equipment and fixtures will be included.

Structural components removed from within the modules will be highly radioactive. These structures must be prepared for shipment off-site in shielded containers or for on-site storage. High level waste requiring offsite storage will probably be compacted to permit easier and more economical packaging. It may also be possible to dilute wastes for on-site low-level storage. To accomplish this, a waste preparation area has been included in the maintenance area. This area would be equipped to further disassemble and cut up the activated structures by totally remote means. A packaging and package decontamination area will also be provided. 


\subsubsection{Blanket Module Changeout Procedure}

We have developed a preliminary removal and changeout procedure for the high temperature blanket. At this stage in the study, the purpose of outlining the procedure is to establish an overall approach and identify problem areas. Timelines are lllustrated in Figs. 4.3-32 and 4.3-33.

Module removal will require remote shutdown and manual and remote disconnect operations. Inftial remote shutdown operations include:

- Plasma off.

- Magnet current rampdown.

- Afterheat removal and structure anneal.

- Systen to atmospheric pressure.

- Install temporary shtelding.

The first wall and adjacent structures will be sufficientiy radioactive to produce significant afterheat for the first day after shutdown. Modules not scheduled for replacement will require $60 \mathrm{hr}$ of annealing at $450^{\circ} \mathrm{C}$ (see Section 4.3.1.8) to lower the ductile-to-brittle transition temperature. Modules being replaced will also be annealed to ease handling. Slowly circulating LIPb will be used to remove afterheat and anneal the structure. Coolant lines will be disconnected remotely because of the tritium hiazard. The joint areas may be bagged to reduce tritiun exposure. Temporary shlelding may be required in certain areas to further reduce radiation exposure.

At least two days after shutdown, activation leveis in the outer shield materials will have decayed enough to allow maintenance teams to enter the reactor central cell area. Six work teams will begin preparing the six modules to be removed. Hands-on operations performed during this time are:

- Drain and purge main coolant systems.

- Disconnect structural attachments.

- Position transhaulers.

- Disconnect main coolant ilnes.

- Disconnect magnet and other service lines. 


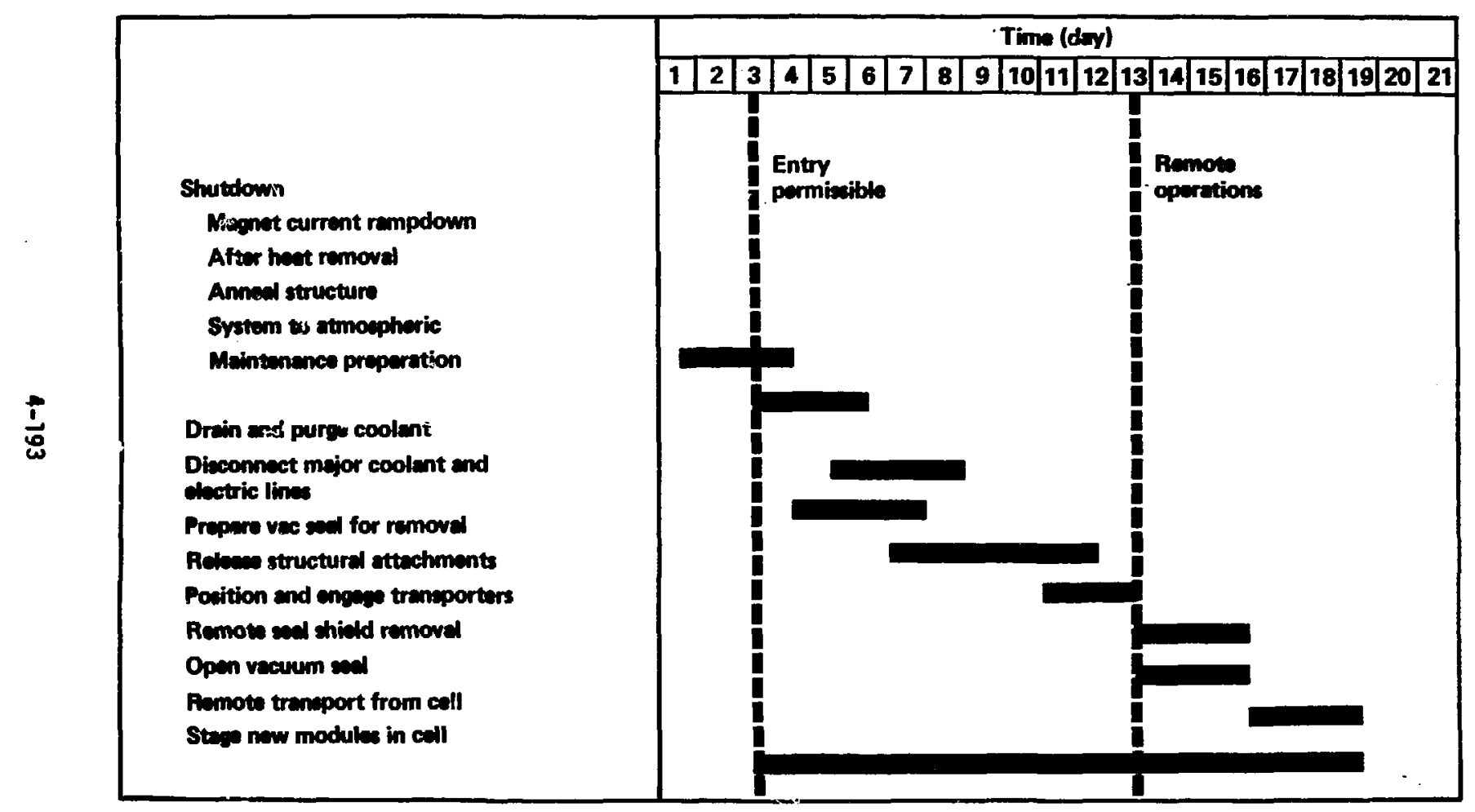

Fig. 4.3-32. Module removal time estimate. 


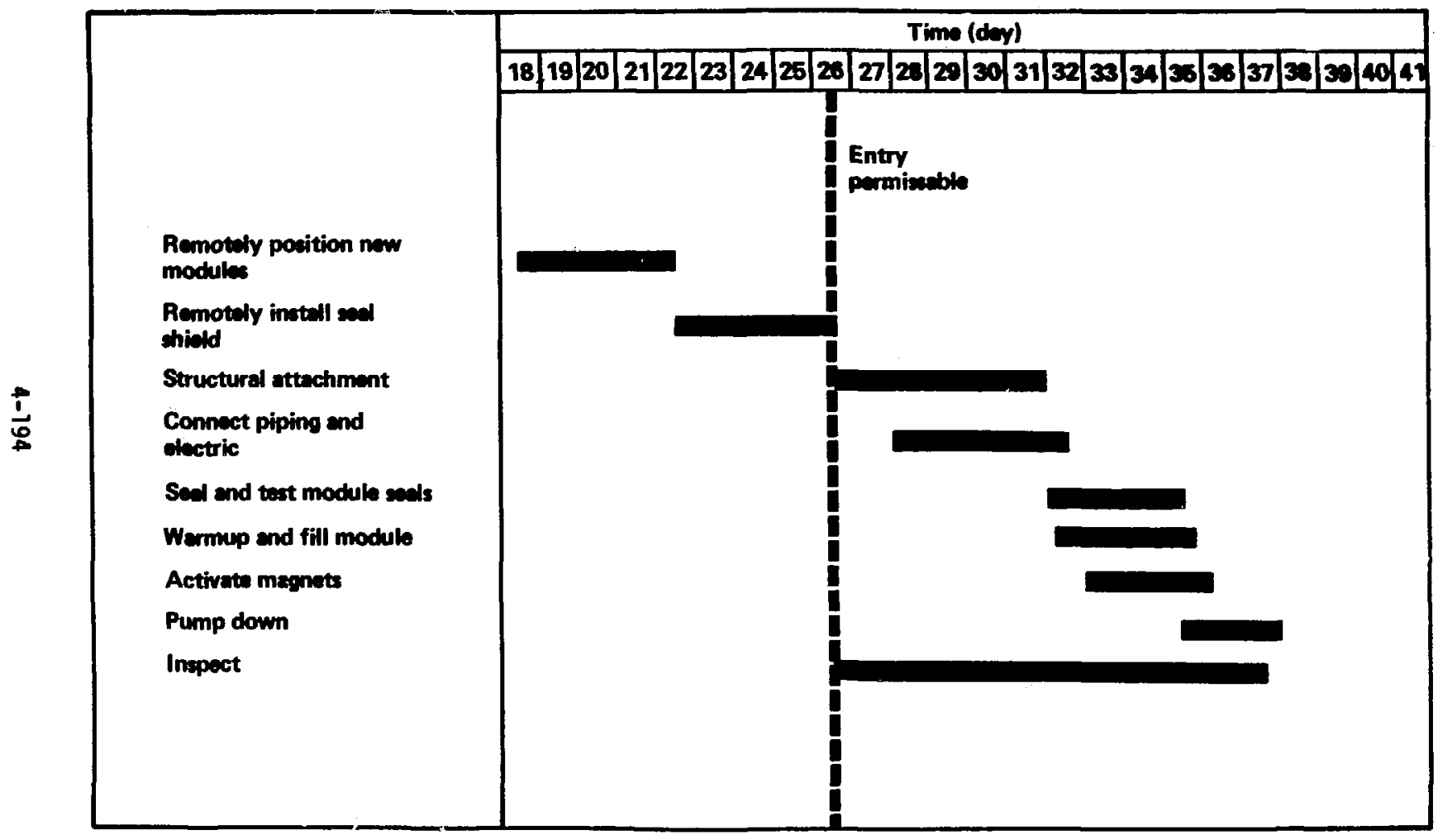

Fig. 4.3-33. Module replacement time estimate. 
The area will then be cleared of personnel so the activated blanket modules can be moved. Remote operations performad before the modules are rolled out include:
- Open vacuum seals.
- Remove seal shields.
- Remove temporary shielding as necesisary.

The modules will then be removed on six transhaulers each and replacement modules w1ll be brought into place. Installation and startup tasks are essentially the reverse of shutdown and removal as follows:

- Rewote operations:

- Install seal shielding;

- Install temporary shtelding.

- Manuar operations:

- Final positioning and clampdown;

- Main coolant lines sealed;

- Connect services;

- Close vacuum seals (remote inside shielding);

- Complete testing of seals and diagnostics.

- Startup :

- Ramp up magnet current;

- Marm up and fill modules;

- Pumpdown;

- Inject plasma.

Future work on central cell maintenance operations will focus on the detalls of the design and procedures, and on interfacing with end-cell and belance-orplant maintenance procedures. For balance-of-plant maintenance, we will need 
systems for removing tritium from the helium and LIPb loops and for removing tritium-contaninated equipwent.

\subsubsection{Equipinent Requirements}

The major equipment in the reactor compartment will be six manipulator/ crame gantries for use on each of the modules to be ramoved. These wlll be sized to for the seai shielding, wich at present is the heaviest object to be handled.

Transporter units for the modules must have approximately 1500 -ton capacity. In addition, the transporters must be remotely controllable. They must also be capable of megotiating a 90-deg turn with the module aboard. This is required to minimize building size. Finally, to precisely align the module in its opening, the transporter must be capable of lateral load movement.

Maintenance hot bays will be equipped with 200-ton bridge cranes to disassemble the components from the modules. Manipulators will be located at appropriate intervals along the bay walls for renote disassembly.

\subsubsection{Transhauler}

The modules will be transported to hot cells for maintenance on the small-rail transhaulers shown in Fig. 4.3-34. These transiaulers, capable of 250 tons of 1ift, are currently used by General Dynamics Electric Bost Division to move submarine components, and in Japan by Kawasaki Heavy Industries to move ship components.

Manufactured by Western Gear at a 1980 cost of $\$ 75 \mathrm{~K}$, the transhaulers are remotely controlled and are capable, along embedded rails, of speeds up to $2 \mathrm{ft} /$ min and a 90 -deg change in direction. Then modified, the speed can increase to $20 \mathrm{ft} / \mathrm{min}$; the orientation of the transhauler to allow the 90-deg change in direction can be accomplished remotely. The six units requiled for each module can be simultaneously remotely operated in all movements. 


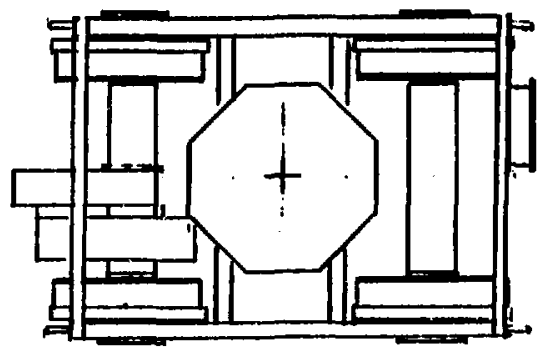

Current decien

MFO - Watarn geer

Cepecity - 260 tons

Diwn - Elcetric $(2 \mathrm{ft} / \mathrm{min})$

Lift - Hydreulic $\left(x_{\mathrm{H}} \mathrm{in} \cdot / \mathrm{min}\right)$

Direction orientution (90-dey rotation-manued)

Cost - \$70K (1900 dollers-60 units)

Modification required

Automete direction orientation

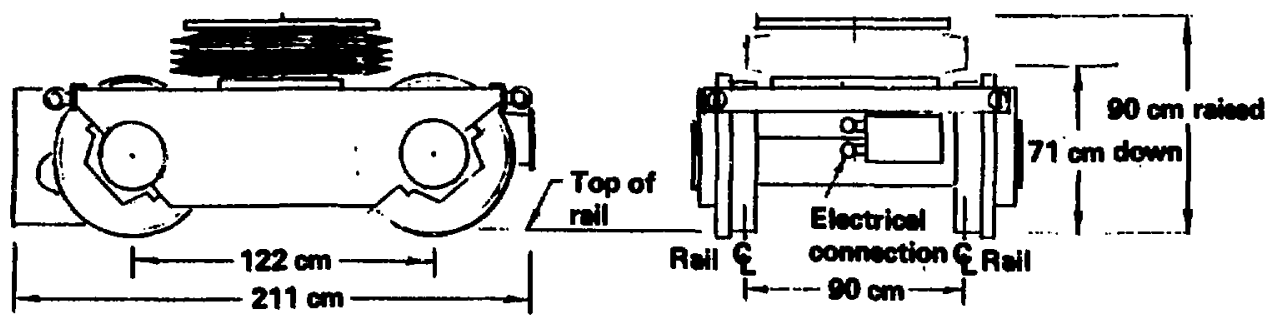

Fig. 4.3-34. Transhauler. 


\subsubsection{Blanket Module Manufacturing}

Minufacturing engineering has been incorporated in the design of the high temperature blanket. This is vital to ensure that the design can be constructed without resorting to high cost or undeveloped techniques. The following components make up the blanket module.

Primary Shield. The shield components can be fabricated with state-ofthe-art manufacturing processes as 11lustrated in F1g. 4.3-35. The inner and outer skins will be plasma arc-cut to near-net dimensions and the oxide surface that is generated by burning will be removed by hand-grinding during fitting for welding. The 2-in.-thick skins will then be rolled to their respective diameters and trimmed to size. Before the inner skin is rolled, the LiPb access ports will be machined into the inboard edge of the skin. The pod attach-rall detalls are machined complete, including weld preparation.

Shield Subassembly. The manacturing procedure for the shield subassembly is also shom in Fig. 4.3-35. The inner skin will be welded together to form a cylinder. On this skin the inner structural rings will be located and welded in place. The outer skin pieces will be attached and welded. The assembly will be complete when the pod ralls have been welded in place. This operation will require a weld fixture to hold the rails in position during the tack and first pass weld. All other operations at this point will use shop and manufacturing alds for support during assembly.

A specialized subcontractor will construct the forged torus ring, which will be delivered complete with edge weld preparation. Two rings that provide roon for welding at assembly will be welded to the inner and outer rins of the forged torus. The torus ring will be ultrasonically inspected before welding, and $x$-ray and dye-penetrant inspection will be performed after welding. A flanged structural ring will be made up of segnented plates and a rolled ring. This welded subassembly forms one of the LiPb walls.

First Nall. Figure 4.3-36 111ustrates the components and assembly of the first wall. The first wall subassembly will contain a bore, LiPb flow diverter and skin. The bore will be rolled and welded to size and then the flow diverter will be resistance-welded to its outer diameter. The flow diverter will be made in segments and the channels will be die-formed. The inner skin will be made up in segnents and resistance-welded to the flow 


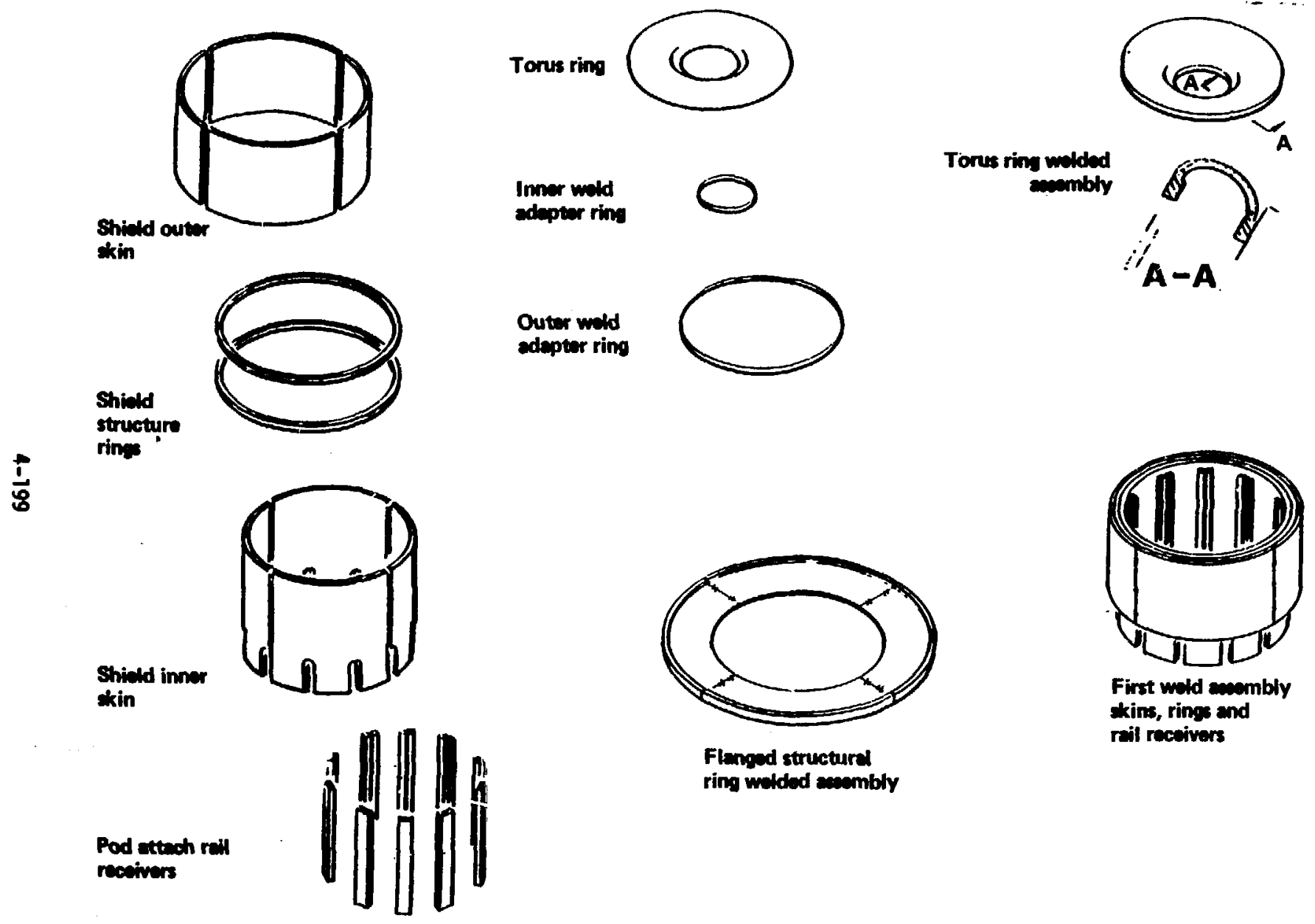

Fig. 4.3-35. Shield support structure fabrication. 


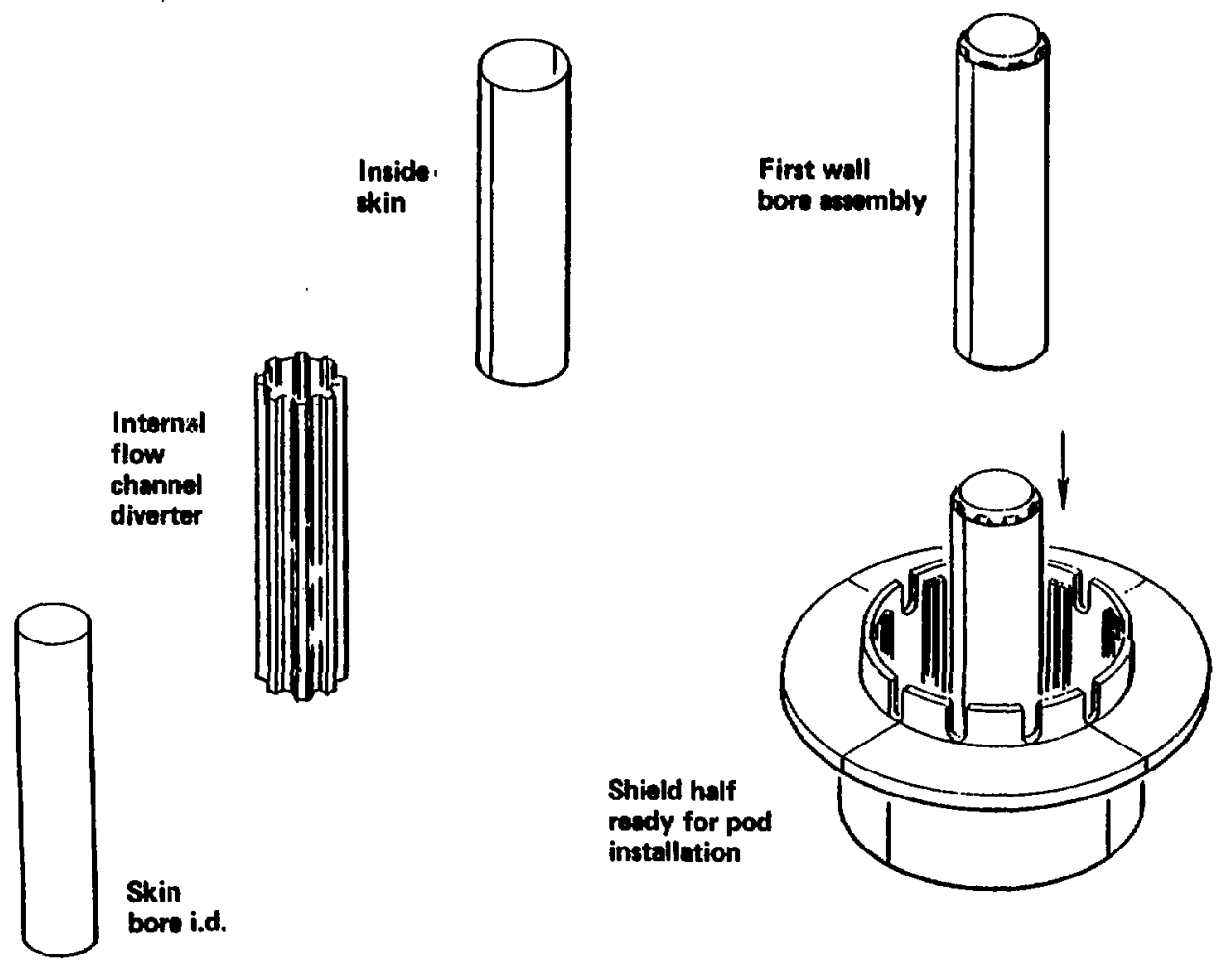

Fig. 4.3-36. First wall assembly. 
diverter and then the longitudinal seams will be welded.

Shield Major Assemblies. Because of weight and stze considerations, an optimum assembly site will be selected before of the subassembltes are further married. Up to this point in the fabrication sequence the components and assemblies will have been bullt "in-plant." The weld assembly for the structural shield ring will recefve the torus ring and flanged ring weld assamblies for welding (see Fig. 4.3-35). One shteld-half wtll then have the first wall assembly positioned and welded in place. This shteld-half will then ready to recetve the pod assemblies (see FIg. 4.3-36).

Siltcon Carbide Components. Cast stlicon carbide is as easy to work with as metal castings. Dimensions are accurate, detafl is prectse, and complex shapes can be accomplished without dffficulty. Generally, cast shapes are used as-produced. The matertal is far too hard to be machined conventionally. Stzes of Indfutdual parts beting produced today may range up to $1.2 \mathrm{~m}$ in length, $1 \mathrm{~m}$ in diameter, and from 0 to $10 \mathrm{~mm}$ in wall thicknesses. Some parts can be made with wall thicknesses of up to $76 \mathrm{~mm}$, depending on the part configuration. Larger parts can be made by temporarfly bonding separate pieces. These bonds must maintain integrity during assembly only and are not required to withstand high temperatures. These paraneters fit the MARS components.

The stlicon carbide pod support panels will be bonded in place on the helfum circulating tubes and this assembly will be bonded to the pod sic skin. After the ends are bonded in place, the pebbles wtll be placed in the cavity. Bonding the top in place wtll complete the silicon carblde assembly (shown in Ftg. 4.3-37).

HT-9 Pod Detalls and Assembly. The pod skin detail will be stretchformed and the end pieces will be die-formed. The upper skin wtil also be die-formed, drilled, and machined to final size. The tubes and collars will be procured components. The pod interface panel wtll be a resistance-welded subassembly. The number of these parts required for MarS would qualify them for rate tooling.

The silicon carbide assembly will have ceramic pedestals and caramic felt bonded to its skin before it is assembled into the HT-9 pod. All the HT-9 detalls will be fftted to the pod and welded in place. After the tubes, 

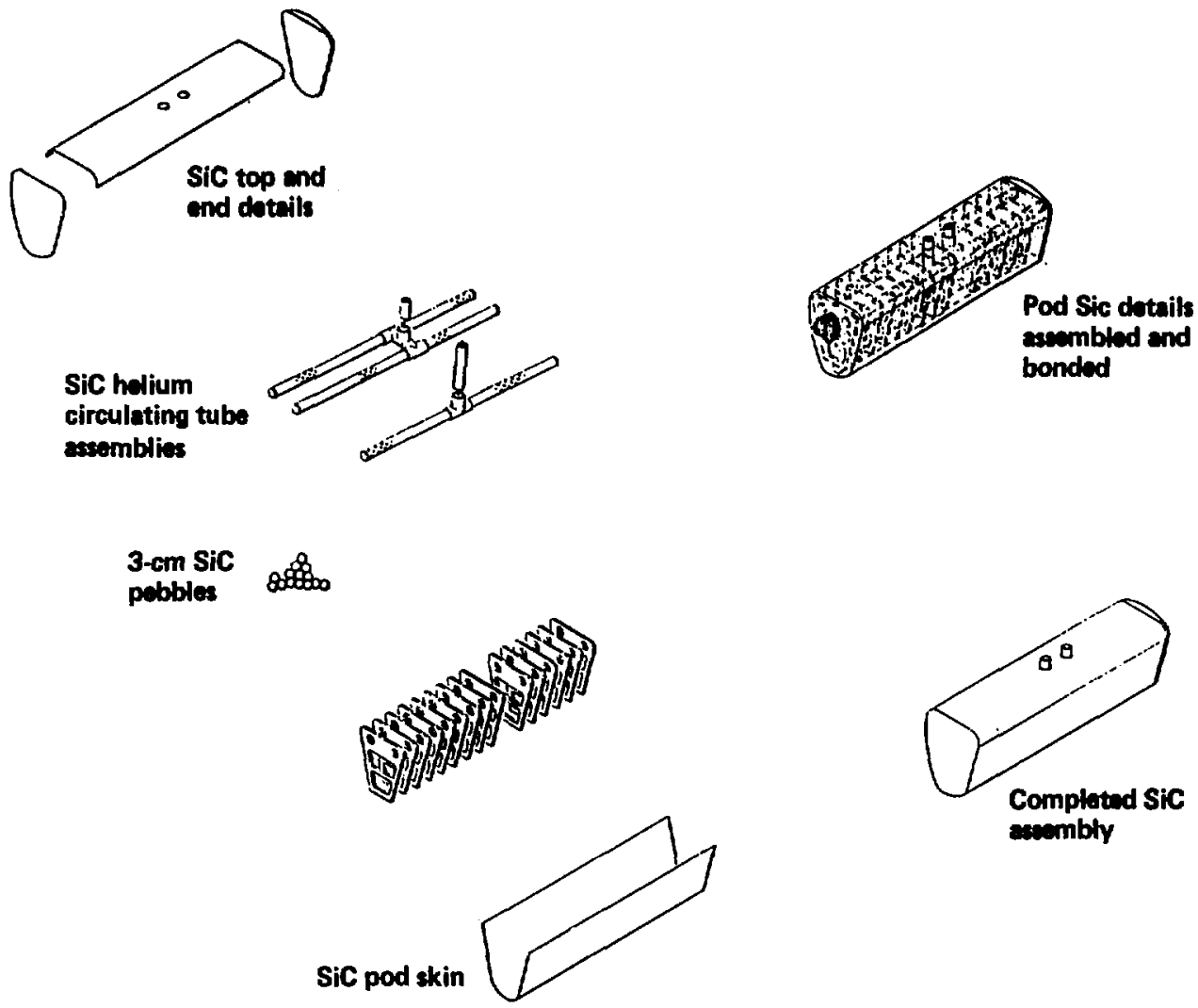

Fig. 4.3-37. Silicon carbide components. 
collars, and doublers are welcied in place, the completed assembly will be heat treated and tested (see Fig. 4.3-38).

Blanket Assembly Major Mating. Each pod assembly will be aligned and lowered in place in the shield rail guides. The pods will be locked in place by inserting LiPb separation plates in the pod rafl key slot. These plates will to be tack-welded in place with each pod. Pod separation panels will be inserted between each pod and tack-welded in place (see Fig. 4.3-39). After all pods are in place, the LiPb separation plates will be welded complete.

The second shield half will be brought to the assembled shteld and lowered in place. To provide for "dom-hand" welding, the mated assemblies will be rotated to horizontal and placed on suftable weld positioner. The two halves will then be welded together at the LiPb separation plate (see Fig. 4.3-40). A close-out weld at the torus ring to first wall is also required.

The Lipt plenum chamber close-out plates, having been previously detalled, rolled, slzed, and drilled, will be ready for installation over the hellum tube roughouts. These plates will be welded to the LIPb sparation plates and the wall of the flanged structure assembly. Weld collars will be placed over the helium tubes and welded to both the close-out plates and the tubes. This closes out the LiPb chamber. See Fig. 4.3-41 for Illustraticii.

The detafls of the helfum manifold consist of tubes, flanged coliar assemblies and sleeves. A box assembly containing the helfum discrnnect is also a part of this assembly. The tubes will be rolled to shape, cut off to size, and ported with oversized holes. The collar assemblies will be made with large skirt doublers attached to a tube that is sized to slip-fit over the helium tubes. The collars will first be slipped over the tubes, wich have excess length in a rough-out conflguratior. The detalled manifolds will be brought to the rough-out tubes and the assembly will be measured to daterwine the required length. The tubes will then be cut to length and weided in place both to the manifold and the tube. After all the ports of the manifolds have been welded, the sleeves will be located over the manifold joints and welded in place. The sleeves are used to provide for manifold alignment tolerances and size mismatch. Leak and pressure tests will be performad when the weiding is completed. Detafls and installation procedures are fllustrated 

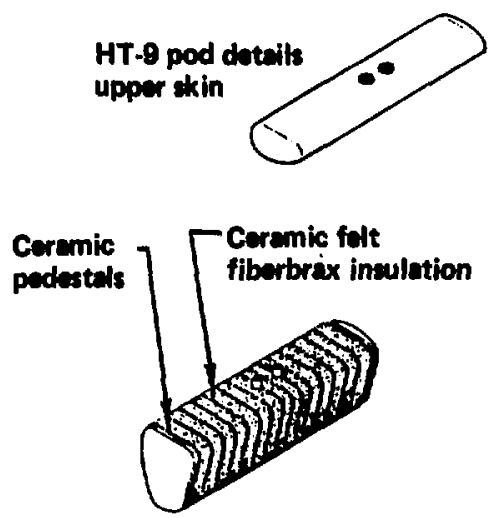

Pod insulation instellation

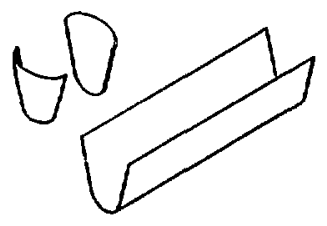

HT.9 pod datails skin and ends

Fig. 4.3-38. Pod assembly.

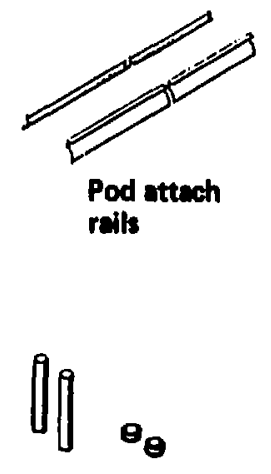

HT-9 tubes and collars

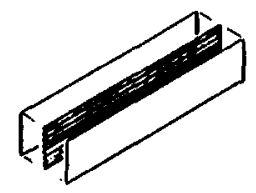

Pod-interfece penel datails

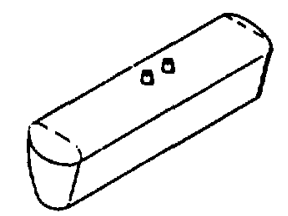

Completed pod nembly (wolded) hant treat

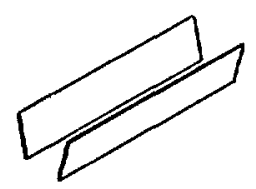

Pod interfece pensils 


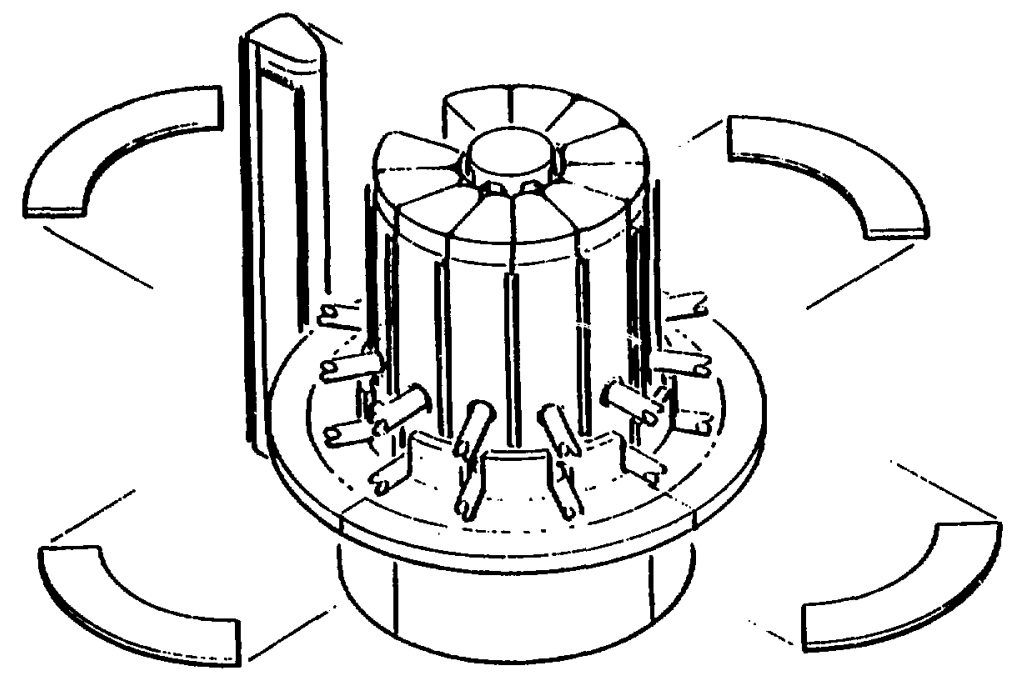

Fig. 4.3-39. Pod installation. 


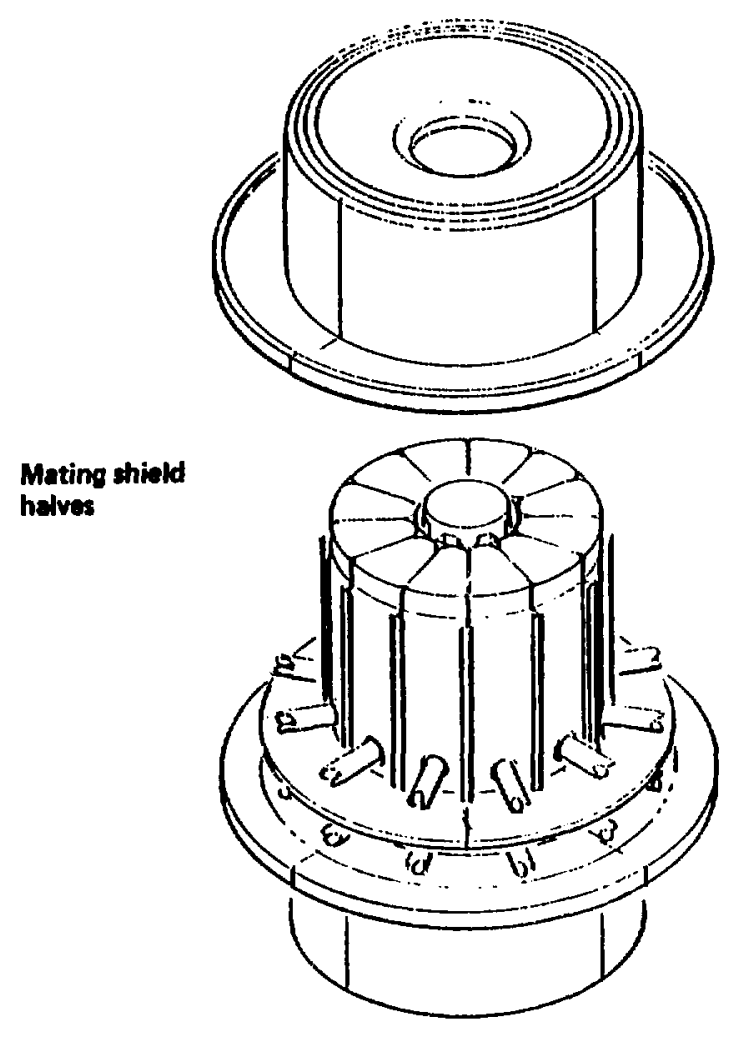

Fig. 4.3-40. Shield half assembly. 
LiPb plenum chember

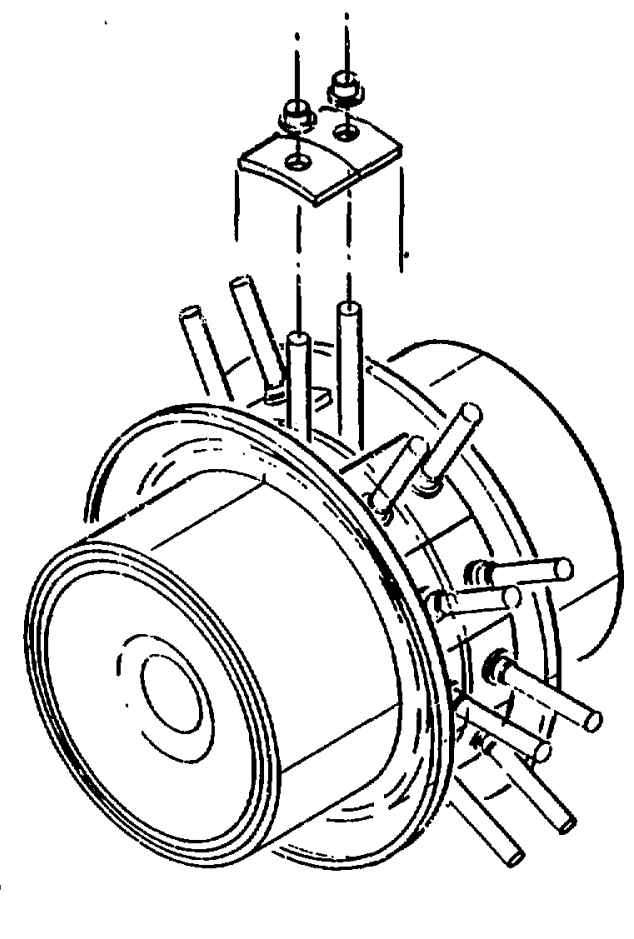

clow-ost plate imstallation

wid and tast

Fig. 4.3-41. Assembly rotated for downhand welding. 
in Fig. 4.3-42. Two sump assemblies will be welded between the helium manifolds for the inlet and outlet of the LiPb.

The blanket and shield will be complete at this point and ready for Installation on the module support structure. 

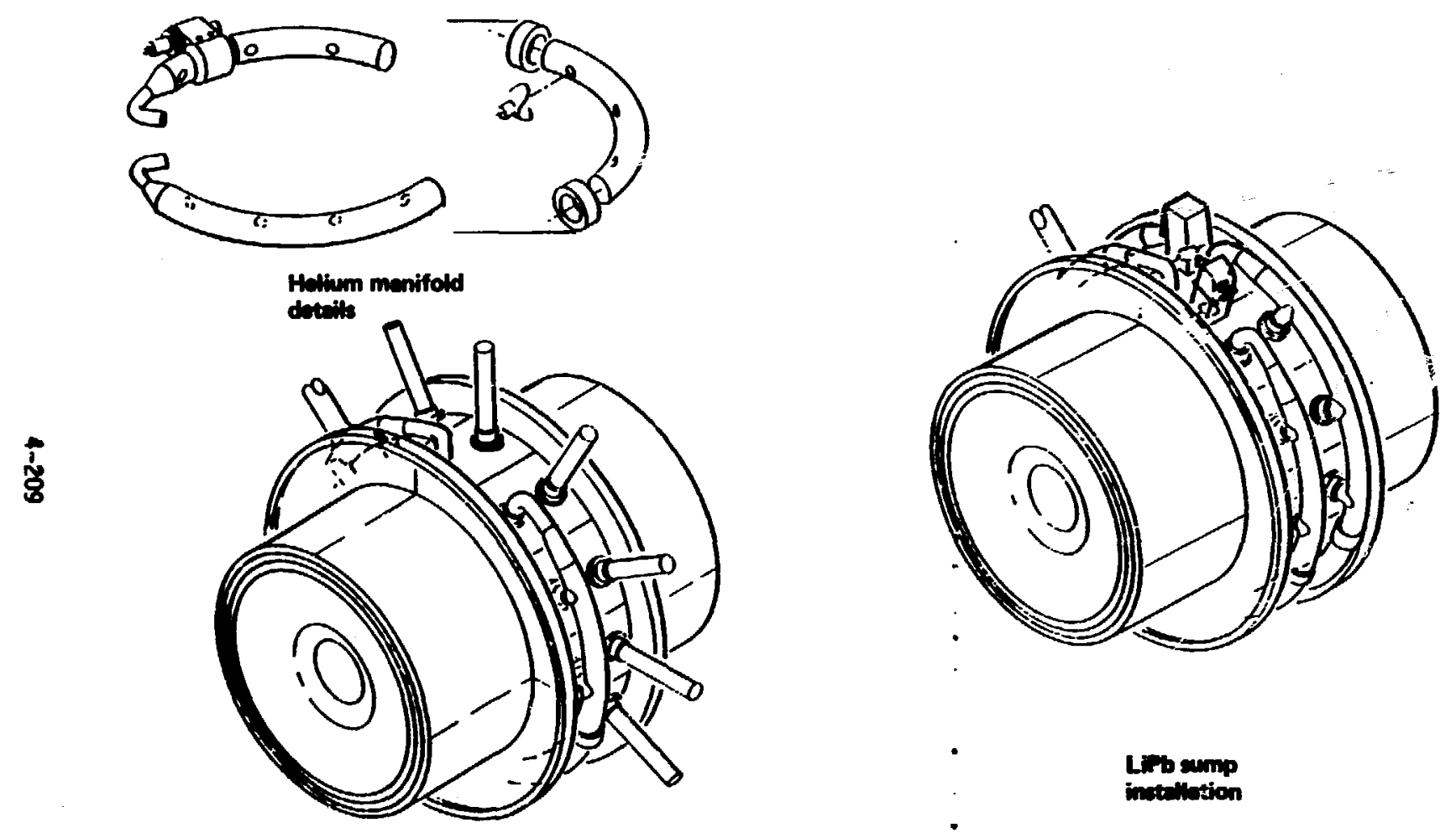

Helium mesifold

inctettetion and ant

Fig. 4.3-42. Helium manifold detailing. 
REFERENCES FOR SECTION 4.3

1. Buckling of Thin-Walled Circular Cylinders, MASA SP-8007 (August 1968).

2. ASHE Boiler and Pressure Vessel Code, Section III, Division 1.

3. H.G. Allen, Analysis and Desion of Structural Sandwich Panels, Pergamon Press, New York.

4. C.O. Bannet and Mrers J.E. Momentum, Heat, and Mass Transfer, Second Edition, McGraw Hill, PP 20B-2II (1\%oz).

5. Hat Transfer and Fluid Flow Data Book, Eeneral Electric Company Corporate Research and Wevelopinont, Schenectady, New York (1980).

6. G.H. Mley, Fustion Energy Conversion, Mmercian Nuclear Society, (1976).

7. F.L. Horn et al., Thermal Conduct ivity Measurements of Insulators for Fuston Blankets, Brookhaven lational taboratory Report BiL-29779, Upton, Now York.

8. M.A. Hoffman, Magnetic Field Effects on the Heat Transfer of Potential Fusion Reactor coolants, Lawrence Livermore kational Laboratory, Livermore, CA, UCRL-73993 (1972).

9. M.A. Hoffman, G.A. Carlson, Calculation Techniques for Estimating the Pressure Losses for Conducting flutd flows in mignetic FIelds, UCRL$51010(1971)$.

10. E.Y. Blum, Effect of Magnetic Field on Heat Transfer in the Turbulent Flow of Conducting Liquid, Institute of Physics and Power, hcadeny of Sciences of the Latv SSR, (Translation) (1967).

11. R.A. Gardner, P.S. Lykondis, Magneto Fluid-Mechanic Pipe Flow in a Transverse Magnetic Field. Parts 1 and $2, "$ Journal of Fluid mechantcs, 47 and 48 (1971).

12. S. Globe, "The Effect of Longitudina? Magnetic Field on Pipe Flow of Mercury," Journal of Heat Transfer, (Novenber 1961).

13. D.S. Kouner, E.Y. Krastl 'nikov, I.6. Panerin, "Experianental Study of the Effect of a Longitudinal Magnetic Field on convective Heat Transfer in a Turbulent Tube Flow of Conducting Liquid, "Magnitnaya. Gidrodinamika, 2, (4) (1966).

14. A.L. Lueffler, A. Mactulattis, M. Huff, MD Round Pipe Flow Experiments. Aerospace Research Laboratories, ARL 67-0236, (1967).

15. B. Badger, et. al., Hitamir-I, A University of Hisconsin Tandem Mirror Reactor Design, UWFOH-400 (1980). 
16. P.F. Tortonelly and J.H. Devan, "Corrosion of Austentic and Ferritic Steals in Static Pb-17 at $\times \mathrm{Li}, "$ in Alloy Development for Irradiation Performance, DOE/ER-0045/8 (Mirch 31, 1982).

17. G.J. Butterworth, Som Inftial Considerations on the Sultability of Ferritic Murtensitic Stainless Steels as First hal and Blanket interials in Fuston Ractors. Euratom/URAEA Fusion Rssociation, CLMR217 (1982).

18. M.M. Ghontem and R.W. Conn, Assessment of Ferritic Steels for SteadyState Fusion Reactors in Proc. of Technical Comittee Mesting and Horkshop on Fusion Reactor Dastgn and Technology, Tokyo, Japan Tuctober 5-16, 1981).

19. J.F. Muncuso, J.A. Spitznagel, R.P. Shogan, and G.M. Jouris, "Irradiation and Annealing of Pressure Vessel Steels," ANS Trans. 38, p325 (1981).

20. M.M. Ghoniem and R.W. Conn "High Temperature Evaluation of Ferritic Steels for Fusion Reactors," Ais Trans. (June 1982).

R.D. Watson,. The Impact- of Inelastic Deformation, Radiation Effects: and Fat fque osmige on Fuston keactor First Wal Lifetime, Ph.D. Thesis, University of Wisconsin (December 1981).

22. D.L. Sinith, et.al., Fusion Reactor Blanket/Shield Design Study, ANL/FPP-79-1 (July 1979).

23. C.S. Caldwell et al., "A Survey of Lithium Compounds for Tritium Breeding Applications", in Proc. of Babcock \& W11cox, 4th Toptcal Meet ing on the Technol ogy of Controlled Muclear fuston (October 1980).

24. C.C. Baker et al., STARF IRE - A Commerctal Tokamak Fusion Power Plant Study, AML/FPP-80-1 (September 1980).

25. A.B. Johnson, Jr., T.J. Kakele, and H.E. Gurwell, BNWL-2097 (August 1976).

26. R.F. O'Mell1, et al., "Convair Thermal Aralyzer Computer Progran No. P45600," 696-0-T-81-737 (June 1980).

27. R.H. Perry and C.H. Chilton, Chemical Engineering Handbook, Fifth Ed, McGraw HIll (1973).

28. B. Lewts and G. von Gelbr, Combustion, Flames, and Explosions in Gases, Acadentc Press, New Tork, P29 (1953).

29. Davison Molecular Steves (4A). Technical Bulletin NS-96-A-272, W.R. Grace and Co., Baltimore, Maryland.

30. E.F. Johnson, "The Use of Molecular Steves for Tritium Control in Fusion Power Plants, In AICHE Sumpostum Sertes on Adsorption and Ion Exchange, 72,(152), p 117-120 (1975). 
31. R.S. Hall, J. Matley, and J. Mcllaughton, "Current Costs of Process Equipment," Chemical Engineering, 89, (7), p 80-116 (April 5, 1982).

32. J. Bartift, Tritfum System Test Assembly (TSTA), Los Alamos Setentific
Laboratory, Los Alamos, 10m iexico (July 1982).

4-212 


\subsection{SOLID BREEDER BLAMKET}

The solfd breeder blanket designed for MRS is helfum-cooled with a water-cooled shield external to the vacuum boundary. The use of a vanadiumbase alloy for breeder-bed components and ferritic steel for the primary structure offers the most practical compromise in meeting operational requirements and achieving a relatively low residual radioactivity.

The central cell module, shown in Fig. 4.4-1, comprises two magnets, a shield assembly, and a blanket assembly. It can be installed and removed from the reactor as a unit except for two small shield assemblies that cover the weld joint between modules. We selected the module size and compositicn based on reasonable weight and fabricable size. The weight of the $6.32-m-1$ ong module is:

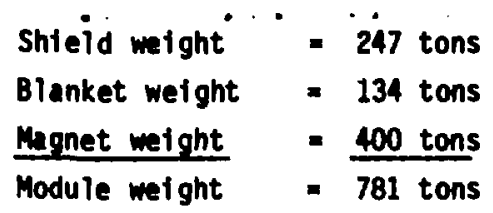

The central cell module geometry is shown in Fig. 4.4-2.

\subsubsection{Blanket Design}

\subsubsection{Design Sumary}

The design principle of the solid breeder blanket is the tube-type heat exchanger. The coolant--heifum--flows over vanadium alloy tubes containing lithium oxide pellets that have been heated by fusion neutrons. This heat exchange produces high-temperature helium that is directly used in a steam generator. The neutrons also react with the lithium to produce tritium in the form of $\mathrm{T}_{2} \mathrm{O}$, which migrates into the helium flow. The tritiun is extracted from the helium loop by processing a slip stream.

Heat generation from neutron deposition in the blanket is largest closest to the piasma and falls off rapidly as the distance from the plasma increases. Therefore, it is necessary to bring the cool helfun directly to the inner wall and direct it radially outwards over the vanadium alloy tubes to the outer wall, as shom in Fig. 4.4-3. The helium ducted to the inner wall is 


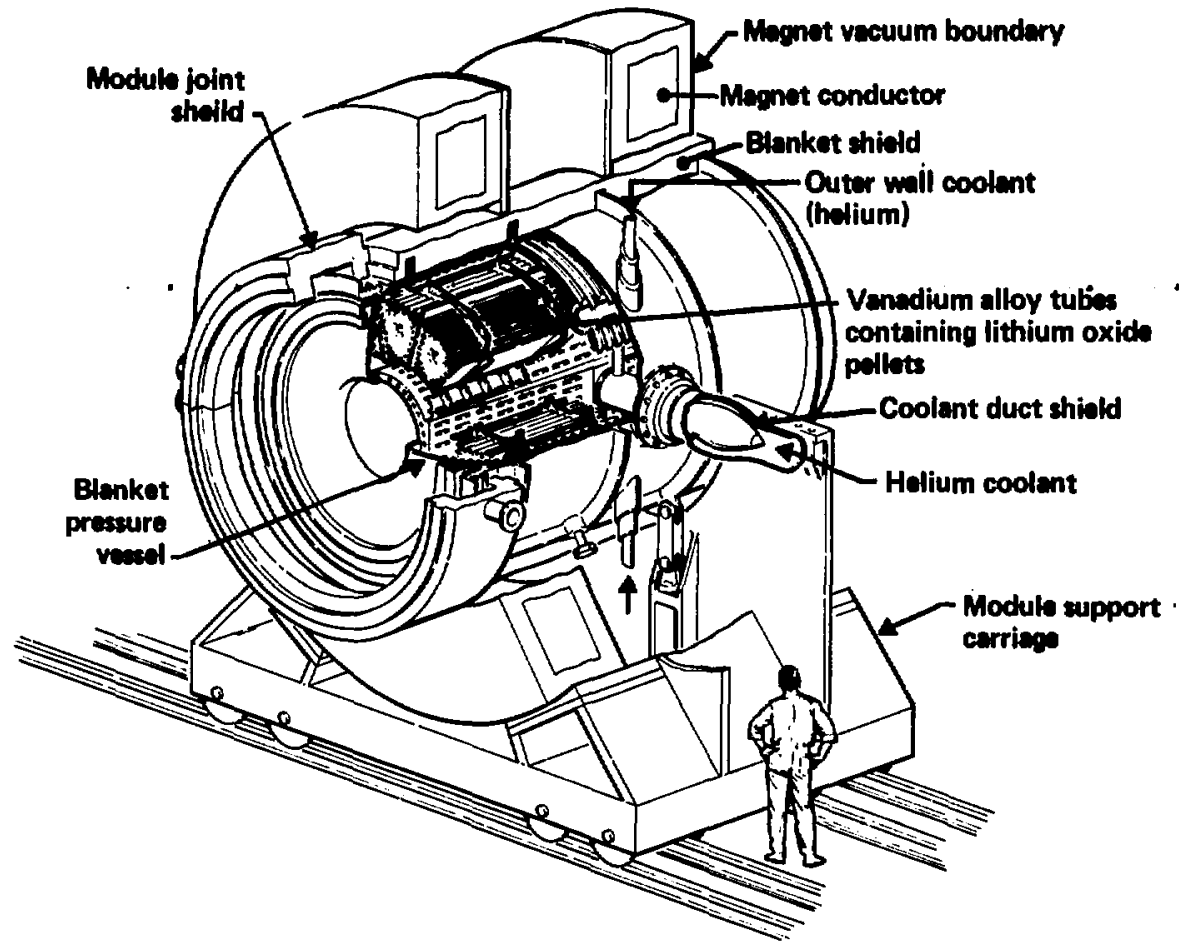

Fig. 4.4-1. Isonetric view of the cantral cell module. 


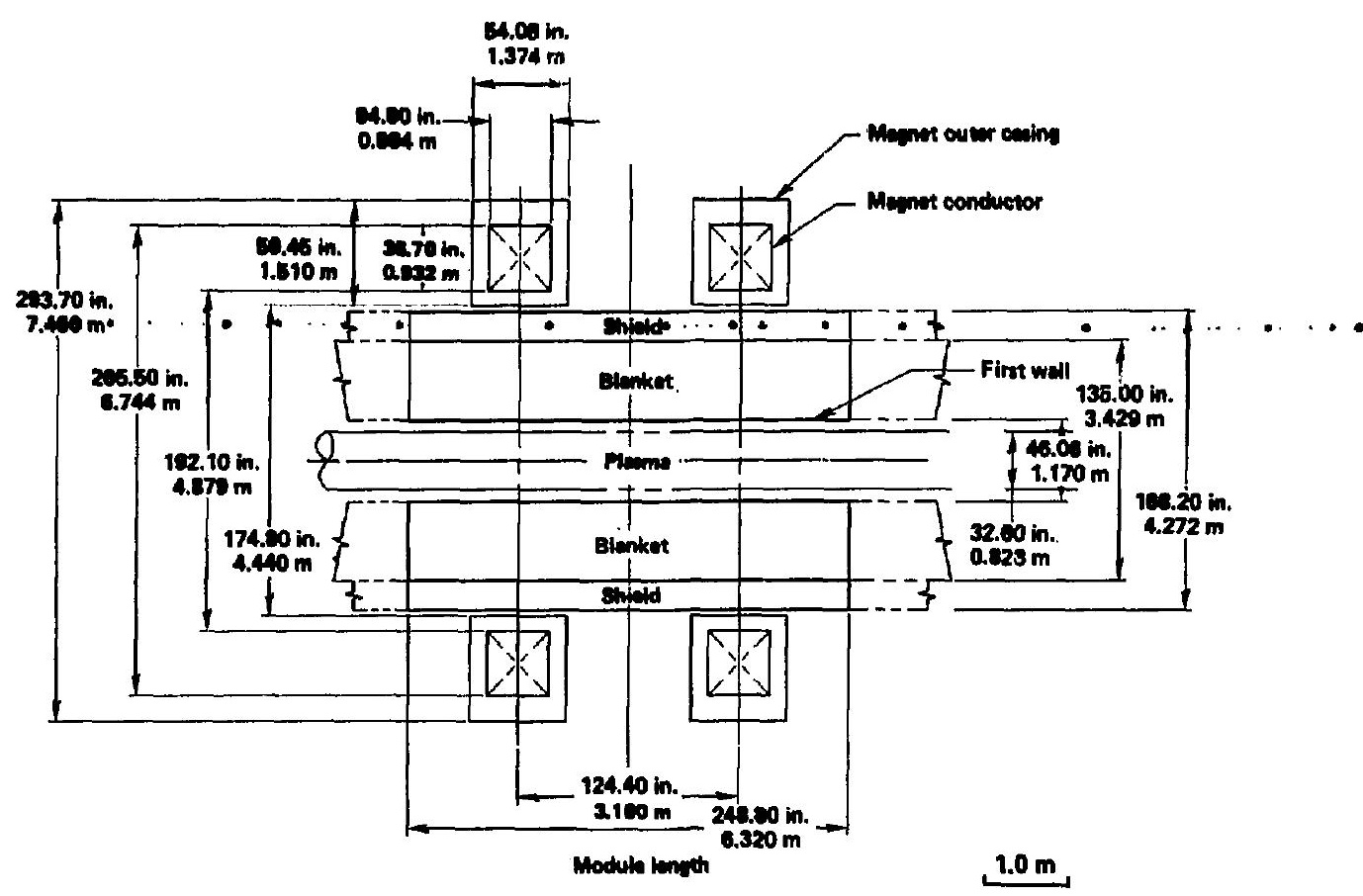

Fig. 4.4-2. MARS central-cell module geometry. 

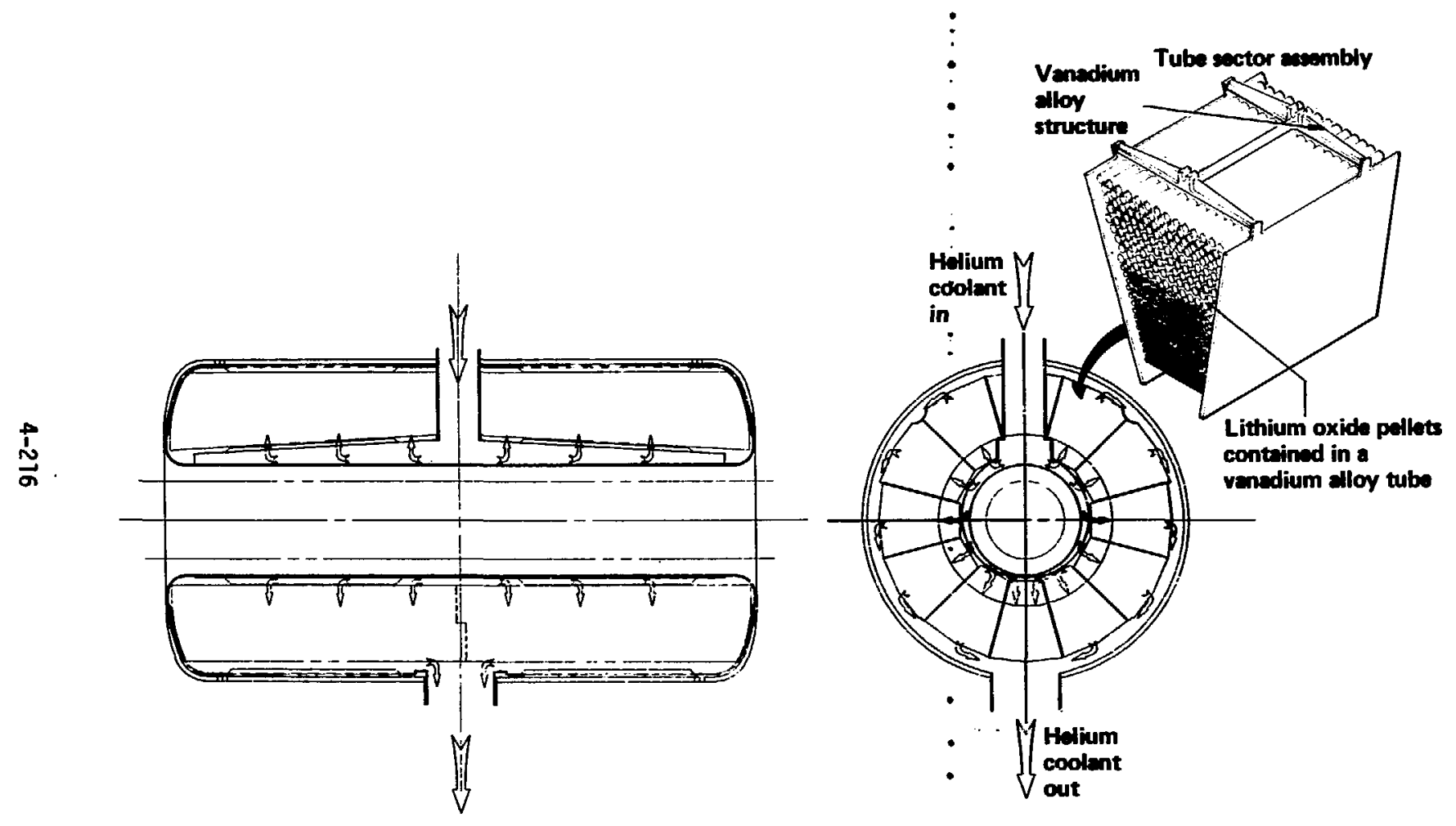

Fig. 4.4-3. Helium coolant flow. 
mantfolded axially to feed a tapered circumferential plenum. The heitum passes through radially outward-facing holes toward the tubes. The Inner wall has circumferential vanes machined into it to control its temperatuire. The outer wall is protected from the outward-flowing hot gas by insulsition and a separate helfum coolant loop (see Section BB of Fig. 4.4-4). The radially flowing helfum is directed circumferentially to flow parallel to the outer wall and exits the blanket via the exhaust duct. A full description of the thermal/hydraulfes is given in Section 4.4.1.4.

\subsubsection{Structural Dasion}

The blanket is contained by an elongated, doughnut-shaped pressure vessel. The internal hellum pressure is 30 atm. F1gure 4.4-4 shows general cross sections of the blanket.

We Have choseh '19thilum 'oxide $\left(\mathrm{L}_{2} \mathrm{O}\right)$ as the breeder' mitertal because it gives an acceptable TBR and energy multiplication $\mathrm{M}_{\text {. The }} \mathrm{LI}_{2} \mathrm{O}$ pellets are fabricated under pressure at an elevated temperature. The pellets will, in all probabllity, tend to break up and will have to be contained. We considered two possible containment materials;

- Silicon carbide (slip cast tubes),

- Vanadium alloy tubes.

The SiC tubes required a wall thickness three times that of the vanadium alloy because of fabrication Iintations. This lowered the $\mathrm{LI}_{2} \mathrm{O}$ packing density, resulting in a marginal TBR. Also, the brittle SIC tubes were difficult to handle during fabrication of the blanket. Although the vanadium alloy would embrittle during reactor operations, its manufactured condition is not brittle; therefore, normal fabrication, assembly, and handling procedures can be used. Because the vanadium alloy offers these advantages over SiC, we decided to use it for the tubes that will contain $\mathrm{LI}_{2} \mathrm{O}$ pellets.

The vanadium alloy tubes vary in size. The smallest diameter tubes are closest to the plasm with the size increasing radially away from the plasma. The variation in diameter is necessary to 11 mit the maximum $\mathrm{Lf}_{2} \mathrm{O}$ temperature to preclude sintering. The tube layout is show in Fig. 4.4-5.

The tubes are supported in loose-fitting holes in two vanadium alloy tube sheets, as shown in FIg, 4.4-6, and are restrained axially at one of the tube 


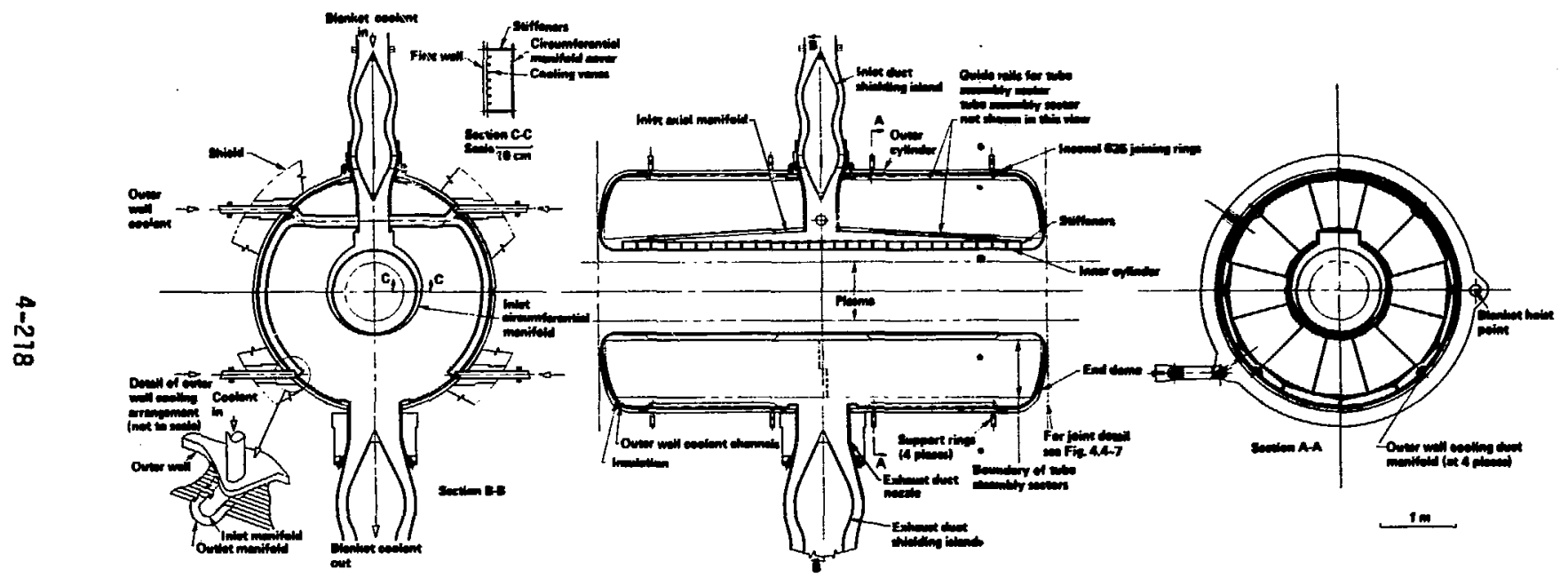

Fig. 4.4-4. Blanket design. 


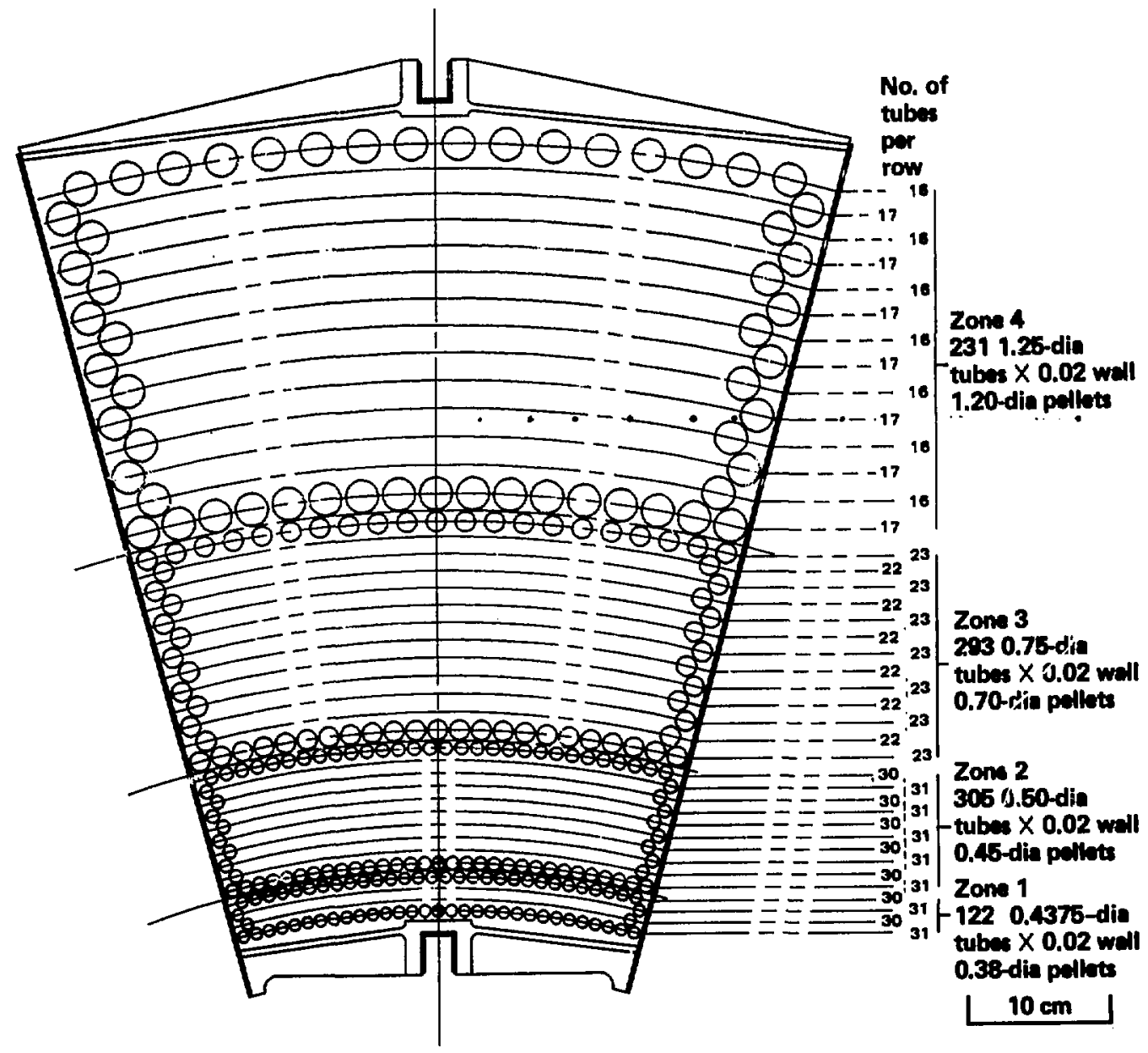

Fig. 4.4-5. Blanket tube geometry. 


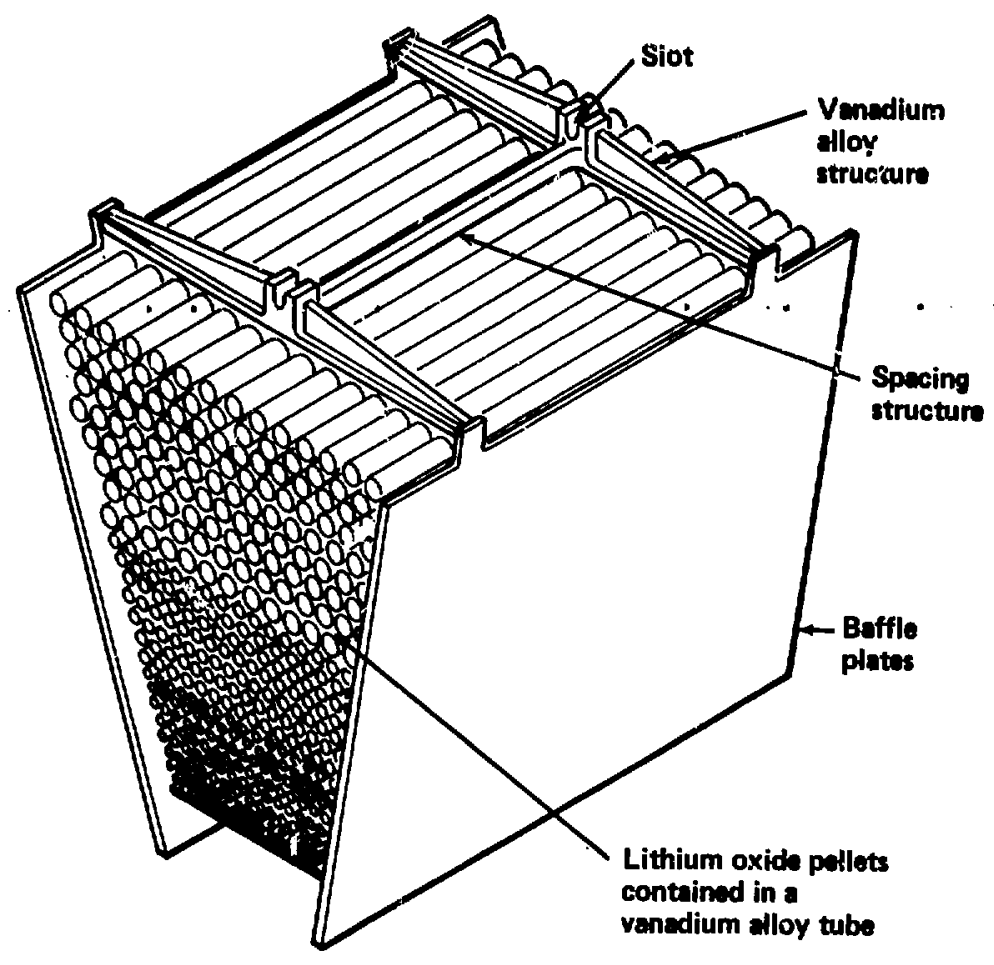

Fig. 4.4-6. Tube sector assembiy. 
sheets. The trapezoldal-shaped tube sheets are connected by a vanasiun alioy spacing structure. At each end of the spacing structure, the tulse sheets contain slots that straddle ralls attached to the outer wall. Vanadium alloy baffle plates are attached to the tube sheets to maintain tice radial direction of the coolant flow. Tinere are 12 tube assembly sectors arranged circumferentially around the plasma. One sector is show in Fig. 4.4-6. The weight of the tube sector assemblies is supported via ralls to support rings attached to the exterior of the outer wall. The blanket is supported at the inner support rings and longltudinal members are attachad to the outer wall from the support points to the adjacent support rings. The design of the blanket supports allows for thermal growth between the support points. One support is rigidly fixed and will react loads in any direction; a second support will react vertical loads and horizontal loads in one direction; the remaining two supports will react vertical loads only.

To allow for thermal and radiation sweliing and creep, the length of the blanket allows for a gap between adfacent modules at initial installation. This gap is almost taken up when the blanket is at its operational temperature. To provide a yacuum wall for plasma containment, a vacuum-tight joint is required, which can make allowance for the relative motion between adjacent modules (see Fig. 4.4-7). A double bellows design is required to contain the helfum coolant. The welded vacuum joint has a configuration that permits it to be welded and cut using remotely operated tools and equipment.

Vanadium alloy $\mathrm{V}-15 \mathrm{Cr}-5 \mathrm{TI}$ was chosen $\mathrm{rat}$ the tubes to contain the $\mathrm{Li}_{2} \mathrm{O}$ pellets and for the structure. It will be necessary to use protective surface coatings to overcome the alloy's susceptibility to surface reactions with tritiun and gaseous impurities in the nelium coolant (e.g. hydrogen, oxygen, nitrogen, and carbon compounds) and with the $\mathrm{LH}_{2} \mathrm{O}$. Because of the long-time high-temperature service conditions, some of these gases will diffuse through the protective surface causing the vanadium alloy substrate to become brictle. Fortunately, this enbrittlement will only be significant at temperatures below the operational range of the blanket. Stress levels are minimal and we have accounten for the relative motions due to differences in thermal expansion of adjacent structures.

The material selecied for the blanket prissure vessel and support stricture is a ferritic steel having the physical, structural, and radiation danage 


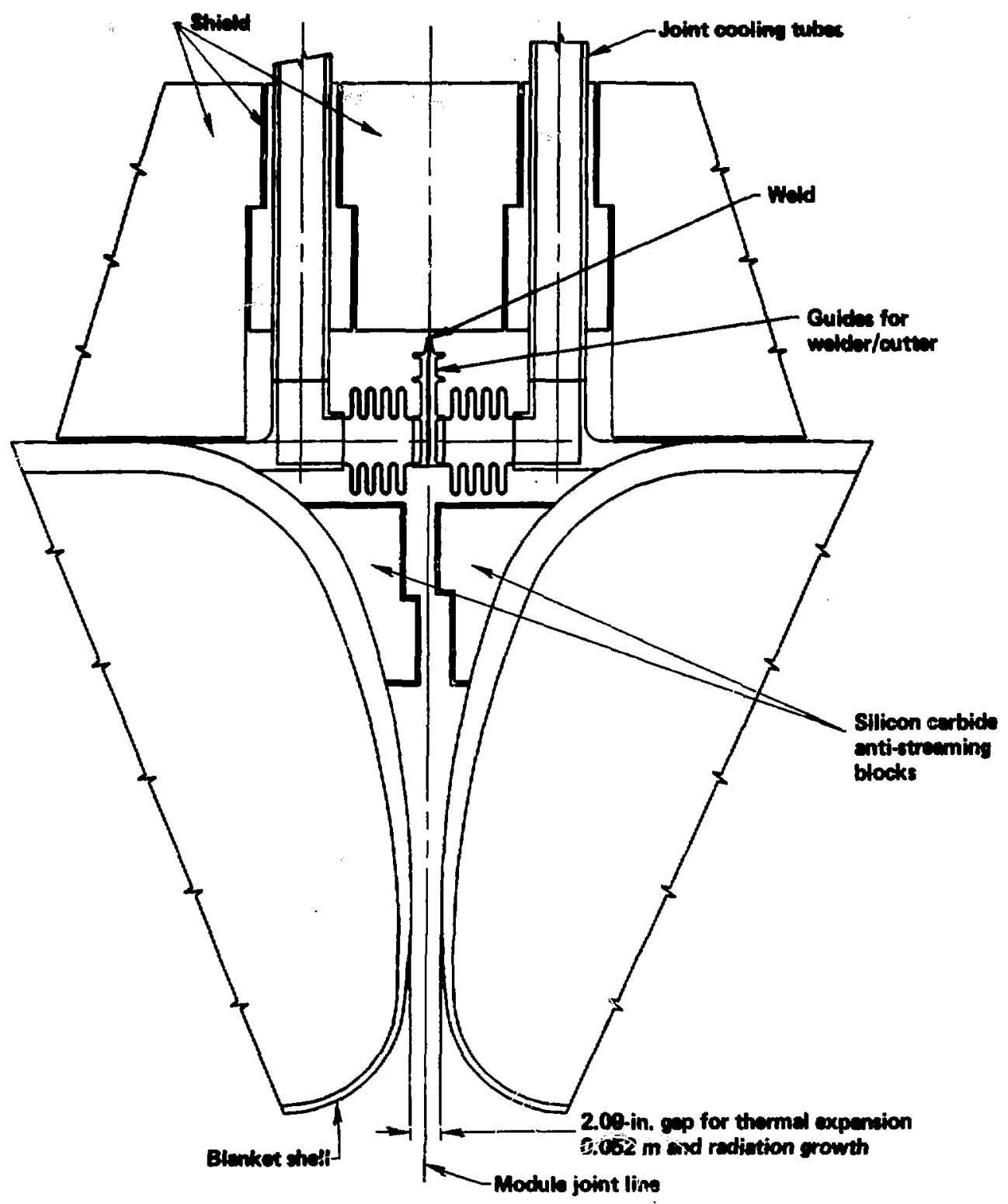

Fig. 4.4-7. Blanket module joint. 
resistance properties of SANDYIK HT-9. The design of the vessel alleviates some of the welding problens of MT-9. However, we expect that development work will lead to improvements in HT-9 fabricability. The service temperature of the pressure vessel was 1 inited to $450^{\circ} \mathrm{C}$ by thermal creep. We have met this temperature liatiation by the using direct cooling and/or insulation. applied the following structural design criteria in the design of the blanket:

The allowbie stress $S_{m}$ (time-independent) is the lowest velue of

$\frac{S_{u}}{3}$ at room temperature,

$\frac{S_{U}}{3}$ at maximum service temperature,

$\frac{2 S_{y}}{3}$ at room temperature, and

$0.9 S_{y}$ at maximum service temperature,

where

$S_{u}=$ ultimate strength of the material and

$S_{y}=$ yield strength of the material.

The allowable stress $S_{t}$ (time-dependent) is the lowest value of

$\frac{2}{3}$ the maximum stress to cause creep rupture in time $t$,

80x of the maximum stress to cause tertiary creep in time $t$, and the mintimen stress to produce 18 strain in time $t$.

The primary allowable mimbrane stress $S_{\text {th }}$ is the lesser vilue of $S_{m}$ and $S_{t}$. For normal operating conditions, the allowable stress limits are

$P_{m}<S_{m t}$ and

$P_{1}+P_{b}<1.5 S_{m}$ or $R_{t} S_{t}$. 
where $k_{t}=1+k_{s}\left(1-\frac{P_{i}}{S_{t}}\right)$,

$K_{3}=0.25$ for shelf-type structures,

$P_{m}=$ gmeral primary mubrane stress,

$P_{1}=$ local primary mambrane stress, and

$P_{b}=$ primary bending stress.

For shell buckling, the static factor of safety shall be not less than 5 .

We designed the solid breeder blanket on the basis of known loads caused by pressure, temperature and dead weight. We have not quancitatively analyzed other design conditions caused by selsmic and magnetic loads.

\subsubsection{Meutronics}

For the solid breeder blanket, the main choices for breeder materials are $\mathrm{Li}_{2} \mathrm{O}$ and $\mathrm{Li}_{7} \mathrm{~Pb}_{2}$. Helium cooling also acts as the purging widiun for the bred tritium. At present, the preferred breeding material is $\mathrm{Li}_{2} \mathrm{O}$, because more established database indicates a favorable upper temperature 11 init of $700^{\circ} \mathrm{C}$. The attractive feature of $\mathrm{LI}_{7} \mathrm{~Pb}_{2}$ is its neutronic properties, with lead providing neutron multiplication; however, the material properties of this alloy are not sufficiently known.

All neutronics analyses used the one-dimensional discrute ordinates code ANISN. They were performed with a 100-neutron/21-ganm group structure from the EPR cross section library (based on ENDF B-IV). The analyses used cylindrical geometry and $A P_{3} S_{8}$ approximation. A distributed isotropic source of 14.1-MeV neutrons was confined to a radius of $43 \mathrm{~cm}$ with the first wall at a $60-\mathrm{cm}$ radius.

To help develop a blanket design with good tritium breeding and energy multiplication properties, we explored the sensitivity of neutronics performance to several design variables. These included blanket thickness, Li-6 enrichment (including variation in enrichment between blanket regions), SIC or graphite reflector, and the introduction of baryllium as a neutron multiplier. The analyses includad the effects of the blanket structure and a shield. One general result was that the active blanket region containing the breeding compound and the reflector or neutron multiplier. would have to be at least $50 \mathrm{~cm}$ thick (including 20 vol $x$ He) to avoid significant energy leakage into the shield (recoverable only as low-grade heat). 
For $\mathrm{LI}_{7} \mathrm{~Pb}_{2}$ blankets, we Investigated the effects of varying the L1-6 fraction from natural to $90 \%$ and the blanket thickness from 30 to $70 \mathrm{~cm}$. We found that for each thickness there is an optimum enrichment that shifts from $45 \%$ at $30 \mathrm{~cm}$ (TER $=1.24$ ) to $35 \%$ at $50 \mathrm{~cm}$ (TBR $=1.44$ ) to $25 \%$ at $70 \mathrm{~cm}$ (TBR = 1.57). Also, the TBR tends to become less sensitive to enrichment as the blanket thickness increases. The total energy deposition in the blanket was found to increase very 11 ttle (from 16.21 to $16.47 \mathrm{mvV} /$ neutron at $15 \%$ enrichment) as the blanket thickness was varted from 30 , to $70 \mathrm{~cm}$, and it showed even less sensitivity to variations in enrichment at a given thickness. These trends, summarized in FIg. 4.4-8, appear to be spectfic to $\mathrm{LI}_{7} \mathrm{~Pb}_{2}$ and are caused by the neutron multiplication properties of lead.

For Li, $\mathrm{L}_{2} \mathrm{O}$ blenkets, we explored the effects of zone enrichment, Inclusion of a STC or graphite reflector, and the use of a beryllium multiplier. As a baseline, considered a blanket with a 50-cm-thick breeding zone containing 80 vol \& $\mathrm{Lt}_{2} \mathrm{O}$ with natural lithium. He obtalned a TBR of 1.32 and an $M$ of 1.18 with 0.64 Mey deposition in the shield. An increase in the $\mathrm{LI}_{2} \mathrm{O}$ region thickness to $60 \mathrm{~cm}$ combined with grading of the LI-6 fraction produced no significant change in the energy deposition but lowered the TBR to 1.24. Replacement of the rear $15 \mathrm{~cm}$ of $\mathrm{Li}_{2} \mathrm{O}$ in this configuration with SIC did not affect these results.

He also examined the partial replacement of $\mathrm{LI}_{2} \mathrm{O}$ with SiC for different moderation characteristics or be for neutron multiplication. The SIC replacement substantially lowered TBR, and slightly reduced $M$, as shown in Fig. 4.4-9(a). In contrast, the partial replacement of $B$ for $L_{1}{ }_{2}$ showed that the $T B R$ as a function of $\mathrm{LI}_{2} \mathrm{O}$ replaceiment rises from 1.26 for 10 vol $\%$ replacement to 2.0 at 60 vol $\%$ replacement. Over the same substitution range, the blanket energy deposition increased fram $16.4 \mathrm{MeV}$ to $21.4 \mathrm{meV} /$ neutron [see Fig. 4.4-9(b)]. We also explored the effect of varying the lithium enrichment (from 10 to 60X) at a given $\mathrm{LI}_{2} \mathrm{O}$ replacement level $(50 \mathrm{vol} \mathrm{x})$. The energy deposition mas unaffected; the TER declined slightly, from 1.91 (10X Li-6) to 1.82 (60X Li-6). In spite of these improvements in performance, a beryllium multiplier uns not carried forward because of comunity concerns over berylliun resource limitations. All of the above analyses include the effects of systcantic cross section errors that overpredict the ${ }^{\prime} L f\left(n, n^{\prime}\right) T$ and $D(n, 2 n)$ reactions. 


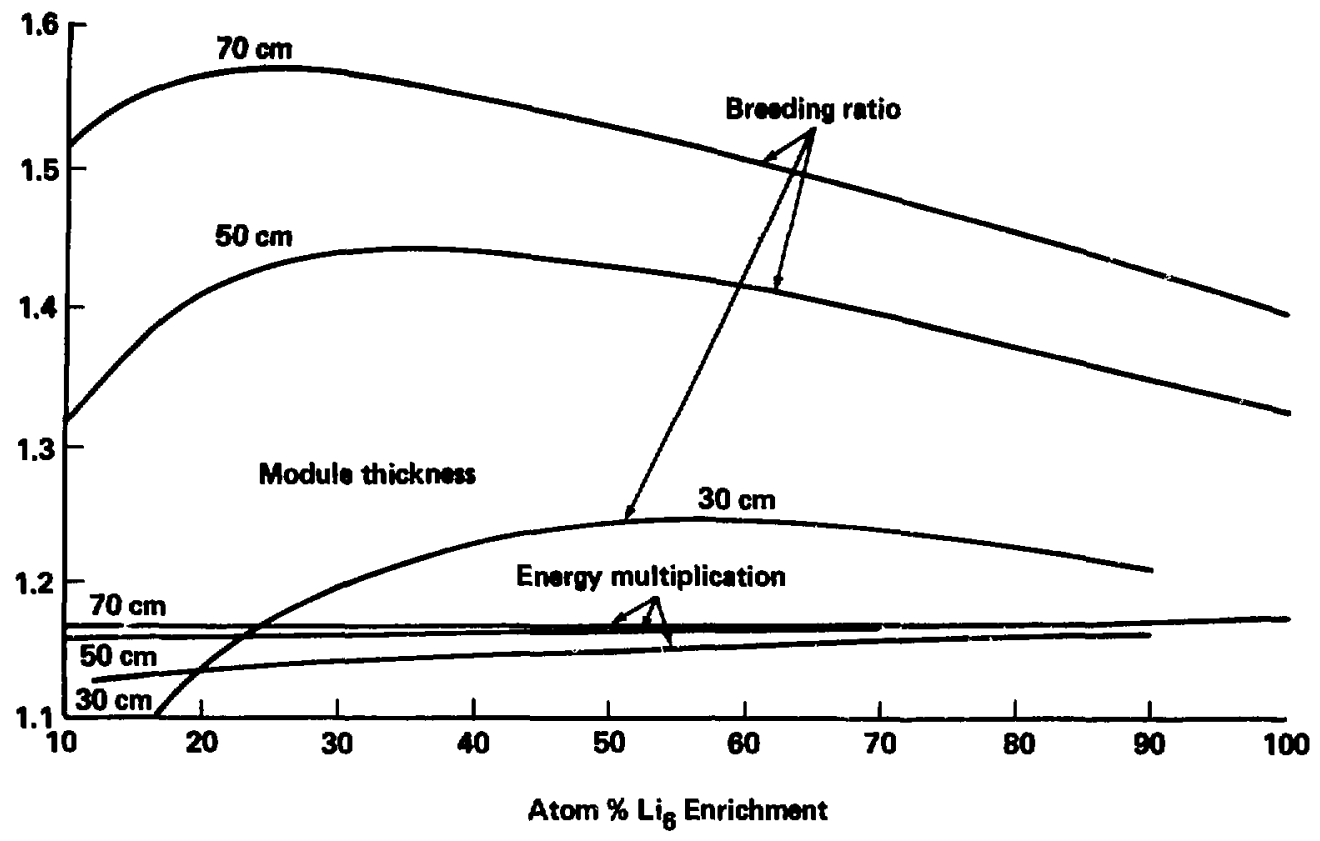

Fig. 4.4-8. Tritium breeding and energy multiplication in Lipp $b_{2}$. 
(a) Silteon centuide

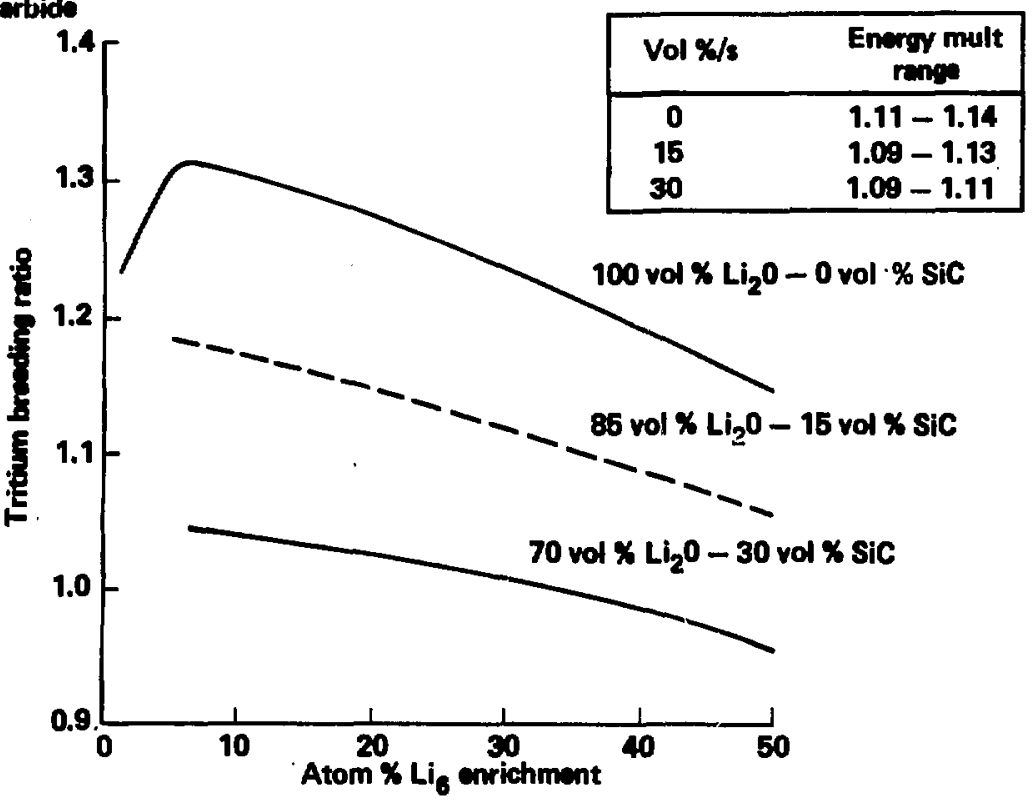

(b) Beryllium

Be vol 8 replacing $\mathrm{Li}_{2} \mathrm{O}\left(50 \% \mathrm{Li}_{6}\right)$

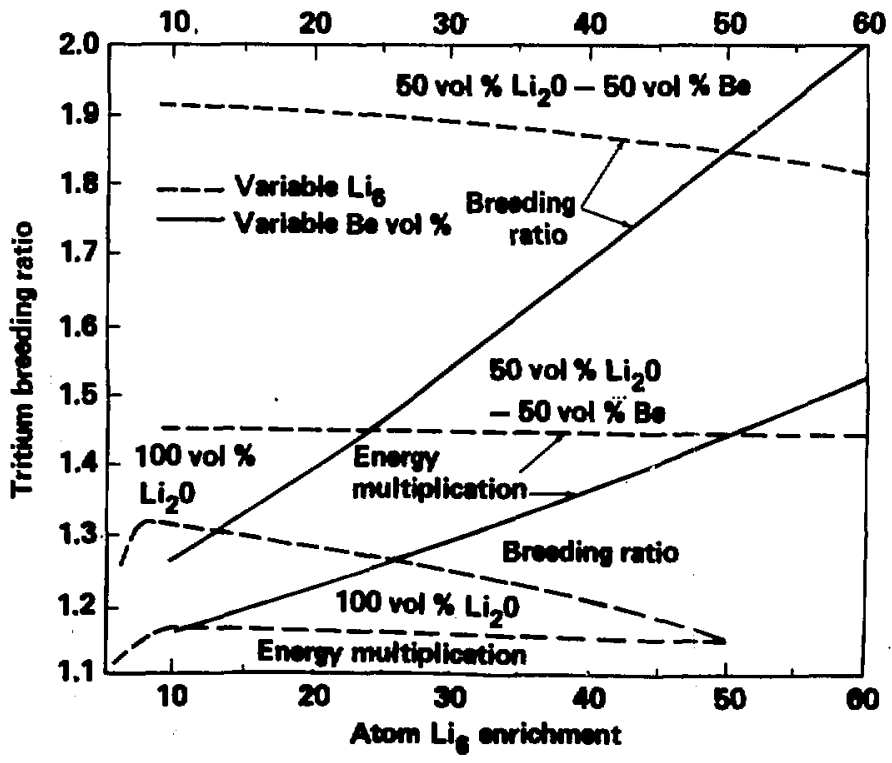

Fig. 4.4-9. Effect of silicon carbide and beryllium on $\mathrm{Li}_{2} \mathrm{O}$ performance. 
Based on these results, we configured a realistic blanket design using $\mathrm{LI}_{2} \mathrm{O}$ with He cooling. The breeding/power conversion region consists of three zones of stacked, thin-walled vanadium alloy tubes filled with $\mathrm{Li}_{2} \mathrm{O}$ pellets (natural lithium), oriented parallel to the plasm axis. The tube dianeter and wall thickness are reduced toward the front to provide for more effective cooling in the region of higher power deposition. The blanket shell, including the first wall, is a stiffened HT-9 structure, also cooled by He. The one-dimensional neutronics representation includes a $60-\mathrm{cm}$ shield and is shown in F1g. 4.4-10. Breeding performance was analyzed with both the EPR library and the LLM. EHOL library, which incorporates a downward correction of the L1-7 $\left(n, n^{\prime}\right) T$ cross section. As shown in Fig. 4.4-10, total energy deposition of $15.64 \mathrm{MeV}$ (excluding the shield) is predicted, together with a TBR of 1.309 based on the EPR library. Use of the ENDL library results in a 27 reduction in the TBR. For this configuration, we evaluated the effect of replacing the vanadium alloy with $\mathrm{SiC}$ and of replacing the $\mathrm{Li}_{2} \mathrm{O}$ with $\mathrm{Li}_{7} \mathrm{~Pb}_{2}$, using either type of tube.

After additional refinenents in the thermal design of the blanket, we partitioned the blanket into four zones of vanadium alloy tubes that contain the $\mathrm{Li}_{2} \mathrm{O}$ (as shown in Fig. 4.4-11). The neutronics results show $M=1.1$ (excluding the shield) and $T B R=1.25$. The inclusion of a steel (Fe-1422) reflector region in the shield did not enhance $M$. Replacing the last breeding zone by 95 vol \% Fe-1422 had the effect of increasing $M$ to 1.15 while lowering TBR to 1.09.

In an attempt to improve $M_{5}$, replaced $\mathrm{Li}_{2} \mathrm{O}$ in the third and fourth breeding zones with $70 \%$ enriched $\mathrm{Li}_{7} \mathrm{~Pb}_{2}$. This produced $M=1.10$ and $T B R=1.25$. Finally, we ex amined the effect of replacing all $\mathrm{LI}_{2} \mathrm{O}$ with $\mathrm{Li}_{7} \mathrm{~Pb}_{2}$, using natural $\mathrm{LI}-6$ for the first two zones and $70 \%$ enrichment for the last two zones. This arrangement also does not produce an improvement in $M$ (1.11), but offers TBR of 1.37 .

In sumary it appears that $\mathrm{LI}_{2} \mathrm{O}$ blankets cannot achieve $M$ greater than 1.1 to 1.15 without a neutron multiplier. The $\mathrm{Li}_{7} \mathrm{~Pb}_{2}$ breeders may provide higher $M_{;}$however, further study and material characterization is required. 


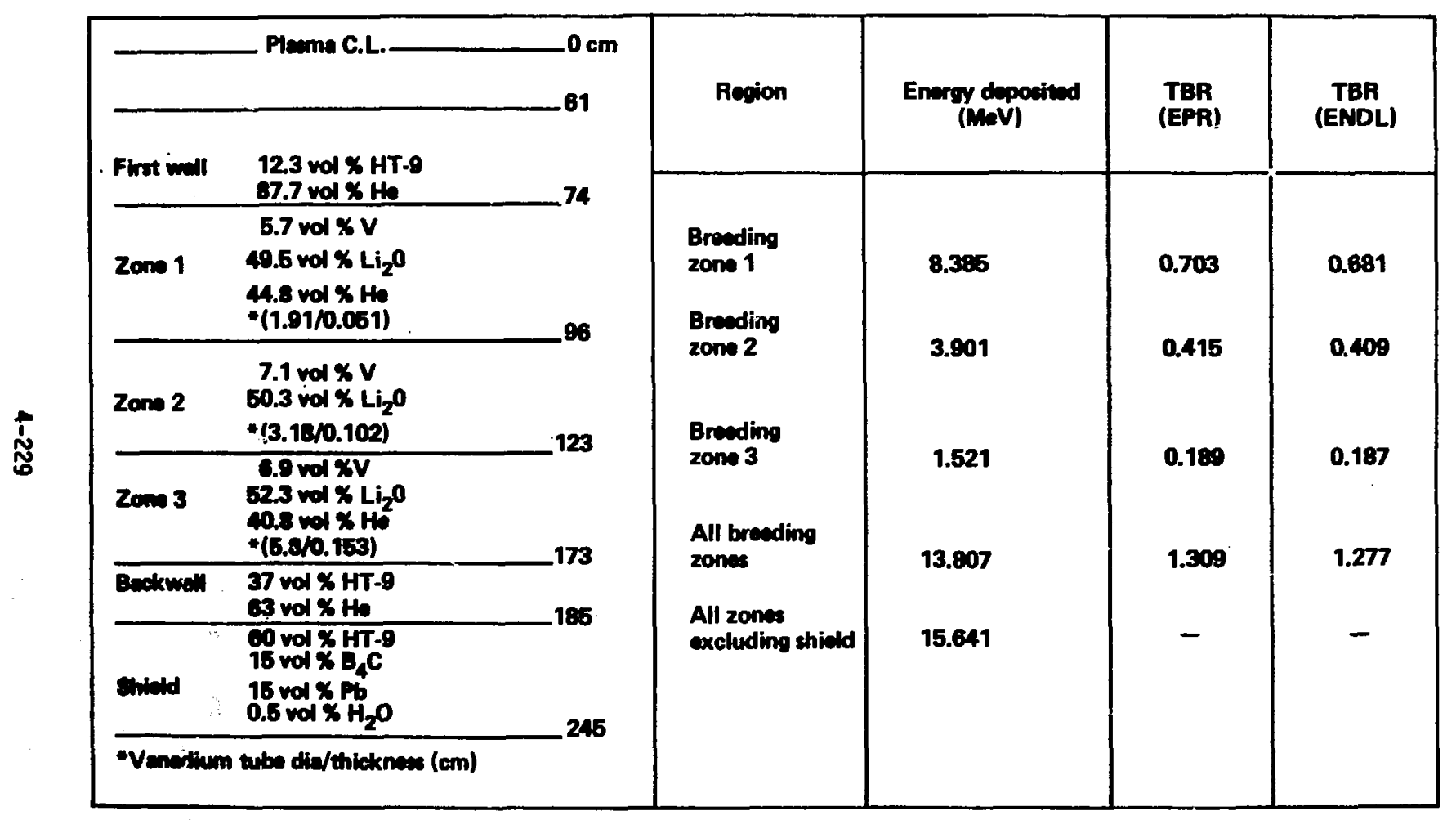

Fig. 4.4-10. $L_{2} 0$ blanket performance with three breeding zones. 


\begin{tabular}{|c|c|c|c|c|c|c|}
\hline \multirow{4}{*}{$\begin{array}{l}0 \mathrm{~cm} \\
40 \\
58.6 \\
60.146 \\
77.40\end{array}$} & \multirow{2}{*}{\multicolumn{2}{|c|}{ Source }} & \multirow{3}{*}{ Zone } & & & \\
\hline & & & & \multirow{2}{*}{$\begin{array}{l}\text { Energy } \\
\text { deposition } \\
(\text { MoV/n) }\end{array}$} & \multirow[t]{2}{*}{$\mathbf{M}$} & \multirow[t]{2}{*}{ TBR } \\
\hline & Void & \multirow{3}{*}{$\begin{array}{l}21 \\
22 \\
23\end{array}$} & & & & \\
\hline & V-alloy 3.545 vol $\%$ & & 21 & 1.808 & 0.128 & \multirow{7}{*}{$\begin{array}{l}0.1874 \\
0.3522 \\
0.4921 \\
0.2150\end{array}$} \\
\hline & $\begin{array}{l}\text { HT.9 } \\
\text { V.elloy }\end{array}$ & & 22 & 0.459 & 0.033 & \\
\hline 82.30 & $\mathrm{Li}_{2} \mathrm{O}$ & \multirow{6}{*}{$\begin{array}{l}24 \\
25 \\
26 \\
27\end{array}$} & $\mathbf{z 4}$ & 4.268 & 0.3027 & \\
\hline & $\begin{array}{l}\mathrm{HT}-9 \\
\mathrm{~V}-\mathrm{elloy} \\
\mathrm{Li}_{2} \mathrm{O}\end{array}$ & & 25 & 4.637 & 0.3269 & \\
\hline 95.20 & $\begin{array}{l}\text { HT-9 } \\
\text { V-elloy }\end{array}$ & & 27 & 0.001 & 0.0000 & \\
\hline \multirow{2}{*}{178.20} & $\mathrm{Li}_{2} \mathrm{O}$ & & $\mathbf{2 8}$ & 0.007 & 0.0005 & \\
\hline & $\begin{array}{l}\text { HT-9 } \\
\text { V-alloy } \\
\mathrm{Li}_{2} \mathrm{O}\end{array}$ & & $\mathbf{2 9}$ & 0.056 & 0.0040 & \\
\hline $\begin{array}{l}156.80- \\
158.236\end{array}$ & $2 \mathrm{rO}_{2}$ & & Subtotal & 15.504 & 1.0997 & \\
\hline \multirow{3}{*}{$\begin{array}{r}165.01 \\
171.60\end{array}$} & V-alloy & 28 & $\mathbf{Z 1 0}$ & 0.075 & 0.0063 & \\
\hline & HT.9 & $\mathbf{2 9}$ & 211 & 0.002 & 0.0001 & \\
\hline & 201.60 Reflector & 210 & Total & 15.581 & $1.105 !$ & 1.2467 \\
\hline \multicolumn{2}{|c|}{231.0 Shield } & 211 & & & & \\
\hline \multicolumn{2}{|c|}{255.0 Void } & & & & & \\
\hline $\begin{array}{l}250.0- \\
300.0-\end{array}$ & Dower and magnet & & & & & \\
\hline
\end{tabular}

Fig. 4.4-11. $\mathrm{Li}_{2} \mathrm{O}$ blanket performance with four breeding zones. 


\subsubsection{Thermal/Hydraulics}

Functional Description. Each blanket module covers $6 \mathrm{~m}$ of the central cell and has single set of helium inlet and outlet ducts. The inlet duct penetrates the blanket core in a radial direction to just behind the first wall, where the gas expands into the first wall channels and travels in a circumferential direction as it absorbs the heat generated in the first wall. As the helium flows around the first wall, it bleeds off through ports Into the chambers holding the breeder. The first 11 passages vary in area so that, as the gas heats up and is bled off, a constant gas flow velocity is maintained. The nelium flow path is illustrated in fig. 4.4-12.

The breeding aterial must be matntained in a temperature band. If the material is too cool, the diffusion coefficient for tritiun will be too low and there will be a large tritium inventory; converseiy, if it is too hot, the material will sinter, wich also inhibits tritium diffusion. In many of the solid breeding materials, this operating temperature range may be quite narrow. For $\mathrm{LI}_{2} \mathrm{O}$, it is presently estinated as 410 to $700^{\circ} \mathrm{C}$. We met this restriction in temperature range by varying the tube diameter in the blanket. With a two-order-of-magnitude variation in volumetric heating rate, the 1-con-diameter inner tubes will be at a temperature close to that of the outer tubes, wich are $3.175 \mathrm{~cm}$ in diameter.

As the gas leaves each breeding region, it flows around the module just Inside of the outer wall and is directed to the outlet duct where it leaves the module.

Thermal Performance. Table 4.4-1 lists the thermal performance parameters of the blanket. Because the total energy deposition is fixed by neutronics, a direct relationship exists between the helfum flow rate and outlet temperature. To maximize power conversion effictency, the helium should be at the highest possible temperature. Because the energy is absorbed in solid materials and transferred to the coolant by conduction and convection, the temperature limits of the blanket materials will limit the maximum helium temperature. The $\mathrm{Li}_{2} \mathrm{O}$ temperature $11 \mathrm{mit}$ is $700^{\circ} \mathrm{C}$. At the rear of the breeding zone, the heating rate has dropped to a point where the temperature gradients, both in the material and across gas flims, are falrly small; the coolant temperature is very close to the $\mathrm{Li}_{2} \mathrm{O}$ linit. The difference in temperature between the breeding tube centerline and the surrounding helium is 


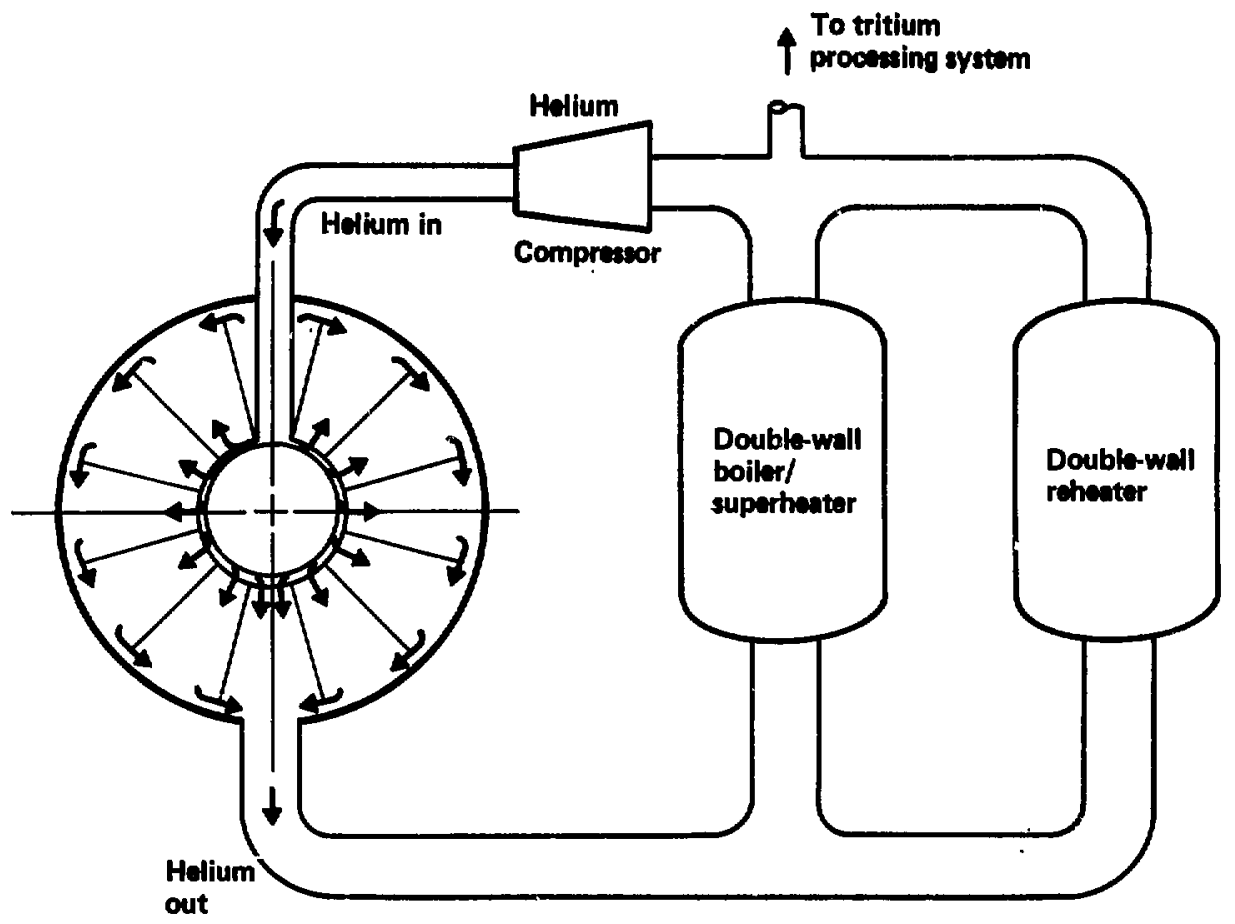

Fig. 4.4-12. Helium coolant flow path. 
Table 4.4-1. Blanket coolant parameters.

\begin{tabular}{ll}
\hline \multicolumn{1}{c}{ Parameter } & Value \\
\hline Helium flow per module $(\mathrm{kg} / \mathrm{s})$ & $79.2^{\mathrm{a}}$ \\
Helium inlet temferature $\left({ }^{\circ} \mathrm{C}\right)$ & 300 \\
Helium outlet temperture $\left({ }^{\circ} \mathrm{C}\right)$ & 630 \\
Helium inlet pressure (atm) & 30 \\
Helium pressure drop (atm) & 2.1 \\
Helium pumping power $($ Mul $)$ & 264 \\
Max first wall temperature $\left({ }^{\circ} \mathrm{C}\right)$ & 464 \\
Min Li ${ }_{2}$ O temperature $\left({ }^{\circ} \mathrm{C}\right)$ & 393 \\
Max Li ${ }_{2} 0$ temperature $\left({ }^{\circ} \mathrm{C}\right)$ & 687 \\
\hline Includes rear wall coolant.
\end{tabular}


only $14^{\circ} \mathrm{C}$ at the outer edge compared to $173^{\circ} \mathrm{C}$ at the inner edge. With a helium inlet temperature of $300^{\circ} \mathrm{C}$, in average nutlet temperature of $630^{\circ} \mathrm{C}$ may be achieved.

Because the helfum flowing around the first wall passages is bled off gradually into the blanket zones, each zone will be receiving progressively higher irlet temperatures, ranging from $300^{\circ} \mathrm{C}$ near the inlet duct to $362^{\circ} \mathrm{C}$ on the opposite side. The mean helium temperature leaving the first wall and entering the breeding zone is $319^{\circ} \mathrm{C}$. As a result, the gas temperature leaving the breeding zones will vary from 611 to $673^{\circ} \mathrm{C}$, with a mean of $630^{\circ} \mathrm{C}$.

ihe first wall temperatures are calculated assuming that the first will is adiabatic on the plasm side. The first wall has a volume of $1.5 .16 \mathrm{~cm}^{3} / \mathrm{cm}^{2}$ of fruntal area and neutronic heating of $41 \mathrm{~W} / \mathrm{cm}^{3}$, resulting in an effective heat flux of $63.4 \mathrm{~W} / \mathrm{cm}^{2}$ of frontal area. Surface heating is caused by plasma radiation and charged-particle interactions and is small for MARS, about $6 \mathrm{~W} / \mathrm{cr}^{2}$. Thus, the error introduced by neglecting surface heating is about $10 \%$.

The first-wall coolant channels vary in cross sectional area to maintain a constant flow velocity of $60 \mathrm{~m} / \mathrm{s}$ (Mach no. = 0.54 ); the film coeffictents are calculated by conventional means. For the purpose of the heat cransfer calculations, we assume that the first wall structure, at any circumferential location, has a uniform temperature on all cooled surfaces; the difference in gas-t?-metal temperature is then equal to the heat deposited at any area divided by the surface area ant film coefficient. To miminize the wall temperatures and reduce the $\pi_{i}$ ial volume (and thus heat deposition) while still maintaining adequate stiffness, fins are iicorporated in the coolant channels as shown in Section CC of Fig. 4.4-4.

With the metal surface temperatures known, the maximum metal temperature is calculated on the basis of an adiabatic wal' on the plasma side and uniform internal heat generation. The wall is $0.48 \mathrm{~cm}$ thick, abso.hs $41 \mathrm{~W} / \mathrm{cm}^{3}$, and has a temperature difference from the front to rear face of $15.7^{\circ} \mathrm{C}$. The maximum temperature gradient is double the overall gradicnt, or $65^{\circ} \mathrm{C} / \mathrm{cm}$, wilch is accep:able based on the thermal stress.

One of our design goals was to limit HT-9 to a maximum temperature of approximately $450^{\circ} \mathrm{C}$. We adjusted the first-wall gas velocity and fin area to approach this goal. We did not feel that further increases in gas velocity were warranted based on increased pressure drop. 
The helium coolant bleeds through ports in the aft portion of the first wall and into the breeding zones. It flows over tubes containing $\mathrm{LI}_{2} \mathrm{O}$, arranged in staggered arrays, para?lel to the plasima axis. The film coefficients are calculated using relationships developed for sross-flow heat exchangers.

The portions of the blanket closer to the plasma have the highest heating and this the tubes must be small (1-cm-diameter) to 1 init the $\mathrm{Li}_{2} \mathrm{O}$ temperature to acceptabis values. As the coolant progresses through the blanket, the heating rate falls off exponentially and the tube diameters may be increased. This design incorporates rour zones contoining tubes with diameters of 1.0 , 1.27, 1.905, and $3.175 \mathrm{~cm}$.

Figure 4.4-13 11 lustrates the predicted temperature profiles for the coolant gas, first wall, vanadium alloy tubes, and $\mathrm{LI}_{2} \mathrm{O}$.

Hydraulic Performance. The hellum pressure drop through the first wall and blanket is 2.1 atm with an inlet pressure of $30 \mathrm{~atm}$. This breaks down as follows:

\begin{tabular}{lc}
$\frac{\text { Item }}{\text { Inlet duct }}$ & Pressure drop (atm) \\
\cline { 2 - 2 } First wall & 1.79 \\
Blanket & 0.07 \\
Outlet duct & 0.01 \\
& $\underline{0.23}$ \\
2.10
\end{tabular}

The bulk of the pressure loss occurs in the inlet duct. The inlet duct penetrates the blanket and it is not practical to have a large duct dianeter with gradual turns and expansion angles. Although in a detalled design every effort would be made to minimize the pressure loss by means of turning vanes, etc., it is still likely that a large portion of the inlet duct velocity head would be lost in the transition from the duct to the first wall. In this analysis we estimated that one full velocity head is lost as a result of the expansion process and another full velocity head is lost in frictional and turbulence effects accrued in distributing the flow uniformly. The pressure loss in the first wall passages is primarily caysed by frictional losses and is relatively small at a velocity of $60 \mathrm{~m} / \mathrm{s}$. 


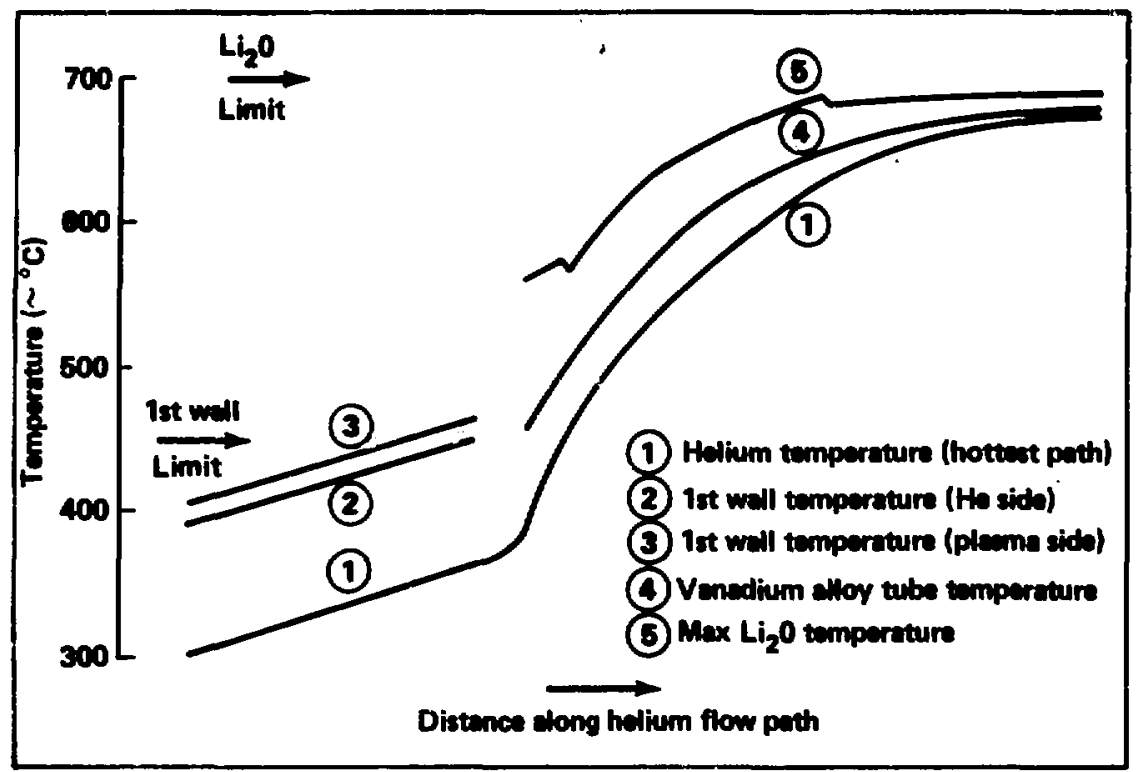

Fig. 4.:-13. Blanket temperature profiles. 
The pressure drop in the blanket is also very rold fraction is high, wich causes the velocity to be low. The highest gas velocity, it the minimum gaps between tubes, is less than $10 \mathrm{~m} / \mathrm{s}$. In fact, it would be desirable to increase this velocity to improve the meat transfer characteristics of the blanket. The min reason for adopting a lower linit on tube-to-tube spacing is that the tubes sre supported in tube sheets that must maintain the structural integrity of the assembly. An alternate design, ihich has yet to be exemined, would use tube bundles with the tubes spiral-wrapped with wire or some other form of spacers to melntain a closer tube spacing. It wight also bemeficial to knurl or etch the cladding to increase heat transfer at a sull expense in pressure drop.

The pressure drop associated with the outlet duct is also relatively sanll. There is little restriction on its diameter and thus the viocities my be kept somemat lamer then the inlet duct and, more inportantly, me have a duct entrance loss that is casier to accomodate efficientily. In this analysis, a prossure loss of one velocity head (referenced to the exit duct velocity) is assumed for the losses assoclated with collecting the flow and directing it into the exit duct.

The total puping power for coolant flow through the first will and blanket is $265 \mathrm{WW}$, or $\mathbf{8 . 6 3}$ of the thermal energy liberated in the blanket. This loss is a strong function of the system operating pressure, as shown in F1g. 4.4-14. The loss could be cut approximately in half by increasing the system pressure to $40 \mathrm{~atm}$. A sinilar study for a high-tcmperature gas-cooled reactor/gas turbine (HTER-GT) system', which has similar components in the power conversion system, reported a baseline system pressure of 78 atm. Their sensitivity study showed little variation in power cost with changing pressure. It is likely that the optimin system pressure is higher than 30 atm; homever, it is doubtful that it would be as high as 80 atm bacause of the large effect on the blanket structure.

Rear Hall Thermal thedraultes. The rear mall is gas cooled by a separate helium strean tapped off the main coolant and reunited with it outside of the blanket. The meutronic energy deposition in the rear will is $0.125 \mathrm{~W} / \mathrm{cm}^{3}$ $\left(0.70 \mathrm{H} / \mathrm{cm}^{2}\right.$ of rear wall surface area) plus $0.44 \mathrm{~W} / \mathrm{cm}^{2}$ heat conduction from the breeding zone. 


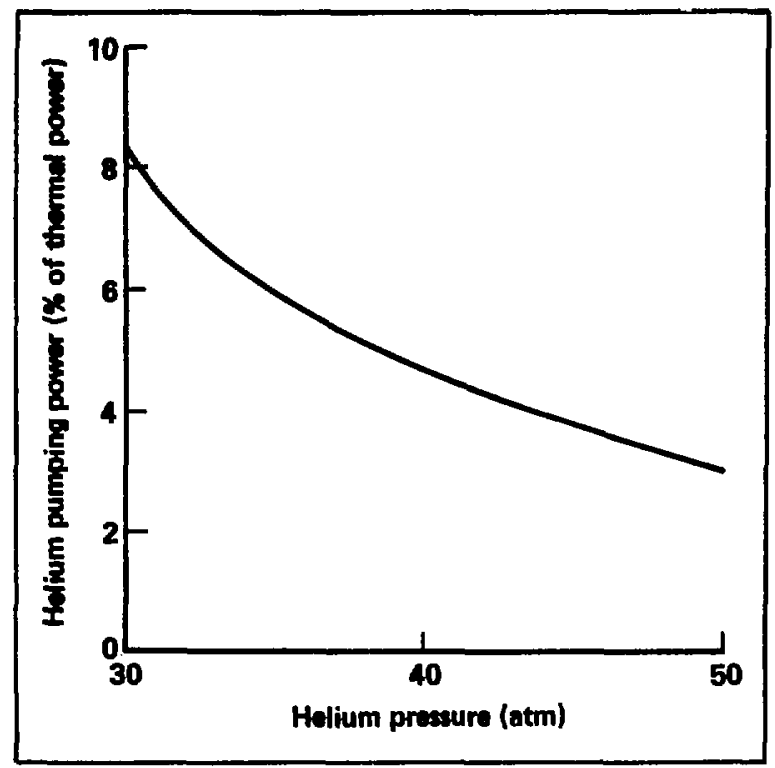

Fig. 4.4-14. Effect of helium pressure on pumping power. 
The coolant is introduced in corrugated passages and extracted at a point $90 \mathrm{deg}$ around the perlphery of the rear wall. The mantfolding is arranged so that the gas in the adjacent passages travels in opposite directions. In this manmer the rear wall is malntained at as constant a temperature as possible. The gas enters at $300^{\circ} \mathrm{C}$ and exits at just under $320^{\circ} \mathrm{C}$; the wall surface is $320^{\circ} \mathrm{C}$. Assuaing that the outer edges of the wall are adiabatic, ine thermal gradient across the rar wall is $5.4^{\circ} \mathrm{C}$. The pressure loss associated with this coolant flow is less than 0.1 tm and the pumping power is less than $0.7 \mathrm{~m}$.

\subsubsection{Radiation Effects On Structural Materials}

The three areas of concern with regard to radiation effects on structural materfals are: change of tensile properties, radiation creep, and shift in ductile-to-brittle transition temperature. Unfortunately, the effects of D-T neutrons on the propertfes of structural materfals have not been axperianentally evaluated at this time. Therefore, it is necessary to use the expertmental database developed with fission neutron, heavy fon, and electron irradtations.

The primary structural material for the solfd breeder blanket is SAbvik HT-9, a chromium-molybdenum ferritic steel. This alloy has been the subject of an extensive experimental evaluation effort by the Fast Breeder Reactor Progrm. Its irradiation response in a fisston environment is very encouraging and we assume that this performance would be maintained in a D-T neutron environment.

An undesirable characteristic of HT-9 is the need for preheat during fusion welding and for a post-weld heat treatment. Heat treatment of assemblies of complex geometry is undesirable and often impractical. For the purpose of this study, the use of dissintlar metal weld lands ande of Inco 625 eliainates the med for post-weld heat treatment of major assemblfes. The Inco 625 alloy, however, suffers a greater loss of ductflity then HT-9 under irradiation and does rot offer a totally satisfactory solution to the protilem.

The tube assembly structure is fabricated from the vanadiun base alloy V-15Cr-5Ti. Although the gemeral techmology of vanadiun base alloys is 11aited, their irradiation response ses ben investigated ${ }^{2}$ and expertimental results indicate on extremely high resistance to vold awelling and no undesirable effects on mechasical preparties. 


\subsubsection{Solld Breeder Performince}

Our primary emphasts la selecting a solid breeder focused on the lithiumbased cerantes such as $\mathrm{LI}_{2} \mathrm{O}, \mathrm{LAAlO}_{2}, \mathrm{LI}_{2} \mathrm{SHO}_{3}, \mathrm{LI}_{4} \mathrm{StO}_{4}, \mathrm{LI}_{2} \mathrm{THO}_{3}$, and $\mathrm{LI}_{2} \mathrm{ZrO}_{3}$. These meterials are not well-characterized; work is in progress under DOE sponsorship to verify that their properties meat design requirewants. The major uncertainties are: (1) the of fect of high temperatures and irradiation on tritiun release, and (2) the effect of impurities on system chemistry (phase change) and tritium release.

For the breeder, we selected $\mathrm{LI}_{2} \mathrm{O}$ as the baseline matertal. It has a high lithium atom density, ressonably high operating temperature, does not require mutron multiplier and is better characterized than the rest of the materials. The maximum operating temperature to preclude sintering is currently estimated to be $700^{\circ} \mathrm{C}$ based on initial experimental data of Yang, et al..$^{3}$ The minimu operating temperature is set at $410^{\circ} \mathrm{C}$ because of LIOT formation.

We have also considered using $\mathrm{Li}_{7} \mathrm{~Pb}_{2}$ as the breeder. It is an intermetallic compound that appears to act as an fonic sait. Preliminary Indications are that tritium is much less soluble in $\mathrm{Li}_{7} \mathrm{~Pb}_{2}$ than in $\mathrm{Li}_{2} \mathrm{O}$. Yang's initial experimental data indicated no wicrostructure change after irradiation at $600^{\circ} \mathrm{C}$. The operating temperature range for $\mathrm{Lt}_{7} \mathrm{~Pb}_{2}$ appears to approach that of $\mathrm{Li}_{2} \mathrm{O}$. In addition, the material is compatible with the present mechanical design of the solid breeder module and could be directly substituted for $\mathrm{LI}_{2} \mathrm{O}$. At this time we are considering $\mathrm{Li}_{7} \mathrm{~Pb}_{2}$ as a backup for the solid breeder while the characterization program for the lithium-based ceramics is being conducted.

\subsubsection{Shield}

\subsubsection{Design Criteria}

The main criterion in configuring the shield for the solid breeder blanket design was to provide adequate radiation protection for the centralcell superconducting magnets. The specific performance requirements imposed were to keep the radiation dose to the magnet insulation (Kapton)--accumulated over 24 FPY--below a generally accepted damage threshold of $5 \times 10^{9}$ rad, to 11 init the peak displacement damage in the copper stabilizer to $2.2 \times 10^{-5} \mathrm{dpa} / \mathrm{FPY}$, 
and to reduce the peak radiation mating in the magnet winding below $0.06 \mathrm{~m} / \mathrm{cm} \mathrm{cm}^{3}$. Another important consideration was to achfeve the required attenuation performance with the minimum shield thickness to minimize magnet bore. We also sought to 11 mit the generation of long-lifed radfoactivity in the shield by selection of the shield materials. Because the blanket design allows only about $0.5 \%$ of the energy to leak into the shield, the requited shield performance was relatively modest. We chose water as the coolant.

\subsubsection{Structural Daston}

The shield is situated radially between the blanket and the magnets to absorb neutron and gamma energy to limit deposition in the magnet. As we stated before, the design of the shield is governed by neutronics and remote handling procedures adopted for removing and replacing the central cell module.

The shield has a radial thickness of $41.5 \mathrm{~cm}$ and a neutronics analysis shows that a volumetric composition of $60 \% \mathrm{HT}-9,15 \% \mathrm{lead}, 15 \% \mathrm{~B} \mathrm{C}_{3}$, and $10 \%$ $\mathrm{H}_{2} \mathrm{O}$ gives a satisfactory performance.

The shield, shown in Fig. 4.4-15, covers two areas. The largest part of the shield, or blanket shield, covers the major portion of the blanket and is made in two pleces with the joint on the horizontal plane parallel to the plasm axis. The lower half of this part of the shteld, attached to the lower carrlage structure, supports the upper half and can remain in position during blanket changeout. The upper half is constructed to permit crane holsting. Both the upper and lower shield halves include penetrations for the blanket outer wall coolant ducts. The lower half has one support point (fixed point) for the blanket and penetrations to allow the other three support links to be attached to the lower carriage structure.

The smaller. part of the shield covers the module joint reglons. The smaller shields are also made in two parts, upper and lower, and are split on the horizontal plane simflar to the blanket shield. The module joint shields are supported by the blanket shield through radial attachment bolts. Each half has penetrations for the module-joint bellows coolant duct. Wan access is requiled to the module joint, the lower half of the shisld 15, lowered to a storage cradle on the floor and the upper half is crane hro,eced. 


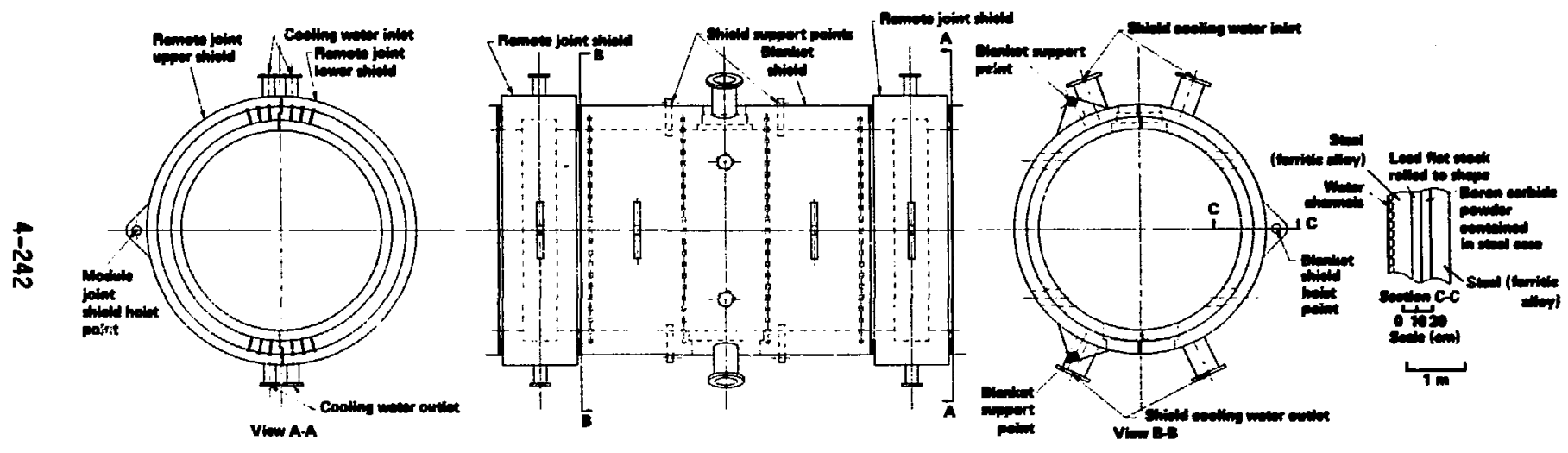

Fig. 4.4-15. Remote joint and blanket shields. 
The shield is cooled by water flowing in channels near the surface closest to the plasma. In the module joint shields, the water iniet opens up into a menifold that feeds channels running circumferentially around the shield half into an exit manifold and outlet pipe. The blanket shield halves are cooled by water piped to a manifold at the center of the blanket. This manifold feeds channels running axially to both ends of the blanket where they are turned 180 deg back to an exit manifold and outlet pipe at the center of the blanket.

The shield is constructed so that the steel forms an annular box to contain rolled slabs of lead held in place by minimum number of studs and steel enclosures that hold the boron carbide powder. A steel cover bolted in place closes off the annular box.

\subsubsection{Meutronics}

We conducted the shield analysis as an integral part of the blanket design using the ANISN code with 100-neutron/21-ganm group structure. We used the EPR cross section library, augmented by data sets from MCKLIB (displacement and activation cross sections). For the radiation iransport analysis, treated this shieid as a homogeneous mixture. In one dasign variant, introduced a $30-\mathrm{cm}$ steel reflector (Fe-1422 with 5 vol $\times \mathrm{H}_{2} \mathrm{O}$ ) between the blanket and the homogenized shield region to increase the energy deposition; however, this was marginally effective and therefore abandoned.

To determine the shield thickness required to satisfy magnet exposure linits, we gemerated parametric results for the various magnet effects vs shield thickness. For these calculations, we used the fluxes exiting from the shield and therefore neglected the attenuation provided by the manet dewar. Based on these analyses we concluded that a $45-\mathrm{cm}-$ thick shleld would satisfy the magnet exposure requirements with adequate margin, as shown in Fig. 4.4-16.

To assess the external dose levels after shwtdown, we analyzed the activity produced in the shield over 2 FPY. All gamm-eaitting radioisotopes with half-lives between $1 \mathrm{hr}$ and $100 \mathrm{yr}$ were included. He considered two options for the 60 vol $x$ structural material: HT-9 and Fe-1422. For the HT-9 shield, we found a shutdown activity par unit of central cell length of $1.754 \times 10^{5} \mathrm{ci} / \mathrm{m}$ dominated by $\mathrm{m}-56$. This excludes an Fe-55 activity of $6.24 \times 10^{4} \mathrm{Ci} / \mathrm{m}$. One day after shutdown, the activity reaches $6.82 \times 10^{4} \mathrm{CI} / \mathrm{a}$ 


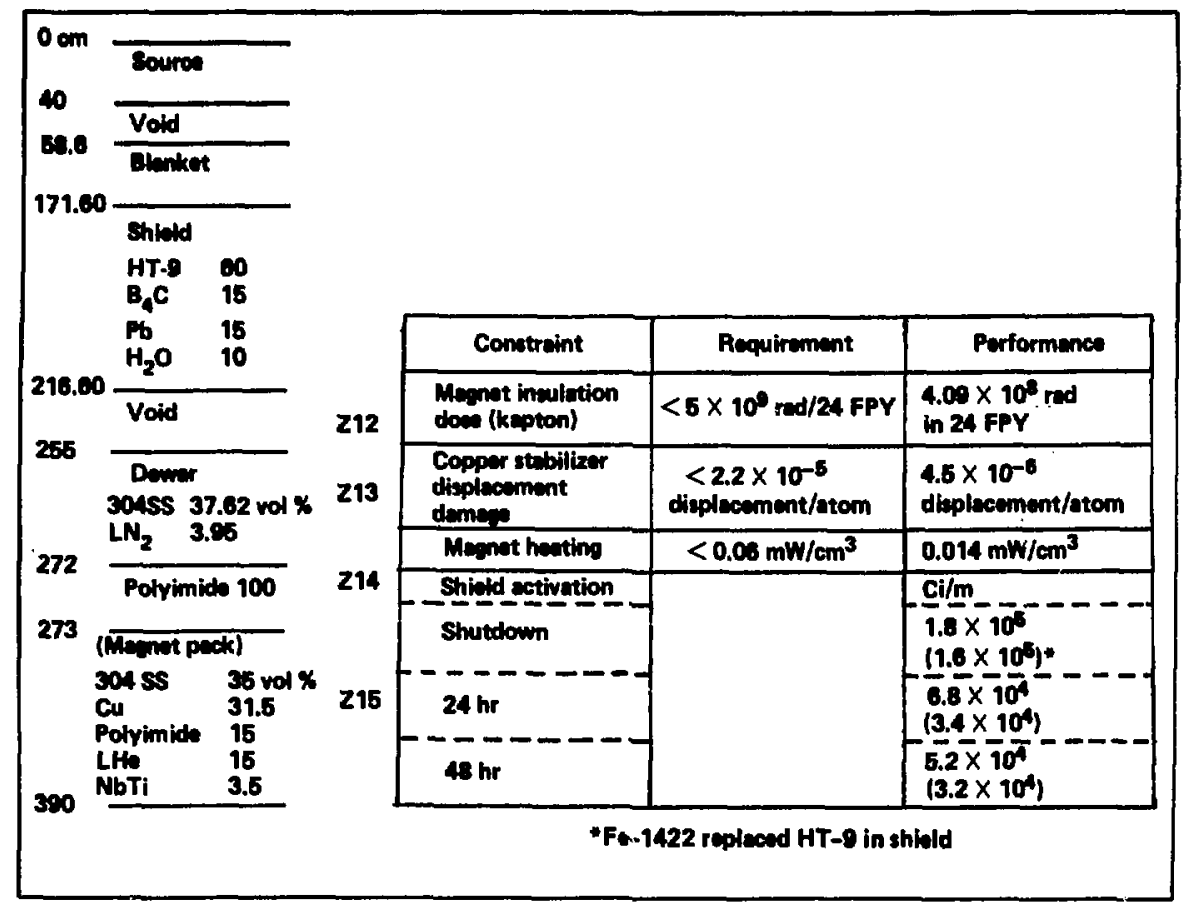

Fig. 4.4-16. Summary of shield design results. 
(now dominated by $W-187$ ); after 2 days it is $5.16 \times 10^{4} \mathrm{Cl} / \mathrm{m}$. With an Fe-1422 structure, the shield activity is $1.59 \times 10^{6} \mathrm{ci} / \mathrm{m}$ at shutdown and is driven by $\mathrm{m}$-56 for the first $16 \mathrm{hr}$. After 1 day, the activity level declines to $3.43 \times 10^{4} \mathrm{Cl} / \mathrm{m}$ (now malnly because of $\mathrm{m}-54$ ) and to $3.17 \times 10^{4} \mathrm{ct} / \mathrm{m}$ after 2 days. These results do not suggest a clear-cut choice between the 2 materla1s. At present, we have chosen HT.-9 as the baseline for the shield, but Fe-1422 is an alternate.

To provide a lower activity alternative to the above shields, ware exploring the use of an aluminum alloy for the shield structure. The magnet protuction requirements can be mot with $150-\mathrm{cm}$ shield, although the safety margin is reduced. For an aluminum alloy shield, the shutdom activity will be mostly due to the 15-hr Ma-24, though alloy additions such as Zn may also contribute. We are curently assessing the post-shutdown radiation doses that these shield options and the shield-transmitted decay radiation from the blanket would presient during inaintenance operations.

We are also in the process of analyzing the design of the shielding plugs needed to suppress streaning between the blanket modules. We are giving special attention to estabifshing the special shielding problems related to the heliu cooling duct and manffold.

\subsubsection{Thermal/Hydraulics}

The total energy deposited in the shield is $15.2 \mathrm{w}$. The shield will be water-cooled; if we assume a $50^{\circ} \mathrm{C}$ temperature rise, the flow required will be 72.711 ter/s for the entire reactor. Although we did not conduct a pressure drop analysts, if we assume a 2-atm pressure drop, then the pumping power will be only $15 \mathrm{~kW}$.

He analyzed thermal profiles in the shield to determine the maxtmum posstble temperature in the lead layer. The analysis was greatly simplified and conservative to ensure that we did not exceed the lead tcmperature ifmits. We assunad that all of the shield energy from the shteld was deposited in the steel and lead zones and that the boron carbide layer was a perfect insulator. On this basis, the maximum lead temperature would be $31^{\circ} \mathrm{C}$ hotter than the inner (water-cooled) face of the shfeld. If the maximum inser-face teaperatures were $80^{\circ} \mathrm{C}$, then the maximin lad temperature would be $111^{\circ} \mathrm{C}$, mich is wil under the allowable linft. 


\subsubsection{Vacuum Seals}

In the solid breeder blanket, the vacuum boundary is not penetrated at any point by coolant pipes. It is a continuous wall containing only weld joints. The critical weld joint is located between the blanket modules; it is designed to take into account the relative growth between adjacent nodules (see Fig. 4.4-7). The module joint has been designed to allow modules to be replaced remotely. The joint can be cut and rewelded by a renotely operated tool similiar to that developed for TFTR.

Wh looked at various mechanical seds but none seemed to offer the vacuum integrity and remote maintenance applicability of the welded joint. A brazed alternative to the welded joint that removes the need for a cutting machine seems practical but it is not yet developed to a point where we can include it in this design.

\subsubsection{Energy Conversion System}

\subsubsection{System Analysis}

The most critical parameter in the energy conversion system is the maximum working flutd temperature, which largely determines the type of thermal-to-electrical conversion cycle and, ultimately, the energy conversion efficlency. For this design, hellum is avallable from the blanket at a mean temperature of $630^{\circ} \mathrm{C}$. This source temperature allows steam to be produced at about $550^{\circ} \mathrm{C}$; thus, we are operating in a region sinilar to that of conventional fossil and HTGR plants. Because of the temperature limits imposed on the blanket by the high temperature sintering characterictics of the $\mathrm{LI}_{2} \mathrm{O}$, we have selected a conventional steam cycle for the power conversion system.

Figure 4.4-17 illustrates the stean cycle efficiencies of various existing and conceptual power plants and a standard steam cycle efficlency curve for a single reheat system. This gives a $42.5 \%$ cycle efficiency at a stean temperature of $550^{\circ} \mathrm{C}$.

Figure 4.4-18 is the flow diagram of the basic power cycle which identifies the major systen components. The cycle is quite conventional, with a single reheat and multiple stages of regenerative feedwater heaters (shown grouped for siaplicity as IP and LP heaters). In this case, steam is 


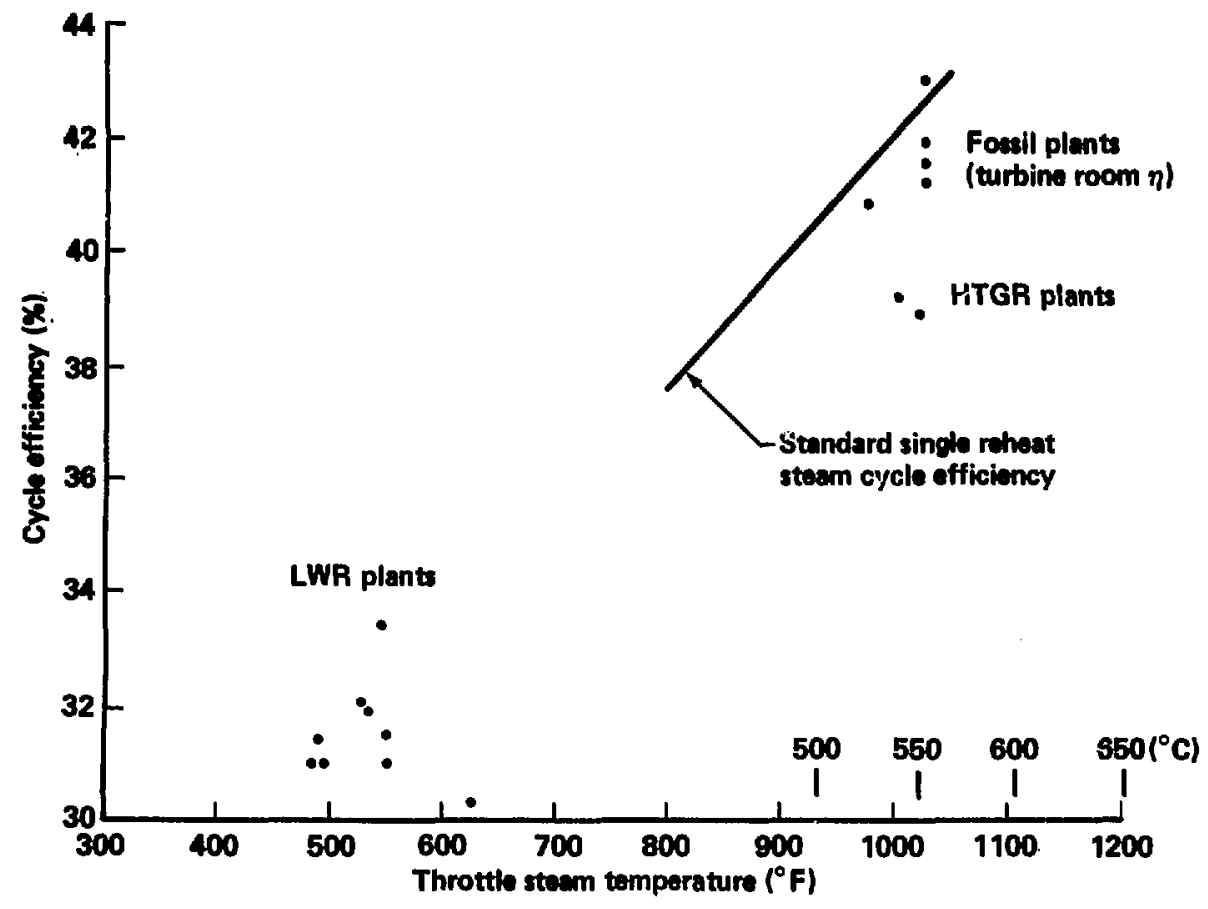

Fig. 4.4-17. Power plant steam cycle efficiencies. 


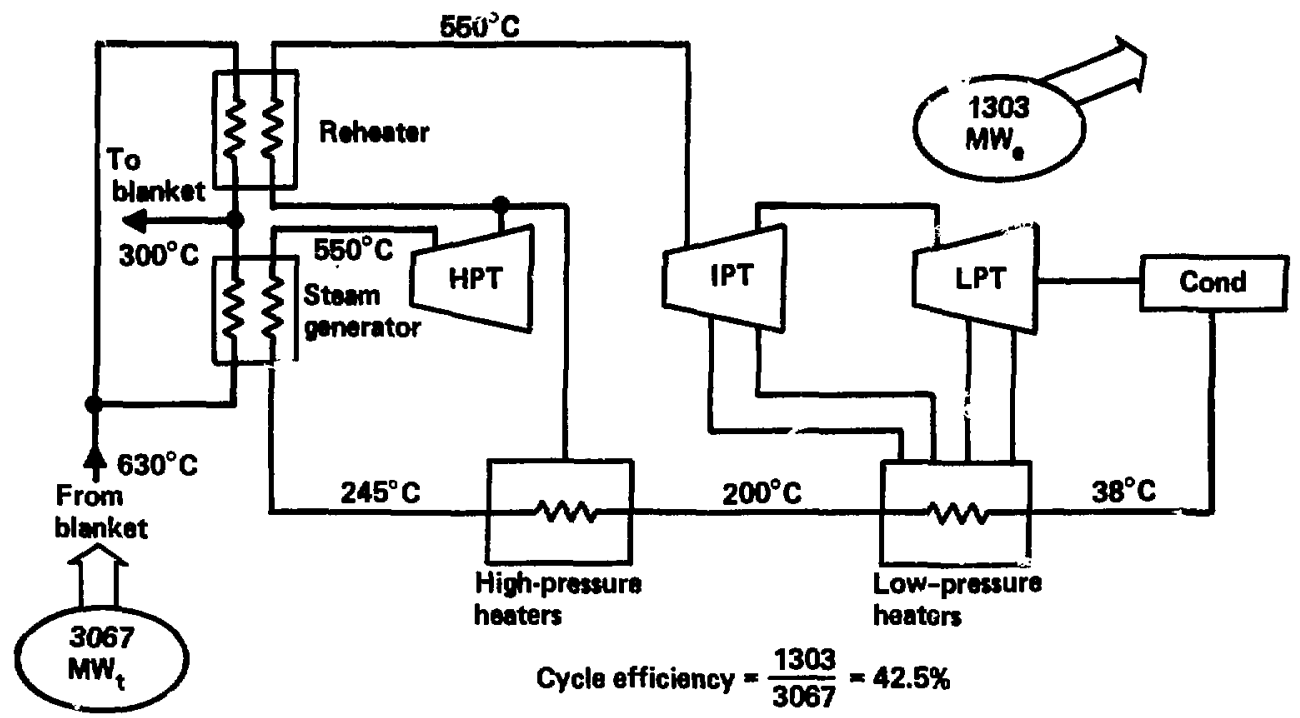

Fig. 4.4-18. Basic power cycle. 
extracted from the intermediate prestiure (IPT) and low pressure (LPT) turbines to supply the feedwater heaters, which is conventional stean cycle practice, and we can select the $42.5 \%$ conversion efficiency noted above.

The MARS system, in addition to the power extracted from the blanket, has $371 \mathrm{~N}_{t}$ of power that must be removed from the direct converter (12\% of the blanket heat). As described in Section 3.6, this energy is extracted with pressurized water at a temperature of $215^{\circ} \mathrm{C}$. The question is whether it is economically feasible to convert this heat to electricity.

The feedwater nust be heated from a temperature of 38 to $245^{\circ} \mathrm{C}$ before entering the steam generator (see Fig. 4.4-18), which is an opportunity io introduce $215^{\circ} \mathrm{C}$ heat source. The required power cycle modifications are: (1) substituting water-to-water heat exchangers for steam-to-water heat exchangers in the feedwater heating system, which should not be complex or expensive, and (2) modifying the IP and LP turbines to handle increased flow resulting from an absence of extraction steam. This modification is considered to be more critical and its extent is not yet known; however, conversations with turbine manufacturers indicated that it is feasible without having a significant effect on turbine efficiency.

The effects of this change were scoped based on the results from a similar study for L.WRs. 4 For MARS, there is energy availabie to perform $78 x$ of the feedwater heating. At this level of feedwater heating, 18\% of the added energy appeared as electricity. In other words, the effic iency of converting the ene:ay in $215^{\circ} \mathrm{C}$ water was $18 \%$.

The cycle shown in Fig. 4.4-19 converts 3067 Mtt from the blanket at 42.5\% and $371 w_{t}$ from the direct converter at 18\%; this results in a total output of $1370 \mathrm{~m} / \mathrm{e}$ and an effective cycle efficiency of $39.8 \%$.

Recovery of the 371 from the direct converter results in an additional output of 67 ; the question that we must address is whether this recovery is worthwhile. If we use a rough direct-cost figure of $\$ 1500$ to $1800 / \mathrm{kWe}$, the modifications required to implement recovery of this direct converter heat could cost between $\$ 100$ and $\$ 120$ million. On this tasis it would seem that recovery of this direct converter heat is economic; however, more study will be required. 


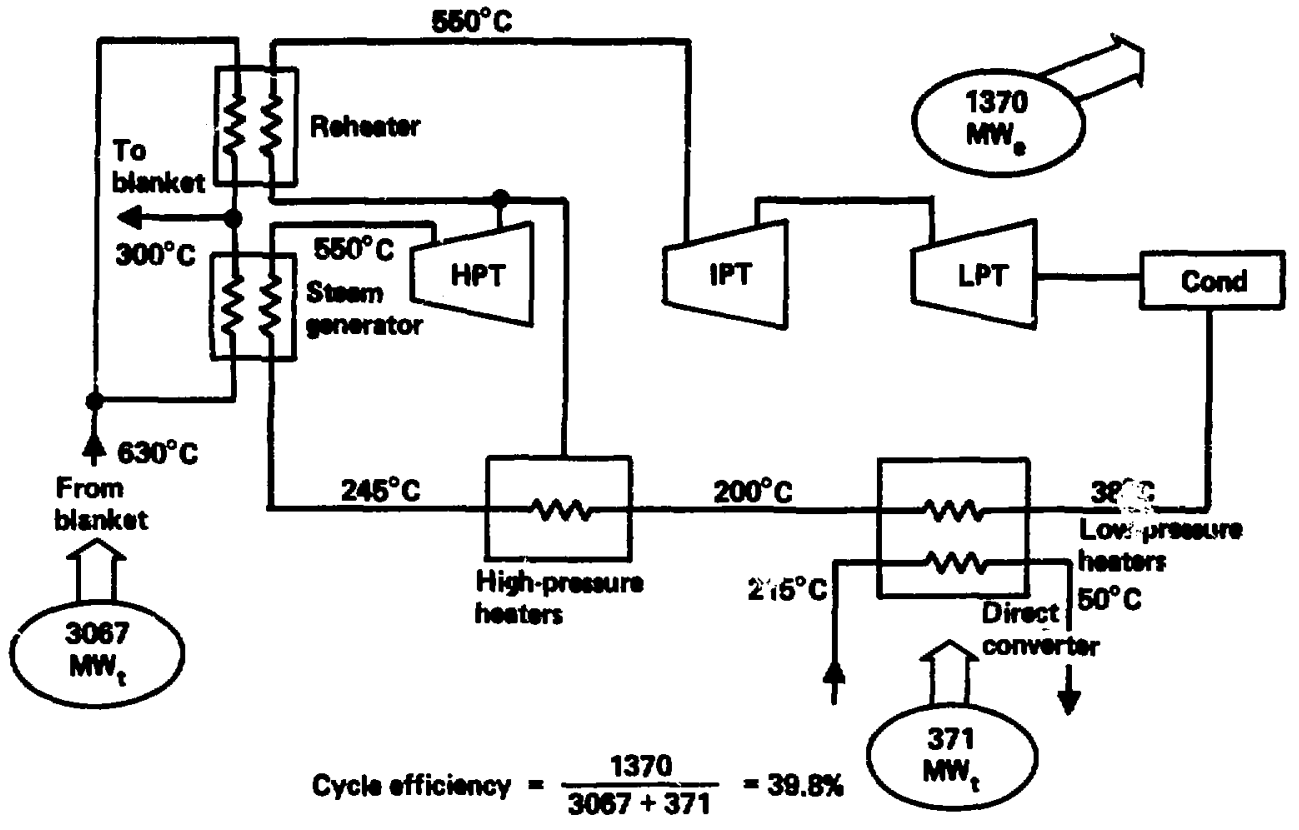

Fig. 4.4-19. Powèr cycle using direct converter heat. 


\subsubsection{Component Description}

Because the power cycle is conventional, we will not discuss the standard components, such as turbines, condensers and feedwater heaters. However, the boiler/superheater and reheater are unique and deserve discusston.

Figure 4.4-20 shows the temperature profiles on the helium and steam sides for both the boller/superheater and the reheater. The heat exchangers are in parallel with a 3000-psig stem supply pressure and a 500-psig reheat pressure.

The multiple-unit boller/superheater transfers 2637 in and the reheater transfers 430 . Reference 5 describes conceptual destgns for the/the heat exchangers up to $900 \mathrm{~m}$. Because the primary helium in each unit will be tritlated, both units must provide adequate barriers to prevent tritium migration into the steam system. He envision that the design will have a concentric tube configuration with extensive metal-to-metal contact to promote good heat transfer, but it will not have a metallurgical bond so that a crack will not propagate from one wall to the other. The gap between tubes will efther be vented to a tritium leak detection device or will contafn in oxidizing gas to convert the tritium to $T_{2} \mathrm{O}$ and thus inhibit its migration through the second mill. Table 4.4-2 lists the basic spectfications for the heat exchangers.

\subsubsection{Blanket Maintenance}

\subsubsection{Maintenance Ph1losophy}

Any mintenance required inside the reactor butlding wille the reactor is operating will be performed rewotely. A few hours after the reactor has been shut down, personnel will be allowed into the reactor bullding to begin disantling equipment outside the shield. The cooling system for the blanket and shleld must be kept operating until the afterheat level is reduced. About 3 days after reactor shut down, the cooling systams can be deactivated and dismantled. From this point on, all operations must be done remotely because the first operation involves removing shield parts. 


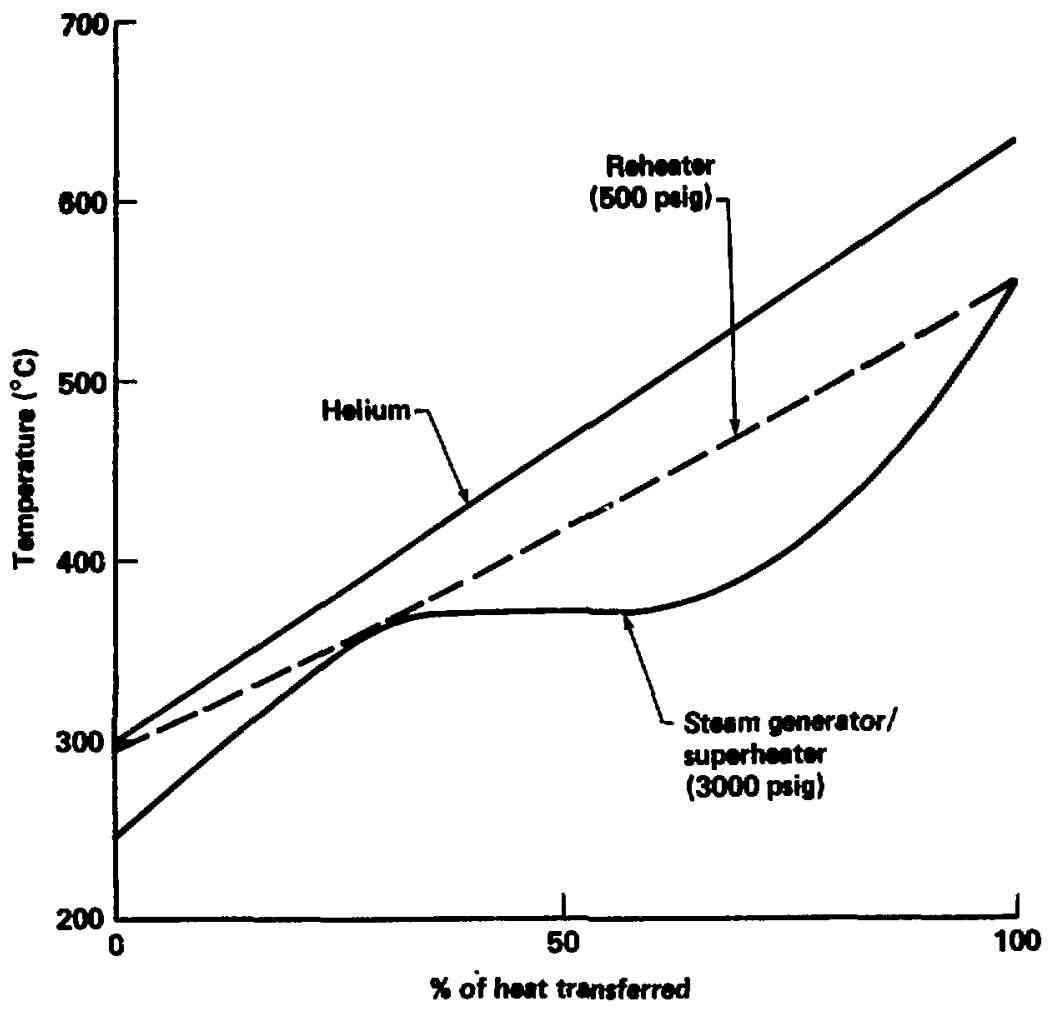

Fig. 4.4-20. Heat exchanger temperature profiles. 
Table 4.4-2. Heat exchanger specification.

\begin{tabular}{llc}
\hline \multicolumn{1}{c}{ Parameter } & Botler/superheater & Reheater \\
\hline Helium inlet temperature $\left({ }^{\circ} \mathrm{C}\right)$ & 630 & 630 \\
Helium outlet temperature $\left({ }^{\circ} \mathrm{C}\right)$ & 300 & 300 \\
Helium flow rate $(\mathrm{kg} / \mathrm{s})$ & 1609 & 371 \\
Helium pressure $(\mathrm{atm})$ & 30 & 30 \\
Feedwatier temperature $\left({ }^{\circ} \mathrm{C}\right)$ & 245 & - \\
Feedwater pressure $($ atm) & 204 & - \\
Steam inlet temperature $\left({ }^{\circ} \mathrm{C}\right)$ & - & 293 \\
Stean outlet temperature $\left({ }^{\circ} \mathrm{C}\right)$ & 550 & 550 \\
Steam flow rate $(\mathrm{kg} / \mathrm{s})$ & 1070 & 963 \\
Total rating (mw/) & 2492 & 575 \\
\hline
\end{tabular}




\subsubsection{Module Changeout Procedure}

As we stated in Section 4.4, the module is defined as two magnets, a shield assembly and a blanket assembly with associated support structure (see F1g. 4.4-1). The total weight of the module is 781 tons.

After all electrical, fluid, and Instrumentation lines have been disconnected, the joint shield segments are removed as shown in Fig. 4.4-21. The lower segments are moved to cradles on the floor and the upper segments crane hoisted to suitable area adjacent to the machins.

The remote welder/cutter, located on the joint ralls, is used to cut the module joint. The bellows in the joint are designed to spring back small amount when the cut is made to provide clearance for module removal. One set of anchor brackets is removed and the module is moved on its rails to the maintenance location. Here the module is held in position by another set of anchor brackets.

A hoisting fixture is attached to one magnet (weighing 200 tons); this assembly is removed by gantry crane to a storage position inside the reactor enclosure. The second magnet is similarly removed and stored. The upper carriage structure is removed as shown in Fig. 4.4-22. The bolts attaching the upper shield to the lower shield are removed and the upper shield lifted off and stored. This now reveals the blanket assembly as shown in Fig. 4.4-23. The four bolts that attach the blanket assembly to its support are removed; the blanket is now ready to be hoisted and lowered onto a cradle trolley on rails adjacent to the door connecting the reactor enclosure with the hot cell. This completes the blanket module removal.

When reassembling the central cell module, the reverse procedure is followed until the replacement module is in the reactor. Before the weld joint is made, the module must be properly aligned by adjusting the anchor brackets, which have remote-controlled vertical and lateral adjusting features. When the module joint has been allgned, the welder cutter is positioned for a triming cut to be made followed by the weld pass. The welder cutter is removed and stored and the joint shield segments bolted in place. Hands-on assembly of the remaining equipment can then proceed. 


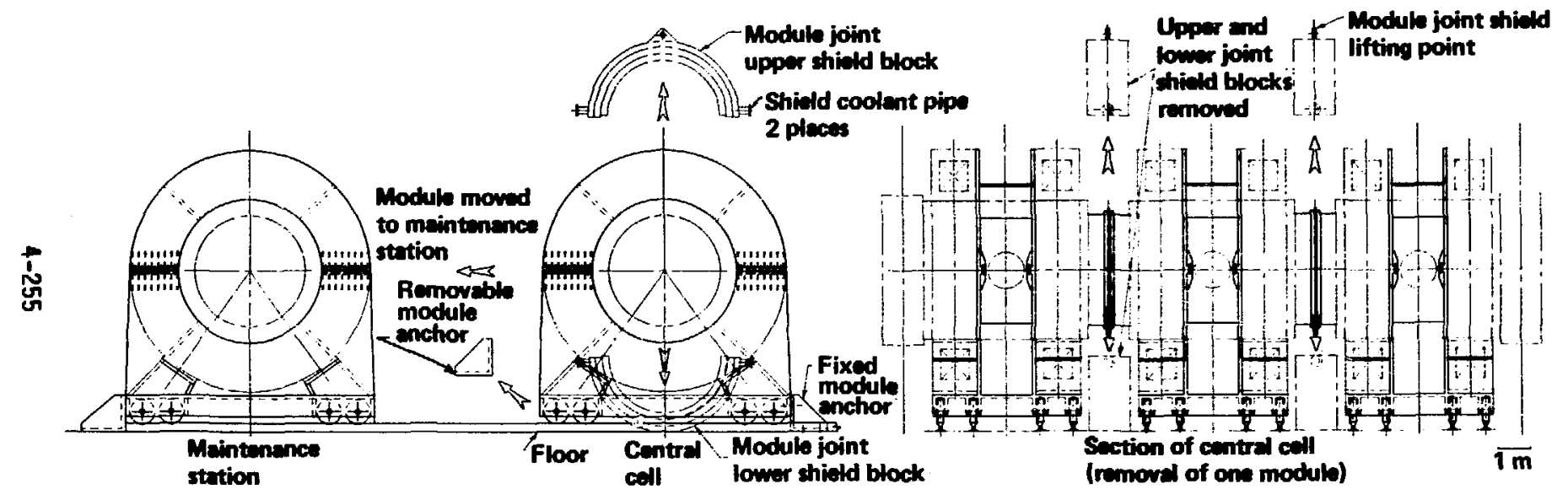

Fig. 4.4-21. Removal of central cell module. 


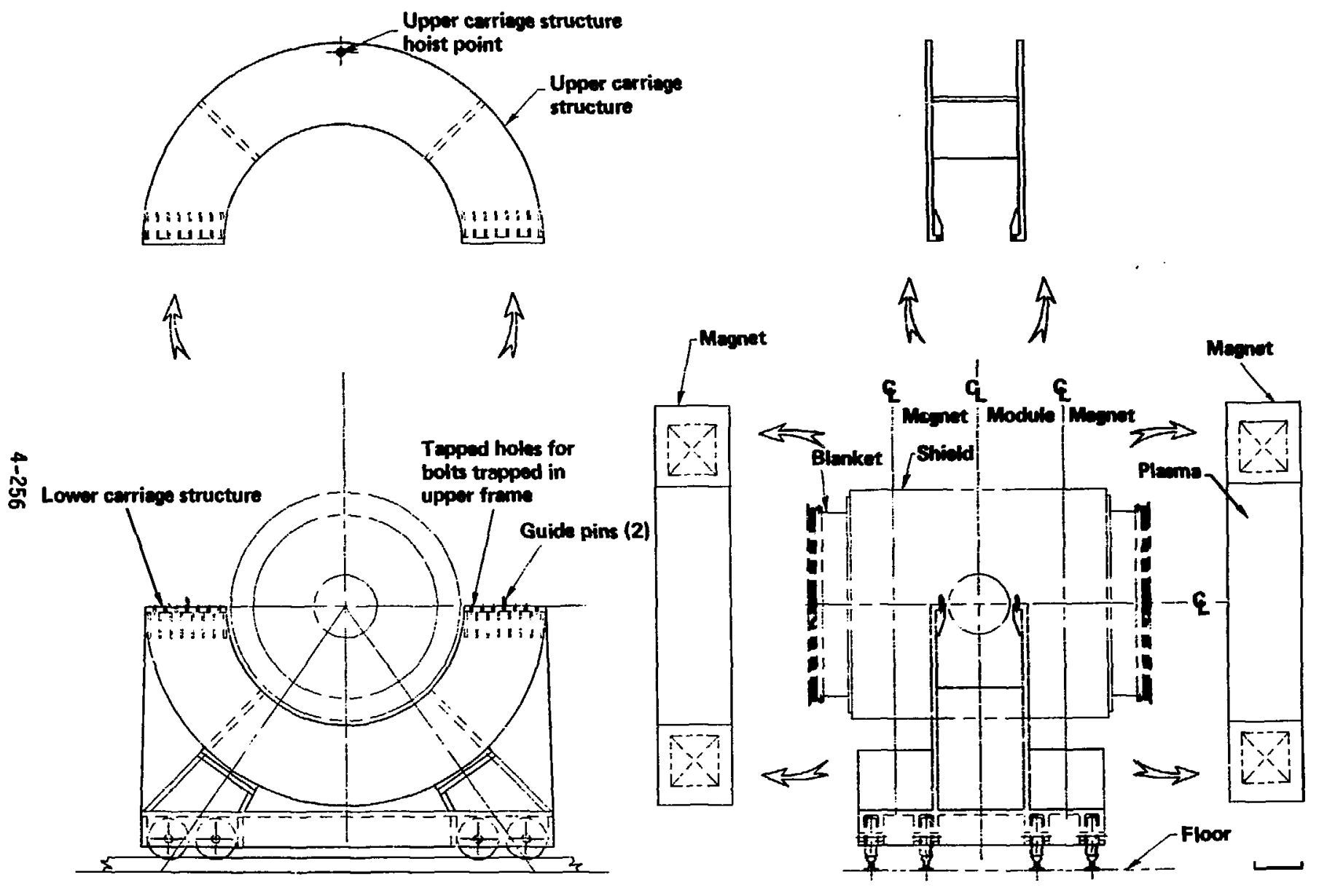

Fig. 4.4-22. Removal of magnet and structure. 


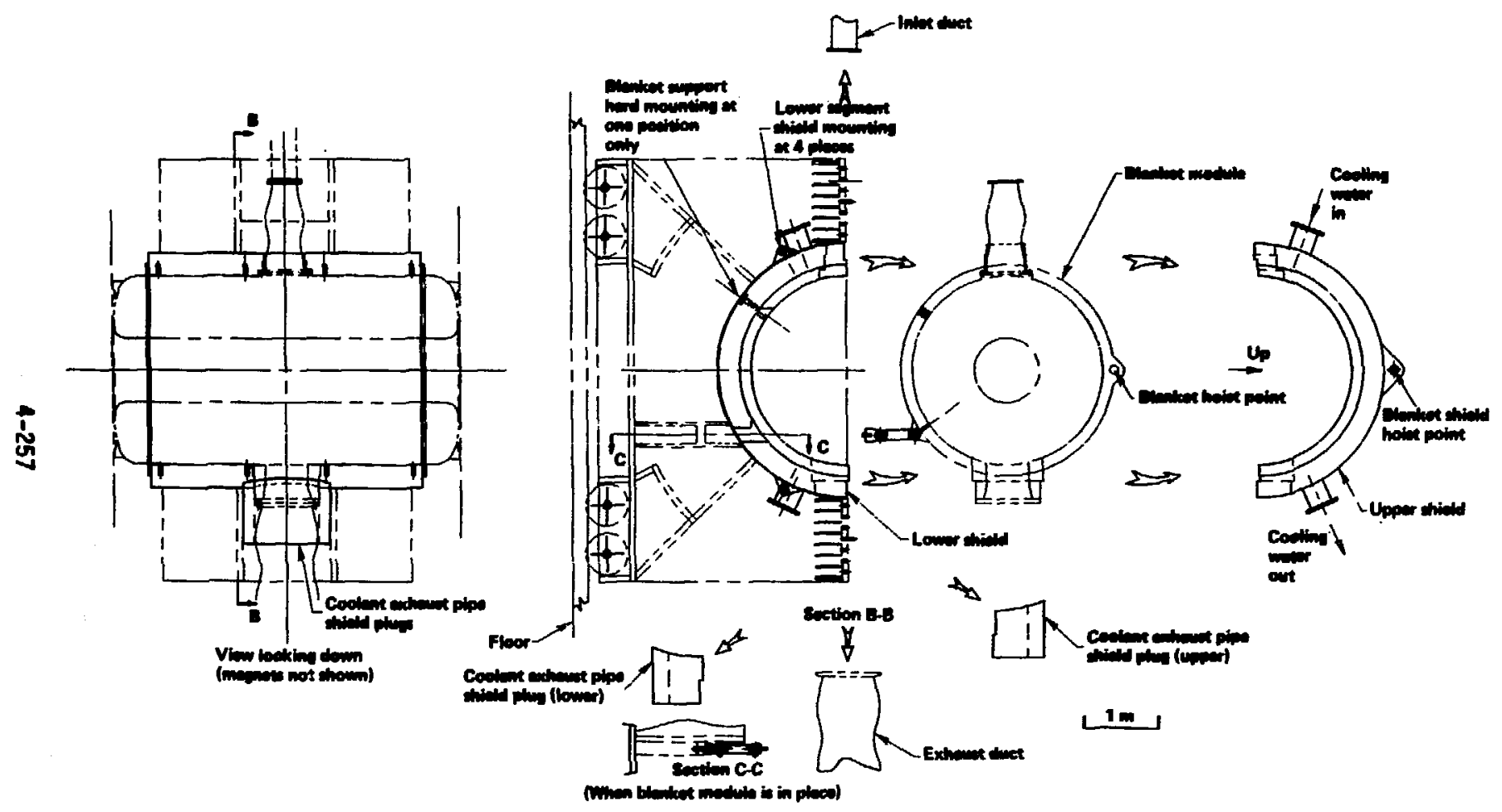

Fig. 4.4-23. Removal of shield and blanket. 


\subsubsection{Blanket Module Manufacturability}

The materials chosen in the construction of the blanket have known fabrication processes and any potential problems are well-understood. The ferritic steel can be mochined, formed, and melded. The weldments require preheating and post-weld heat treatment. The vanadium alloy can be machined and welded in a manner simtlar to that of titantum.

The ferritic alloy inner cylinders are shear-formed, have the grooves machined, the sections welded together, and the short joining rings of Inconel 625 weldad to each end. The circumferential plenum sections are welded in place and then the inlet axtal manifold is installed. The tube assembly sector ralls are welded in position to complete the assembly of the inner cylinder.

The outer cylinder is also ferritic steel; the cylinder sections are rolled and welded and the sections are welded together to form the outer cylinders. Joining rings of Inconel 625 are added to the inner and outer cylinders and the end domes to enable final closing welds to be made without pre-weld heating or post-weld heat treatment. Cutouts are made for the inlet and outlet ducts; the inlet manifold reinforcing ring is welded in position. The support rings are now welded in place, and the outer wall cooling channels and ducts are welded to the inside of the wall and the inlet duct connecting piece. The exhaust duct nozzle is fabricated and welded to the outer cylinder. The tube sector assembly rails are welded in place to complete the outer cylinder assembly.

The end domes are cut from flat ferritic alloy plate and are then tapermachined and spun-formed to shape. Inconel 625 joining rings are welded to both the inner and outer cylindrical flanges. The SiC-filled antistreaming blocks are attached to the outside and the wall cooling channels and ducts are attached to the outside to complete the assembly.

The outer and inner wall assemblies are positioned with the inlet-duct connecting piece in place and cylinder axis vertical. The two pleces are flxtured rigidly together so that one layer of the tube assembly sectors can be inserted from the top, located, and pinned. Now the end dome can be positioned on top, located, and welded to both cylinders. The assembly is now inverted and the remaining layer of tube assembly sectors are inserted, 
located, and pinned in position. The blanket can now be closed by positioning and welding the second end dore.

All welds can be $x$-rayed except the final end dome welds. These can be liquid-penetrant inspected after the root pass and at completion.

REFERENCES FOR SECTION 4.4

1. L. L. Kammerzell and J. W. Read, HTGR-GT Systems Optimization Studtes, ASME BO-WA/GT-3.

2. R. Gold, D. Harrold, R. Mmon, R. Buckman, and R. Svedberg, Technical Assessment of Vanadium and Vanadium-Basse Alloys for Fusion Reactor Applications, DOE Report CO0-4540-1 Vol II (1978).

3. L. Yang, et al.. "Irradiation Study of Lithium Compound Samples for Tritium Breeding Application," J. of Nuclar Materials, 103 \& 104 , p 585 (1981).

4. R. F. Benenati and J. R. Powel1, "The SOAR (Solar Assisted Reactor) Power System," in Proc. of AMS Annual Meeting, At lanta, GA (June 1979).

5. T. H. Van Halen, C. F. McDonald, R. B. Creek, Heat Exchinger Designs for Gas Turbine HTGR Power Plant, ASIE Paper No. 79-WA/GT-2. 


\title{
MIRROR ADVANCED REACTOR STUDY--INTERIM DESIGN REPORT CONTR IBUTING AUTHORS
}

\author{
Lawrence Livermore National Laboratory \\ W. L. Barr \\ B. M. Boghosian \\ B. G. Logan, C. D. Henning, and G. A. Carlson \\ R. S. Devoto \\ G. W. Hamilton \\ R. W. Moir \\ B. M. Johnson \\ W. S. Neef \\ D. W. Dorn \\ W. N. Kumai \\ R. C. Maninger \\ D. H. Berwald \\ R. B. Campbell \\ A. J. Cole \\ B. A. Flanders \\ TRW Energy Development Group \\ J. D. Gordon and J. A. Maniscalco \\ S. A. Freije J. R. Ogren \\ N. M. Ghoniem (UCLA) S. L. Salem \\ D. M. Goebel Y. Saito \\ S. C. Mortenson R. H. Whitley \\ General Dynamics/Convair Division \\ J. F. Parmer and R. W. Baldi \\ J. L. Saunders D. J. Tait \\ K. L. Agarwa 1 \\ R. Dharmarajan \\ M. W. Liggett \\ C. A. Sink \\ J. D. Sutliff \\ W. E. Toffolo \\ C. M. Powers \\ R. A. Sutton \\ J. I. Valerio \\ EBASCO Services, Inc. \\ H. Audi \\ R. Hensler \\ N. Young

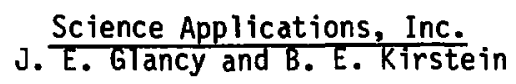 \\ University of Wisconsin--Madison \\ H. M. Attaya \\ K. R. Audenaerde \\ M. L. Corradini \\ G. A. Emmert \\ L. A. E1-Guebaly \\ J. - H. Huang \\ E. M. Larsen \\ G. L. Kulcinski and L. J. Perkins \\ Y.-T. Li \\ C. W. Maynard \\ K. E. Plute \\ R. A. Sanders \\ J. F. Santarius \\ M. E. Sawan \\ J. C. Yu \\ J. E. Scharer \\ D. C. Schluderberg \\ (Babcock and Wi icox) \\ I. N. Sviatos lavsky \\ D. -K. Sze \\ W. F. Vogelsang \\ A. M. White \\ Grumman Aerospace Corporation \\ V. Calia \\ I. Clarkson \\ L. Deutsch \\ J. Erickson \\ R. Botwin, R. H. Bullis, and R. J. Herbermann \\ S. Fixler \\ T. Luzzi \\ R. Micich \\ M. Ross i \\ D. Sedgley \\ M. Stauber \\ P. Suh \\ Energy Technology Engineering Center (ETEC) \\ N. J. Hoffman
}

